\title{
Site Specific Advisory Board Initiative Evaluation Survey Results Supplementary Appendix: Summary of Individual Site Results
}

August 1996

The U.S. Department of Energy

Office of Environmental Management 


\section{DISCLAIMER}

Portions of this document may be illegible in electronic image products. Images are produced from the best available original document. 


\section{DISCLAIMER}

This report was prepared as an account of work sponsored by an agency of the United States Government. Neither the United States Government nor any agency thereof, nor any of their employees, makes any warranty, express or implied, or assumes any legal liability or responsibility for the accuracy, completeness, or usefulness of any information, apparatus, product, or process disclosed, or represents that its use would not infringe privately owned rights. Reference herein to any specific commercial product, process, or service by trade name, trademark, manufacturer, or otherwise does not necessarily constitute or imply its endorsement, recommendation, or favoring by the United States Government or any agency thereof. The views and opinions of authors expressed herein do not necessarily state or reflect those of the United States Government or any agency thereof. 


\section{Site Specific Advisory Board Initiative Evaluation \\ Survey Results, Supplementary Appendix: \\ Summary of Individual Site Results}

\section{INTRODUCTION}

This Appendix presents results of the Site-Specific Advisory Board (SSAB) Initiative for each of the 11 sites that participated in the survey. These individual results are a supplement to the June 1996 Summary Report which presented overall survey results.

Results are presented in 11 sections, arranged alphabetically by site. Each section includes a series of figures and tables that parallel those presented in the Summary Report. To facilitate comparison, figures are presented both for the individual site and for the overall long survey. The sequence of sections is:
- $\quad$ Fernald
- Hanford
- Idaho
- Los Alamos
- Monticello
- Nevada
- Pantex
- $\quad$ Rocky Flats
- $\quad$ St. Louis
- Sandia

- Savannah River 
Fernald Site Specific Advisory Board Evaluation Survey Results

SSAB Supplementary Appendix

August 1996

S-2 
Figure 1a. Fernald

Goal 1. Establish processes and procedures to provide an effective forum

for exchange of information and viewpoints regarding DOE site issues

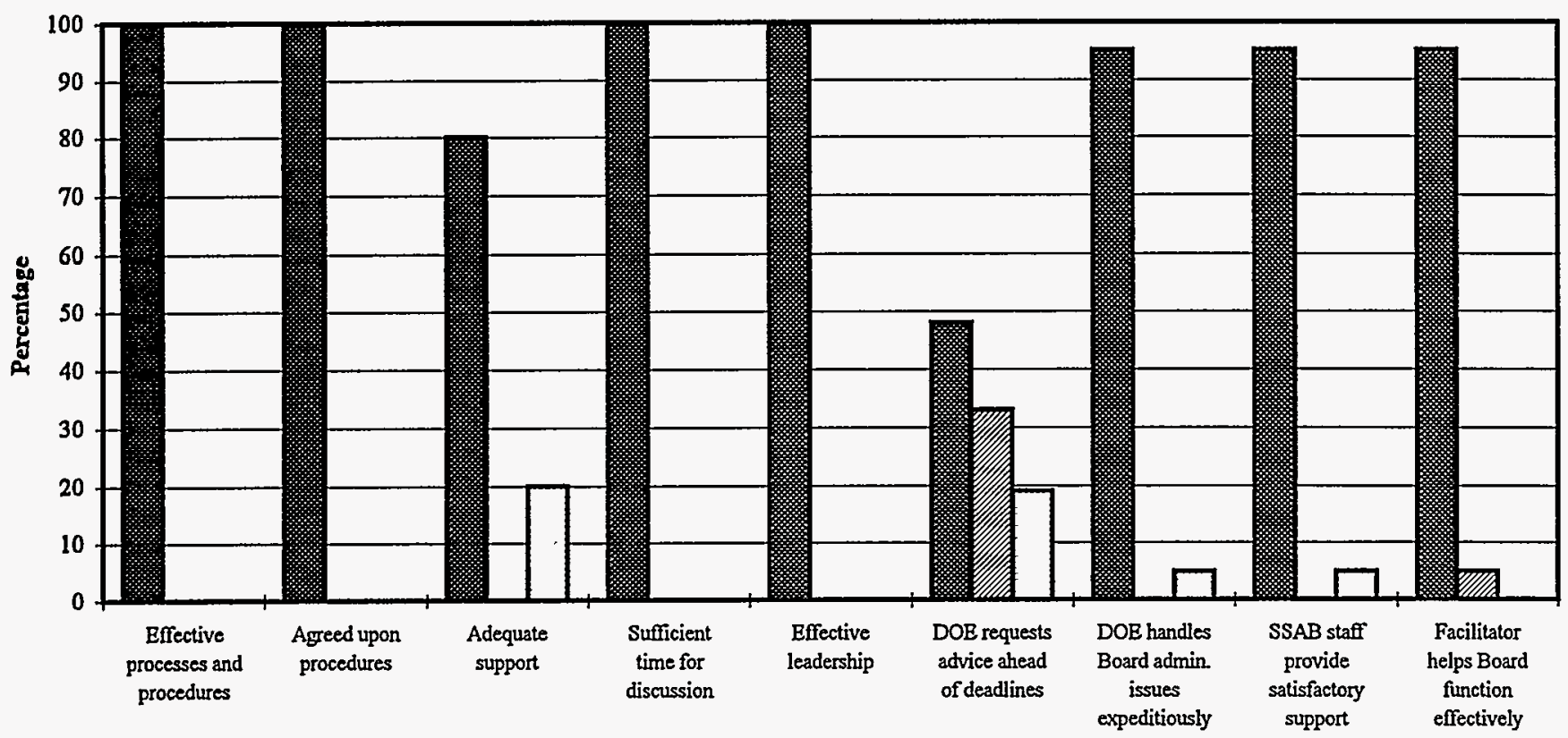

Agree (\%) Neither Agree Nor Disagree/Don't Know (\%) DDisagree (\%)

Figure 1b. Long Survey: Entire Sample

Goal 1. Establish processes and procedures to provide an effective forum for exchange of information and viewpoints regarding DOE site issues

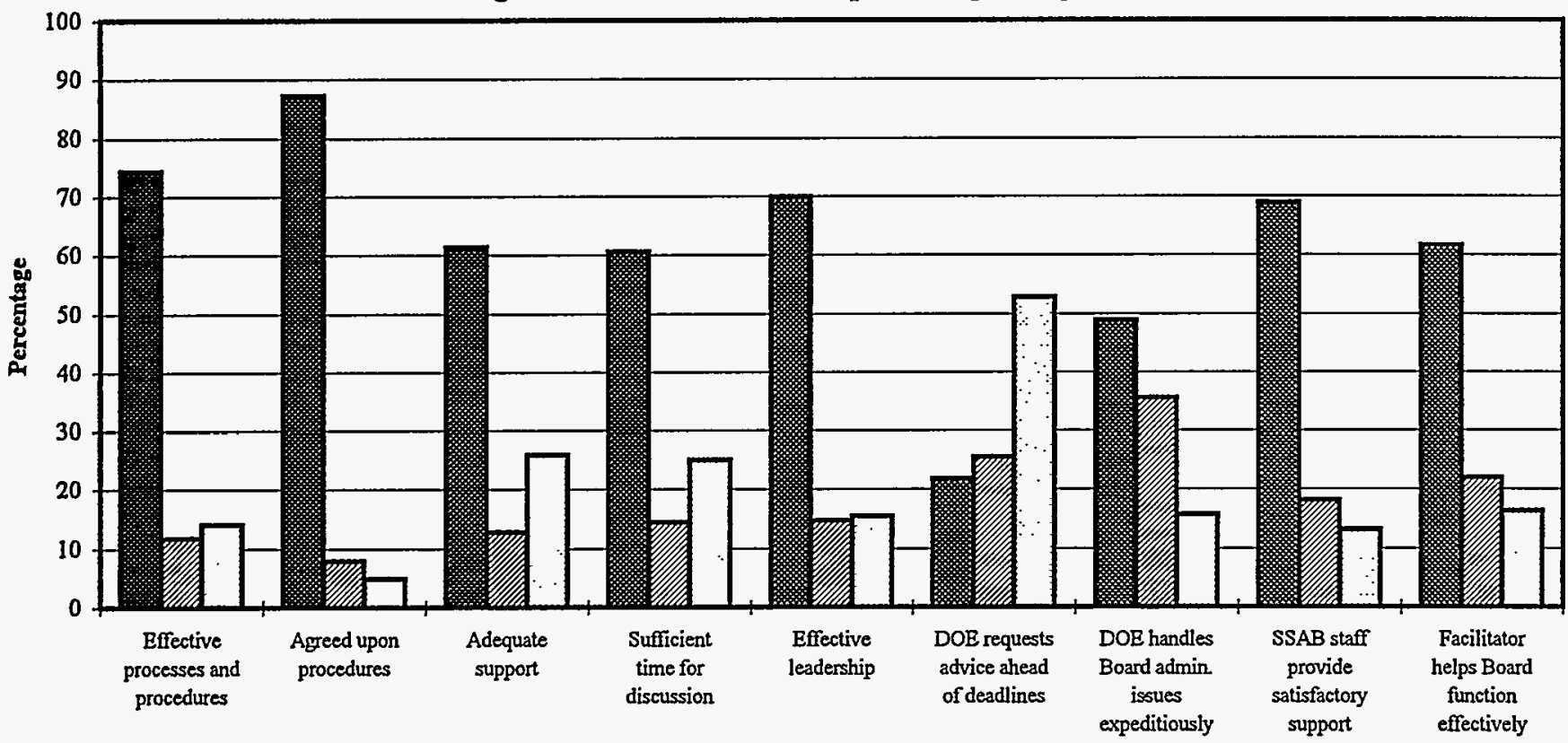

Agree (\%) ENeither Agree Nor Disagree/Don't Know (\%) DDisagree (\%)

SSAB Supplementary Appendix

August 1996 
Figure 2a. Fernald

Goal 2. Facilitate interaction and exchange of information and viewpoints regarding $\mathrm{DOE}$ site issues

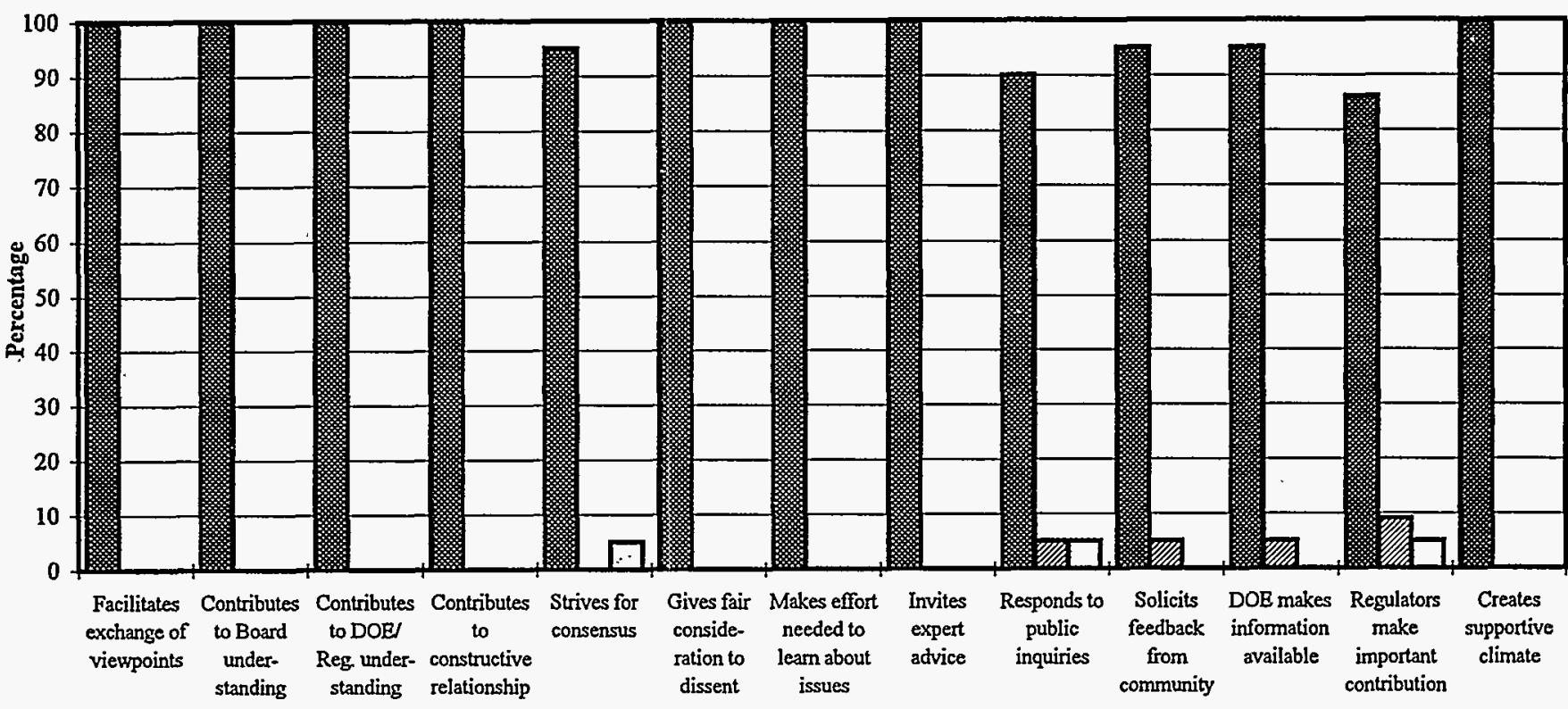

Agree (\%) Weither Agree Nor Disagree/Don't Know (\%) घDisagree (\%)

Figure 2b. Long Survey: Entire Sample

Goal 2. Facilitate interaction and exchange of information and viewpoints regarding DOE site issues

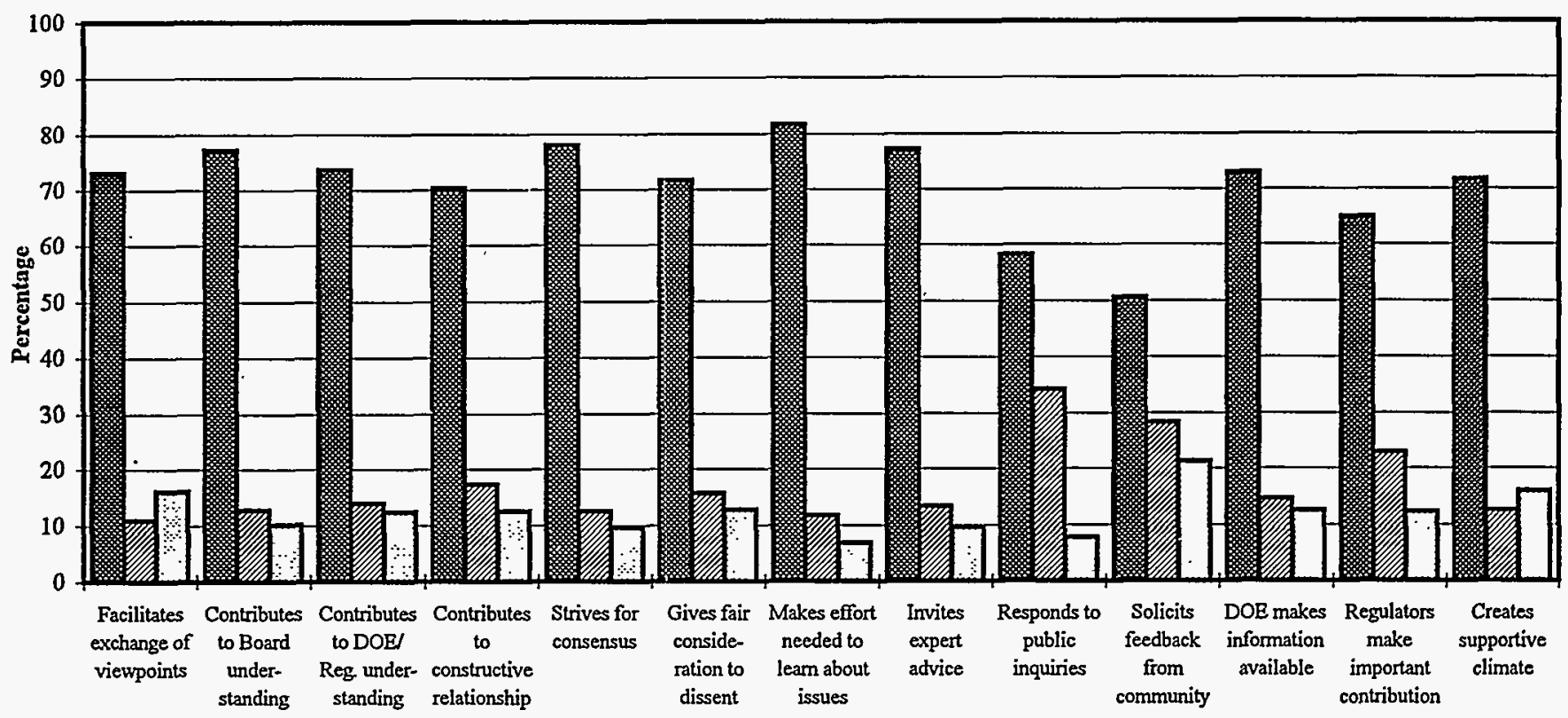

Agree (\%) Weither Agree Nor Disagree/Don't Know (\%) DDisagree (\%)

SSAB Supplementary Appendix

August 1996 
Figure 3a. Fernald

Goal 3. Provide useful advice and/or recommendations to DOE

(and regulators, where appropriate)

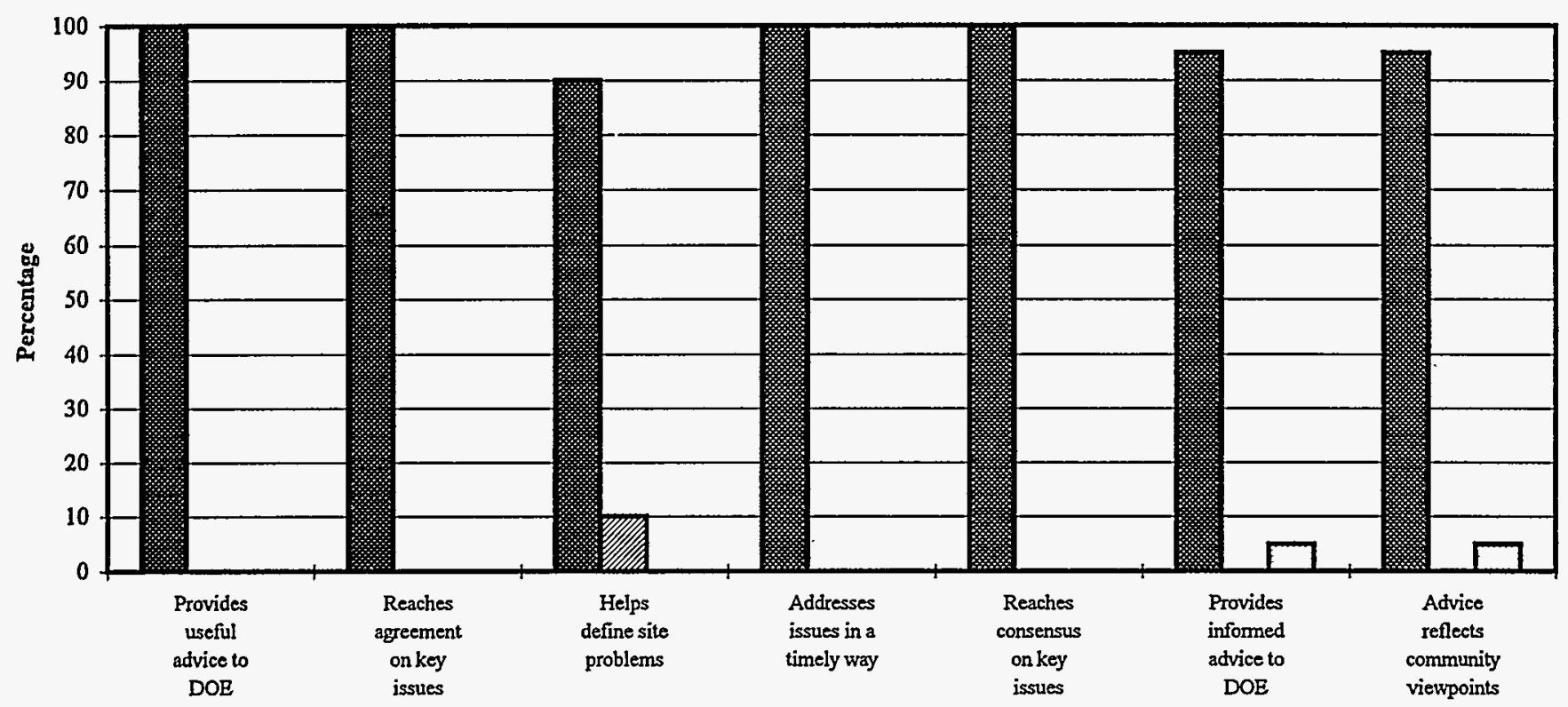

Agree (\%) Neither Agree Nor Disagree/Don't Know (\%) 口Disagree (\%)

Figure 3b. Long Survey: Entire Sample

Goal 3. Provide useful advice and/or recommendations to DOE

(and regulators, where appropriate)

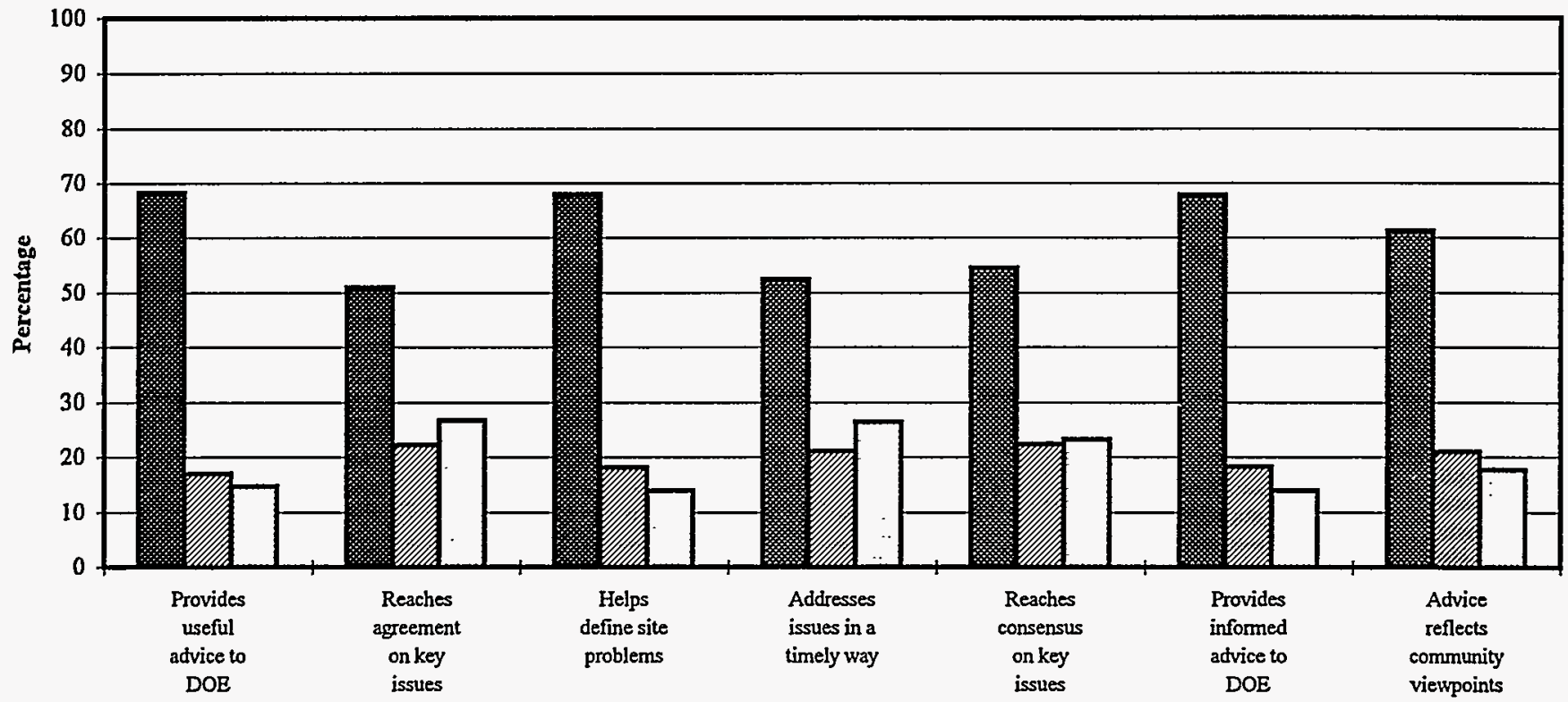

Agree (\%) ENeither Agree Nor Disagree/Dont' Know (\%) DDisagree (\%)

SSAB Supplementary Appendix

August 1996 
Figure 4a. Fernald

Goal 4. Improve DOE's (and where applicable, regulators') site decisions and decision making process

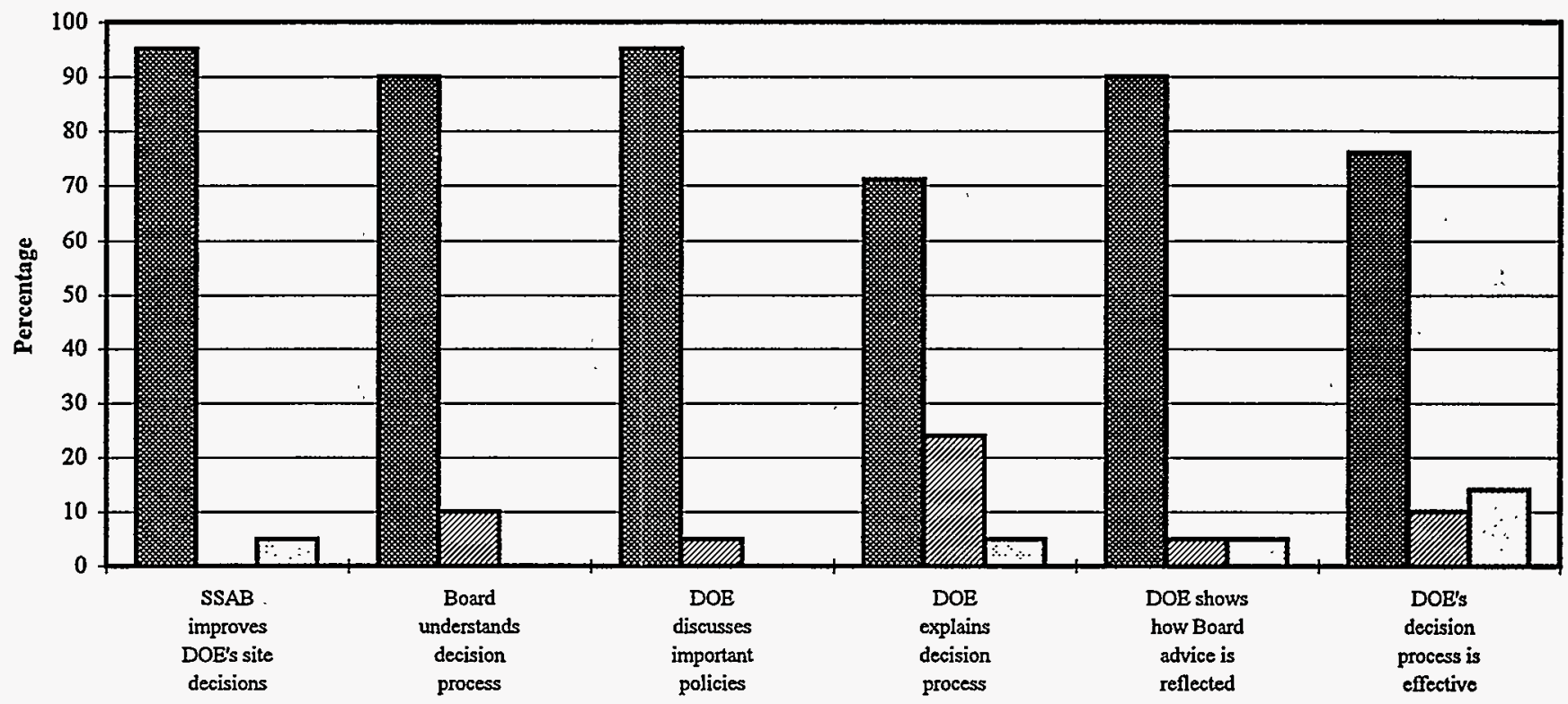

圆 Agree (\%) Neither Agree Nor Disagree/Don't Know (\%) DDisagree (\%)

Figure 4b. Long Survey: Entire Sample

Goal 4. Improve DOE's (and where applicable, regulators') site decisions and decision making process

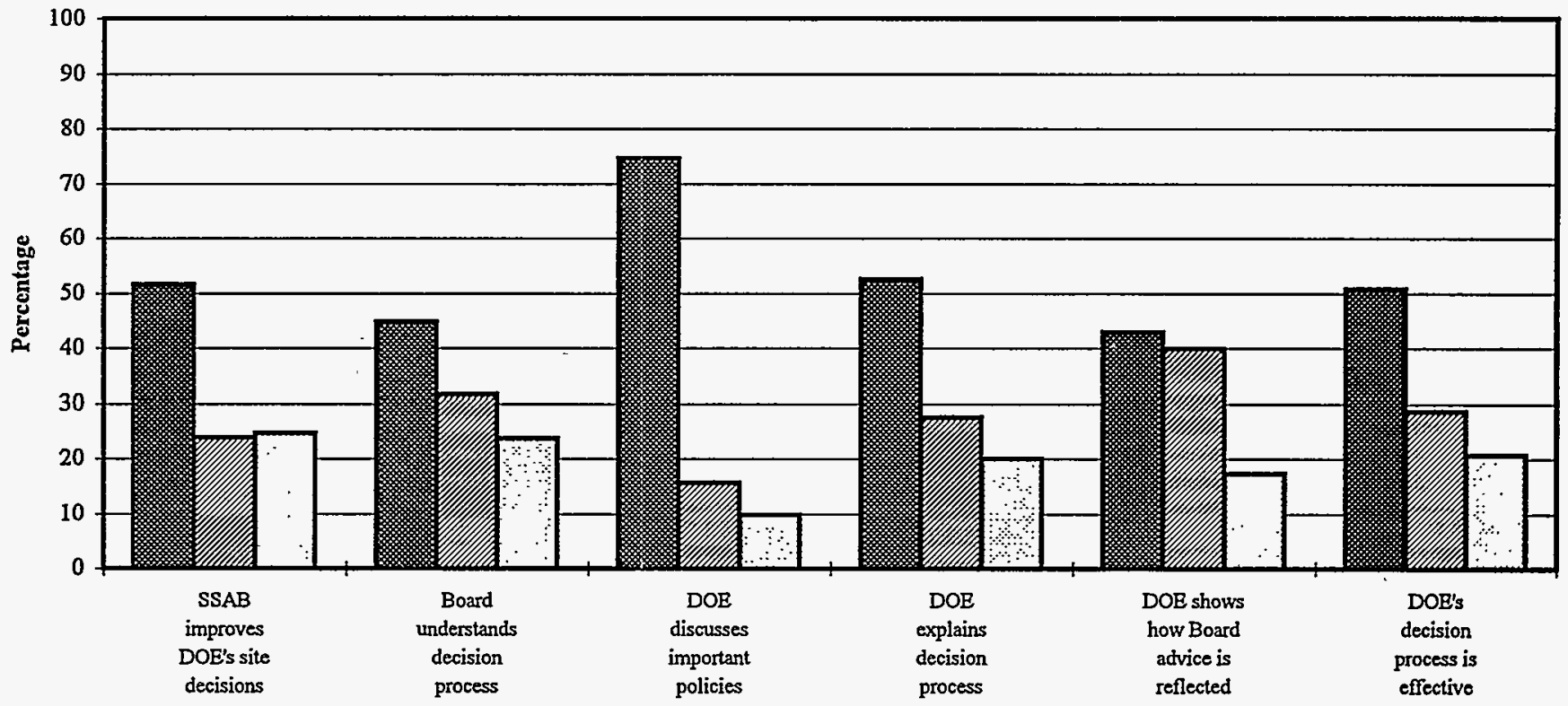

Agree (\%) שNeither Agree Nor Disagree/Don't Know (\%) DDisagree (\%)

SSAB Supplementary Appendix 
Figure 5a. Fernald

Goal 5. Lead to more acceptable actions

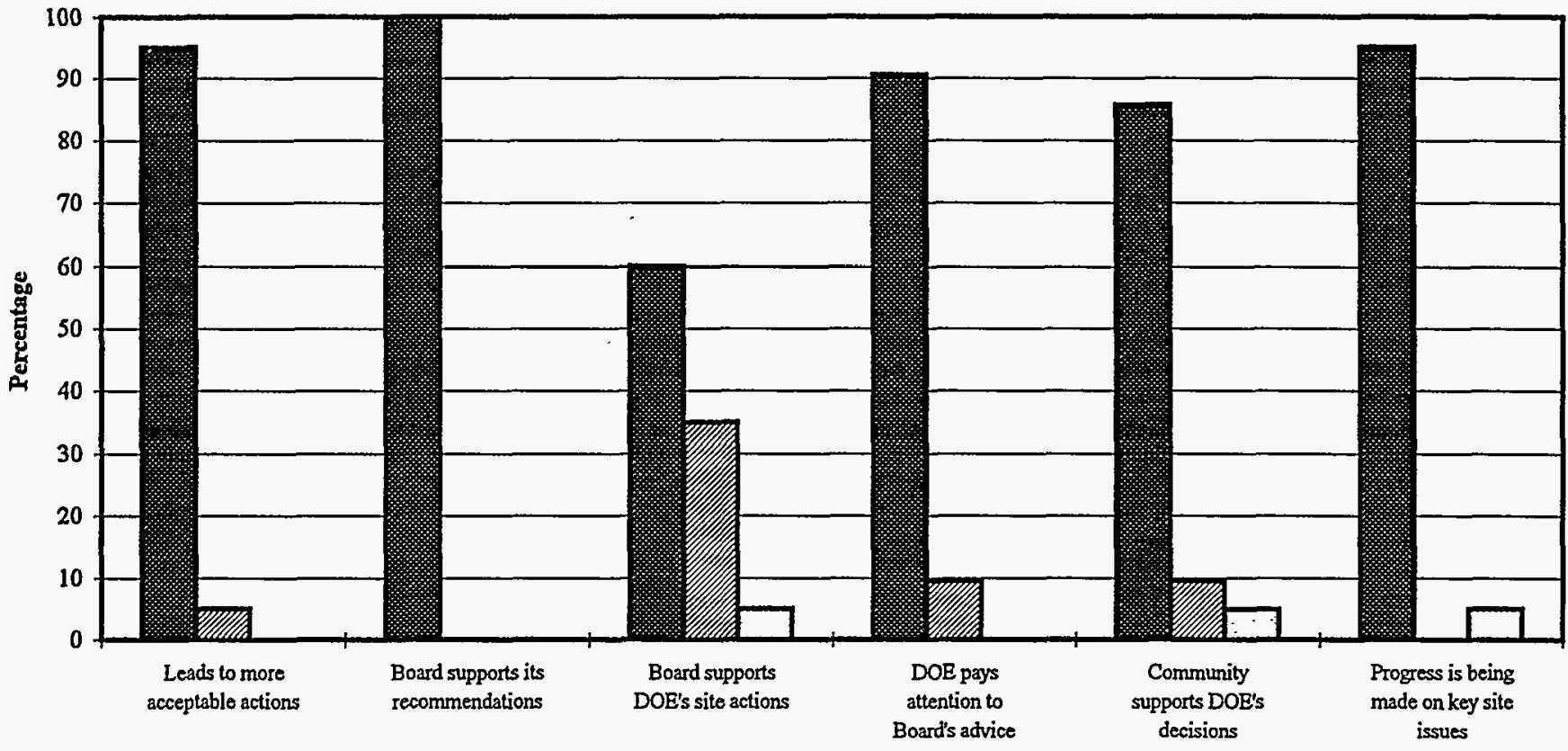

Agree (\%) Weither Agree Nor Disagree/Don't Know (\%) DDisagree (\%)

Figure 5b. Long Survey: Entire Sample

Goal 5. Lead to more acceptable actions

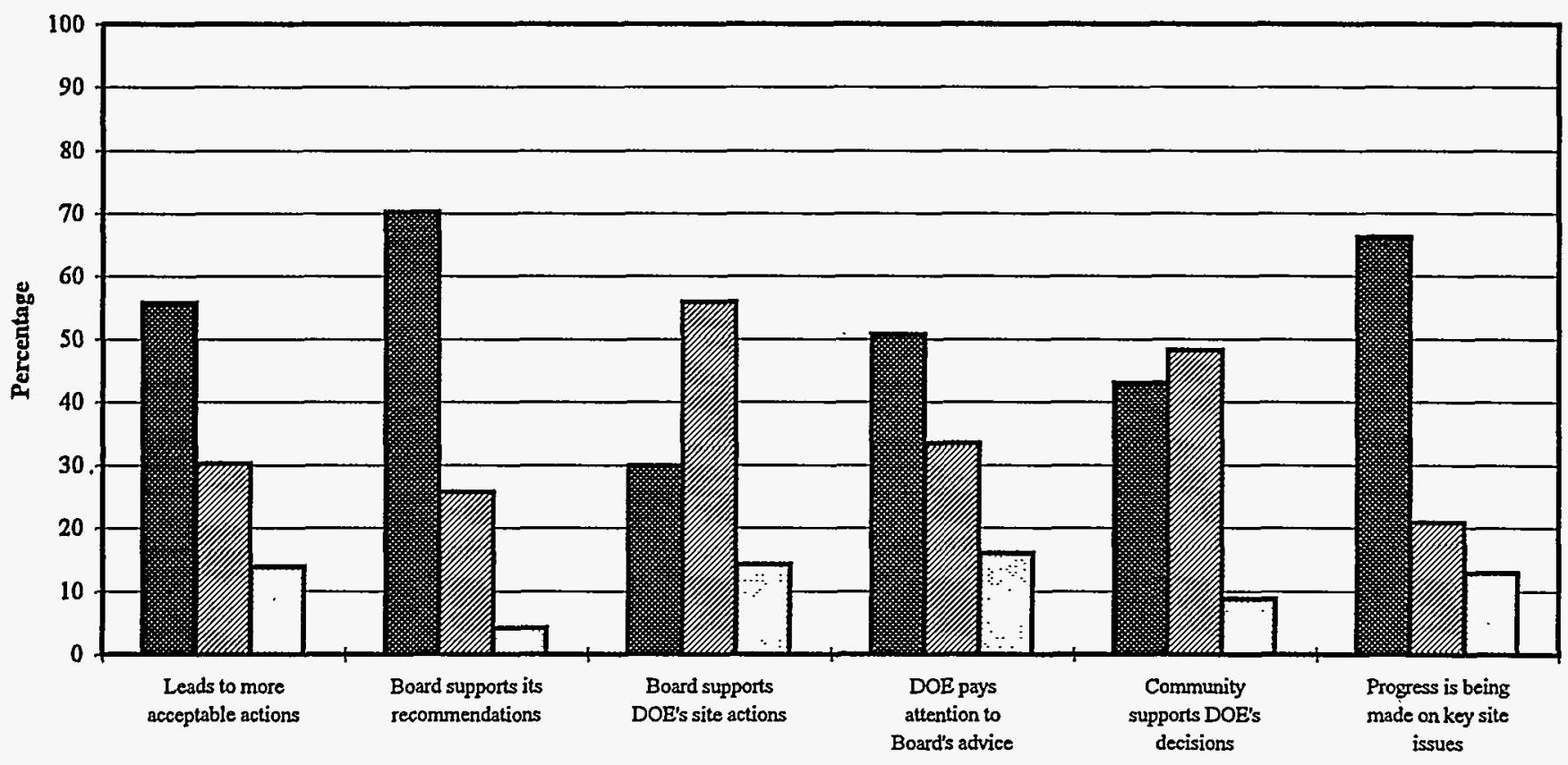

Agree (\%) DNeither Agree Nor Disagree/Don't Know (\%) QDDisagree (\%)

SSAB Supplementary Appendix

August 1996 
Figure 6a. Fernald

Goal 6. Contribute to trust and confidence in DOE

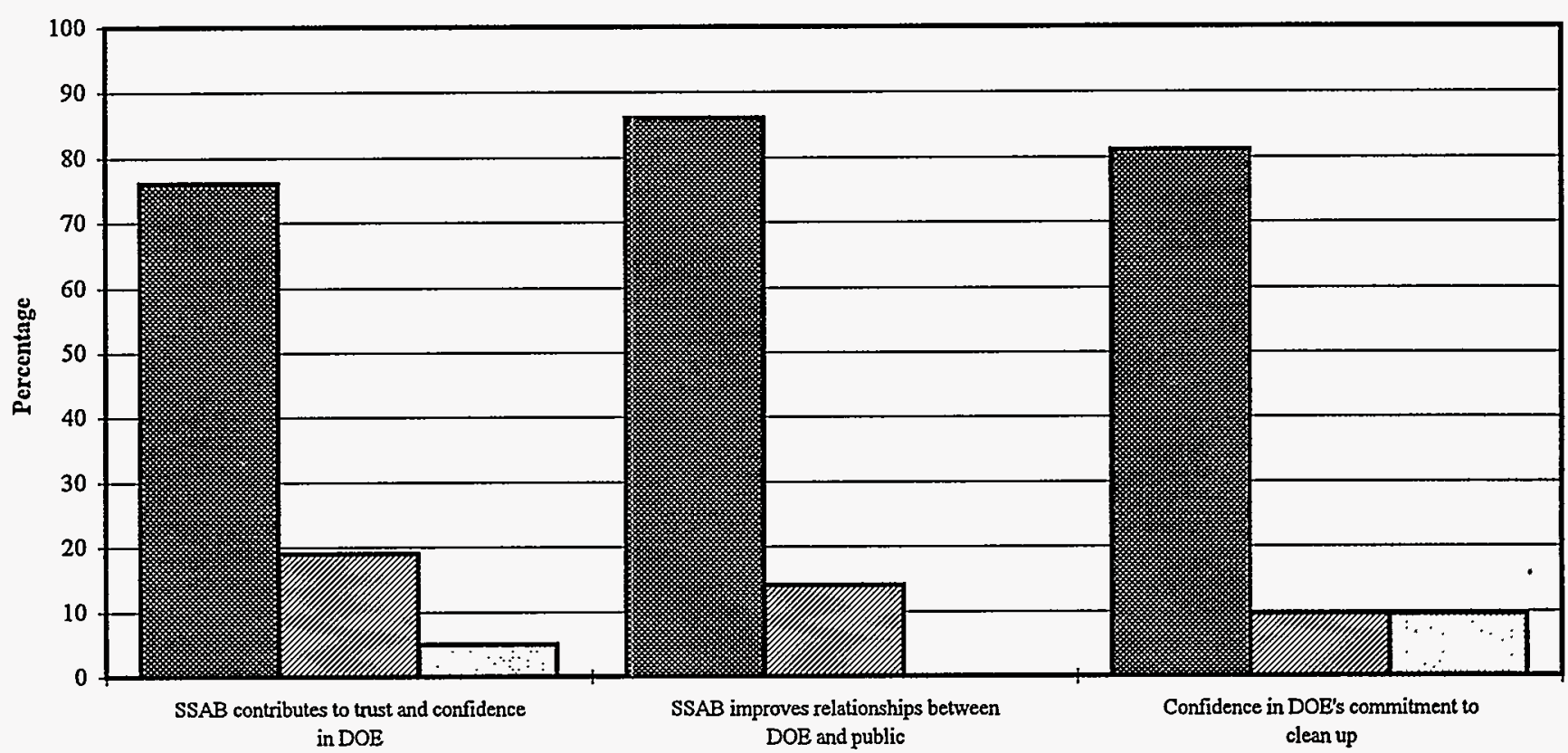

Agree (\%) $\mathbf{D}$ Neither Agree Nor Disagree/Don't Know (\%) DDisagree (\%)

Figure 6b. Long Survey: Entire Sample

Goal 6. Contribute to trust and confidence in DOE

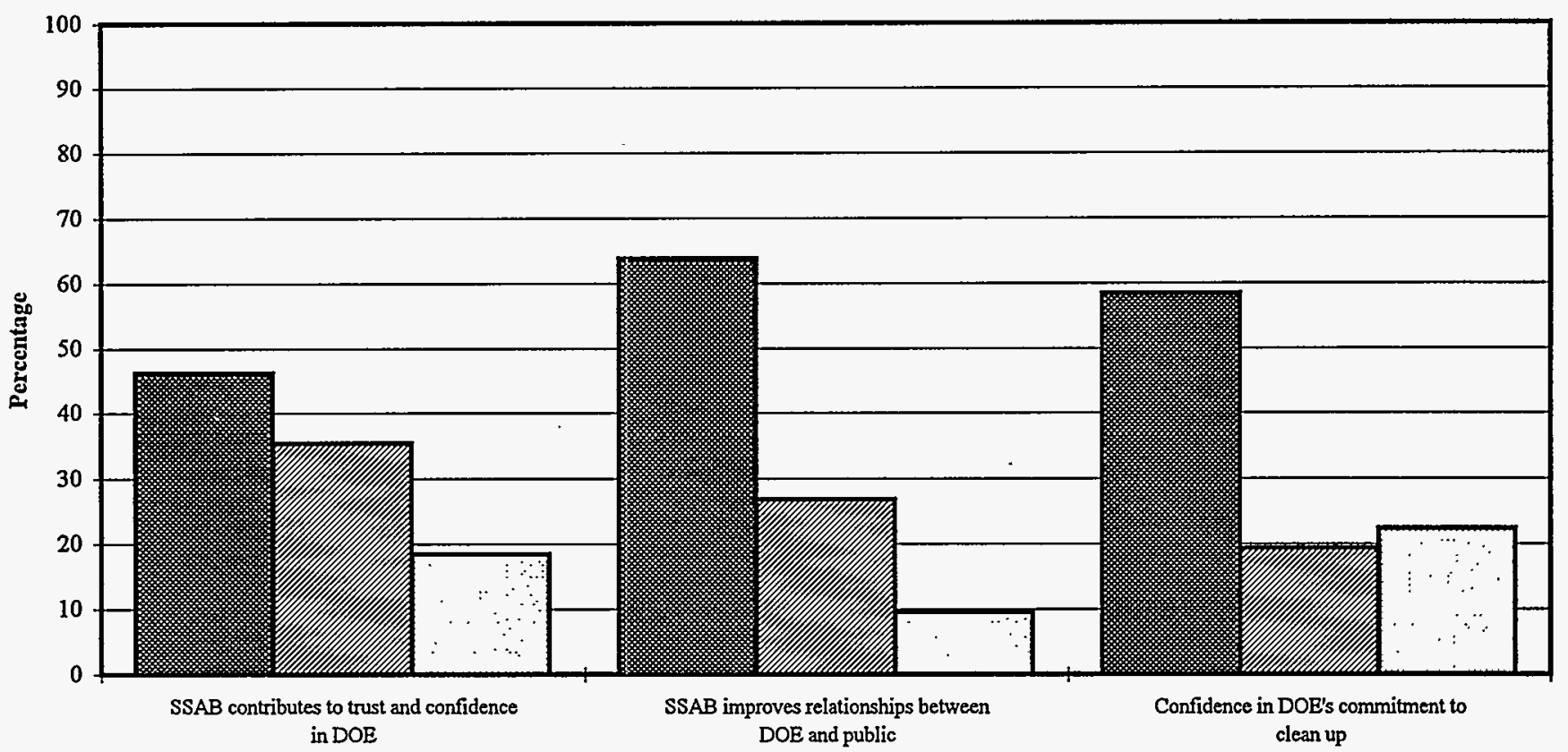

Agree (\%) ENeither Agree Nor Disagree/Don't Know (\%) 口Disagree (\%)

SSAB Supplementary Appendix

August 1996 
Figure 7a. Fernald

Public awareness

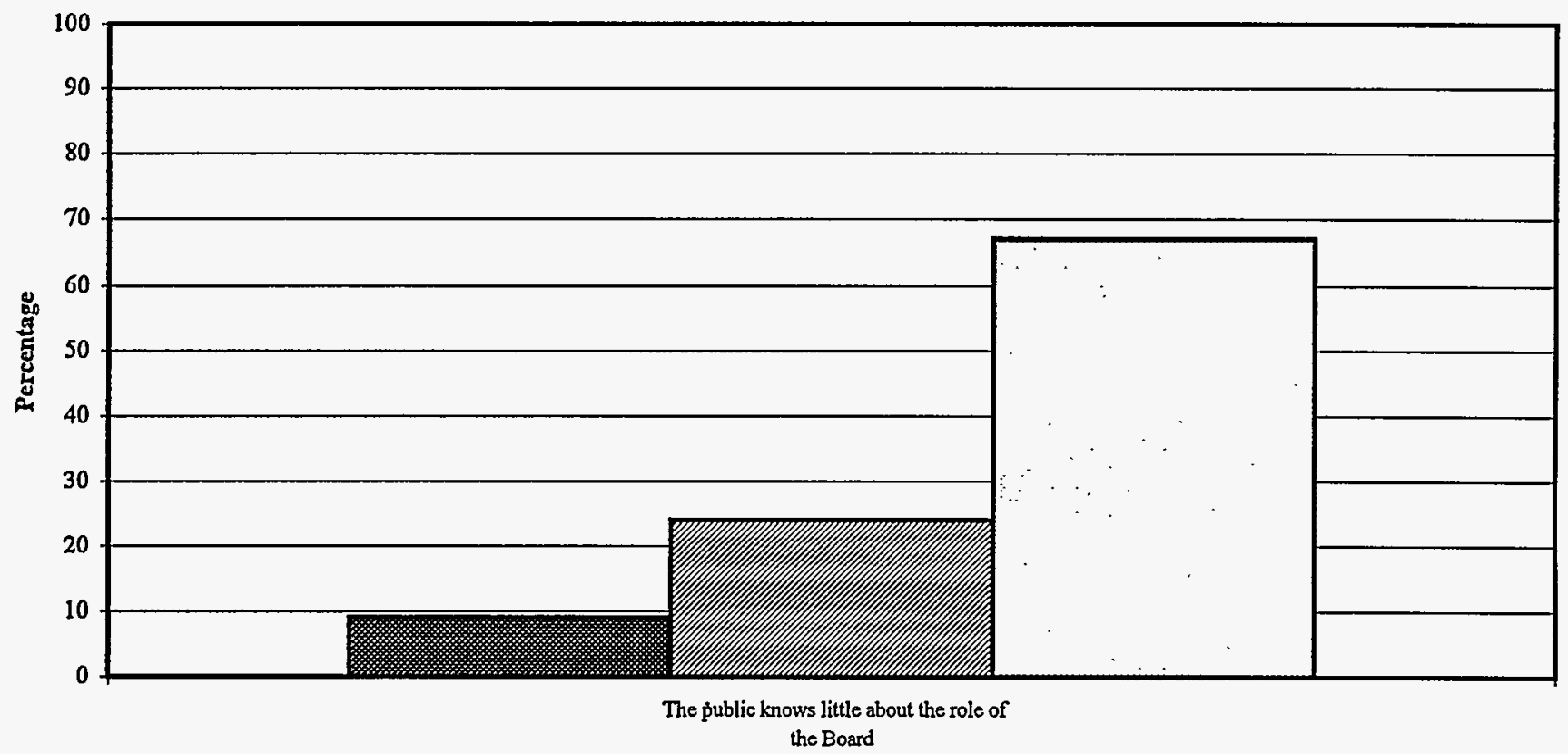

EAgree (\%) WNeither Agree Nor Disagree/Don't Know (\%) DDisagree (\%)

Figure 7b. Long Survey: Entire Sample

Public awareness

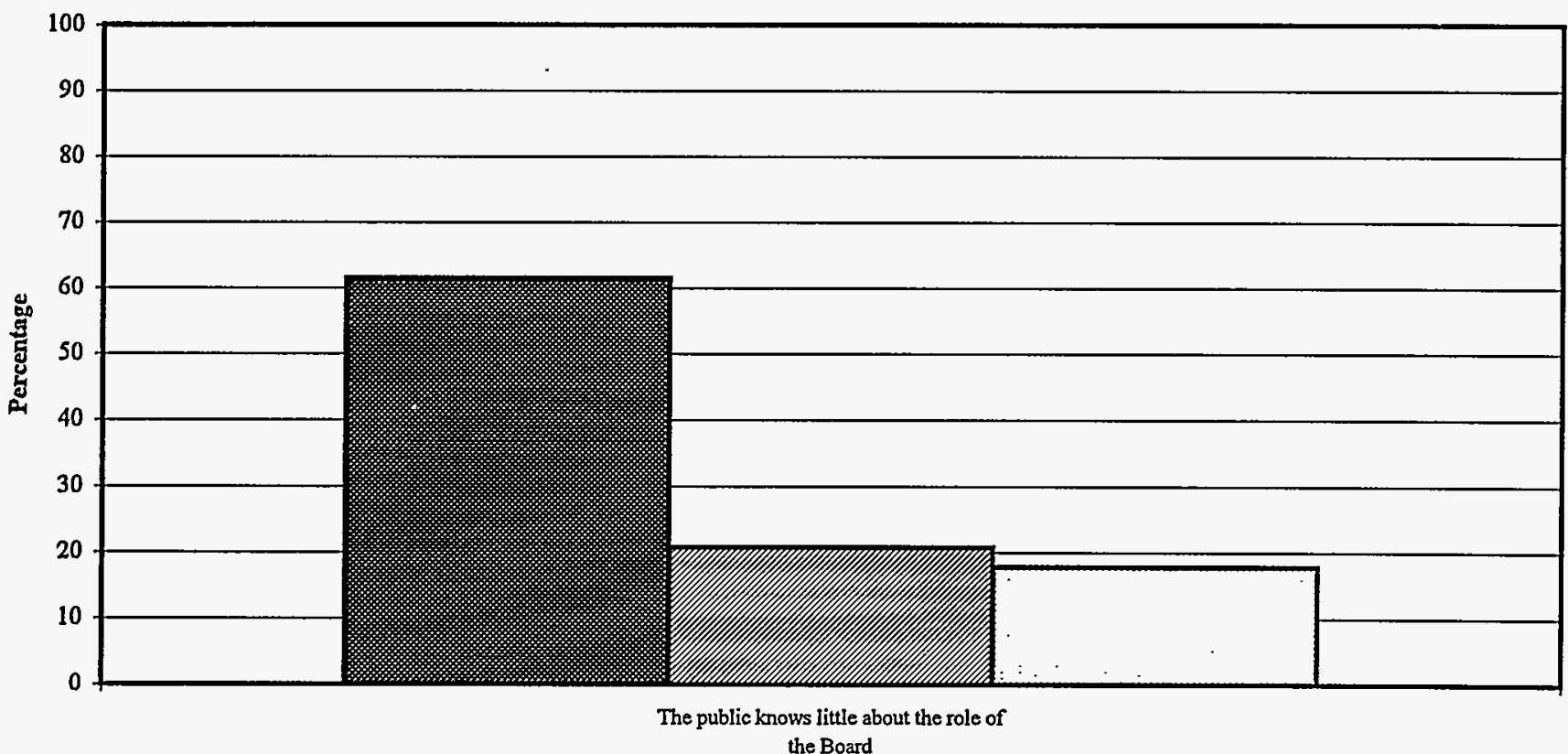

Agree (\%) Deither Agree Nor Disagree/Don't Know (\%) DDisagree (\%) 
Figure 8a. Fernald

DOE-HQ involvement with the SSAB Initiative

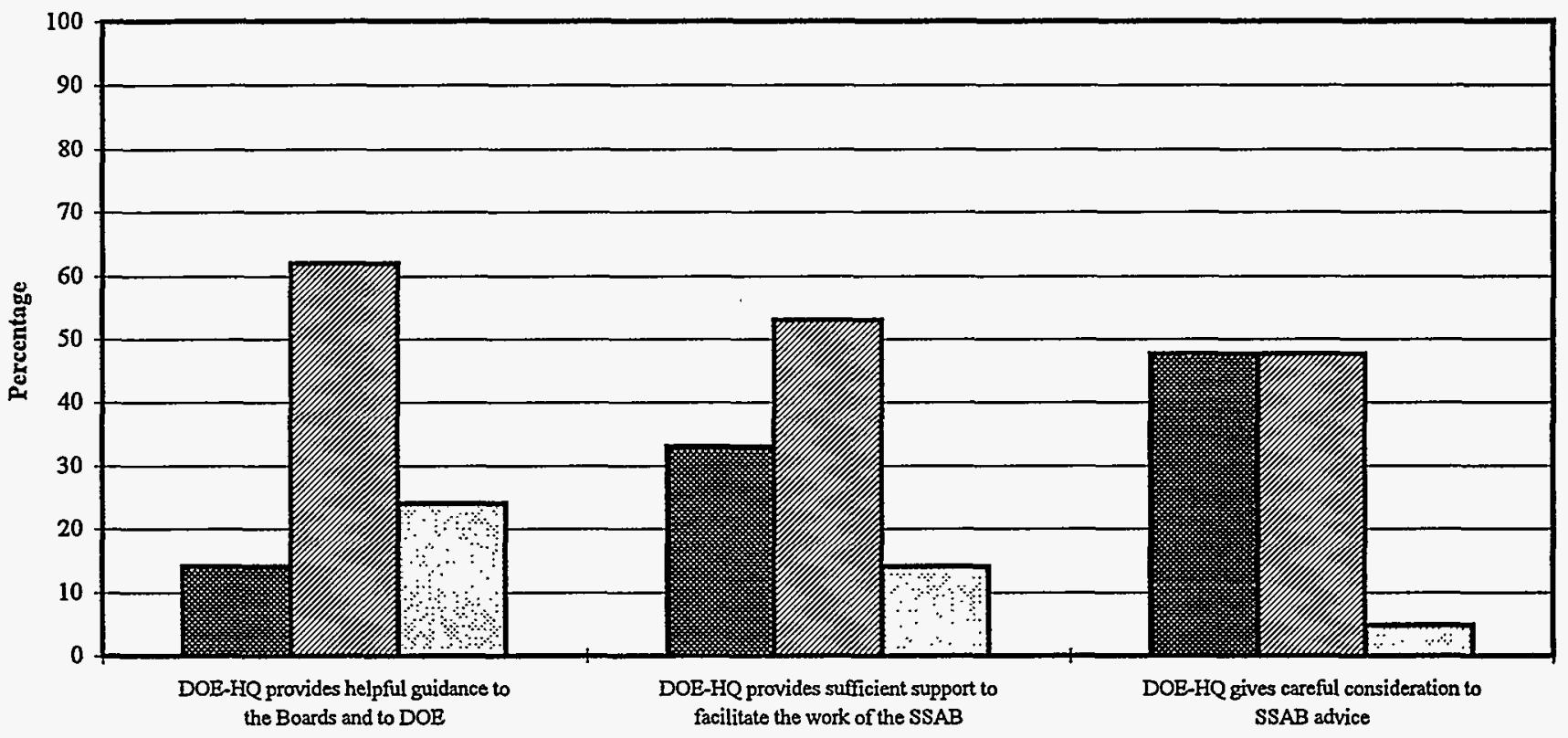

Agree (\%) Weither Agree Nor Disagree/Don't Know (\%) DDisagree (\%)

Figure 8b. Long Survey: Entire Sample

DOE-HQ involvement with the SSAB Initiative

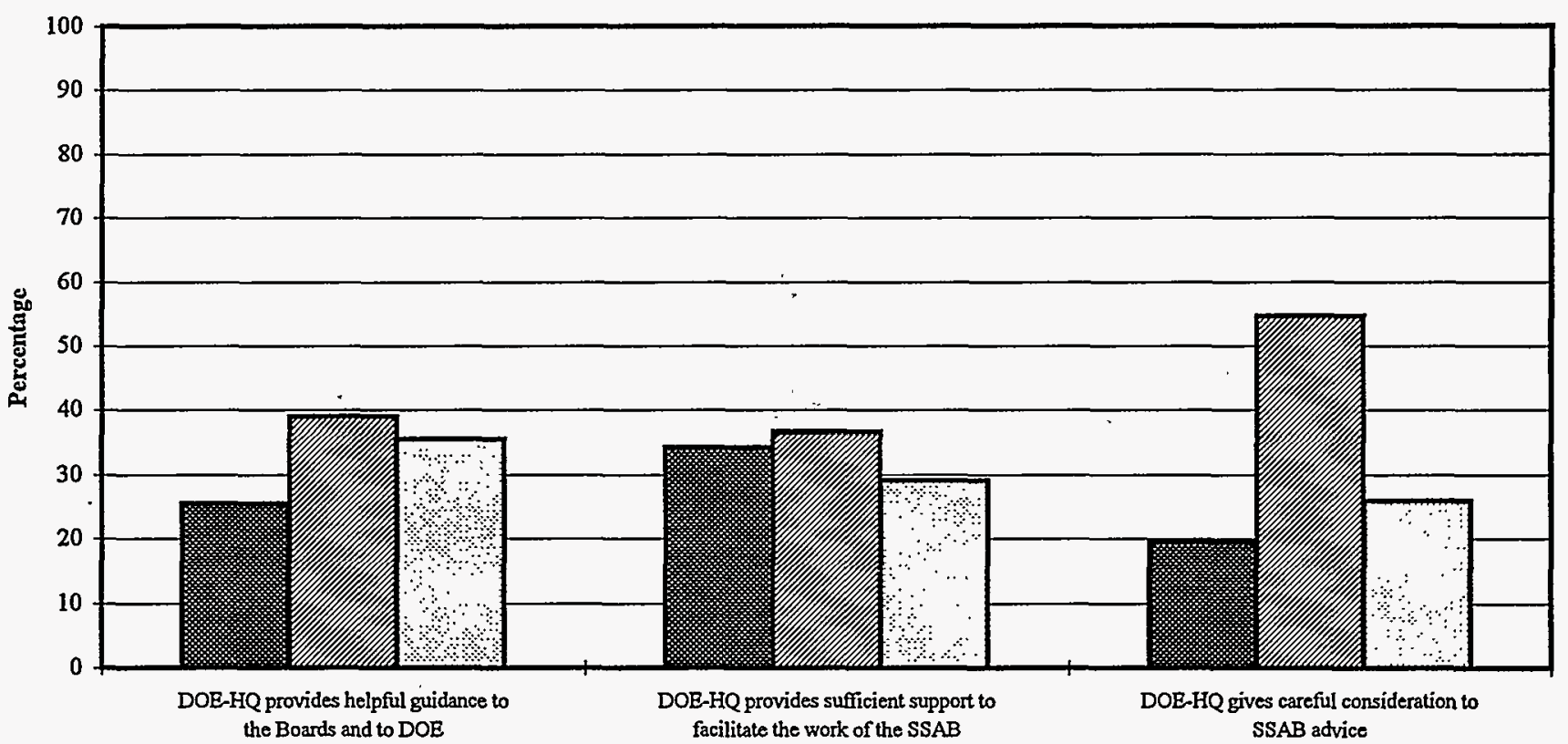

A Agree (\%) ENeither Agree Nor Disagree/Don't Know (\%) 口Disagree (\%)

SSAB Supplementary Appendix

August 1996 
Figure 9a. Fernald

Personal experience with the SSAB Initiative

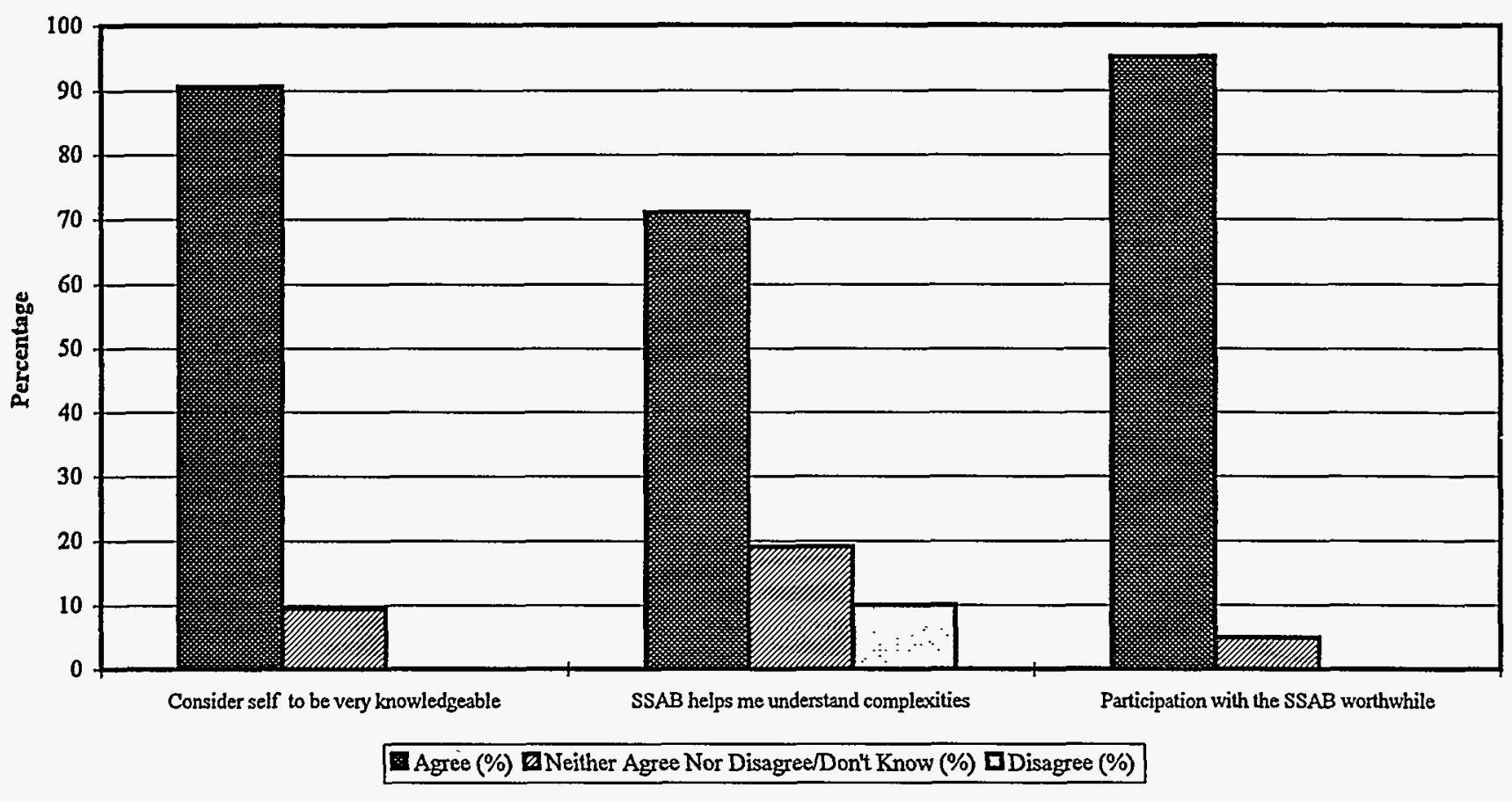

Figure 9b. Long Survey: Entire Sample Personal experience with the SSAB Initiative

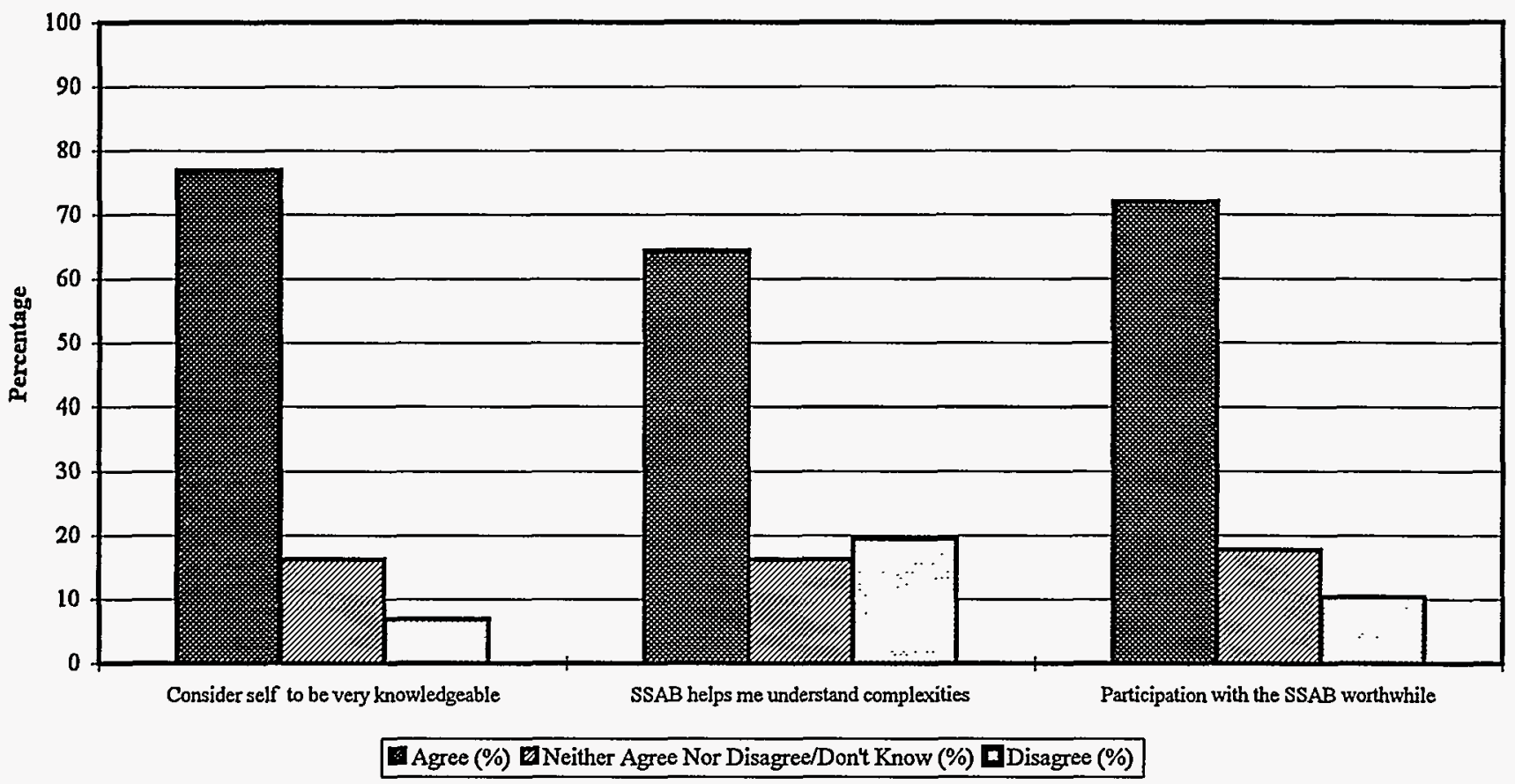

SSAB Supplementary Appendix 
Figure 10a. Fernald

Group/Team work skills

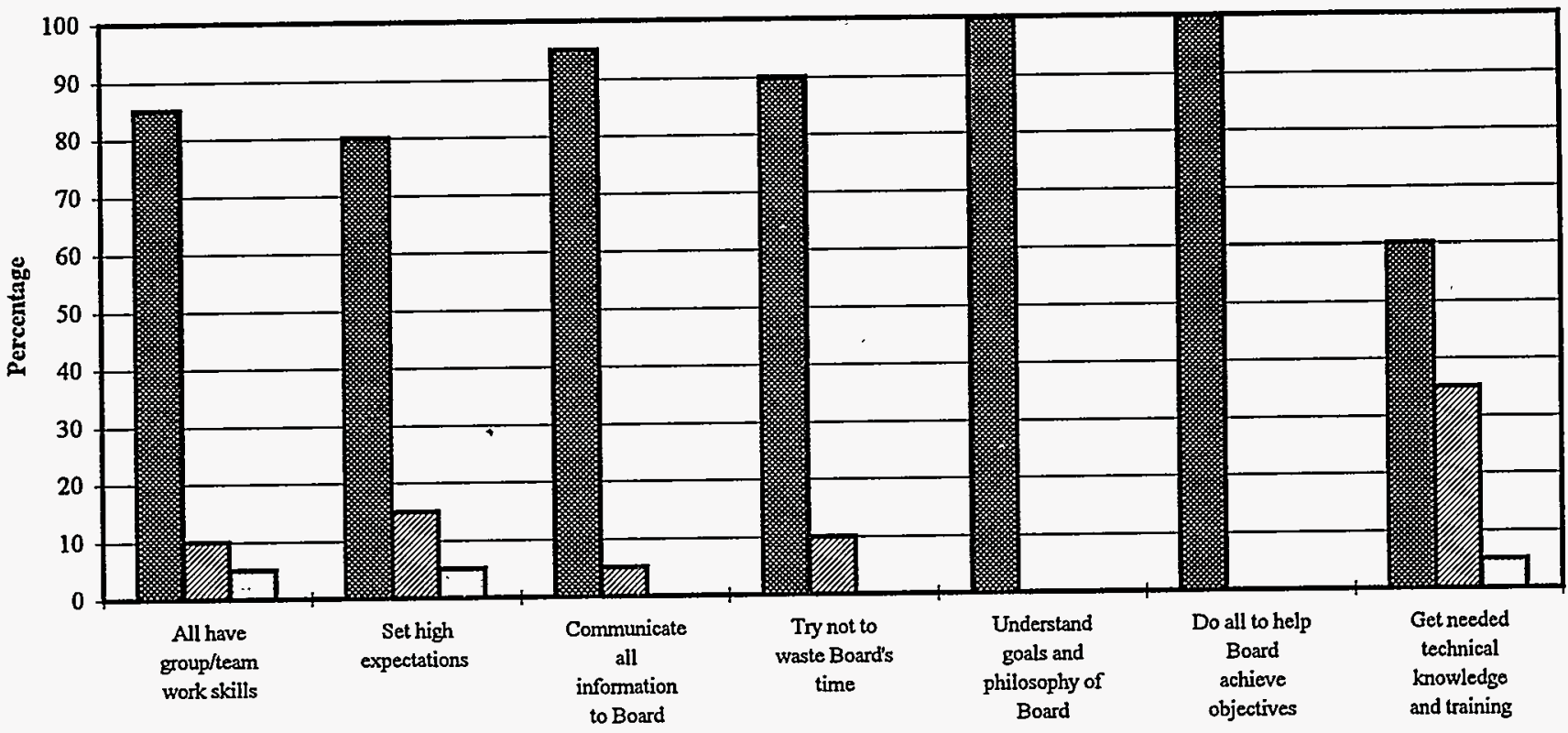

Agree (\%) ENeither Agree Nor Disagree/Don't Know (\%) DDisagree (\%)

Figure 10b. Long Survey: Entire Sample

Group/Team work skills

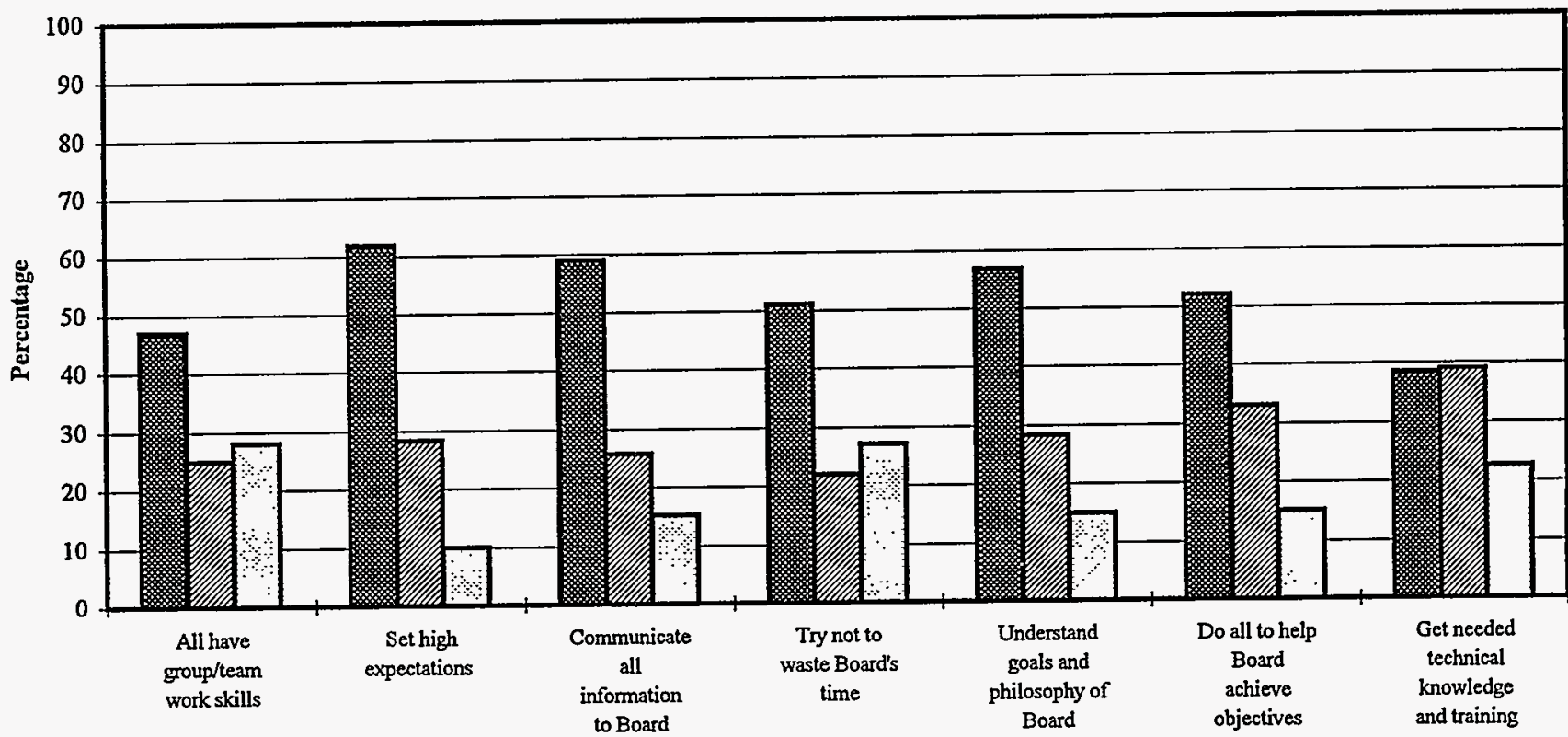

QAgree (\%) Deither Agree Nor Disagree/Don't Know (\%) DDisagree (\%)

SSAB Supplementary Appendix

August 1996 
Figure 11a. Fernald

Working relations

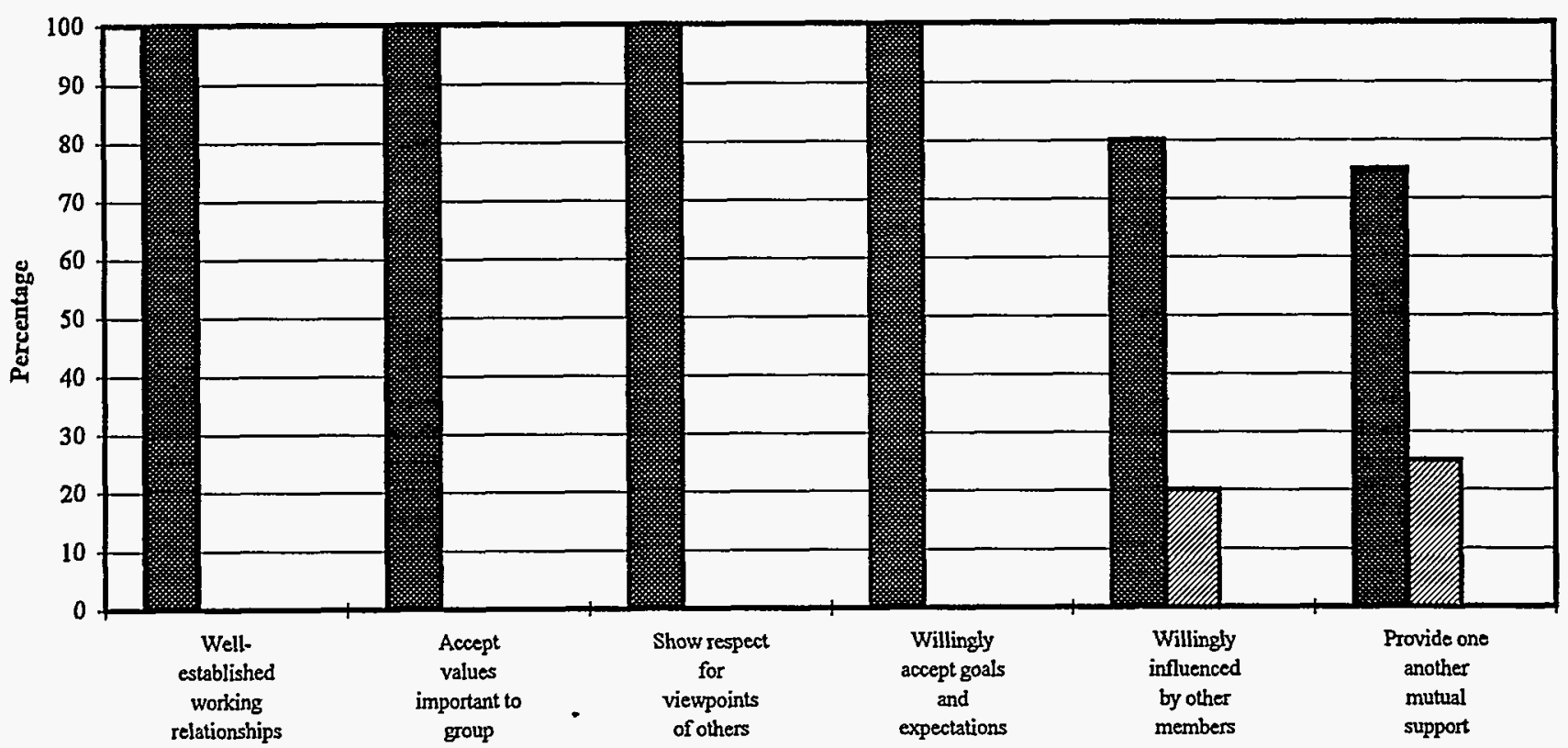

A Agree (\%) BNeither Agree Nor Disagree/Don't Know (\%) DDisagree (\%)

Figure 11b. Long Survey: Entire Sample

Working relations

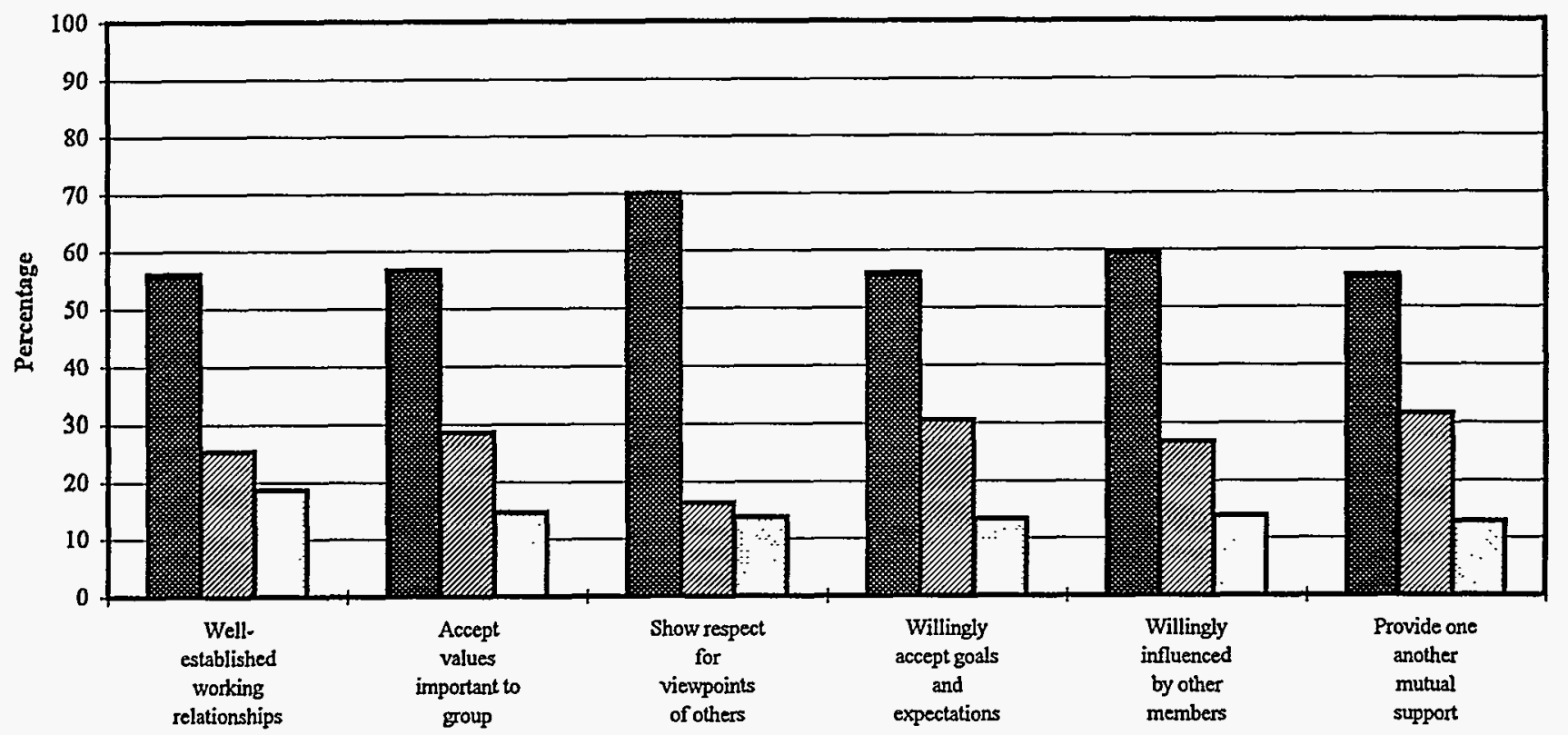

Agree (\%) DNeither Agree Nor Disagree/Don't Know (\%) DDisagree (\%)

SSAB Supplementary Appendix

August 1996 
Figure 12a. Fernald

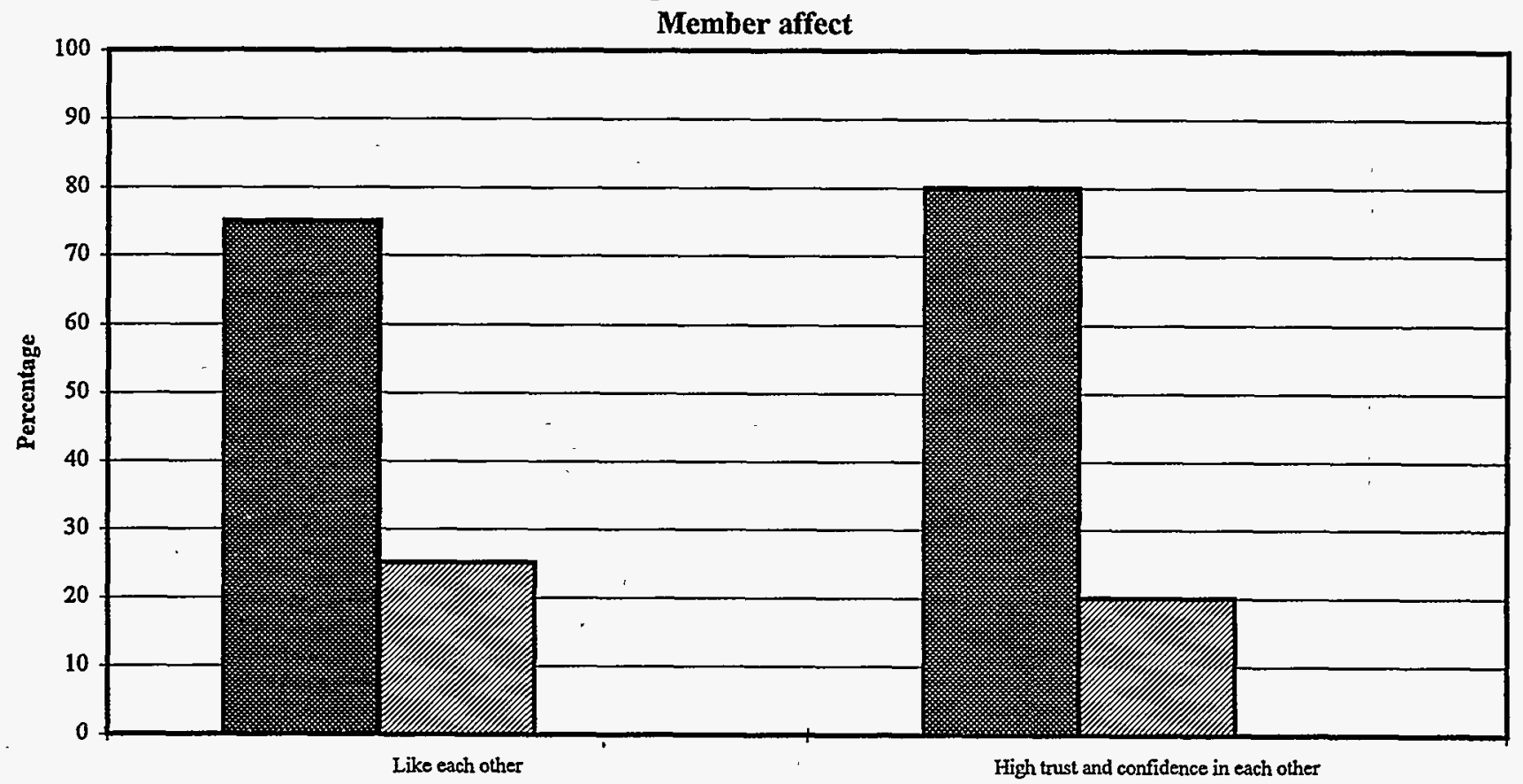

Agree (\%) Neither Agree Nor Disagree/Dont' Know (\%) DDisagree (\%)

Figure 12b. Long Survey: Entire Sample

Member affect

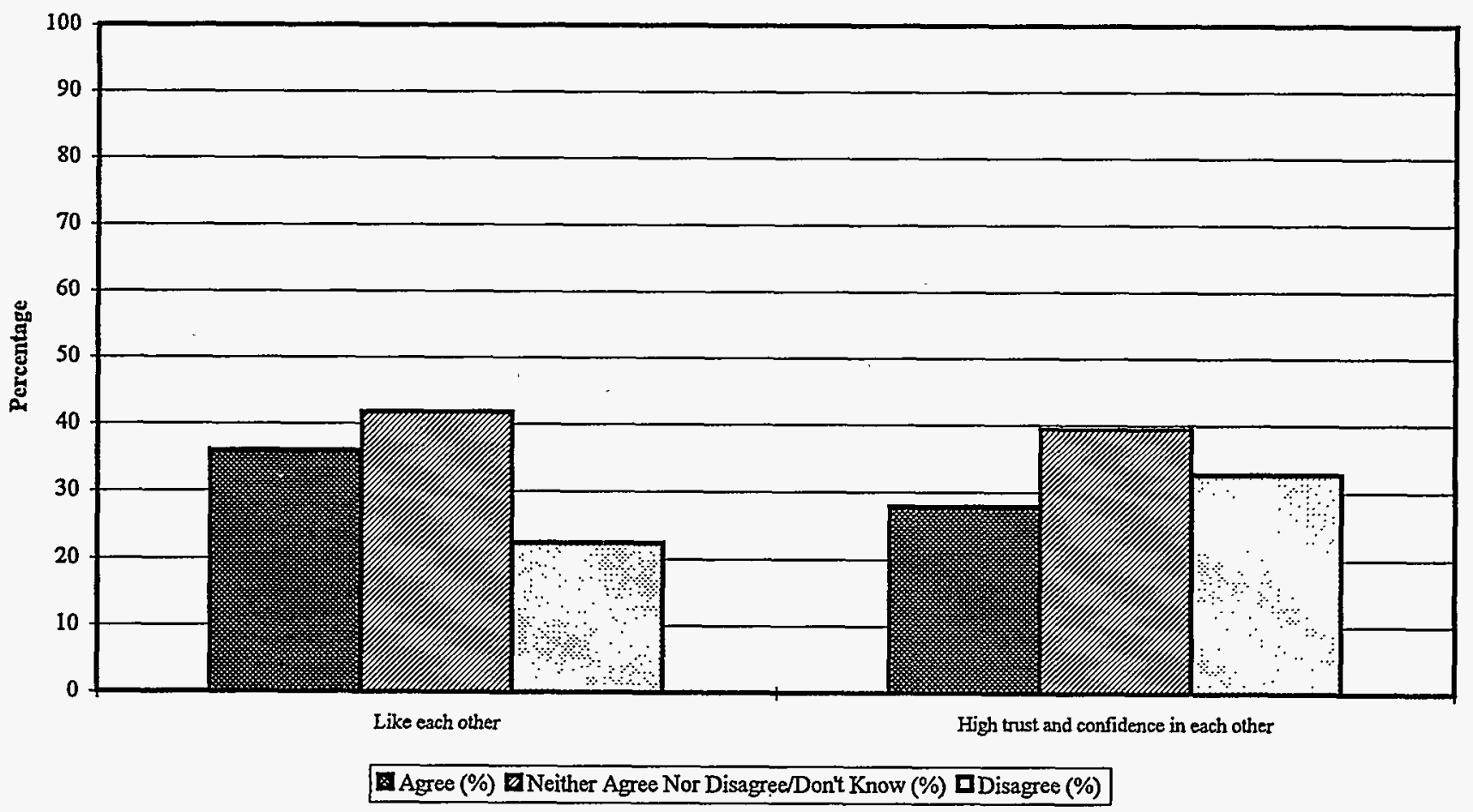

SSAB Supplementary Appendix

August 1996 
Figure 13a. Fernald

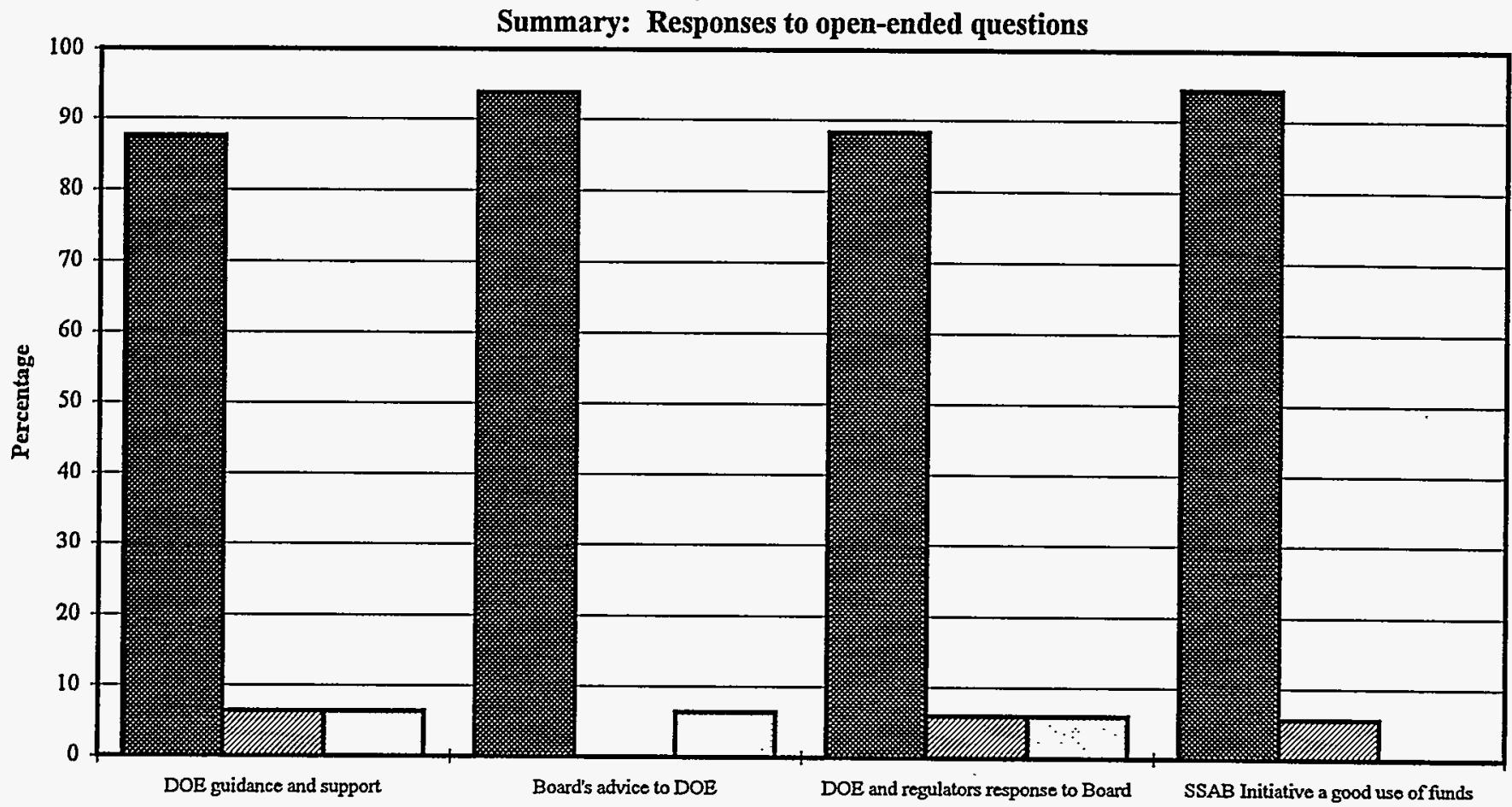

Yes, Good (\%) ENeutral, Conditional, No Opinion (\%) DNo, Not Good (\%)

Figure 13b. Long Survey: Entire Sample

Summary: Responses to open-ended questions

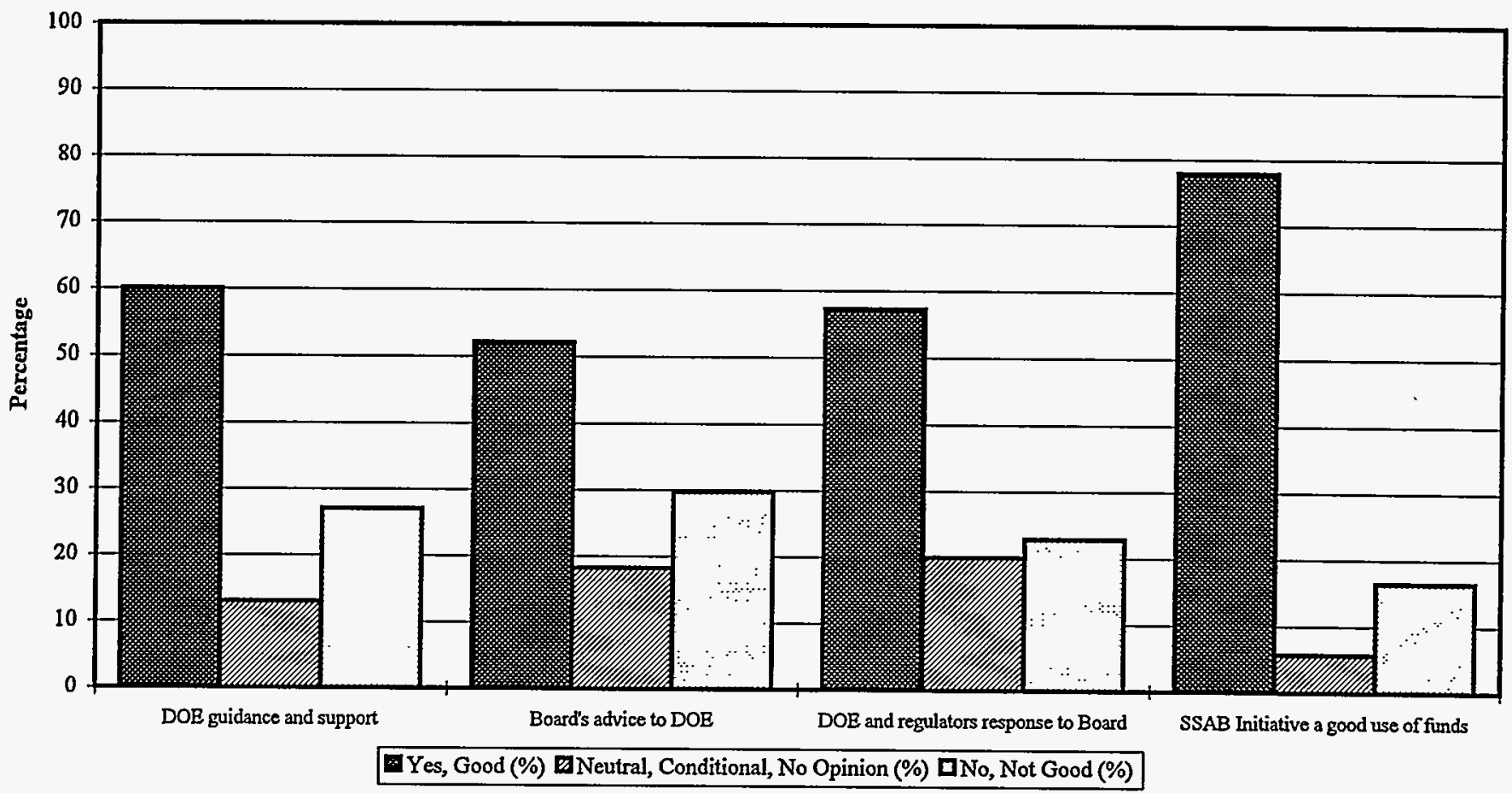

SSAB Supplementary Appendix

August 1996 
Figure 14a. Fernald

Summary: Combined responses for SSAB Initiative goals (Means)

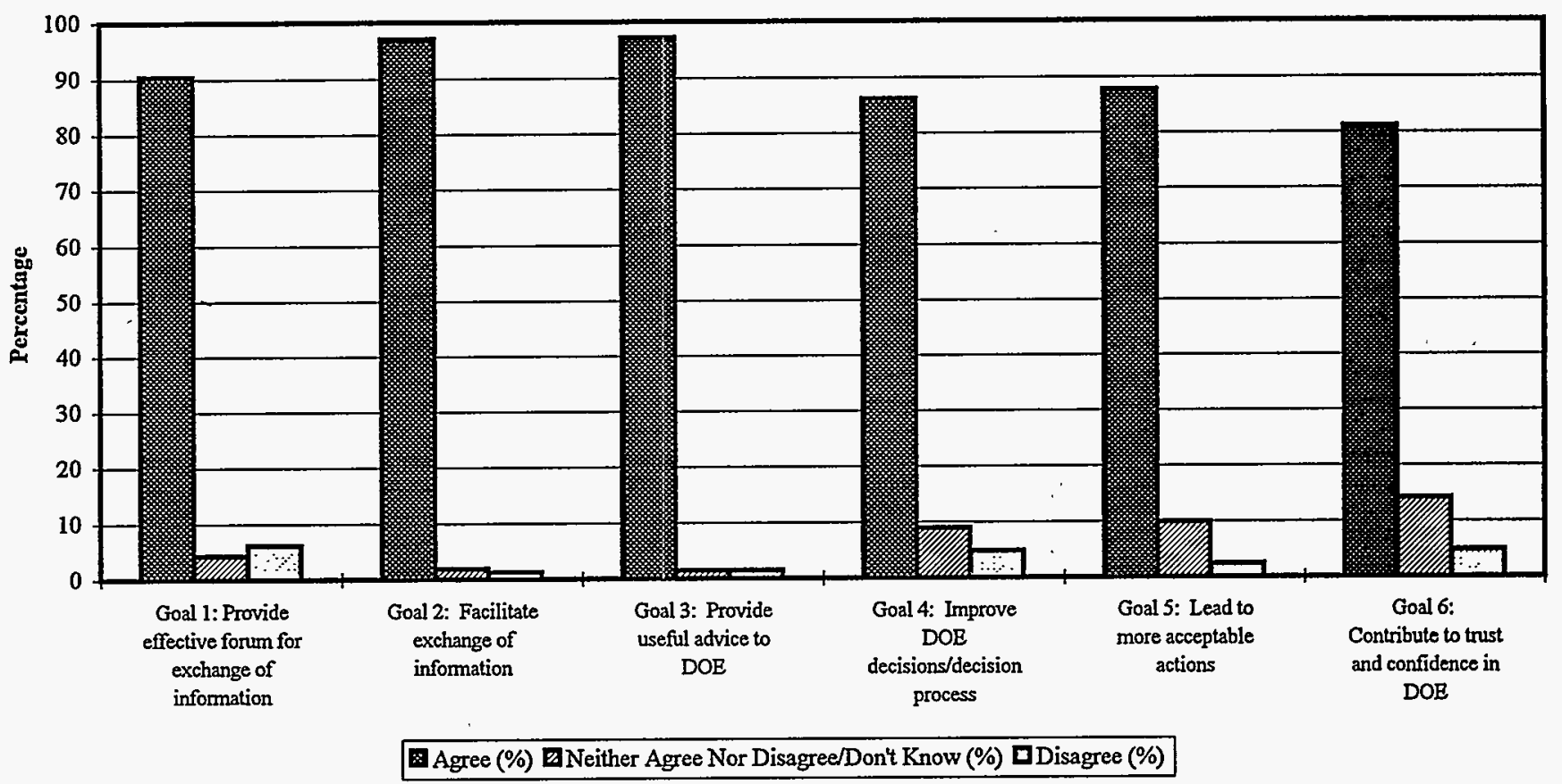

Figure 14b. Long Survey: Entire Sample

Summary: Combined responses for SSAB Initiative goals (Means)

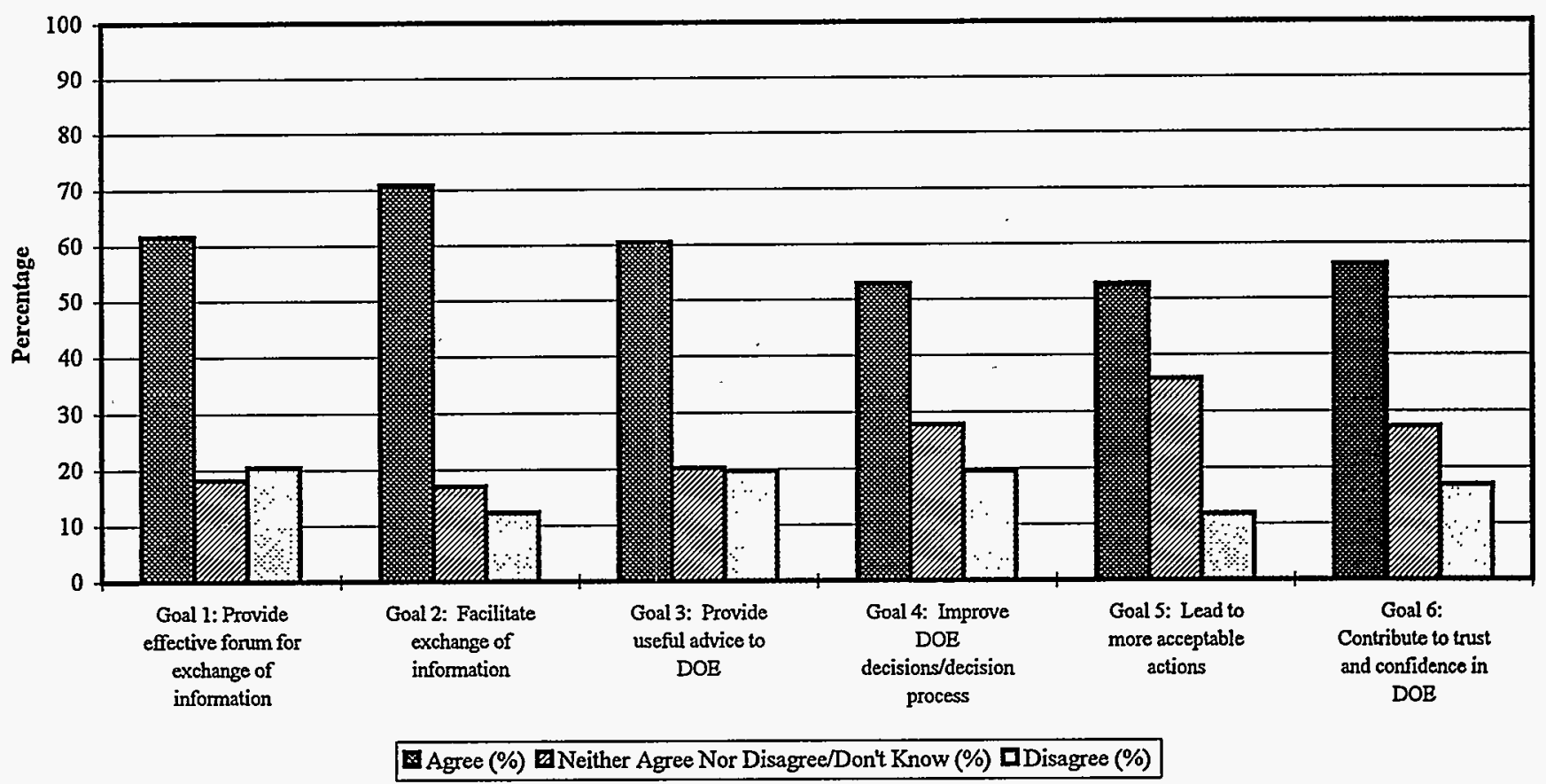

SSAB Supplementary Appendix

August 1996 
Figure 15a. Fernald

Summary: Combined responses on DOE-HQ involvement,

Personal experience, Group/Team work skills,

Working relations, Member affect (Means)

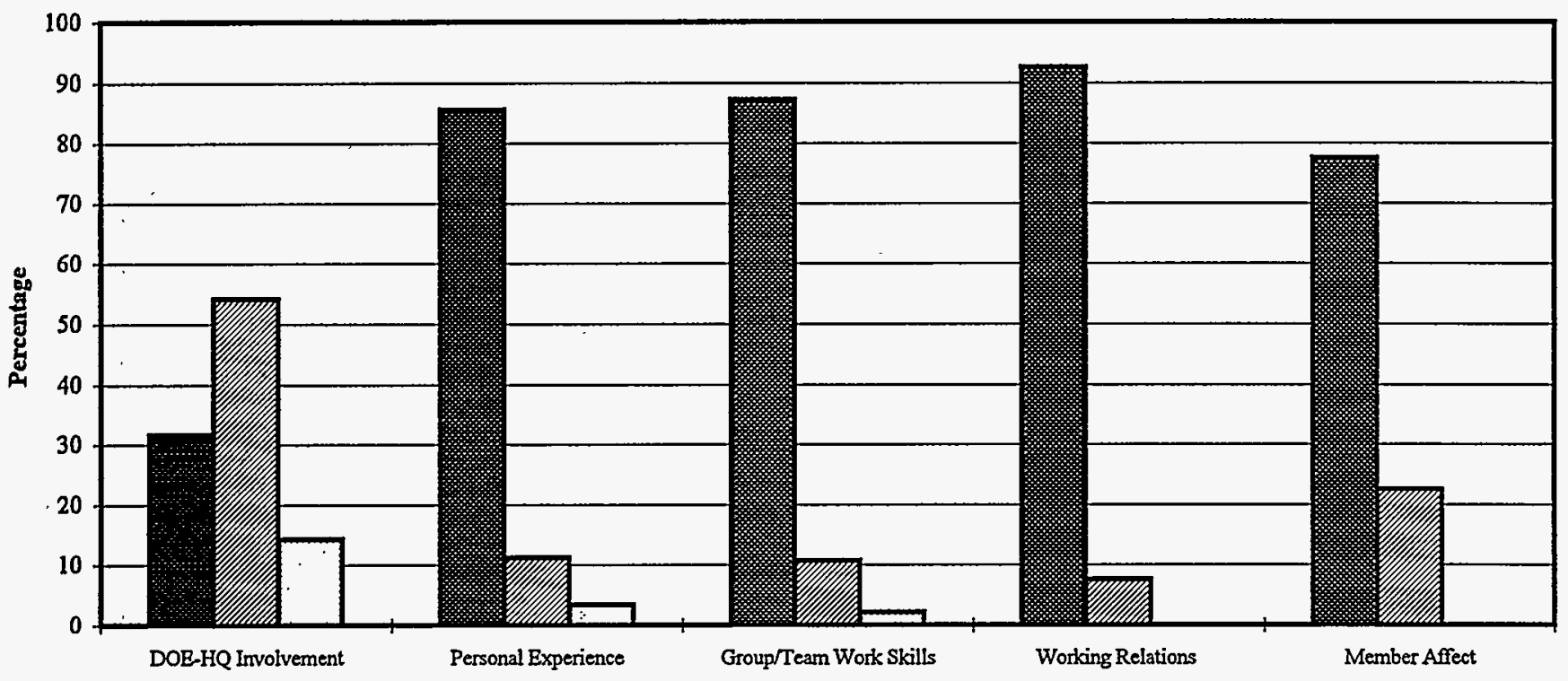

Agree (\%) Neither Agree Nor Disagree/Don't Know (\%) D Disagree (\%)

Figure 15b. Long Survey: Entire Sample

Summary: Combined responses on DOE-HQ involvement,

Personal experience, Group/Team work skills,

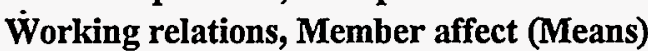

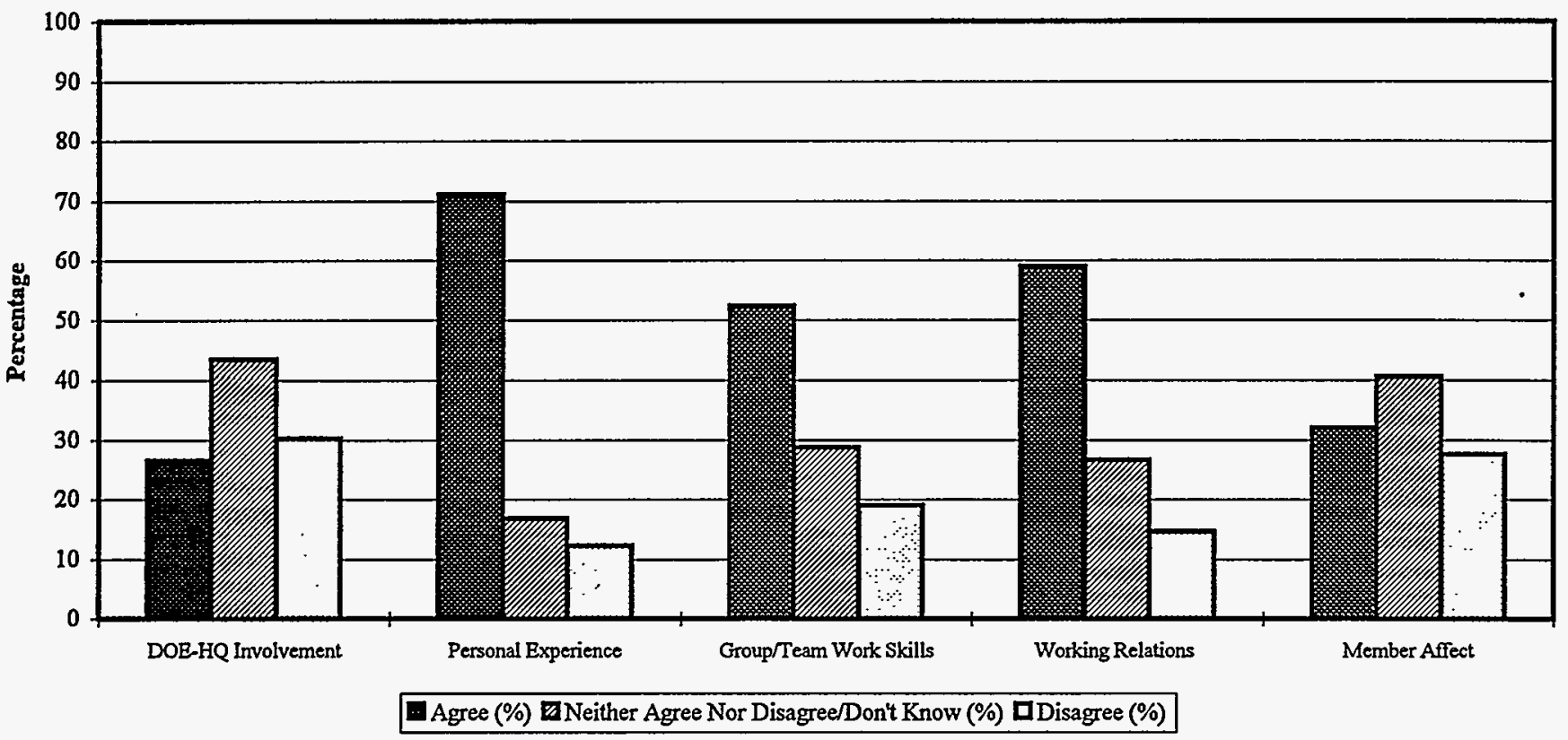

SSAB Supplementary Appendix

August 1996 
Table 1.

Goal 1. Establish processes and procedures to provide an effective forum for exchange of information and viewpoints regarding DOE site issues

\begin{tabular}{|c|c|c|c|c|c|c|c|c|}
\hline \multirow[t]{2}{*}{ Fernald } & \multicolumn{2}{|c|}{ Agree } & \multicolumn{2}{|c|}{$\begin{array}{l}\text { Neither/ } \\
\text { Don't Know }\end{array}$} & \multicolumn{2}{|c|}{ Disagree } & \multicolumn{2}{|c|}{ Total } \\
\hline & $\mathrm{N}$ & $\%$ & $N$ & $\%$ & $\mathrm{~N}$ & $\%$ & $\mathrm{~N}$ & $\%$ \\
\hline $\begin{array}{l}\text { The Board has established processes and } \\
\text { procedures for the effective exchange of } \\
\text { information }\end{array}$ & 21 & $100.0 \%$ & 0 & $0 \%$ & 0 & $0 \%$ & 21 & $100 \%$ \\
\hline $\begin{array}{l}\text { The Board has agreed-upon procedures to } \\
\text { operate the board }\end{array}$ & 20 & $100.0 \%$ & 0 & $0 \%$ & 0 & $0 \%$ & 20 & $100 \%$ \\
\hline $\begin{array}{l}\text { The Board has adequate support to allow } \\
\text { it to focus on substantive issues (R) * }\end{array}$ & 16 & $80.0 \%$ & 0 & $0 \%$ & 4 & $20.0 \%$ & 20 & $100 \%$ \\
\hline $\begin{array}{l}\text { The Board provides sufficient time for } \\
\text { discussion of issues }\end{array}$ & 20 & $100.0 \%$ & 0 & $0 \%$ & 0 & $0 \%$ & 20 & $100 \%$ \\
\hline The Board has effective leadership & 20 & $100.0 \%$ & 0 & $0 \%$ & 0 & $0 \%$ & 20 & $100 \%$ \\
\hline $\begin{array}{l}\text { DOE requests Board advice far enough } \\
\text { ahead of decision deadlines }(\mathrm{R})^{*}\end{array}$ & 10 & $47.6 \%$ & 7 & $33.3 \%$ & 4 & $19.0 \%$ & 21 & $100 \%$ \\
\hline $\begin{array}{l}\text { DOE handles Board administrative issues } \\
\text { expeditiously }(\mathrm{R})^{*}\end{array}$ & 20 & $95.2 \%$ & 0 & $0 \%$ & 1 & $4.8 \%$ & 21 & $100 \%$ \\
\hline $\begin{array}{l}\text { The SSAB staff provide satisfactory } \\
\text { support }\end{array}$ & 20 & $95.2 \%$ & 0 & $0 \%$ & 1 & $4.8 \%$ & 21 & $100 \%$ \\
\hline $\begin{array}{l}\text { The facilitator for the Board has helped } \\
\text { the Board function effectively }\end{array}$ & 20 & $95.2 \%$ & 1 & $4.8 \%$ & 0 & $0 \%$ & 21 & $100 \%$ \\
\hline
\end{tabular}

* (R) denotes a question that was asked in the negative form. For ease of review, these questions and data have been reversed in this table and are stated in positive form.

SSAB Supplementary Appendix

August 1996 
Table 2.

Goal 2. Facilitate interaction and exchange of information and viewpoints regarding DOE site issues

\begin{tabular}{|c|c|c|c|c|c|c|c|c|}
\hline \multirow[t]{2}{*}{ Fernald } & \multicolumn{2}{|c|}{ Agree } & \multicolumn{2}{|c|}{$\begin{array}{l}\text { Neither/ } \\
\text { Don't Know }\end{array}$} & \multicolumn{2}{|c|}{ Disagree } & \multicolumn{2}{|c|}{ Total } \\
\hline & $N$ & $\%$ & $N$ & $\%$ & $\mathrm{~N}$ & $\%$ & $\mathrm{~N}$ & $\%$ \\
\hline $\begin{array}{l}\text { The SSAB facilitates effective exchange } \\
\text { of viewpoints on site issues }\end{array}$ & 21 & $100.0 \%$ & 0.00 & $0 \%$ & 0 & $0 \%$ & 21 & $100 \%$ \\
\hline $\begin{array}{l}\text { The SSAB contributes to Board members' } \\
\text { understanding of the basis for key site } \\
\text { decisions }\end{array}$ & 21 & $100.0 \%$ & 0 & $0 \%$ & 0 & $0 \%$ & 21 & $100 \%$ \\
\hline $\begin{array}{l}\text { The SSAB contributes to DOE and } \\
\text { regulators' understanding of the public's } \\
\text { viewpoints on key site decisions }\end{array}$ & 21 & $100.0 \%$ & 0 & $0 \%$ & 0 & $0 \%$ & 21 & $100 \%$ \\
\hline $\begin{array}{l}\text { The SSAB contributes to a constructive } \\
\text { working relationship among the } \\
\text { participants }\end{array}$ & 21 & $100.0 \%$ & 0 & $0 \%$ & 0 & $0 \%$ & 21 & $100 \%$ \\
\hline The SSAB strives for consensus & 20 & $95.2 \%$ & 0 & $0 \%$ & 1 & $4.8 \%$ & 21 & $100 \%$ \\
\hline $\begin{array}{l}\text { The SSAB gives fair consideration to } \\
\text { dissenting opinions }\end{array}$ & 21 & $100.0 \%$ & 0 & $0 \%$ & 0 & $0 \%$ & 21 & $100 \%$ \\
\hline $\begin{array}{l}\text { The Board has made the effort needed to } \\
\text { learn about site issues }\end{array}$ & 20 & $100.0 \%$ & 0 & $0 \%$ & 0 & $0 \%$ & 20 & $100 \%$ \\
\hline $\begin{array}{l}\text { The Board invites expert advice into its } \\
\text { discussion on key policy issues }\end{array}$ & 20 & $100.0 \%$ & 0 & $0 \%$ & 0 & $0 \%$ & 20 & $100 \%$ \\
\hline $\begin{array}{l}\text { The Board responds to public inquiries } \\
\text { and comments about its decisions }\end{array}$ & 18 & $90.0 \%$ & 1 & $5.0 \%$ & 1 & $5.0 \%$ & 20 & $100 \%$ \\
\hline $\begin{array}{l}\text { The Board solicits feedback from the } \\
\text { community on its work }\end{array}$ & 19 & $95.0 \%$ & 1 & $5.0 \%$ & 0 & $0 \%$ & 20 & $100 \%$ \\
\hline $\begin{array}{l}\text { DOE makes information on key site issues } \\
\text { readily available to the Board }\end{array}$ & 20 & $95.2 \%$ & 1 & $4.8 \%$ & 0 & $0 \%$ & 21 & $100 \%$ \\
\hline $\begin{array}{l}\text { The regulators make important } \\
\text { contributions to the Board's work }\end{array}$ & 18 & $85.7 \%$ & 2 & $9.5 \%$ & 1 & $4.8 \%$ & 21 & $100 \%$ \\
\hline $\begin{array}{l}\text { The SSAB creates a climate supportive of } \\
\text { differing viewpoints }(\mathrm{R})^{*}\end{array}$ & 21 & $100.0 \%$ & 0 & $0 \%$ & 0 & $0 \%$ & 21 & $100 \%$ \\
\hline
\end{tabular}

* (R) denotes a question that was asked in the negative form. For ease of review, these questions and data have been reversed in this table and are stated in positive form.

SSAB Supplementary Appendix

August 1996

S-19 
Table 3.

Goal 3. Provide useful advice and/or recommendations to DOE (and regulators, where appropriate).

\begin{tabular}{|c|c|c|c|c|c|c|c|c|}
\hline \multirow[t]{2}{*}{ Fernald } & \multicolumn{2}{|c|}{ Agree } & \multicolumn{2}{|c|}{$\begin{array}{c}\text { Neither/ } \\
\text { Don't Know }\end{array}$} & \multicolumn{2}{|c|}{ Disagree } & \multicolumn{2}{|c|}{ Total } \\
\hline & $\mathrm{N}$ & $\%$ & $\mathrm{~N}$ & $\%$ & $\mathrm{~N}$ & $\%$ & $N$ & $\%$ \\
\hline $\begin{array}{l}\text { The Board provides useful advice to DOE } \\
\text { (and regulators, where appropriate) (R) * }\end{array}$ & 20 & $100.0 \%$ & 0 & $0 \%$ & 0 & $0 \%$ & 20 & $100 \%$ \\
\hline $\begin{array}{l}\text { The SSAB reaches agreement about } \\
\text { prioritization of key site issues for which } \\
\text { advice is sought }(\mathrm{R}){ }^{*}\end{array}$ & 21 & $100.0 \%$ & 0 & $0 \%$ & 0 & $0 \%$ & 21 & $100 \%$ \\
\hline The Board helps define site problems & 18 & $90.0 \%$ & 2 & $10.0 \%$ & 0 & $0 \%$ & 20 & $100 \%$ \\
\hline $\begin{array}{l}\text { The Board addresses issues in a timely } \\
\text { manner (R) * }\end{array}$ & 20 & $100.0 \%$ & 0 & $0 \%$ & 0 & $0 \%$ & 20 & $100 \%$ \\
\hline $\begin{array}{l}\text { The Board reaches consensus on key site } \\
\text { issues (R) * }\end{array}$ & 20 & $100.0 \%$ & 0 & $0 \%$ & 0 & $0 \%$ & 20 & $100 \%$ \\
\hline $\begin{array}{l}\text { The Board provides informed advice to } \\
\text { DOE (and regulators, where appropriate) }\end{array}$ & 19 & $95.0 \%$ & 0 & $0 \%$ & 1 & $5.0 \%$ & 20 & $100 \%$ \\
\hline $\begin{array}{l}\text { The Board provides advice that reflects } \\
\text { the viewpoints and priorities of the } \\
\text { community }\end{array}$ & 19 & $95.0 \%$ & 0 & $0 \%$ & 1 & $5.0 \%$ & 20 & $100 \%$ \\
\hline
\end{tabular}

* (R) denotes a question that was asked in the negative form. For ease of review, these questions and data have been reversed in this table and are stated in positive form. 
Table 4.

Goal 4. Improve DOE's (and where applicable, regulators') site decisions and decision making process

\begin{tabular}{|c|c|c|c|c|c|c|c|c|}
\hline \multirow[t]{2}{*}{ Fernald } & \multicolumn{2}{|c|}{ Agree } & \multicolumn{2}{|c|}{$\begin{array}{l}\text { Neither/ } \\
\text { Don't Know }\end{array}$} & \multicolumn{2}{|c|}{ Disagree } & \multicolumn{2}{|c|}{ Total } \\
\hline & $\mathrm{N}$ & $\%$ & $\mathrm{~N}$ & $\%$ & $N$ & $\%$ & $\mathrm{~N}$ & $\%$ \\
\hline $\begin{array}{l}\text { The SSAB has improved DOE's site } \\
\text { decisions }(\mathrm{R})^{*}\end{array}$ & 20 & $95.2 \%$ & 0 & $0 \%$ & 1 & $4.8 \%$ & 21 & $100 \%$ \\
\hline $\begin{array}{l}\text { The Board understands the decision } \\
\text { making process at [a particular] site }\end{array}$ & 18 & $90.0 \%$ & 2 & $10.0 \%$ & 0 & $0 \%$ & 20 & $100 \%$ \\
\hline $\begin{array}{l}\text { DOE discusses important policies } \\
\text { affecting site decisions with the Board }\end{array}$ & 20 & $95.2 \%$ & 1 & $4.8 \%$ & 0 & $0 \%$ & 21 & $100 \%$ \\
\hline $\begin{array}{l}\text { DOE has explained to the Board its site } \\
\text { decision making process }\end{array}$ & 15 & $71.4 \%$ & 5 & $23.8 \%$ & 1 & $4.8 \%$ & 21 & $100 \%$ \\
\hline $\begin{array}{l}\text { The DOE shows how Board advice is } \\
\text { reflected in site decisions }\end{array}$ & 19 & $90.5 \%$ & 1 & $4.8 \%$ & 1 & $4.8 \%$ & 21 & $100 \%$ \\
\hline $\begin{array}{l}\text { The decision making process used by } \\
\text { DOE at [a particular] site is effective in } \\
\text { furthering site clean-up (R) } *\end{array}$ & 16 & $76.2 \%$ & 2 & $9.5 \%$ & 3 & $14.3 \%$ & 21 & $100 \%$ \\
\hline
\end{tabular}

* $(\mathrm{R})$ denotes a question that was asked in the negative form. For ease of review, these questions and data have been reversed in this table and are stated in positive form.

SSAB Supplementary Appendix

August 1996 
Table 5.

Goal 5. Lead to more acceptable actions

\begin{tabular}{|c|c|c|c|c|c|c|c|c|}
\hline \multirow[t]{2}{*}{ Fernald } & \multicolumn{2}{|c|}{ Agree } & \multicolumn{2}{|c|}{$\begin{array}{l}\text { Neither/ } \\
\text { Don't Know }\end{array}$} & \multicolumn{2}{|c|}{ Disagree } & \multicolumn{2}{|c|}{ Total } \\
\hline & $\mathbf{N}$ & $\%$ & $\mathrm{~N}$ & $\%$ & $N$ & $\%$ & $N$ & $\%$ \\
\hline $\begin{array}{l}\text { The SSAB leads to more acceptable site } \\
\text { decisions }\end{array}$ & 20 & $95.2 \%$ & 1 & $4.8 \%$ & 0 & $0 \%$ & 21 & $100 \%$ \\
\hline $\begin{array}{l}\text { The Board supports the recommendations } \\
\text { it gives DOE }\end{array}$ & 20 & $100.0 \%$ & 0 & $0 \%$ & 0 & $0 \%$ & 20 & $100 \%$ \\
\hline The Board supports DOE's site actions & 12 & $60.0 \%$ & 7 & $35.0 \%$ & 1 & $5.0 \%$ & 20 & $100 \%$ \\
\hline $\begin{array}{l}\text { The DOE pays attention to the Board's } \\
\text { advice on key site issues (R) * }\end{array}$ & 19 & $90.5 \%$ & 2 & $9.5 \%$ & 0 & $0 \%$ & 21 & $100 \%$ \\
\hline $\begin{array}{l}\text { There is support in the community for } \\
\text { DOE's site decisions that have SSAB } \\
\text { input }\end{array}$ & 18 & $85.7 \%$ & 2 & $9.5 \%$ & 1 & $4.8 \%$ & 21 & $100 \%$ \\
\hline Progress is being made on key site issues & 20 & $95.2 \%$ & 0 & $0 \%$ & 1 & $4.8 \%$ & 21 & $100 \%$ \\
\hline
\end{tabular}

* $(\mathrm{R})$ denotes a question that was asked in the negative form. For ease of review, these questions and data have been reversed in this table and are stated in positive form. 
Table 6.

Goal 6. Contribute to trust and confidence in DOE

\begin{tabular}{|c|c|c|c|c|c|c|c|c|}
\hline \multirow[t]{2}{*}{ Fernald } & \multicolumn{2}{|c|}{ Agree } & \multicolumn{2}{|c|}{$\begin{array}{c}\text { Neither/ } \\
\text { Don't Know }\end{array}$} & \multicolumn{2}{|c|}{ Disagree } & \multicolumn{2}{|c|}{ Total } \\
\hline & $\mathrm{N}$ & $\%$ & $\mathrm{~N}$ & $\%$ & $\mathrm{~N}$ & $\%$ & $N$ & $\%$ \\
\hline $\begin{array}{l}\text { The SSAB contributes to trust and } \\
\text { confidence in DOE }\end{array}$ & 16 & $76.2 \%$ & 4 & $19.0 \%$ & 1 & $4.8 \%$ & 21 & $100 \%$ \\
\hline $\begin{array}{l}\text { Relationships between DOE and the public } \\
\text { have improved since the formation of the } \\
\text { SSAB }\end{array}$ & 18 & $85.7 \%$ & 3 & $14.3 \%$ & 0 & $0 \%$ & 21 & $100 \%$ \\
\hline $\begin{array}{l}\text { DOE is committed to clean up [a } \\
\text { particular] site }\end{array}$ & 17 & $81.0 \%$ & 2 & $9.5 \%$ & 2 & $9.5 \%$ & 21 & $100 \%$ \\
\hline
\end{tabular}


Table 7.

Public awareness

\begin{tabular}{llllllllll}
\hline \multicolumn{1}{c}{ Fernald } & Agree & & $\begin{array}{c}\text { Neither/ } \\
\text { Don't Know }\end{array}$ & Disagree & & Total \\
& $\mathrm{N}$ & $\%$ & $\mathrm{~N}$ & $\%$ & $\mathrm{~N}$ & $\%$ & $\mathrm{~N}$ & $\%$ \\
\hline $\begin{array}{l}\text { The public knows little about the role of } \\
\text { the Board }\end{array}$ & 2 & $9.5 \%$ & 5 & $23.8 \%$ & 14 & $66.7 \%$ & 21 & $100 \%$ \\
\hline
\end{tabular}

Table 8.

SSAB Initiative is a good use of funds

\begin{tabular}{|c|c|c|c|c|c|c|c|c|}
\hline \multirow[t]{2}{*}{ Fernald } & \multicolumn{2}{|c|}{ Yes, Good } & \multicolumn{2}{|c|}{$\begin{array}{c}\text { Neutral, } \\
\text { Conditional, } \\
\text { No Opinion }\end{array}$} & \multicolumn{2}{|c|}{$\begin{array}{c}\text { No, } \\
\text { Not Good }\end{array}$} & \multicolumn{2}{|c|}{ Total } \\
\hline & $\mathrm{N}$ & $\%$ & $\mathrm{~N}$ & $\%$ & $N$ & $\%$ & $\mathbf{N}$ & $\%$ \\
\hline SSAB Initiative is a good use of funds & 17 & $94.4 \%$ & 1 & $5.6 \%$ & 0 & $0 \%$ & 18 & $100 \%$ \\
\hline
\end{tabular}


Table 9.

DOE-HQ involvement with the SSAB Initiative

\begin{tabular}{|c|c|c|c|c|c|c|c|c|}
\hline \multirow[t]{2}{*}{ Fernald } & \multicolumn{2}{|c|}{ Agree } & \multicolumn{2}{|c|}{$\begin{array}{l}\text { Neither/ } \\
\text { Don't Know }\end{array}$} & \multicolumn{2}{|c|}{ Disagree } & \multicolumn{2}{|c|}{ Total } \\
\hline & $\mathrm{N}$ & $\%$ & $N$ & $\%$ & $N$ & $\%$ & $N$ & $\%$ \\
\hline $\begin{array}{l}\text { DOE-HQ provides helpful guidance to the } \\
\text { Boards and to DOE }\end{array}$ & 3 & $14.3 \%$ & 13 & $61.9 \%$ & 5 & $23.8 \%$ & 21 & $100 \%$ \\
\hline $\begin{array}{l}\text { DOE-HQ provides sufficient support to } \\
\text { facilitate the work of the SSAB }\end{array}$ & 7 & $33.3 \%$ & 11 & $52.4 \%$ & 3 & $14.3 \%$ & 21 & $100 \%$ \\
\hline $\begin{array}{l}\text { DOE-HQ gives careful consideration to } \\
\text { SSAB advice in its decisions }\end{array}$ & 10 & $47.6 \%$ & 10 & $47.6 \%$ & 1 & $4.8 \%$ & 21 & $100 \%$ \\
\hline
\end{tabular}


Table 10.

Personal experience with the SSAB Initiative

\begin{tabular}{|c|c|c|c|c|c|c|c|c|}
\hline \multirow[t]{2}{*}{ Fernald } & \multicolumn{2}{|c|}{ Agree } & \multicolumn{2}{|c|}{$\begin{array}{l}\text { Neither/ } \\
\text { Don't Know }\end{array}$} & \multicolumn{2}{|c|}{ Disagree } & \multicolumn{2}{|c|}{ Total } \\
\hline & $\mathrm{N}$ & $\%$ & $\mathrm{~N}$ & $\%$ & $\mathrm{~N}$ & $\%$ & $\mathrm{~N}$ & $\%$ \\
\hline $\begin{array}{l}\text { I consider myself to be very } \\
\text { knowledgeable about site issues }\end{array}$ & 19 & $90.5 \%$ & 2 & $9.5 \%$ & 0 & $0 \%$ & 21 & $100 \%$ \\
\hline $\begin{array}{l}\text { Because of the SSAB, I better understand } \\
\text { the complexities of site clean-up }\end{array}$ & 15 & $71.4 \%$ & 4 & $19.0 \%$ & 2 & $9.5 \%$ & 21 & $100 \%$ \\
\hline $\begin{array}{l}\text { I feel that my participation in the SSAB } \\
\text { has been worthwhile }(\mathrm{R}) *\end{array}$ & 20 & $95.2 \%$ & 1 & $4.8 \%$ & 0 & $0 \%$ & 21 & $100 \%$ \\
\hline
\end{tabular}

* (R) denotes a question that was asked in the negative form. For ease of review, these questions and data have been reversed in this table and are stated in positive form. 
Table 11.

Group/Team work skills

\begin{tabular}{|c|c|c|c|c|c|c|c|c|}
\hline \multirow[t]{2}{*}{ Fernald } & \multicolumn{2}{|c|}{ Agree } & \multicolumn{2}{|c|}{$\begin{array}{l}\text { Neither/ } \\
\text { Don't Know }\end{array}$} & \multicolumn{2}{|c|}{ Disagree } & \multicolumn{2}{|c|}{ Total } \\
\hline & $\mathrm{N}$ & $\%$ & $\mathrm{~N}$ & $\%$ & $N$ & $\%$ & $N$ & $\%$ \\
\hline All have group/team work skills & 17 & $85.0 \%$ & 2 & $10.0 \%$ & 1 & $5.0 \%$ & 20 & $100 \%$ \\
\hline $\begin{array}{l}\text { Have developed well-established working } \\
\text { relationships with each other }\end{array}$ & 20 & $100.0 \%$ & 0 & $0 \%$ & 0 & $0 \%$ & 20 & $100 \%$ \\
\hline Like each other & 15 & $75.0 \%$ & 5 & $25.0 \%$ & 0 & $0 \%$ & 20 & $100 \%$ \\
\hline $\begin{array}{l}\text { Have a high degree of trust and } \\
\text { confidence in each other }\end{array}$ & 16 & $80.0 \%$ & 4 & $20.0 \%$ & 0 & $0 \%$ & 20 & $100 \%$ \\
\hline $\begin{array}{l}\text { Accept the values that are important to the } \\
\text { group }\end{array}$ & 20 & $100.0 \%$ & 0 & $0 \%$ & 0 & $0 \%$ & 20 & $100 \%$ \\
\hline Show respect for the viewpoints of others & 20 & $100.0 \%$ & 0 & $0 \%$ & 0 & $0 \%$ & 20 & $100 \%$ \\
\hline $\begin{array}{l}\text { Accept willingly the goals and } \\
\text { expectations for the Board }\end{array}$ & 20 & $100.0 \%$ & 0 & $0 \%$ & 0 & $0 \%$ & 20 & $100 \%$ \\
\hline $\begin{array}{l}\text { Set high expectations for what they } \\
\text { believe they can accomplish }\end{array}$ & 16 & $80.0 \%$ & 3 & $15.0 \%$ & 1 & $5.0 \%$ & 20 & $100 \%$ \\
\hline $\begin{array}{l}\text { Communicate fully and frankly to the } \\
\text { Board all relevant and valuable } \\
\text { information }\end{array}$ & 19 & $95.0 \%$ & 1 & $5.0 \%$ & 0 & $0 \%$ & 20 & $100 \%$ \\
\hline $\begin{array}{l}\text { Try not to waste the Board's time with } \\
\text { irrelevant material or communications }\end{array}$ & 18 & $90.0 \%$ & 2 & $10.0 \%$ & 0 & $0 \%$ & 20 & $100 \%$ \\
\hline $\begin{array}{l}\text { Are willing to be influenced by other } \\
\text { Board members about new ideas and } \\
\text { methods }\end{array}$ & 16 & $80.0 \%$ & 4 & $20.0 \%$ & 0 & $0 \%$ & 20 & $100 \%$ \\
\hline $\begin{array}{l}\text { Clearly understand the goals and } \\
\text { philosophy of the Board's operation }\end{array}$ & 20 & $100.0 \%$ & 0 & $0 \%$ & 0 & $0 \%$ & 20 & $100 \%$ \\
\hline $\begin{array}{l}\text { Do all they can to help the Board achieve } \\
\text { its objectives }\end{array}$ & 20 & $100.0 \%$ & 0 & $0 \%$ & 0 & $0 \%$ & 20 & $100 \%$ \\
\hline $\begin{array}{l}\text { Get the technical knowledge and training } \\
\text { in group skills they need }\end{array}$ & 12 & $60.0 \%$ & 7 & $35.0 \%$ & 1 & $5.0 \%$ & 20 & $100 \%$ \\
\hline Provide one another mutual help & 15 & $75.0 \%$ & 5 & $25.0 \%$ & 0 & $0 \%$ & 20 & $100 \%$ \\
\hline
\end{tabular}

SSAB Supplementary Appendix 
Hanford Site Specific Advisory Board Evaluation Survey Results 
Figure 1a. Hanford

Goal 1. Establish processes and procedures to provide an effective forum for exchange of information and viewpoints regarding DOE site issues

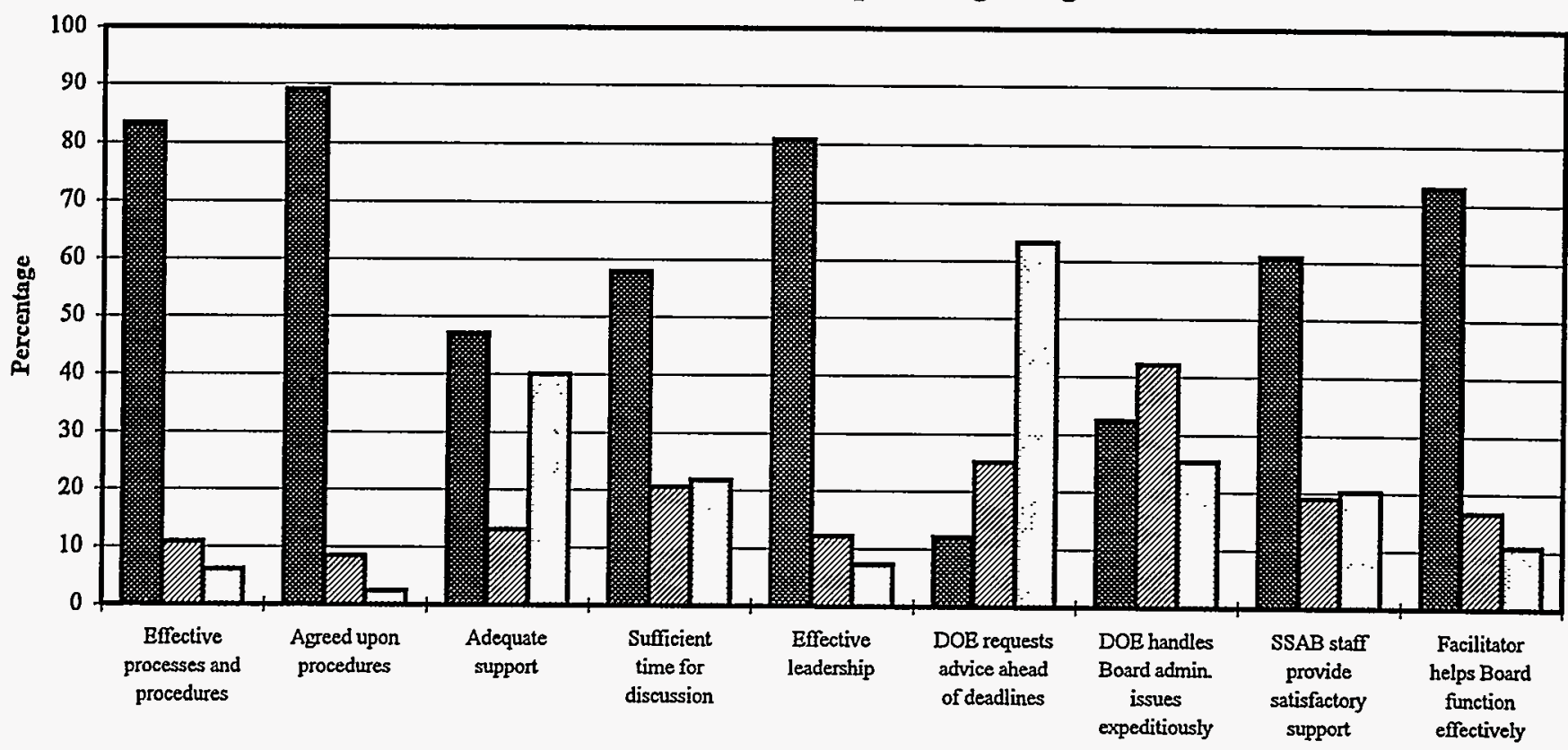

Agree (\%) Weither Agree Nor Disagree/Don't Know (\%) DDisagree (\%)

Figure 1b. Long Survey: Entire Sample

Goal 1. Establish processes and procedures to provide an effective forum for exchange of information and viewpoints regarding DOE site issues

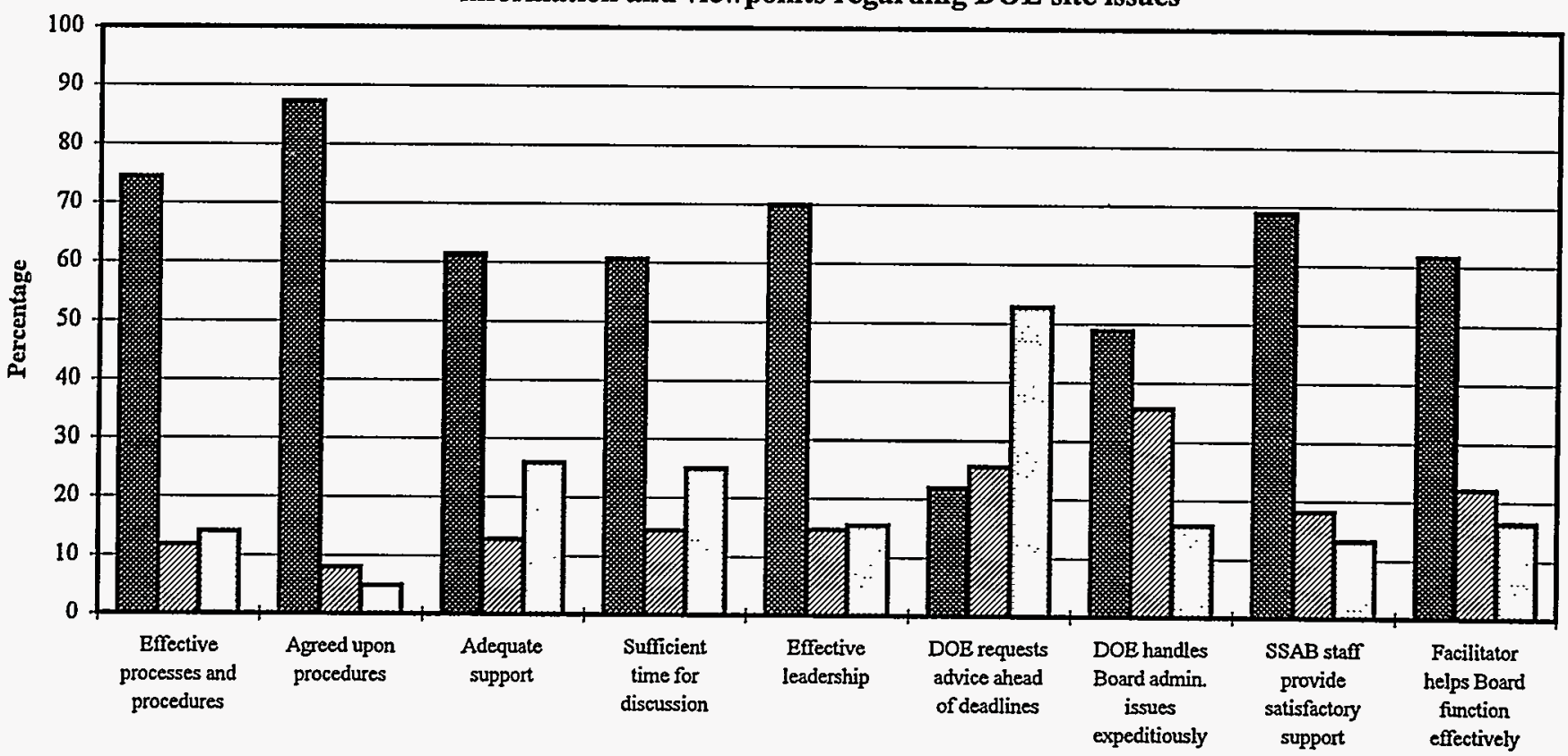

国Agree (\%) Neither Agree Nor Disagree/Don't Know (\%) 口Disagree (\%)

SSAB Supplementary Appendix

August 1996 
Figure 2a. Hanford

Goal 2. Facilitate interaction and exchange of information and viewpoints regarding $\mathrm{DOE}$ site issues

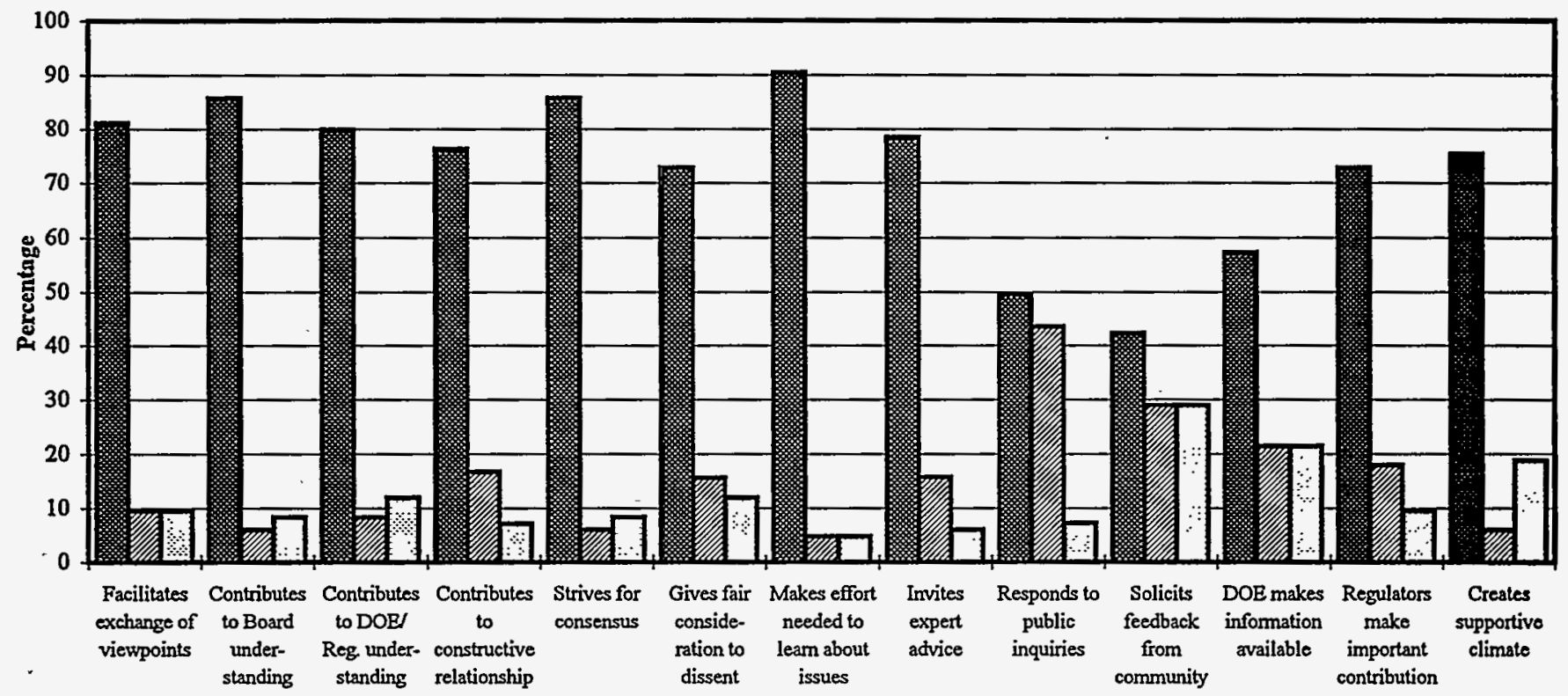

OAgree (\%) Weither Agree Nor Disagree/Don't Know (\%) DDisagree (\%)

Figure 2b. Long Survey: Entire Sample

Goal 2. Facilitate interaction and exchange of information and viewpoints regarding DOE site issues

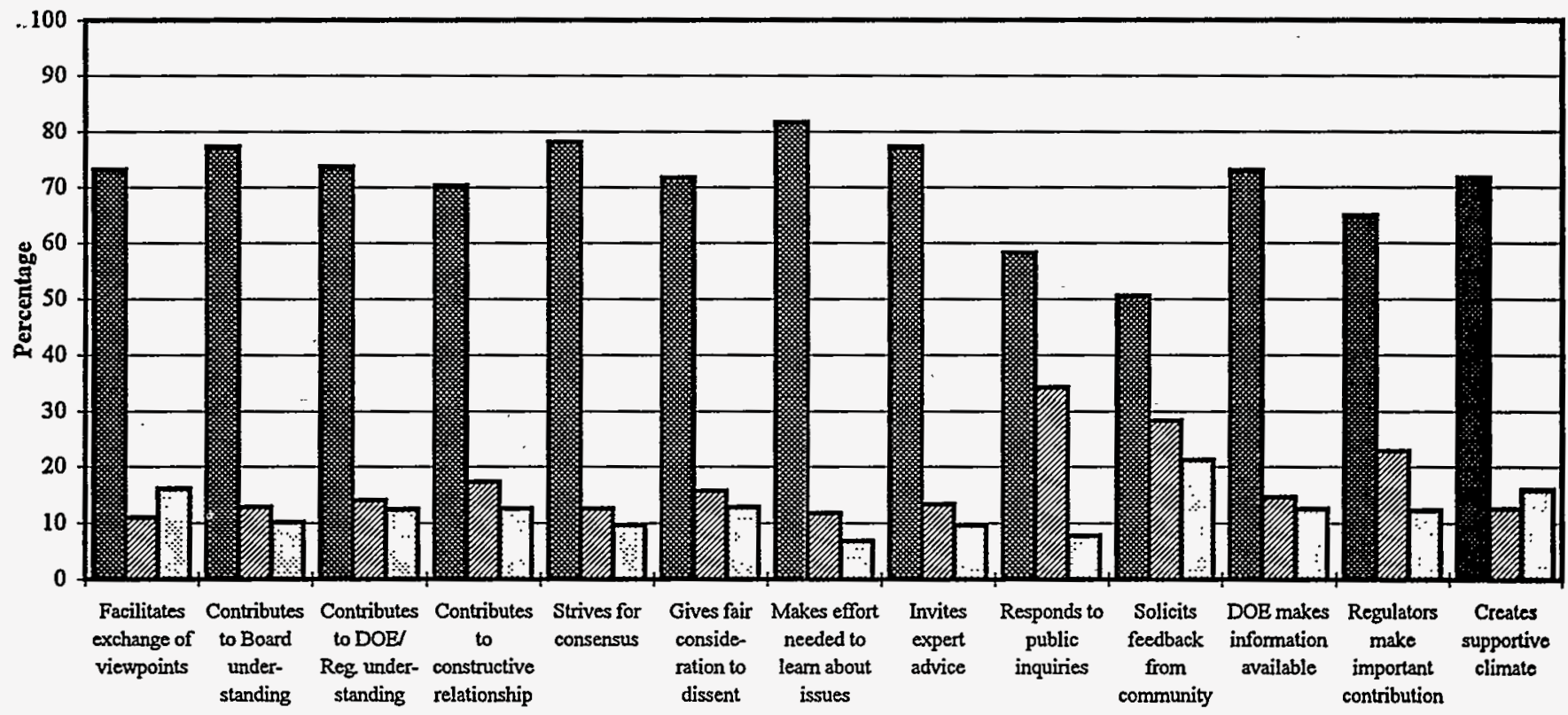

Agree (\%) Neither Agree Nor Disagree/Don't Know (\%) DDisagree (\%)

SSAB Supplementary Appendix

August 1996 
Figure 3a. Hanford

Goal 3. Provide useful advice and/or recommendations to DOE

(and regulators, where appropriate)

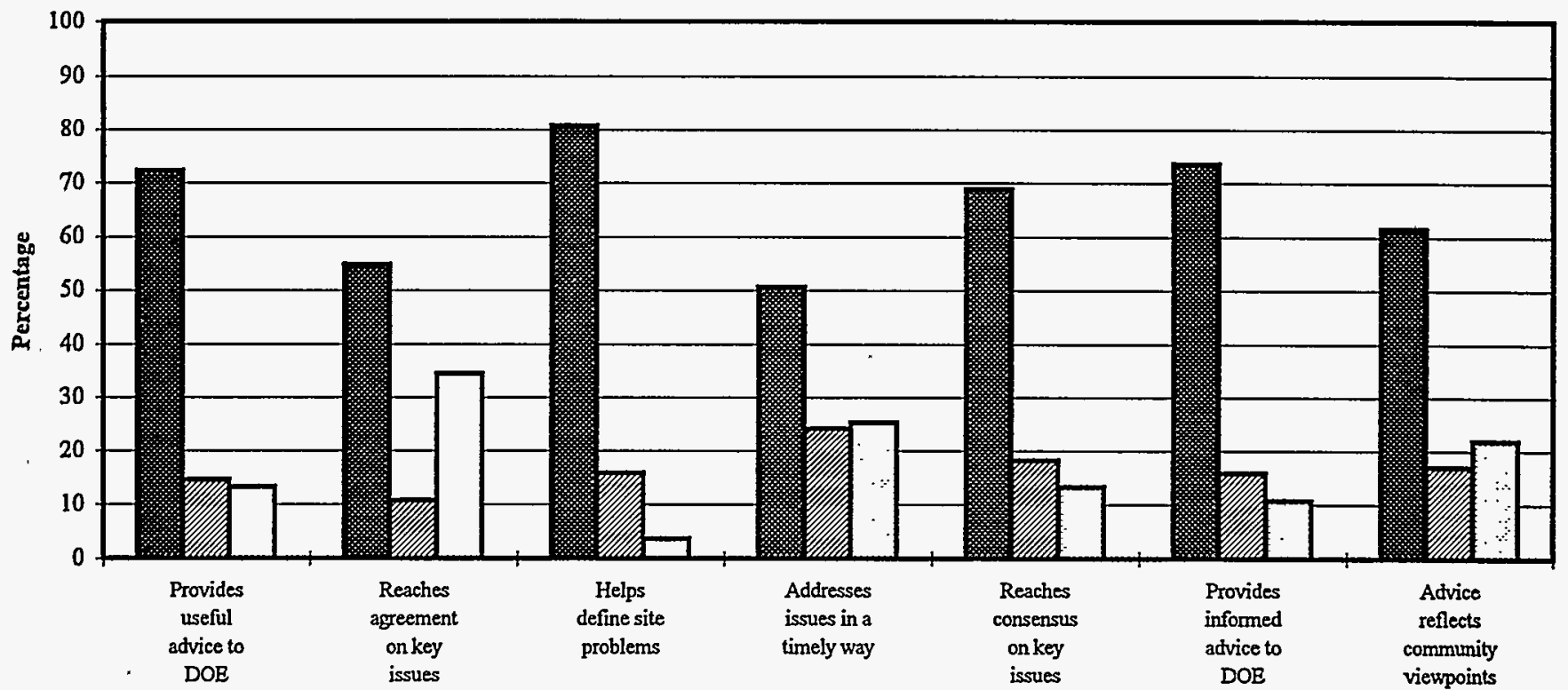

Agree (\%) Deither Agree Nor Disagree/Don't Know (\%) DDisagree (\%)

Figure 3b. Long Survey: Entire Sample

Goal 3. Provide useful advice and/or recommendations to DOE

(and regulators, where appropriate)

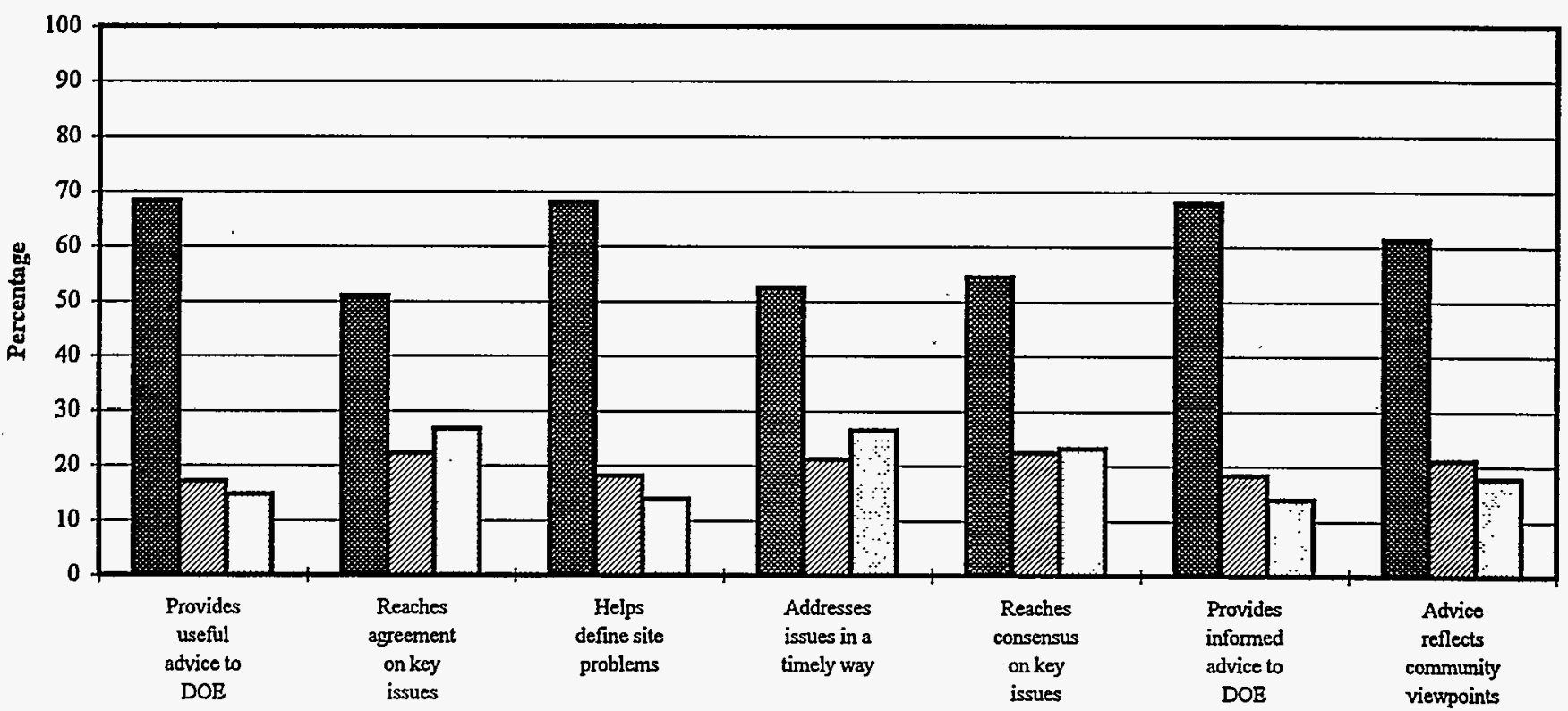

Agree (\%) EINNeither Agree Nor Disagree/Don't Know (\%) DDisagree (\%)

SSAB Supplementary Appendix

August 1996 
Figure 4a. Hanford

Goal 4. Improve DOE's (and where applicable, regulators') site decisions and decision making process

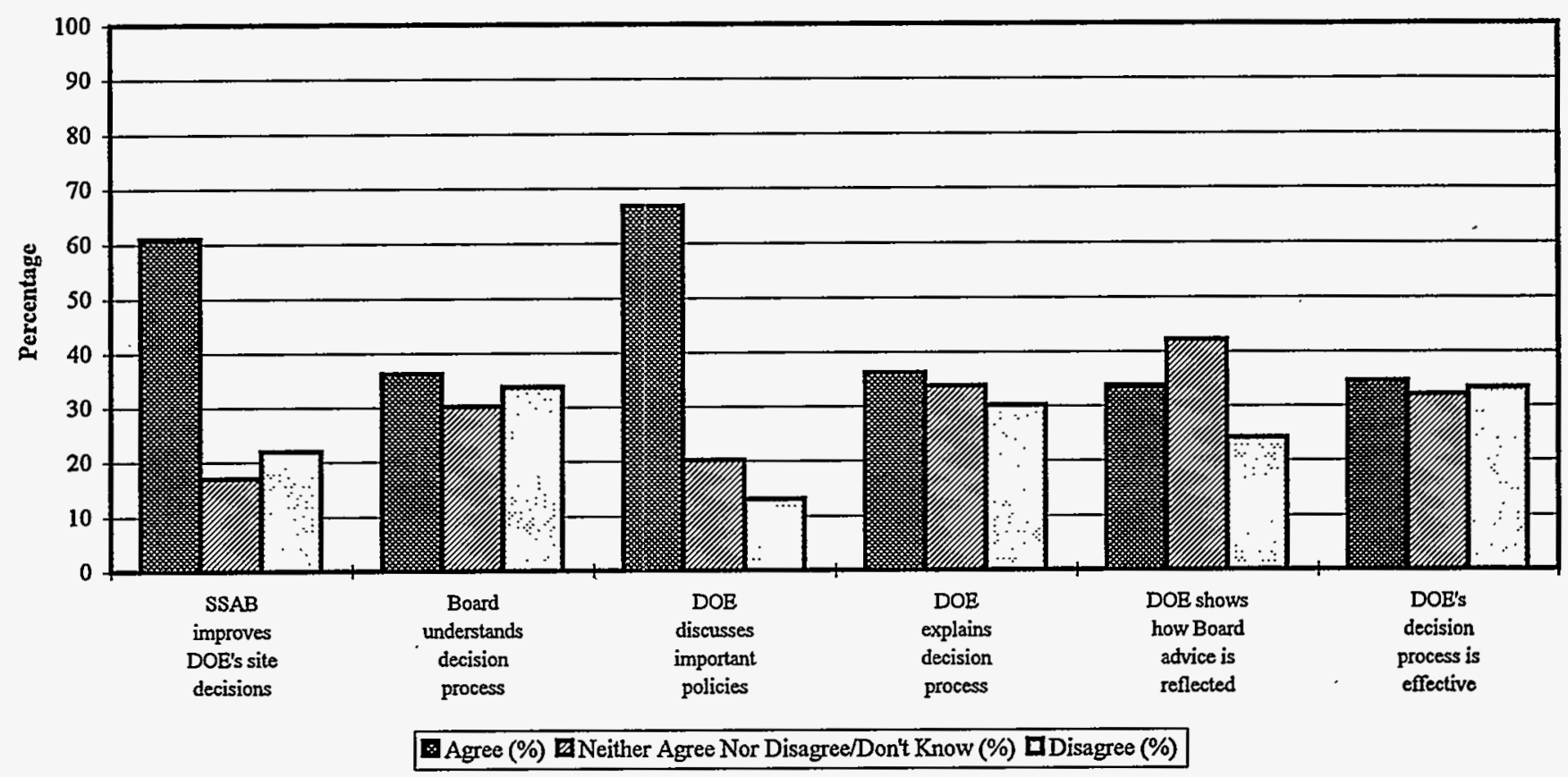

Figure 4b. Long Survey: Entire Sample

Goal 4. Improve DOE's (and where applicable, regulators')

site decisions and decision making process

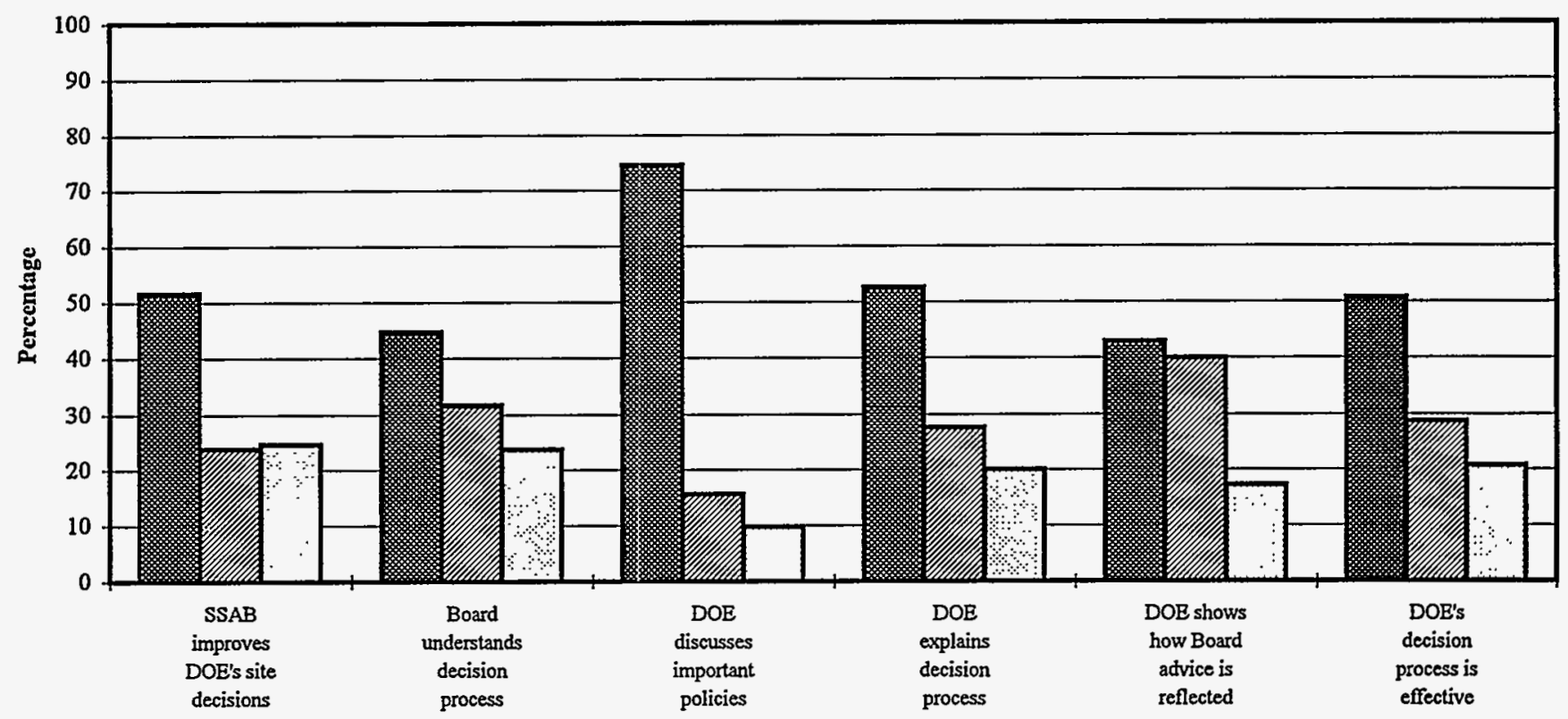

Pagree (\%) WNeither Agree Nor Disagree/Don't Know (\%) Disagree (\%)

SSAB Supplementary Appendix 
Figure 5a. Hanford

Goal 5. Lead to more acceptable actions

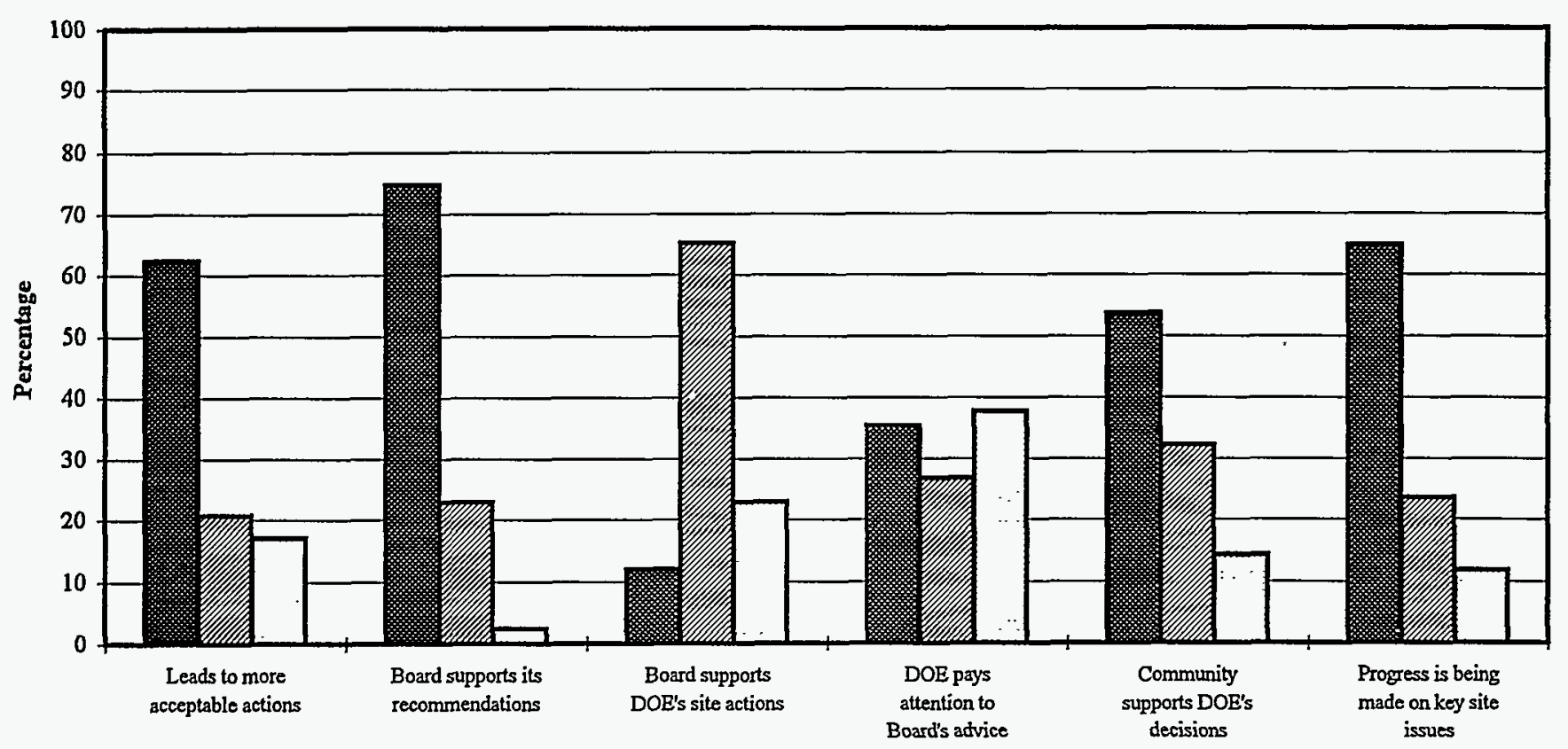

E Agree (\%) 7 Neither Agree Nor Disagree/Don't Know (\%) DDisagree (\%)

Figure 5b. Long Survey: Entire Sample

Goal 5. Lead to more acceptable actions

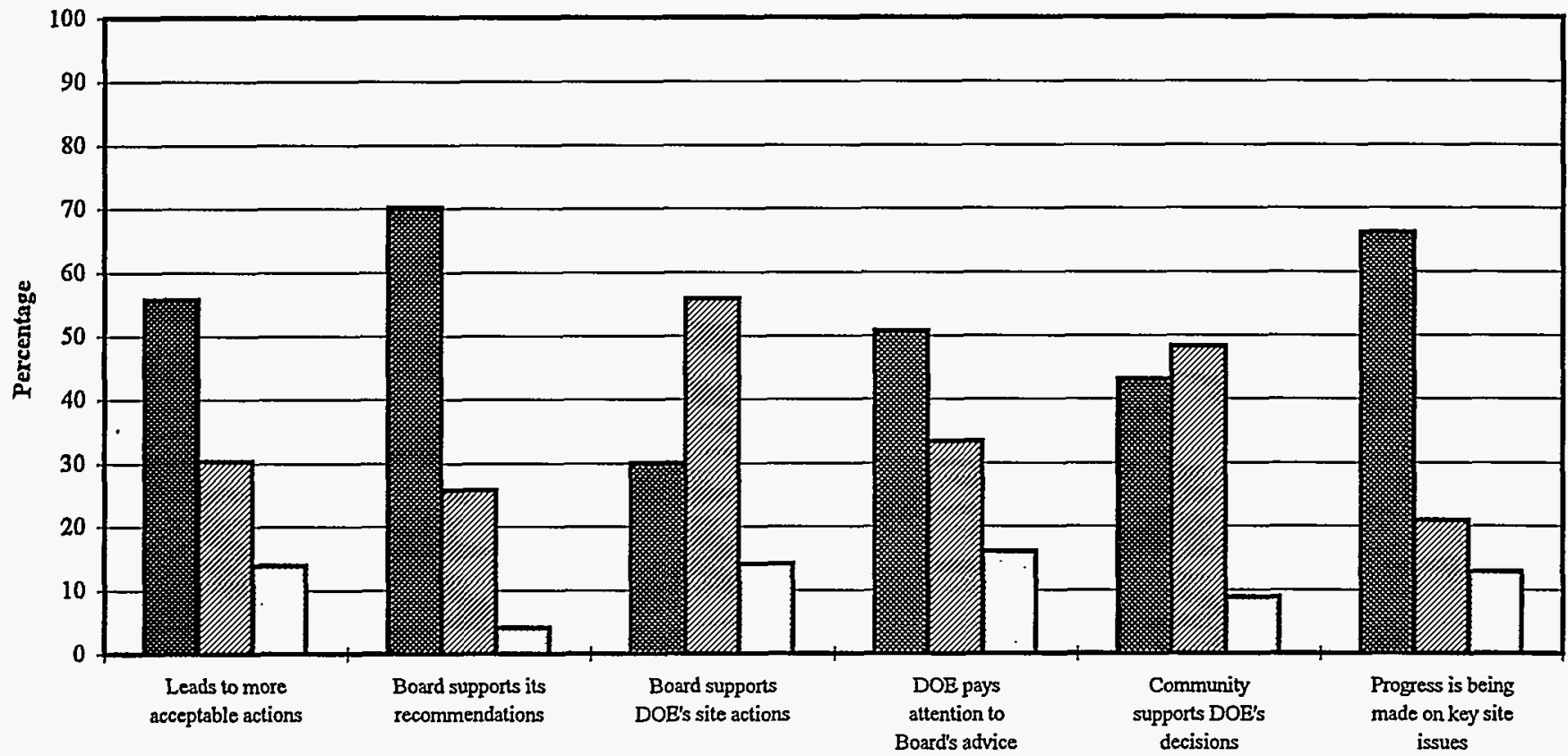

Agree (\%) Weither Agree Nor Disagree/Don't Know (\%) DDisagree (\%)

SSAB Supplementary Appendix

August 1996 
Figure 6a. Hanford

Goal 6. Contribute to trust and confidence in DOE

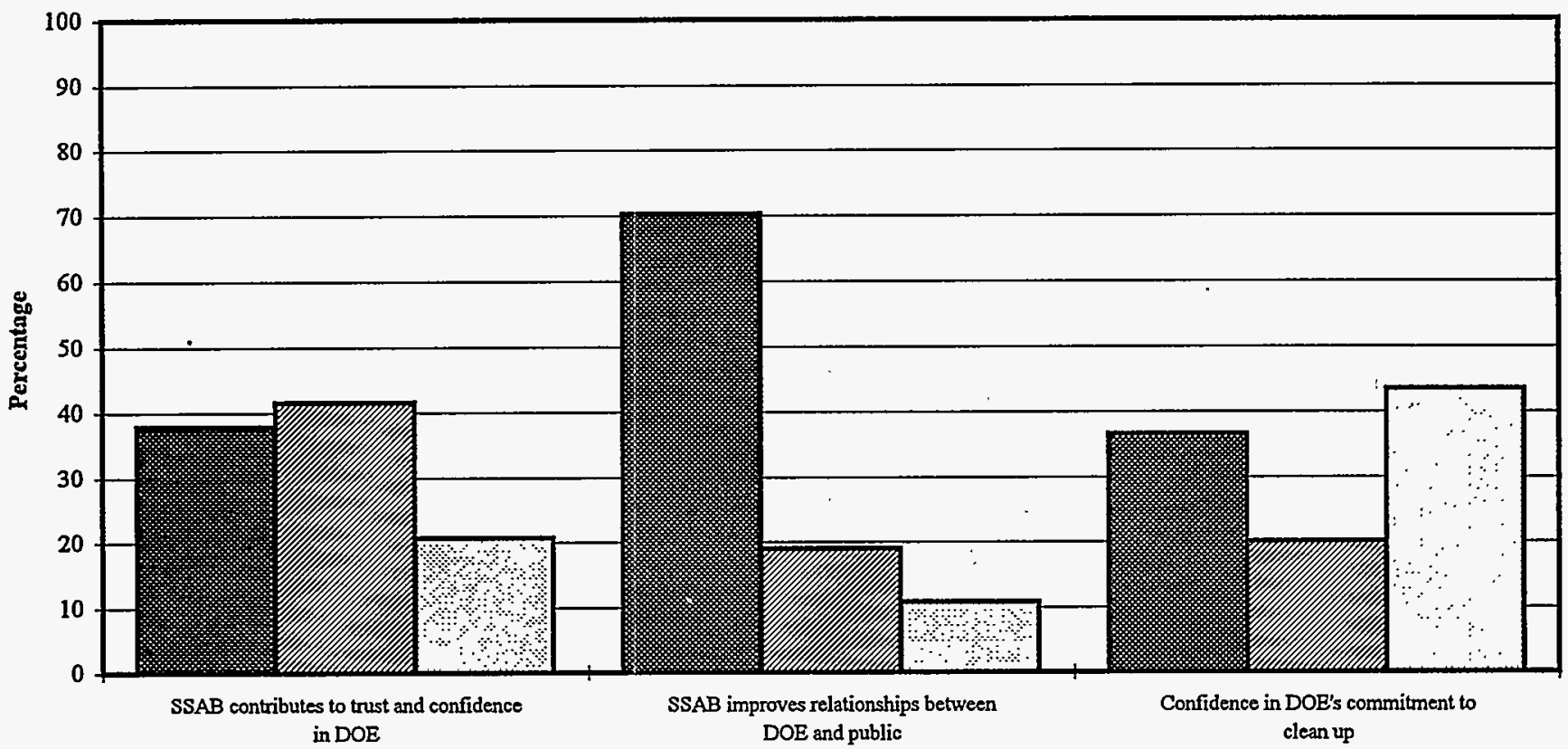

Agree (\%) Deither Agree Nor Disagree/Don't Know (\%) DDisagree (\%)

Figure $6 \mathrm{~b}$. Long Survey: Entire Sample

Goal 6. Contribute to trust and confidence in DOE

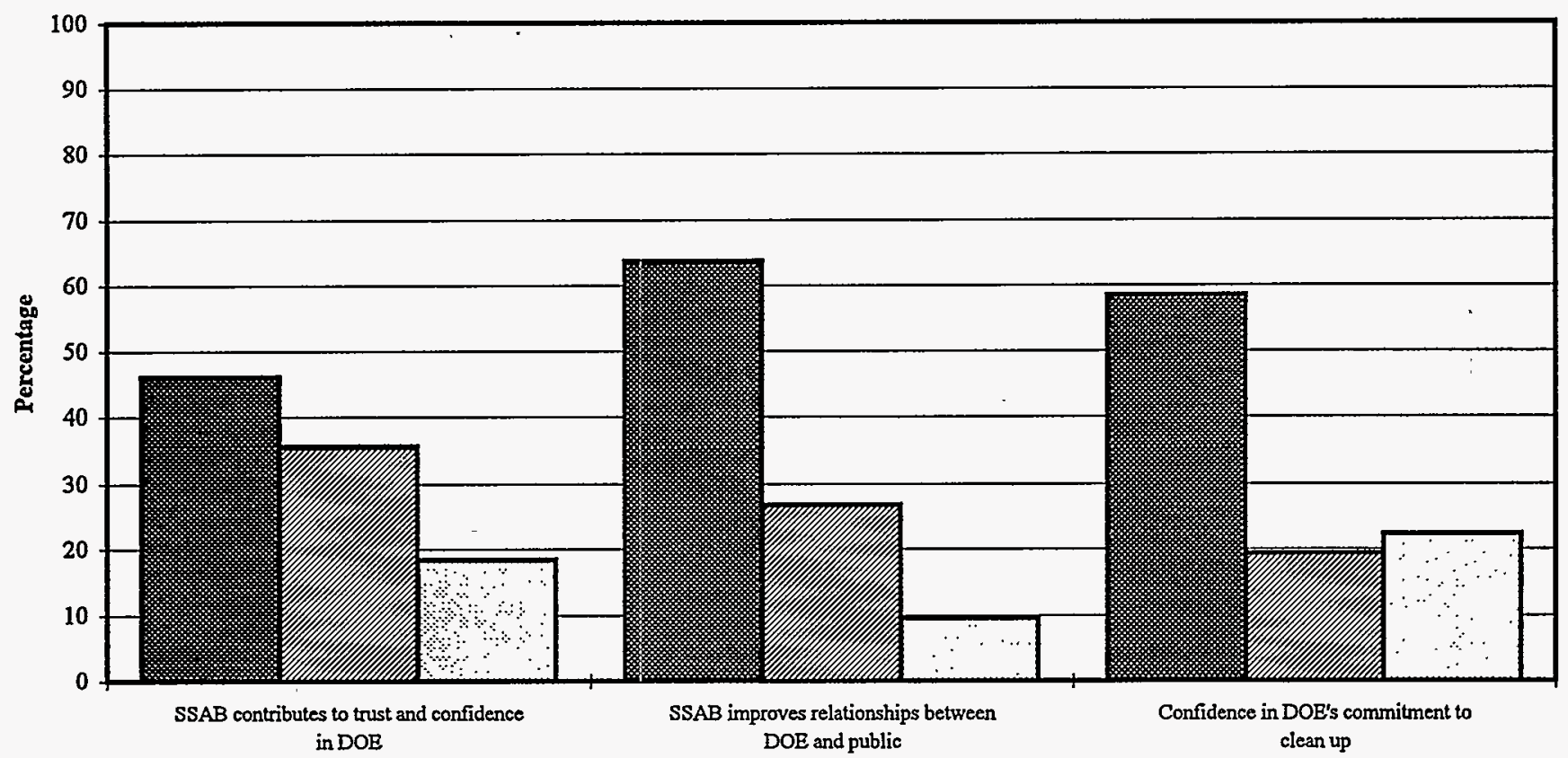

Agree (\%) Deither Agree Nor Disagree/Don't Know (\%) DDisagree (\%)

SSAB Supplementary Appendix

August 1996 
Figure 7a. Hanford

Public awareness

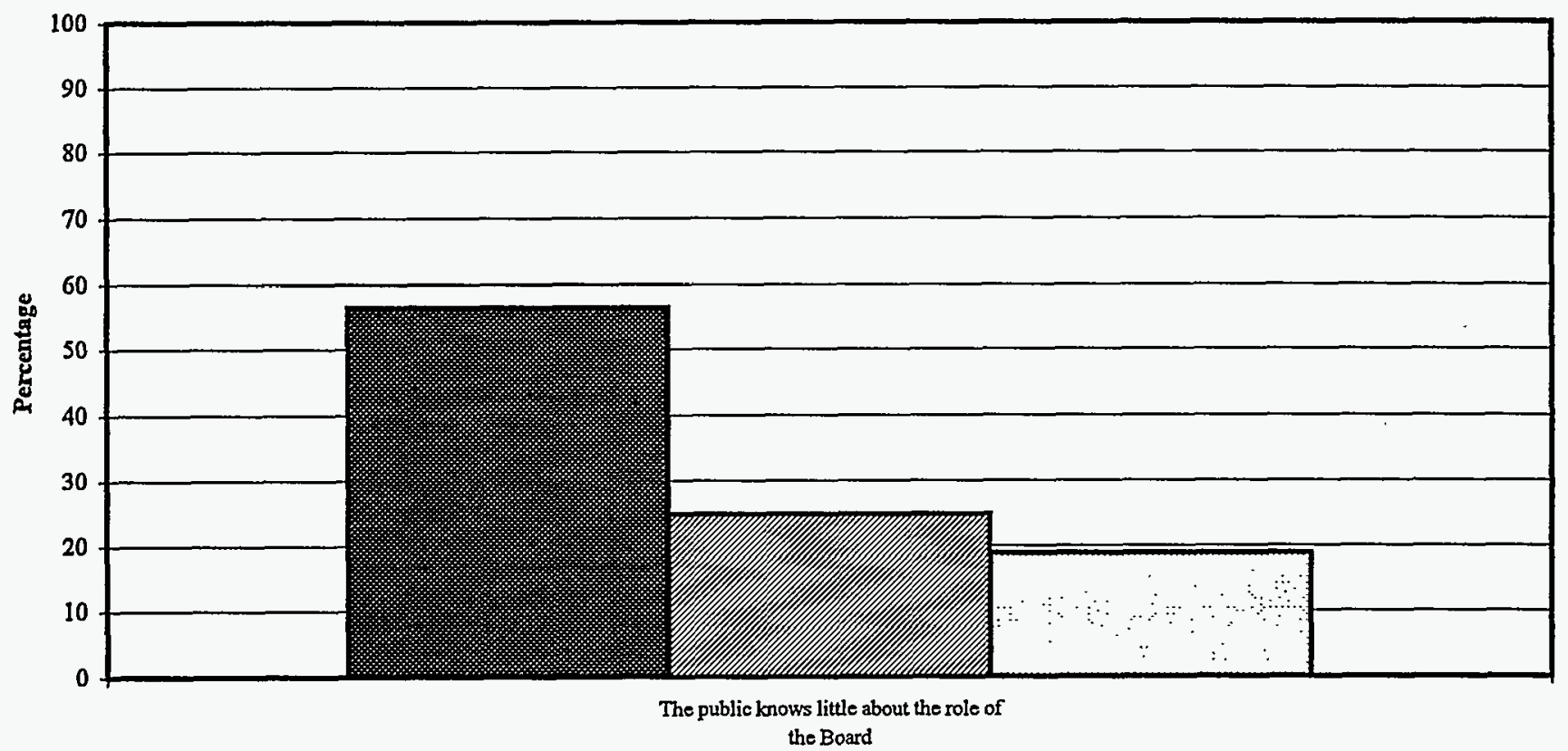

Agree (\%) Weither Agree Nor Disagree/Don't Know (\%) DDisagree (\%)

Figure 7b. Long Survey: Entire Sample

Public awareness

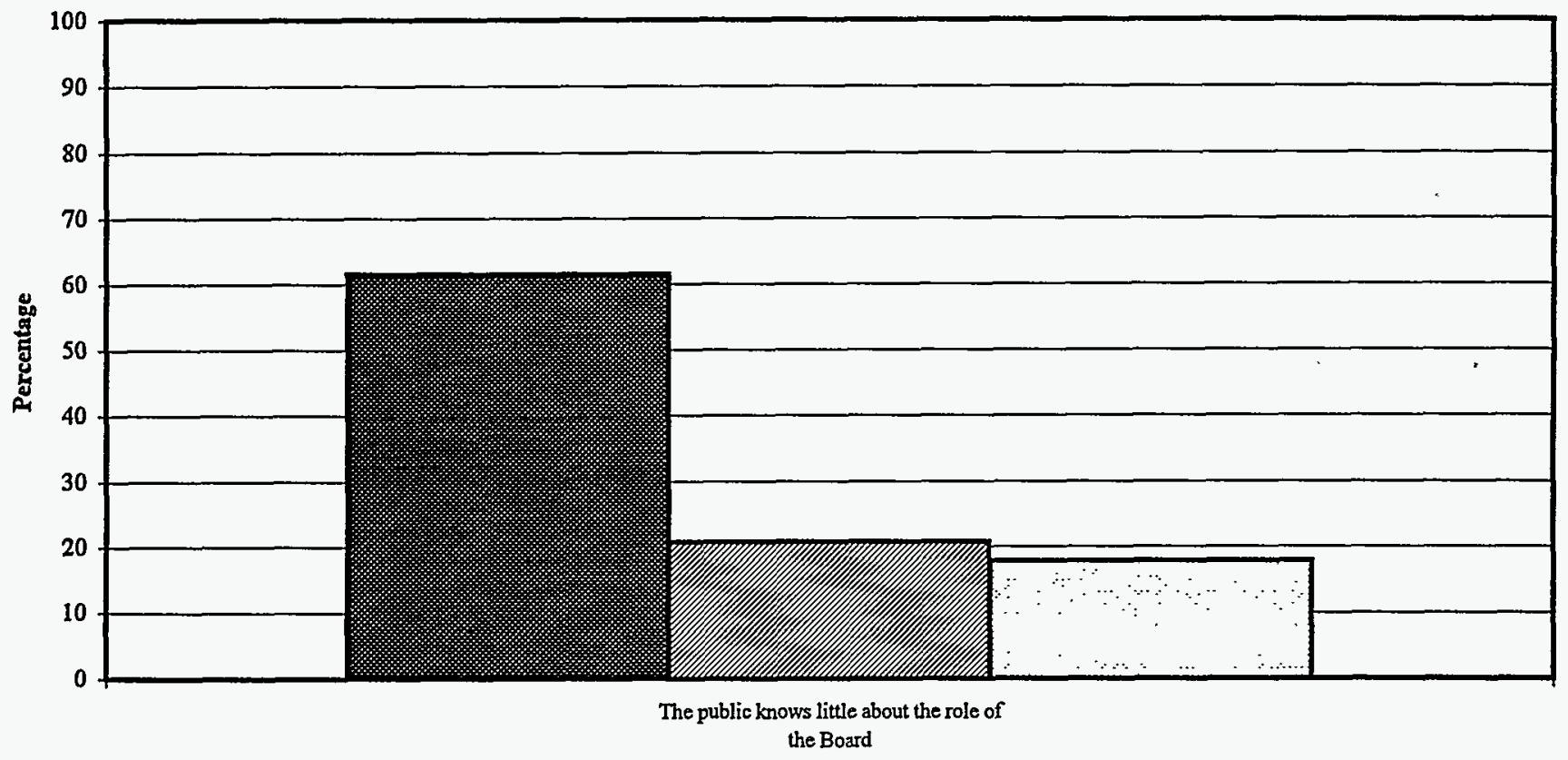

Agree (\%) Weither Agree Nor Disagree/Don't Know (\%) DDisagree (\%)

SSAB Supplementary Appendix

August 1996 
Figure 8a. Hanford

DOE-HQ involvement with the SSAB Initiative

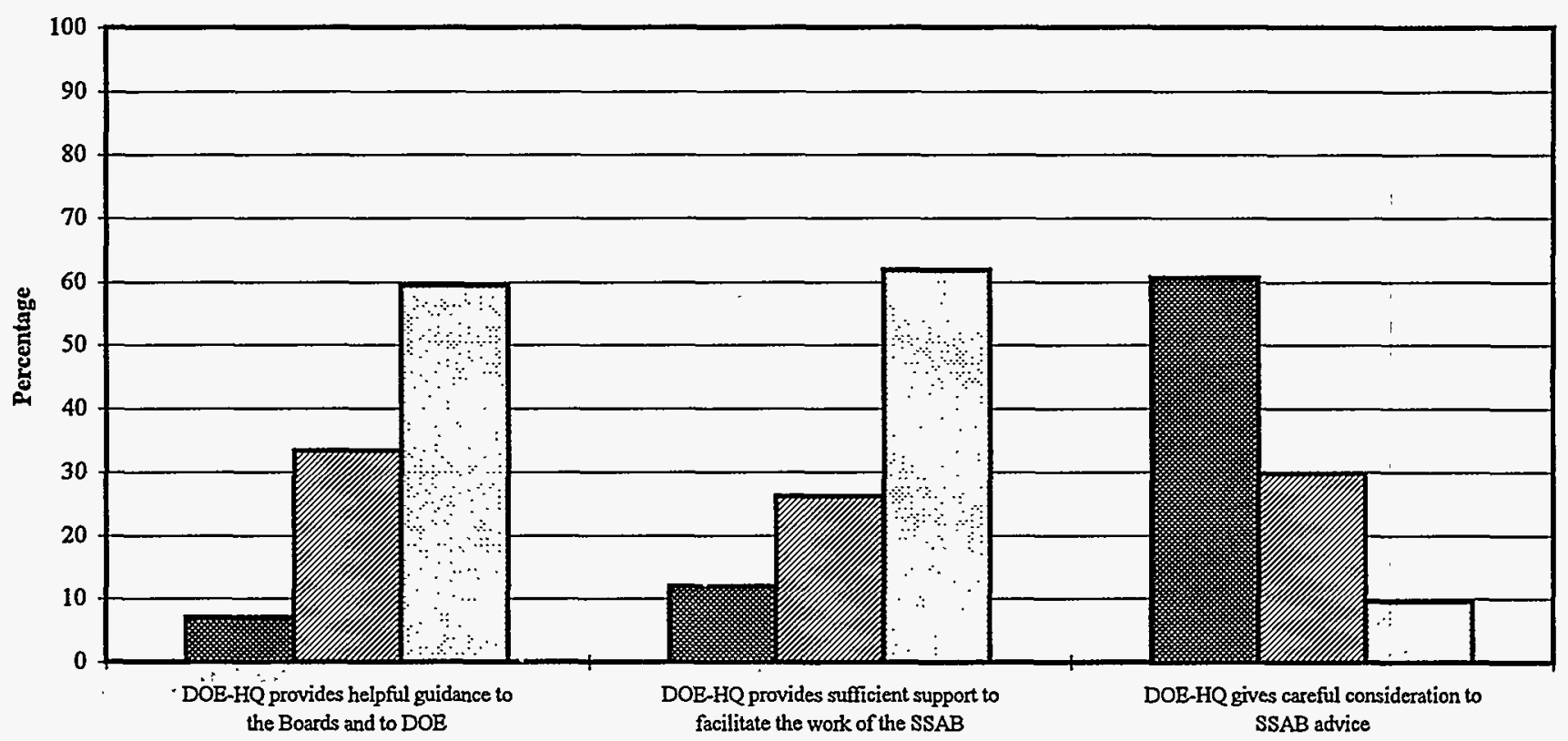

Agree (\%) Neither Agree Nor Disagree/Don't Know (\%) 口Disagree (\%)

Figure 8b. Long Survey: Entire Sample

DOE-HQ involvement with the SSAB Initiative

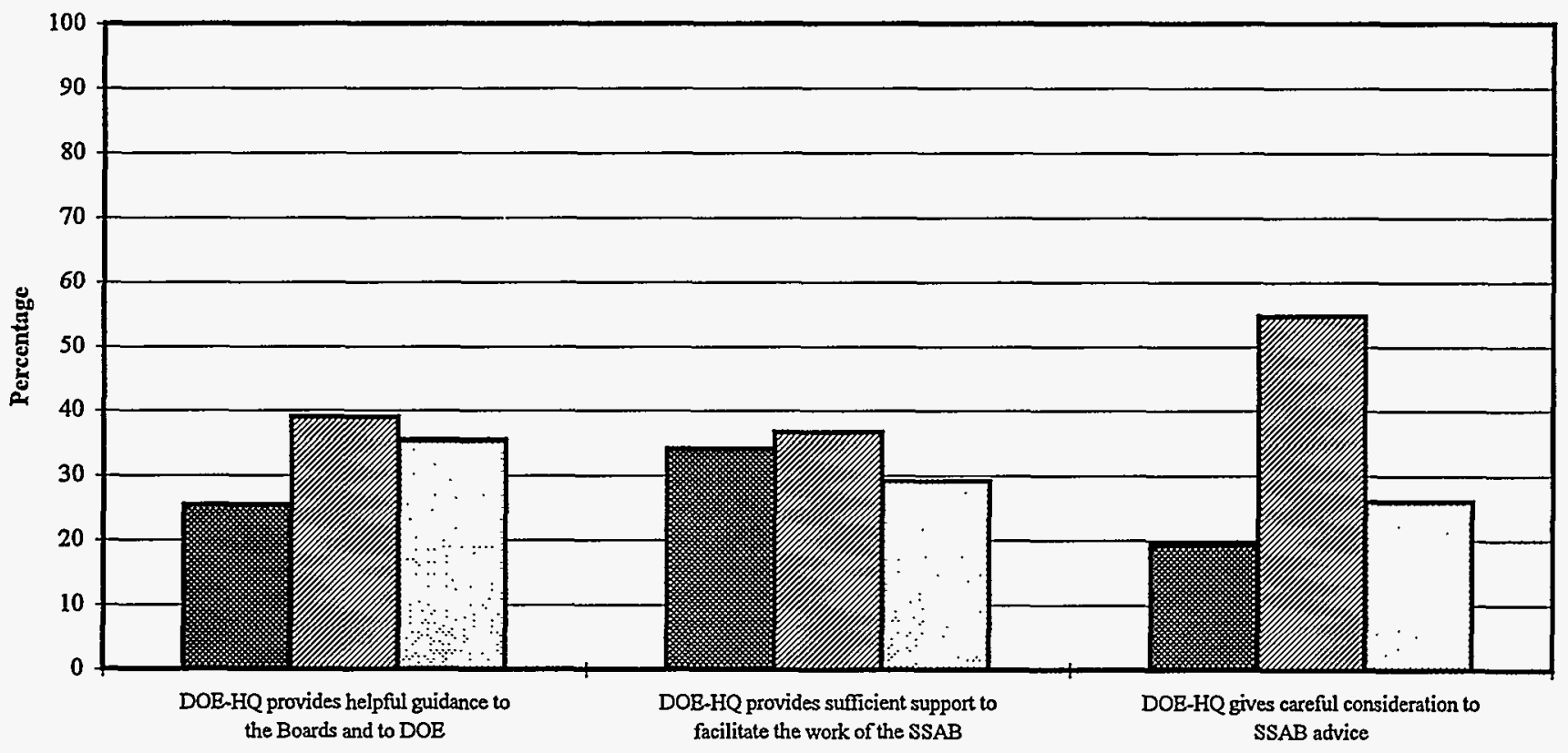

Agree (\%) E Neither Agree Nor Disagree/Don't Know (\%) DDisagree (\%)

SSAB Supplementary Appendix

August 1996 
Figure 9a. Hanford

Personal experience with the SSAB Initiative

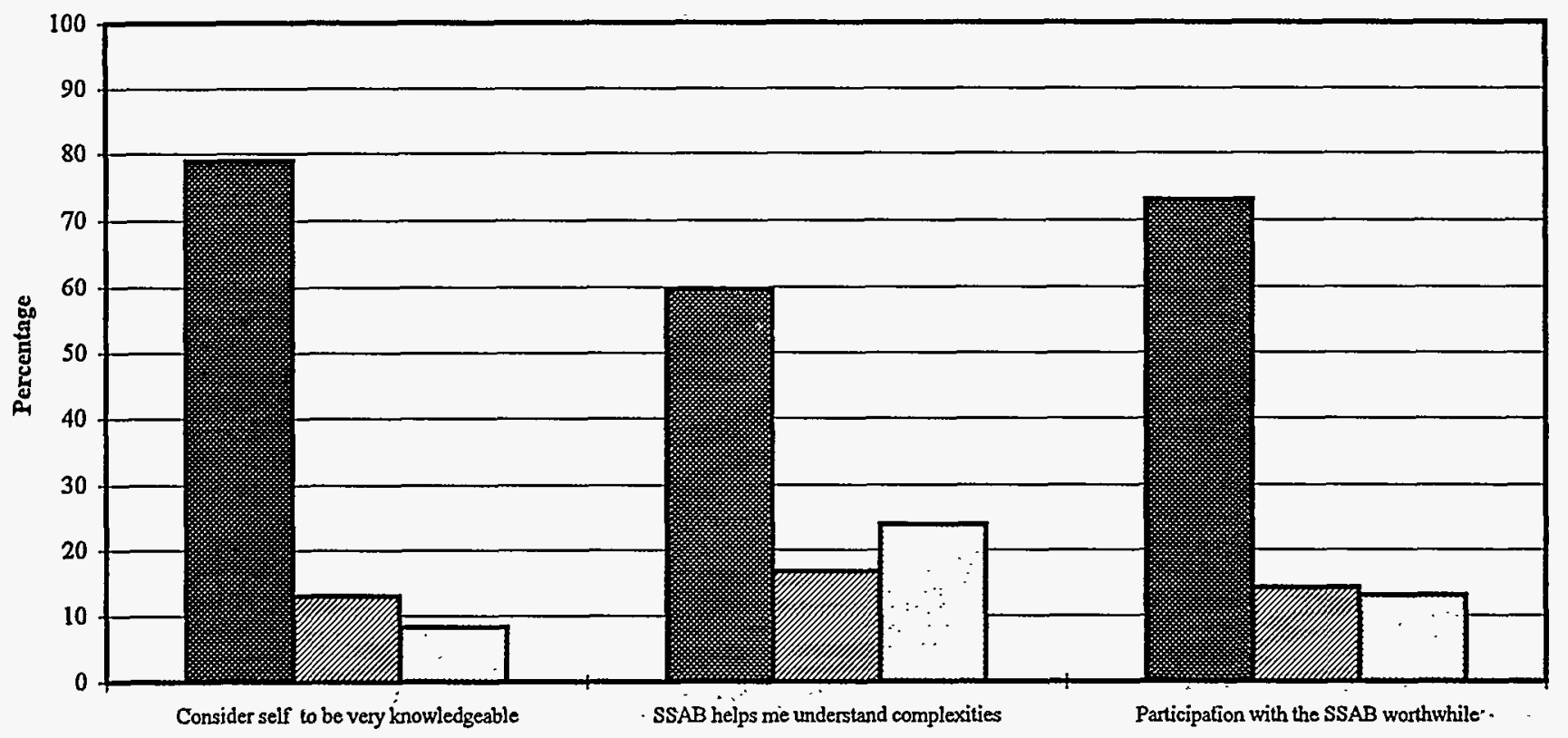

EAgree (\%) ENeither Agree Nor Disagree/Don't Know (\%) DDisagree (\%)

Figure 9b. Long Survey: Entire Sample

Personal experience with the SSAB Initiative

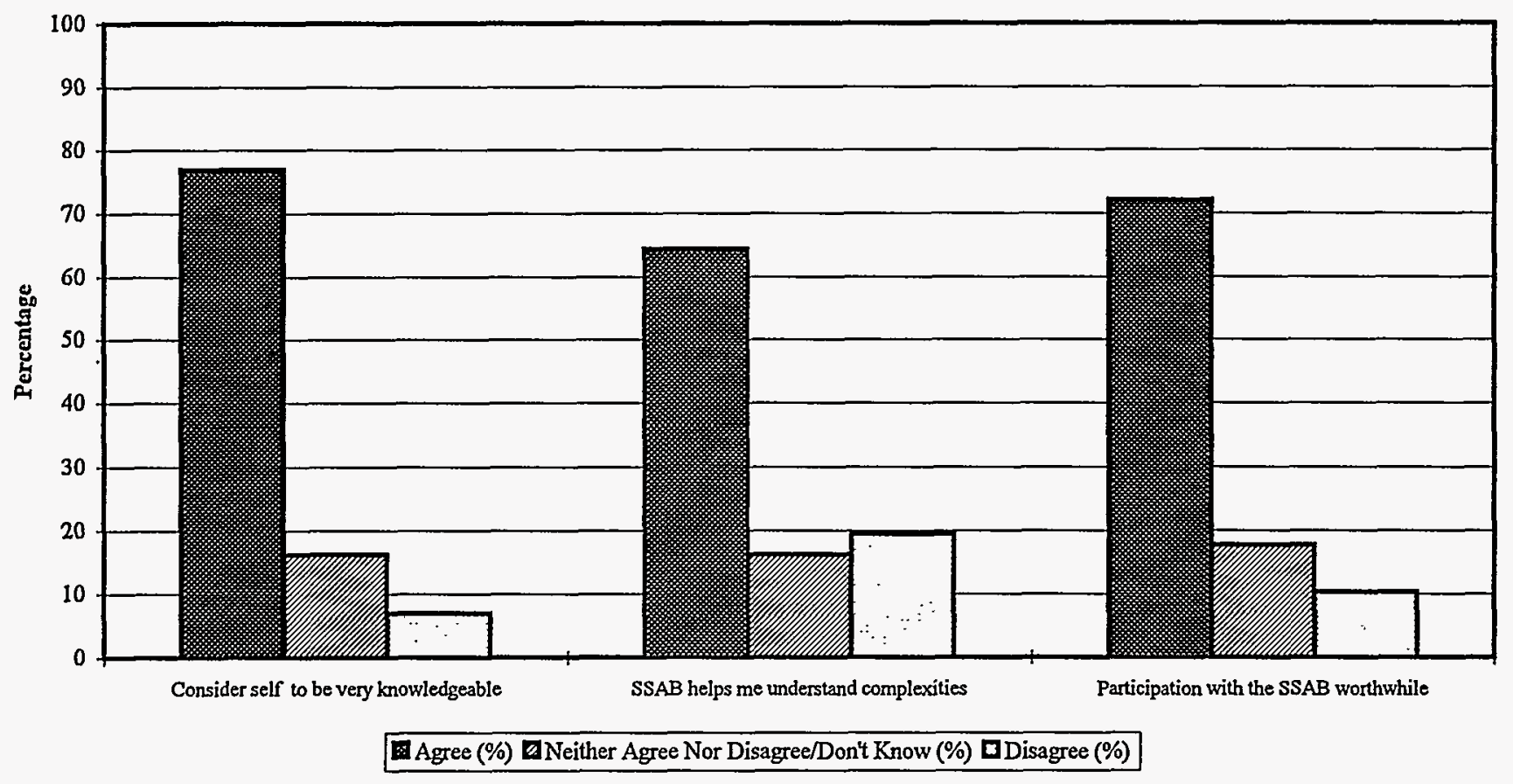

SSAB Supplementary Appendix 
Figure 10a. Hanford

Group/Team work skills

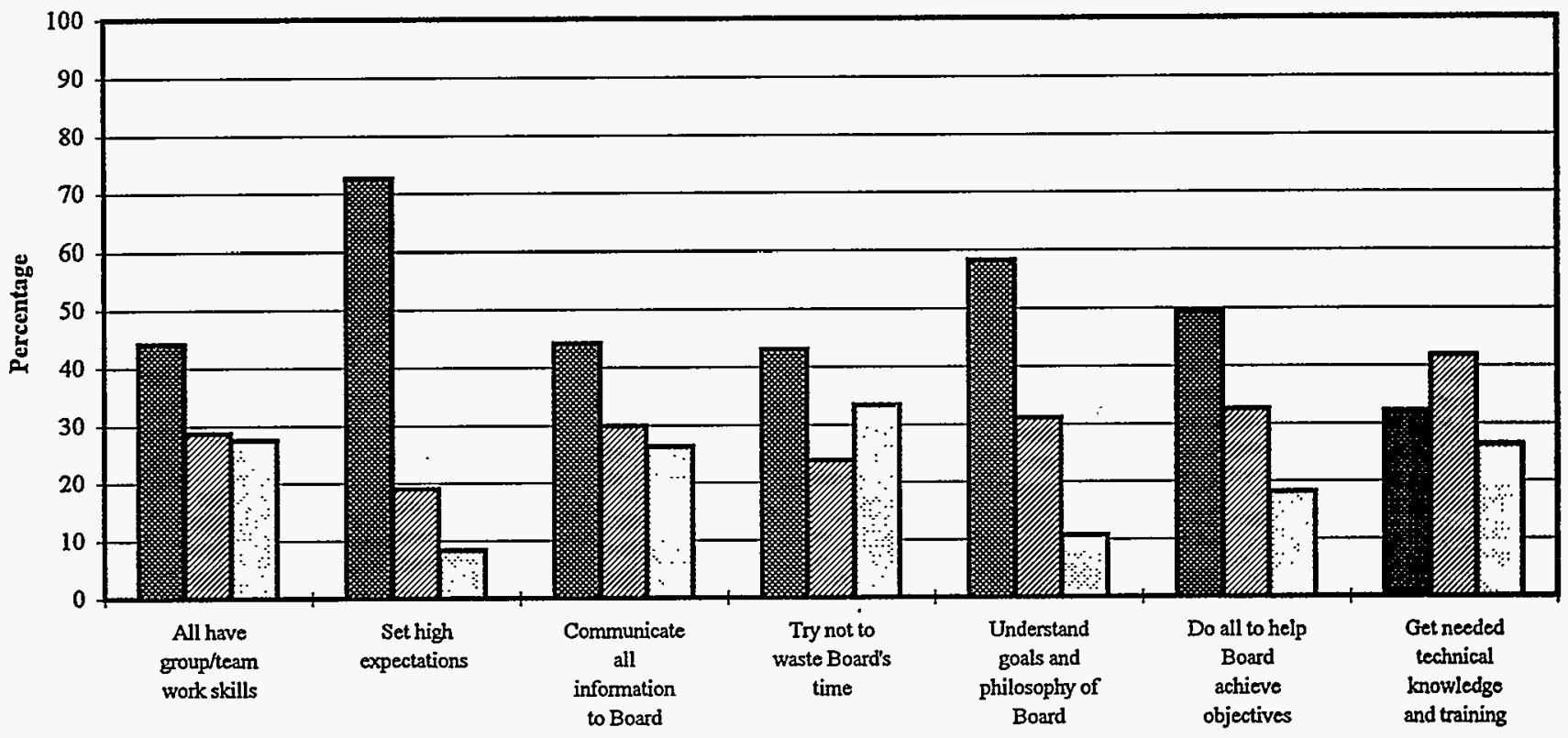

Agree (\%) Neither Agree Nor Disagree/Don't Know (\%) DDisagree (\%)

Figure 10b. Long Survey: Entire Sample

Group/Team work skills

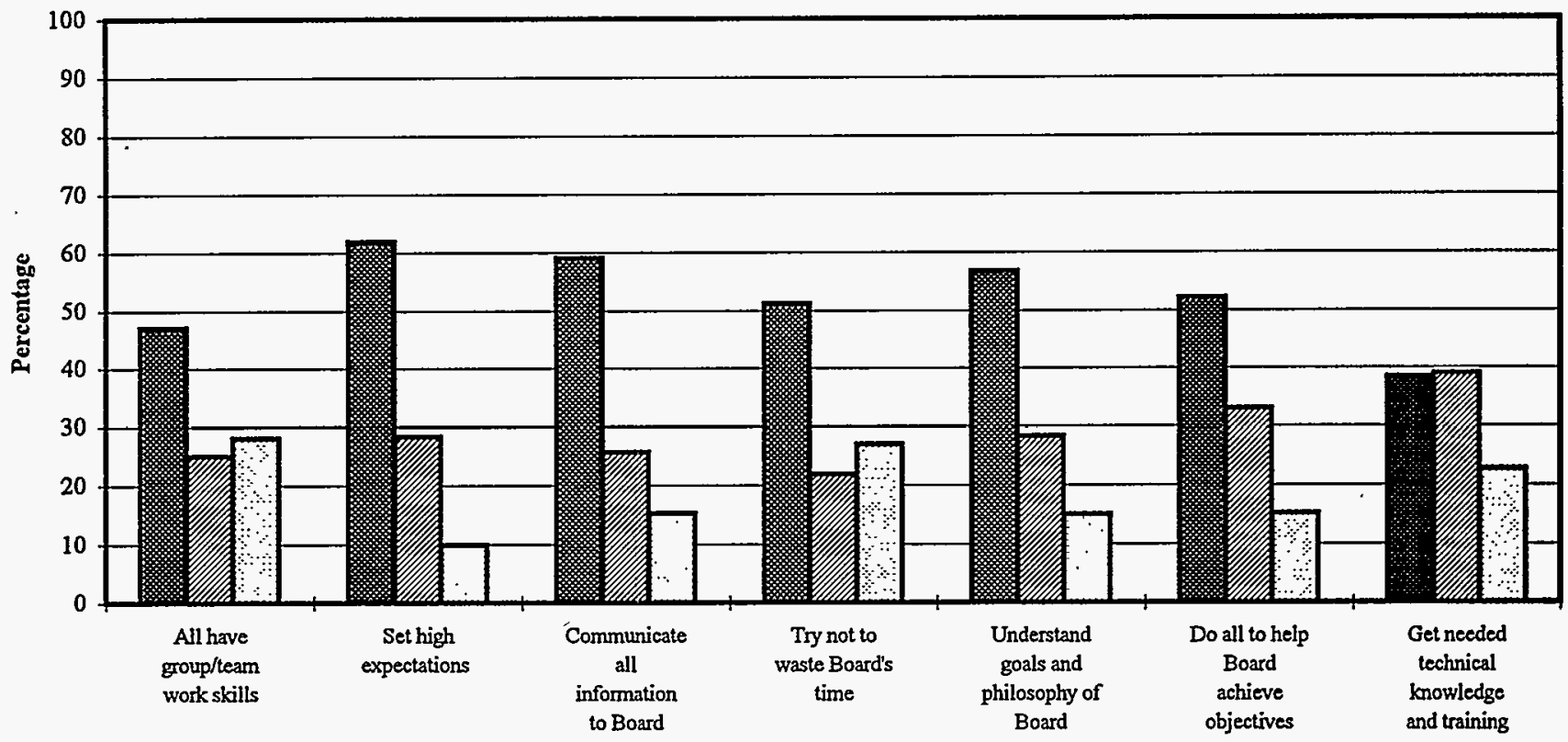

Agree (\%) Weither Agree Nor Disagree/Don't Know (\%) DDisagree (\%)

SSAB Supplementary Appendix

August 1996 
Figure 11a. Hanford

Working relations

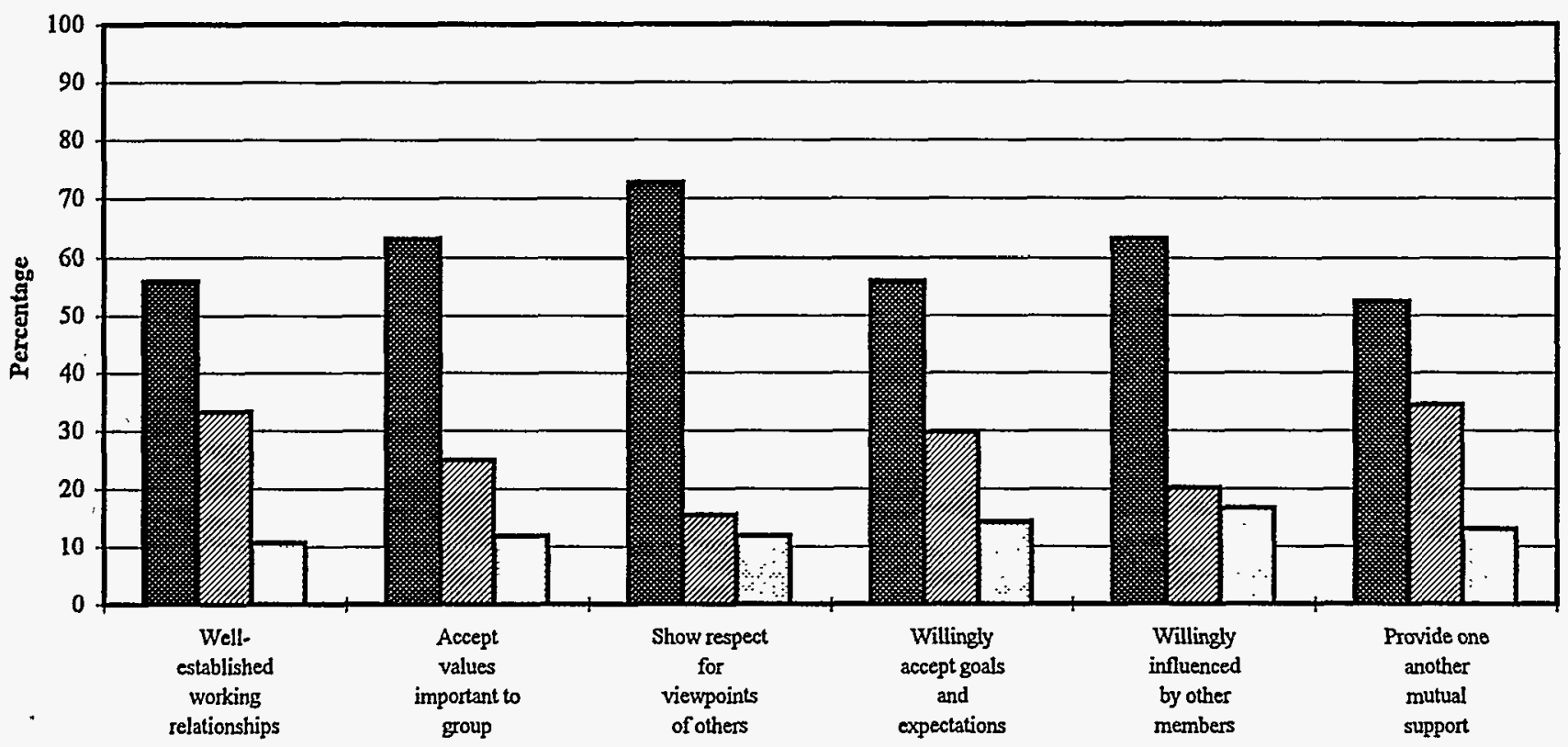

Agree (\%) ENeither Agree Nor Disagree/Don't Know (\%) DDisagree (\%)

Figure 11b. Long Survey: Entire Sample

Working relations

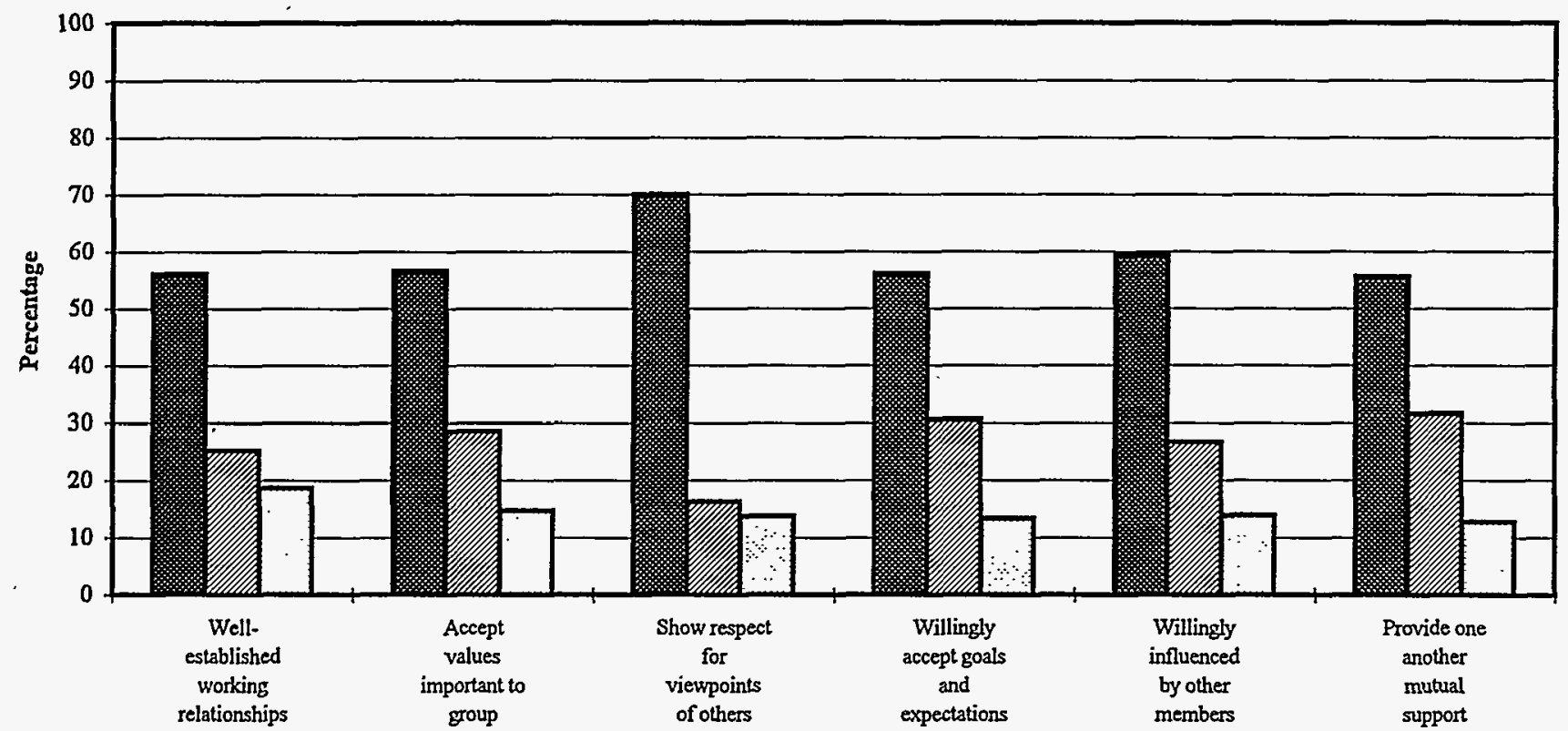

Agree (\%) Deither Agree Nor Disagree/Don't Know (\%) DDisagree (\%)

SSAB Supplementary Appendix

August 1996 
Figure 12a. Hanford

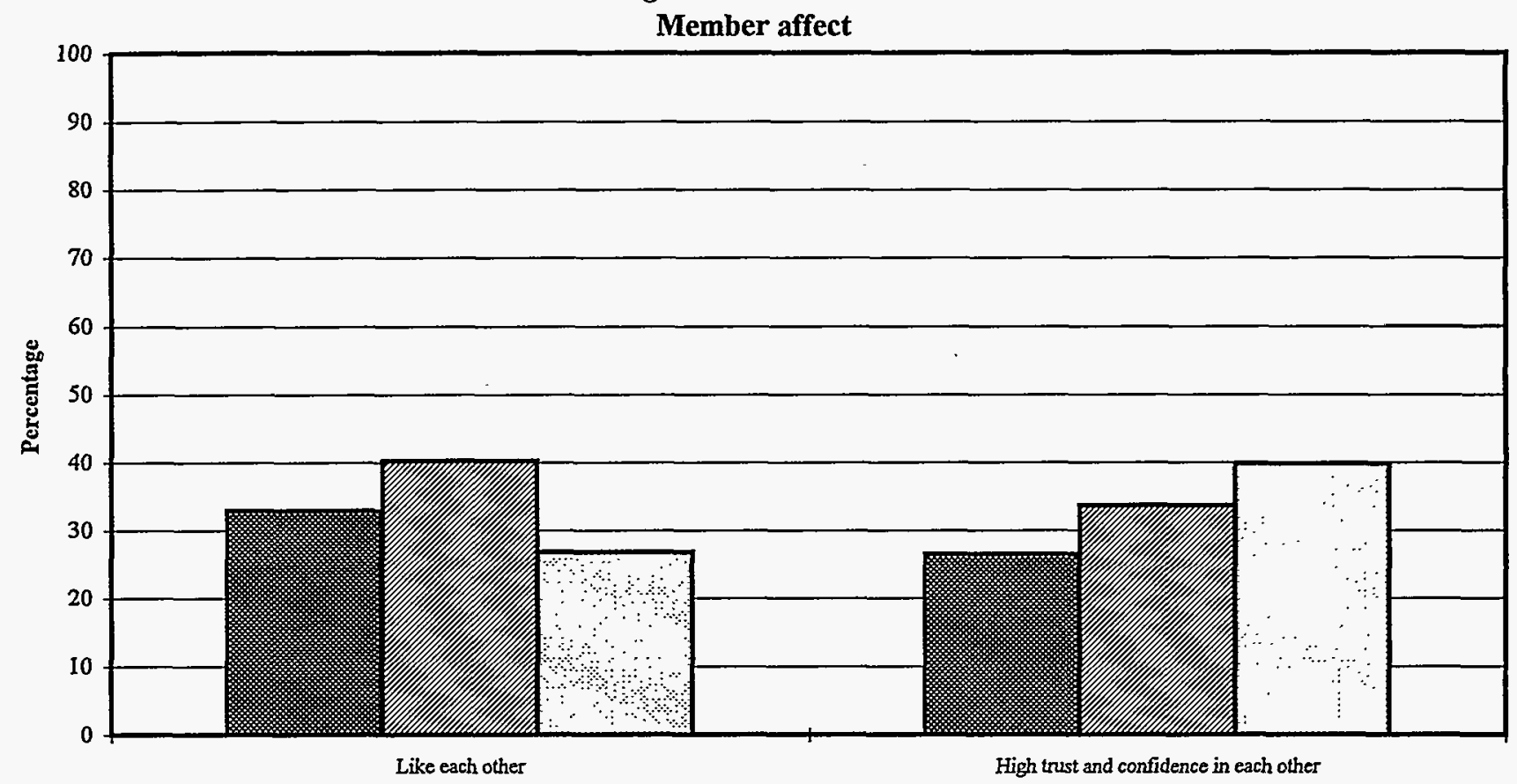

Agree (\%) QNeither Agree Nor Disagree/Don't Know (\%) DDisagree (\%)

Figure 12b. Long Survey: Entire Sample

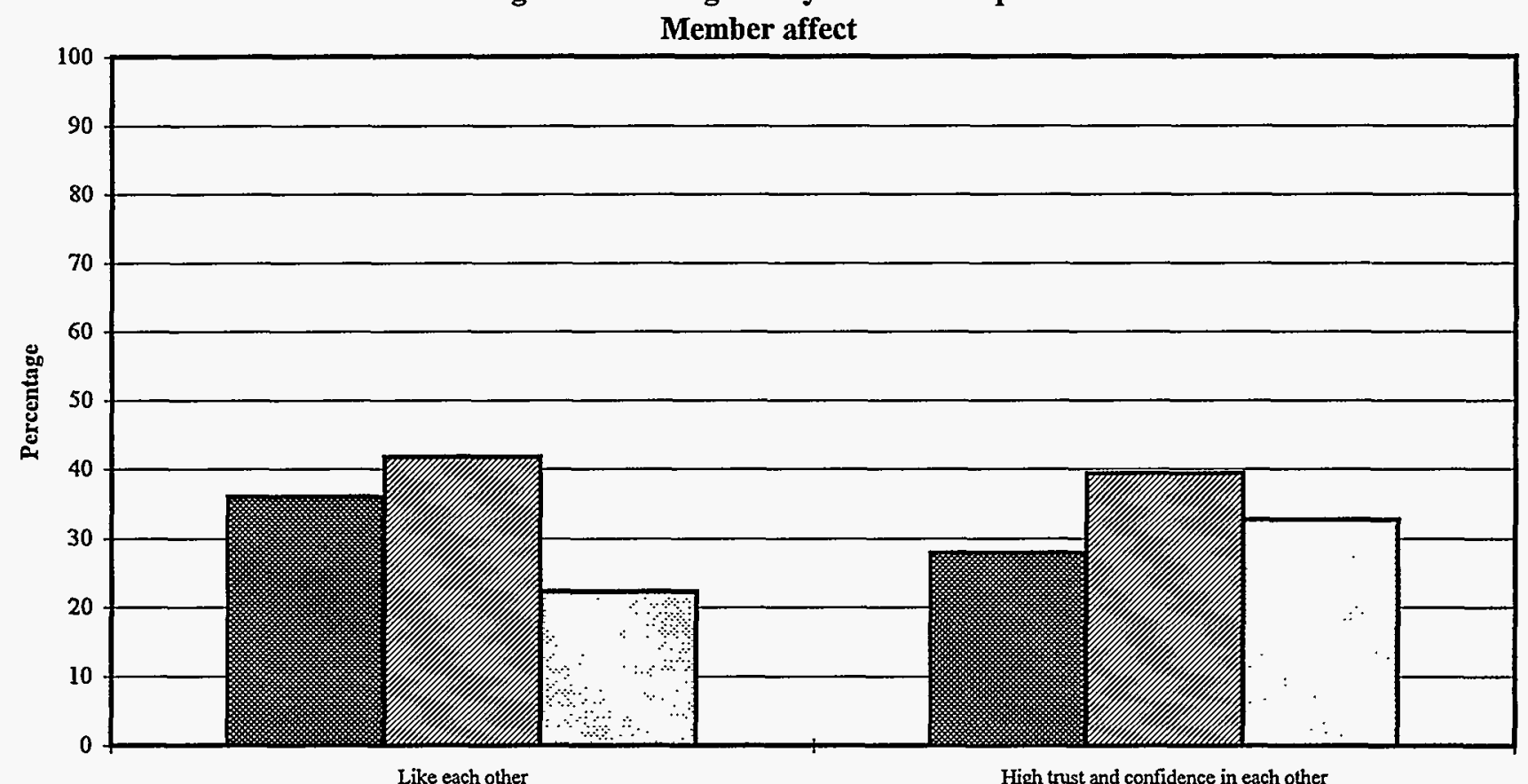

F Agree (\%) Neither Agree Nor Disagree/Dontt Know (\%) DDisagree (\%)

SSAB Supplementary Appendix

August 1996 
Figure 13a. Hanford

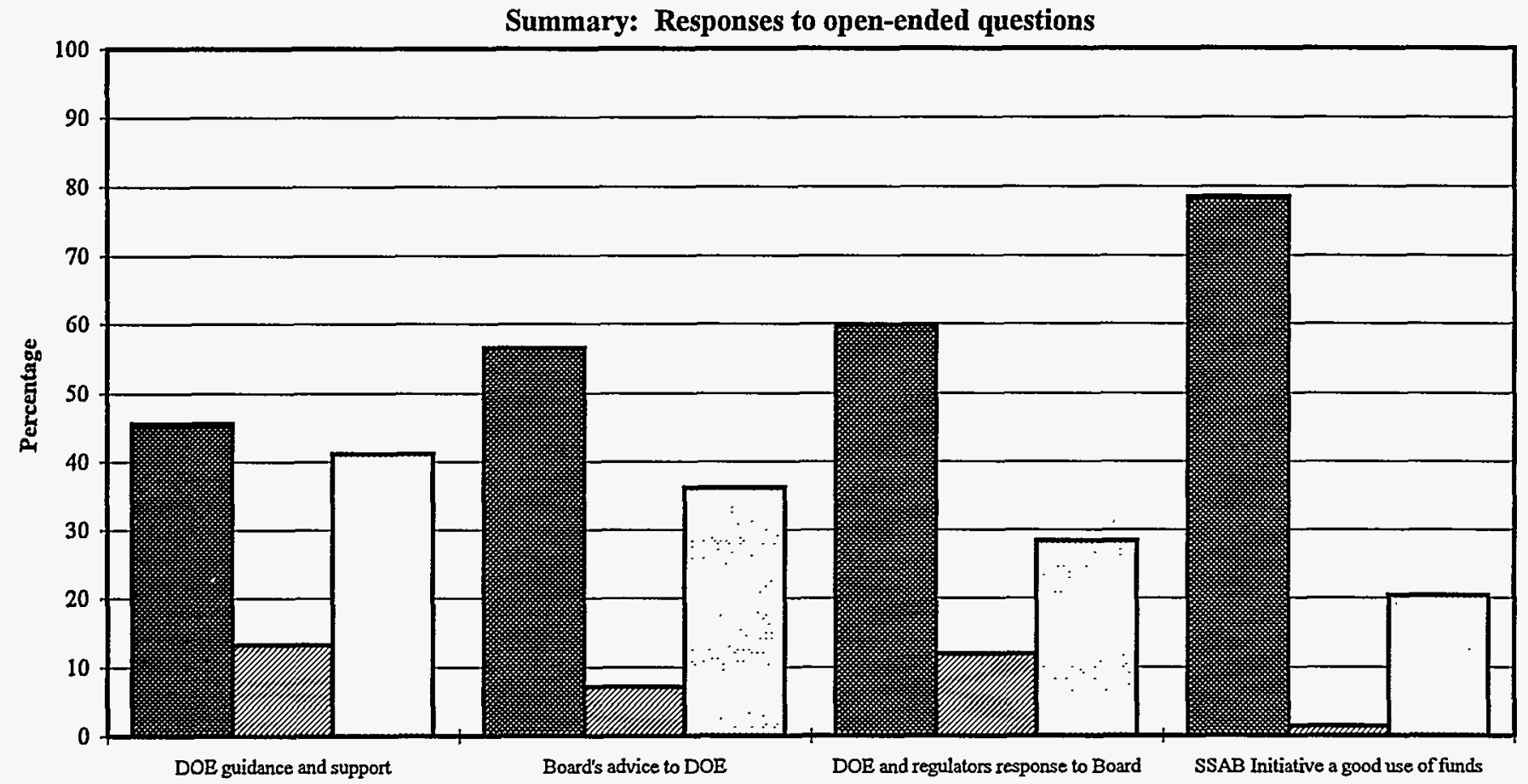

Yes, Good (\%) שaNeutral, Conditional, No Opinion (\%) $\mathbf{E}$ No, Not Good (\%)

Figure 13b. Long Survey: Entire Sample

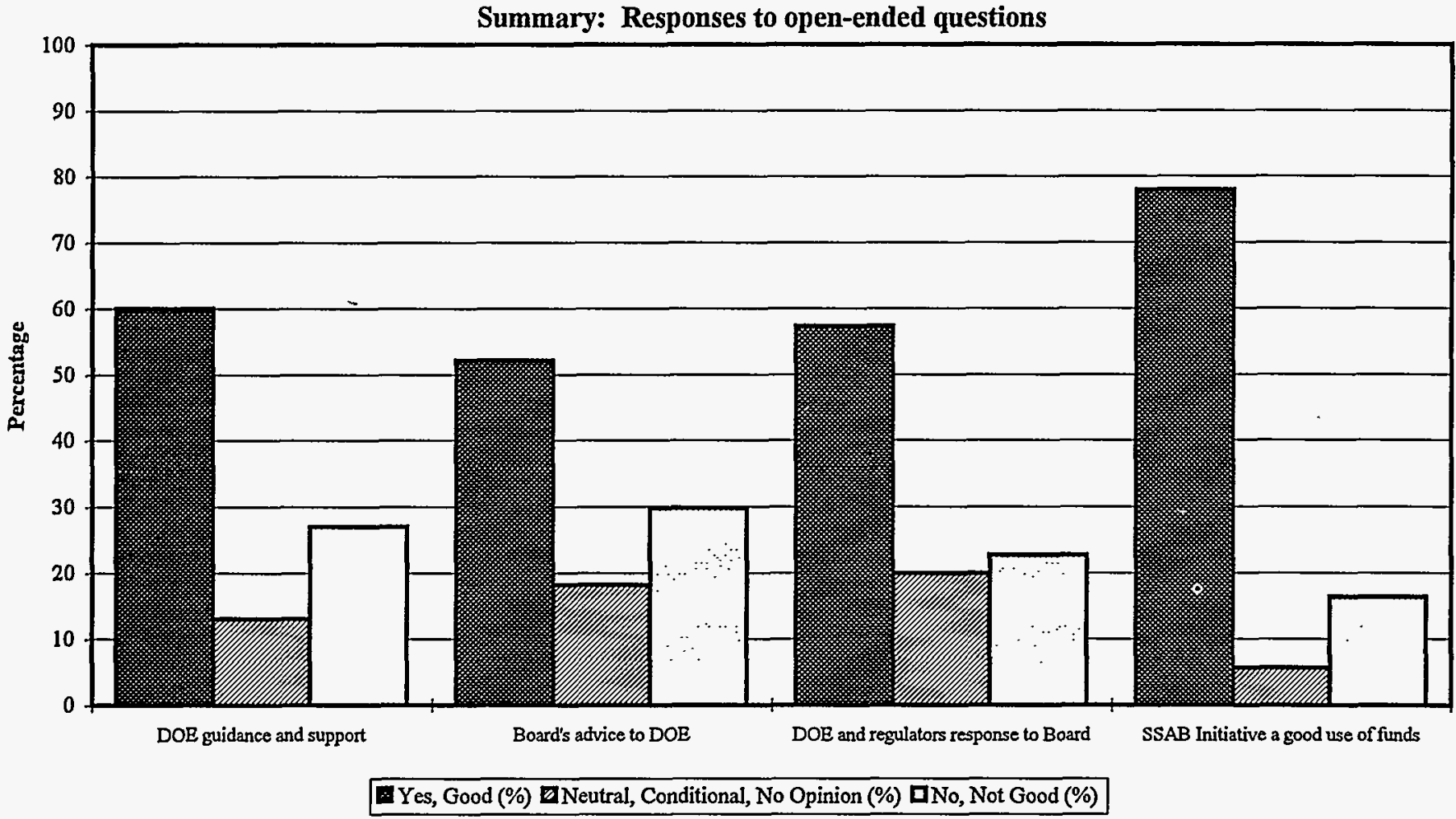

SSAB Supplementary Appendix

August 1996 
Figure 14a. Hanford

Summary: Combined responses for SSAB Initiative goals (Means)

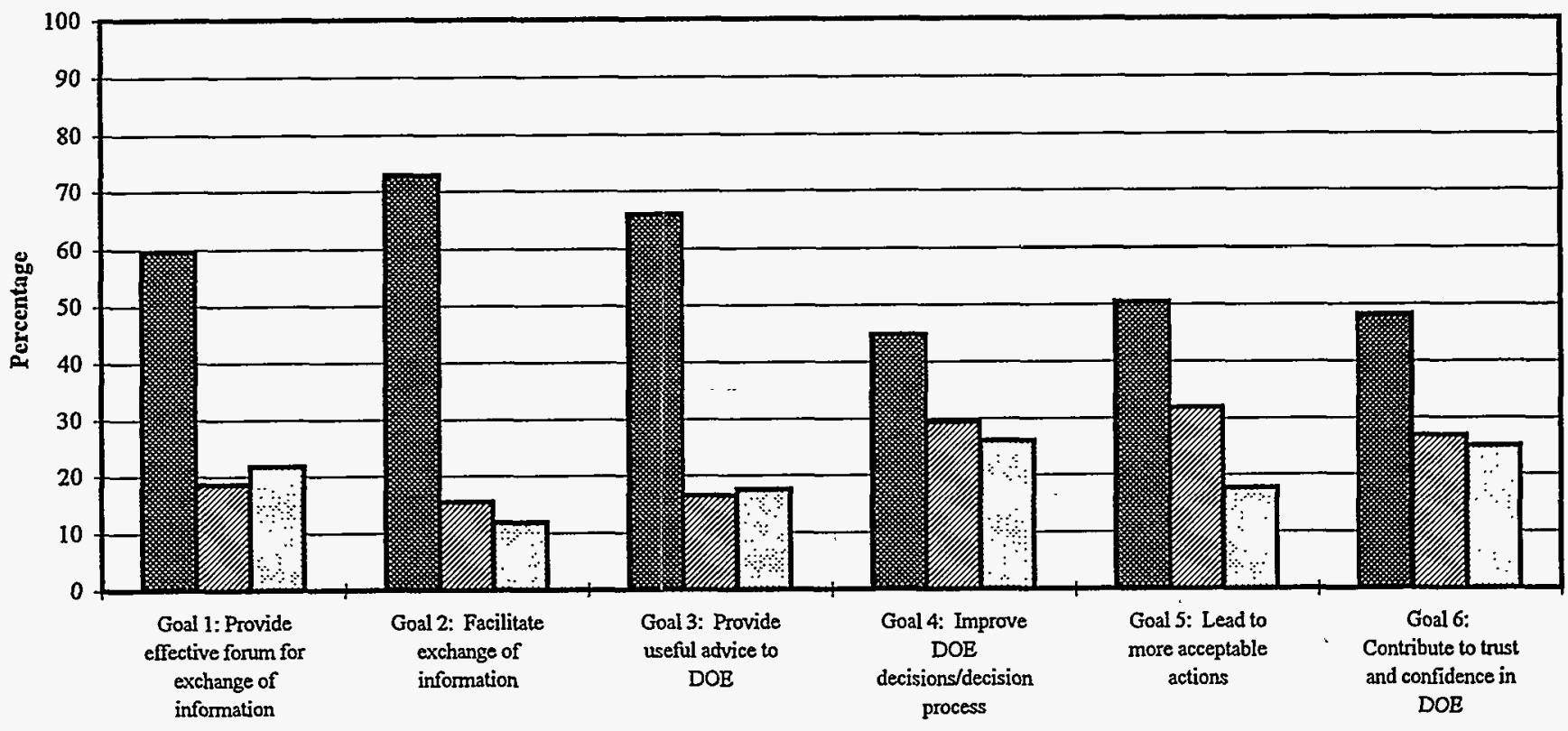

Agree (\%) ENeither Agree Nor Disagree/Don't Know (\%) DDisagree (\%)

Figure 14b. Long Survey: Entire Sample

Summary: Combined responses for SSAB Initiative goals (Means)

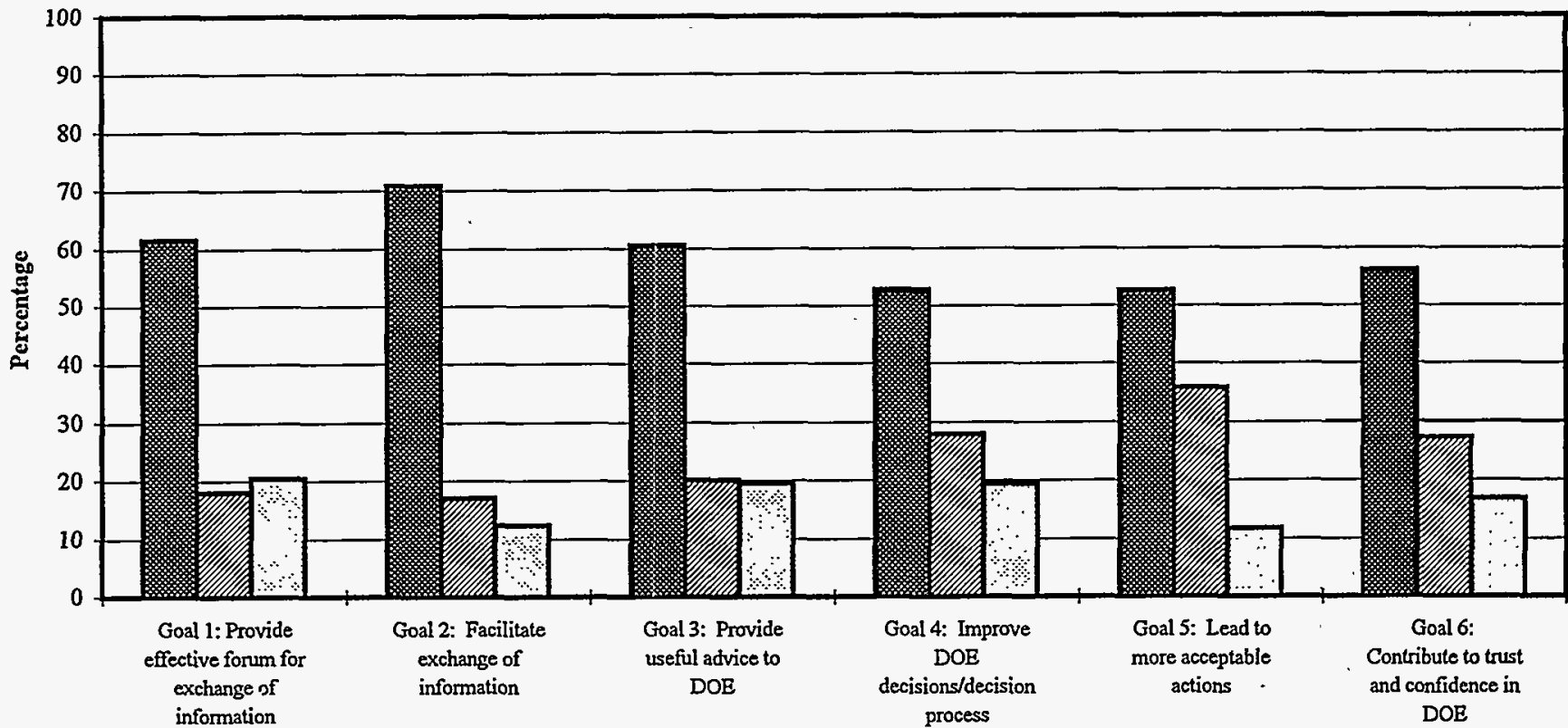

I Agree (\%) WNeither Agree Nor Disagree/Don't Know (\%) DDisagree (\%)

SSAB Supplementary Appendix

August 1996 
Figure 15a. Hanford

Summary: Combined responses on DOE-HQ involvement,

Personal experience, Group/Team work skills,

Working relations, Member affect (Means)

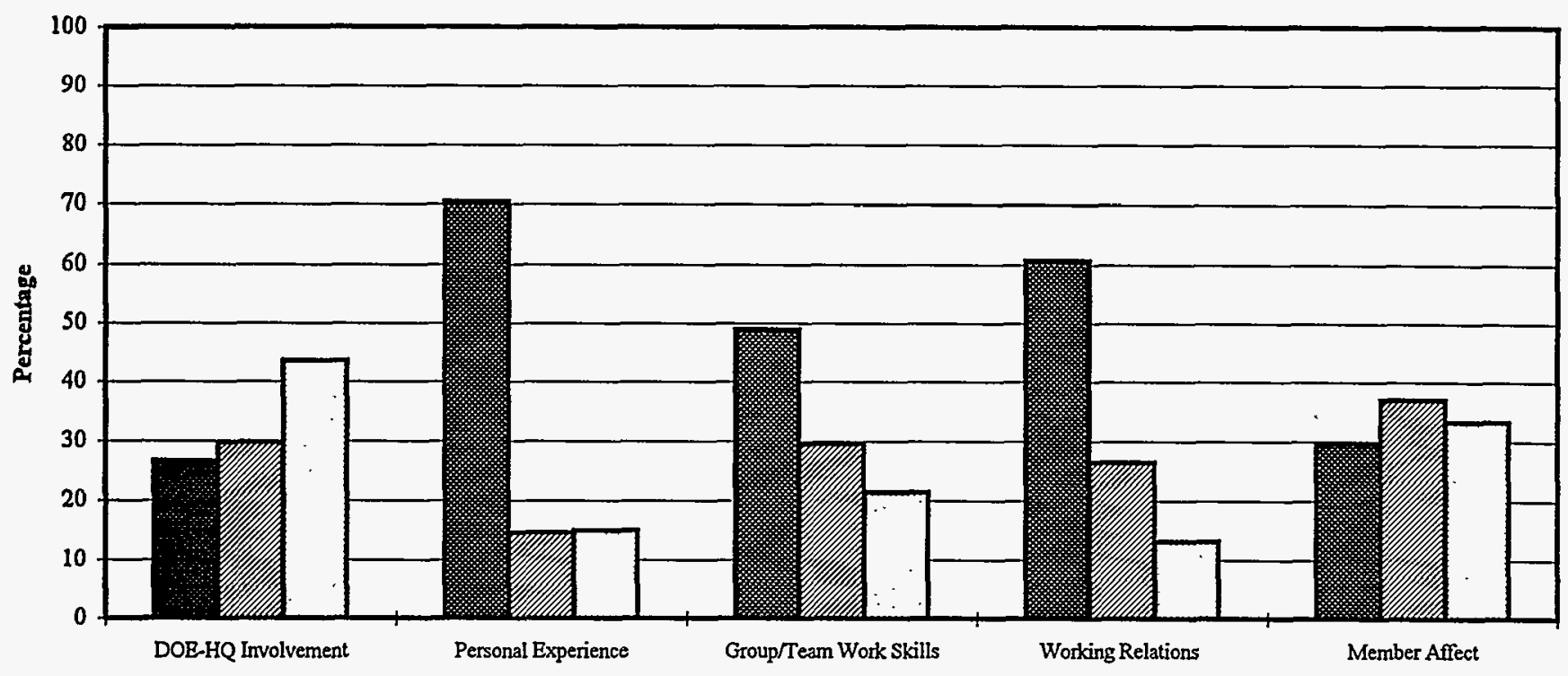

Agree (\%) Deither Agree Nor Disagree/Don't Know (\%) DDisagree (\%)

Figure 15b. Long Survey: Entire Sample

Summary: Combined responses on DOE-HQ involvement, Personal experience, Group/Team work skills,

Working relations, Member affect (Means)

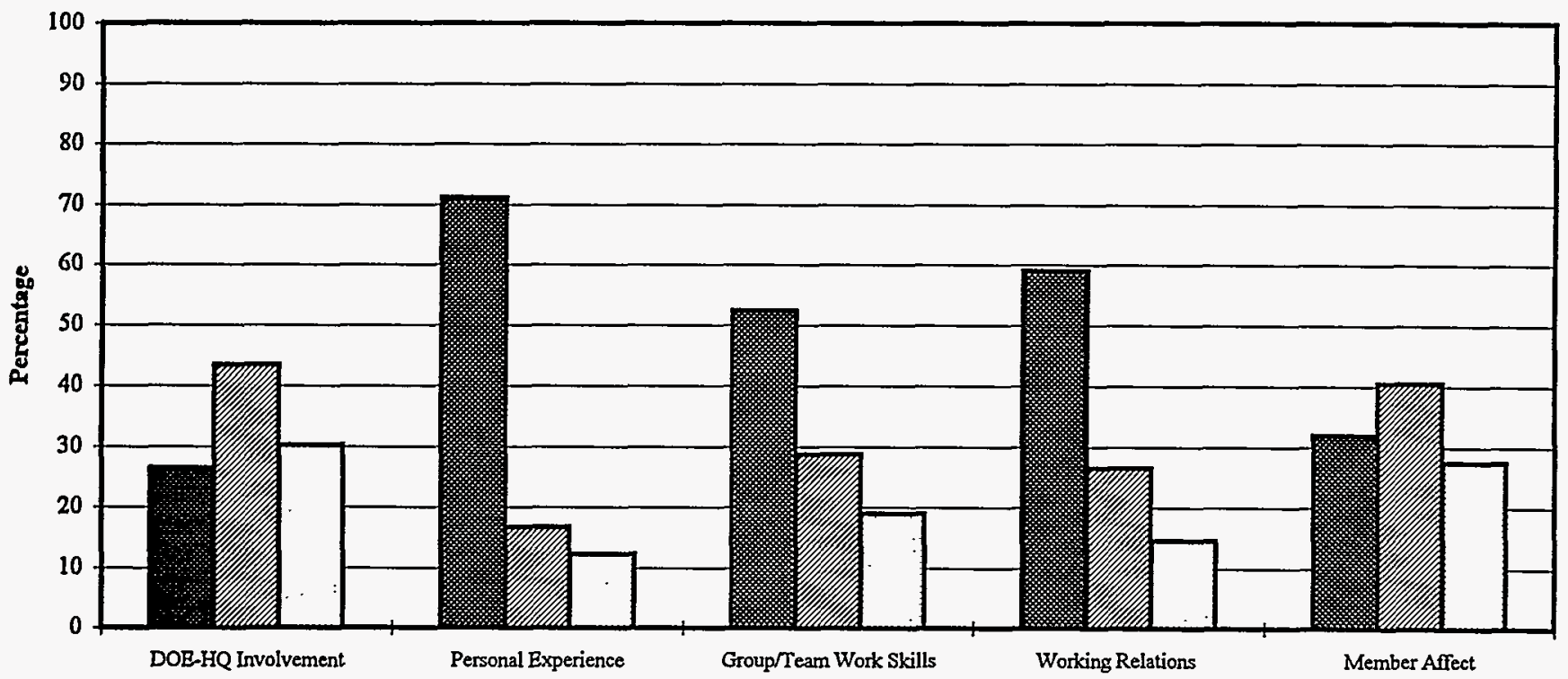

Agree (\%) 8 Neither Agree Nor Disagree/Don't Know (\%) DDisagree (\%)

SSAB Supplementary Appendix

August 1996 
Table 1.

Goal 1. Establish processes and procedures to provide an effective forum for exchange of information and viewpoints regarding DOE site issues

\begin{tabular}{|c|c|c|c|c|c|c|c|c|}
\hline \multirow[t]{2}{*}{ Hanford } & \multicolumn{2}{|c|}{ Agree } & \multicolumn{2}{|c|}{$\begin{array}{c}\text { Neither/ } \\
\text { Don't Know }\end{array}$} & \multicolumn{2}{|c|}{ Disagree } & \multicolumn{2}{|c|}{ Total } \\
\hline & $N$ & $\%$ & $\mathrm{~N}$ & $\%$ & $\mathrm{~N}$ & $\%$ & $\mathrm{~N}$ & $\%$ \\
\hline $\begin{array}{l}\text { The Board has established processes and } \\
\text { procedures for the effective exchange of } \\
\text { information }\end{array}$ & 70 & $83.3 \%$ & 9 & $10.7 \%$ & 5 & $6.0 \%$ & 84 & $100 \%$ \\
\hline $\begin{array}{l}\text { The Board has agreed-upon procedures to } \\
\text { operate the board }\end{array}$ & 74 & $89.2 \%$ & 7 & $8.4 \%$ & 2 & $2.4 \%$ & 83 & $100 \%$ \\
\hline $\begin{array}{l}\text { The Board has adequate support to allow } \\
\text { it to focus on substantive issues }(\mathrm{R}) *\end{array}$ & 39 & $47.0 \%$ & 11 & $13.3 \%$ & 33 & $39.8 \%$ & 83 & $100 \%$ \\
\hline $\begin{array}{l}\text { The Board provides sufficient time for } \\
\text { discussion of issues }\end{array}$ & 48 & $57.8 \%$ & 17 & $20.5 \%$ & 18 & $21.7 \%$ & 83 & $100 \%$ \\
\hline The Board has effective leadership & 67 & $80.7 \%$ & 10 & $12.0 \%$ & 6 & $7.2 \%$ & 83 & $100 \%$ \\
\hline $\begin{array}{l}\text { DOE requests Board advice far enough } \\
\text { ahead of decision deadlines }(R) *\end{array}$ & 10 & $11.9 \%$ & 21 & $25.0 \%$ & 53 & $63.1 \%$ & 84 & $100 \%$ \\
\hline $\begin{array}{l}\text { DOE handles Board administrative issues } \\
\text { expeditiously (R) * }\end{array}$ & 27 & $32.5 \%$ & 35 & $42.2 \%$ & 21 & $25.3 \%$ & 83 & $100 \%$ \\
\hline $\begin{array}{l}\text { The SSAB staff provide satisfactory } \\
\text { support }\end{array}$ & 51 & $60.7 \%$ & 16 & $19.0 \%$ & 17 & $20.2 \%$ & 84 & $100 \%$ \\
\hline $\begin{array}{l}\text { The facilitator for the Board has helped } \\
\text { the Board function effectively }\end{array}$ & 62 & $72.9 \%$ & 14 & $16.5 \%$ & 9 & $10.6 \%$ & 85 & $100 \%$ \\
\hline
\end{tabular}

* (R) denotes a question that was asked in the negative form. For ease of review, these questions and data have been reversed in this table and are stated in positive forms.

SSAB Supplementary Appendix

August 1996 
Table 2.

Goal 2. Facilitate interaction and exchange of information and viewpoints regarding DOE site issues

\begin{tabular}{|c|c|c|c|c|c|c|c|c|}
\hline \multirow[t]{2}{*}{ Hanford } & \multicolumn{2}{|c|}{ Agree } & \multicolumn{2}{|c|}{$\begin{array}{c}\text { Neither/ } \\
\text { Don't Know }\end{array}$} & \multicolumn{2}{|c|}{ Disagree } & \multicolumn{2}{|c|}{ Total } \\
\hline & $N$ & $\%$ & $\mathrm{~N}$ & $\%$ & $N$ & $\%$ & $\mathrm{~N}$ & $\%$ \\
\hline $\begin{array}{l}\text { The SSAB facilitates effective exchange } \\
\text { of viewpoints on site issues }\end{array}$ & 68 & $81.0 \%$ & 8 & $9.5 \%$ & 8 & $9.5 \%$ & 84 & $100 \%$ \\
\hline $\begin{array}{l}\text { The SSAB contributes to Board members' } \\
\text { understanding of the basis for key site } \\
\text { decisions }\end{array}$ & 72 & $85.7 \%$ & 5 & $6.0 \%$ & 7 & $8.3 \%$ & 84 & $100 \%$ \\
\hline $\begin{array}{l}\text { The SSAB contributes to DOE and } \\
\text { regulators' understanding of the public's } \\
\text { viewpoints on key site decisions }\end{array}$ & 67 & $79.8 \%$ & 7 & $8.3 \%$ & 10 & $11.9 \%$ & 84 & $100 \%$ \\
\hline $\begin{array}{l}\text { The SSAB contributes to a constructive } \\
\text { working relationship among the } \\
\text { participants }\end{array}$ & 64 & $76.2 \%$ & 14 & $16.7 \%$ & 6 & $7.1 \%$ & 84 & $100 \%$ \\
\hline The SSAB strives for consensus & 72 & $85.7 \%$ & 5 & $6.0 \%$ & 7 & $8.3 \%$ & 84 & $100 \%$ \\
\hline $\begin{array}{l}\text { The SSAB gives fair consideration to } \\
\text { dissenting opinions }\end{array}$ & 61 & $72.6 \%$ & 13 & $15.5 \%$ & 10 & $11.9 \%$ & 84 & $100 \%$ \\
\hline $\begin{array}{l}\text { The Board has made the effort needed to } \\
\text { learn about site issues }\end{array}$ & 75 & $90.4 \%$ & 4 & $4.8 \%$ & 4 & $4.8 \%$ & 83 & $100 \%$ \\
\hline $\begin{array}{l}\text { The Board invites expert advice into its } \\
\text { discussion on key policy issues }\end{array}$ & 65 & $78.3 \%$ & 13 & $15.7 \%$ & 5 & $6.0 \%$ & 83 & $100 \%$ \\
\hline $\begin{array}{l}\text { The Board responds to public inquiries } \\
\text { and comments about its decisions }\end{array}$ & 41 & $49.4 \%$ & 36 & $43.4 \%$ & 6 & $7.2 \%$ & 83 & $100 \%$ \\
\hline $\begin{array}{l}\text { The Board solicits feedback from the } \\
\text { community on its work }\end{array}$ & 35 & $42.2 \%$ & 24 & $28.9 \%$ & 24 & $28.9 \%$ & 83 & $100 \%$ \\
\hline $\begin{array}{l}\text { DOE makes information on key site issues } \\
\text { readily available to the Board }\end{array}$ & 48 & $57.1 \%$ & 18 & $21.4 \%$ & 18 & $21.4 \%$ & 84 & $100 \%$ \\
\hline $\begin{array}{l}\text { The regulators make important } \\
\text { contributions to the Board's work }\end{array}$ & 61 & $72.6 \%$ & 15 & $17.9 \%$ & 8 & $9.5 \%$ & 84 & $100 \%$ \\
\hline $\begin{array}{l}\text { The SSAB creates a climate supportive of } \\
\text { differing viewpoints }(\mathrm{R})^{*}\end{array}$ & 64 & $75.3 \%$ & 5 & $5.9 \%$ & 16 & $18.8 \%$ & 85 & $100 \%$ \\
\hline
\end{tabular}

* (R) denotes a question that was asked in the negative form. For ease of review, these questions and data have been reversed in this table and are stated in positive forms.

SSAB Supplementary Appendix

August 1996 
Table 3.

Goal 3. Provide useful advice and/or recommendations to DOE (and regulators, where appropriate)

\begin{tabular}{|c|c|c|c|c|c|c|c|c|}
\hline \multirow[t]{2}{*}{ Hanford } & \multicolumn{2}{|c|}{ Agree } & \multicolumn{2}{|c|}{$\begin{array}{l}\text { Neither/ } \\
\text { Don't Know }\end{array}$} & \multicolumn{2}{|c|}{ Disagree } & \multicolumn{2}{|c|}{ Total } \\
\hline & $\mathrm{N}$ & $\%$ & $\mathbf{N}$ & $\%$ & $N$ & $\%$ & $N$ & $\%$ \\
\hline $\begin{array}{l}\text { The Board provides useful advice to DOE } \\
\text { (and regulators, where appropriate) }(R)^{*}\end{array}$ & 60 & $72.3 \%$ & 12 & $14.5 \%$ & 11 & $13.3 \%$ & 83 & $100 \%$ \\
\hline $\begin{array}{l}\text { The SSAB reaches agreement about } \\
\text { prioritization of key site issues for which } \\
\text { advice is sought }(\mathrm{R}) *\end{array}$ & 46 & $54.8 \%$ & 9 & $10.7 \%$ & 29 & $34.5 \%$ & 84 & $100 \%$ \\
\hline The Board helps define site problems & 67 & $80.7 \%$ & 13 & $15.7 \%$ & 3 & $3.6 \%$ & 83 & $100 \%$ \\
\hline $\begin{array}{l}\text { The Board addresses issues in a timely } \\
\text { manner (R)* }\end{array}$ & 42 & $50.6 \%$ & 20 & $24.1 \%$ & 21 & $25.3 \%$ & 83 & $100 \%$ \\
\hline $\begin{array}{l}\text { The Board reaches consensus on key site } \\
\text { issues }(R)^{*}\end{array}$ & 57 & $68.7 \%$ & 15 & $18.1 \%$ & 11 & $13.3 \%$ & 83 & $100 \%$ \\
\hline $\begin{array}{l}\text { The Board provides informed advice to } \\
\text { DOE (and regulators, where appropriate) }\end{array}$ & 61 & $73.5 \%$ & 13 & $15.7 \%$ & 9 & $10.8 \%$ & 83 & $100 \%$ \\
\hline $\begin{array}{l}\text { The Board provides advice that reflects } \\
\text { the viewpoints and priorities of the } \\
\text { community }\end{array}$ & 51 & $61.4 \%$ & 14 & $16.9 \%$ & 18 & $21.7 \%$ & 83 & $100 \%$ \\
\hline
\end{tabular}

* (R) denotes a question that was asked in the negative form. For ease of review, these questions and data have been reversed in this table and are stated in positive forms.

SSAB Supplementary Appendix August 1996 
Table 4.

Goal 4. Improve DOE's (and where applicable, regulators') site decisions and decision making process

\begin{tabular}{|c|c|c|c|c|c|c|c|c|}
\hline \multirow[t]{2}{*}{ Hanford } & \multicolumn{2}{|c|}{ Agree } & \multicolumn{2}{|c|}{$\begin{array}{l}\text { Neither/ } \\
\text { Don't Know }\end{array}$} & \multicolumn{2}{|c|}{ Disagree } & \multicolumn{2}{|c|}{ Total } \\
\hline & $N$ & $\%$ & $\mathbf{N}$ & $\%$ & $\mathrm{~N}$ & $\%$ & $\mathrm{~N}$ & $\%$ \\
\hline $\begin{array}{l}\text { The SSAB has improved DOE's site } \\
\text { decisions (R) * }\end{array}$ & 50 & $61.0 \%$ & 14 & $17.1 \%$ & 18 & $22.0 \%$ & 82 & $100 \%$ \\
\hline $\begin{array}{l}\text { The Board understands the decision } \\
\text { making process at [a particular] site }\end{array}$ & 30 & $36.1 \%$ & 25 & $30.1 \%$ & 28 & $33.7 \%$ & 83 & $100 \%$ \\
\hline $\begin{array}{l}\text { DOE discusses important policies } \\
\text { affecting site decisions with the Board }\end{array}$ & 56 & $66.7 \%$ & 17 & $20.2 \%$ & 11 & $13.1 \%$ & 84 & $100 \%$ \\
\hline $\begin{array}{l}\text { DOE has explained to the Board its site } \\
\text { decision making process }\end{array}$ & 30 & $36.1 \%$ & 28 & $33.7 \%$ & 25 & $30.1 \%$ & 83 & $100 \%$ \\
\hline $\begin{array}{l}\text { The DOE shows how Board advice is } \\
\text { reflected in site decisions }\end{array}$ & 28 & $33.7 \%$ & 35 & $42.2 \%$ & 20 & $24.1 \%$ & 83 & $100 \%$ \\
\hline $\begin{array}{l}\text { The decision making process used by } \\
\text { DOE at [a particular] site is effective in } \\
\text { furthering site clean-up (R) * }\end{array}$ & 29 & $34.5 \%$ & 27 & $32.1 \%$ & 28 & $33.3 \%$ & 84 & $100 \%$ \\
\hline
\end{tabular}

* (R) denotes a question that was asked in the negative form. For ease of review, these questions and data have been reversed in this table and are stated in positive forms. 
Table 5.

Goal 5. Lead to more acceptable actions

\begin{tabular}{|c|c|c|c|c|c|c|c|c|}
\hline \multirow[t]{2}{*}{ Hanford } & \multicolumn{2}{|c|}{ Agree } & \multicolumn{2}{|c|}{$\begin{array}{l}\text { Neitherl } \\
\text { Don't Know }\end{array}$} & \multicolumn{2}{|c|}{ Disagree } & \multicolumn{2}{|c|}{ Total } \\
\hline & $\mathrm{N}$ & $\%$ & $\mathrm{~N}$ & $\%$ & $\mathrm{~N}$ & $\%$ & $\mathrm{~N}$ & $\%$ \\
\hline $\begin{array}{l}\text { The SSAB leads to more acceptable site } \\
\text { decisions }\end{array}$ & 51 & $62.2 \%$ & 17 & $20.7 \%$ & 14 & $17.1 \%$ & 82 & $100 \%$ \\
\hline $\begin{array}{l}\text { The Board supports the recommendations } \\
\text { it gives DOE }\end{array}$ & 62 & $74.7 \%$ & 19 & $22.9 \%$ & 2 & $2.4 \%$ & 83 & $100 \%$ \\
\hline The Board supports DOE's site actions & 10 & $12.0 \%$ & 54 & $65.1 \%$ & 19 & $22.9 \%$ & 83 & $100 \%$ \\
\hline $\begin{array}{l}\text { The DOE pays attention to the Board's } \\
\text { advice on key site issues (R) * }\end{array}$ & 29 & $35.4 \%$ & 22 & $26.8 \%$ & 31 & $37.8 \%$ & 82 & $100 \%$ \\
\hline $\begin{array}{l}\text { There is support in the community for } \\
\text { DOE's site decisions that have SSAB } \\
\text { input }\end{array}$ & 45 & $53.6 \%$ & 27 & $32.1 \%$ & 12 & $14.3 \%$ & 84 & $100 \%$ \\
\hline Progress is being made on key site issues & 55 & $64.7 \%$ & 20 & $23.5 \%$ & 10 & $11.8 \%$ & 85 & $100 \%$ \\
\hline
\end{tabular}

* (R) denotes a question that was asked in the negative form. For ease of review, these questions and data have been reversed in this table and are stated in positive forms.

SSAB Supplementary Appendix 
Table 6.

Goal 6. Contribute to trust and confidence in DOE

\begin{tabular}{|c|c|c|c|c|c|c|c|c|}
\hline \multirow[t]{2}{*}{ Hanford } & \multicolumn{2}{|c|}{ Agree } & \multicolumn{2}{|c|}{$\begin{array}{c}\text { Neither/ } \\
\text { Don't Know }\end{array}$} & \multicolumn{2}{|c|}{ Disagree } & \multicolumn{2}{|c|}{ Total } \\
\hline & $N$ & $\%$ & $N$ & $\%$ & $N$ & $\%$ & $N$ & $\%$ \\
\hline $\begin{array}{l}\text { The SSAB contributes to trust and } \\
\text { confidence in DOE }\end{array}$ & 31 & $37.8 \%$ & 34 & $41.5 \%$ & 17 & $20.7 \%$ & 82 & $100 \%$ \\
\hline $\begin{array}{l}\text { Relationships between DOE and the public } \\
\text { have improved since the formation of the } \\
\text { SSAB }\end{array}$ & 59 & $70.2 \%$ & 16 & $19.0 \%$ & 9 & $10.7 \%$ & 84 & $100 \%$ \\
\hline $\begin{array}{l}\text { DOE is committed to clean up [a } \\
\text { particular] site }\end{array}$ & 31 & $36.5 \%$ & 17 & $20.0 \%$ & 37 & $43.5 \%$ & 85 & $100 \%$ \\
\hline
\end{tabular}


Table 7.

Public awareness

\begin{tabular}{|c|c|c|c|c|c|c|c|c|}
\hline \multirow[t]{2}{*}{ Hanford } & \multicolumn{2}{|c|}{ Agree } & \multicolumn{2}{|c|}{$\begin{array}{c}\text { Neither/ } \\
\text { Don't Know }\end{array}$} & \multicolumn{2}{|c|}{ Disagree } & \multicolumn{2}{|c|}{ Total } \\
\hline & $N$ & $\%$ & $\mathrm{~N}$ & $\%$ & $\mathrm{~N}$ & $\%$ & $N$ & $\%$ \\
\hline $\begin{array}{l}\text { The public knows little about the role of } \\
\text { the Board }\end{array}$ & 48 & $56.5 \%$ & 21 & $24.7 \%$ & 16 & $18.8 \%$ & 85 & $100 \%$ \\
\hline
\end{tabular}

Table 8.

SSAB Initiative is a good use of funds

\begin{tabular}{ccccccccc}
\hline Hanford & Yes, Good & $\begin{array}{c}\text { Neutral, } \\
\text { Conditional, } \\
\text { No Opinion }\end{array}$ & $\begin{array}{c}\text { No, } \\
\text { Not Good }\end{array}$ & Total \\
& $\mathrm{N}$ & $\%$ & $\mathrm{~N}$ & $\%$ & $\mathrm{~N}$ & $\%$ & $\mathrm{~N}$ & $\%$ \\
\hline SSAB Initiative is a good use of funds & 58 & $78.4 \%$ & 1 & $1.4 \%$ & 15 & $20.3 \%$ & 74 & $100 \%$ \\
\hline
\end{tabular}

SSAB Supplementary Appendix 
Table 9.

DOE-HQ involvement with the SSAB Initiative

\begin{tabular}{|c|c|c|c|c|c|c|c|c|}
\hline \multirow[t]{2}{*}{ Hanford } & \multicolumn{2}{|c|}{ Agree } & \multicolumn{2}{|c|}{$\begin{array}{c}\text { Neither/ } \\
\text { Don't Know }\end{array}$} & \multicolumn{2}{|c|}{ Disagree } & \multicolumn{2}{|c|}{ Total } \\
\hline & $N$ & $\%$ & $\mathrm{~N}$ & $\%$ & $N$ & $\%$ & $\mathrm{~N}$ & $\%$ \\
\hline $\begin{array}{l}\text { DOE-HQ provides helpful guidance to the } \\
\text { Boards and to DOE }\end{array}$ & 6 & $7.1 \%$ & 28 & $33.3 \%$ & 50 & $59.5 \%$ & 84 & $100 \%$ \\
\hline $\begin{array}{l}\text { DOE-HQ provides sufficient support to } \\
\text { facilitate the work of the SSAB }\end{array}$ & 10 & $11.9 \%$ & 22 & $26: 2 \%$ & 52 & $61.9 \%$ & 84 & $100 \%$ \\
\hline $\begin{array}{l}\text { DOE-HQ gives careful consideration to } \\
\text { SSAB advice in its decisions }\end{array}$ & 8 & $9.5 \%$ & 25 & $29.8 \%$ & 51 & $60.7 \%$ & 84 & $100 \%$ \\
\hline
\end{tabular}


Table 10.

Personal experience with the SSAB Initiative

\begin{tabular}{|c|c|c|c|c|c|c|c|c|}
\hline \multirow[t]{2}{*}{ Hanford } & \multicolumn{2}{|c|}{ Agree } & \multicolumn{2}{|c|}{$\begin{array}{c}\text { Neither/ } \\
\text { Don't Know }\end{array}$} & \multicolumn{2}{|c|}{ Disagree } & \multicolumn{2}{|c|}{ Total } \\
\hline & $N$ & $\%$ & $\mathrm{~N}$ & $\%$ & $N$ & $\%$ & $N$ & $\%$ \\
\hline $\begin{array}{l}\text { I consider myself to be very } \\
\text { knowledgeable about site issues }\end{array}$ & 67 & $78.8 \%$ & 11 & $12.9 \%$ & 7 & $8.2 \%$ & 85 & $100 \%$ \\
\hline $\begin{array}{l}\text { Because of the SSAB, I better understand } \\
\text { the complexities of site clean-up }\end{array}$ & 50 & $59.5 \%$ & 14 & $16.7 \%$ & 20 & $23.8 \%$ & 84 & $100 \%$ \\
\hline $\begin{array}{l}\text { I feel that my participation in the SSAB } \\
\text { has been worthwhile }(\mathrm{R}) *\end{array}$ & 62 & $72.9 \%$ & 12 & $14.1 \%$ & 11 & $12.9 \%$ & 85 & $100 \%$ \\
\hline
\end{tabular}

* (R) denotes a question that was asked in the negative form. For ease of review, these questions and data have been reversed in this table and are stated in positive forms. 
Table 11.

Group/Team work skills

\begin{tabular}{|c|c|c|c|c|c|c|c|c|}
\hline \multirow[t]{2}{*}{ Hanford } & \multicolumn{2}{|c|}{ Agree } & \multicolumn{2}{|c|}{$\begin{array}{l}\text { Neither/ } \\
\text { Don't Know }\end{array}$} & \multicolumn{2}{|c|}{ Disagree } & \multicolumn{2}{|c|}{ Total } \\
\hline & $\mathrm{N}$ & $\%$ & $\mathrm{~N}$ & $\%$ & $N$ & $\%$ & $N$ & $\%$ \\
\hline All have group/team work skills & 37 & $44.0 \%$ & 24 & $28.6 \%$ & 23 & $27.4 \%$ & 84 & $100 \%$ \\
\hline $\begin{array}{l}\text { Have developed well-established working } \\
\text { relationships with each other }\end{array}$ & 47 & $56.0 \%$ & 28 & $33.3 \%$ & 9 & $10.7 \%$ & 84 & $100 \%$ \\
\hline Like each other & 27 & $32.9 \%$ & 33 & $40.2 \%$ & 22 & $26.8 \%$ & 82 & $100 \%$ \\
\hline $\begin{array}{l}\text { Have a high degree of trust and } \\
\text { confidence in each other }\end{array}$ & 22 & $26.5 \%$ & 28 & $33.7 \%$ & 33 & $39.8 \%$ & 83 & $100 \%$ \\
\hline $\begin{array}{l}\text { Accept the values that are important to the } \\
\text { group }\end{array}$ & 53 & $63.1 \%$ & 21 & $25.0 \%$ & 10 & $11.9 \%$ & 84 & $100 \%$ \\
\hline Show respect for the viewpoints of others & 61 & $72.6 \%$ & 13 & $15.5 \%$ & 10 & $11.9 \%$ & 84 & $100 \%$ \\
\hline $\begin{array}{l}\text { Accept willingly the goals and } \\
\text { expectations for the Board }\end{array}$ & 47 & $56.0 \%$ & 25 & $29.8 \%$ & 12 & $14.3 \%$ & 84 & $100 \%$ \\
\hline $\begin{array}{l}\text { Set high expectations for what they } \\
\text { believe they can accomplish }\end{array}$ & 61 & $72.6 \%$ & 16 & $19.0 \%$ & 7 & $8.3 \%$ & 84 & $100 \%$ \\
\hline $\begin{array}{l}\text { Communicate fully and frankly to the } \\
\text { Board all relevant and valuable } \\
\text { information }\end{array}$ & 37 & $44.0 \%$ & 25 & $29.8 \%$ & 22 & $26.2 \%$ & 84 & $100 \%$ \\
\hline $\begin{array}{l}\text { Try not to waste the Board's time with } \\
\text { irrelevant material or communications }\end{array}$ & 36 & $42.9 \%$ & 20 & $23.8 \%$ & 28 & $33.3 \%$ & 84 & $100 \%$ \\
\hline $\begin{array}{l}\text { Are willing to be influenced by other } \\
\text { Board members about new ideas and } \\
\text { methods }\end{array}$ & 53 & $63.1 \%$ & 17 & $20.2 \%$ & 14 & $16.7 \%$ & 84 & $100 \%$ \\
\hline $\begin{array}{l}\text { Clearly understand the goals and } \\
\text { philosphy of the Board's operation }\end{array}$ & 49 & $58.3 \%$ & 26 & $31.0 \%$ & 9 & $10.7 \%$ & 84 & $100 \%$ \\
\hline $\begin{array}{l}\text { Do all they can to help the Board achieve } \\
\text { its objectives }\end{array}$ & 41 & $49.4 \%$ & 27 & $32.5 \%$ & 15 & $18.1 \%$ & 83 & $100 \%$ \\
\hline $\begin{array}{l}\text { Get the technical knowledge and training } \\
\text { in group skills they need }\end{array}$ & 27 & $32.1 \%$ & 35 & $41.7 \%$ & 22 & $26.2 \%$ & 84 & $100 \%$ \\
\hline Provide one another mutual help & 44 & $52.4 \%$ & 29 & $34.5 \%$ & 11 & $13.1 \%$ & 84 & $100 \%$ \\
\hline
\end{tabular}

SSAB Supplementary Appendix

August 1996 
Idaho Site Specific Advisory Board

Evaluation Survey Results

SSAB Supplementary Appendix

August 1996

S-54 
Figure 1a. Idaho

Goal 1. Establish processes and procedures to provide an effective forum for exchange of information and viewpoints regarding DOE site issues

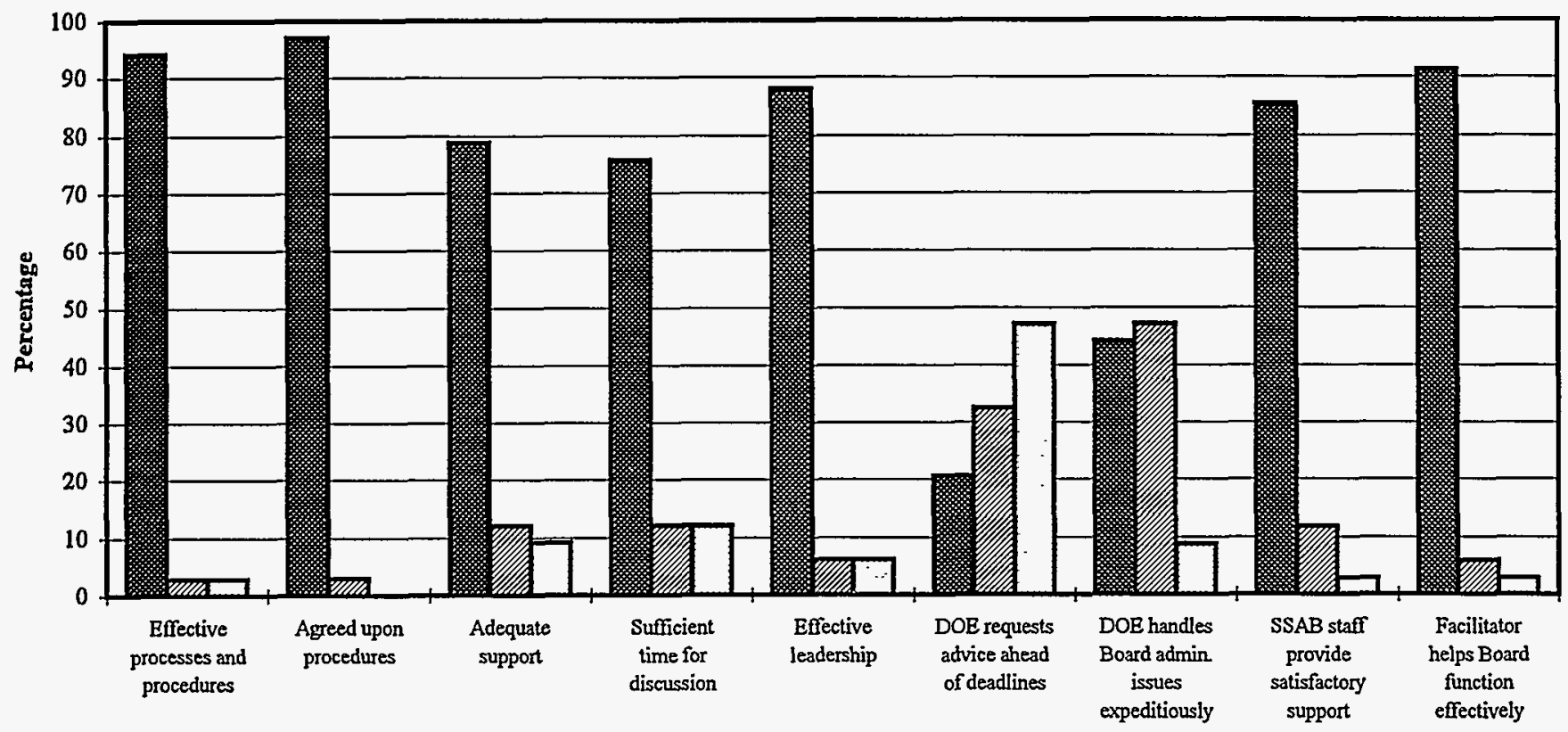

Agree (\%) ER Neither Agree Nor Disagree/Don't Know (\%) DDisagree (\%)

Figure 1b. Long Survey: Entire Sample

Goal 1. Establish processes and procedures to provide an effective forum for exchange of information and viewpoints regarding DOE site issues

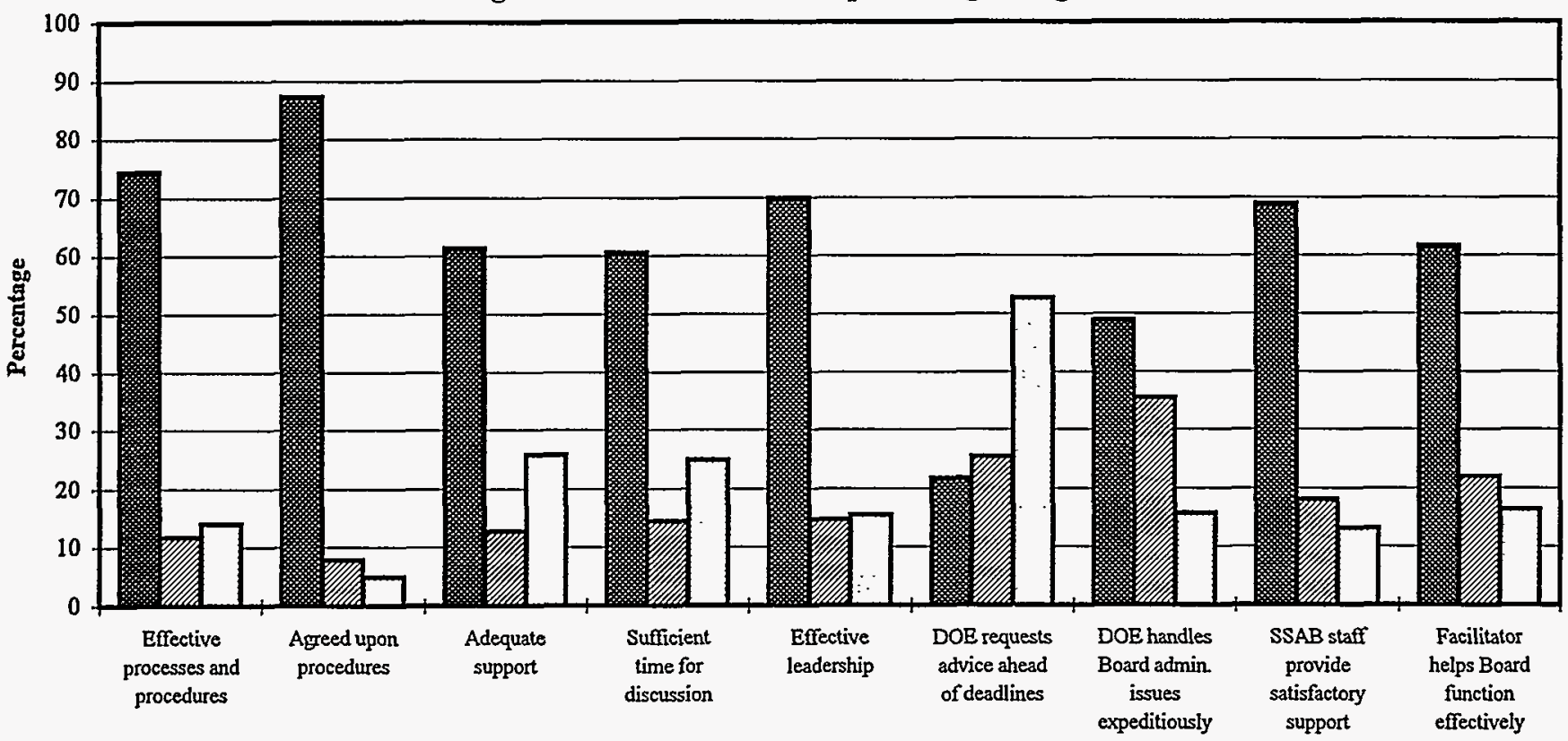

Agree (\%) $\mathbf{E}$ Neither Agree Nor Disagree/Don't Know (\%) DDisagree (\%)

SSAB Supplementary Appendix

August 1996 
Figure 2a. Idaho

Goal 2. Facilitate interaction and exchange of information and viewpoints regarding DOE site issues

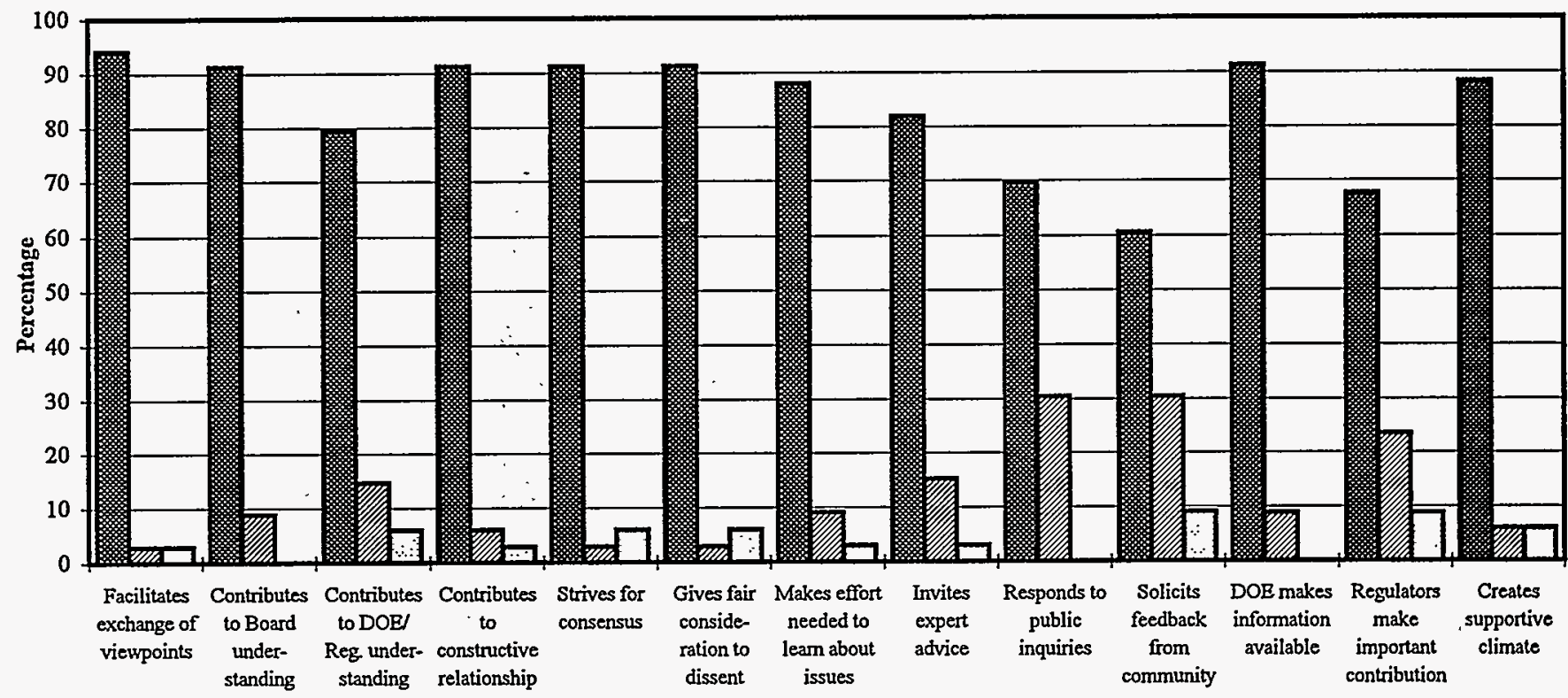

Agree (\%) ENeither Agree Nor Disagree/Don't Know (\%) QDisagree (\%)

Figure 2b. Long Survey: Entire Sample

Goal 2. Facilitate interaction and exchange of information and viewpoints regarding DOE site issues

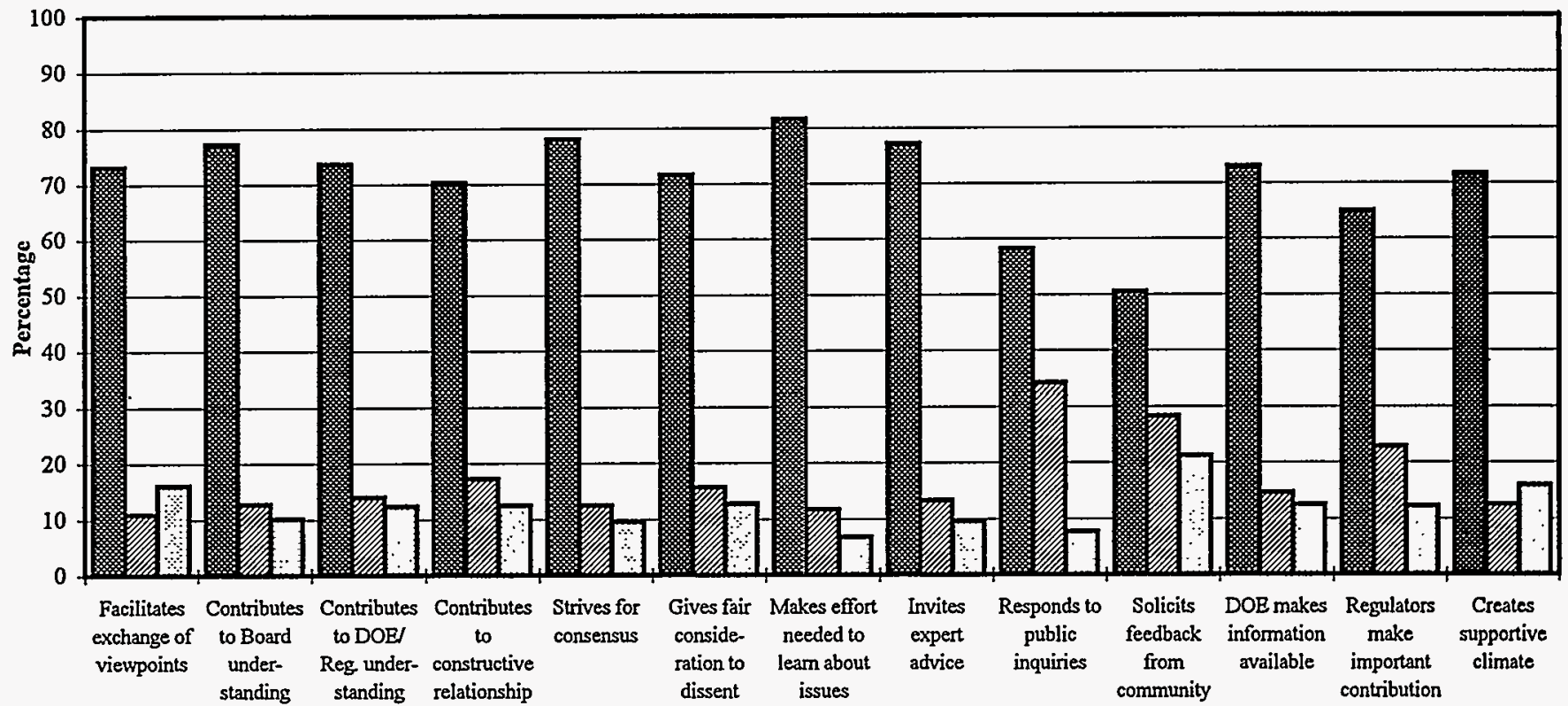

Agree (\%) Neither Agree Nor Disagree/Don't Know (\%) DDisagree (\%)

SSAB Supplementary Appendix

August 1996 
Figure 3a. Idaho

Goal 3. Provide useful advice and/or recommendations to DOE

(and regulators, where appropriate)

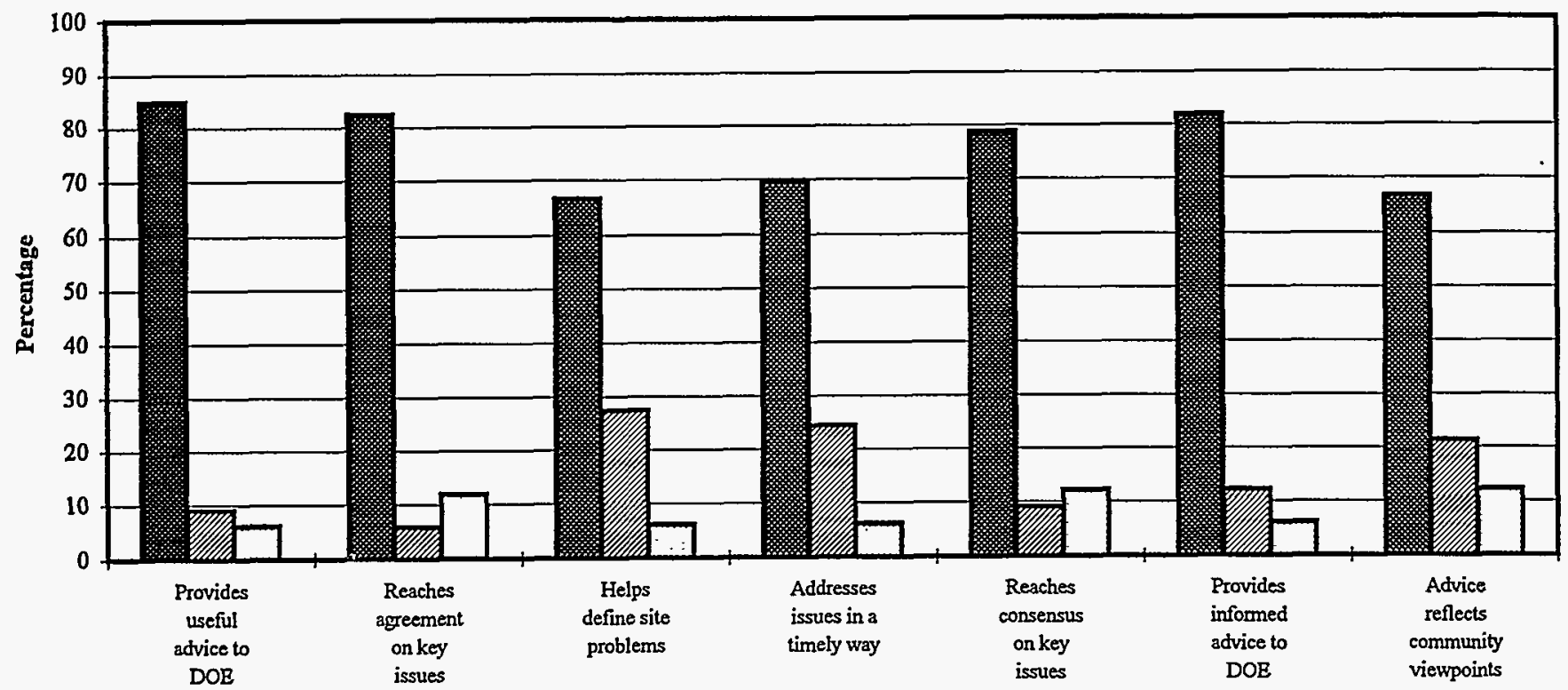

Agree (\%) Neither Agree Nor Disagree/Don't Know (\%) DDisagree (\%)

Figure 3b. Long Survey: Entire Sample

Goal 3. Provide useful advice and/or recommendations to DOE

(and regulators, where appropriate)

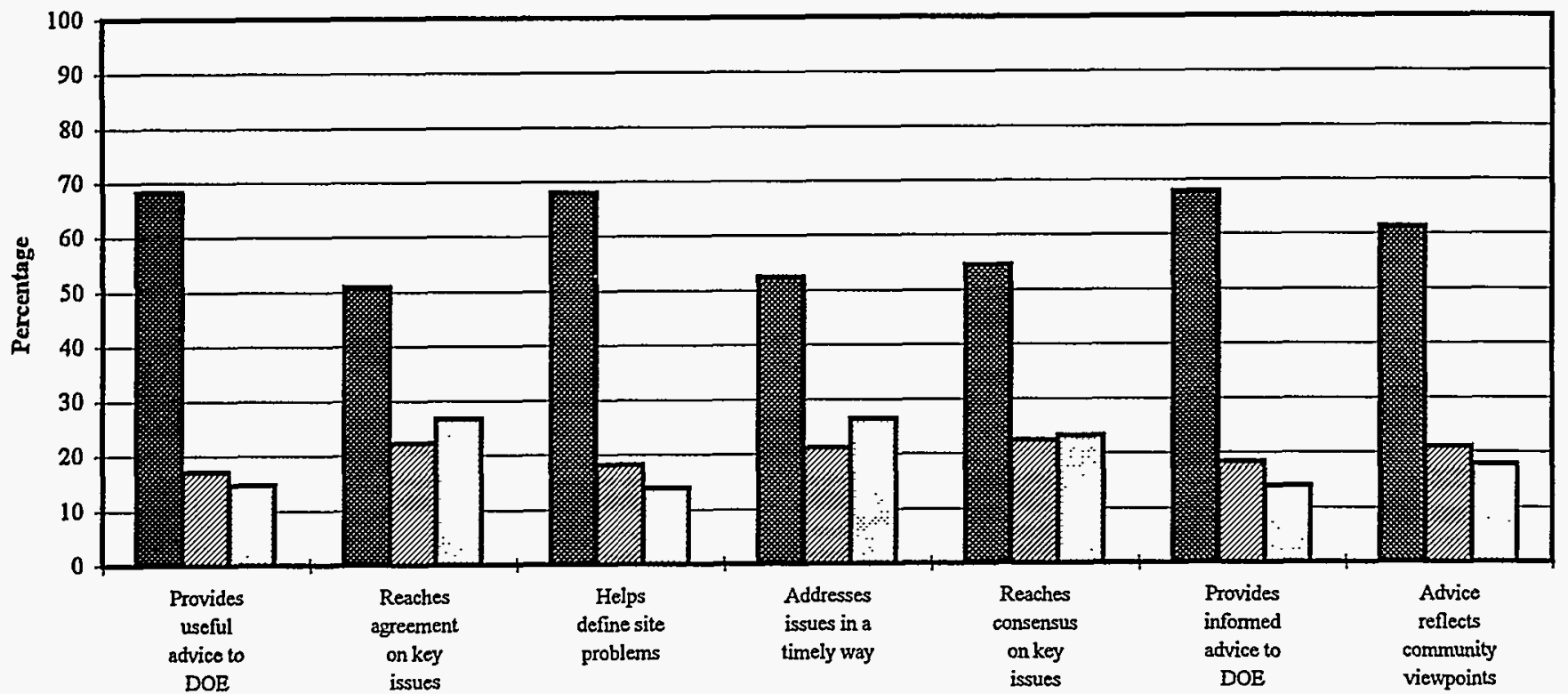

E Agree (\%) घNeither Agree Nor Disagree/Dont Know (\%) DDisagree (\%)

SSAB Supplementary Appendix

August 1996 
Figure 4a. Idaho

Goal 4. Improve DOE's (and where applicable, regulators') site decisions and decision making process

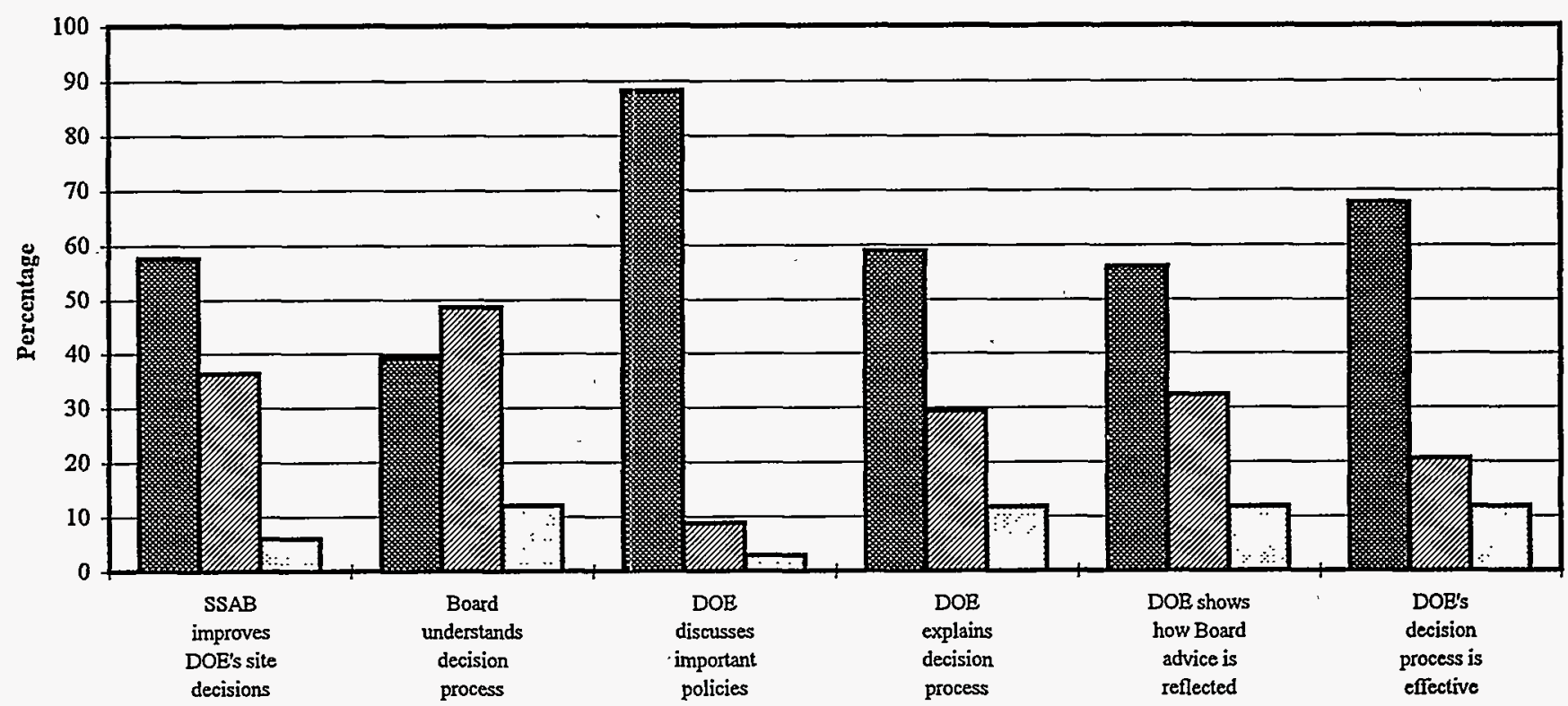

Agree (\%) DNeither Agree Nor Disagree/Don't Know (\%) DDisagree (\%)

Figure 4b. Long Survey: Entire Sample

Goal 4. Improve DOE's (and where applicable, regulators') site decisions and decision making process

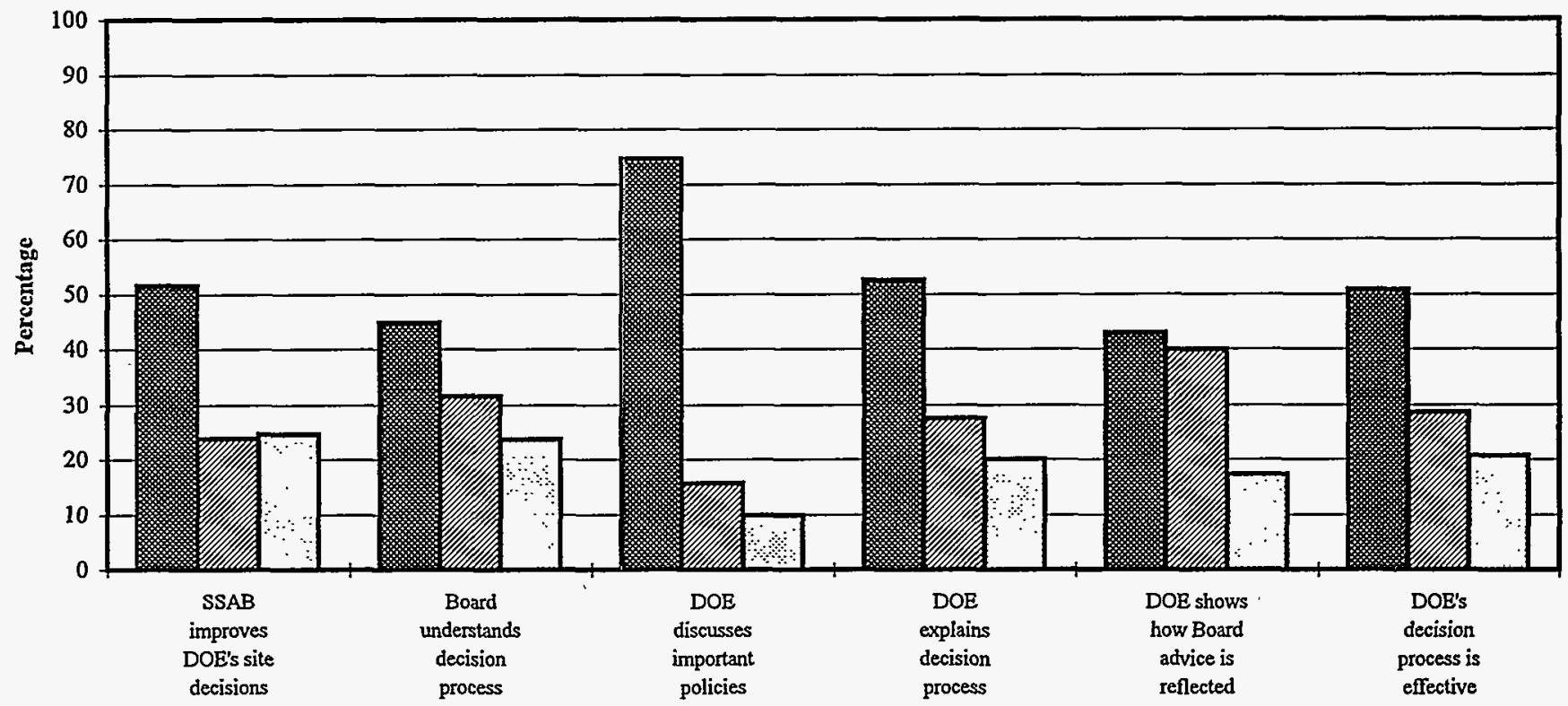

A Agree (\%) Weither Agree Nor Disagree/Don't Know (\%) DDisagree (\%)

SSAB Supplementary Appendix

August 1996 
Figure 5a. Idaho

Goal 5. Lead to more acceptable actions

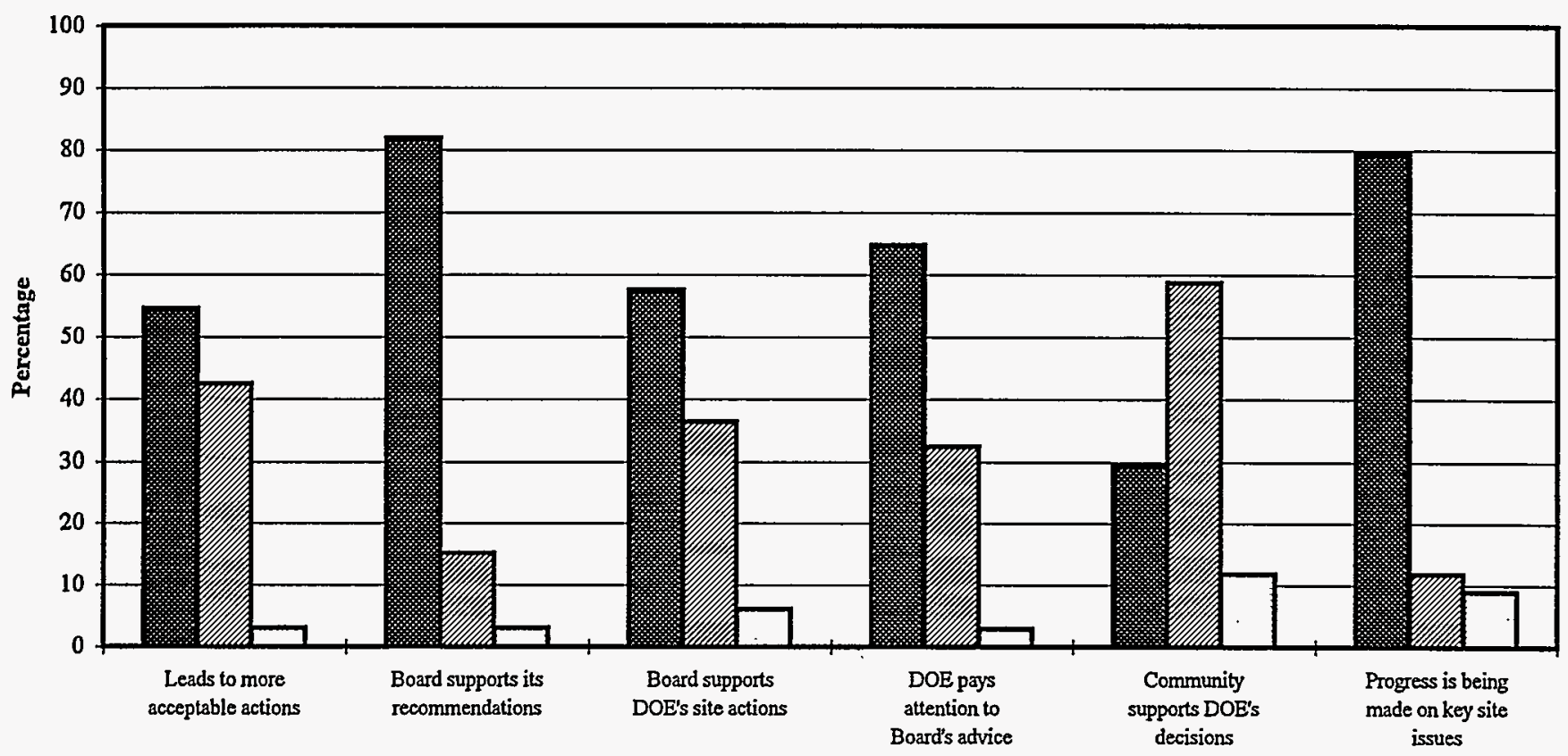

Agree (\%) ENeither Agree Nor Disagree/Don't Know (\%) DDisagree (\%)

Figure 5b. Long Survey: Entire Sample

Goal 5. Lead to more acceptable actions

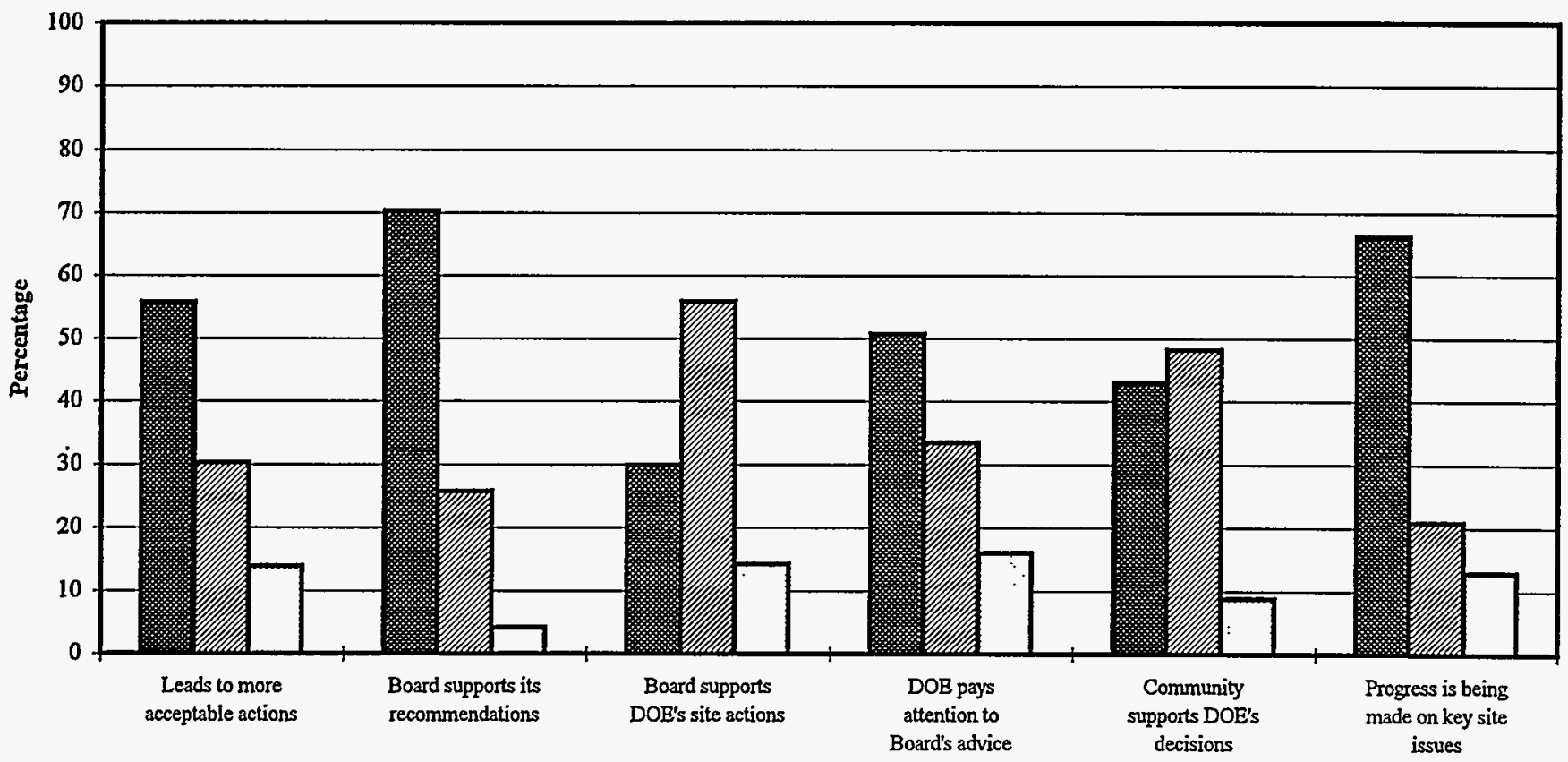

Agree (\%) Weither Agree Nor Disagree/Don't Know (\%) DDisagree (\%)

SSAB Supplementary Appendix

August 1996 
Figure 6a. Idaho

Goal 6. Contribute to trust and confidence in DOE

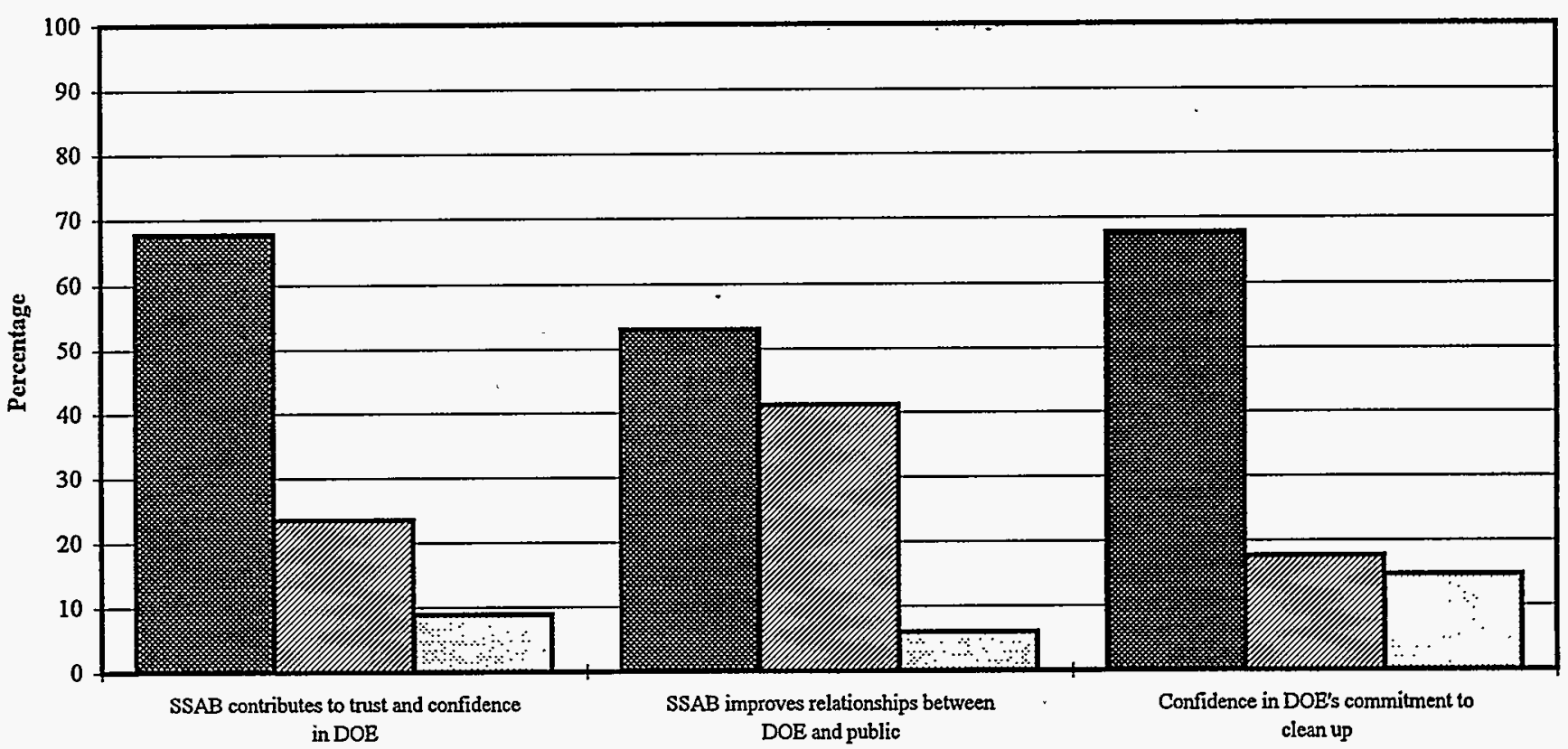

E Agree (\%) Weither Agree Nor Disagree/Don't Know (\%) DDisagree (\%)

Figure 6b. Long Survey: Entire Sample

Goal 6. Contribute to trust and confidence in DOE

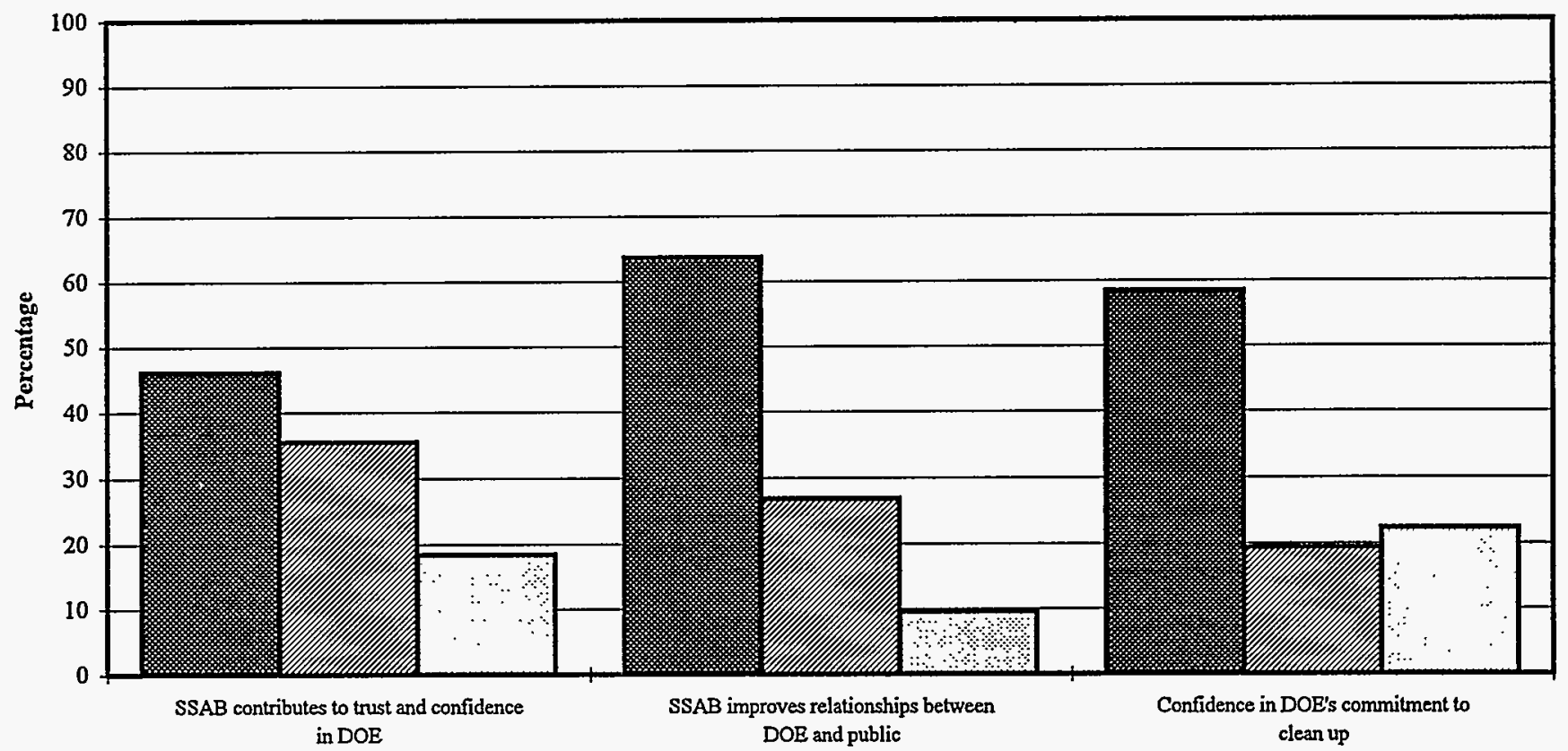

I. Agree (\%) Deither Agree Nor Disagree/Don't Know (\%) Disagree (\%)

SSAB Supplementary Appendix

August 1996 
Figure 7a. Idaho

Public awareness

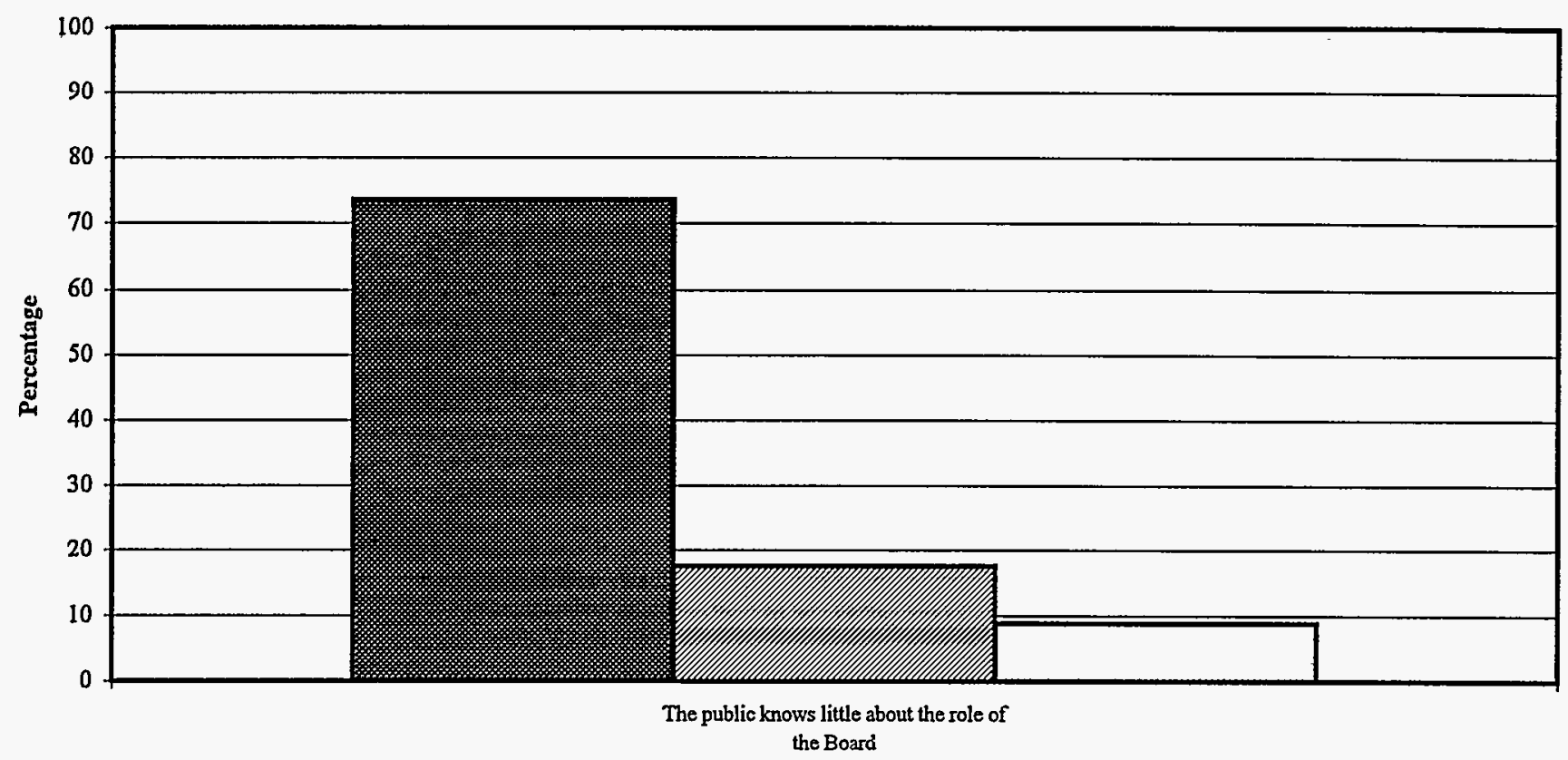

Agree (\%) Deither Agree Nor Disagree/Don't Know (\%) पDisagree (\%)

Figure 7b. Long Survey: Entire Sample

Public awareness

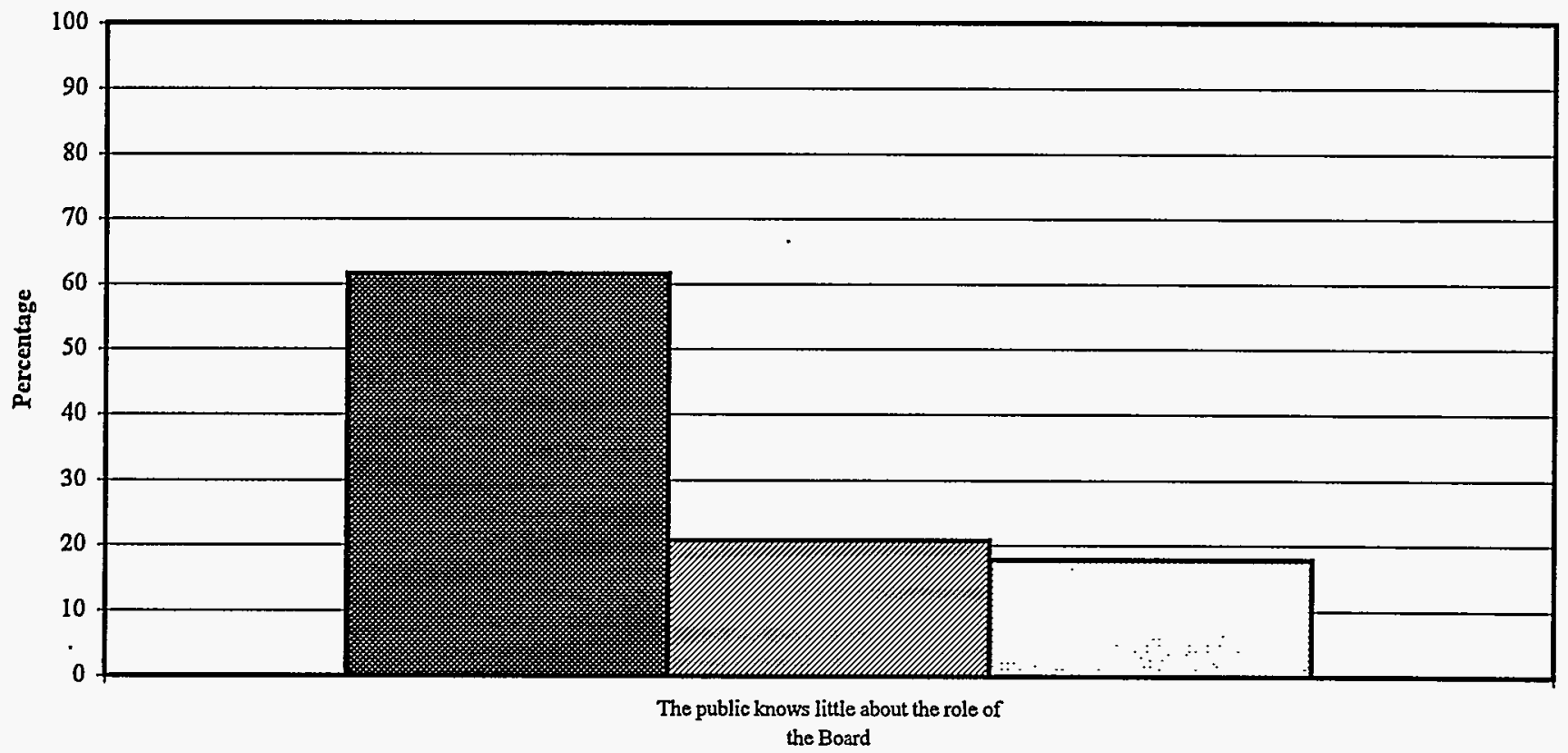

Agree (\%) ENeither Agree Nor Disagree/Don't Know (\%) DDisagree (\%)

SSAB Supplementary Appendix 
Figure 8a. Idaho

DOE-HQ involvement with the SSAB Initiative

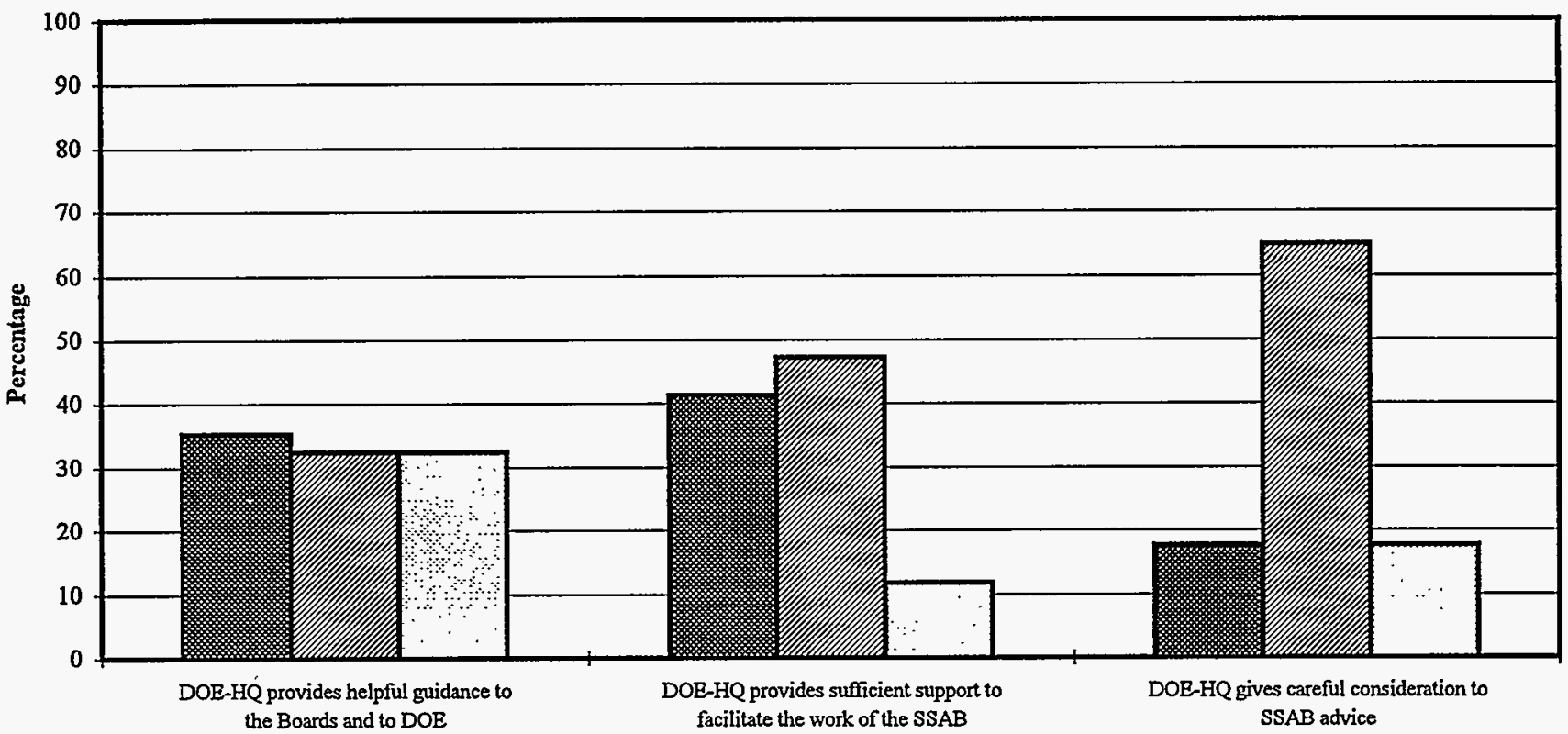

Agree (\%) ENeither Agree Nor Disagree/Don't Know (\%) DDisagree (\%)

Figure 8b. Long Survey: Entire Sample DOE-HQ involvement with the SSAB Initiative

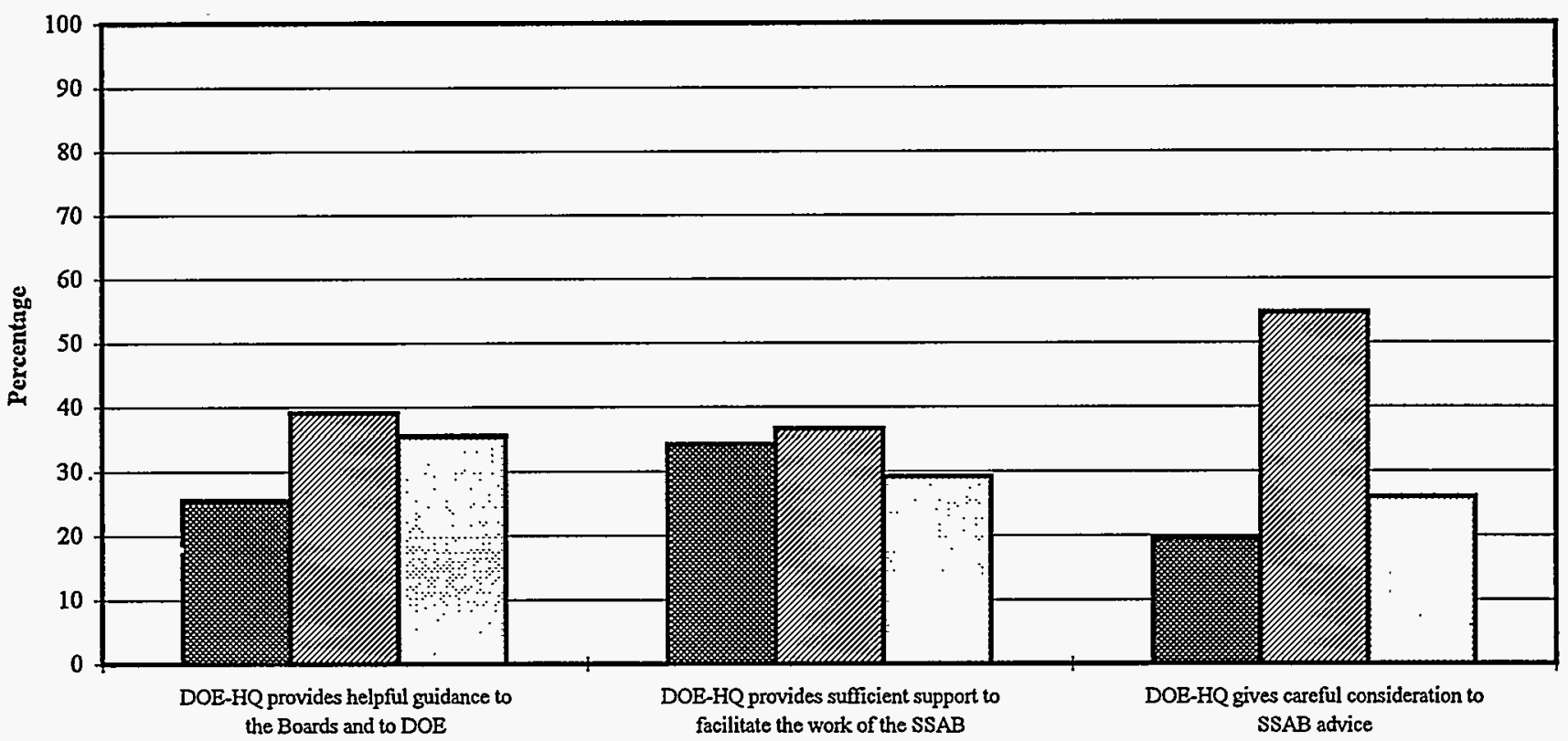

Agree (\%) DNeither Agree Nor Disagree/Don't Know (\%) DDisagree (\%)

SSAB Supplementary Appendix August 1996 
Figure 9a. Idaho

Personal experience with the SSAB Initiative

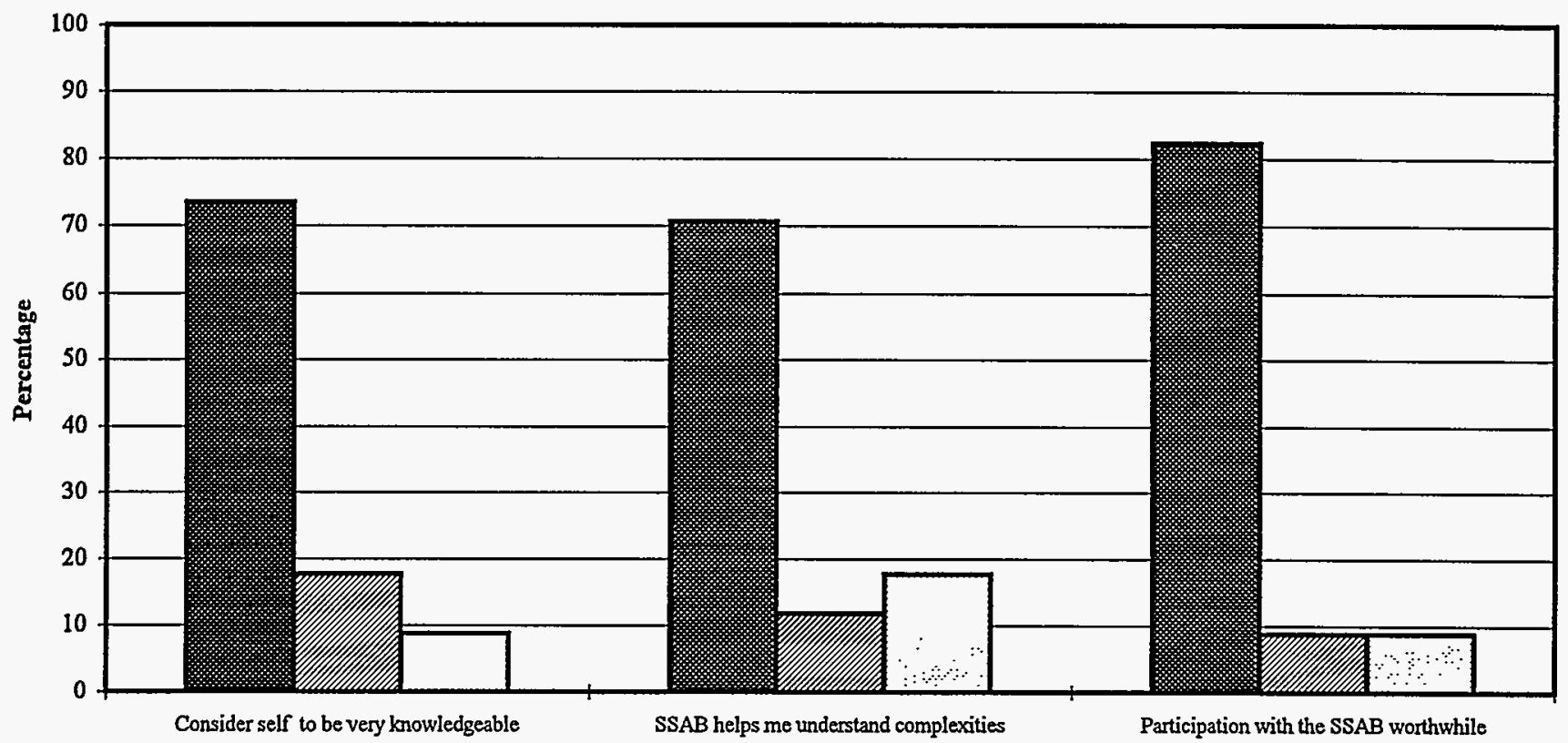

Agree (\%) ENeither Agree Nor Disagree/Don't Know (\%) DDisagree (\%)

Figure 9b. Long Survey: Entire Sample Personal experience with the SSAB Initiative

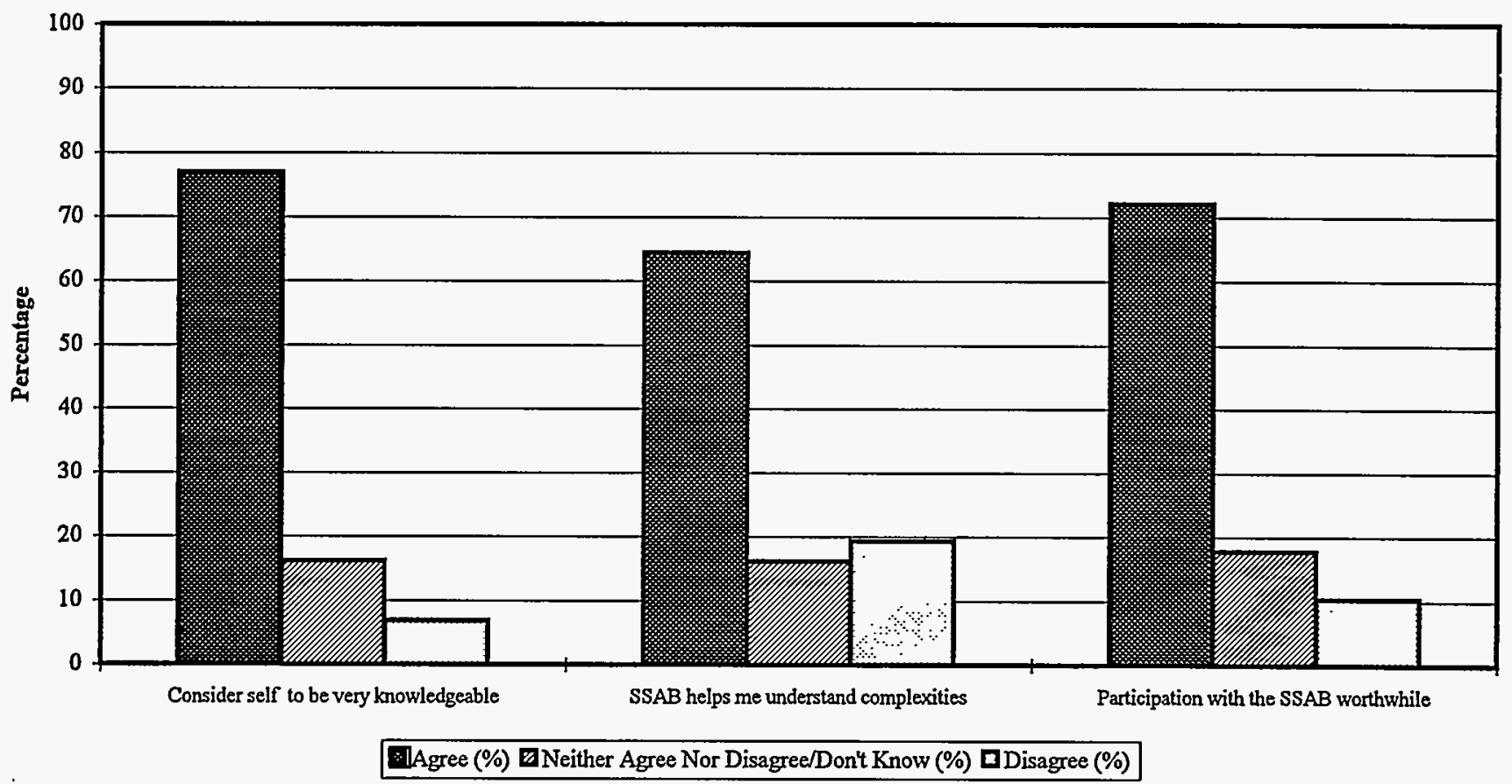

SSAB Supplementary Appendix 
Figure 10a. Idaho

Group/Team work skills

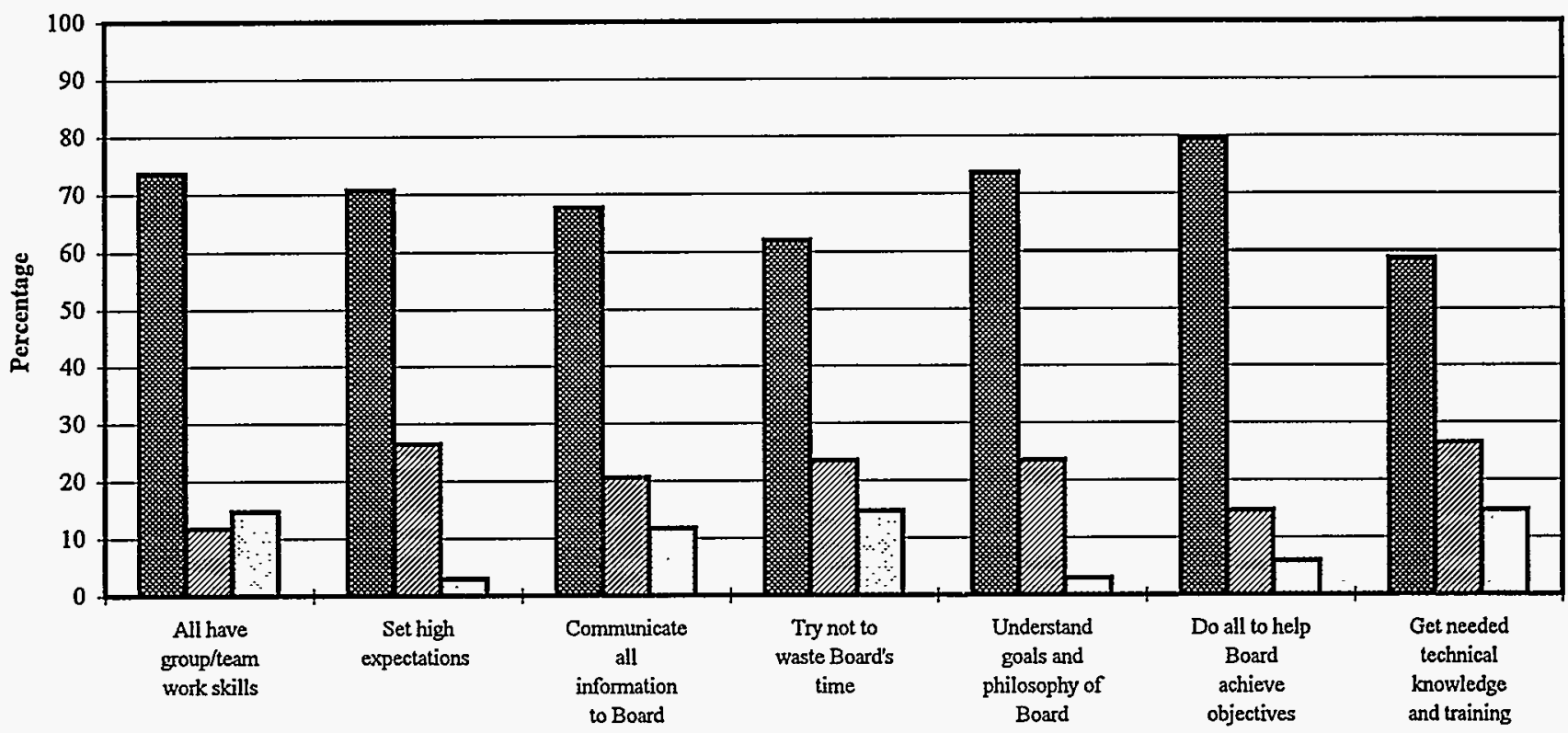

Agree (\%) ENeither Agree Nor Disagree/Don't Know (\%) DDisagree (\%)

Figure 10b. Long Survey: Entire Sample

Group/Team work skills

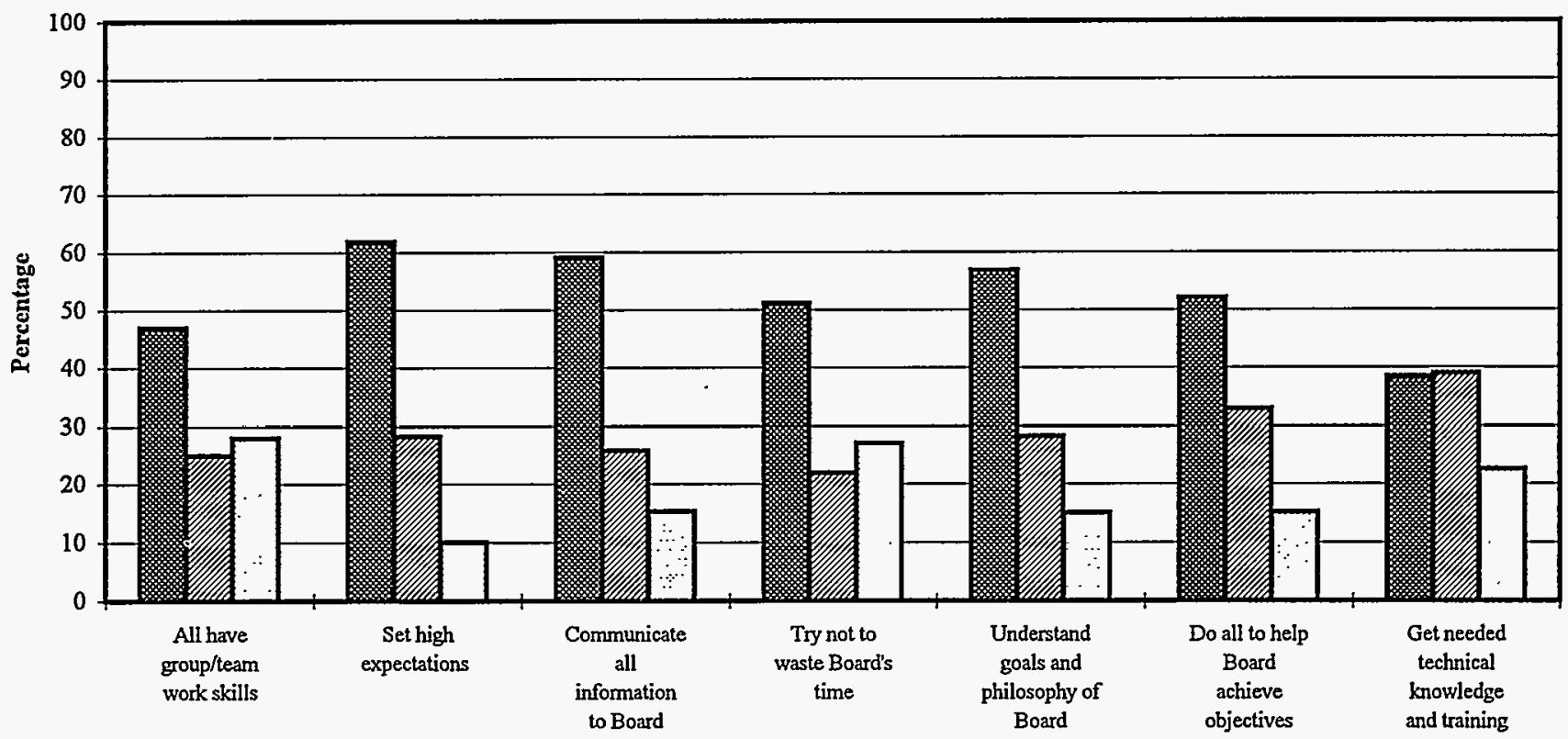

Agree (\%) WNeither Agree Nor Disagree/Don't Know (\%) DDisagree (\%)

SSAB Supplementary Appendix

August 1996 
Figure 11a. Idaho

Working relations

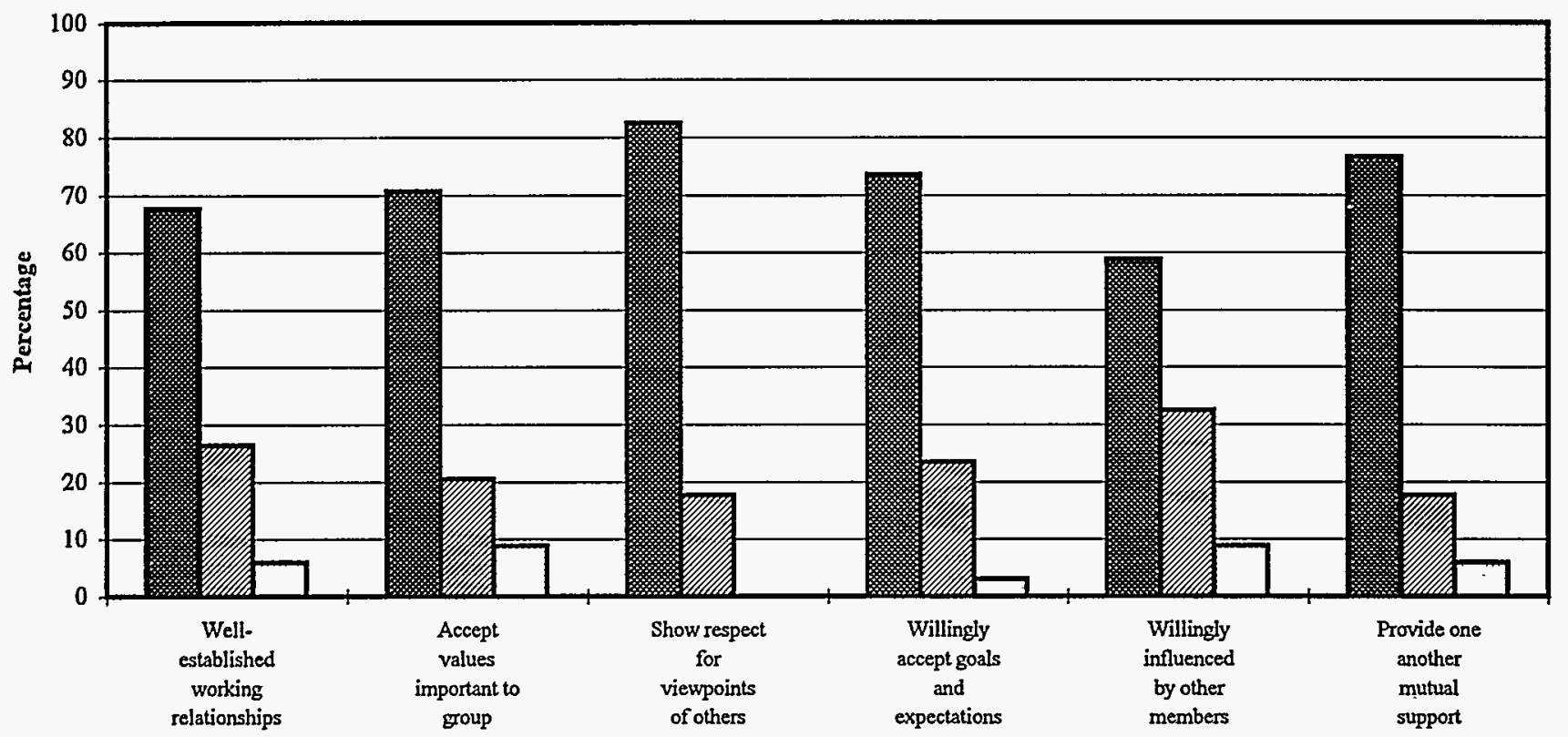

Agree (\%) ENeither Agree Nor Disagree/Don't Know (\%) DDisagree (\%)

Figure 11b. Long Survey: Entire Sample

Working relations

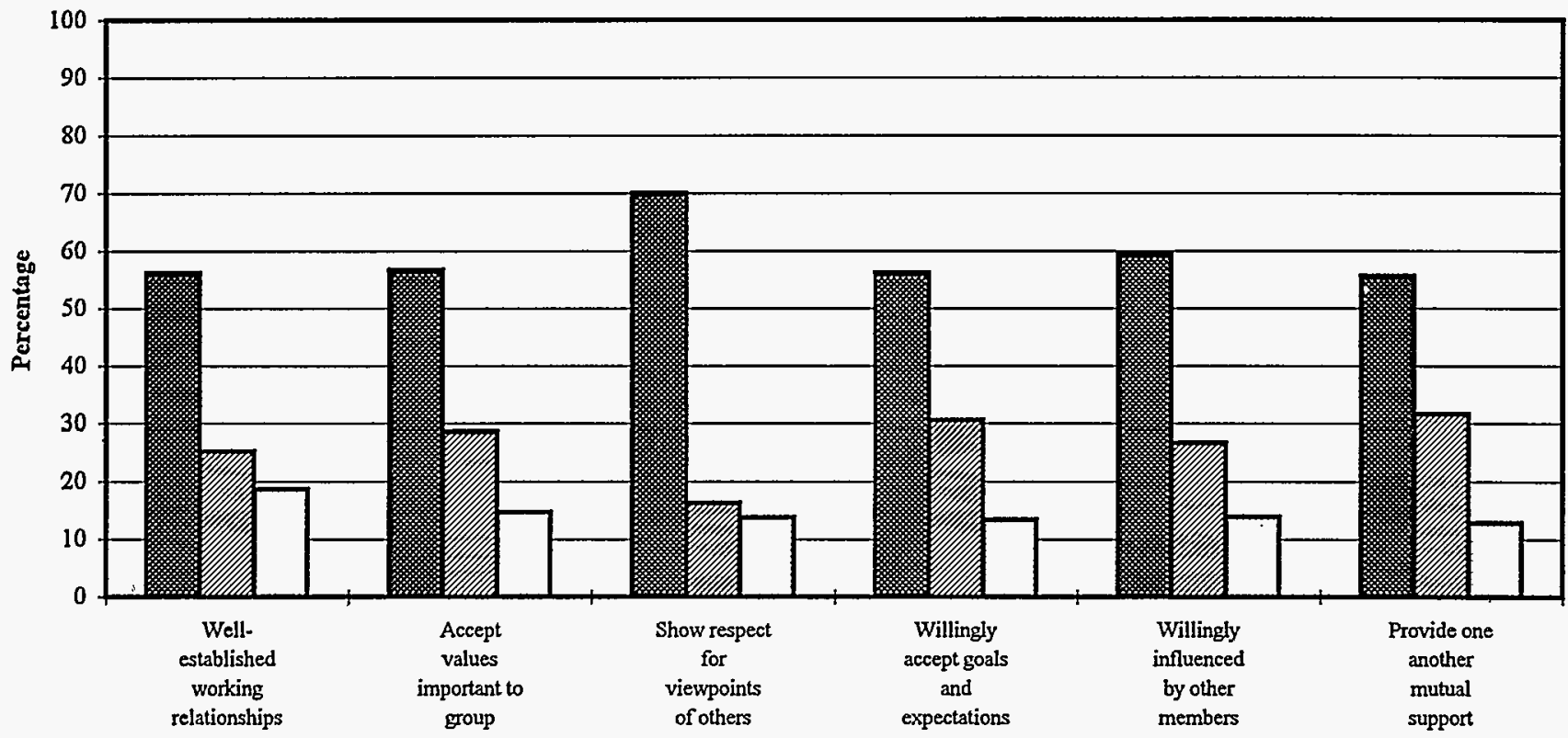

Agree (\%) Deither Agree Nor Disagree/Dont Know (\%) DDisagree (\%)

SSAB Supplementary Appendix 
Figure 12a. Idaho

Member affect

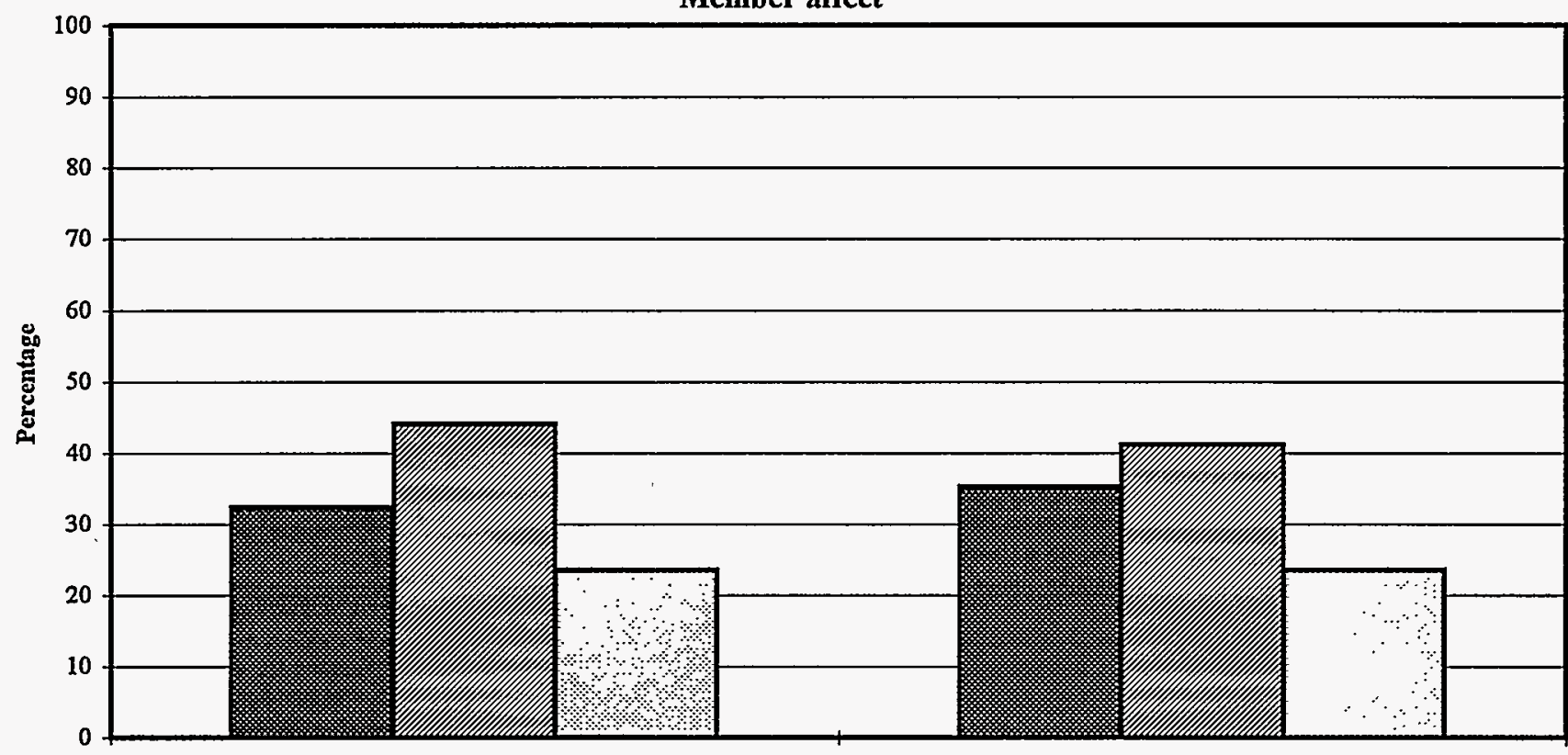

Like each other

High trust and confidence in each other

G Agree (\%) WNeither Agree Nor Disagree/Don't Know (\%) DDisagree (\%)

Figure 12b. Long Survey: Entire Sample

Member affect

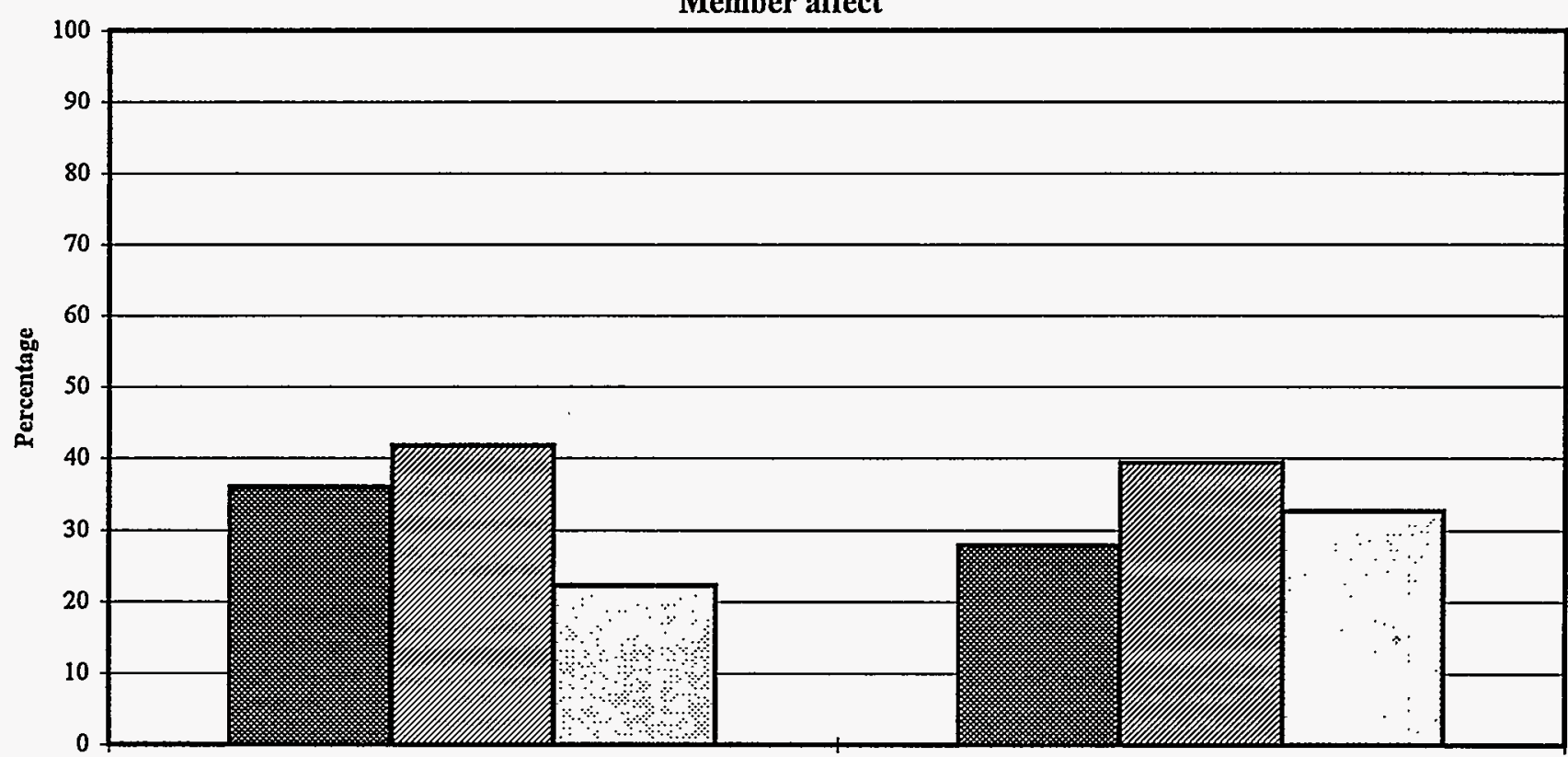

Like each other

High trust and confidence in each other

Agree (\%) Neither Agree Nor Disagree/Don't Know (\%) DDisagree (\%)

SSAB Supplementary Appendix

August 1996 
Figure 13a. Idaho

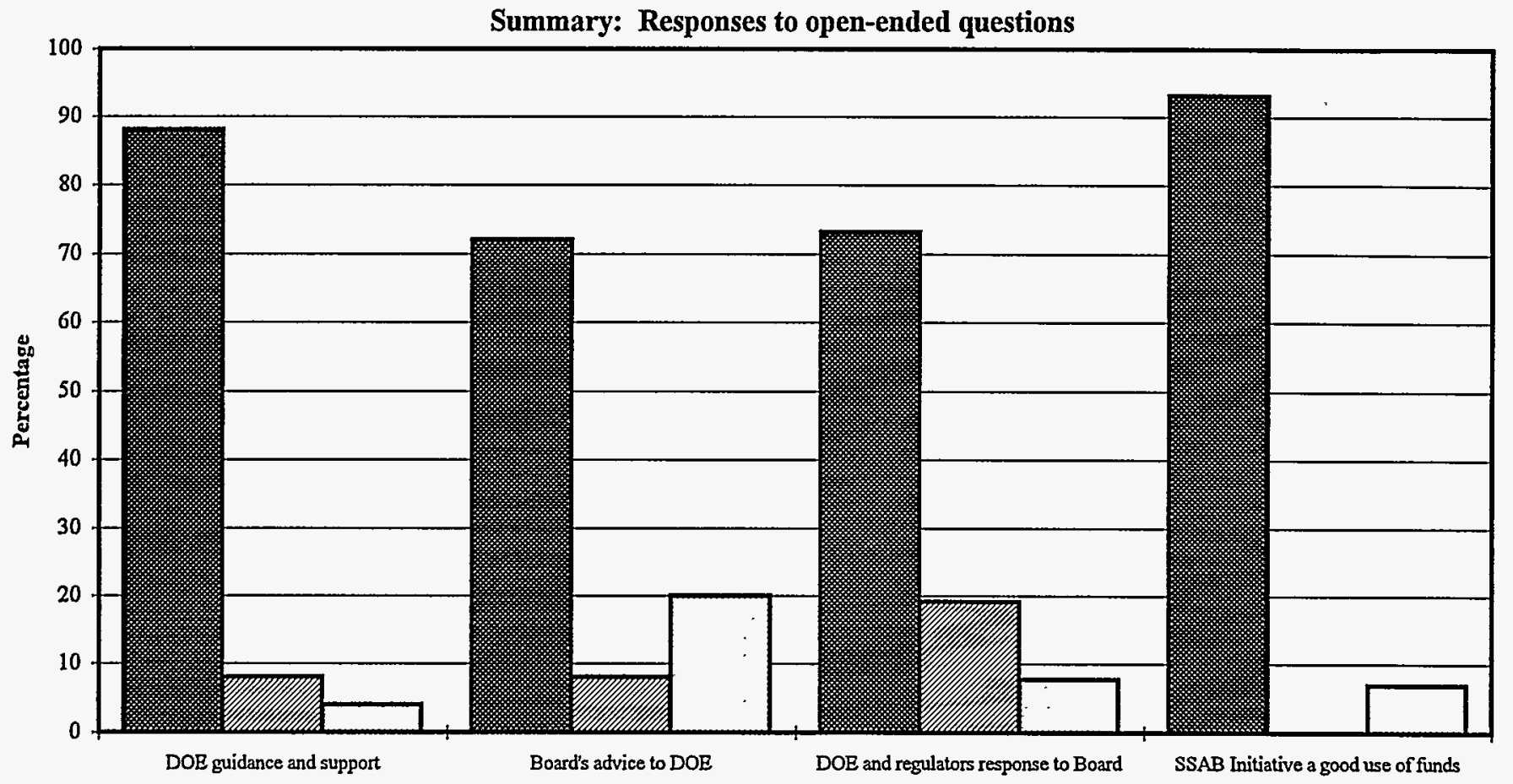

EYes, Good (\%) Neutral, Conditional, No Opinion (\%) DNo, Not Good (\%)

Figure 13b. Long Survey: Entire Sample

Summary: Responses to open-ended questions

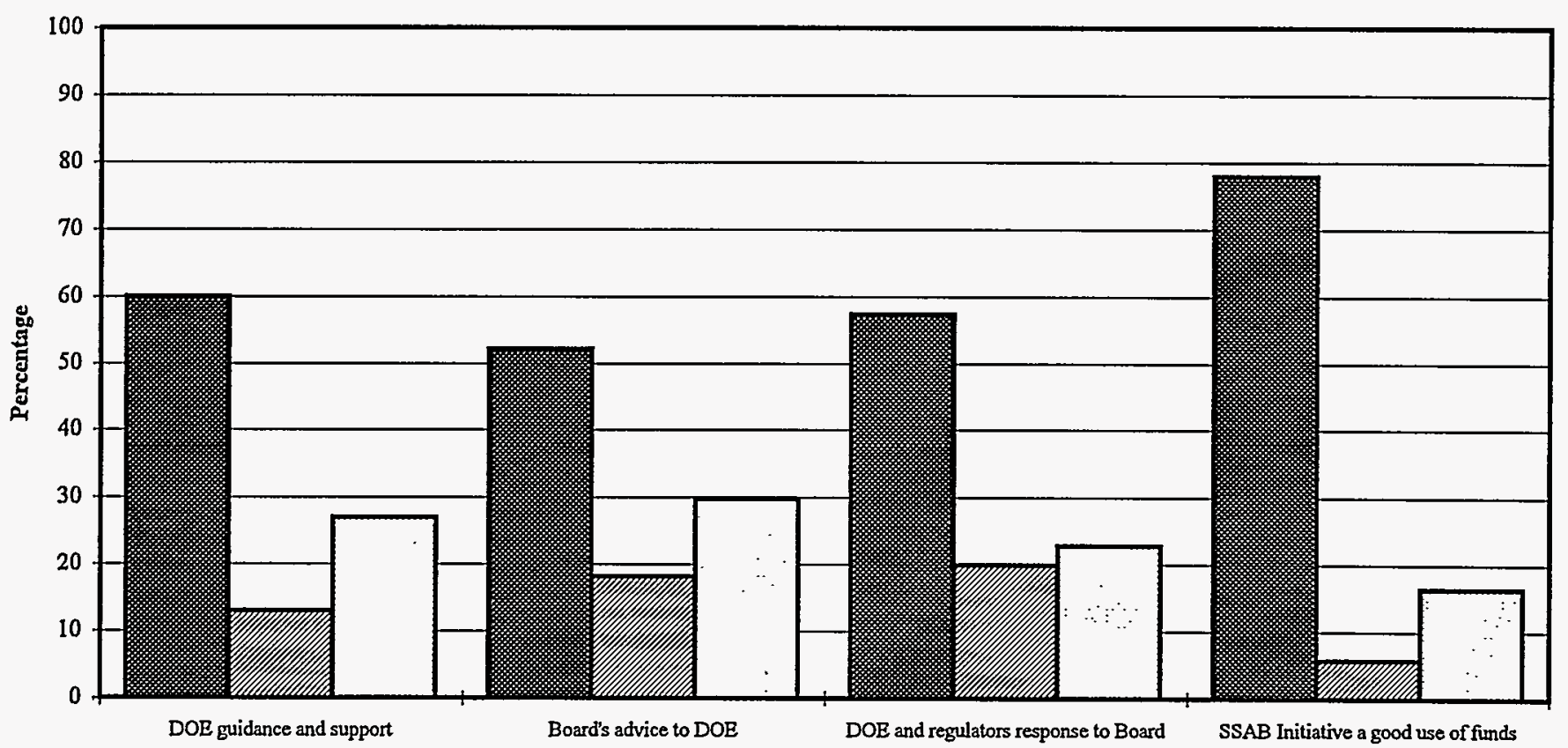

W Yes, Good (\%) DNeutral, Conditional, No Opinion (\%) DNo, Not Good (\%)

SSAB Supplementary Appendix

August 1996 
Figure 14a. Idaho

Summary: Combined responses for SSAB Initiative goals (Means)

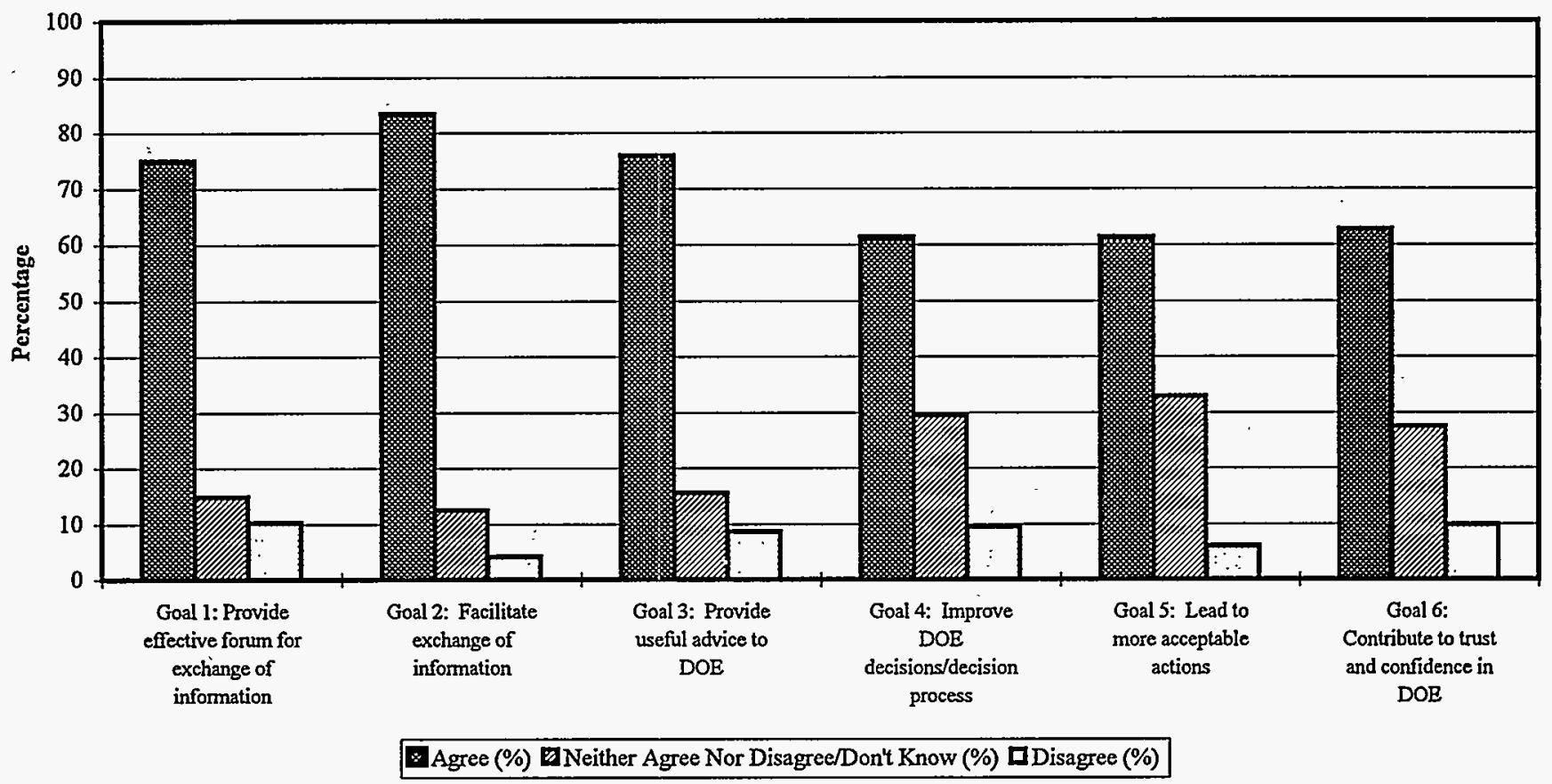

Figure 14b. Long Survey: Entire Sample

Summary: Combined responses for SSAB Initiative goals (Means)

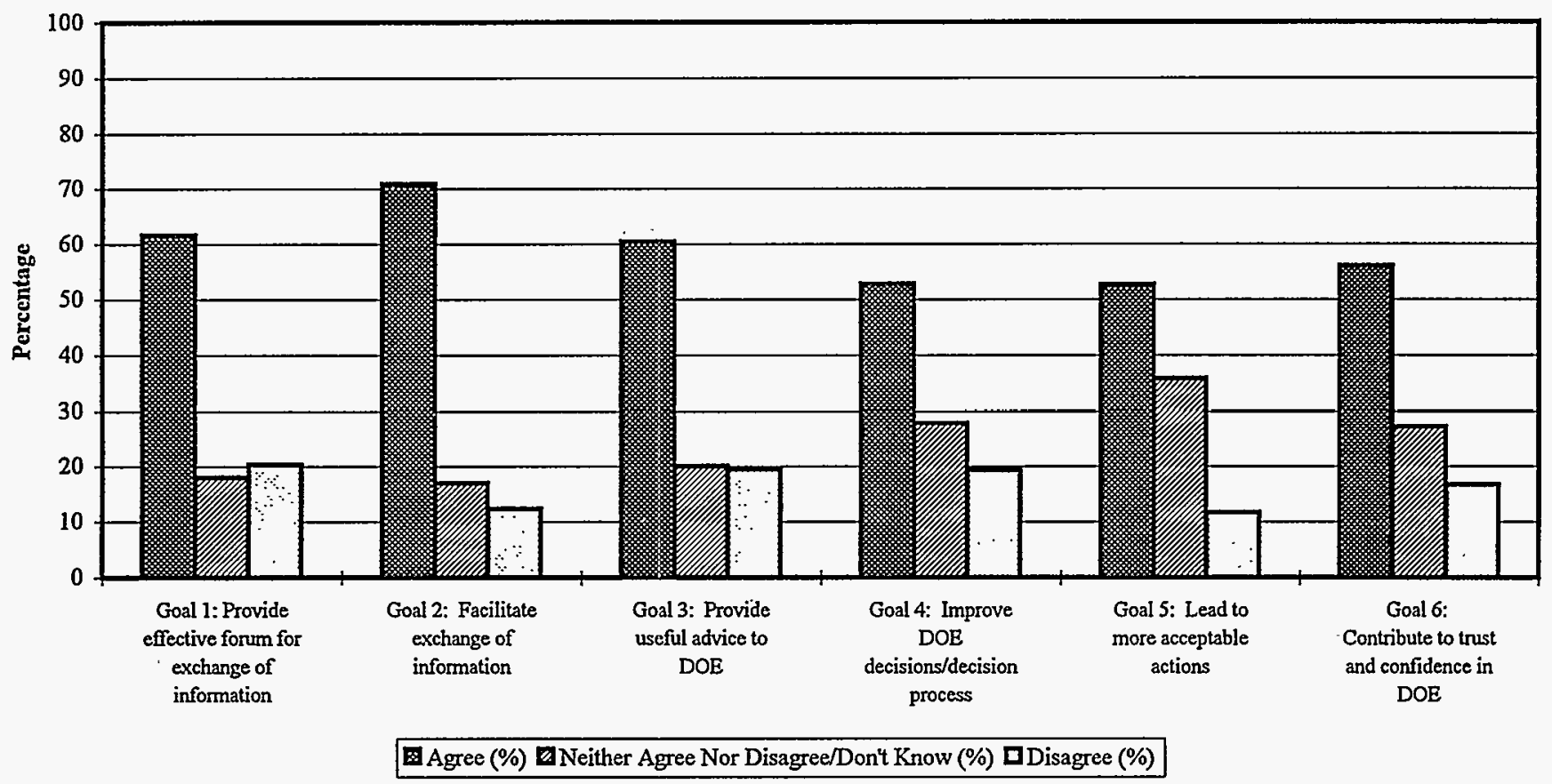

SSAB Supplementary Appendix 
Figure 15a. Idaho

Summary: Combined responses on DOE-HQ involvement,

Personal experience, Group/Team work skills,

Working relations, Member affect (Means)

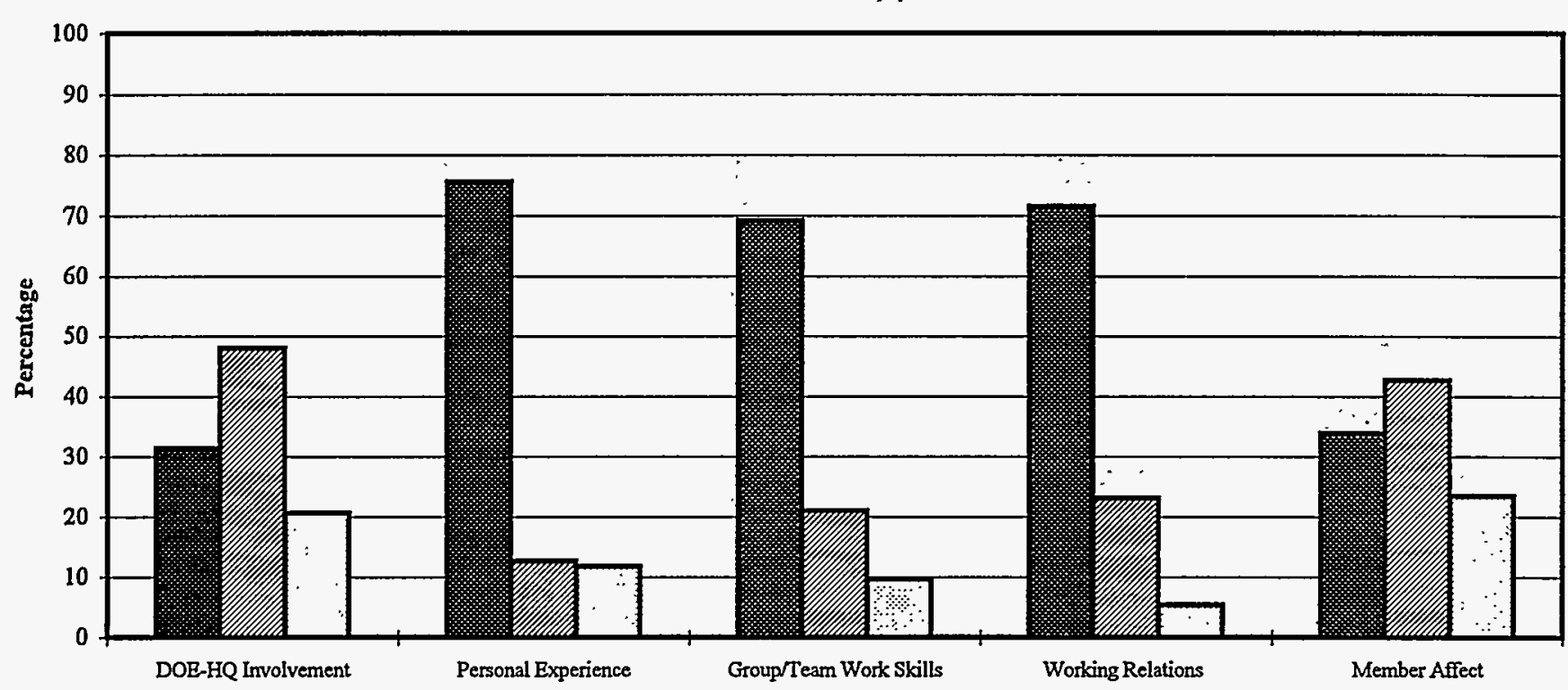

Agree (\%) ENeither Agree Nor Disagree/Don't Know (\%) DDisagree (\%)

Figure 15b. Long Survey: Entire Sample

Summary: Combined responses on DOE-HQ involvement,

Personal experience, Group/Team work skills,

Working relations, Member affect (Means)

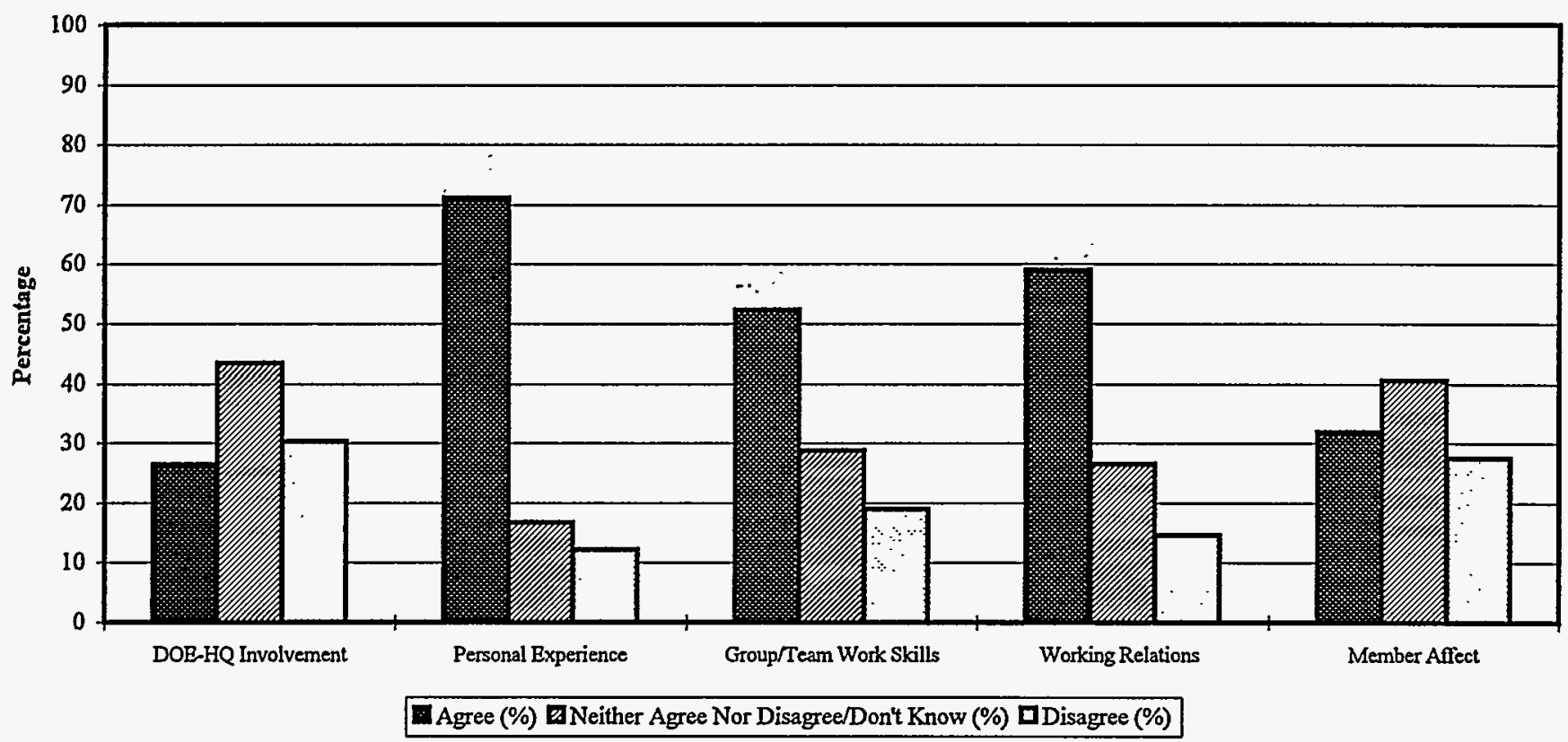

SSAB Supplementary Appendix 
Table 1.

Goal 1. Establish processes and procedures to provide an effective forum for exchange of information and viewpoints regarding DOE site issues

\begin{tabular}{|c|c|c|c|c|c|c|c|c|}
\hline \multirow[t]{2}{*}{ Idaho } & \multicolumn{2}{|c|}{ Agree } & \multicolumn{2}{|c|}{$\begin{array}{l}\text { Neither/ } \\
\text { Don't Know }\end{array}$} & \multicolumn{2}{|c|}{ Disagree } & \multicolumn{2}{|c|}{ Total } \\
\hline & $\mathbf{N}$ & $\%$ & $\mathbf{N}$ & $\%$ & $\mathrm{~N}$ & $\%$ & $\mathrm{~N}$ & $\%$ \\
\hline $\begin{array}{l}\text { The Board has established processes and } \\
\text { procedures for the effective exchange of } \\
\text { information }\end{array}$ & 32 & $94.1 \%$ & 1 & $2.9 \%$ & 1 & $2.9 \%$ & 34 & $100 \%$ \\
\hline $\begin{array}{l}\text { The Board has agreed-upon procedures to } \\
\text { operate the board }\end{array}$ & 32 & $97.0 \%$ & 1 & $3.0 \%$ & 0 & $0 \%$ & 33 & $100 \%$ \\
\hline $\begin{array}{l}\text { The Board has adequate support to allow } \\
\text { it to focus on substantive issues (R) } *\end{array}$ & 26 & $78.8 \%$ & 4 & $12.1 \%$ & 3 & $9.1 \%$ & 33 & $100 \%$ \\
\hline $\begin{array}{l}\text { The Board provides sufficient time for } \\
\text { discussion of issues }\end{array}$ & 25 & $75.8 \%$ & 4 & $12.1 \%$ & 4 & $12.1 \%$ & 33 & $100 \%$ \\
\hline The Board has effective leadership & 29 & $87.9 \%$ & 2 & $6.1 \%$ & 2 & $6.1 \%$ & 33 & $100 \%$ \\
\hline $\begin{array}{l}\text { DOE requests Board advice far enough } \\
\text { ahead of decision deadlines }(R) *\end{array}$ & 7 & $20.6 \%$ & 11 & $32.4 \%$ & 16 & $47.1 \%$ & 34 & $100 \%$ \\
\hline $\begin{array}{l}\text { DOE handles Board administrative issues } \\
\text { expeditiously (R) * }\end{array}$ & 15 & $44.1 \%$ & 16 & $47.1 \%$ & 3 & $8.8 \%$ & 34 & $100 \%$ \\
\hline $\begin{array}{l}\text { The SSAB staff provide satisfactory } \\
\text { support }\end{array}$ & 29 & $85.3 \%$ & 4 & $11.8 \%$ & 1 & $2.9 \%$ & 34 & $100 \%$ \\
\hline $\begin{array}{l}\text { The facilitator for the Board has helped } \\
\text { the Board function effectively }\end{array}$ & 31 & $91.2 \%$ & 2 & $5.9 \%$ & 1 & $2.9 \%$ & 34 & $100 \%$ \\
\hline
\end{tabular}

* (R) denotes a question that was asked in the negative form. For ease of review, these questions and data have been reversed in this table and are stated in positive forms.

SSAB Supplementary Appendix

August 1996 
Table 2.

Goal 2. Facilitate interaction and exchange of information and viewpoints regarding DOE site issues

\begin{tabular}{|c|c|c|c|c|c|c|c|c|}
\hline \multirow[t]{2}{*}{ Idaho } & \multicolumn{2}{|c|}{ Agree } & \multicolumn{2}{|c|}{$\begin{array}{l}\text { Neither/ } \\
\text { Don't Know }\end{array}$} & \multicolumn{2}{|c|}{ Disagree } & \multicolumn{2}{|c|}{ Total } \\
\hline & $\mathrm{N}$ & $\%$ & $\mathrm{~N}$ & $\%$ & $\mathrm{~N}$ & $\%$ & $\mathrm{~N}$ & $\%$ \\
\hline $\begin{array}{l}\text { The SSAB facilitates effective exchange } \\
\text { of viewpoints on site issues }\end{array}$ & 32 & $94.1 \%$ & 1 & $2.9 \%$ & 1 & $2.9 \%$ & 34 & $100 \%$ \\
\hline $\begin{array}{l}\text { The SSAB contributes to Board members' } \\
\text { understanding of the basis for key site } \\
\text { decisions }\end{array}$ & 31 & $91.2 \%$ & 3 & $8.8 \%$ & 0 & $0 \%$ & 34 & $100 \%$ \\
\hline $\begin{array}{l}\text { The SSAB contributes to DOE and } \\
\text { regulators' understanding of the public's } \\
\text { viewpoints on key site decisions }\end{array}$ & 27 & $79.4 \%$ & 5 & $14.7 \%$ & 2 & $5.9 \%$ & 34 & $100 \%$ \\
\hline $\begin{array}{l}\text { The SSAB contributes to a constructive } \\
\text { working relationship among the } \\
\text { participants }\end{array}$ & 31 & $91.2 \%$ & 2 & $5.9 \%$ & 1 & $2.9 \%$ & 34 & $100 \%$ \\
\hline The SSAB strives for consensus & 31 & $91.2 \%$ & 1 & $2.9 \%$ & 2 & $5.9 \%$ & 34 & $100 \%$ \\
\hline $\begin{array}{l}\text { The SSAB gives fair consideration to } \\
\text { dissenting opinions }\end{array}$ & 31 & $91.2 \%$ & 1 & $2.9 \%$ & 2 & $5.9 \%$ & 34 & $100 \%$ \\
\hline $\begin{array}{l}\text { The Board has made the effort needed to } \\
\text { learn about site issues }\end{array}$ & 29 & $87.9 \%$ & 3 & $9.1 \%$ & 1 & $3.0 \%$ & 33 & $100 \%$ \\
\hline $\begin{array}{l}\text { The Board invites expert advice into its } \\
\text { discussion on key policy issues }\end{array}$ & 27 & $81.8 \%$ & 5 & $15.2 \%$ & 1 & $3.0 \%$ & 33 & $100 \%$ \\
\hline $\begin{array}{l}\text { The Board responds to public inquiries } \\
\text { and comments about its decisions }\end{array}$ & 23 & $69.7 \%$ & 10 & $30.3 \%$ & 0 & $0 \%$ & 33 & $100 \%$ \\
\hline $\begin{array}{l}\text { The Board solicits feedback from the } \\
\text { community on its work }\end{array}$ & 20 & $60.6 \%$ & 10 & $30.3 \%$ & 3 & $9.1 \%$ & 33 & $100 \%$ \\
\hline $\begin{array}{l}\text { DOE makes information on key site issues } \\
\text { readily available to the Board }\end{array}$ & 31 & $91.2 \%$ & 3 & $8.8 \%$ & 0 & $0 \%$ & 34 & $100 \%$ \\
\hline $\begin{array}{l}\text { The regulators make important } \\
\text { contributions to the Board's work }\end{array}$ & 23 & $67.6 \%$ & 8 & $23.5 \%$ & 3 & $8.8 \%$ & 34 & $100 \%$ \\
\hline $\begin{array}{l}\text { The SSAB creates a climate supportive of } \\
\text { differing viewpoints }(\mathrm{R}) *\end{array}$ & 30 & $88.2 \%$ & 2 & $5.9 \%$ & 2 & $5.9 \%$ & 34 & $100 \%$ \\
\hline
\end{tabular}

* (R) denotes a question that was asked in the negative form. For ease of review, these questions and data have been reversed in this table and are stated in positive forms.

SSAB Supplementary Appendix

August 1996 
Table 3.

Goal 3. Provide useful advice and/or recommendations to DOE (and regulators, where appropriate)

\begin{tabular}{|c|c|c|c|c|c|c|c|c|}
\hline \multirow[t]{2}{*}{ Idaho } & \multicolumn{2}{|c|}{ Agree } & \multicolumn{2}{|c|}{$\begin{array}{l}\text { Neither/ } \\
\text { Don't Know }\end{array}$} & \multicolumn{2}{|c|}{ Disagree } & \multicolumn{2}{|c|}{ Total } \\
\hline & $\mathrm{N}$ & $\%$ & $N$ & $\%$ & $N$ & $\%$ & $\mathrm{~N}$ & $\%$ \\
\hline $\begin{array}{l}\text { The Board provides useful advice to DOE } \\
\text { (and regulators, where appropriate) (R) * }\end{array}$ & 28 & $84.8 \%$ & 3 & $9.1 \%$ & 2 & $6.1 \%$ & 33 & $100 \%$ \\
\hline $\begin{array}{l}\text { The SSAB reaches agreement about } \\
\text { prioritization of key site issues for which } \\
\text { advice is sought }(\mathrm{R})^{*}\end{array}$ & 28 & $82.4 \%$ & 2 & $5.9 \%$ & 4 & $11.8 \%$ & 34 & $100 \%$ \\
\hline The Board helps define site problems & 22 & $66.7 \%$ & 9 & $27.3 \%$ & 2 & $6.1 \%$ & 33 & $100 \%$ \\
\hline $\begin{array}{l}\text { The Board addresses issues in a timely } \\
\text { manner }(R)^{*}\end{array}$ & 23 & $69.7 \%$ & 8 & $24.2 \%$ & 2 & $6.1 \%$ & 33 & $100 \%$ \\
\hline $\begin{array}{l}\text { The Board reaches consensus on key site } \\
\text { issues (R) * }\end{array}$ & 26 & $78.8 \%$ & 3 & $9.1 \%$ & 4 & $12.1 \%$ & 33 & $100 \%$ \\
\hline $\begin{array}{l}\text { The Board provides informed advice to } \\
\text { DOE (and regulators, where appropriate) }\end{array}$ & 27 & $81.8 \%$ & 4 & $12.1 \%$ & 2 & $6.1 \%$ & 33 & $100 \%$ \\
\hline $\begin{array}{l}\text { The Board provides advice that reflects } \\
\text { the viewpoints and priorities of the } \\
\text { community }\end{array}$ & 22 & $66.7 \%$ & 7 & $21.2 \%$ & 4 & $12.1 \%$ & 33 & $100 \%$ \\
\hline
\end{tabular}

* (R) denotes a question that was asked in the negátive form. For ease of review, these questions and data have been reversed in this table and are stated in positive forms.

SSAB Supplementary Appendix 
Table 4.

Goal 4. Improve DOE's (and where applicable, regulators') site decisions and decision making process

\begin{tabular}{|c|c|c|c|c|c|c|c|c|}
\hline \multirow[t]{2}{*}{ Idaho } & \multicolumn{2}{|c|}{ Agree } & \multicolumn{2}{|c|}{$\begin{array}{c}\text { Neither/ } \\
\text { Don't Know }\end{array}$} & \multicolumn{2}{|c|}{ Disagree } & \multicolumn{2}{|c|}{ Total } \\
\hline & $N$ & $\%$ & $N$ & $\%$ & $N$ & $\%$ & $N$ & $\%$ \\
\hline $\begin{array}{l}\text { The SSAB has improved DOE's site } \\
\text { decisions (R) * }\end{array}$ & 19 & $57.6 \%$ & 12 & $36.4 \%$ & 2 & $6.1 \%$ & 33 & $100 \%$ \\
\hline $\begin{array}{l}\text { The Board understands the decision } \\
\text { making process at [a particular] site }\end{array}$ & 13 & $39.4 \%$ & 16 & $48.5 \%$ & 4 & $12.1 \%$ & 33 & $100 \%$ \\
\hline $\begin{array}{l}\text { DOE discusses important policies } \\
\text { affecting site decisions with the Board }\end{array}$ & 30 & $88.2 \%$ & 3 & $8.8 \%$ & 1 & $2.9 \%$ & 34 & $100 \%$ \\
\hline $\begin{array}{l}\text { DOE has explained to the Board its site } \\
\text { decision making process }\end{array}$ & 20 & $58.8 \%$ & 10 & $29.4 \%$ & 4 & $11.8 \%$ & 34 & $100 \%$ \\
\hline $\begin{array}{l}\text { The DOE shows how Board advice is } \\
\text { reflected in site decisions }\end{array}$ & 19 & $55.9 \%$ & 11 & $32.4 \%$ & 4 & $11.8 \%$ & 34 & $100 \%$ \\
\hline $\begin{array}{l}\text { The decision making process used by } \\
\text { DOE at [a particular] site is effective in } \\
\text { furthering site clean-up }(\mathrm{R}) *\end{array}$ & 23 & $67.6 \%$ & 7 & $20.6 \%$ & 4 & $11.8 \%$ & 34 & $100 \%$ \\
\hline
\end{tabular}

* (R) denotes a question that was asked in the negative form. For ease of review, these questions and data have been reversed in this table and are stated in positive forms. 
Table 5.

Goal 5. Lead to more acceptable actions

\begin{tabular}{|c|c|c|c|c|c|c|c|c|}
\hline \multirow[t]{2}{*}{ Idaho } & \multicolumn{2}{|c|}{ Agree } & \multicolumn{2}{|c|}{$\begin{array}{l}\text { Neither/ } \\
\text { Don't Know }\end{array}$} & \multicolumn{2}{|c|}{ Disagree } & \multicolumn{2}{|c|}{ Total } \\
\hline & $N$ & $\%$ & $\mathrm{~N}$ & $\%$ & $\mathrm{~N}$ & $\%$ & $\mathrm{~N}$ & $\%$ \\
\hline $\begin{array}{l}\text { The SSAB leads to more acceptable site } \\
\text { decisions }\end{array}$ & 18 & $54.5 \%$ & 14 & $42.4 \%$ & 1 & $3.0 \%$ & 33 & $100 \%$ \\
\hline $\begin{array}{l}\text { The Board supports the recommendations } \\
\text { it gives DOE }\end{array}$ & 27 & $81.8 \%$ & 5 & $15.2 \%$ & 1 & $3.0 \%$ & 33 & $100 \%$ \\
\hline The Board supports DOE's site actions & 19 & $57.6 \%$ & 12 & $36.4 \%$ & 2 & $6.1 \%$ & 33 & $100 \%$ \\
\hline $\begin{array}{l}\text { The DOE pays attention to the Board's } \\
\text { advice on key site issues }(R)^{*}\end{array}$ & 22 & $64.7 \%$ & 11 & $32.4 \%$ & 1 & $2.9 \%$ & 34 & $100 \%$ \\
\hline $\begin{array}{l}\text { There is support in the community for } \\
\text { DOE's site decisions that have SSAB } \\
\text { input }\end{array}$ & 10 & $29.4 \%$ & 20 & $58.8 \%$ & 4 & $11.8 \%$ & 34 & $100 \%$ \\
\hline Progress is being made on key site issues & 27 & $79.4 \%$ & 4 & $11.8 \%$ & 3 & $8.8 \%$ & 34 & $100 \%$ \\
\hline
\end{tabular}

* (R) denotes a question that was asked in the negative form. For ease of review, these questions and data have been reversed in this table and are stated in positive forms. 


\section{Table 6.}

Goal 6. Contribute to trust and confidence in DOE

\begin{tabular}{|c|c|c|c|c|c|c|c|c|}
\hline \multirow[t]{2}{*}{ Idaho } & \multicolumn{2}{|c|}{ Agree } & \multicolumn{2}{|c|}{$\begin{array}{l}\text { Neither/ } \\
\text { Don't Know }\end{array}$} & \multicolumn{2}{|c|}{ Disagree } & \multicolumn{2}{|c|}{ Total } \\
\hline & $\mathrm{N}$ & $\%$ & $N$ & $\%$ & $\mathrm{~N}$ & $\%$ & $\mathrm{~N}$ & $\%$ \\
\hline $\begin{array}{l}\text { The SSAB contributes to trust and } \\
\text { confidence in DOE }\end{array}$ & 23 & $67.6 \%$ & 8 & $23.5 \%$ & 3 & $8.8 \%$ & 34 & $100 \%$ \\
\hline $\begin{array}{l}\text { Relationships between DOE and the public } \\
\text { have improved since the formation of the } \\
\text { SSAB }\end{array}$ & 18 & $52.9 \%$ & 14 & $41.2 \%$ & 2 & $5.9 \%$ & 34 & $100 \%$ \\
\hline $\begin{array}{l}\text { DOE is committed to clean up [a } \\
\text { particular] site }\end{array}$ & 23 & $67.6 \%$ & 6 & $17.6 \%$ & 5 & $14.7 \%$ & 34 & $100 \%$ \\
\hline
\end{tabular}


Table 7.

Public awareness

\begin{tabular}{|c|c|c|c|c|c|c|c|c|}
\hline \multirow[t]{2}{*}{ Idaho } & \multicolumn{2}{|c|}{ Agree } & \multicolumn{2}{|c|}{$\begin{array}{c}\text { Neither/ } \\
\text { Don't Know }\end{array}$} & \multicolumn{2}{|c|}{ Disagree } & \multicolumn{2}{|c|}{ Total } \\
\hline & $\mathrm{N}$ & $\%$ & $N$ & $\%$ & $N$ & $\%$ & $N$ & $\%$ \\
\hline $\begin{array}{l}\text { The public knows little about the role of } \\
\text { the Board }\end{array}$ & 25 & $73.5 \%$ & 6 & $17.6 \%$ & 3 & $8.8 \%$ & 34 & $100 \%$ \\
\hline
\end{tabular}

Table 8.

SSAB Initiative is a good use of funds

\begin{tabular}{ccccccccc}
\hline Idaho & Yes, Good & $\begin{array}{c}\text { Neutral, } \\
\text { Conditional, } \\
\text { No Opinion }\end{array}$ & $\begin{array}{c}\text { No, } \\
\text { Not Good }\end{array}$ & Total \\
& $\mathrm{N}$ & $\%$ & $\mathrm{~N}$ & $\%$ & $\mathrm{~N}$ & $\%$ & $\mathrm{~N}$ & $\%$ \\
\hline SSAB Initiative is a good use of funds & 27 & $93.1 \%$ & 0 & $0 \%$ & 2 & $6.9 \%$ & 29 & $100 \%$ \\
\hline
\end{tabular}


Table 9.

DOE-HQ involvement with the SSAB Initiative

\begin{tabular}{|c|c|c|c|c|c|c|c|c|}
\hline \multirow[t]{2}{*}{ Idaho } & \multicolumn{2}{|c|}{ Agree } & \multicolumn{2}{|c|}{$\begin{array}{c}\text { Neither/ } \\
\text { Don't Know }\end{array}$} & \multicolumn{2}{|c|}{ Disagree } & \multicolumn{2}{|c|}{ Total } \\
\hline & $\mathrm{N}$ & $\%$ & $\mathrm{~N}$ & $\%$ & $\mathrm{~N}$ & $\%$ & $\mathrm{~N}$ & $\%$ \\
\hline $\begin{array}{l}\text { DOE-HQ provides helpful guidance to the } \\
\text { Boards and to DOE }\end{array}$ & 12 & $35.3 \%$ & 11 & $32.4 \%$ & 11 & $32.4 \%$ & 34 & $100 \%$ \\
\hline $\begin{array}{l}\text { DOE-HQ provides sufficient support to } \\
\text { facilitate the work of the SSAB }\end{array}$ & 14 & $41.2 \%$ & 16 & $47.1 \%$ & 4 & $11.8 \%$ & 34 & $100 \%$ \\
\hline $\begin{array}{l}\text { DOE-HQ gives careful consideration to } \\
\text { SSAB advice in its decisions }\end{array}$ & 6 & $17.6 \%$ & 22 & $64.7 \%$ & 6 & $17.6 \%$ & 34 & $100 \%$ \\
\hline
\end{tabular}


Table 10.

Personal experience with the SSAB Initiative

\begin{tabular}{|c|c|c|c|c|c|c|c|c|}
\hline \multirow[t]{2}{*}{ Idaho } & \multicolumn{2}{|c|}{ Agree } & \multicolumn{2}{|c|}{$\begin{array}{l}\text { Neither/ } \\
\text { Don't Know }\end{array}$} & \multicolumn{2}{|c|}{ Disagree } & \multicolumn{2}{|c|}{ Total } \\
\hline & $\mathrm{N}$ & $\%$ & $\mathbf{N}$ & $\%$ & $N$ & $\%$ & $\mathrm{~N}$ & $\%$ \\
\hline $\begin{array}{l}\text { I consider myself to be very } \\
\text { knowledgeable about site issues }\end{array}$ & 25 & $73.5 \%$ & 6 & $17.6 \%$ & 3 & $8.8 \%$ & 34 & $100 \%$ \\
\hline $\begin{array}{l}\text { Because of the SSAB, I better understand } \\
\text { the complexities of site clean-up }\end{array}$ & 24 & $70.6 \%$ & 4 & $11.8 \%$ & 6 & $17.6 \%$ & 34 & $100 \%$ \\
\hline $\begin{array}{l}\text { I feel that my participation in the SSAB } \\
\text { has been worthwhile }(\mathrm{R}) *\end{array}$ & 28 & $82.4 \%$ & 3 & $8.8 \%$ & 3 & $8.8 \%$ & 34 & $100 \%$ \\
\hline
\end{tabular}

* (R) denotes a question that was asked in the negative form. For ease of review, these questions and data have been reversed in this table and are stated in positive forms. 
Table 11.

Group/Team work șkills

\begin{tabular}{|c|c|c|c|c|c|c|c|c|}
\hline \multirow[t]{2}{*}{ Idaho } & \multicolumn{2}{|c|}{ Agree } & \multicolumn{2}{|c|}{$\begin{array}{c}\text { Neither/ } \\
\text { Don't Know }\end{array}$} & \multicolumn{2}{|c|}{ Disagree } & \multicolumn{2}{|c|}{ Total } \\
\hline & $\mathrm{N}$ & $\%$ & $\mathrm{~N}$ & $\%$ & $\mathrm{~N}$ & $\%$ & $\mathbf{N}$ & $\%$ \\
\hline All have group/team work skills & 25 & $73.5 \%$ & 4 & $11.8 \%$ & 5 & $14.7 \%$ & 34 & $100 \%$ \\
\hline $\begin{array}{l}\text { Have developed well-established working } \\
\text { relationships with each other }\end{array}$ & 23 & $67.6 \%$ & 9 & $26.5 \%$ & 2 & $5.9 \%$ & 34 & $100 \%$ \\
\hline Like each other & 11 & $32.4 \%$ & 15 & $44.1 \%$ & 8 & $23.5 \%$ & 34 & $100 \%$ \\
\hline $\begin{array}{l}\text { Have a high degree of trust and } \\
\text { confidence in each other }\end{array}$ & 12 & $35.3 \%$ & 14 & $41.2 \%$ & 8 & $23.5 \%$ & 34 & $100 \%$ \\
\hline $\begin{array}{l}\text { Accept the values that are important to the } \\
\text { group }\end{array}$ & 24 & $70.6 \%$ & 7 & $20.6 \%$ & 3 & $8.8 \%$ & 34 & $100 \%$ \\
\hline Show respect for the viewpoints of others & 28 & $82.4 \%$ & 6 & $17.6 \%$ & 0 & $0 \%$ & 34 & $100 \%$ \\
\hline $\begin{array}{l}\text { Accept willingly the goals and } \\
\text { expectations for the Board }\end{array}$ & 25 & $73.5 \%$ & 8 & $23.5 \%$ & 1 & $2.9 \%$ & 34 & $100 \%$ \\
\hline $\begin{array}{l}\text { Set high expectations for what they } \\
\text { believe they can accomplish }\end{array}$ & 24 & $70.6 \%$ & 9 & $26.5 \%$ & 1 & $2.9 \%$ & 34 & $100 \%$ \\
\hline $\begin{array}{l}\text { Communicate fully and frankly to the } \\
\text { Board all relevant and valuable } \\
\text { information }\end{array}$ & 23 & $67.6 \%$ & 7 & $20.6 \%$ & 4 & $11.8 \%$ & 34 & $100 \%$ \\
\hline $\begin{array}{l}\text { Try not to waste the Board's time with } \\
\text { irrelevant material or communications }\end{array}$ & 21 & $61.8 \%$ & 8 & $23.5 \%$ & 5 & $14.7 \%$ & 34 & $100 \%$ \\
\hline $\begin{array}{l}\text { Are willing to be influenced by other } \\
\text { Board members about new ideas and } \\
\text { methods }\end{array}$ & 20 & $58.8 \%$ & 11 & $32.4 \%$ & 3 & $8.8 \%$ & 34 & $100 \%$ \\
\hline $\begin{array}{l}\text { Clearly understand the goals and } \\
\text { philosphy of the Board's operation }\end{array}$ & 25 & $73.5 \%$ & 8 & $23.5 \%$ & 1 & $2.9 \%$ & 34 & $100 \%$ \\
\hline $\begin{array}{l}\text { Do all they can to help the Board achieve } \\
\text { its objectives }\end{array}$ & 27 & $79.4 \%$ & 5 & $14.7 \%$ & 2 & $5.9 \%$ & 34 & $100 \%$ \\
\hline $\begin{array}{l}\text { Get the technical knowledge and training } \\
\text { in group skills they need }\end{array}$ & 20 & $58.8 \%$ & 9 & $26.5 \%$ & 5 & $14.7 \%$ & 34 & $100 \%$ \\
\hline Provide one another mutual help & 26 & $76.5 \%$ & 6 & $17.6 \%$ & 2 & $5.9 \%$ & 34 & $100 \%$ \\
\hline
\end{tabular}

SSAB Supplementary Appendix

August 1996 
Los Alamos Site Specific Advisory Board Evaluation Survey Results

SSAB Supplementary Appendix 
Figure 1a. Los Alamos

Goal 1. Establish processes and procedures to provide an effective forum

for exchange of information and viewpoints regarding DOE site issues

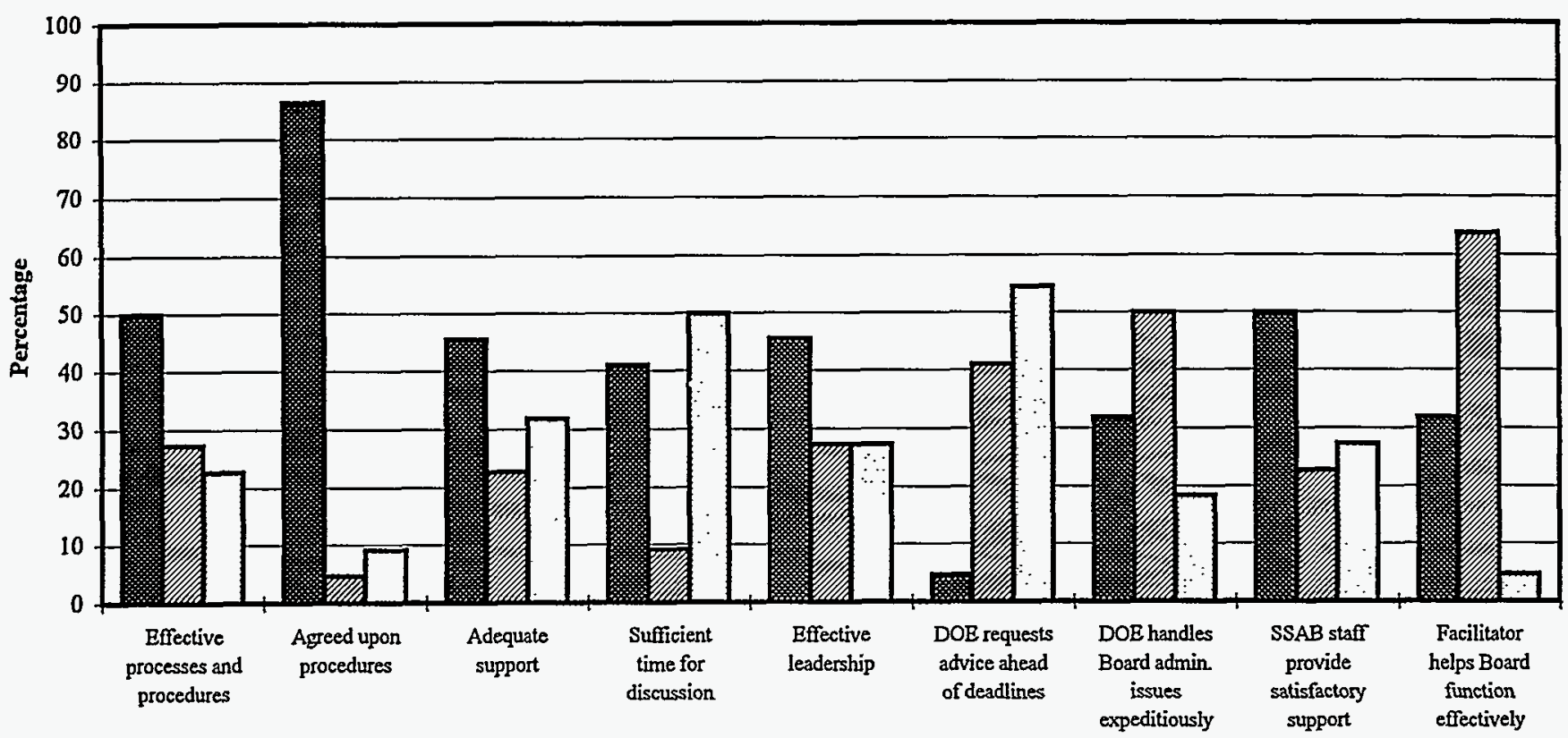

Agree (\%) BNeither Agree Nor Disagree/Don't Know (\%) DDisagree (\%)

Figure 1b. Long Survey: Entire Sample

Goal 1. Establish processes and procedures to provide an effective forum for exchange of information and viewpoints regarding DOE site issues

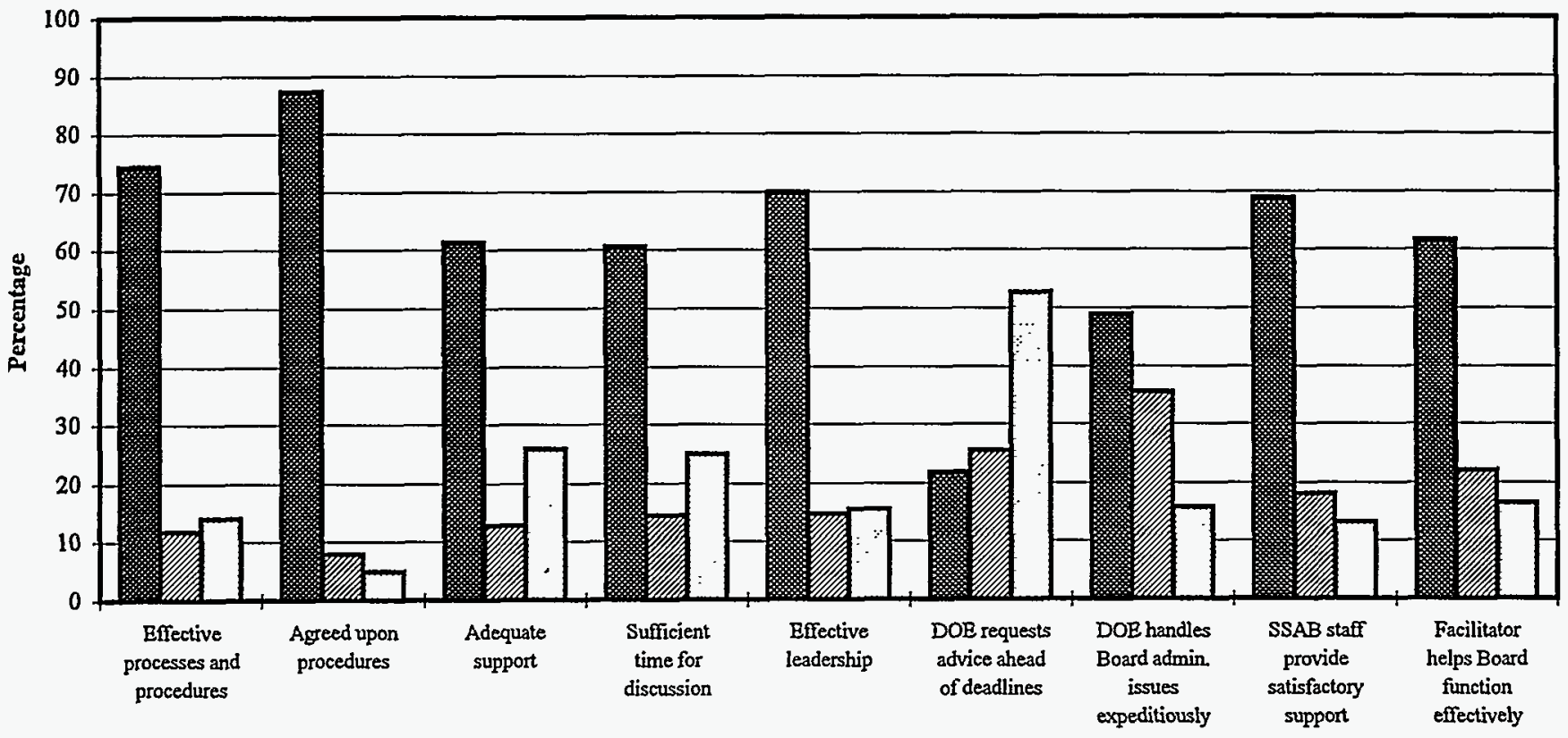

A Agree (\%) ENeither Agree Nor Disagree/Don't Know (\%) DDisagree (\%)

SSAB Supplementary Appendix

August 1996 
Figure 2a. Los Alamos

Goal 2. Facilitate interaction and exchange of information and viewpoints regarding $\mathrm{DOE}$ site issues

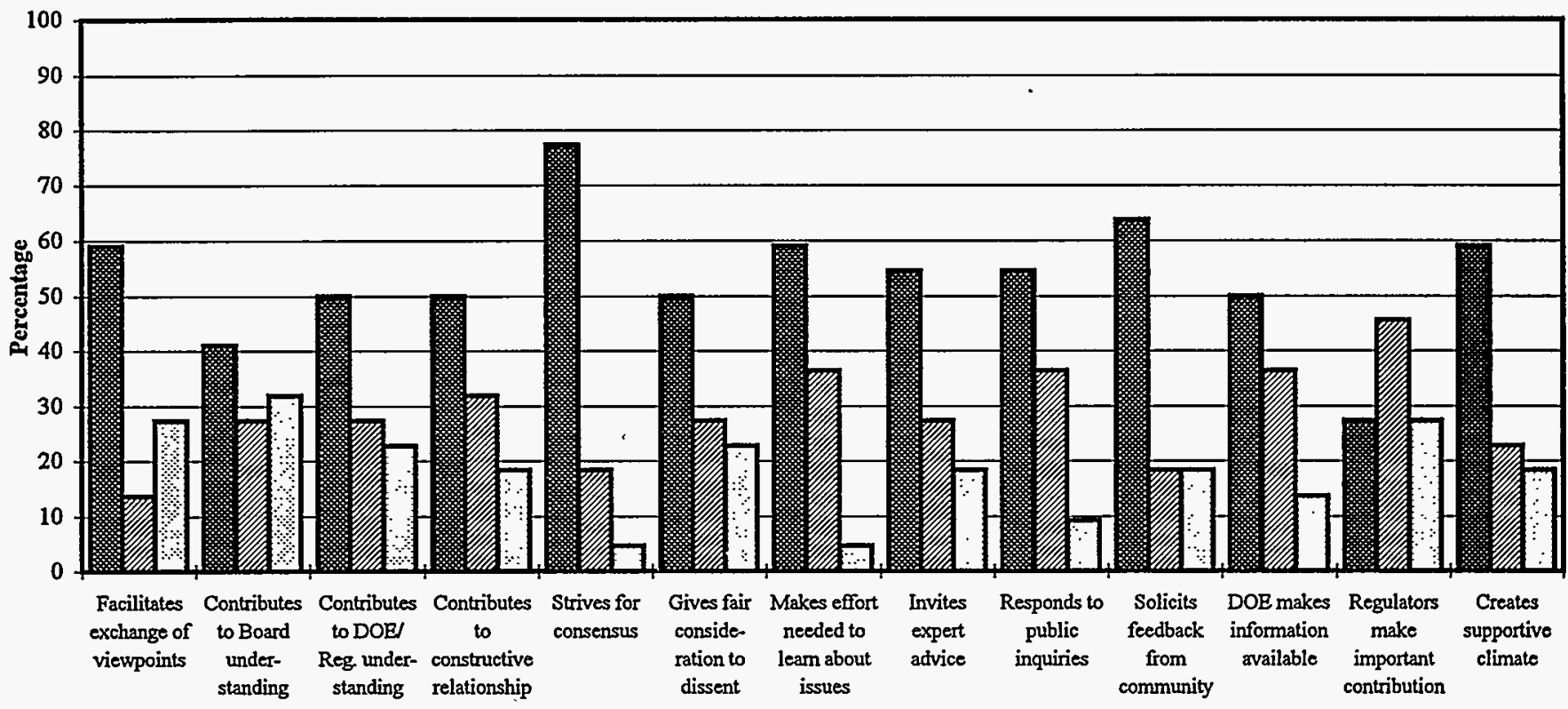

WAgree (\%) DNeither Agree Nor Disagree/Dont Know (\%) DDisagree (\%)

Figure 2b. Long Survey: Entire Sample

Goal 2. Facilitate interaction and exchange of information and viewpoints regarding $\mathrm{DOE}$ site issues

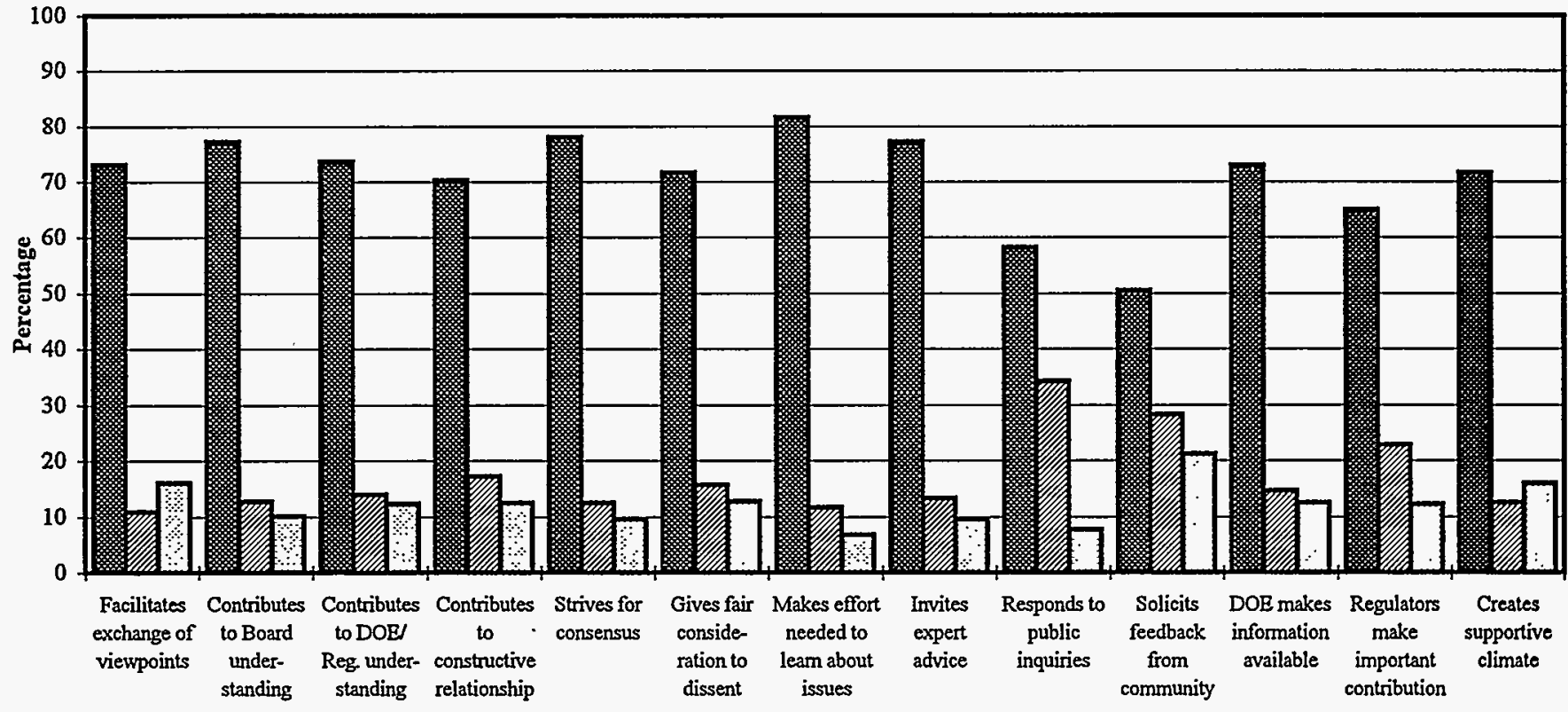

Q Agree (\%) Deither Agree Nor Disagree/Don't Know (\%) DDisagree (\%) 
Figure 3a. Los Alamos

Goal 3. Provide useful advice and/or recommendations to DOE

(and regulators, where appropriate)

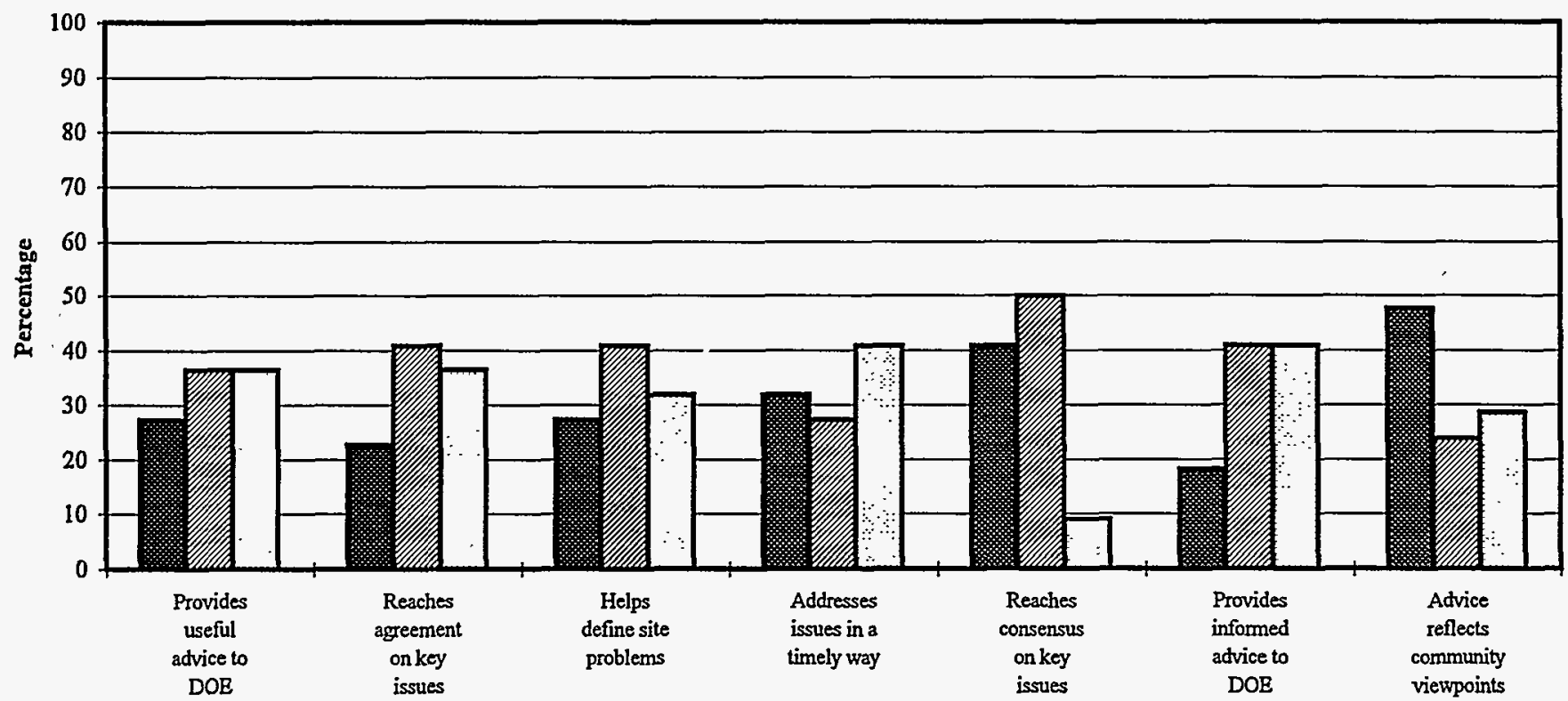

Agree (\%) Neither Agree Nor Disagree/Don't Know (\%) DDisagree (\%)

Figure 3b. Long Survey: Entire Sample

Goal 3. Provide useful advice and/or recommendations to DOE

(and regulators, where appropriate)

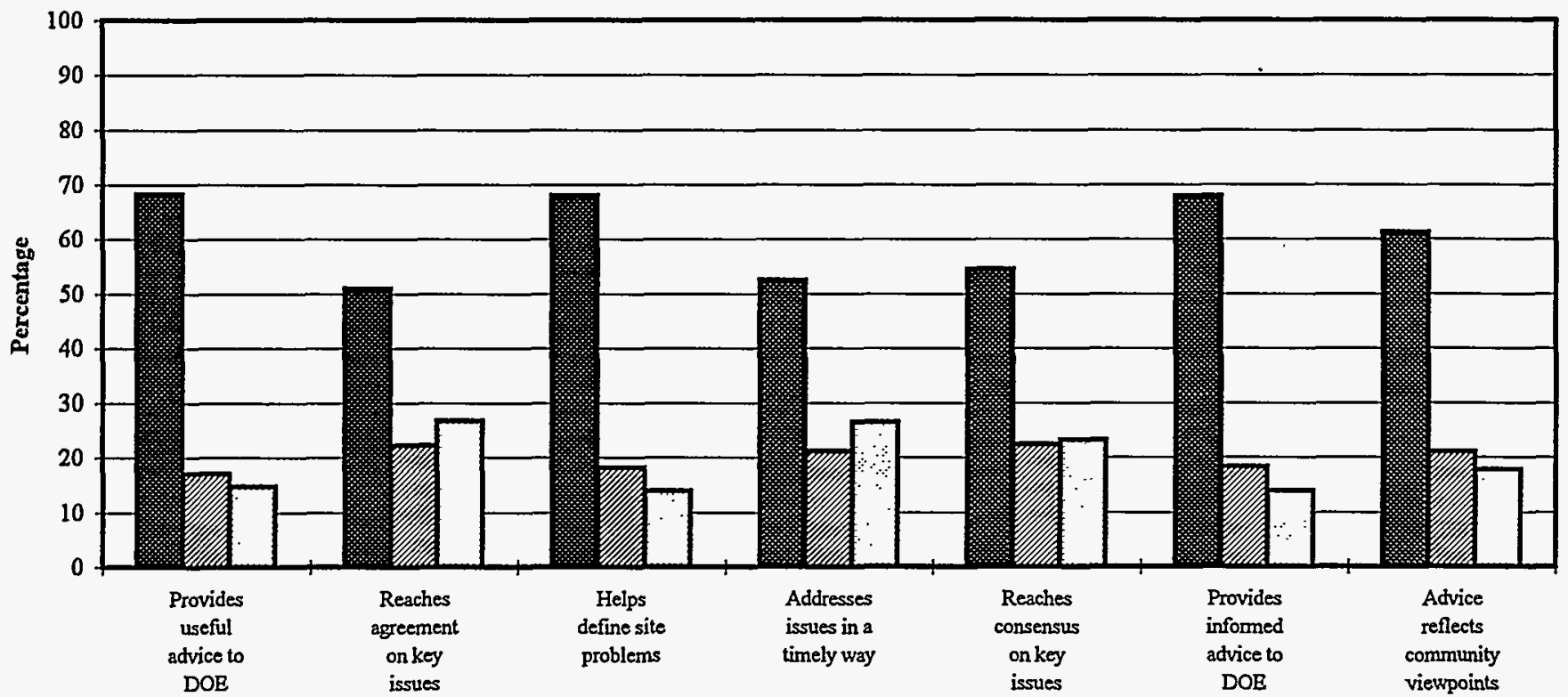

Agree (\%) $\mathbf{E}$ Neither Agree Nor Disagree/Don't Know (\%) DDisagree (\%)

SSAB Supplementary Appendix 
Figure 4a. Los Alamos

Goal 4. Improve DOE's (and where applicable, regulators') site decisions and decision making process

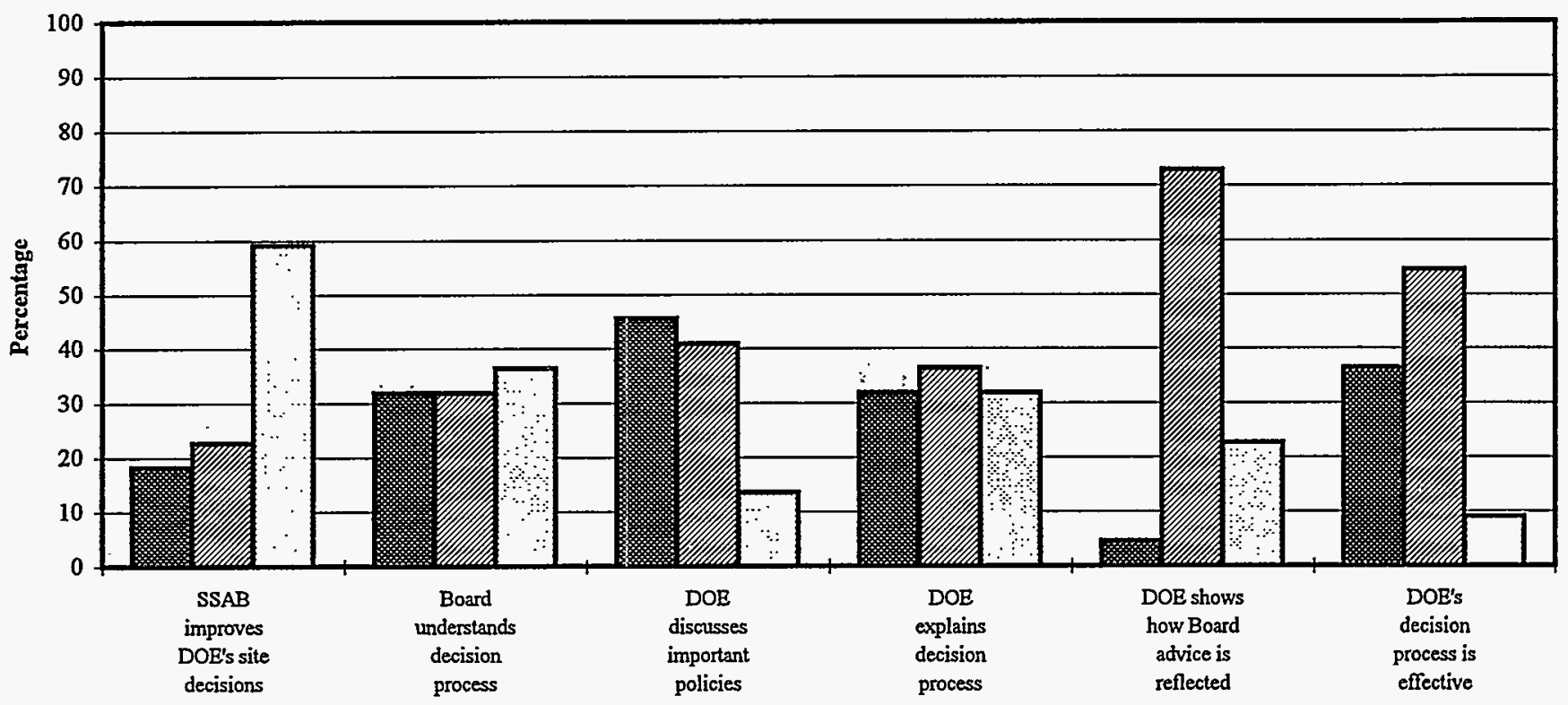

Agree (\%) Deither Agree Nor Disagree/Don't Know (\%) DDisagree (\%)

Figure 4b. Long Survey: Entire Sample

Goal 4. Improve DOE's (and where applicable, regulators') site decisions and decision making process

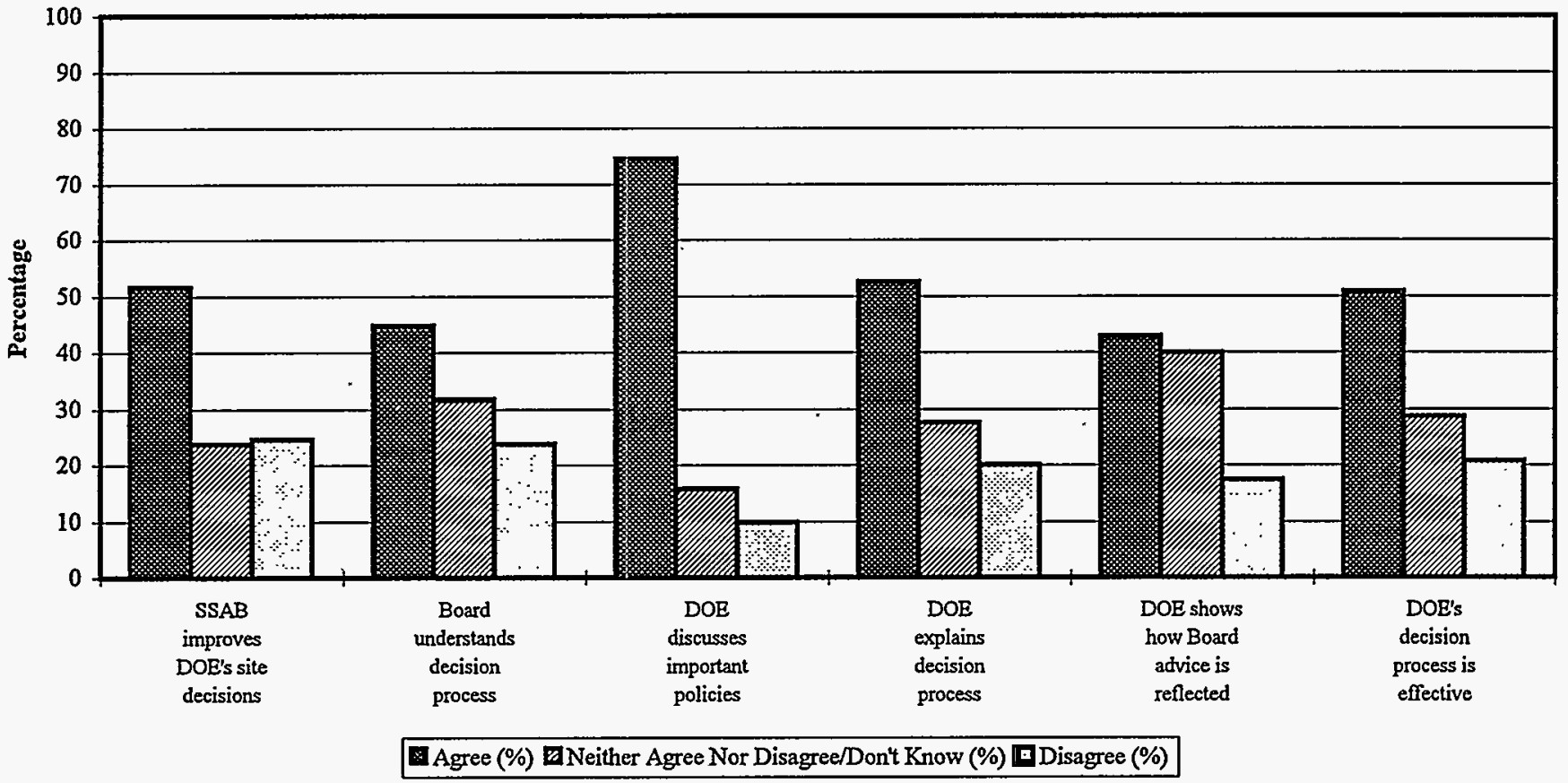

SSAB Supplementary Appendix 
Figure 5a. Los Alamos

Goal 5. Lead to more acceptable actions

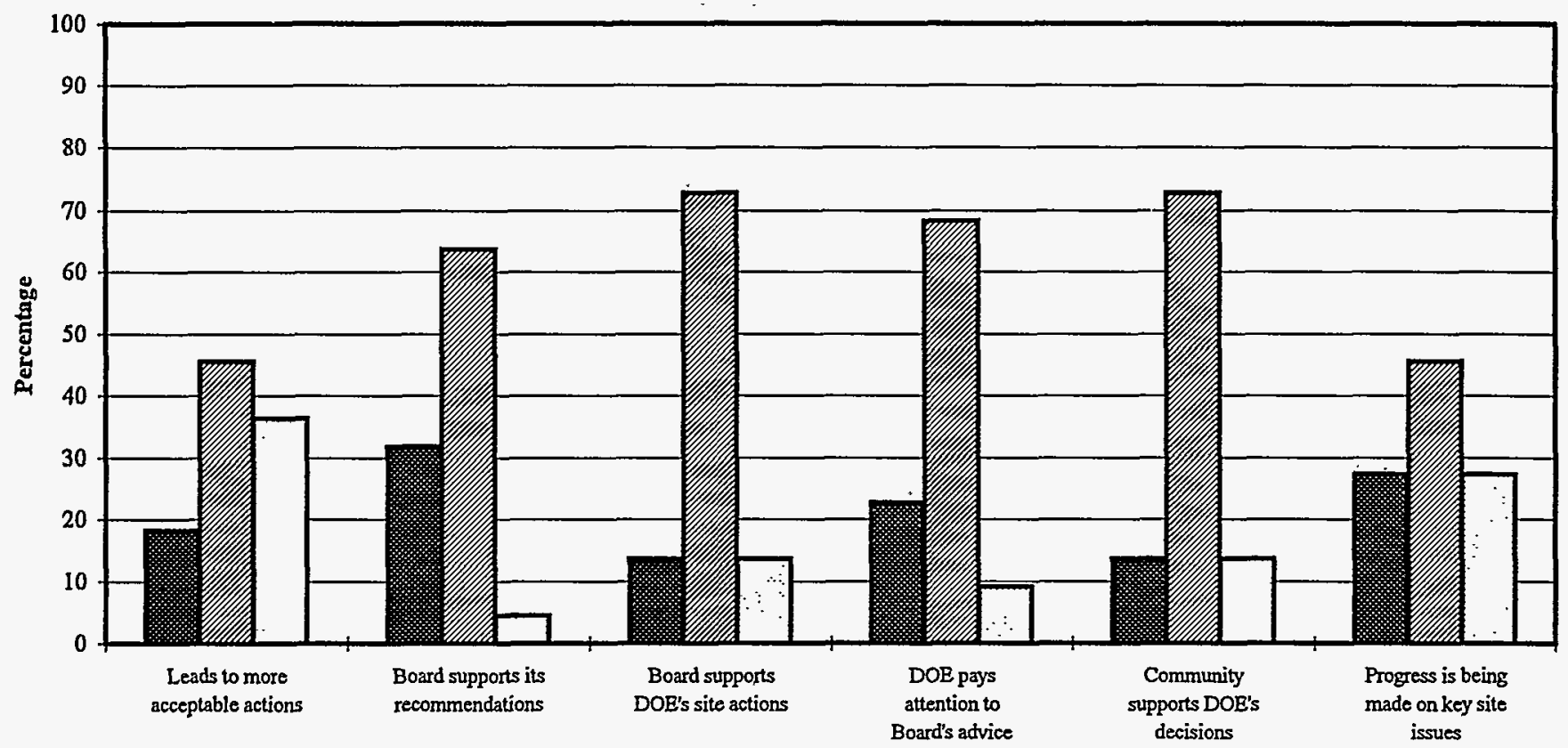

Agree (\%) ש Neither Agree Nor Disagree/Don't Know (\%) DDisagree (\%)

Figure 5b. Long Survey: Entire Sample

Goal 5. Lead to more acceptable actions

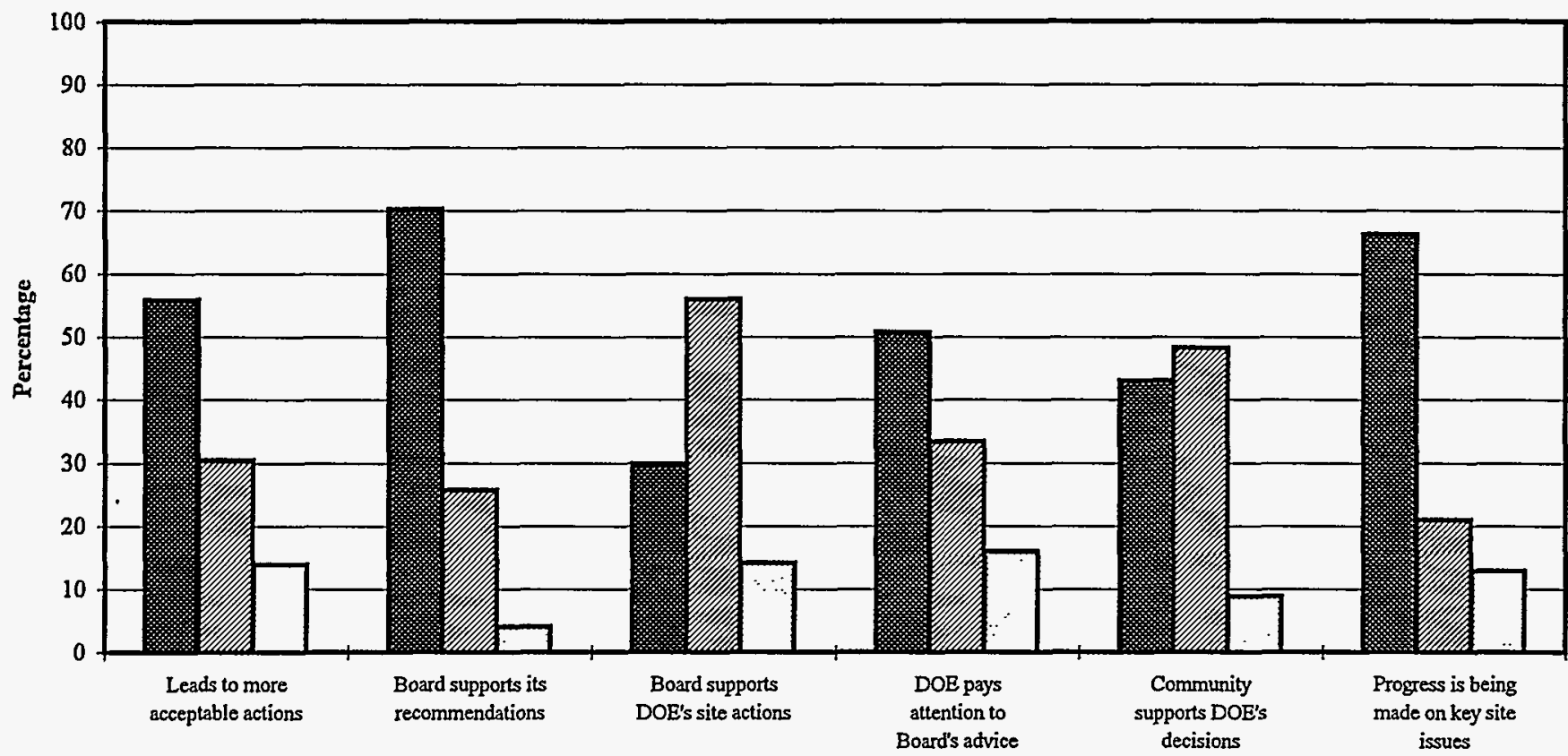

A Agree (\%) ZNeither Agree Nor Disagree/Don't Know (\%) पDisagree (\%)

SSAB Supplementary Appendix

August 1996 
Figure 6a. Los Alamos

Goal 6. Contribute to trust and confidence in DOE

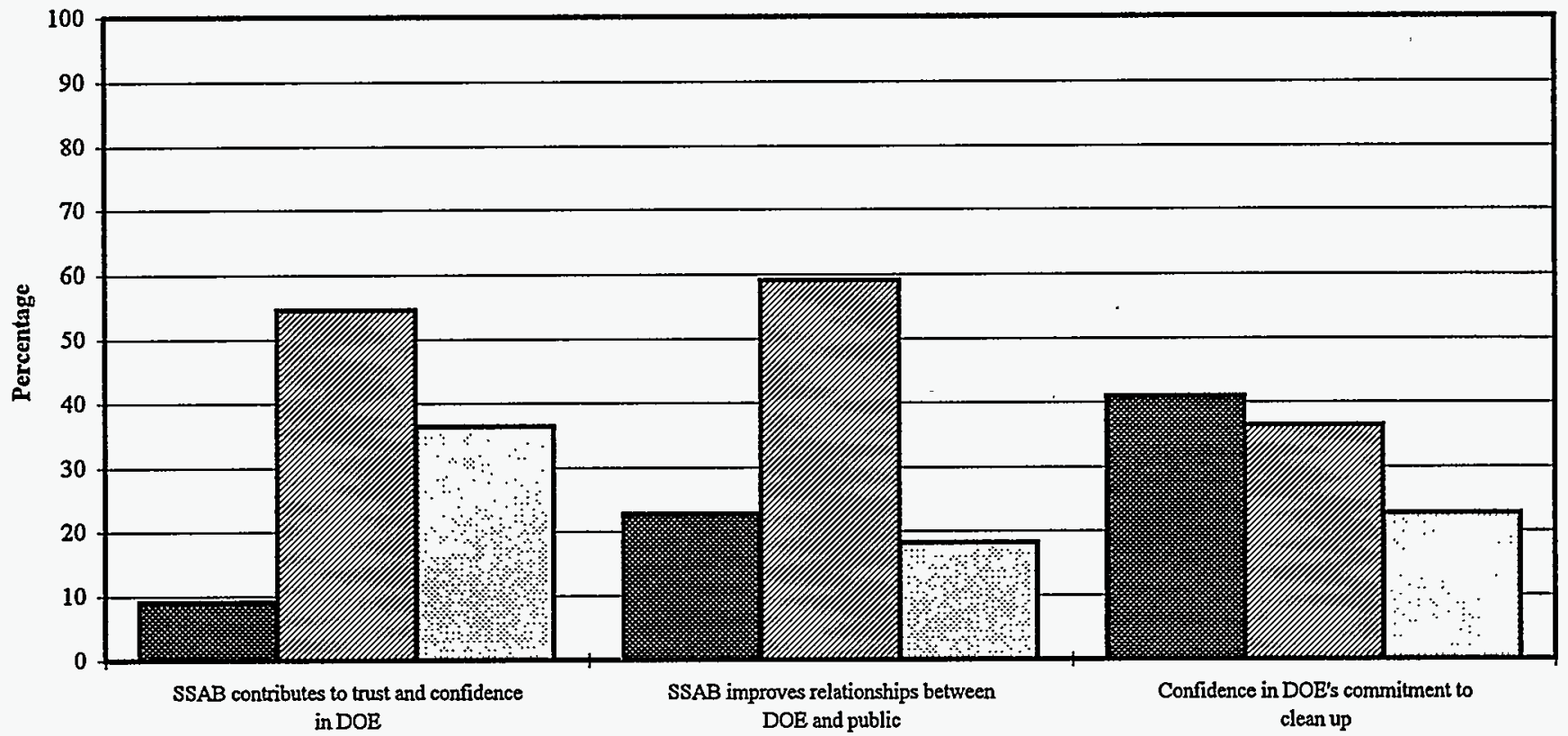

A Agree (\%) BNeither Agree Nor Disagree/Dont' Know (\%) DDisagree (\%)

Figure 6b. Long Survey: Entire Sample

Goal 6. Contribute to trust and confidence in DOE

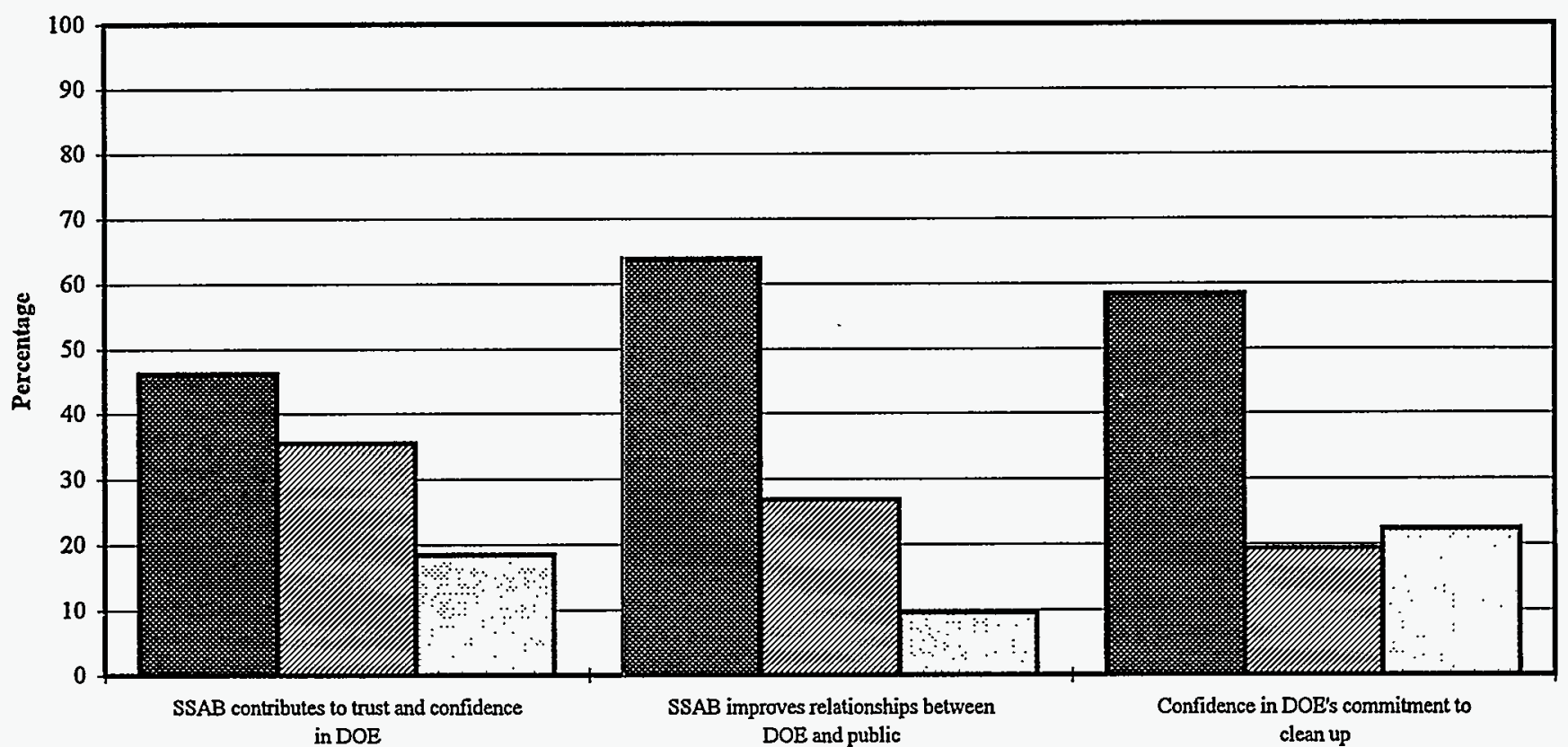

DAgree (\%) DNeither Agree Nor Disagree/Don't Know (\%) DDisagree (\%)

SSAB Supplementary Appendix

August 1996 
Figure 7a. Los Alamos

Public awareness

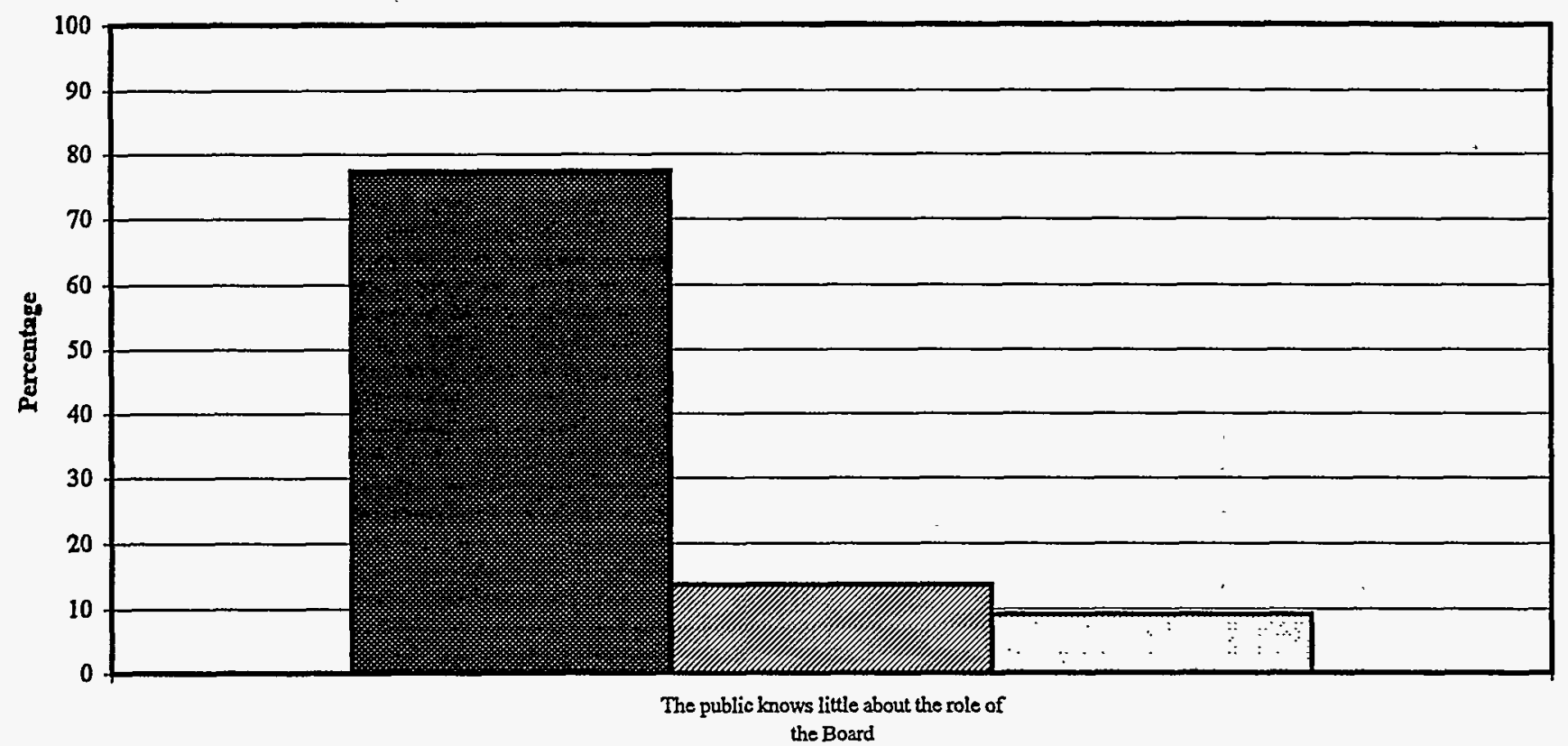

Agree (\%) DNeither Agree Nor Disagree/Don't Know (\%) QDisagree (\%)

Figure 7b. Long Survey: Entire Sample

Public awareness

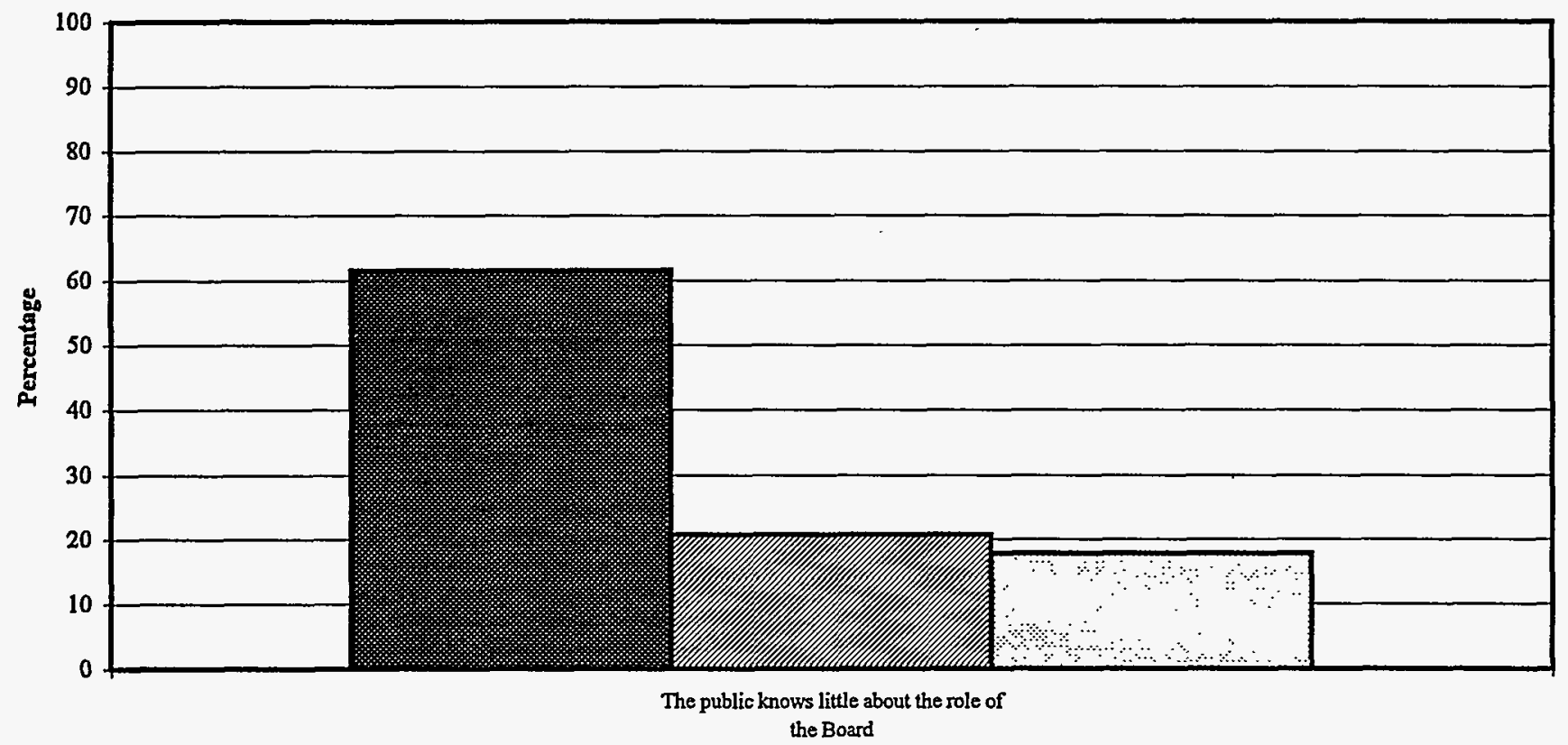

Agree (\%) Weither Agree Nor Disagree/Don't Know (\%) DDisagree (\%)

SSAB Supplementary Appendix

August 1996 
Figure 8a. Los Alamos

DOE-HQ involvement with the SSAB Initiative

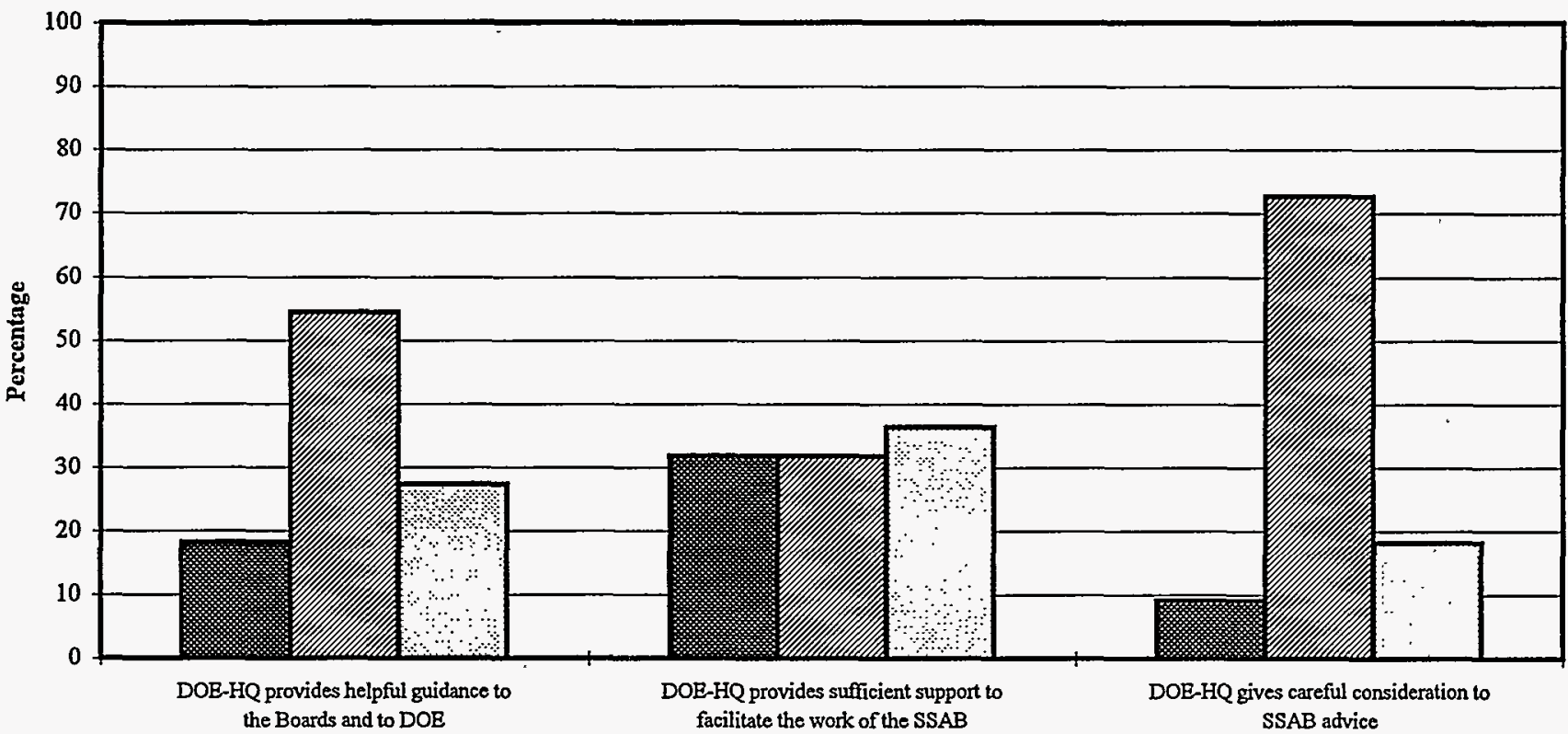

Agree (\%) ENeither Agree Nor Disagree/Don't Know (\%) DDisagree (\%)

Figure 8b. Long Survey: Entire Sample DOE-HQ involvement with the SSAB Initiative

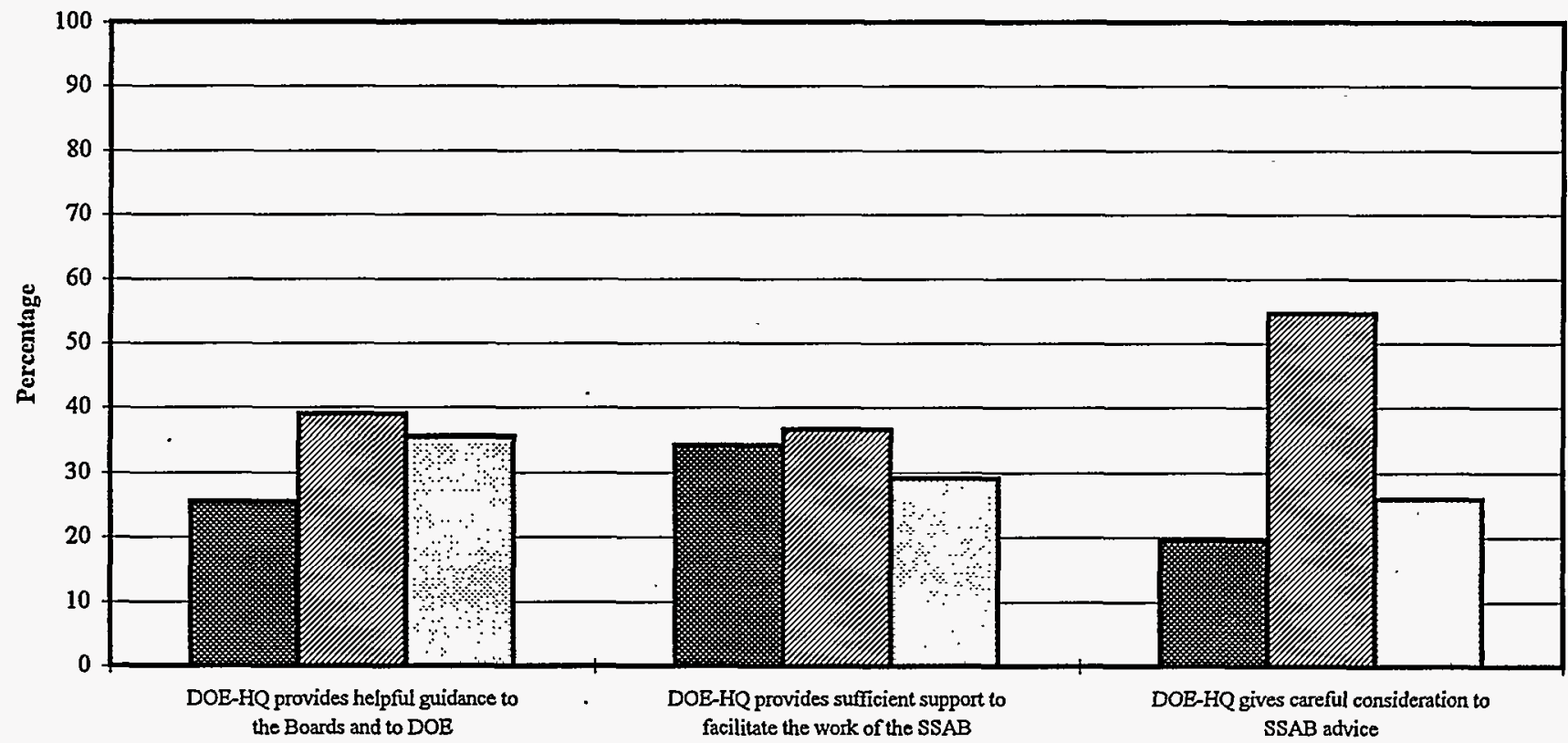

Agree (\%) Weither Agree Nor Disagree/Don't Know (\%) 口Disagree (\%)

SSAB Supplementary Appendix

August 1996 
Figure 9a. Los Alamos

Personal experience with the SSAB Initiative

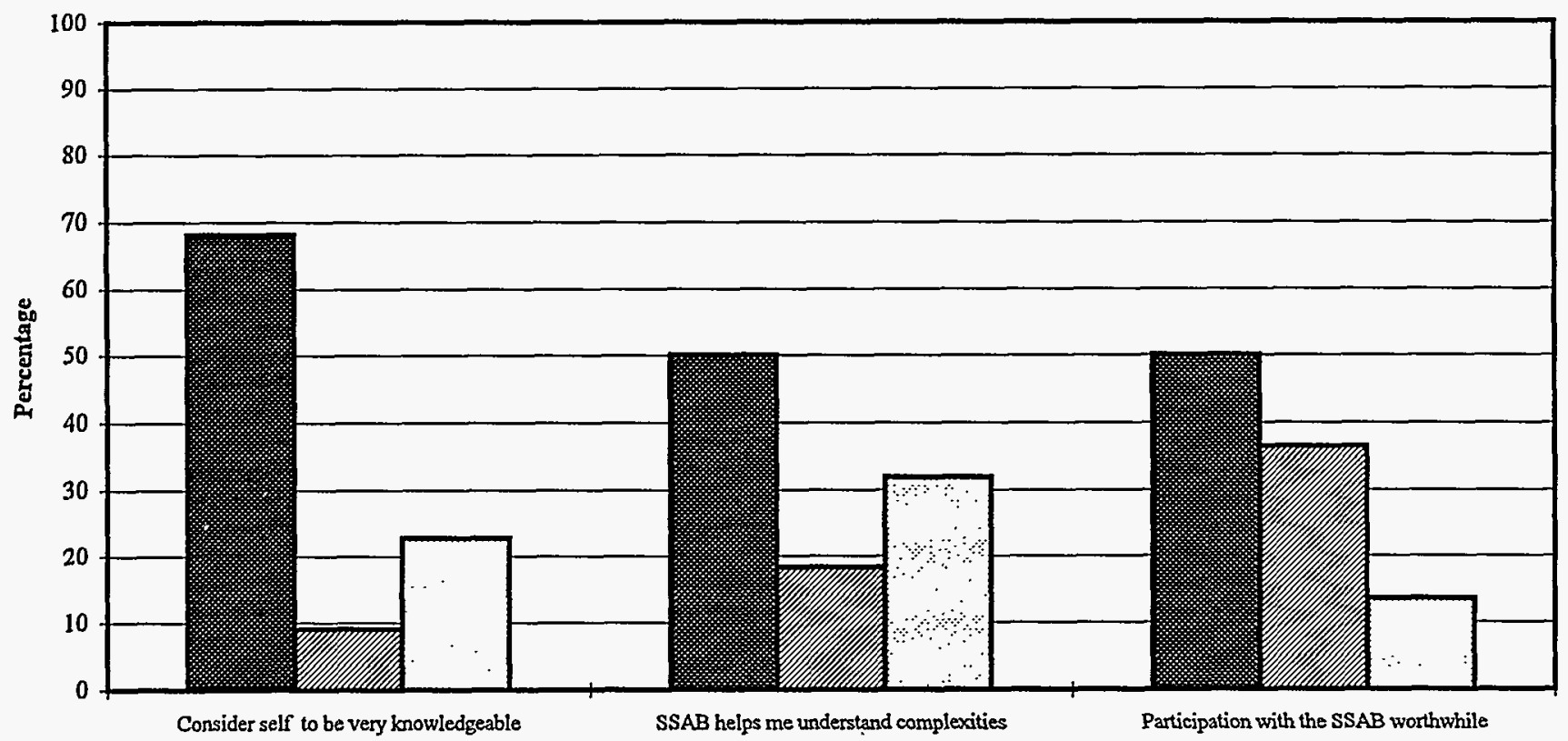

Agree (\%) Neither Agree Nor Disagree/Dont' Know (\%) D Disagree (\%)

Figure 9b. Long Survey: Entire Sample Personal experience with the SSAB Initiative

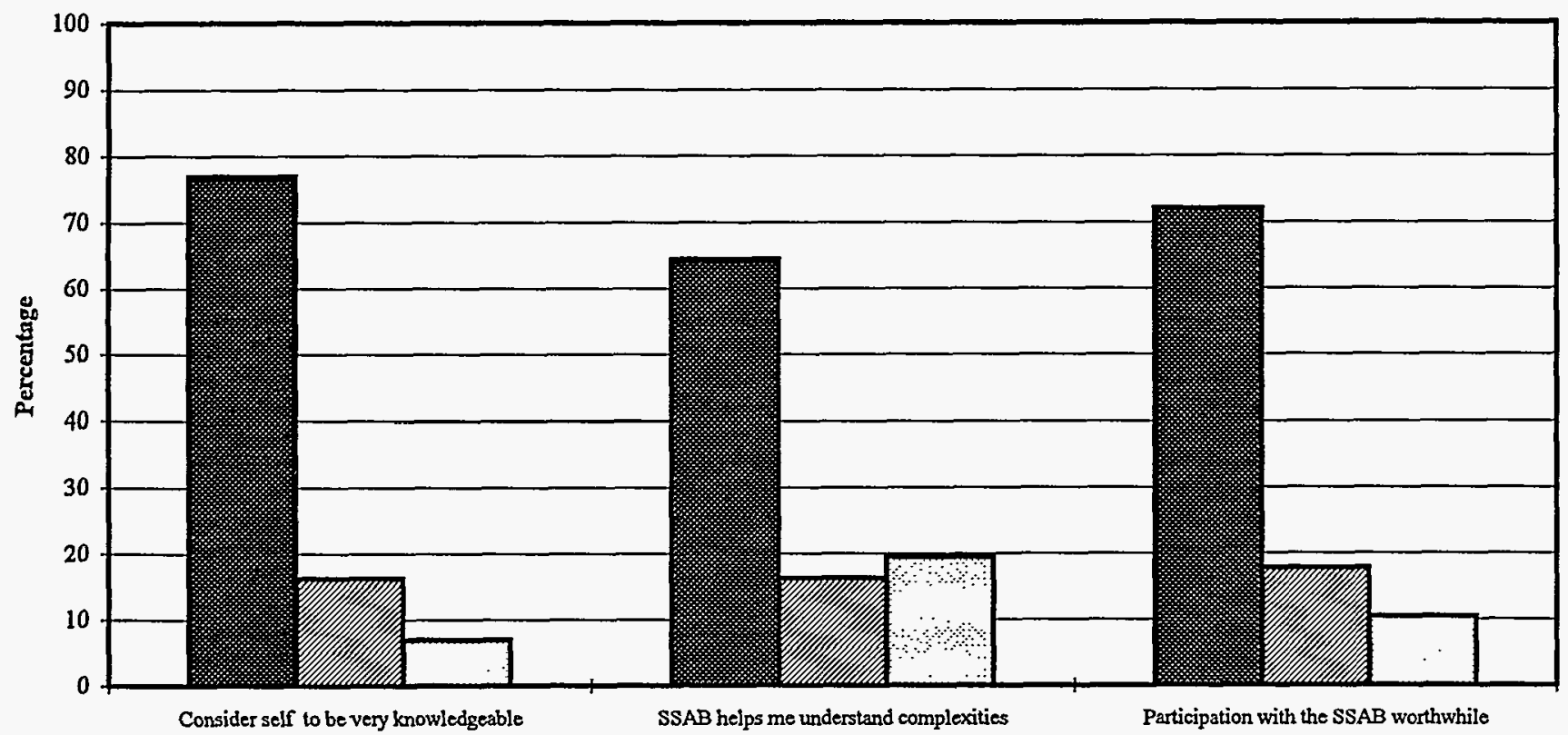

Agree (\%) Weither Agree Nor Disagree/Don't Know (\%) DDisagree (\%)

SSAB Supplementary Appendix 
Figure 10a. Los Alamos

Group/Team work skills

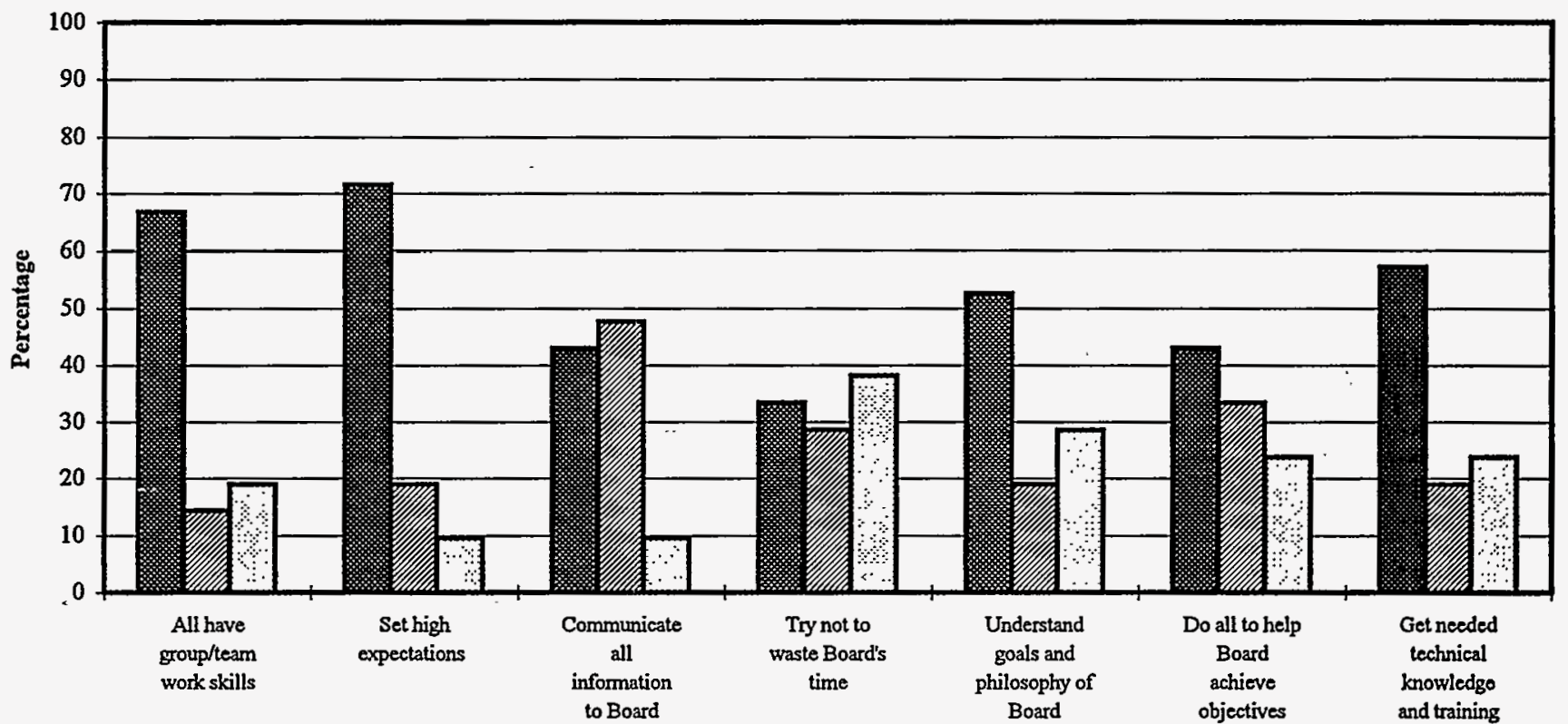

Agree (\%) Neither Agree Nor Disagree/Don't Know (\%) DDisagree (\%)

Figure 10b. Long Survey: Entire Sample

Group/Team work skills

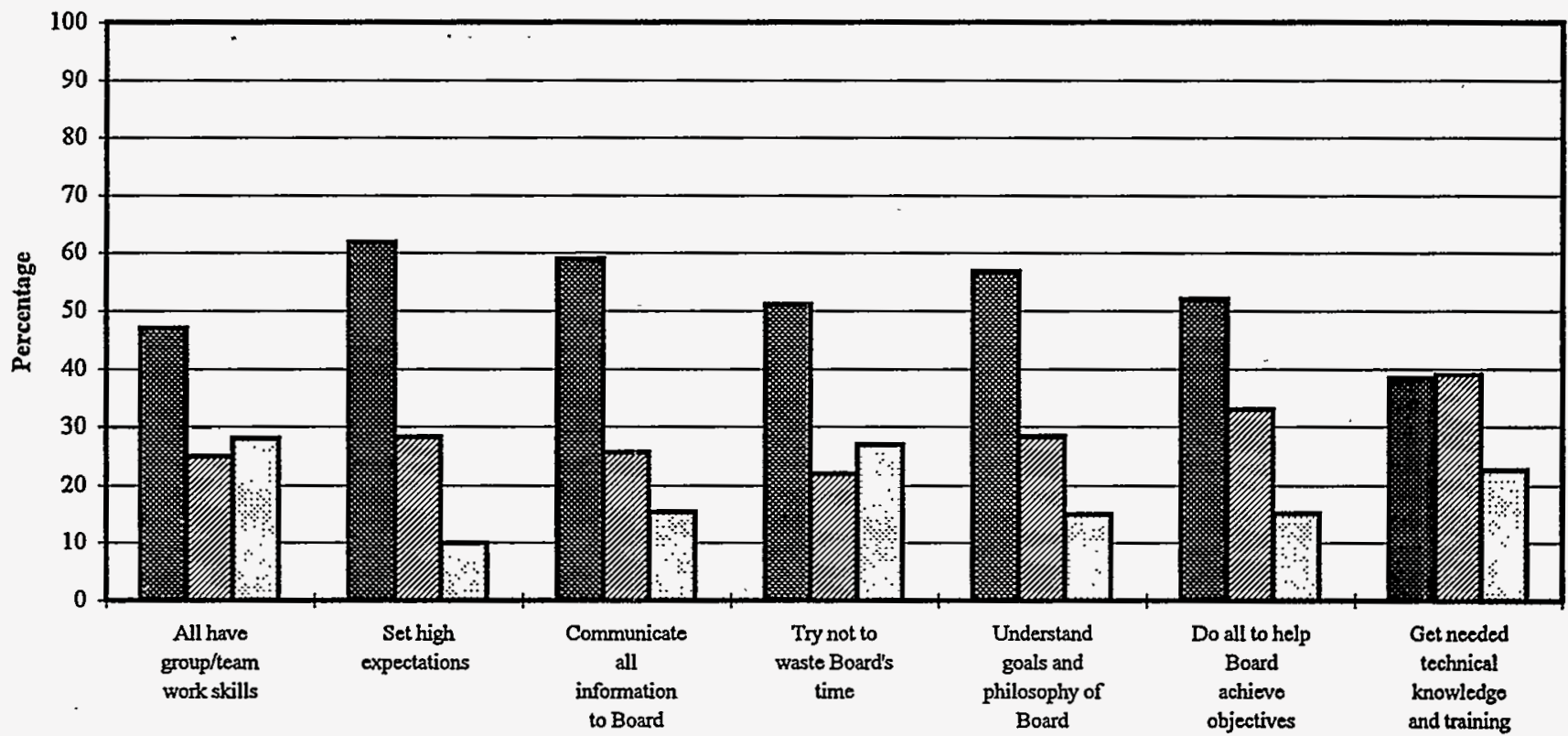

Agree (\%) WNeither Agree Nor Disagree/Don't Know (\%) DDisagree (\%)

SSAB Supplementary Appendix

August 1996 
Figure 11a. Los Alamos

Working relations

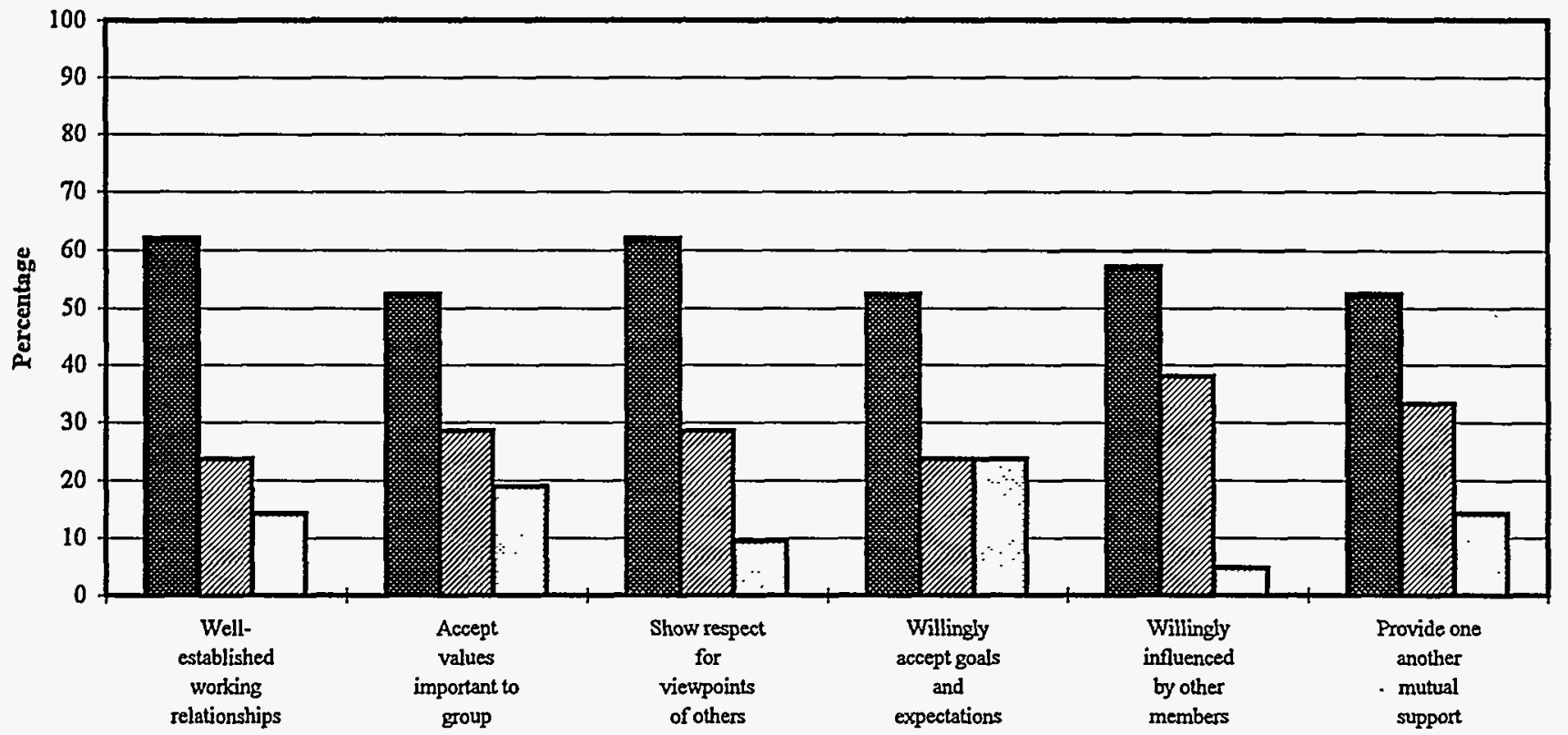

Agree (\%) DNeither Agree Nor Disagree/Don't Know (\%) DDisagree (\%)

Figure 11b. Long Survey: Entire Sample Working relations

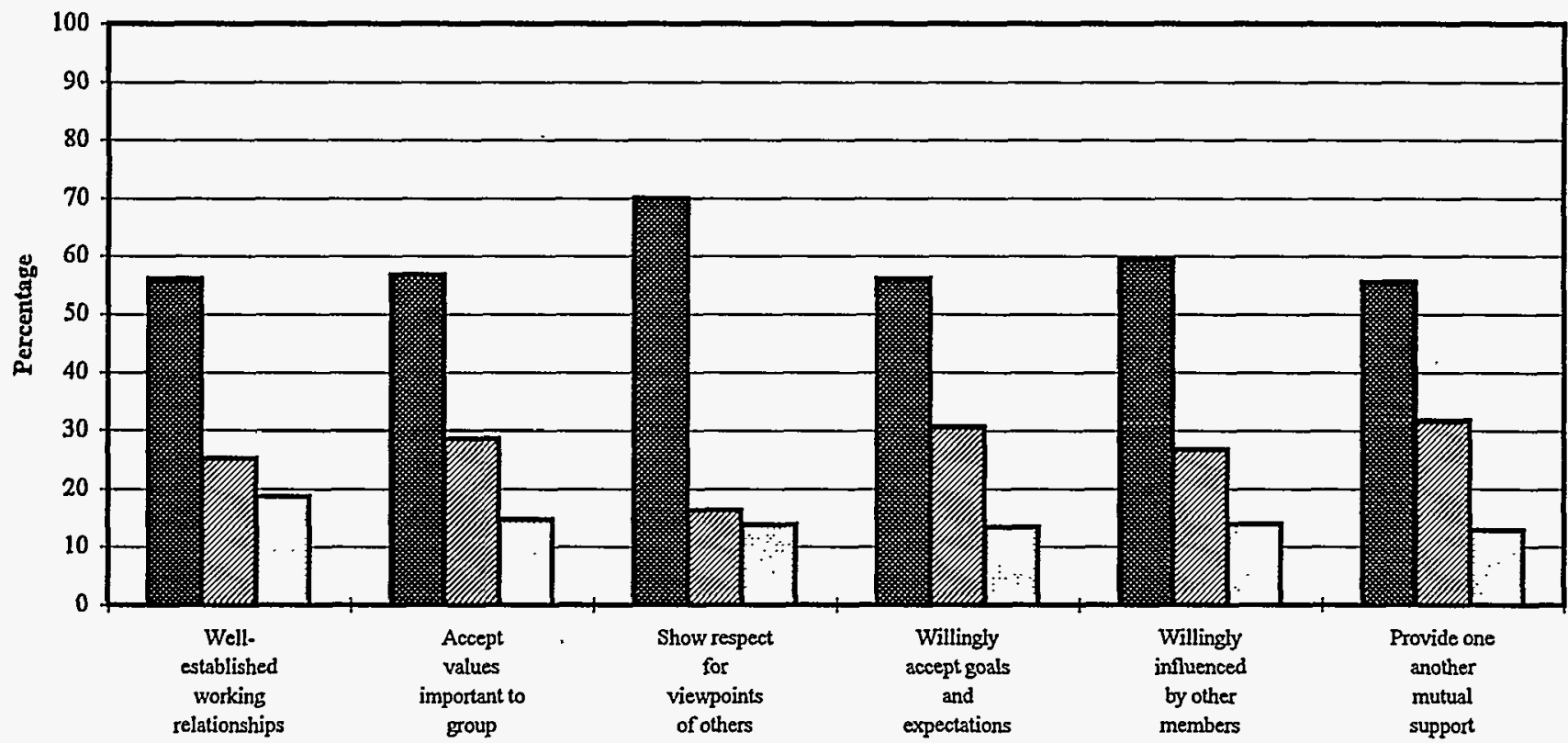

Agree (\%) שNeither Agree Nor Disagree/Don't Know (\%) DDisagree (\%)

SSAB Supplementary Appendix 
Figure 12a. Los Alamos

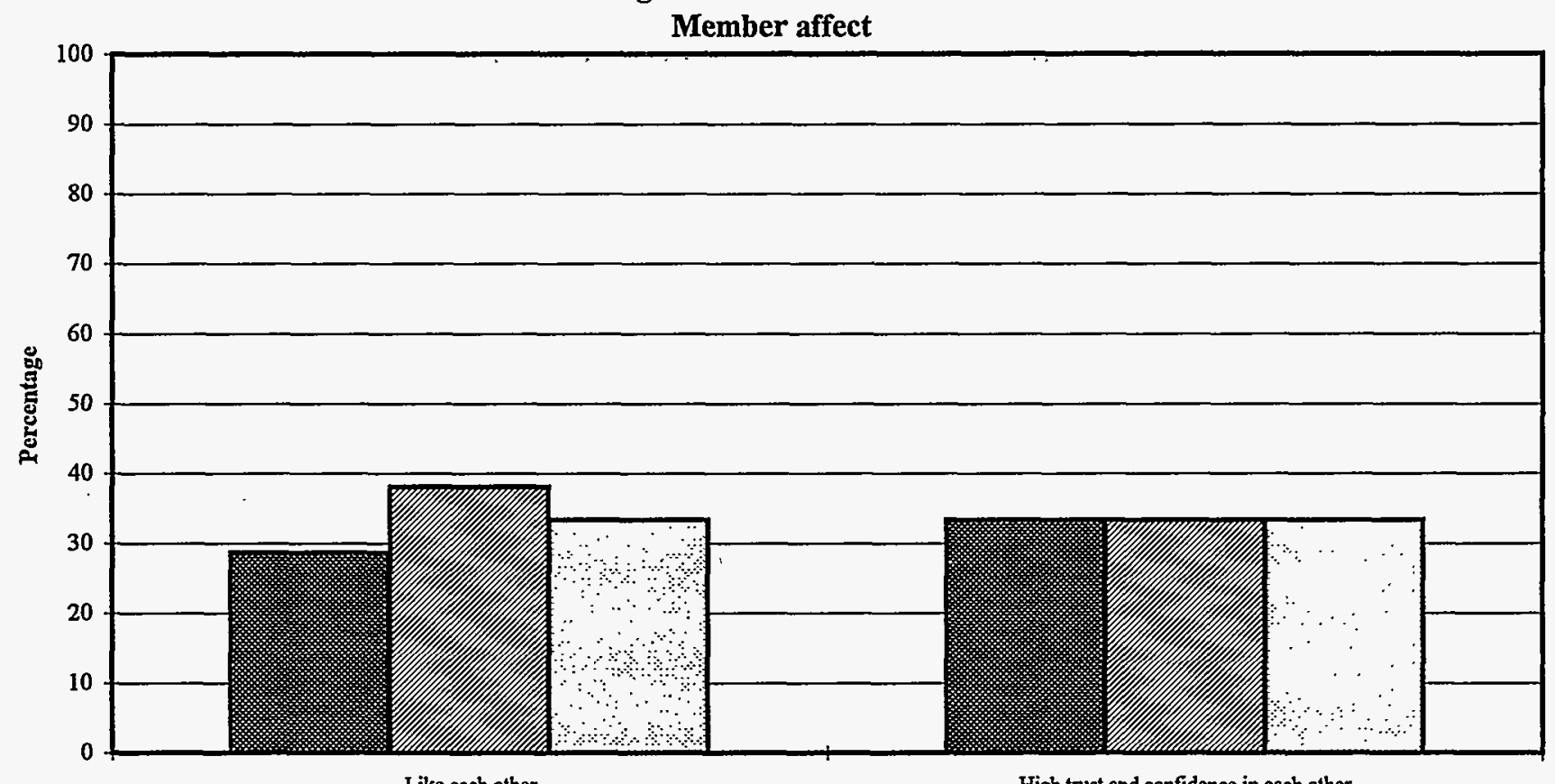

Like each other

High trust and confidence in each other

Agree (\%) Neither Agree Nor Disagree/Don't Know (\%) DDisagree (\%)

Figure 12b. Long Survey: Entire Sample Member affect

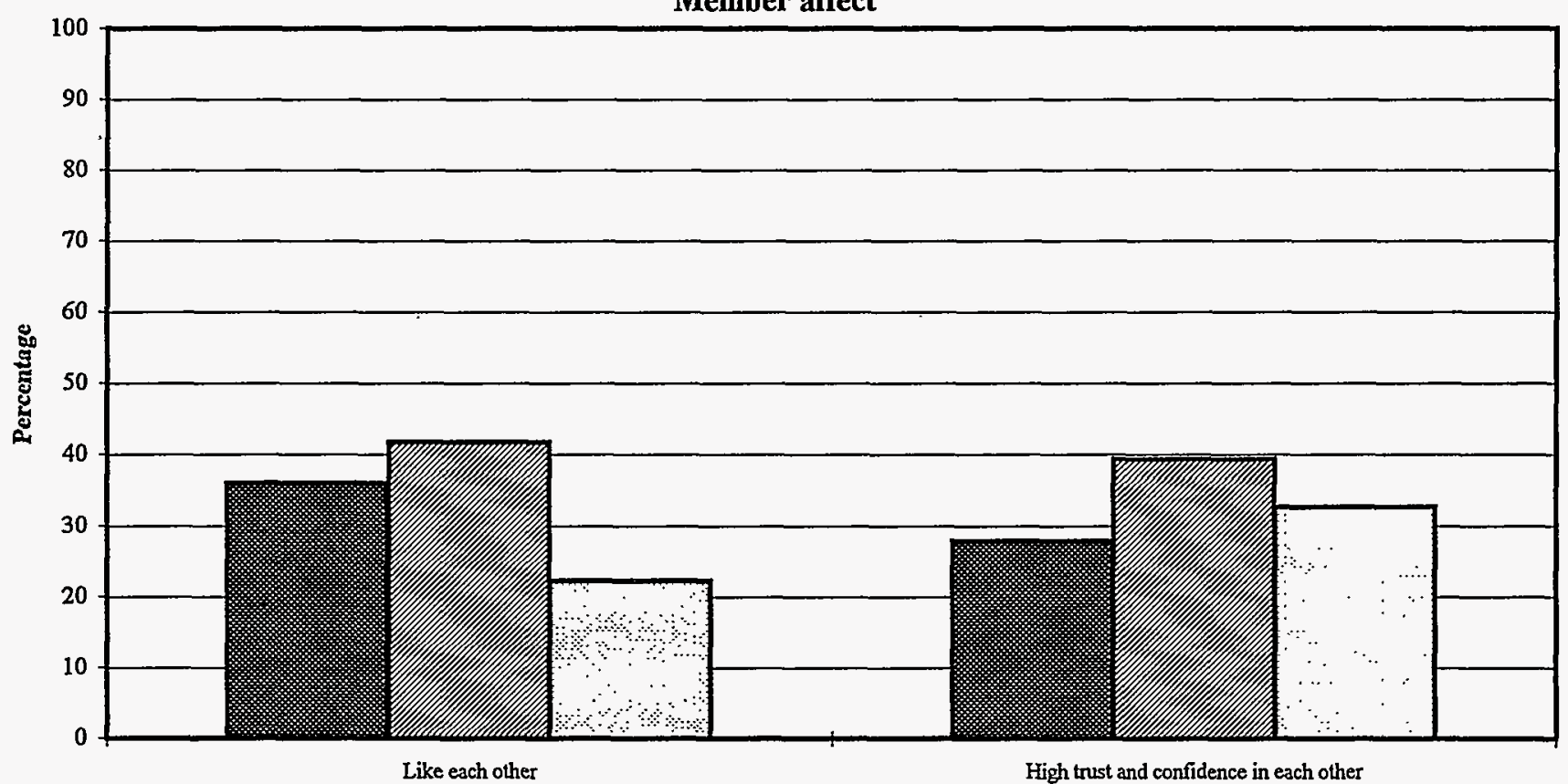

․ㅡㄹ Agree (\%) Neither Agree Nor Disagree/Don't Know (\%) DDisagree (\%)

SSAB Supplementary Appendix

August 1996 
Figure 13a. Los Alamos

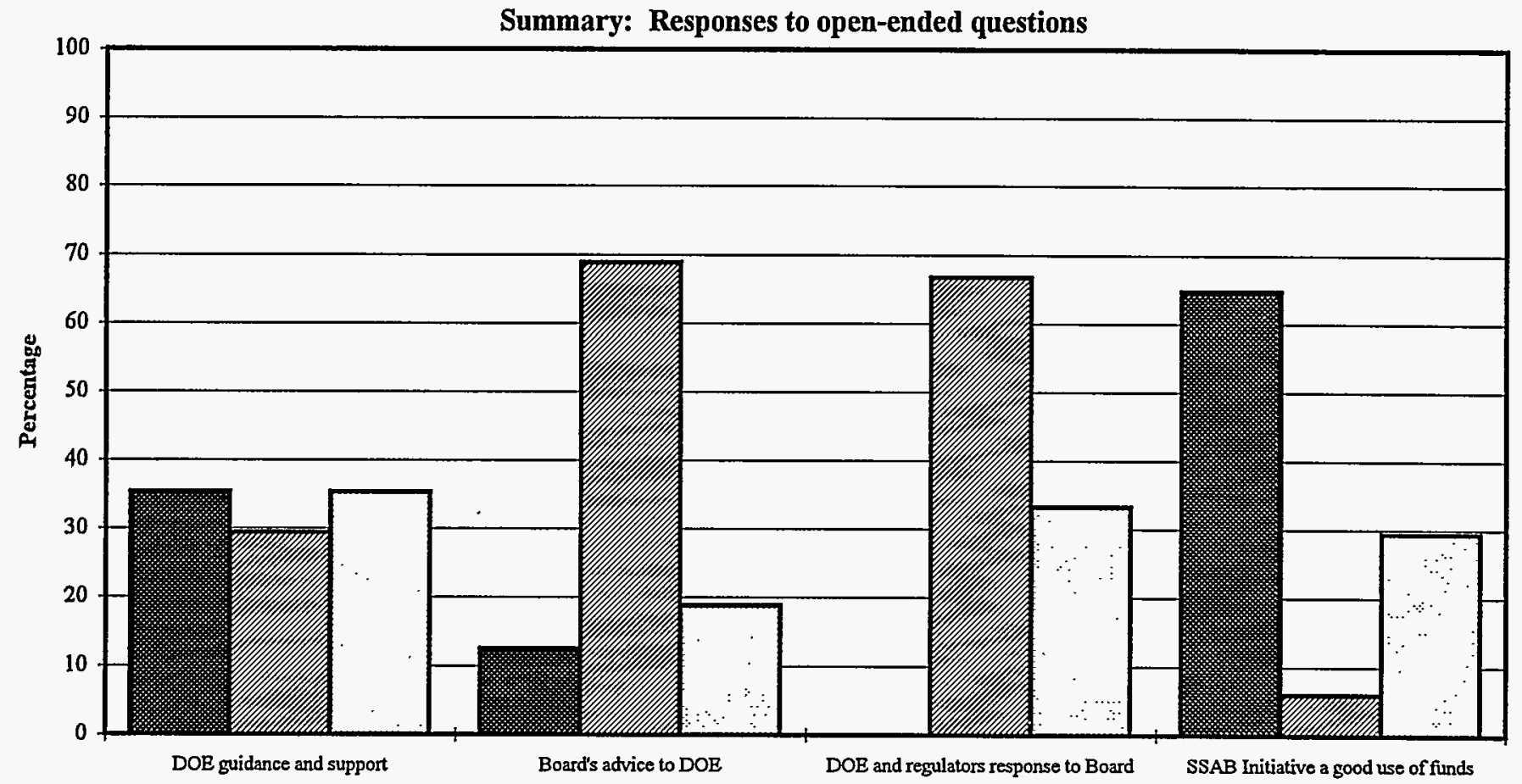

Yes, Good (\%) Weutral, Conditional, No Opinion (\%) DNo, Not Good (\%)

Figure 13b. Long Survey: Entire Sample

Summary: Responses to open-ended questions

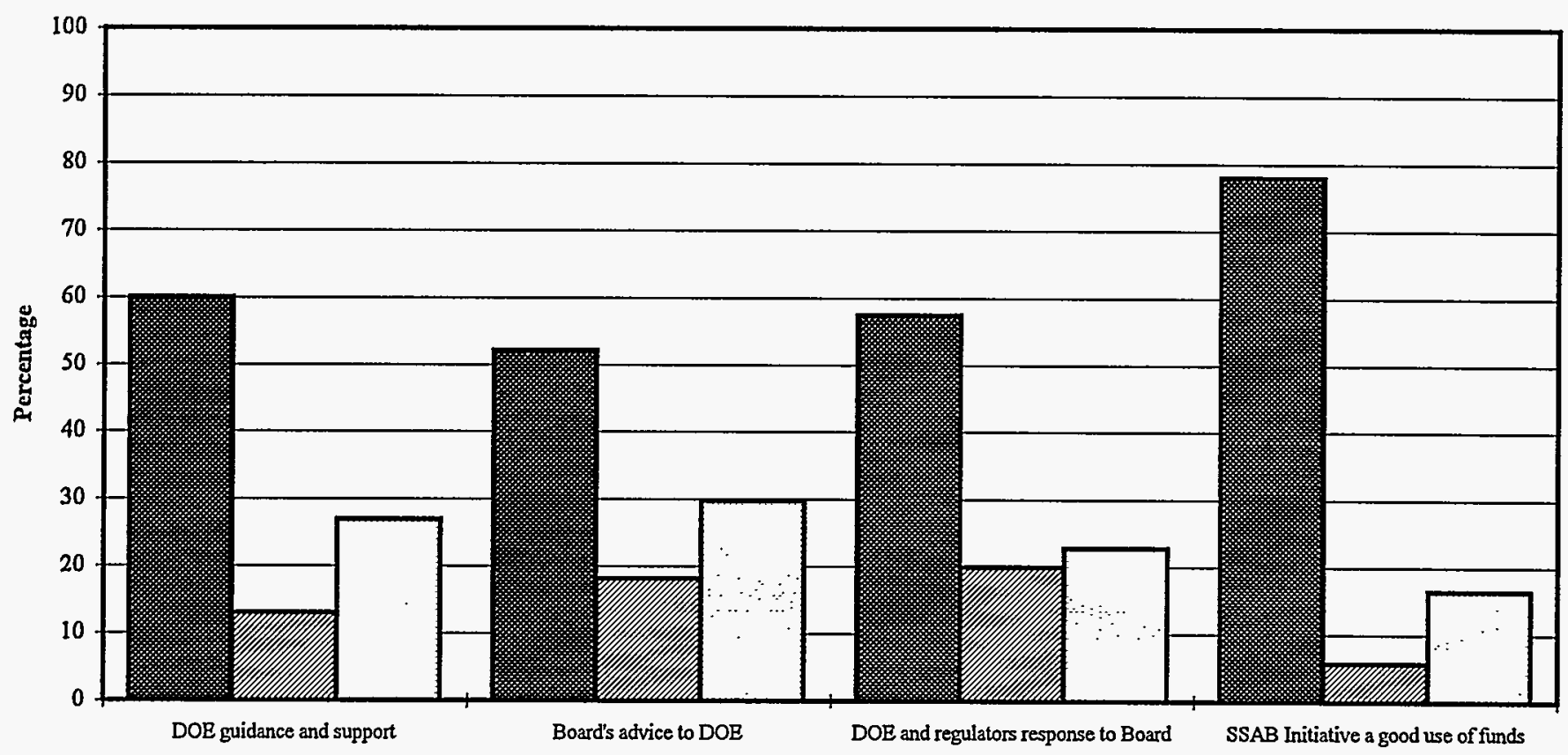

W Yes, Good (\%) Neutral, Conditional, No Opinion (\%) DNo, Not Good (\%)

SSAB Supplementary Appendix

August 1996 
Figure 14a. Los Alamos

Summary: Combined responses for SSAB Initiative goals (Means)

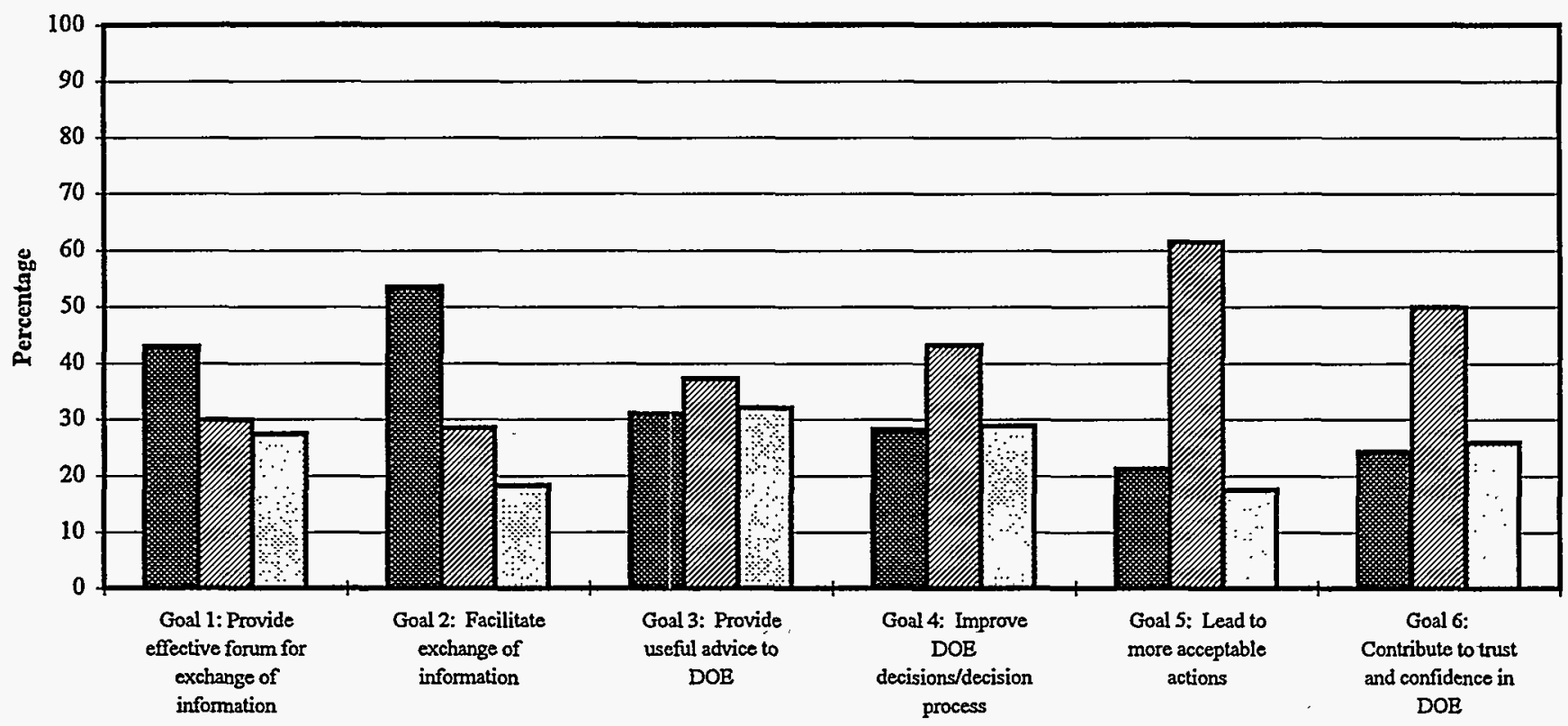

Agree (\%) ENeither Agree Nor Disagree/Don't Know (\%) DDisagree (\%)

Figure 14b. Long Survey: Entire Sample

Summary: Combined responses for SSAB Initiative goals (Means)

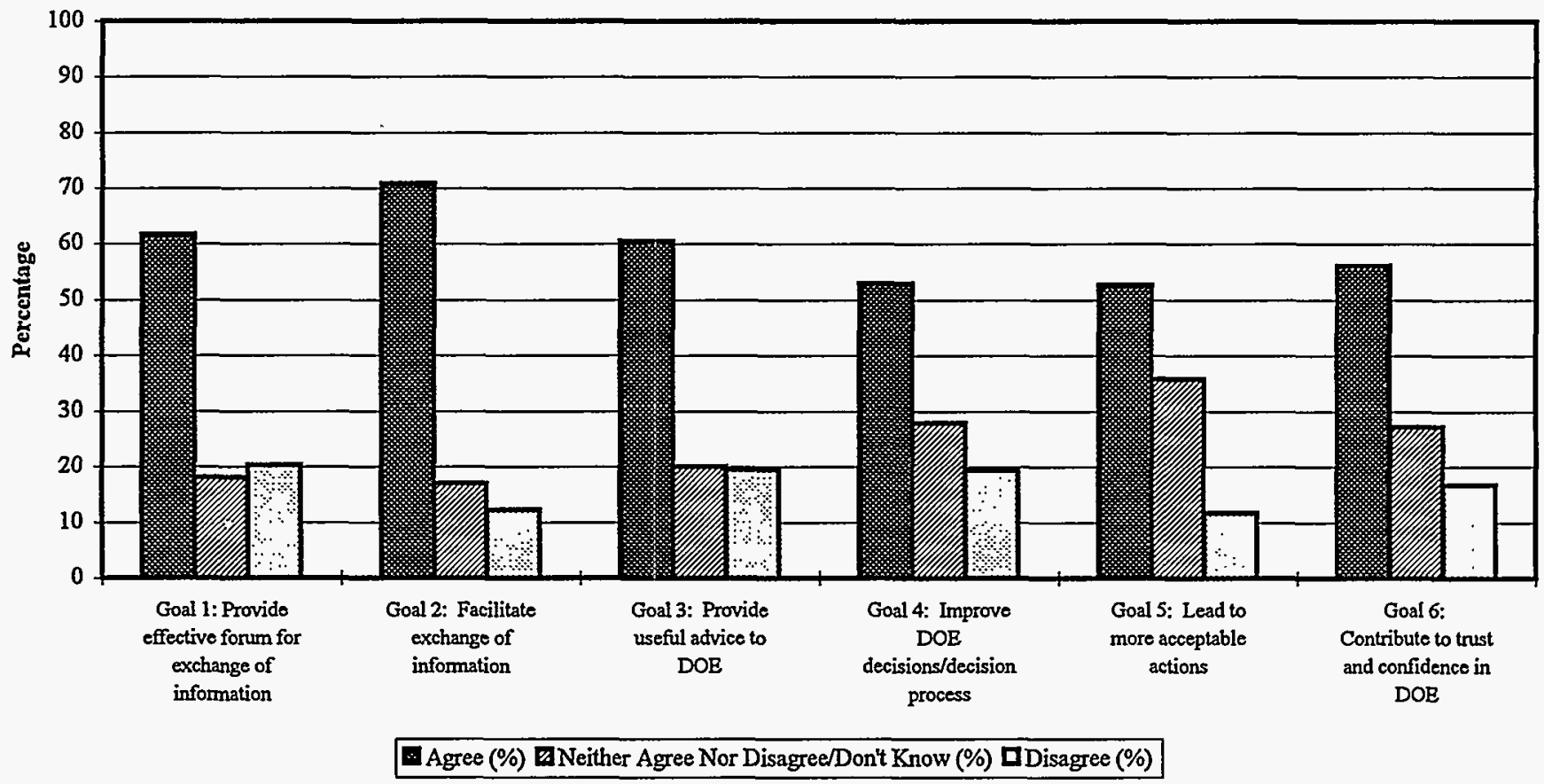

SSAB Supplementary Appendix

August 1996 
Figure 15a. Los Alamos

Summary: Combined responses on DOE-HQ involvement,

Personal experience, Group/Team work skills,

Working relations, Member affect (Means)

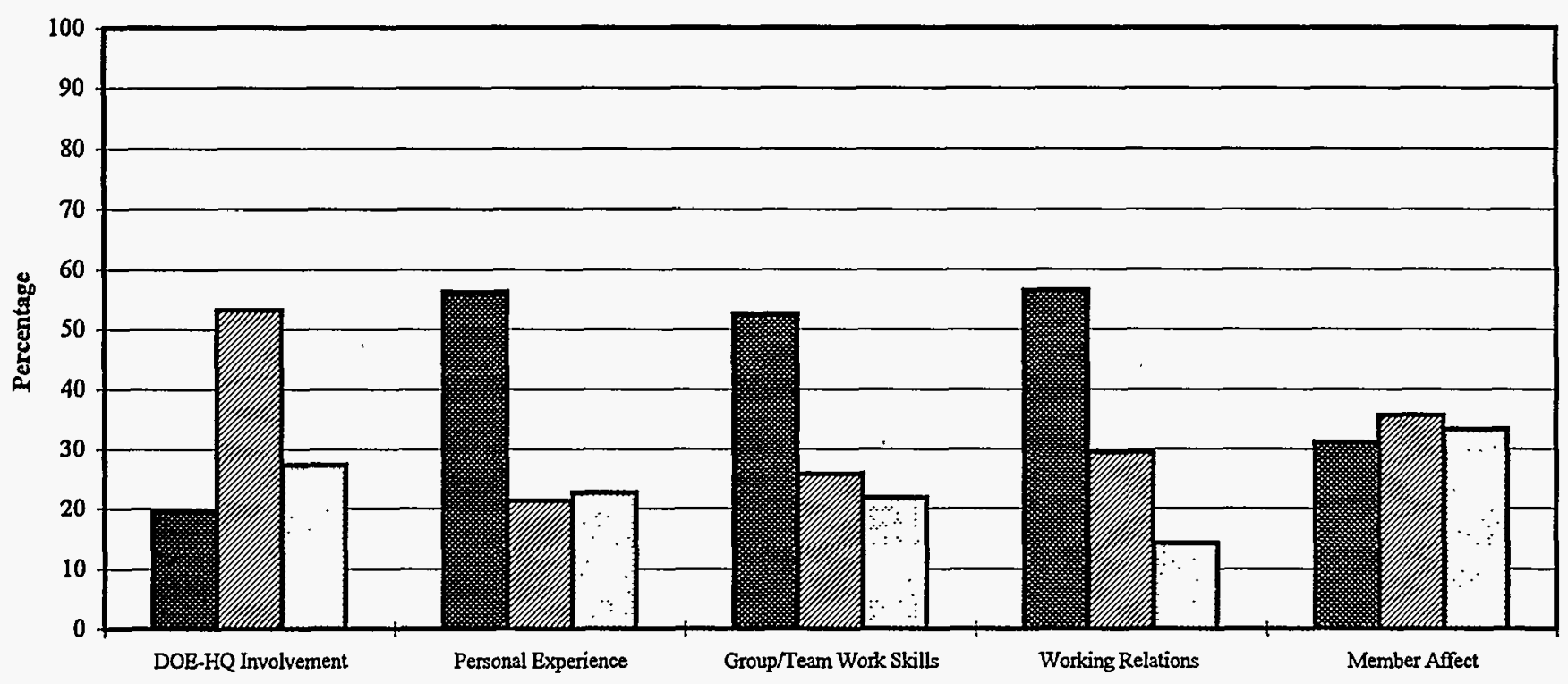

Agree (\%) Neither Agree Nor Disagree/Don't Know (\%) DDisagree (\%)

Figure 15b. Long Survey: Entire Sample

Summary: Combined responses on DOE-HQ involvement,

Personal experience, Group/Team work skills,

Working relations, Member affect (Means)

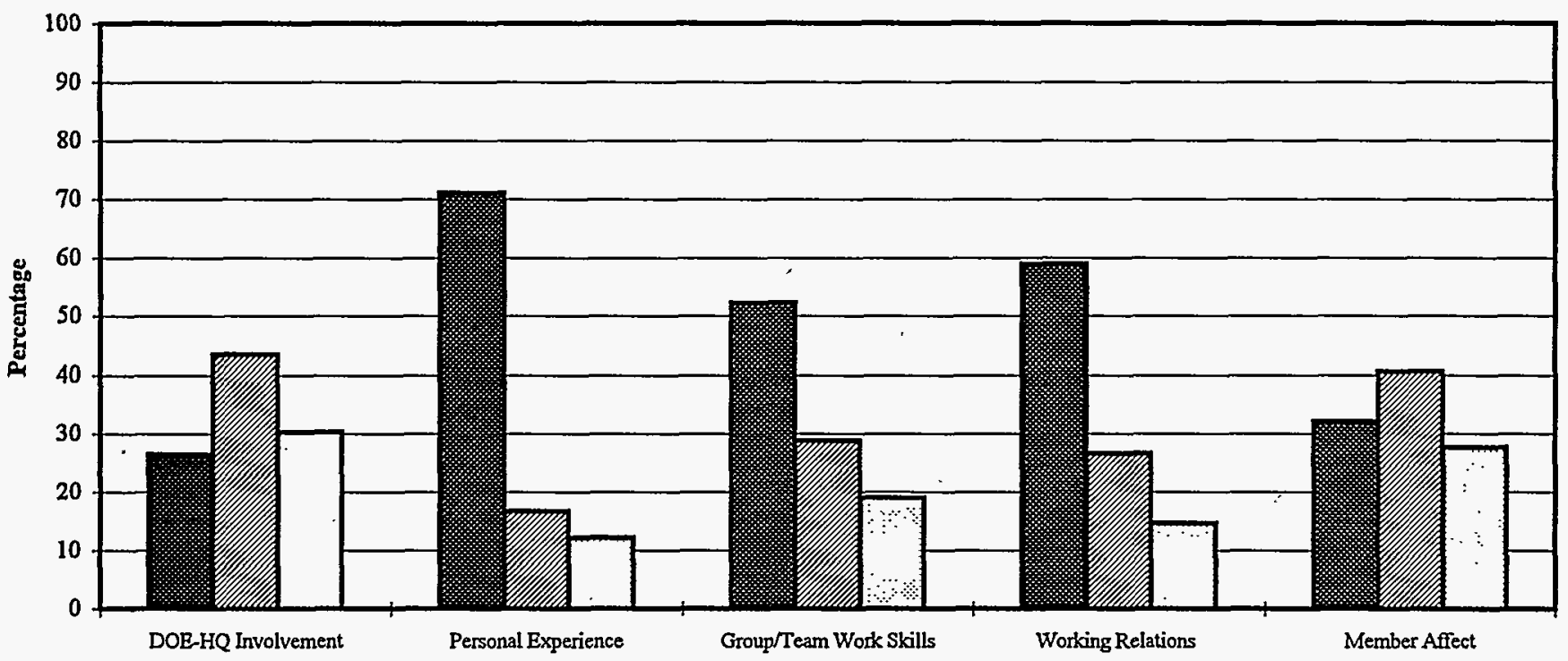

Agree (\%) DNeither Agree Nor Disagree/Don't Know (\%) DDisagree (\%)

SSAB Supplementary Appendix

August 1996 
Table 1.

Goal 1. Establish processes and procedures to provide an effective forum for exchange of information and viewpoints regarding $\mathrm{DOE}$ site issues

\begin{tabular}{|c|c|c|c|c|c|c|c|c|}
\hline \multirow[t]{2}{*}{ Los Alamos } & \multicolumn{2}{|c|}{ Agree } & \multicolumn{2}{|c|}{$\begin{array}{l}\text { Neither/ } \\
\text { Don't Know }\end{array}$} & \multicolumn{2}{|c|}{ Disagree } & \multicolumn{2}{|c|}{ Total } \\
\hline & $N$ & $\%$ & $N$ & $\%$ & $N$ & $\%$ & $\mathrm{~N}$ & $\%$ \\
\hline $\begin{array}{l}\text { The Board has established processes and } \\
\text { procedures for the effective exchange of } \\
\text { information }\end{array}$ & 11 & $50.0 \%$ & 6 & $27.3 \%$ & 5 & $22.7 \%$ & 22 & $100 \%$ \\
\hline $\begin{array}{l}\text { The Board has agreed-upon procedures to } \\
\text { operate the board }\end{array}$ & 19 & $86.4 \%$ & 1 & $4.5 \%$ & 2 & $9.1 \%$ & 22 & $100 \%$ \\
\hline $\begin{array}{l}\text { The Board has adequate support to allow } \\
\text { it to focus on substantive issues (R) * }\end{array}$ & 10 & $45.5 \%$ & 5 & $22.7 \%$ & 7 & $31.8 \%$ & 22 & $100 \%$ \\
\hline $\begin{array}{l}\text { The Board provides sufficient time for } \\
\text { discussion of issues }\end{array}$ & 9 & $40.9 \%$ & 2 & $9.1 \%$ & 11 & $50.0 \%$ & 22 & $100 \%$ \\
\hline The Board has effective leadership & 10 & $45.5 \%$ & 6 & $27.3 \%$ & 6 & $27.3 \%$ & 22 & $100 \%$ \\
\hline $\begin{array}{l}\text { DOE requests Board advice far enough } \\
\text { ahead of decision deadlines }(R)^{*}\end{array}$ & 1 & $4.5 \%$ & 9 & $40.9 \%$ & 12 & $54.5 \%$ & 22 & $100 \%$ \\
\hline $\begin{array}{l}\text { DOE handles Board administrative issues } \\
\text { expeditiously (R) * }\end{array}$ & 7 & $31.8 \%$ & 11 & $50.0 \%$ & 4 & $18.2 \%$ & 22 & $100 \%$ \\
\hline $\begin{array}{l}\text { The SSAB staff provide satisfactory } \\
\text { support }\end{array}$ & 11 & $50.0 \%$ & 5 & $22.7 \%$ & 6 & $27.3 \%$ & 22 & $100 \%$ \\
\hline $\begin{array}{l}\text { The facilitator for the Board has helped } \\
\text { the Board function effectively }\end{array}$ & 7 & $31.8 \%$ & 14 & $63.6 \%$ & 1 & $4.5 \%$ & 22 & $100 \%$ \\
\hline
\end{tabular}

* (R) denotes a question that was asked in the negative form. For ease of review, these questions and data have been reversed in this table and are stated in positive forms.

SSAB Supplementary Appendix

August 1996 
Table 2.

Goal 2. Facilitate interaction and exchange of information and viewpoints regarding DOE site issues

\begin{tabular}{|c|c|c|c|c|c|c|c|c|}
\hline \multirow[t]{2}{*}{ Los Alamos } & \multicolumn{2}{|c|}{ Agree } & \multicolumn{2}{|c|}{$\begin{array}{l}\text { Neither/ } \\
\text { Don't Know }\end{array}$} & \multicolumn{2}{|c|}{ Disagree } & \multicolumn{2}{|c|}{ Total } \\
\hline & $\mathrm{N}$ & $\%$ & $N$ & $\%$ & $\mathrm{~N}$ & $\%$ & $\mathrm{~N}$ & $\%$ \\
\hline $\begin{array}{l}\text { The SSAB facilitates effective exchange } \\
\text { of viewpoints on site issues }\end{array}$ & 13 & $59.1 \%$ & 3 & $13.6 \%$ & 6 & $27.3 \%$ & 22 & $100 \%$ \\
\hline $\begin{array}{l}\text { The SSAB contributes to Board members' } \\
\text { understanding of the basis for key site } \\
\text { decisions }\end{array}$ & 9 & $40.9 \%$ & 6 & $27.3 \%$ & 7 & $31.8 \%$ & 22 & $100 \%$ \\
\hline $\begin{array}{l}\text { The SSAB contributes to DOE and } \\
\text { regulators' understanding of the public's } \\
\text { viewpoints on key site decisions }\end{array}$ & 11 & $50.0 \%$ & 6 & $27.3 \%$ & 5 & $22.7 \%$ & 22 & $100 \%$ \\
\hline $\begin{array}{l}\text { The SSAB contributes to a constructive } \\
\text { working relationship among the } \\
\text { participants }\end{array}$ & 11 & $50.0 \%$ & 7 & $31.8 \%$ & 4 & $18.2 \%$ & 22 & $100 \%$ \\
\hline The SSAB strives for consensus & 17 & $77.3 \%$ & 4 & $18.2 \%$ & 1 & $4.5 \%$ & 22 & $100 \%$ \\
\hline $\begin{array}{l}\text { The SSAB gives fair consideration to } \\
\text { dissenting opinions }\end{array}$ & 11 & $50.0 \%$ & 6 & $27.3 \%$ & 5 & $22.7 \%$ & 22 & $100 \%$ \\
\hline $\begin{array}{l}\text { The Board has made the effort needed to } \\
\text { learn about site issues }\end{array}$ & 13 & $59.1 \%$ & 8 & $36.4 \%$ & 1 & $4.5 \%$ & 22 & $100 \%$ \\
\hline $\begin{array}{l}\text { The Board invites expert advice into its } \\
\text { discussion on key policy issues }\end{array}$ & 12 & $54.5 \%$ & 6 & $27.3 \%$ & 4 & $18.2 \%$ & 22 & $100 \%$ \\
\hline $\begin{array}{l}\text { The Board responds to public inquiries } \\
\text { and comments about its decisions }\end{array}$ & 12 & $54.5 \%$ & 8 & $36.4 \%$ & 2 & $9.1 \%$ & 22 & $100 \%$ \\
\hline $\begin{array}{l}\text { The Board solicits feedback from the } \\
\text { community on its work }\end{array}$ & 14 & $63.6 \%$ & 4 & $18.2 \%$ & 4 & $18.2 \%$ & 22 & $100 \%$ \\
\hline $\begin{array}{l}\text { DOE makes information on key site issues } \\
\text { readily available to the Board }\end{array}$ & 11 & $50.0 \%$ & 8 & $36.4 \%$ & 3 & $13.6 \%$ & 22 & $100 \%$ \\
\hline $\begin{array}{l}\text { The regulators make important } \\
\text { contributions to the Board's work }\end{array}$ & 6 & $27.3 \%$ & 10 & $45.5 \%$ & 6 & $27.3 \%$ & 22 & $100 \%$ \\
\hline $\begin{array}{l}\text { The SSAB creates a climate supportive of } \\
\text { differing viewpoints }(\mathrm{R}) *\end{array}$ & 13 & $59.1 \%$ & 5 & $22.7 \%$ & 4 & $18.2 \%$ & 22 & $100 \%$ \\
\hline
\end{tabular}

* (R) denotes a question that was asked in the negative form. For ease of review, these questions and data have been reversed in this table and are stated in positive forms.

SSAB Supplementary Appendix

August 1996 
Table 3.

Goal 3. Provide useful advice and/or recommendations to DOE (and regulators, where appropriate)

\begin{tabular}{|c|c|c|c|c|c|c|c|c|}
\hline \multirow[t]{2}{*}{ Los Alamos } & \multicolumn{2}{|c|}{ Agree } & \multicolumn{2}{|c|}{$\begin{array}{l}\text { Neither/ } \\
\text { Don't Know }\end{array}$} & \multicolumn{2}{|c|}{ Disagree } & \multicolumn{2}{|c|}{ Total } \\
\hline & $\mathbf{N}$ & $\%$ & $N$ & $\%$ & $N$ & $\%$ & $\mathrm{~N}$ & $\%$ \\
\hline $\begin{array}{l}\text { The Board provides useful advice to DOE } \\
\text { (and regulators, where appropriate) (R) * }\end{array}$ & 6 & $27.3 \%$ & 8 & $36.4 \%$ & 8 & $36.4 \%$ & 22 & $100 \%$ \\
\hline $\begin{array}{l}\text { The SSAB reaches agreement about } \\
\text { prioritization of key site issues for which } \\
\text { advice is sought }(\mathrm{R})^{*}\end{array}$ & 5 & $22.7 \%$ & 9 & $40.9 \%$ & 8 & $36.4 \%$ & 22 & $100 \%$ \\
\hline The Board helps define site problems & 6 & $27.3 \%$ & 9 & $40.9 \%$ & 7 & $31.8 \%$ & 22 & $100 \%$ \\
\hline $\begin{array}{l}\text { The Board addresses issues in a timely } \\
\text { manner }(\mathrm{R}) *\end{array}$ & 7 & $31.8 \%$ & 6 & $27.3 \%$ & 9 & $40.9 \%$ & 22 & $100 \%$ \\
\hline $\begin{array}{l}\text { The Board reaches consensus on key site } \\
\text { issues (R) * }\end{array}$ & 9 & $40.9 \%$ & 11 & $50.0 \%$ & 2 & $9.1 \%$ & 22 & $100 \%$ \\
\hline $\begin{array}{l}\text { The Board provides informed advice to } \\
\text { DOE (and regulators, where appropriate) }\end{array}$ & 4 & $18.2 \%$ & 9 & $40.9 \%$ & 9 & $40.9 \%$ & 22 & $100 \%$ \\
\hline $\begin{array}{l}\text { The Board provides advice that reflects } \\
\text { the viewpoints and priorities of the } \\
\text { community }\end{array}$ & 10 & $47.6 \%$ & 5 & $23.8 \%$ & 6 & $28.6 \%$ & 21 & $100 \%$ \\
\hline
\end{tabular}

* (R) denotes a question that was asked in the negative form. For ease of review, these questions and data have been reversed in this table and are stated in positive forms. 
Table 4.

Goal 4. Improve DOE's (and where applicable, regulators') site decisions and decision making process

\begin{tabular}{|c|c|c|c|c|c|c|c|c|}
\hline \multirow[t]{2}{*}{ Los Alamos } & \multicolumn{2}{|c|}{ Agree } & \multicolumn{2}{|c|}{$\begin{array}{c}\text { Neither/ } \\
\text { Don't Know }\end{array}$} & \multicolumn{2}{|c|}{ Disagree } & \multicolumn{2}{|c|}{ Total } \\
\hline & $\mathrm{N}$ & $\%$ & $\mathrm{~N}$ & $\%$ & $\mathrm{~N}$ & $\%$ & $\mathrm{~N}$ & $\%$ \\
\hline $\begin{array}{l}\text { The SSAB has improved DOE's site } \\
\text { decisions (R) * }\end{array}$ & 4 & $18.2 \%$ & 5 & $22.7 \%$ & 13 & $59.1 \%$ & 22 & $100 \%$ \\
\hline $\begin{array}{l}\text { The Board understands the decision } \\
\text { making process at [a particular] site }\end{array}$ & 7 & $31.8 \%$ & 7 & $31.8 \%$ & 8 & $36.4 \%$ & 22 & $100 \%$ \\
\hline $\begin{array}{l}\text { DOE discusses important policies } \\
\text { affecting site decisions with the Board }\end{array}$ & 10 & $45.5 \%$ & 9 & $40.9 \%$ & 3 & $13.6 \%$ & 22 & $100 \%$ \\
\hline $\begin{array}{l}\mathrm{DOE} \text { has explained to the Board its site } \\
\text { decision making process }\end{array}$ & 7 & $31.8 \%$ & 8 & $36.4 \%$ & 7 & $31.8 \%$ & 22 & $100 \%$ \\
\hline $\begin{array}{l}\text { The DOE shows how Board advice is } \\
\text { reflected in site decisions }\end{array}$ & 1 & $4.5 \%$ & 16 & $72.7 \%$ & 5 & 22.7 & 22 & $100 \%$ \\
\hline $\begin{array}{l}\text { The decision making process used by } \\
\text { DOE at [a particular] site is effective in } \\
\text { furthering site clean-up (R) }{ }^{*}\end{array}$ & 8 & $36.4 \%$ & 12 & $54.5 \%$ & 2 & $9.1 \%$ & 22 & $100 \%$ \\
\hline
\end{tabular}

* (R) denotes a question that was asked in the negative form. For ease of review, these questions and data have been reversed in this table and are stated in positive forms. 
Table 5.

Goal 5. Lead to more acceptable actions

\begin{tabular}{|c|c|c|c|c|c|c|c|c|}
\hline \multirow[t]{2}{*}{ Los Alamos } & \multicolumn{2}{|c|}{ Agree } & \multicolumn{2}{|c|}{$\begin{array}{c}\text { Neither/ } \\
\text { Don't Know }\end{array}$} & \multicolumn{2}{|c|}{ Disagree } & \multicolumn{2}{|c|}{ Total } \\
\hline & $\mathrm{N}$ & $\%$ & $\mathrm{~N}$ & $\%$ & $\mathrm{~N}$ & $\%$ & $\mathrm{~N}$ & $\%$ \\
\hline $\begin{array}{l}\text { The SSAB leads to more acceptable site } \\
\text { decisions }\end{array}$ & 4 & $18.2 \%$ & 10 & $45.5 \%$ & 8 & $36.4 \%$ & 22 & $100 \%$ \\
\hline $\begin{array}{l}\text { The Board supports the recommendations } \\
\text { it gives DOE }\end{array}$ & 7 & $31.8 \%$ & 14 & $63.6 \%$ & 1 & $4.5 \%$ & 22 & $100 \%$ \\
\hline The Board supports DOE's site actions & 3 & $13.6 \%$ & 16 & $72.7 \%$ & 3 & $13.6 \%$ & 22 & $100 \%$ \\
\hline $\begin{array}{l}\text { The DOE pays attention to the Board's } \\
\text { advice on key site issues (R) * }\end{array}$ & 5 & $22.7 \%$ & 15 & $68.2 \%$ & 2 & $9.1 \%$ & 22 & $100 \%$ \\
\hline $\begin{array}{l}\text { There is support in the community for } \\
\text { DOE's site decisions that have SSAB } \\
\text { input }\end{array}$ & 3 & $13.6 \%$ & 16 & $72.7 \%$ & 3 & $13.6 \%$ & 22 & $100 \%$ \\
\hline Progress is being made on key site issues & 6 & $27.3 \%$ & 10 & $45.5 \%$ & 6 & $27.3 \%$ & 22 & $100 \%$ \\
\hline
\end{tabular}

* (R) denotes a question that was asked in the negative form. For ease of review, these questions and data have been reversed in this table and are stated in positive forms. 
Table 6.

Goal 6. Contribute to trust and confidence in DOE

\begin{tabular}{|c|c|c|c|c|c|c|c|c|}
\hline \multirow[t]{2}{*}{ Los Alamos } & \multicolumn{2}{|c|}{ Agree } & \multicolumn{2}{|c|}{$\begin{array}{c}\text { Neither/ } \\
\text { Don't Know }\end{array}$} & \multicolumn{2}{|c|}{ Disagree } & \multicolumn{2}{|c|}{ Total } \\
\hline & $\mathrm{N}$ & $\%$ & $\mathrm{~N}$ & $\%$ & $\mathrm{~N}$ & $\%$ & $N$ & $\%$ \\
\hline $\begin{array}{l}\text { The SSAB contributes to trust and } \\
\text { confidence in DOE }\end{array}$ & 2 & $9.1 \%$ & 12 & $54.5 \%$ & 8 & $36.4 \%$ & 22 & $100 \%$ \\
\hline $\begin{array}{l}\text { Relationships between DOE and the public } \\
\text { have improved since the formation of the } \\
\text { SSAB }\end{array}$ & 5 & $22.7 \%$ & 13 & $59.1 \%$ & 4 & $18.2 \%$ & 22 & $100 \%$ \\
\hline $\begin{array}{l}\mathrm{DOE} \text { is committed to clean up [a } \\
\text { particular] site }\end{array}$ & 9 & $40.9 \%$ & 8 & $36.4 \%$ & 5 & $22.7 \%$ & 22 & $100 \%$ \\
\hline
\end{tabular}


Table 7.

Public awareness

\begin{tabular}{llllllllll}
\hline \multicolumn{1}{c}{ Los Alamos } & Agree & $\begin{array}{c}\text { Neither/ } \\
\text { Don't Know }\end{array}$ & Disagree & Total \\
& $\mathrm{N}$ & $\%$ & $\mathrm{~N}$ & $\%$ & $\mathrm{~N}$ & $\%$ & $\mathrm{~N}$ & $\%$ \\
\hline $\begin{array}{l}\text { The public knows little about the role of } \\
\text { the Board }\end{array}$ & 17 & $77.3 \%$ & 3 & $13.6 \%$ & 2 & $9.1 \%$ & 22 & $100 \%$ \\
\hline
\end{tabular}

Table 8.

SSAB Initiative is a good use of funds

\begin{tabular}{|c|c|c|c|c|c|c|c|c|}
\hline \multirow[t]{2}{*}{ Los Alamos } & \multicolumn{2}{|c|}{ Yes, Good } & \multicolumn{2}{|c|}{$\begin{array}{c}\text { Neutral, } \\
\text { Conditional, } \\
\text { No Opinion }\end{array}$} & \multicolumn{2}{|c|}{$\begin{array}{c}\text { No, } \\
\text { Not Good }\end{array}$} & \multicolumn{2}{|c|}{ Total } \\
\hline & $\mathrm{N}$ & $\%$ & $\mathrm{~N}$ & $\%$ & $\mathrm{~N}$ & $\%$ & $\mathrm{~N}$ & $\%$ \\
\hline SSAB Initiative is a good use of funds & 11 & $64.7 \%$ & 1 & $5.9 \%$ & 5 & $29.4 \%$ & 17 & $100 \%$ \\
\hline
\end{tabular}

SSAB Supplementary Appendix

August 1996 
Table 9.

DOE-HQ involvement with the SSAB Initiative

\begin{tabular}{|c|c|c|c|c|c|c|c|c|}
\hline \multirow[t]{2}{*}{ Los Alamos } & \multicolumn{2}{|c|}{ Agree } & \multicolumn{2}{|c|}{$\begin{array}{l}\text { Neither/ } \\
\text { Don't Know }\end{array}$} & \multicolumn{2}{|c|}{ Disagree } & \multicolumn{2}{|c|}{ Total } \\
\hline & $N$ & $\%$ & $N$ & $\%$ & $N$ & $\%$ & $\mathrm{~N}$ & $\%$ \\
\hline $\begin{array}{l}\text { DOE-HQ provides helpful guidance to the } \\
\text { Boards and to DOE }\end{array}$ & 4 & $18.2 \%$ & 12 & $54.5 \%$ & 6 & $27.3 \%$ & 22 & $100 \%$ \\
\hline $\begin{array}{l}\text { DOE-HQ provides sufficient support to } \\
\text { facilitate the work of the SSAB }\end{array}$ & 7 & $31.8 \%$ & 7 & $31.8 \%$ & 8 & $36.4 \%$ & 22 & $100 \%$ \\
\hline $\begin{array}{l}\text { DOE-HQ gives careful consideration to } \\
\text { SSAB advice in its decisions }\end{array}$ & 2 & $9.1 \%$ & 16 & $72.7 \%$ & 4 & $18.2 \%$ & 22 & $100 \%$ \\
\hline
\end{tabular}


Table 10.

Personal experience with the SSAB Initiative

\begin{tabular}{|c|c|c|c|c|c|c|c|c|}
\hline \multirow[t]{2}{*}{ Los Alamos } & \multicolumn{2}{|c|}{ Agree } & \multicolumn{2}{|c|}{$\begin{array}{c}\text { Neither/ } \\
\text { Don't Know }\end{array}$} & \multicolumn{2}{|c|}{ Disagree } & \multicolumn{2}{|c|}{ Total } \\
\hline & $\mathrm{N}$ & $\%$ & $\mathrm{~N}$ & $\%$ & $\mathrm{~N}$ & $\%$ & $\mathbf{N}$ & $\%$ \\
\hline $\begin{array}{l}\text { I consider myself to be very } \\
\text { knowledgeable about site issues }\end{array}$ & 15 & $68.2 \%$ & 2 & $9.1 \%$ & 5 & $22.7 \%$ & 22 & $100 \%$ \\
\hline $\begin{array}{l}\text { Because of the SSAB, I better understand } \\
\text { the complexities of site clean-up }\end{array}$ & 11 & $50.0 \%$ & 4 & $18.2 \%$ & 7 & $31.8 \%$ & 22 & $100 \%$ \\
\hline $\begin{array}{l}\text { I feel that my participation in the SSAB } \\
\text { has been worthwhile }(\mathrm{R}) *\end{array}$ & 11 & $50.0 \%$ & 8 & $36.4 \%$ & 3 & $13.6 \%$ & 22 & $100 \%$ \\
\hline
\end{tabular}

* (R) denotes a question that was asked in the negative form. For ease of review, these questions and data have been reversed in this table and are stated in positive forms.

SSAB Supplementary Appendix 
Table 11.

Group/Team work skills

\begin{tabular}{|c|c|c|c|c|c|c|c|c|}
\hline \multirow[t]{2}{*}{ Los Alamos } & \multicolumn{2}{|c|}{ Agree } & \multicolumn{2}{|c|}{$\begin{array}{l}\text { Neither/ } \\
\text { Don't Know }\end{array}$} & \multicolumn{2}{|c|}{ Disagree } & \multicolumn{2}{|c|}{ Total } \\
\hline & $\mathrm{N}$ & $\%$ & $\mathrm{~N}$ & $\%$ & $\mathrm{~N}$ & $\%$ & $N$ & $\%$ \\
\hline All have group/team work skills & 14 & $66.7 \%$ & 3 & $14.3 \%$ & 4 & $19.0 \%$ & 21 & $100 \%$ \\
\hline $\begin{array}{l}\text { Have developed well-established working } \\
\text { relationships with each other }\end{array}$ & 13 & $61.9 \%$ & 5 & $23.8 \%$ & 3 & $14.3 \%$ & 21 & $100 \%$ \\
\hline Like each other & 6 & $28.6 \%$ & 8 & $38.1 \%$ & 7 & $33.3 \%$ & 21 & $100 \%$ \\
\hline $\begin{array}{l}\text { Have a high degree of trust and } \\
\text { confidence in each other }\end{array}$ & 7 & $33.3 \%$ & 7 & $33.3 \%$ & 7 & $33.3 \%$ & 21 & $100 \%$ \\
\hline $\begin{array}{l}\text { Accept the values that are important to the } \\
\text { group }\end{array}$ & 11 & $52.4 \%$ & 6 & $28.6 \%$ & 4 & $19.0 \%$ & 21 & $100 \%$ \\
\hline Show respect for the viewpoints of others & 13 & $61.9 \%$ & 6 & $28.6 \%$ & 2 & $9.5 \%$ & 21 & $100 \%$ \\
\hline $\begin{array}{l}\text { Accept willingly the goals and } \\
\text { expectations for the Board }\end{array}$ & 11 & $52.4 \%$ & 5 & $23.8 \%$ & 5 & $23.8 \%$ & 21 & $100 \%$ \\
\hline $\begin{array}{l}\text { Set high expectations for what they } \\
\text { believe they can accomplish }\end{array}$ & 15 & $71.4 \%$ & 4 & $19.0 \%$ & 2 & $9.5 \%$ & 21 & $100 \%$ \\
\hline $\begin{array}{l}\text { Communicate fully and frankly to the } \\
\text { Board all relevant and valuable } \\
\text { information }\end{array}$ & 9 & $42.9 \%$ & 10 & $47.6 \%$ & 2 & $9.5 \%$ & 21 & $100 \%$ \\
\hline $\begin{array}{l}\text { Try not to waste the Board's time with } \\
\text { irrelevant material or communications }\end{array}$ & 7 & $33.3 \%$ & 6 & $28.6 \%$ & 8 & $38.1 \%$ & 21 & $100 \%$ \\
\hline $\begin{array}{l}\text { Are willing to be influenced by other } \\
\text { Board members about new ideas and } \\
\text { methods }\end{array}$ & 12 & $57.1 \%$ & 8 & $38.1 \%$ & 1 & $4.8 \%$ & 21 & $100 \%$ \\
\hline $\begin{array}{l}\text { Clearly understand the goals and } \\
\text { philosphy of the Board's operation }\end{array}$ & 11 & $52.4 \%$ & 4 & $19.0 \%$ & 6 & $28.6 \%$ & 21 & $100 \%$ \\
\hline $\begin{array}{l}\text { Do all they can to help the Board achieve } \\
\text { its objectives }\end{array}$ & 9 & $42.9 \%$ & 7 & $33.3 \%$ & 5 & $23.8 \%$ & 21 & $100 \%$ \\
\hline $\begin{array}{l}\text { Get the technical knowledge and training } \\
\text { in group skills they need }\end{array}$ & 12 & $57.1 \%$ & 4 & $19.0 \%$ & 5 & $23.8 \%$ & $21^{\circ}$ & $100 \%$ \\
\hline Provide one another mutual help & 11 & $52.4 \%$ & 7 & $33.3 \%$ & 3 & $14.3 \%$ & 21 & $100 \%$ \\
\hline
\end{tabular}

SSAB Supplementary Appendix

August 1996 
Monticello Site Specific Advisory Board Evaluation Survey Results

SSAB Supplementary Appendix August 1996 
Figure 1a. Monticello

Goal 1. Establish processes and procedures to provide an effective forum for exchange of information and viewpoints regarding DOE site issues

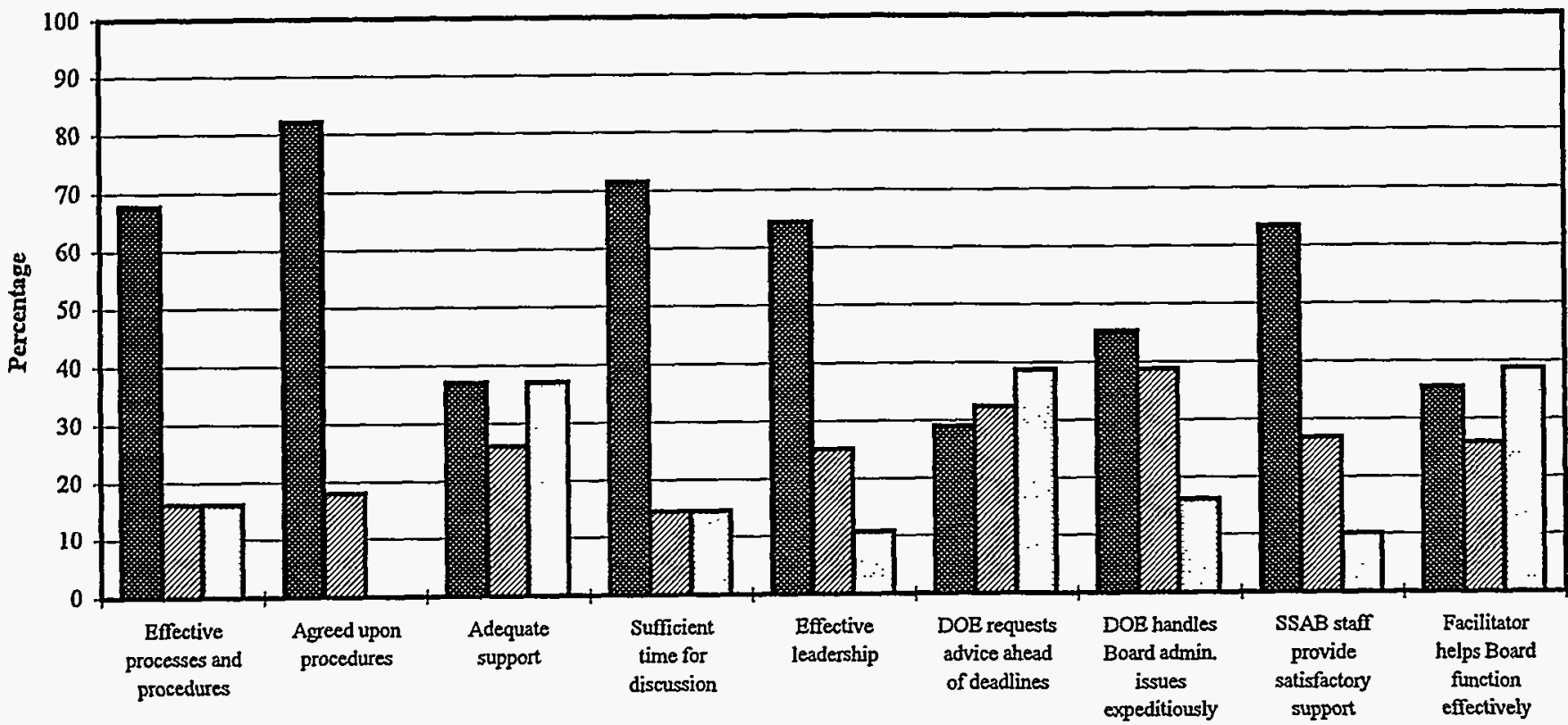

Agree (\%) DNeither Agree Nor Disagree/Don't Know (\%) DDisagree (\%)

Figure 1b. Long Survey: Entire Sample

Goal 1. Establish processes and procedures to provide an effective forum for exchange of information and viewpoints regarding DOE site issues

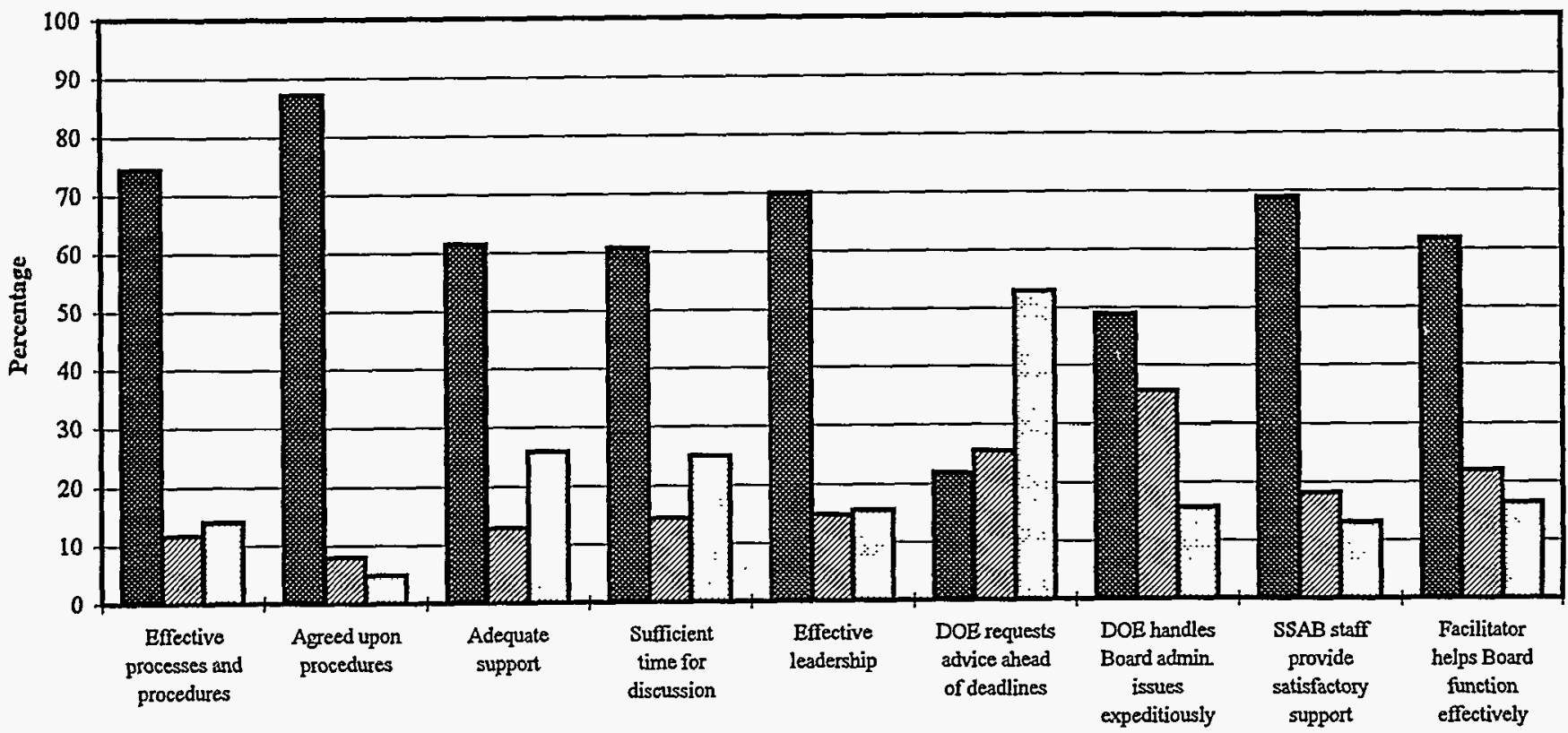

Agree (\%) Deither Agree Nor Disagree/Don't Know (\%) DDisagree (\%)

SSAB Supplementary Appendix

August 1996 
Figure 2a. Monticello

Goal 2. Facilitate interaction and exchange of information and viewpoints regarding $\mathrm{DOE}$ site issues

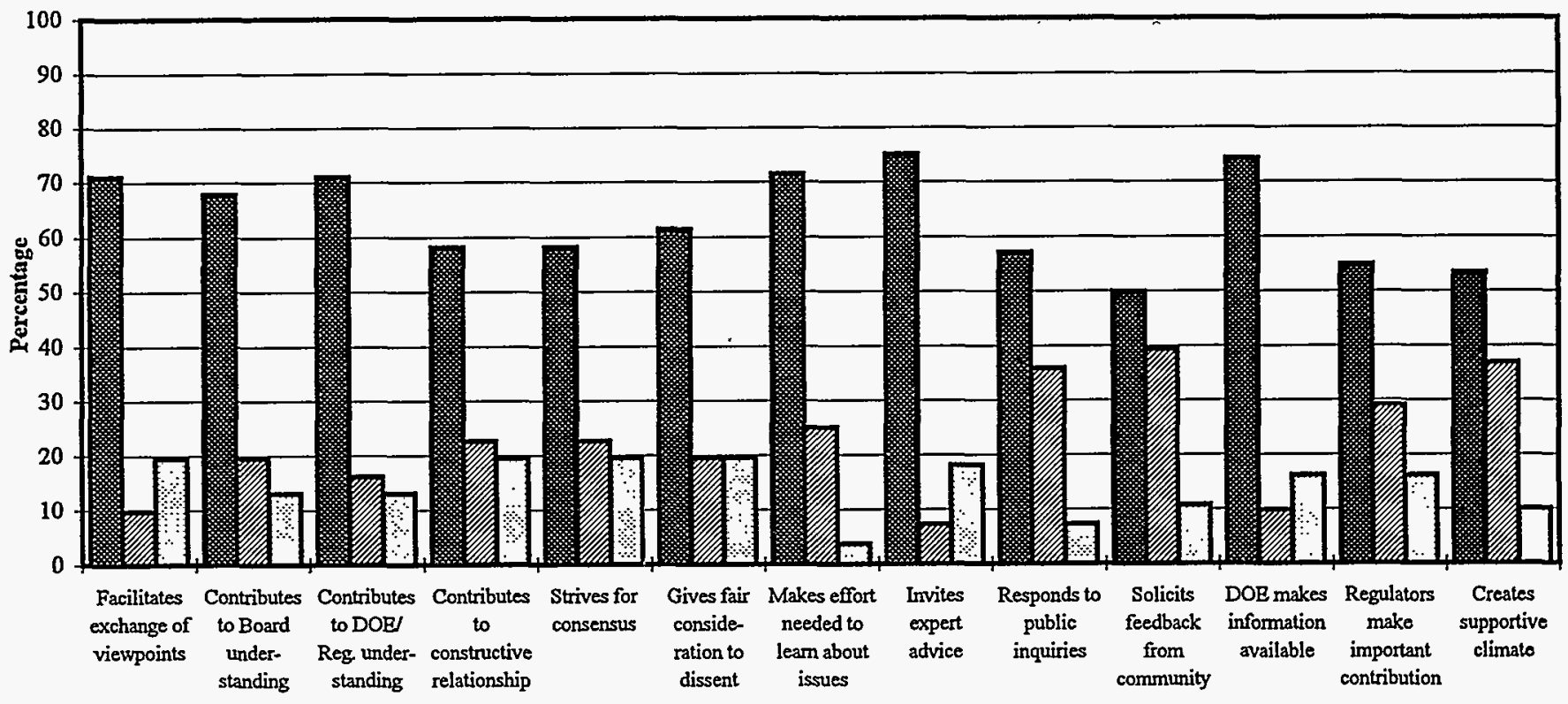

Agree (\%) DNeither Agree Nor Disagree/Don't Know (\%) DDisagree (\%)

Figure 2b. Long Survey: Entire Sample

Goal 2. Facilitate interaction and exchange of information and viewpoints regarding DOE site issues

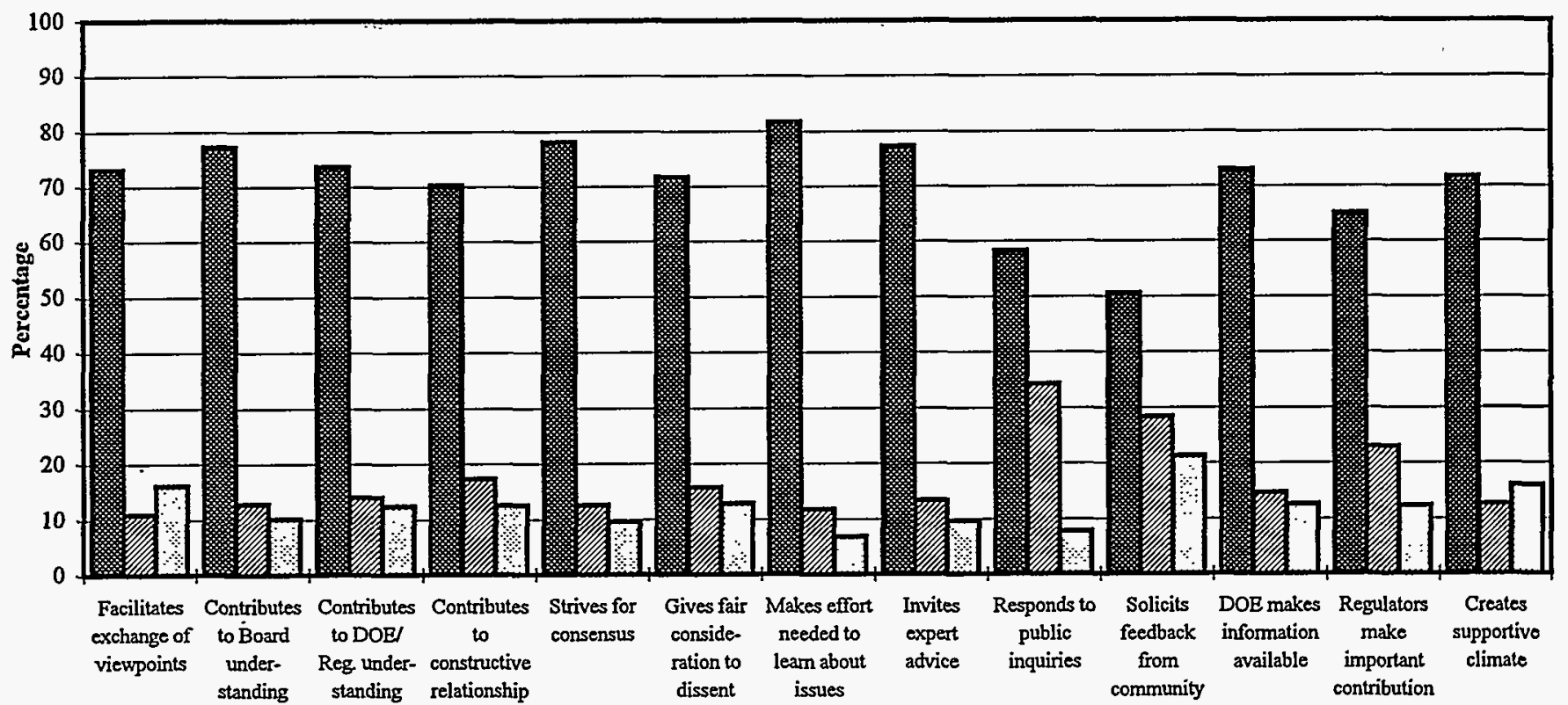

A Agree (\%) DNeither Agree Nor Disagree/Don't Know (\%) DDisagree (\%)

SSAB Supplementary Appendix

August 1996 
Figure 3a. Monticello

Goal 3. Provide useful advice and/or recommendations to DOE

(and regulators, where appropriate)

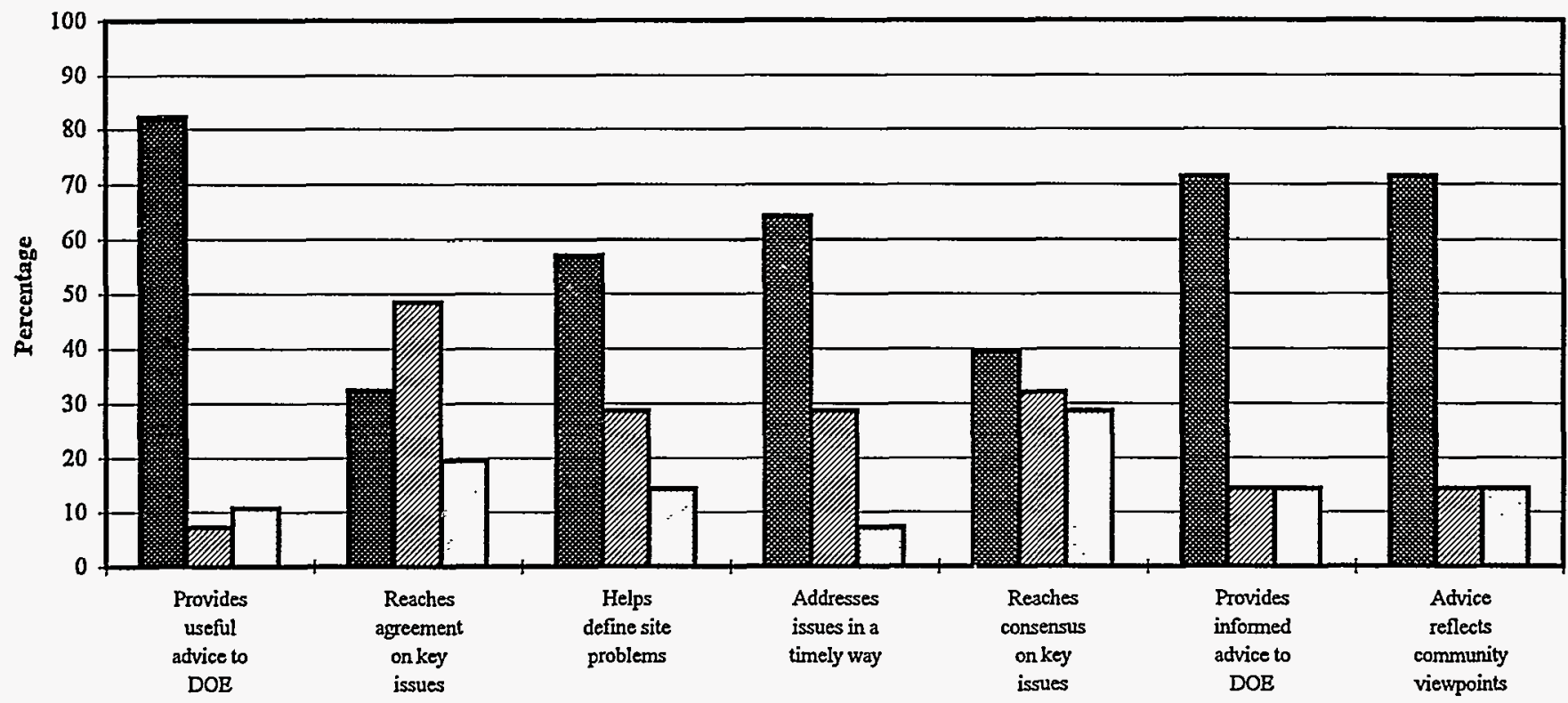

Agree (\%) Q Weither Agree Nor Disagree/Don't Know (\%) DDisagree (\%)

Figure 3b. Long Survey: Entire Sample

Goal 3. Provide useful advice. and/or recommendations to DOE

(and regulators, where appropriate)

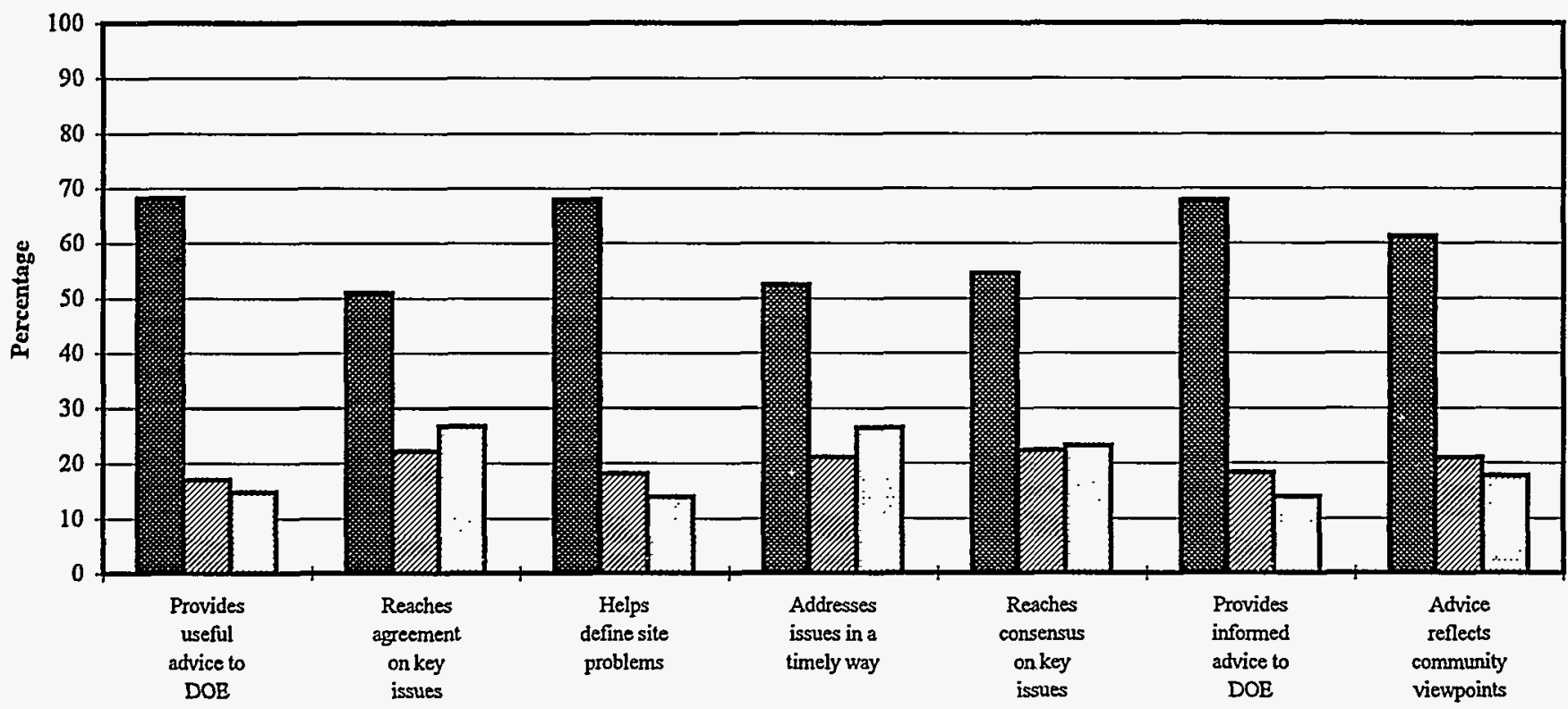

Agree (\%) DNeither Agree Nor Disagree/Don't Know (\%) D Disagree (\%)

SSAB Supplementary Appendix

August 1996 
Figure 4a. Monticello

Goal 4. Improve DOE's (and where applicable, regulators') site decisions and decision making process

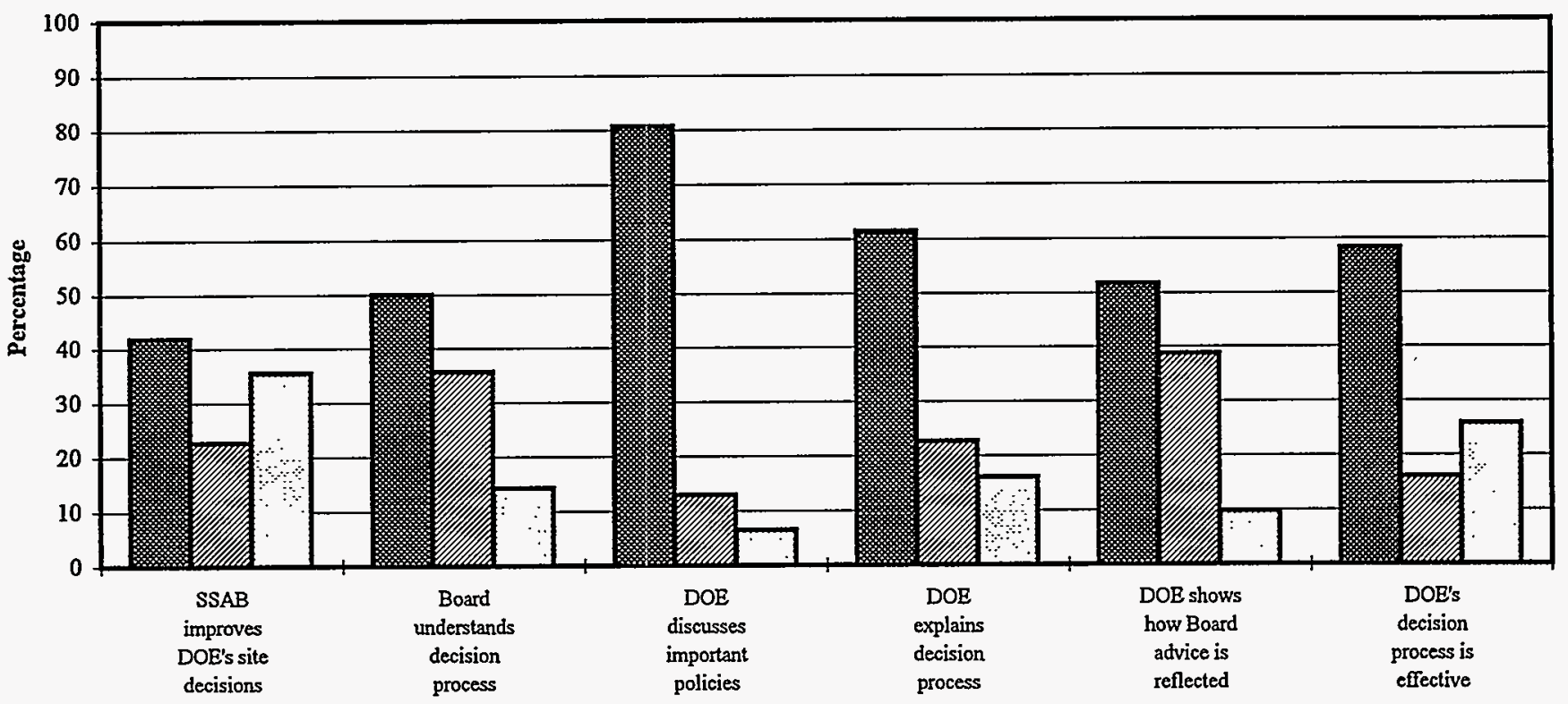

Agree (\%) Neither Agree Nor Disagree/Dont Know (\%) DDisagree (\%)

Figure 4b. Long Survey: Entire Sample

Goal 4. Improve DOE's (and where applicable, regulators') site decisions and decision making process

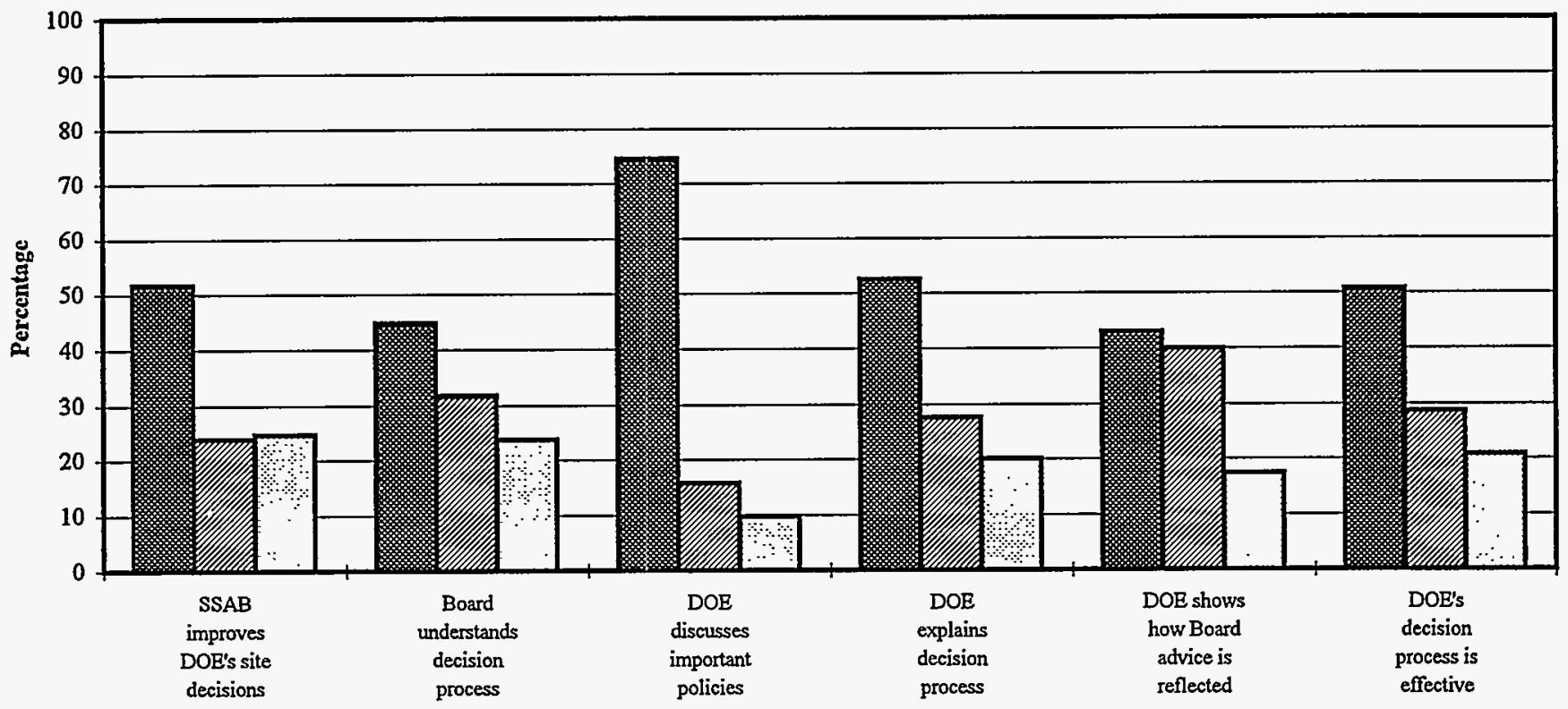

Agree (\%) Neither Agree Nor Disagree/Don't Know (\%) DDisagree (\%)

SSAB Supplementary Appendix August 1996 
Figure 5a. Monticello

Goal 5. Lead to more acceptable actions

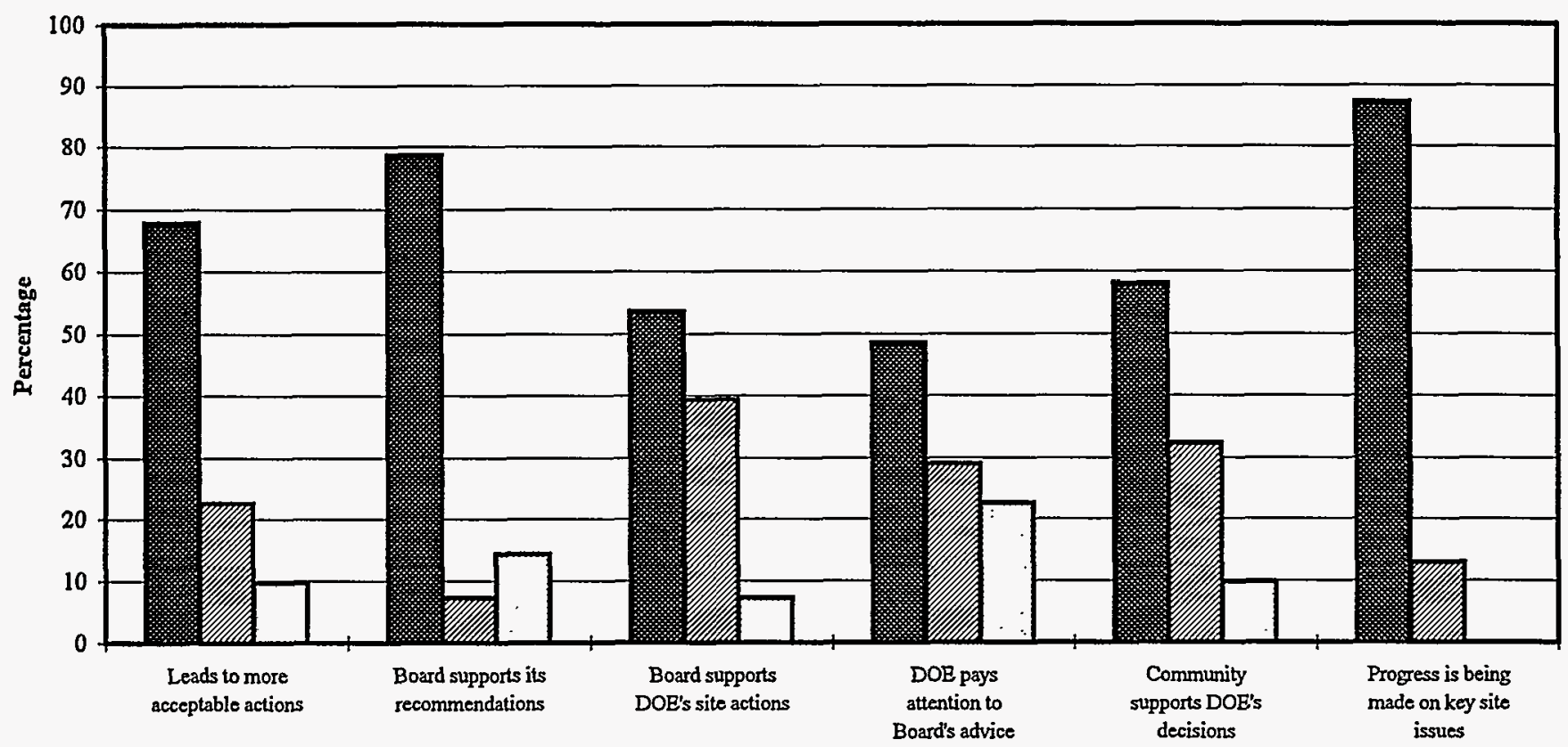

EAgree (\%) ENeither Agree Nor Disagree/Don't Know (\%) DDisagree (\%)

Figure 5b. Long Survey: Entire Sample

Goal 5. Lead to more acceptable actions

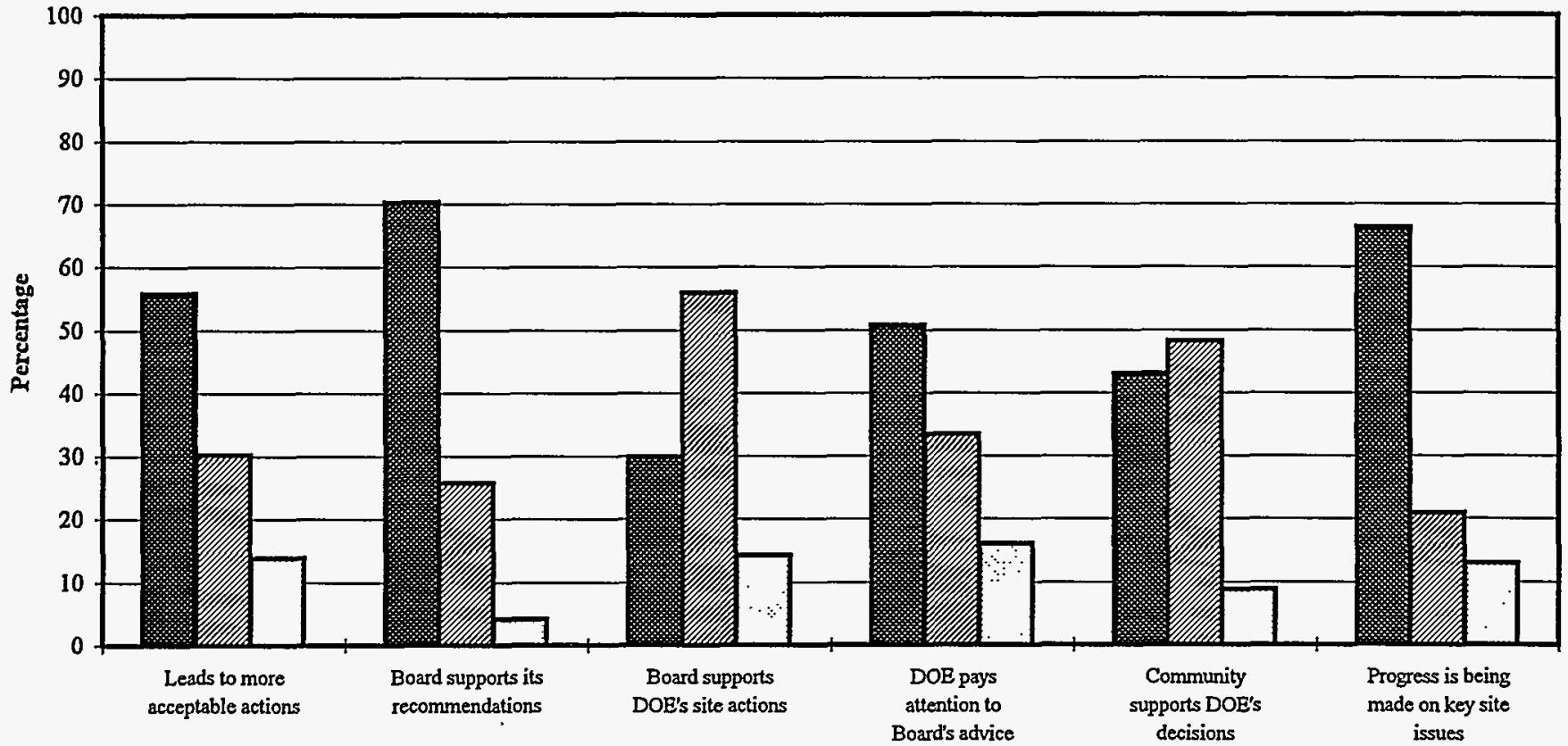

DAgree (\%) DNeither Agree Nor Disagree/Don't Know (\%) DDisagree (\%)

SSAB Supplementary Appendix

August 1996 
Figure 6a. Monticello

Goal 6. Contribute to trust and confidence in DOE

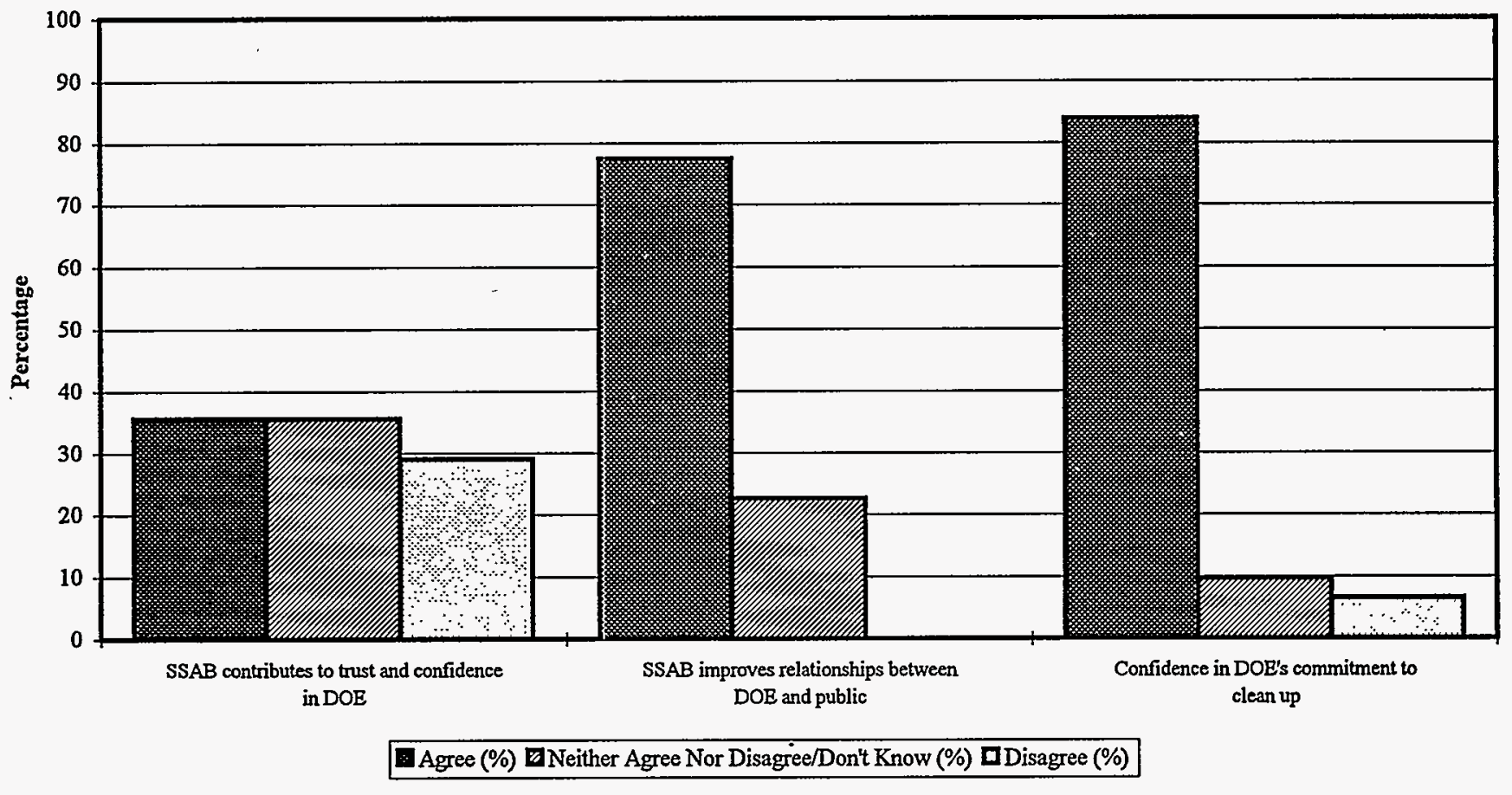

Figure 6b. Long Survey: Entire Sample

Goal 6. Contribute to trust and confidence in DOE

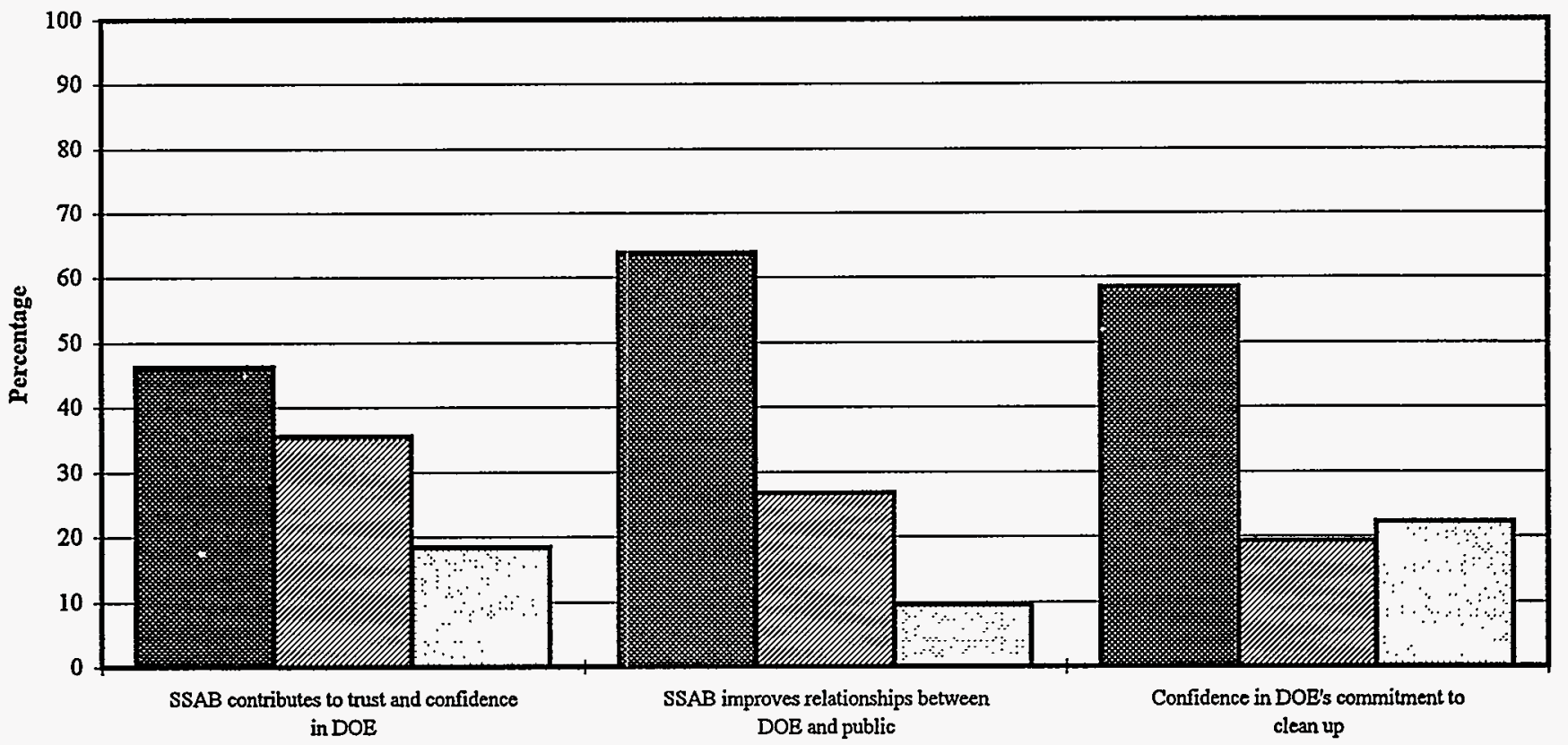

Agree (\%) $D$ Neither Agree Nor Disagree/Don't Know (\%) DDisagree (\%)

SSAB Supplementary Appendix

August 1996 
Figure 7a. Monticello

Public awareness

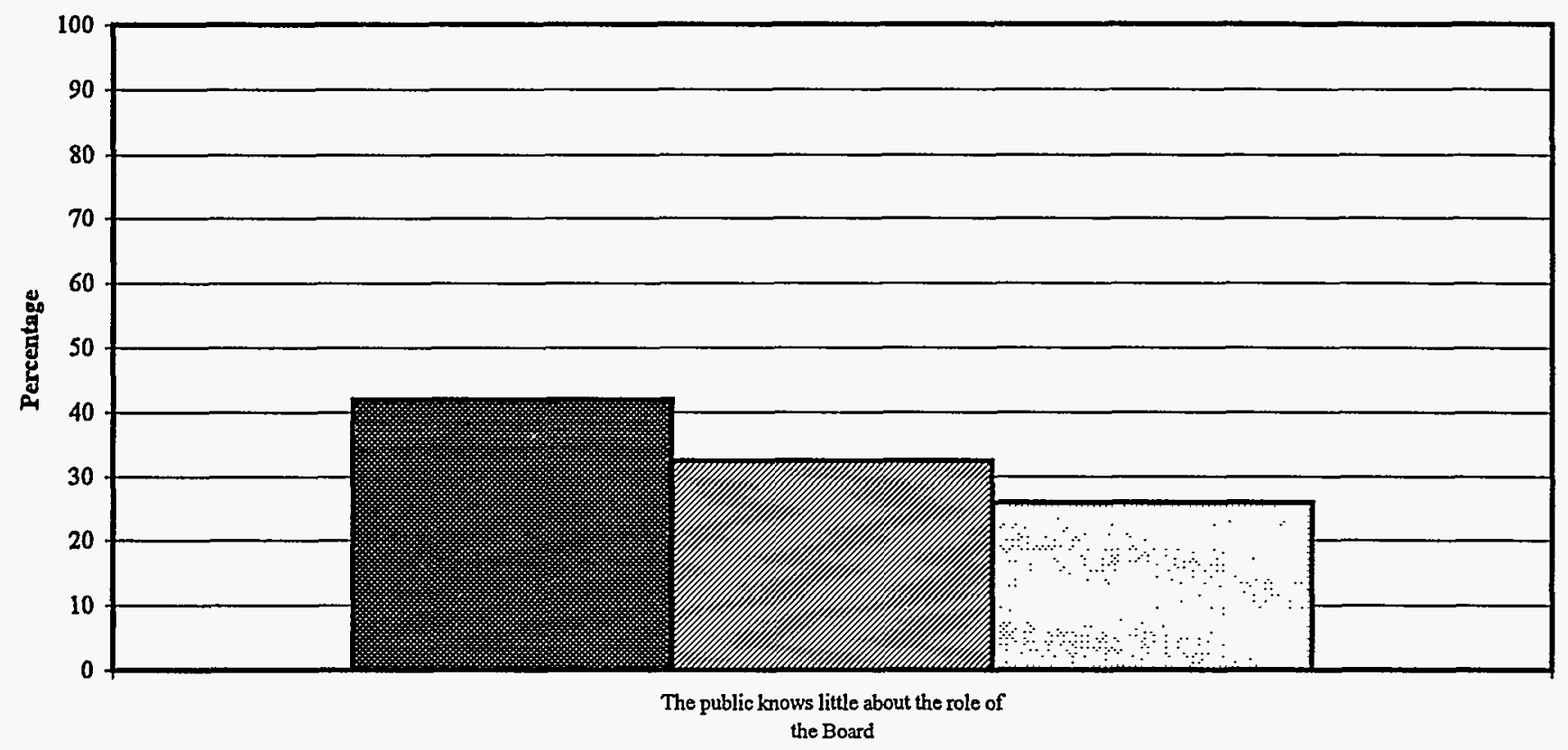

Agree (\%) Neither Agree Nor Disagree/Don't Know (\%) DDisagree (\%)

Figure 7b. Long Survey: Entire Sample

Public awareness

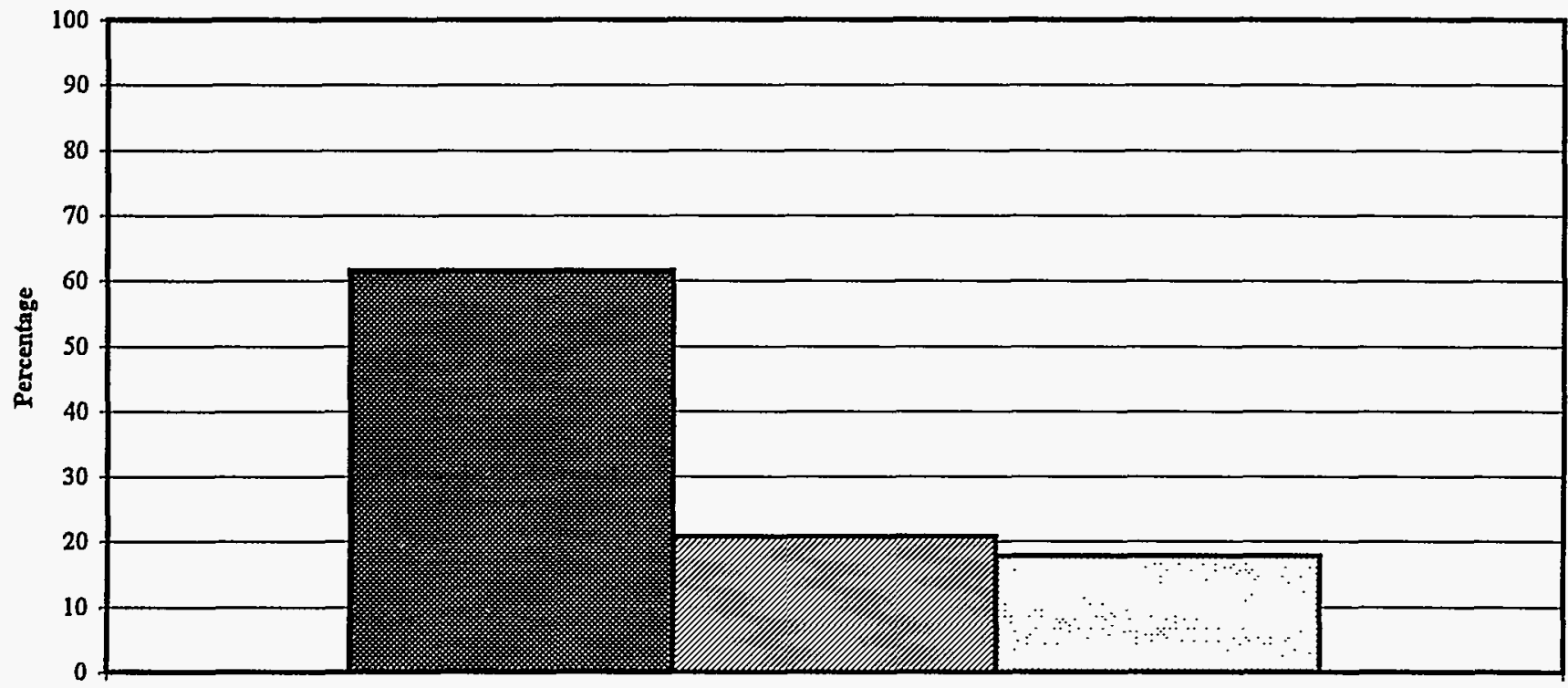

The public knows little about the role of the Board

A Agree (\%) ENeither Agree Nor Disagree/Don't Know (\%) DDisagree (\%)

SSAB Supplementary Appendix

August 1996 
Figure 8a. Monticello

DOE-HQ involvement with the SSAB Initiative

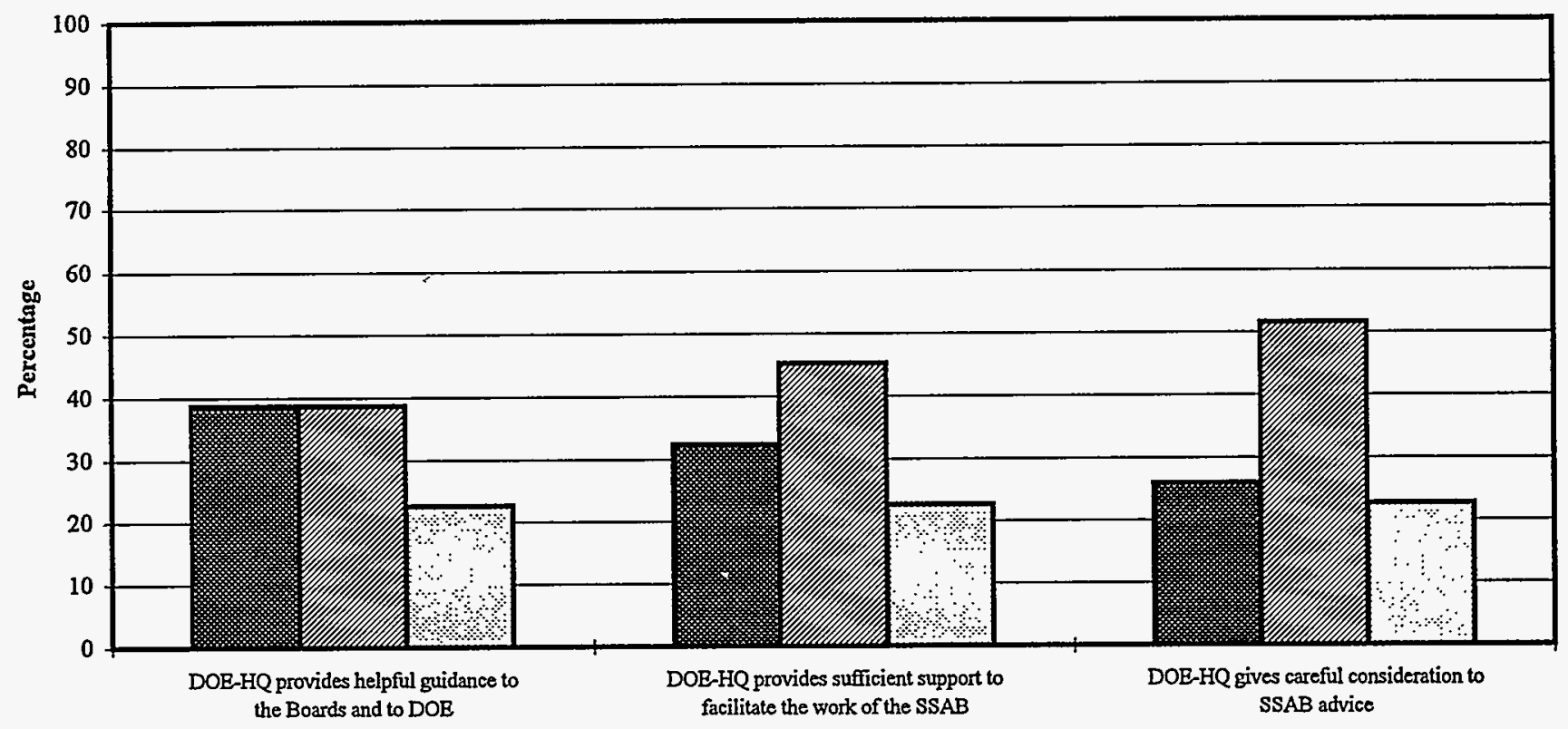

Agree (\%) Neither Agree Nor Disagree/Dont Know (\%) DDisagree (\%)

Figure 8b. Long Survey: Entire Sample

DOE-HQ involvement with the SSAB Initiative

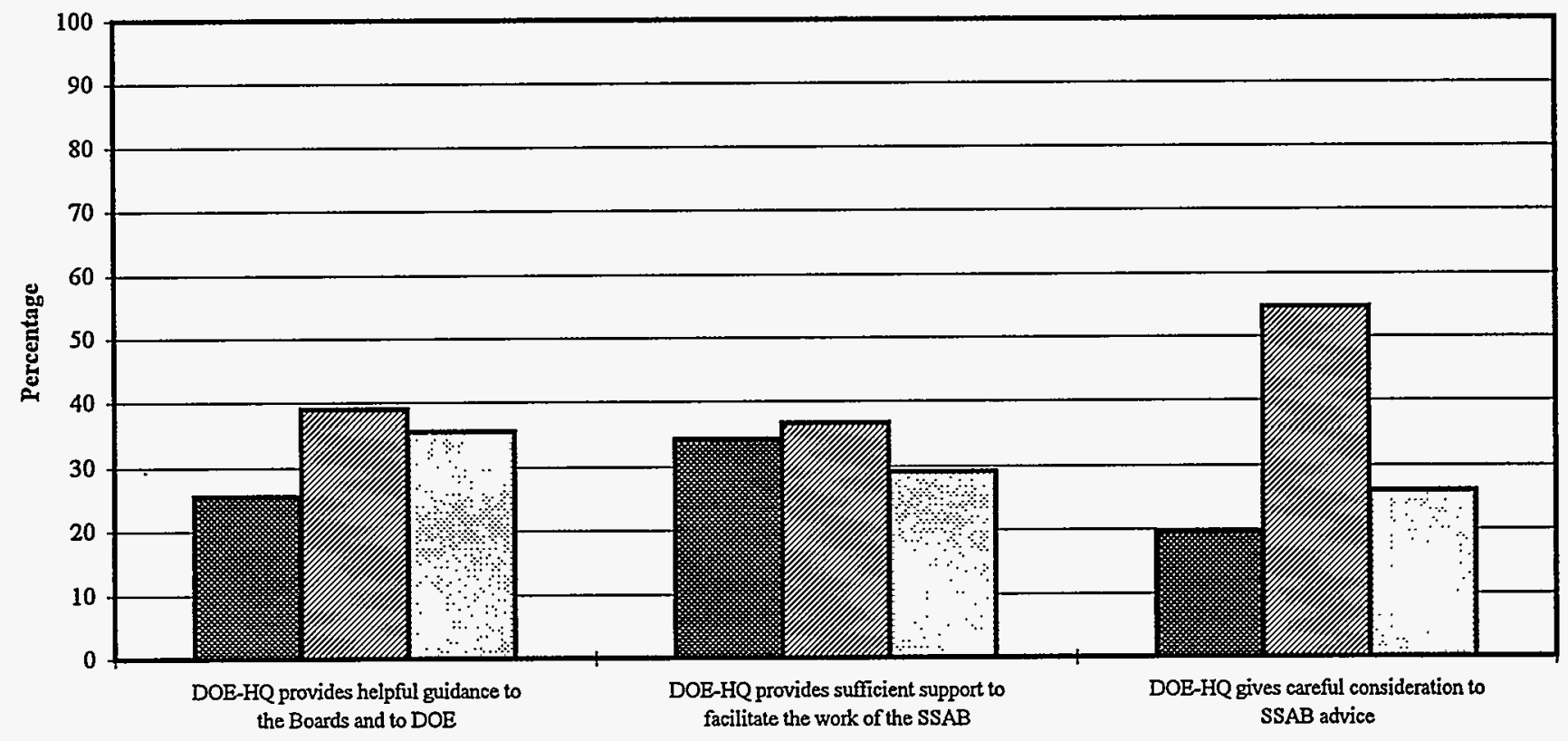

Q Agree (\%) ENeither Agree Nor Disagree/Don't Know (\%) DDisagree (\%)

SSAB Supplementary Appendix 
Figure 9a. Monticello

Personal experience with the SSAB Initiative

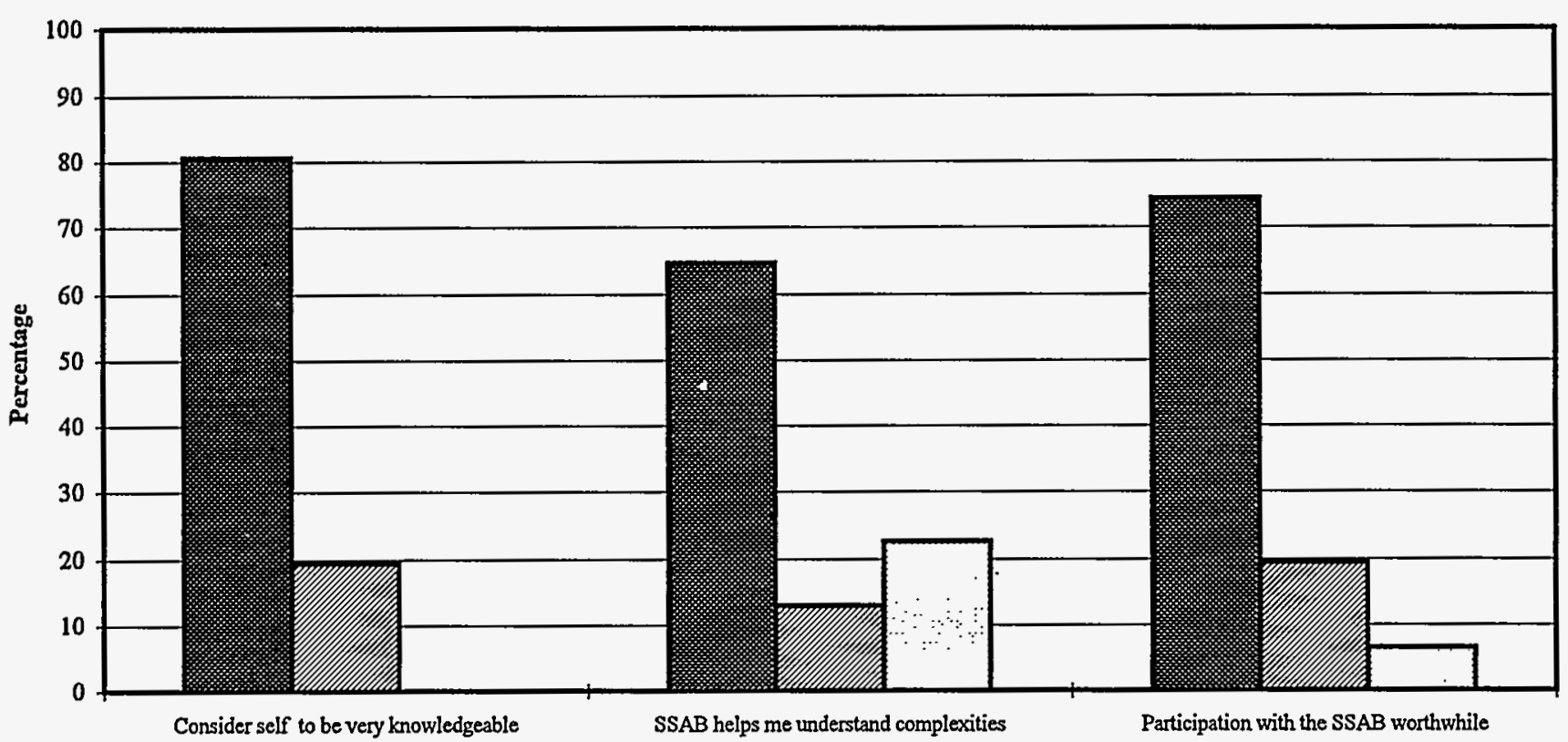

EAgree (\%) BNeither Agree Nor Disagree/Don't Know (\%) DDisagree (\%)

Figure 9b. Long Survey: Entire Sample

Personal experience with the SSAB Initiative

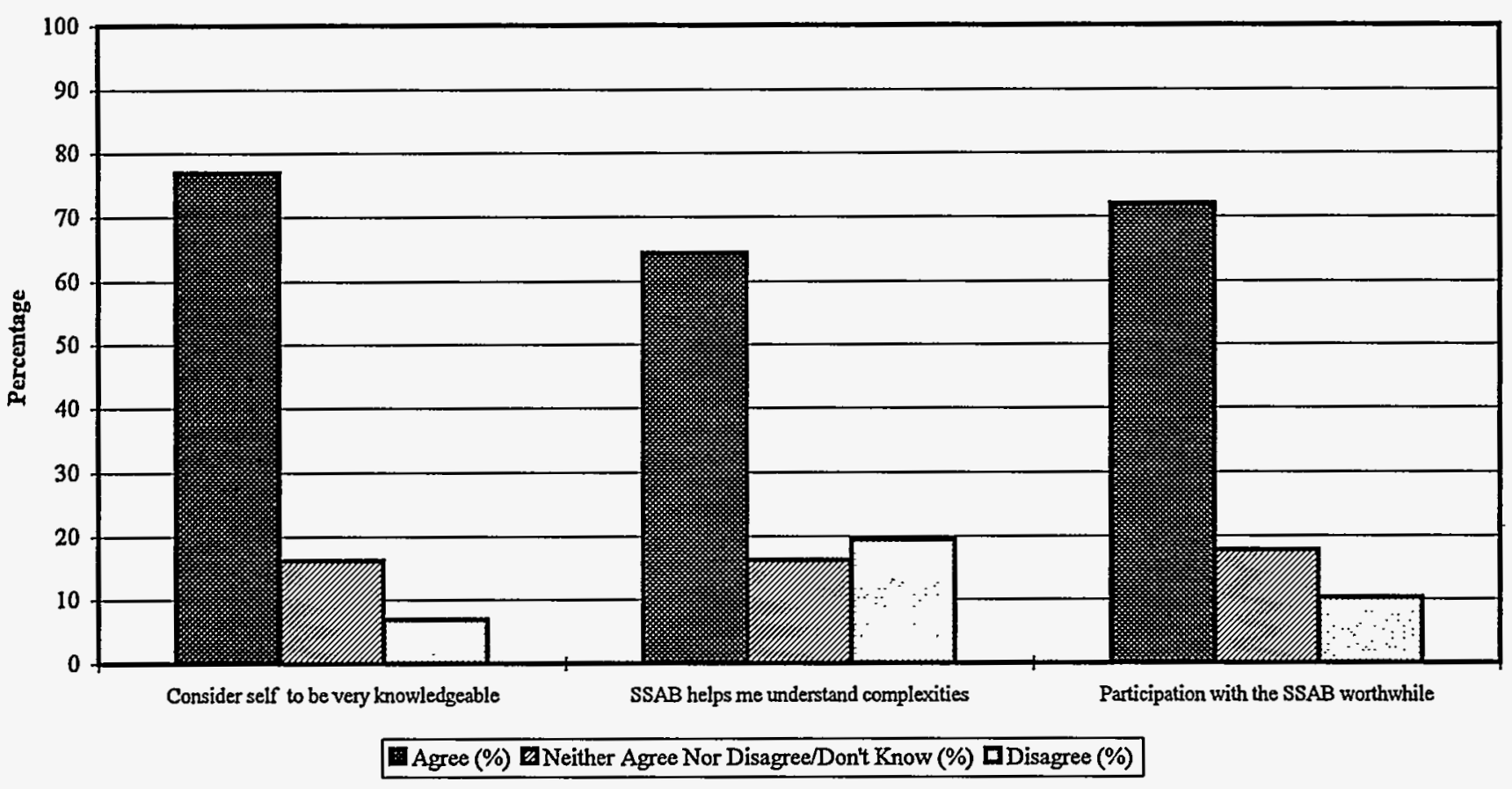

SSAB Supplementary Appendix

August 1996 
Figure 10a. Monticello

Group/Team work skills

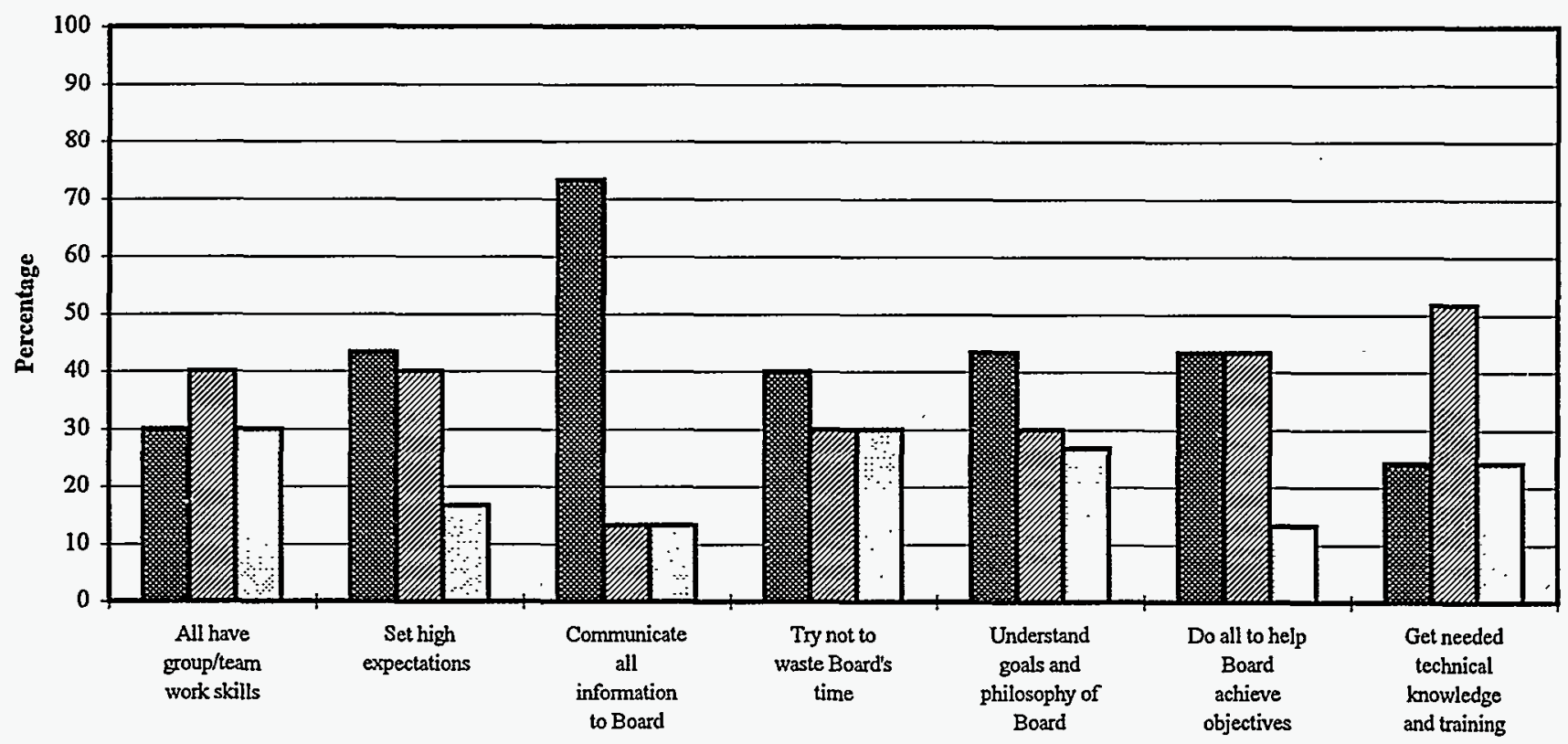

F Agree (\%) $\mathbf{Z}$ Neither Agree Nor Disagree/Don't Know (\%) DDisagree (\%)

Figure 10b. Long Survey: Entire Sample

Group/Team work skills

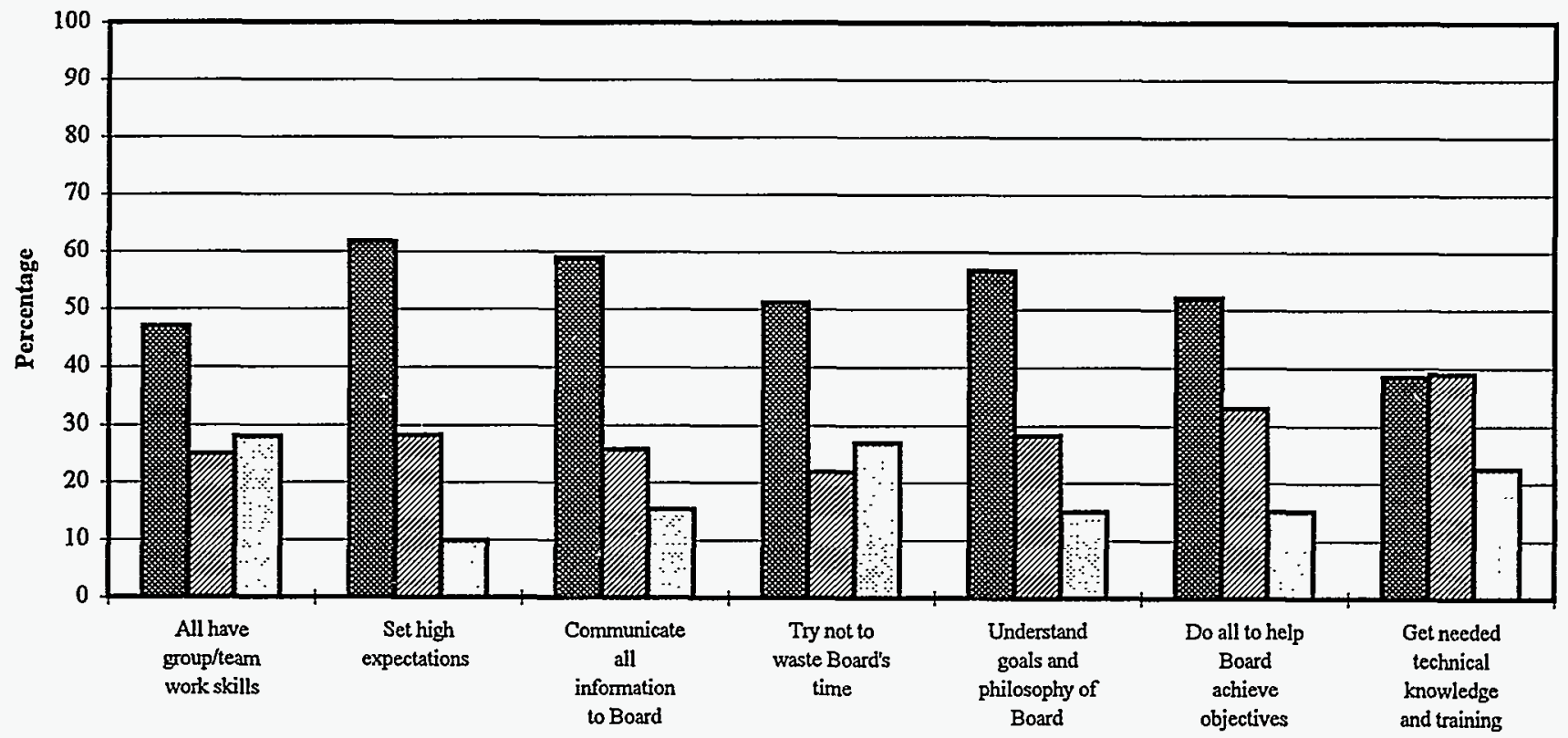

SAgree (\%) Deither Agree Nor Disagree/Don't Know (\%) DDisagree (\%)

SSAB Supplementary Appendix

August 1996 
Figure 11a. Monticello

Working relations

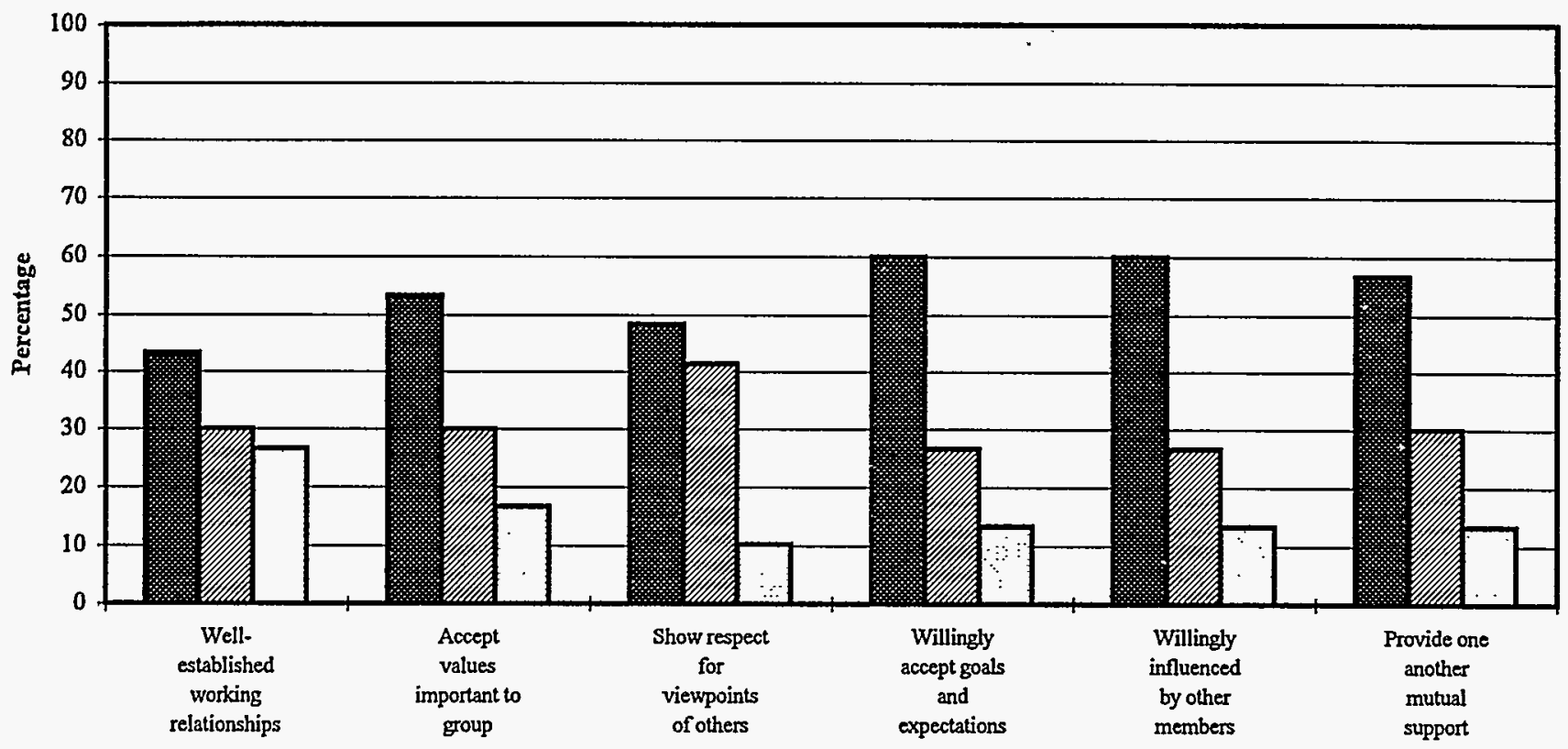

Agree (\%) E Neither Agree Nor Disagree/Dont Know (\%) DDisagree (\%)

Figure 11b. Long Survey: Entire Sample

Working relations

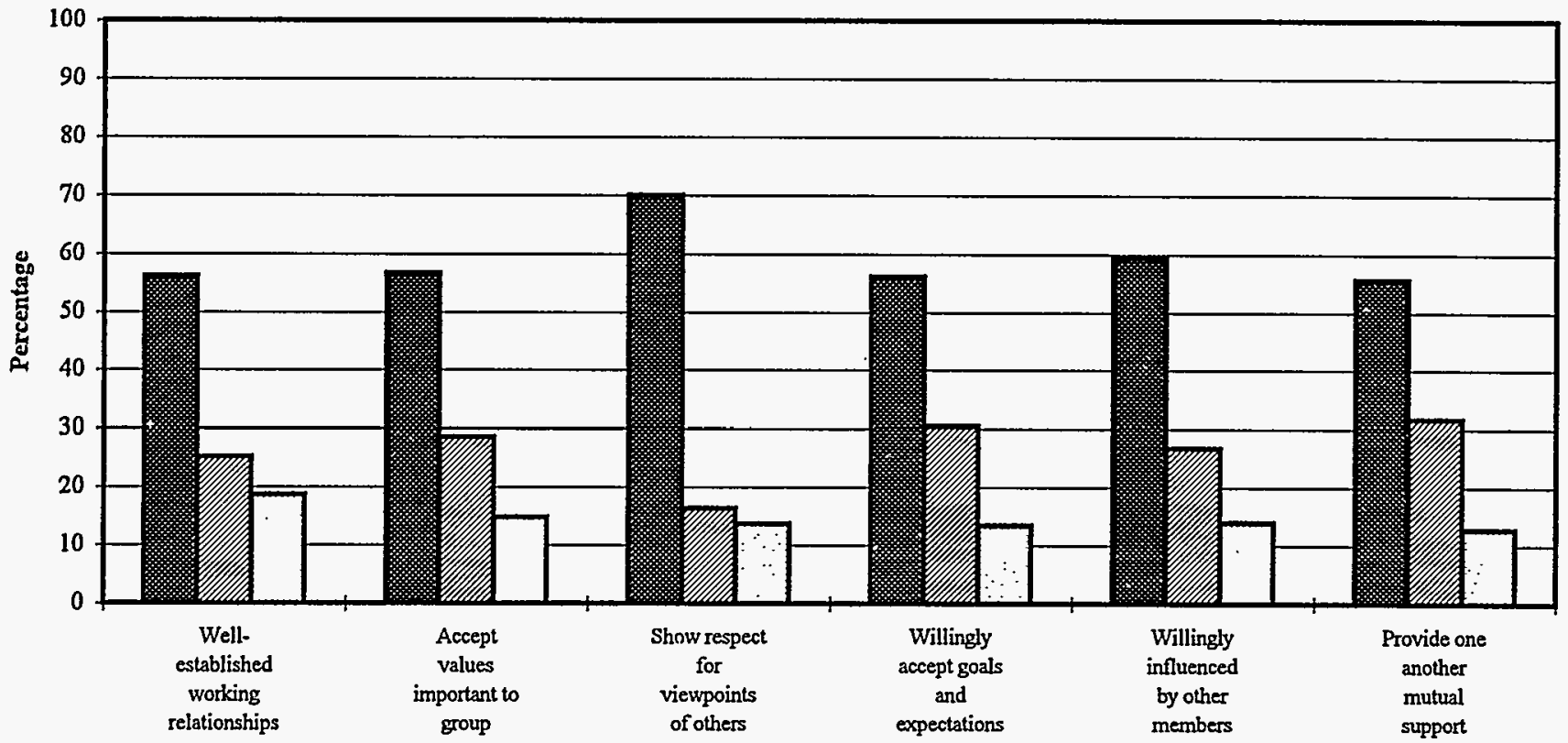

DAgree (\%) QNeither Agree Nor Disagree/Don't Know (\%) D Disagree (\%)

SSAB Supplementary Appendix

August 1996 
Figure 12a. Monticello

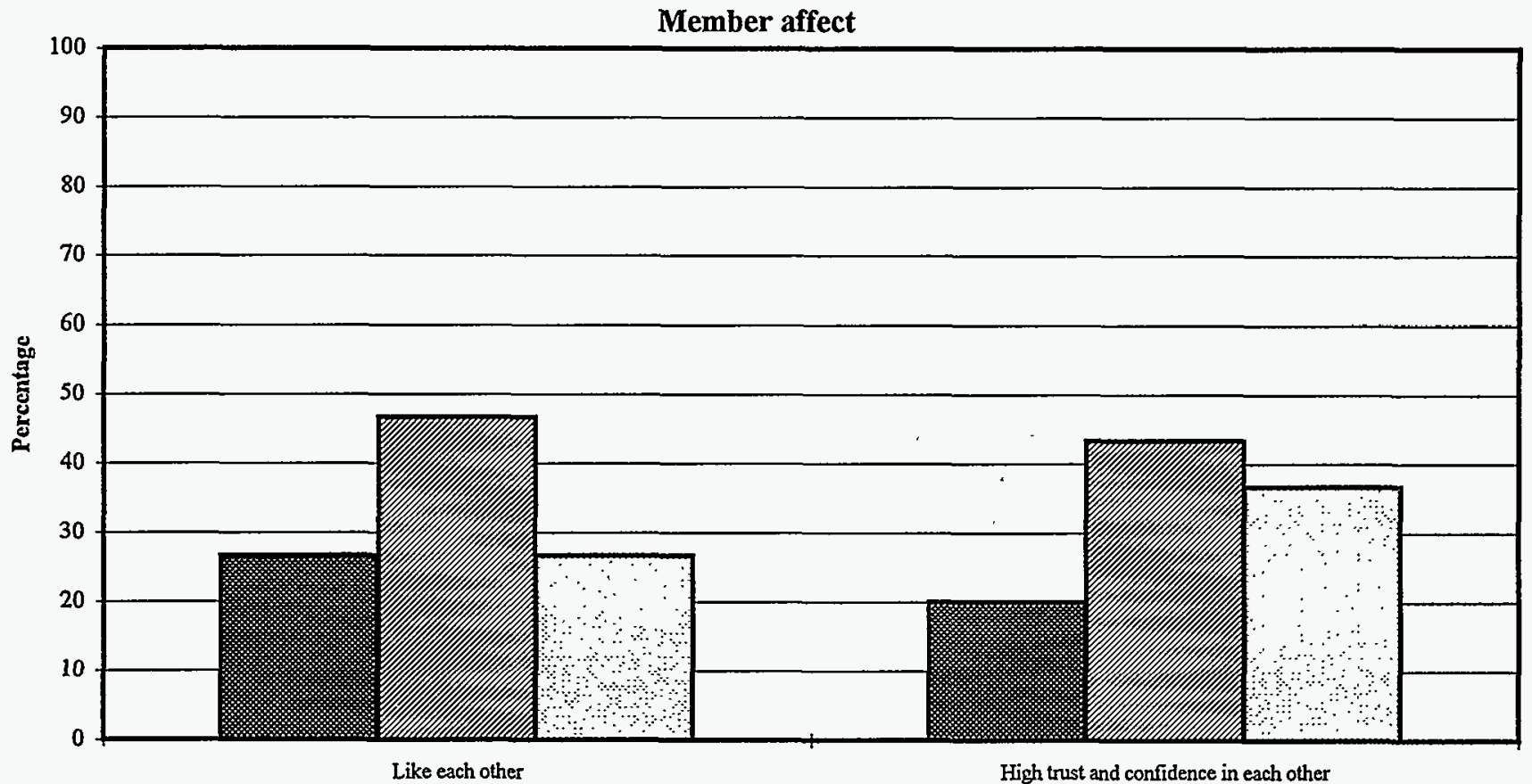

High trust and confidence in each other

T. Agree (\%) Neither Agree Nor Disagree/Don't Know (\%) DDisagree (\%)

Figure 12b. Long Survey: Entire Sample

Member affect

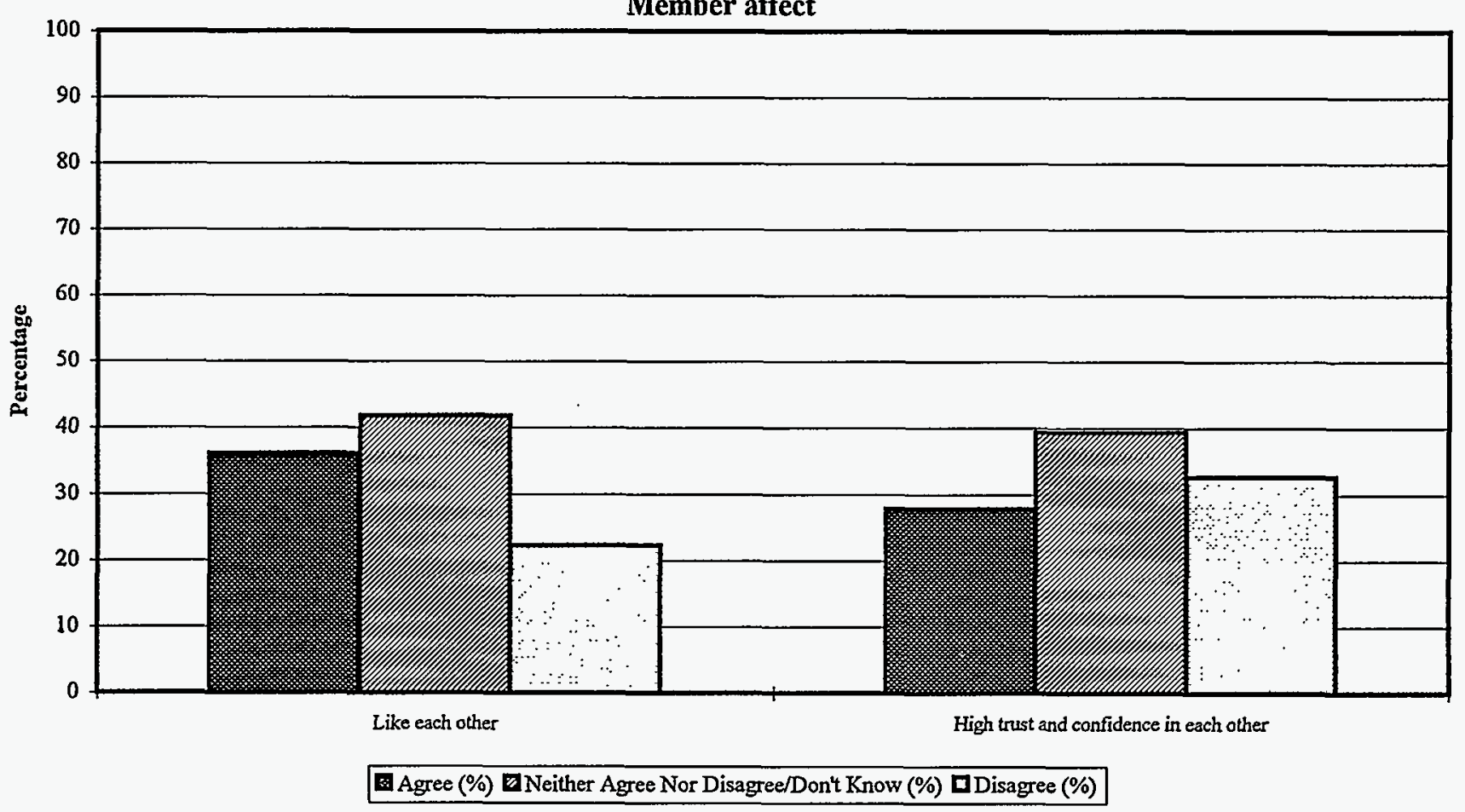

SSAB Supplementary Appendix

August 1996 
Figure 13a. Monticello

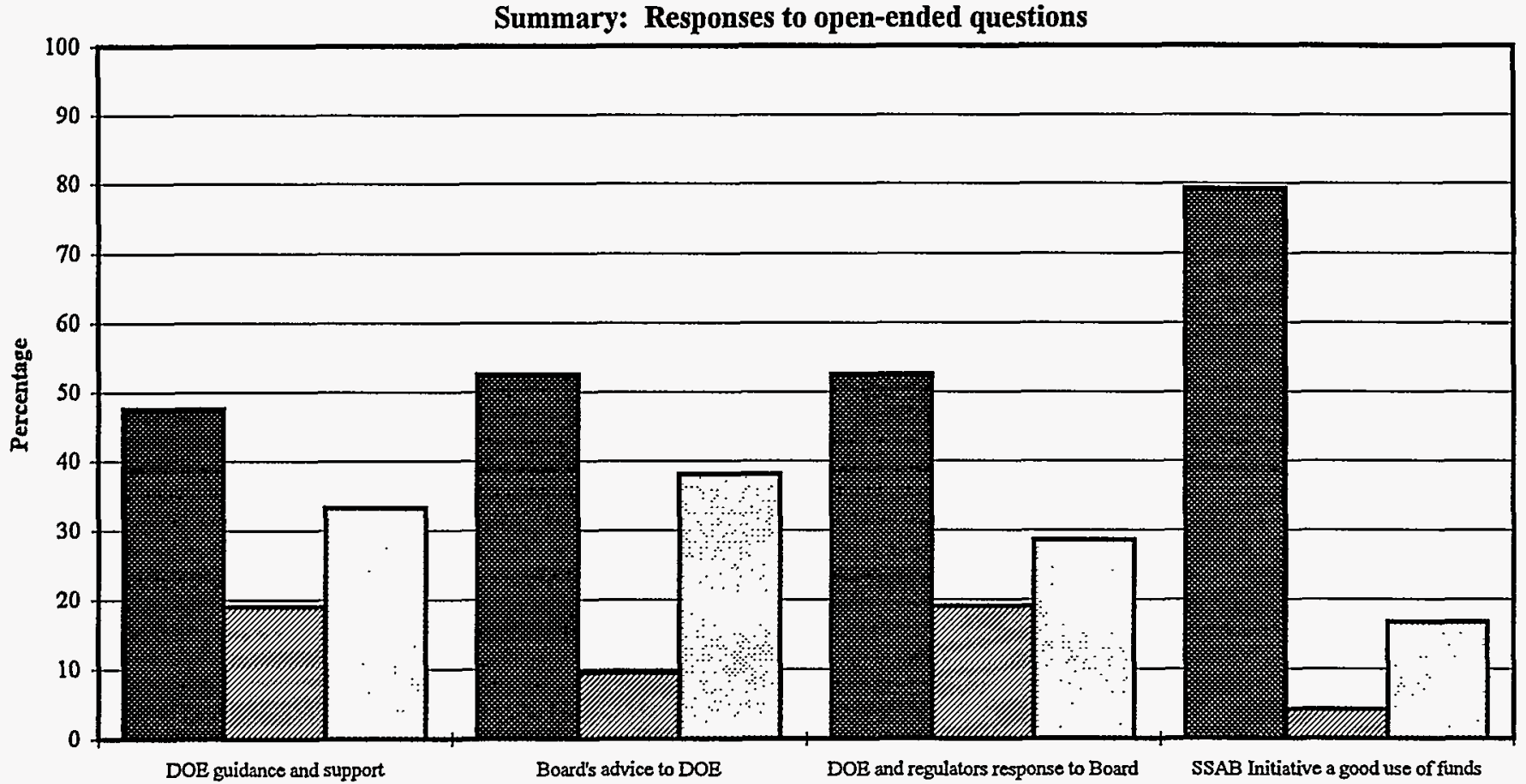

Yes, Good (\%) Neutral, Conditional, No Opinion (\%) DNo, Not Good (\%)

Figure 13b. Long Survey: Entire Sample

Summary: Responses to open-ended questions

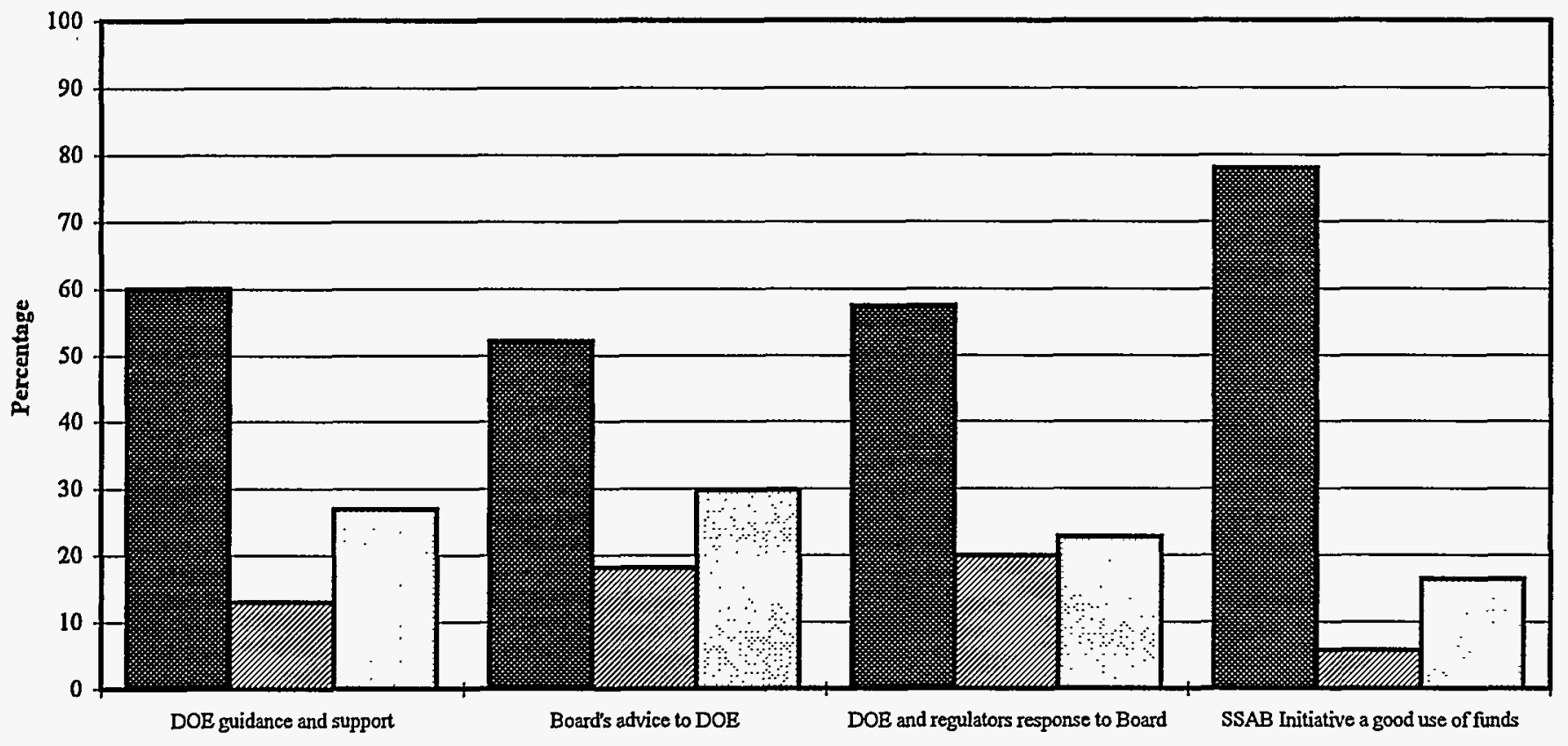

QYes, Good (\%) DNeutral, Conditional, No Opinion (\%) DNo, Not Good (\%)

SSAB Supplementary Appendix

August 1996 
Figure 14a. Monticello

Summary: Combined responses for SSAB Initiative goals (Means)

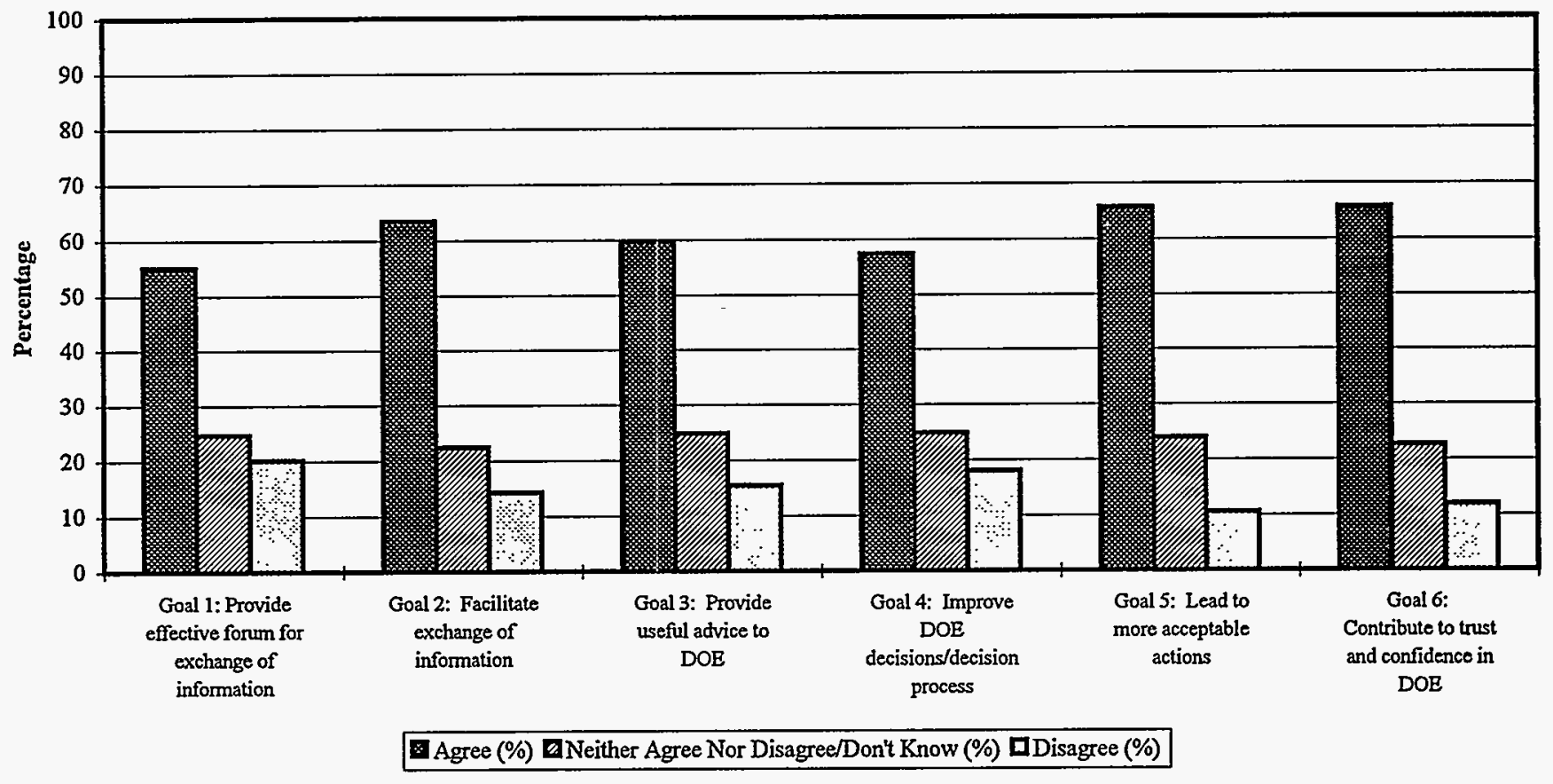

Figure 14b. Long Survey: Entire Sample

Summary: Combined responses for SSAB Initiative goals (Means)

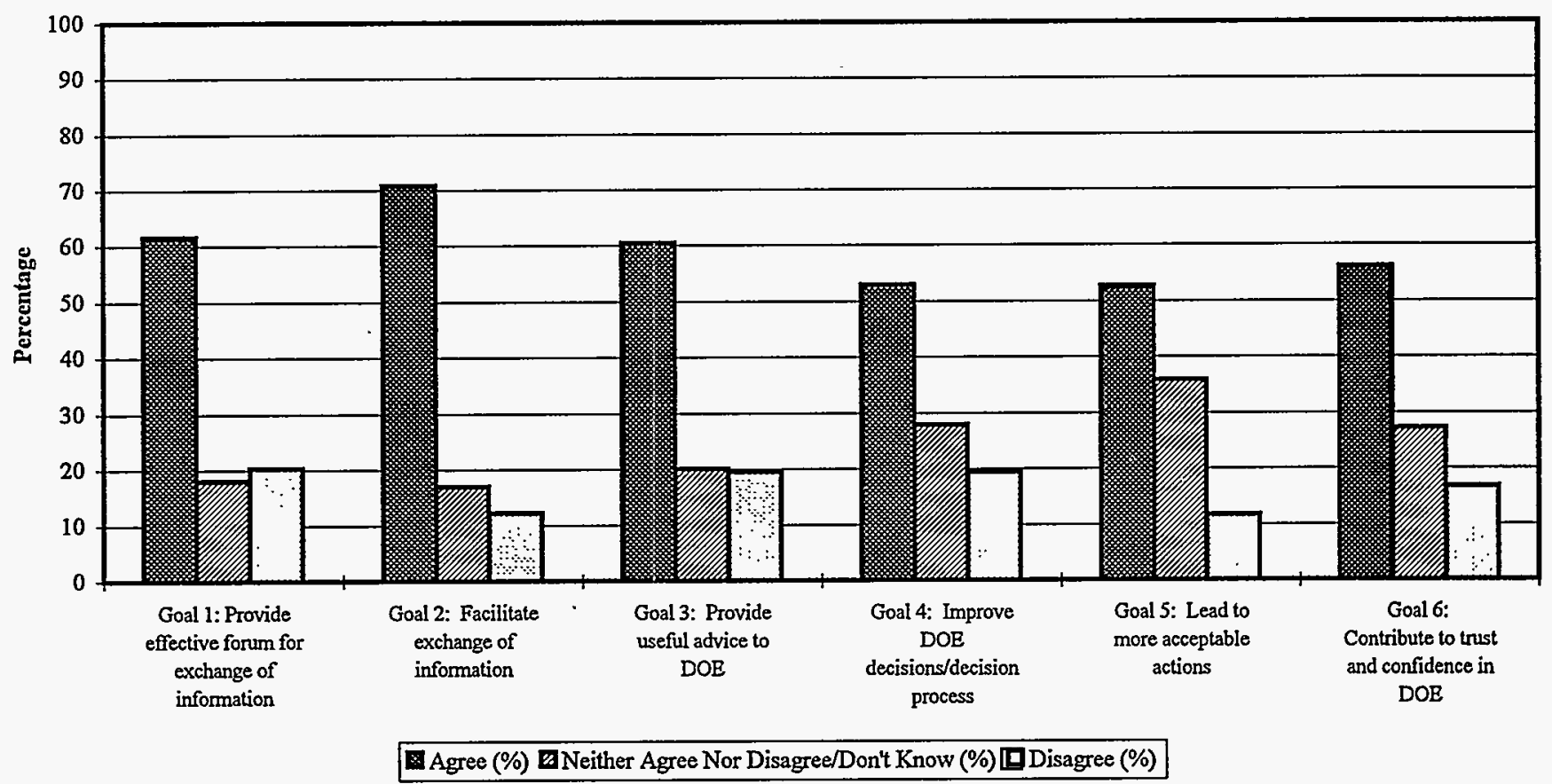

SSAB Supplementary Appendix

August 1996 
Figure 15a. Monticello

Summary: Combined responses on DOE-HQ involvement,

Personal experience, Group/Team work skills,

Working relations, Member affect (Means)

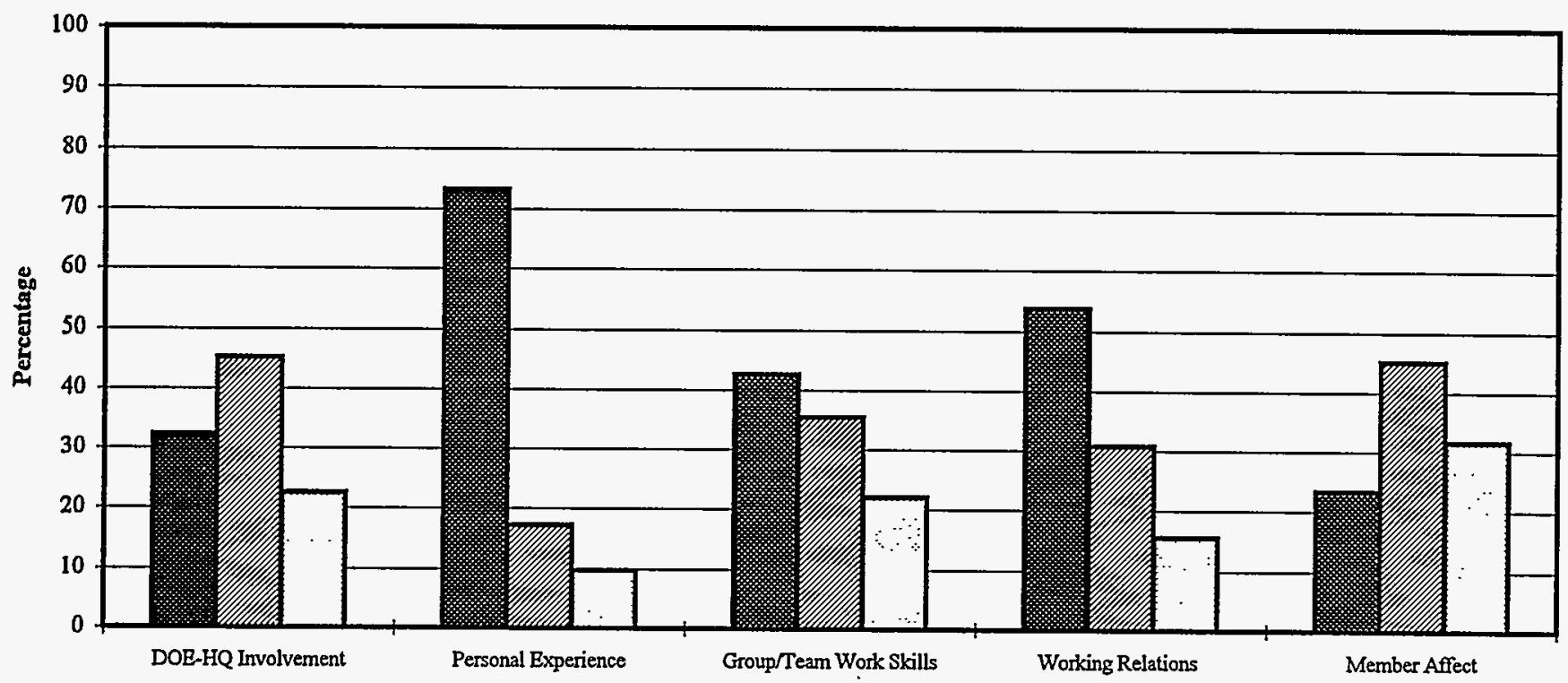

Agree (\%) Deither Agree Nor Disagree/Don't Know (\%) DDisagree (\%)

Figure 15b. Long Survey: Entire Sample

Summary: Combined responses on DOE-HQ involvement,

Personal experience, Group/Team work skills,

Working relations, Member affect (Means)

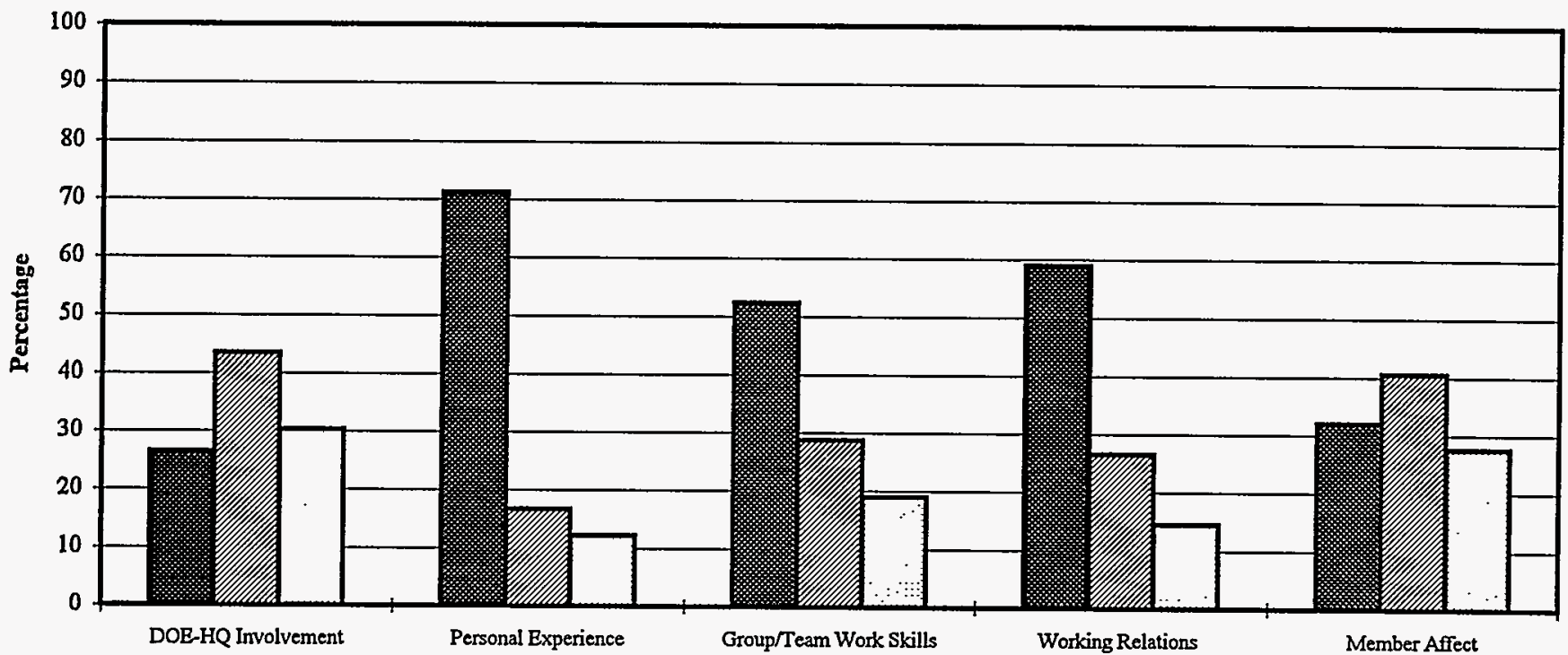

W Agree (\%) Deither Agree Nor Disagree/Don't Know (\%) DDisagree (\%)

SSAB Supplementary Appendix

August 1996 
Table 1.

Goal 1. Establish processes and procedures to provide an effective forum for exchange of information and viewpoints regarding DOE site issues

\begin{tabular}{|c|c|c|c|c|c|c|c|c|}
\hline \multirow[t]{2}{*}{ Monticello } & \multicolumn{2}{|c|}{ Agree } & \multicolumn{2}{|c|}{$\begin{array}{l}\text { Neither/ } \\
\text { Don't Know }\end{array}$} & \multicolumn{2}{|c|}{ Disagree } & \multicolumn{2}{|c|}{ Total } \\
\hline & $\mathbf{N}$ & $\%$ & $\mathrm{~N}$ & $\%$ & $\mathrm{~N}$ & $\%$ & $\mathrm{~N}$ & $\%$ \\
\hline $\begin{array}{l}\text { The Board has established processes and } \\
\text { procedures for the effective exchange of } \\
\text { information }\end{array}$ & 21 & $67.7 \%$ & 5 & $16.1 \%$ & 5 & $16.1 \%$ & 31 & $100 \%$ \\
\hline $\begin{array}{l}\text { The Board has agreed-upon procedures to } \\
\text { operate the board }\end{array}$ & 23 & $82.1 \%$ & 5 & $17.9 \%$ & 0 & $0 \%$ & 28 & $100 \%$ \\
\hline $\begin{array}{l}\text { The Board has adequate support to allow } \\
\text { it to focus on substantive issues }(R)^{*}\end{array}$ & 10 & $37.0 \%$ & 7 & $25.9 \%$ & 10 & $37.0 \%$ & 27 & $100 \%$ \\
\hline $\begin{array}{l}\text { The Board provides sufficient time for } \\
\text { discussion of issues }\end{array}$ & 20 & $71.4 \%$ & 4 & $14.3 \%$ & 4 & $14.3 \%$ & 28 & $100 \%$ \\
\hline The Board has effective leadership & 18 & $64.3 \%$ & 7 & $25.0 \%$ & 3 & $10.7 \%$ & 28 & $100 \%$ \\
\hline $\begin{array}{l}\text { DOE requests Board advice far enough } \\
\text { ahead of decision deadlines }(\mathrm{R})^{*}\end{array}$ & 9 & $29.0 \%$ & 10 & $32.3 \%$ & 12 & $38.7 \%$ & 31 & $100 \%$ \\
\hline $\begin{array}{l}\text { DOE handles Board administrative issues } \\
\text { expeditiously (R) * }\end{array}$ & 14 & $45.2 \%$ & 12 & $38.7 \%$ & 5 & $16.1 \%$ & 31 & $100 \%$ \\
\hline $\begin{array}{l}\text { The SSAB staff provide satisfactory } \\
\text { support }\end{array}$ & 19 & $63.3 \%$ & 8 & $26.7 \%$ & 3 & $10.0 \%$ & 30 & $100 \%$ \\
\hline $\begin{array}{l}\text { The facilitator for the Board has helped } \\
\text { the Board function effectively }\end{array}$ & 11 & $35.5 \%$ & 8 & $25.8 \%$ & 12 & $38.7 \%$ & 31 & $100 \%$ \\
\hline
\end{tabular}

* (R) denotes a question that was asked in the negative form. For ease of review, these questions and data have been reversed in this table and are stated in positive forms.

SSAB Supplementary Appendix

August 1996 
Table 2.

Goal 2. Facilitate interaction and exchange of information and viewpoints regarding DOE site issues

\begin{tabular}{|c|c|c|c|c|c|c|c|c|}
\hline \multirow[t]{2}{*}{ Monticello } & \multicolumn{2}{|c|}{ Agree } & \multicolumn{2}{|c|}{$\begin{array}{c}\text { Neither/ } \\
\text { Don't Know }\end{array}$} & \multicolumn{2}{|c|}{ Disagree } & \multicolumn{2}{|c|}{ Total } \\
\hline & $N$ & $\%$ & $\mathrm{~N}$ & $\%$ & $N$ & $\%$ & $\mathrm{~N}$ & $\%$ \\
\hline $\begin{array}{l}\text { The SSAB facilitates effective exchange } \\
\text { of viewpoints on site issues }\end{array}$ & 22 & $71.0 \%$ & 3 & $9.7 \%$ & 6 & $19.4 \%$ & 31 & $100 \%$ \\
\hline $\begin{array}{l}\text { The SSAB contributes to Board members' } \\
\text { understanding of the basis for key site } \\
\text { decisions }\end{array}$ & 21 & $677 \%$ & 6 & $19.4 \%$ & 4 & $12.9 \%$ & 31 & $100 \%$ \\
\hline $\begin{array}{l}\text { The SSAB contributes to DOE and } \\
\text { regulators' understanding of the public's } \\
\text { viewpoints on key site decisions }\end{array}$ & 22 & $71.0 \%$ & 5 & $16.1 \%$ & 4 & $12.9 \%$ & 31 & $100 \%$ \\
\hline $\begin{array}{l}\text { The SSAB contributes to a constructive } \\
\text { working relationship among the } \\
\text { participants }\end{array}$ & 18 & $58.1 \%$ & 7 & $22.6 \%$ & 6 & $19.4 \%$ & 31 & $100 \%$ \\
\hline The SSAB strives for consensus & 18 & $58.1 \%$ & 7 & $22.6 \%$ & 6 & $19.4 \%$ & 31 & $100 \%$ \\
\hline $\begin{array}{l}\text { The SSAB gives fair consideration to } \\
\text { dissenting opinions }\end{array}$ & 19 & $61.3 \%$ & 6 & $19.4 \%$ & 6 & $19.4 \%$ & 31 & $100 \%$ \\
\hline $\begin{array}{l}\text { The Board has made the effort needed to } \\
\text { learn about site issues }\end{array}$ & 20 & $71.4 \%$ & 7 & $25.0 \%$ & 1 & $3.6 \%$ & 28 & $100 \%$ \\
\hline $\begin{array}{l}\text { The Board invites expert advice into its } \\
\text { discussion on key policy issues }\end{array}$ & 21 & $75.0 \%$ & 2 & $7.1 \%$ & 5 & $17.9 \%$ & 28 & $100 \%$ \\
\hline $\begin{array}{l}\text { The Board responds to public inquiries } \\
\text { and comments about its decisions }\end{array}$ & 16 & $57.1 \%$ & 10 & $35.7 \%$ & 2 & $7.1 \%$ & 28 & $100 \%$ \\
\hline $\begin{array}{l}\text { The Board solicits feedback from the } \\
\text { community on its work }\end{array}$ & 14 & $50.0 \%$ & 11 & $39.3 \%$ & 3 & $10.7 \%$ & 28 & $100 \%$ \\
\hline $\begin{array}{l}\text { DOE makes information on key site issues } \\
\text { readily available to the Board }\end{array}$ & 23 & $74.2 \%$ & 3 & $9.7 \%$ & 5 & $16.1 \%$ & 31 & $100 \%$ \\
\hline $\begin{array}{l}\text { The regulators make important } \\
\text { contributions to the Board's work }\end{array}$ & 17 & $54.8 \%$ & 9 & $29.0 \%$ & 5 & $16.1 \%$ & 31 & $100 \%$ \\
\hline $\begin{array}{l}\text { The SSAB creates a climate supportive of } \\
\text { differing viewpoints }(R) *\end{array}$ & 16 & $53.3 \%$ & 11 & $36.7 \%$ & 3 & $10.0 \%$ & 30 & $100 \%$ \\
\hline
\end{tabular}

* (R) denotes a question that was asked in the negative form. For ease of review, these questions and data have been reversed in this table and are stated in positive forms.

SSAB Supplementary Appendix

August 1996 
Table 3.

Goal 3. Provide useful advice and/or recommendations to DOE (and regulators, where appropriate)

\begin{tabular}{|c|c|c|c|c|c|c|c|c|}
\hline \multirow[t]{2}{*}{ Monticello } & \multicolumn{2}{|c|}{ Agree } & \multicolumn{2}{|c|}{$\begin{array}{c}\text { Neither/ } \\
\text { Don't Know }\end{array}$} & \multicolumn{2}{|c|}{ Disagree } & \multicolumn{2}{|c|}{ Total } \\
\hline & $\mathrm{N}$ & $\%$ & $\mathbf{N}$ & $\%$ & $\mathrm{~N}$ & $\%$ & $N$ & $\%$ \\
\hline $\begin{array}{l}\text { The Board provides useful advice to DOE } \\
\text { (and regulators, where appropriate) }(R)^{*}\end{array}$ & 23 & $82.1 \%$ & 2 & $7.1 \%$ & 3 & $10.7 \%$ & 28 & $100 \%$ \\
\hline $\begin{array}{l}\text { The SSAB reaches agreement about } \\
\text { prioritization of key site issues for which } \\
\text { advice is sought }(R)\end{array}$ & 10 & $32.3 \%$ & 15 & $48.4 \%$ & 6 & $19.4 \%$ & 31 & $100 \%$ \\
\hline The Board helps define site problems & 16 & $57.1 \%$ & 8 & $28.6 \%$ & 4 & $14.3 \%$ & 28 & $100 \%$ \\
\hline $\begin{array}{l}\text { The Board addresses issues in a timely } \\
\text { manner }(\mathrm{R}) *\end{array}$ & 18 & $64.3 \%$ & 8 & $28.6 \%$ & 2 & $7.1 \%$ & 28 & $100 \%$ \\
\hline $\begin{array}{l}\text { The Board reaches consensus on key site } \\
\text { issues (R) * }\end{array}$ & 11 & $39.3 \%$ & 9 & $32.1 \%$ & 8 & $28.6 \%$ & 28 & $100 \%$ \\
\hline $\begin{array}{l}\text { The Board provides informed advice to } \\
\text { DOE (and regulators, where appropriate) }\end{array}$ & 0 & $71.4 \%$ & 4 & $14.3 \%$ & 4 & $14.3 \%$ & 28 & $100 \%$ \\
\hline $\begin{array}{l}\text { The Board provides advice that reflects } \\
\text { the viewpoints and priorities of the } \\
\text { community }\end{array}$ & 20 & $71.4 \%$ & 4 & $14.3 \%$ & 4 & $14.3 \%$ & 28 & $100 \%$ \\
\hline
\end{tabular}

* (R) denotes a question that was asked in the negative form. For ease of review, these questions and data have been reversed in this table and are stated in positive forms.

SSAB Supplementary Appendix August 1996 


\section{Table 4.}

Goal 4. Improve DOE's (and where applicable, regulators') site decisions and decision making process

\begin{tabular}{|c|c|c|c|c|c|c|c|c|}
\hline \multirow[t]{2}{*}{ Monticello } & \multicolumn{2}{|c|}{ Agree } & \multicolumn{2}{|c|}{$\begin{array}{c}\text { Neither/ } \\
\text { Don't Know }\end{array}$} & \multicolumn{2}{|c|}{ Disagree } & \multicolumn{2}{|c|}{ Total } \\
\hline & $\mathrm{N}$ & $\%$ & $N$ & $\%$ & $\mathrm{~N}$ & $\%$ & $\mathrm{~N}$ & $\%$ \\
\hline $\begin{array}{l}\text { The SSAB has improved DOE's site } \\
\text { decisions }(\mathrm{R})^{*}\end{array}$ & 13 & $41.9 \%$ & 7 & $22.6 \%$ & 11 & $35.5 \%$ & 31 & $100 \%$ \\
\hline $\begin{array}{l}\text { The Board understands the decision } \\
\text { making process at [a particular] site }\end{array}$ & 14 & $50.0 \%$ & 10 & $35.7 \%$ & 4 & $14.3 \%$ & 28 & $100 \%$ \\
\hline $\begin{array}{l}\text { DOE discusses important policies } \\
\text { affecting site decisions with the Board }\end{array}$ & 25 & $80.6 \%$ & 4 & $12.9 \%$ & 2 & $6.5 \%$ & 31 & $100 \%$ \\
\hline $\begin{array}{l}\text { DOE has explained to the Board its site } \\
\text { decision making process }\end{array}$ & 19 & $61.3 \%$ & 7 & $22.6 \%$ & 5 & $16.1 \%$ & 31 & $100 \%$ \\
\hline $\begin{array}{l}\text { The DOE shows how Board advice is } \\
\text { reflected in site decisions }\end{array}$ & 16 & $51.6 \%$ & 12 & $38.7 \%$ & 3 & $9.7 \%$ & 31 & $100 \%$ \\
\hline $\begin{array}{l}\text { The decision making process used by } \\
\text { DOE at [a particular] site is effective in } \\
\text { furthering site clean-up (R) }{ }^{*}\end{array}$ & 18 & $58.1 \%$ & 5 & $16.1 \%$ & 8 & $25.8 \%$ & 31 & $100 \%$ \\
\hline
\end{tabular}

* (R) denotes a question that was asked in the negative form. For ease of review, these questions and data have been reversed in this table and are stated in positive forns. 
Table 5.

Goal 5. Lead to more acceptable actions

\begin{tabular}{|c|c|c|c|c|c|c|c|c|}
\hline \multirow[t]{2}{*}{ Monticello } & \multicolumn{2}{|c|}{ Agree } & \multicolumn{2}{|c|}{$\begin{array}{l}\text { Neither/ } \\
\text { Don't Know }\end{array}$} & \multicolumn{2}{|c|}{ Disagree } & \multicolumn{2}{|c|}{ Total } \\
\hline & $\mathrm{N}$ & $\%$ & $N$ & $\%$ & $\mathrm{~N}$ & $\%$ & $N$ & $\%$ \\
\hline $\begin{array}{l}\text { The SSAB leads to more acceptable site } \\
\text { decisions }\end{array}$ & 21 & $67.7 \%$ & 7 & $22.6 \%$ & 3 & $9.7 \%$ & 31 & $100 \%$ \\
\hline $\begin{array}{l}\text { The Board supports the recommendations } \\
\text { it gives DOE }\end{array}$ & 22 & $78.6 \%$ & 2 & $7.1 \%$ & 4 & $14.3 \%$ & 28 & $100 \%$ \\
\hline The Board supports DOE's site actions & 15 & $53.6 \%$ & 11 & $39.3 \%$ & 2 & $7.1 \%$ & 28 & $100 \%$ \\
\hline $\begin{array}{l}\text { The DOE pays attention to the Board's } \\
\text { advice on key site issues }(\mathrm{R}) *\end{array}$ & 15 & $48.4 \%$ & 9 & $29.0 \%$ & 7 & $22.6 \%$ & 31 & $100 \%$ \\
\hline $\begin{array}{l}\text { There is support in the community for } \\
\text { DOE's site decisions that have SSAB } \\
\text { input }\end{array}$ & 18 & $58.1 \%$ & 10 & $32.3 \%$ & 3 & $9.7 \%$ & 31 & $100 \%$ \\
\hline Progress is being made on key site issues & 27 & $87.1 \%$ & 4 & $12.9 \%$ & 0 & $0 \%$ & 31 & $100 \%$ \\
\hline
\end{tabular}

* (R) denotes a question that was asked in the negative form. For ease of review, these questions and data have been reversed in this table and are stated in positive forms.

SSAB Supplementary Appendix 
Table 6.

Goal 6. Contribute to trust and confidence in DOE

\begin{tabular}{|c|c|c|c|c|c|c|c|c|}
\hline \multirow[t]{2}{*}{ Monticello } & \multicolumn{2}{|c|}{ Agree } & \multicolumn{2}{|c|}{$\begin{array}{c}\text { Neither/ } \\
\text { Don't Know }\end{array}$} & \multicolumn{2}{|c|}{ Disagree } & \multicolumn{2}{|c|}{ Total } \\
\hline & $N$ & $\%$ & $\mathrm{~N}$ & $\%$ & $\mathrm{~N}$ & $\%$ & $N$ & $\%$ \\
\hline $\begin{array}{l}\text { The SSAB contributes to trust and } \\
\text { confidence in DOE }\end{array}$ & 11 & $35.5 \%$ & 11 & $35.5 \%$ & 9 & $29.0 \%$ & 31 & $100 \%$ \\
\hline $\begin{array}{l}\text { Relationships between DOE and the public } \\
\text { have improved since the formation of the } \\
\text { SSAB }\end{array}$ & 24 & $77.4 \%$ & 7 & $22.6 \%$ & 0 & $0 \%$ & 31 & $100 \%$ \\
\hline $\begin{array}{l}\text { DOE is committed to clean up [a } \\
\text { particular] site }\end{array}$ & 26 & $83.9 \%$ & 3 & $9.7 \%$ & 2 & $6.5 \%$ & 31 & $100 \%$ \\
\hline
\end{tabular}


Table 7.

Public awareness

\begin{tabular}{lllllllllll}
\hline \multicolumn{1}{c}{ Monticello } & Agree & & $\begin{array}{c}\text { Neither/ } \\
\text { Don't Know }\end{array}$ & Disagree & \multicolumn{2}{c}{ Total } \\
& $\mathrm{N}$ & $\%$ & $\mathrm{~N}$ & $\%$ & $\mathrm{~N}$ & $\%$ & $\mathrm{~N}$ & $\%$ \\
\hline $\begin{array}{l}\text { The public knows little about the role of } \\
\text { the Board }\end{array}$ & 13 & $41.9 \%$ & 10 & $32.3 \%$ & 8 & $25.8 \%$ & 31 & $100 \%$ \\
\hline
\end{tabular}

Table 8.

SSAB Initiative is a good use of funds

\begin{tabular}{|c|c|c|c|c|c|c|c|c|}
\hline \multirow[t]{2}{*}{ Monticello } & \multicolumn{2}{|c|}{ Yes, Good } & \multicolumn{2}{|c|}{$\begin{array}{l}\text { Neutral, } \\
\text { Conditional, } \\
\text { No Opinion }\end{array}$} & \multicolumn{2}{|c|}{$\begin{array}{c}\text { No, } \\
\text { Not Good }\end{array}$} & \multicolumn{2}{|c|}{ Total } \\
\hline & $\mathrm{N}$ & $\%$ & $N$ & $\%$ & $\mathrm{~N}$ & $\%$ & $N$ & $\%$ \\
\hline SSAB Initiative is a good use of funds & 19 & $79.2 \%$ & 1 & $16.7 \%$ & 4 & $4.2 \%$ & 24 & $100 \%$ \\
\hline
\end{tabular}

SSAB Supplementary Appendix 
Table 9.

DOE-HQ involvement with the SSAB Initiative

\begin{tabular}{|c|c|c|c|c|c|c|c|c|}
\hline \multirow[t]{2}{*}{ Monticello } & \multicolumn{2}{|c|}{ Agree } & \multicolumn{2}{|c|}{$\begin{array}{c}\text { Neither/ } \\
\text { Don't Know }\end{array}$} & \multicolumn{2}{|c|}{ Disagree } & \multicolumn{2}{|c|}{ Total } \\
\hline & $N$ & $\%$ & $N$ & $\%$ & $\mathrm{~N}$ & $\%$ & $\mathrm{~N}$ & $\%$ \\
\hline $\begin{array}{l}\text { DOE-HQ provides helpful guidance to the } \\
\text { Boards and to DOE }\end{array}$ & 12 & $38.7 \%$ & 12 & $38.7 \%$ & 7 & $22.6 \%$ & 31 & $100 \%$ \\
\hline $\begin{array}{l}\text { DOE-HQ provides sufficient support to } \\
\text { facilitate the work of the SSAB }\end{array}$ & 10 & $32.3 \%$ & 14 & $45.2 \%$ & 7 & $22.6 \%$ & 31 & $100 \%$ \\
\hline $\begin{array}{l}\text { DOE-HQ gives careful consideration to } \\
\text { SSAB advice in its decisions }\end{array}$ & 8 & $25.8 \%$ & 16 & $51.6 \%$ & 7 & $22.6 \%$ & 31 & $100 \%$ \\
\hline
\end{tabular}


Table 10.

Personal experience with the SSAB Initiative

\begin{tabular}{|c|c|c|c|c|c|c|c|c|}
\hline \multirow[t]{2}{*}{ Monticello } & \multicolumn{2}{|c|}{ Agree } & \multicolumn{2}{|c|}{$\begin{array}{c}\text { Neither/ } \\
\text { Don't Know }\end{array}$} & \multicolumn{2}{|c|}{ Disagree } & \multicolumn{2}{|c|}{ Total } \\
\hline & $N$ & $\%$ & $N$ & $\%$ & $\mathrm{~N}$ & $\%$ & $N$ & $\%$ \\
\hline $\begin{array}{l}\text { I consider myself to be very } \\
\text { knowledgeable about site issues }\end{array}$ & 25 & $80.6 \%$ & 6 & $19.4 \%$ & 0 & $0 \%$ & 31 & $100 \%$ \\
\hline $\begin{array}{l}\text { Because of the SSAB, I better understand } \\
\text { the complexities of site clean-up }\end{array}$ & 20 & $64.5 \%$ & 4 & $12.9 \%$ & 7 & $22.6 \%$ & 31 & $100 \%$ \\
\hline $\begin{array}{l}\text { I feel that my participation in the SSAB } \\
\text { has been worthwhile }(\mathrm{R}){ }^{*}\end{array}$ & 23 & $74.2 \%$ & 6 & $19.4 \%$ & 2 & $6.5 \%$ & 31 & $100 \%$ \\
\hline
\end{tabular}

* $(\mathrm{R})$ denotes a question that was asked in the negative form. For ease of review, these questions and data have been reversed in this table and are stated in positive forms.

SSAB Supplementary Appendix 
Table 11.

Group/Team work skills

\begin{tabular}{|c|c|c|c|c|c|c|c|c|}
\hline \multirow[t]{2}{*}{ Monticello } & \multicolumn{2}{|c|}{ Agree } & \multicolumn{2}{|c|}{$\begin{array}{l}\text { Neither/ } \\
\text { Don't Know }\end{array}$} & \multicolumn{2}{|c|}{ Disagree } & \multicolumn{2}{|c|}{ Total } \\
\hline & $\mathrm{N}$ & $\%$ & $\mathbf{N}$ & $\%$ & $\mathrm{~N}$ & $\%$ & $\mathbf{N}$ & $\%$ \\
\hline All have group/team work skills & 9 & $30.0 \%$ & 12 & $40.0 \%$ & 9 & $30.0 \%$ & 30 & $100 \%$ \\
\hline $\begin{array}{l}\text { Have developed well-established working } \\
\text { relationships with each other }\end{array}$ & 13 & $43.3 \%$ & 9 & $30.0 \%$ & 8 & $26.7 \%$ & 30 & $100 \%$ \\
\hline Like each other & 8 & $26.7 \%$ & 14 & $46.7 \%$ & 8 & $26.7 \%$ & 30 & $100 \%$ \\
\hline $\begin{array}{l}\text { Have a high degree of trust and } \\
\text { confidence in each other }\end{array}$ & 6 & $20.0 \%$ & 13 & $43.3 \%$ & 11 & $36.7 \%$ & 30 & $100 \%$ \\
\hline $\begin{array}{l}\text { Accept the values that are important to the } \\
\text { group }\end{array}$ & 16 & $53.3 \%$ & 9 & $30.0 \%$ & 5 & $16.7 \%$ & 30 & $100 \%$ \\
\hline Show respect for the viewpoints of others & 14 & $48.3 \%$ & 12 & $41.4 \%$ & 3 & $10.3 \%$ & 29 & $100 \%$ \\
\hline $\begin{array}{l}\text { Accept willingly the goals and } \\
\text { expectations for the Board }\end{array}$ & 18 & $60.0 \%$ & 8 & $26.7 \%$ & 4 & $13.3 \%$ & 30 & $100 \%$ \\
\hline $\begin{array}{l}\text { Set high expectations for what they } \\
\text { believe they can accomplish }\end{array}$ & 13 & $43.3 \%$ & 12 & $40.0 \%$ & 5 & $16.7 \%$ & 30 & $100 \%$ \\
\hline $\begin{array}{l}\text { Communicate fully and frankly to the } \\
\text { Board all relevant and valuable } \\
\text { information }\end{array}$ & 22 & $73.3 \%$ & 4 & $13.3 \%$ & 4 & $13.3 \%$ & 30 & $100 \%$ \\
\hline $\begin{array}{l}\text { Try not to waste the Board's time with } \\
\text { irrelevant material or communications }\end{array}$ & 12 & $40.0 \%$ & 9 & $30.0 \%$ & 9 & $30.0 \%$ & 30 & $100 \%$ \\
\hline $\begin{array}{l}\text { Are willing to be influenced by other } \\
\text { Board members about new ideas and } \\
\text { methods }\end{array}$ & 18 & $60.0 \%$ & 8 & $26.7 \%$ & 4 & $13.3 \%$ & 30 & $100 \%$ \\
\hline $\begin{array}{l}\text { Clearly understand the goals and } \\
\text { philosphy of the Board's operation }\end{array}$ & 13 & $43.3 \%$ & 9 & $30.0 \%$ & 8 & $26.7 \%$ & 30 & $100 \%$ \\
\hline $\begin{array}{l}\text { Do all they can to help the Board achieve } \\
\text { its objectives }\end{array}$ & 13 & $43.3 \%$ & 13 & $43.3 \%$ & 4 & $13.3 \%$ & 30 & $100 \%$ \\
\hline $\begin{array}{l}\text { Get the technical knowledge and training } \\
\text { in group skills they need }\end{array}$ & 7 & $24.1 \%$ & 15 & $51.7 \%$ & 7 & $24.1 \%$ & 29 & $100 \%$ \\
\hline Provide one another mutual help & 17 & $56.7 \%$ & 9 & $30.0 \%$ & 4 & $13.3 \%$ & 30 & $100 \%$ \\
\hline
\end{tabular}

SSAB Supplementary Appendix

August 1996

S-131 
Nevada Site Specific Advisory Board Evaluation Survey Results 
Figure 1a. Nevada

Goal 1. Establish processes and procedures to provide an effective forum

for exchange of information and viewpoints regarding DOE site issues

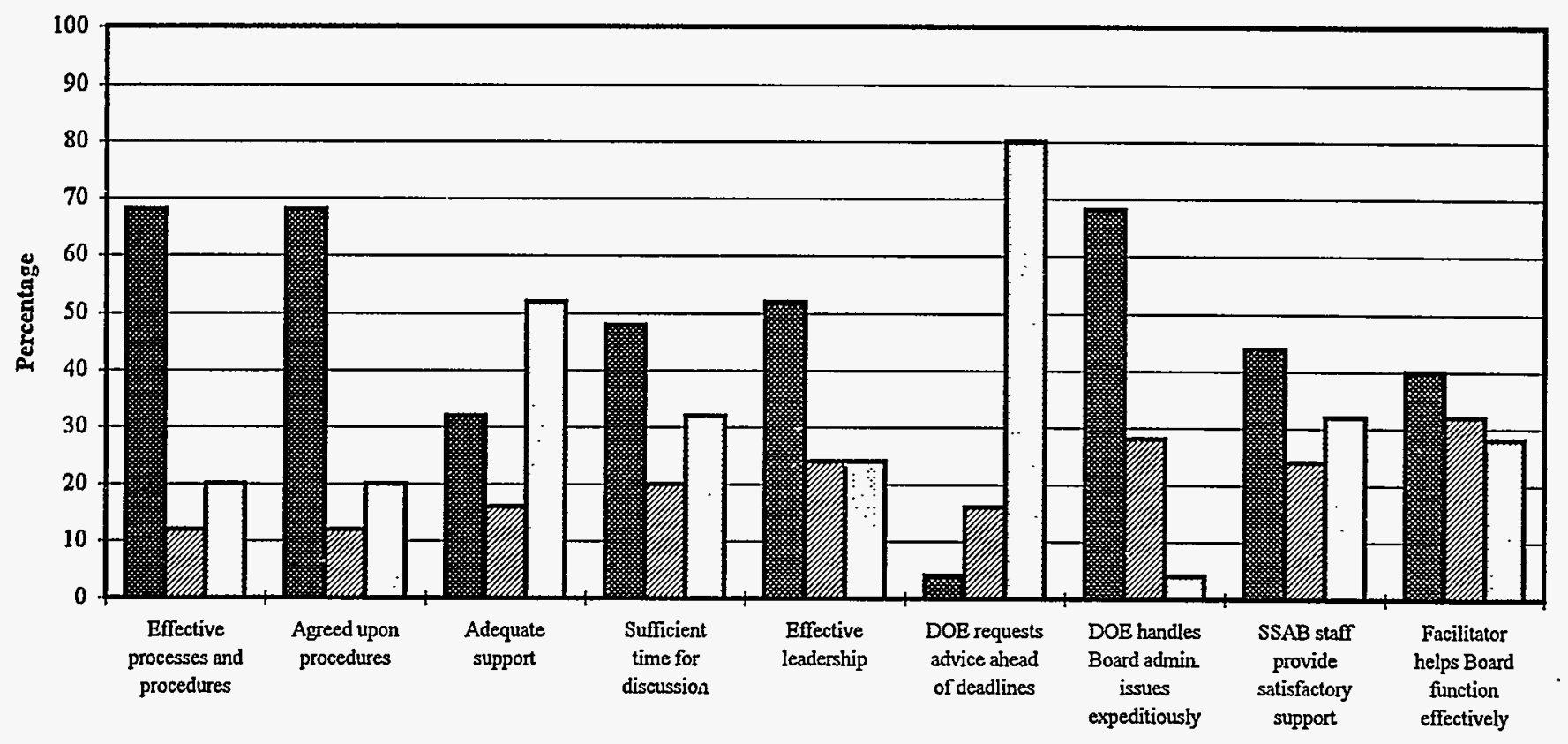

Agree (\%) DNeither Agree Nor Disagree/Don't Know (\%) DDisagree (\%)

Figure 1b. Long Survey: Entire Sample

Goal 1. Establish processes and procedures to provide an effective forum for exchange of information and viewpoints regarding DOE site issues

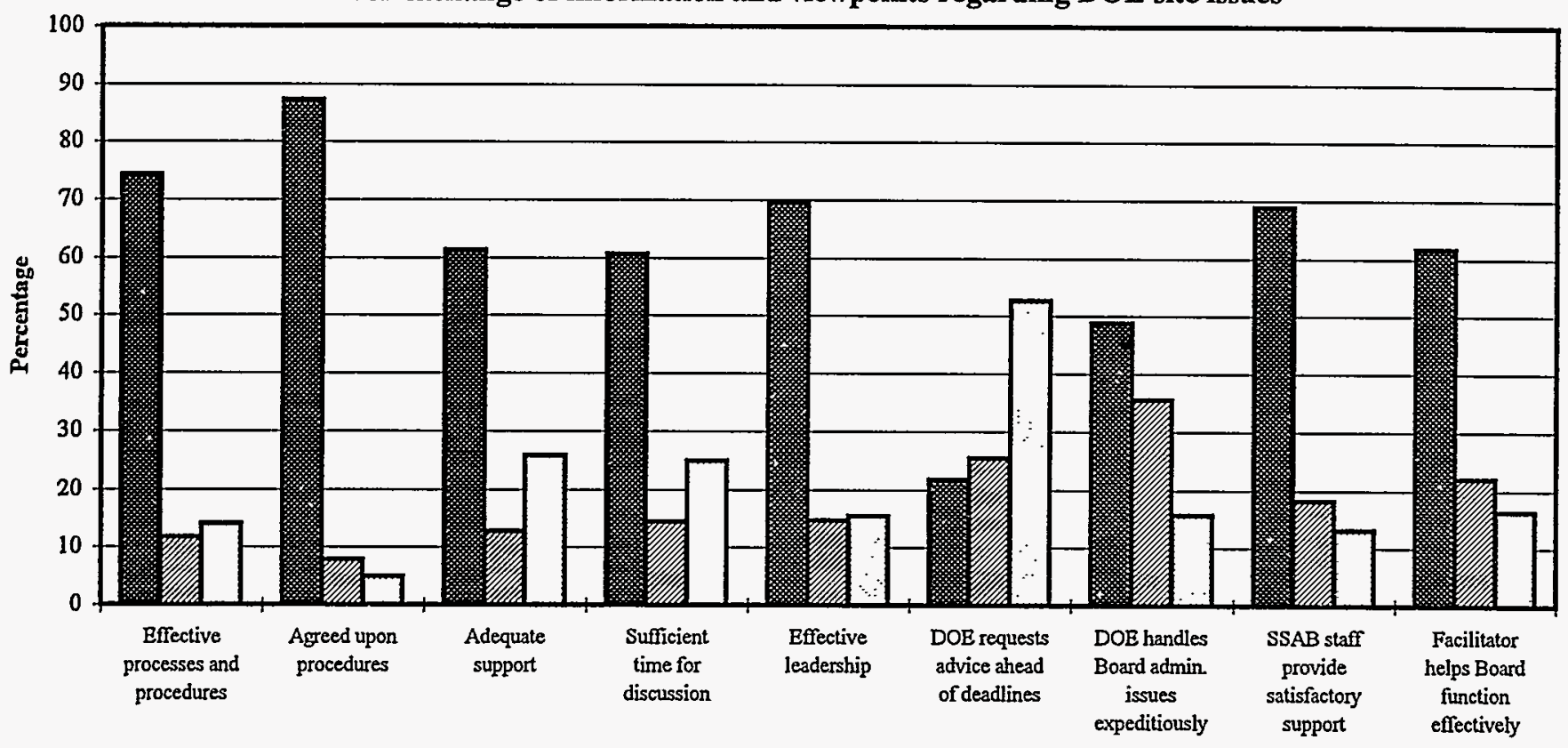

EAgree (\%) Neither Agree Nor Disagree/Dont Know (\%) DDisagree (\%)

SSAB Supplementary Appendix 
Figure 2a. Nevada

Goal 2. Facilitate interaction and exchange of information and viewpoints regarding DOE site issues

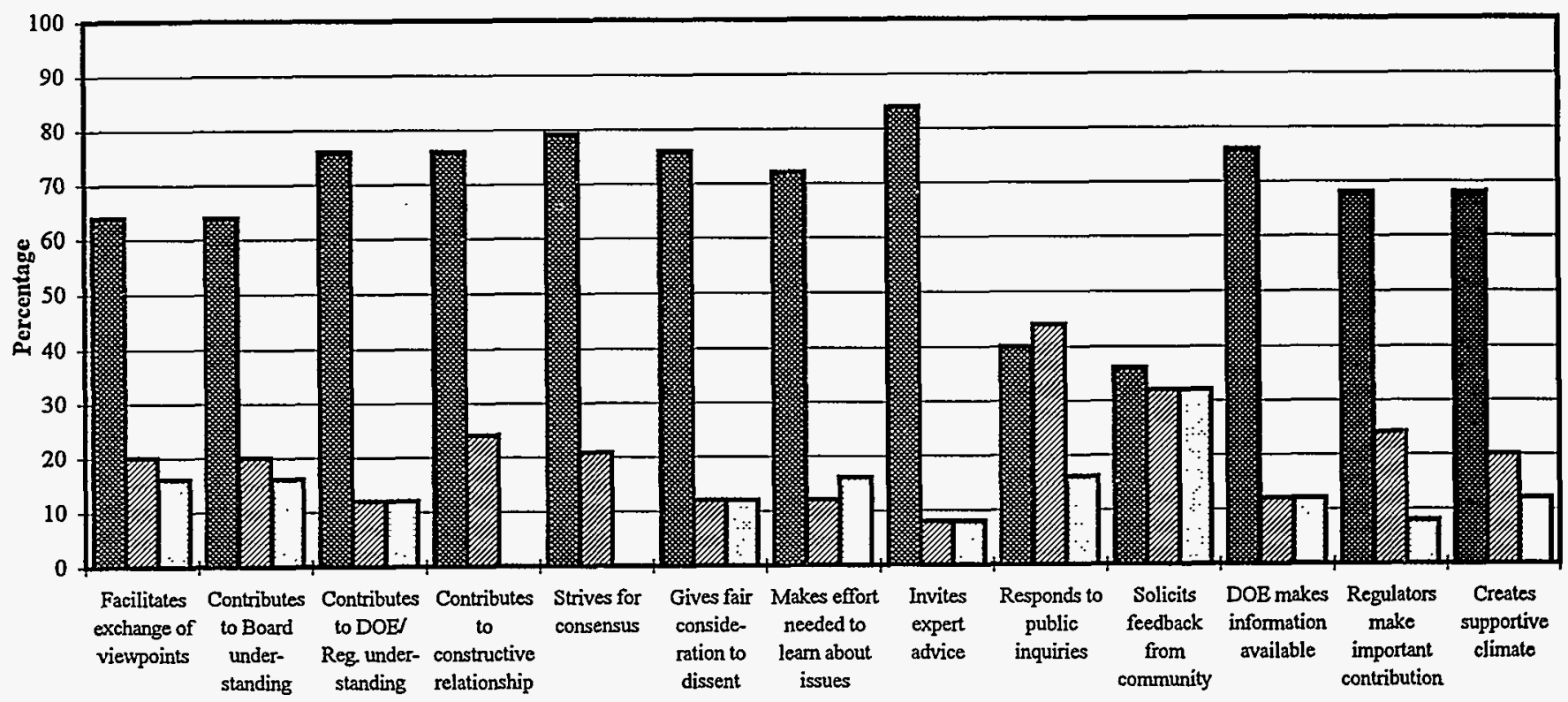

E Agree (\%) ENeither Agree Nor Disagree/Don't Know (\%) DDisagree (\%)

Figure 2b. Long Survey: Entire Sample

Goal 2. Facilitate interaction and exchange of information and viewpoints regarding $\mathrm{DOE}$ site issues

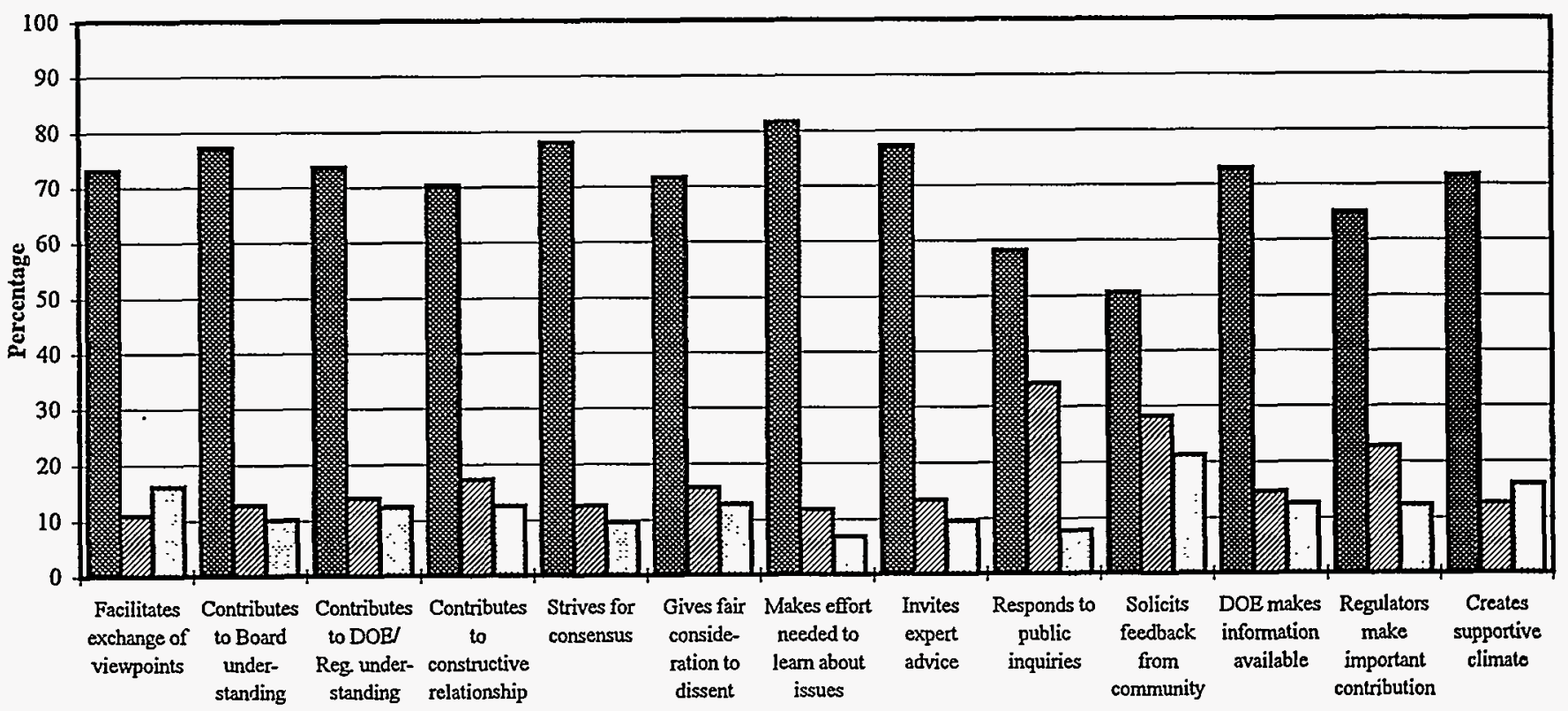

P Agree (\%) $\mathbf{E}$ Neither Agree Nor Disagree/Don't Know (\%) DDisagree (\%)

SSAB Supplementary Appendix

August 1996 
Figure 3a. Nevada

Goal 3. Provide useful advice and/or recommendations to DOE

(and regulators, where appropriate)

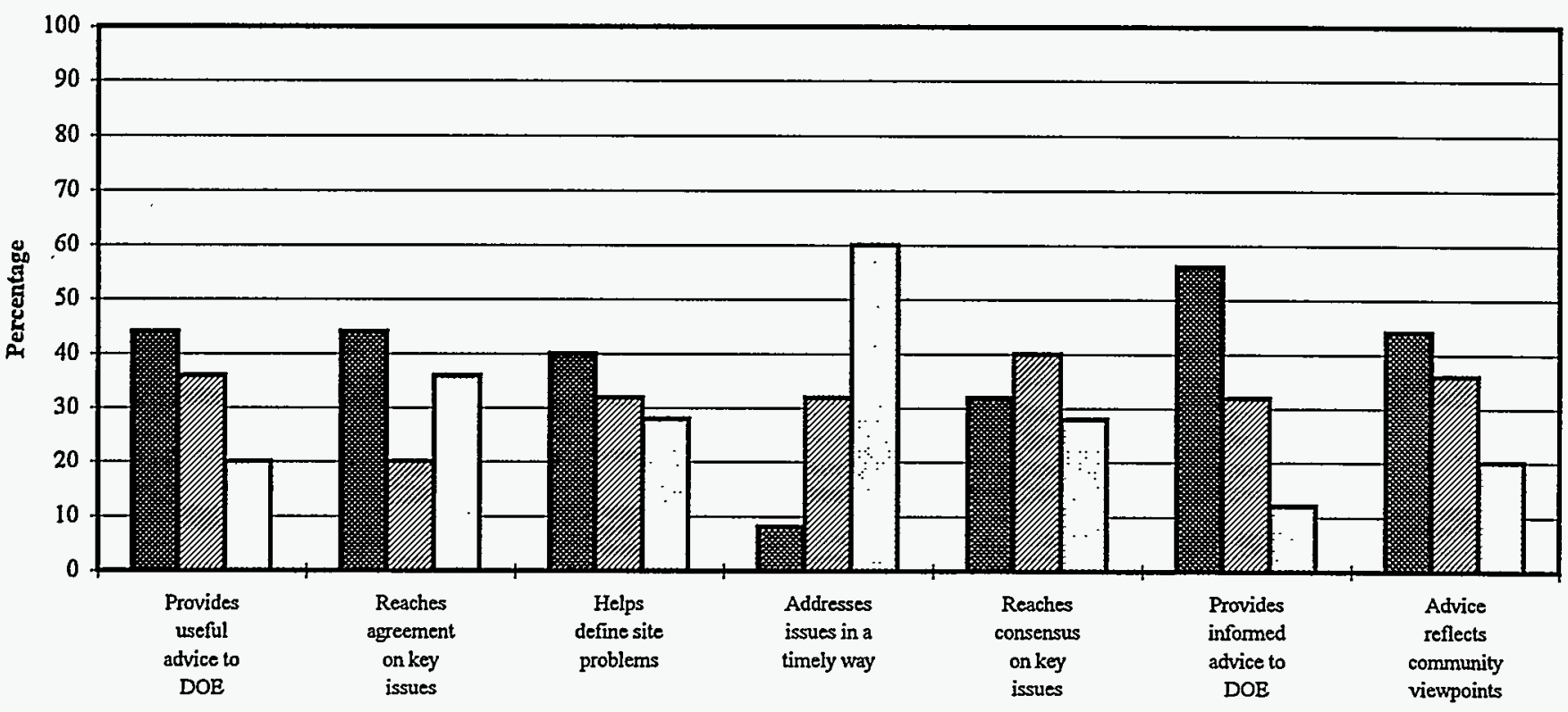

Agree (\%) DNeither Agree Nor Disagree/Dont Know (\%) 口Disagree (\%)

Figure 3b. Long Survey: Entire Sample

Goal 3. Provide useful advice and/or recommendations to DOE

(and regulators, where appropriate)

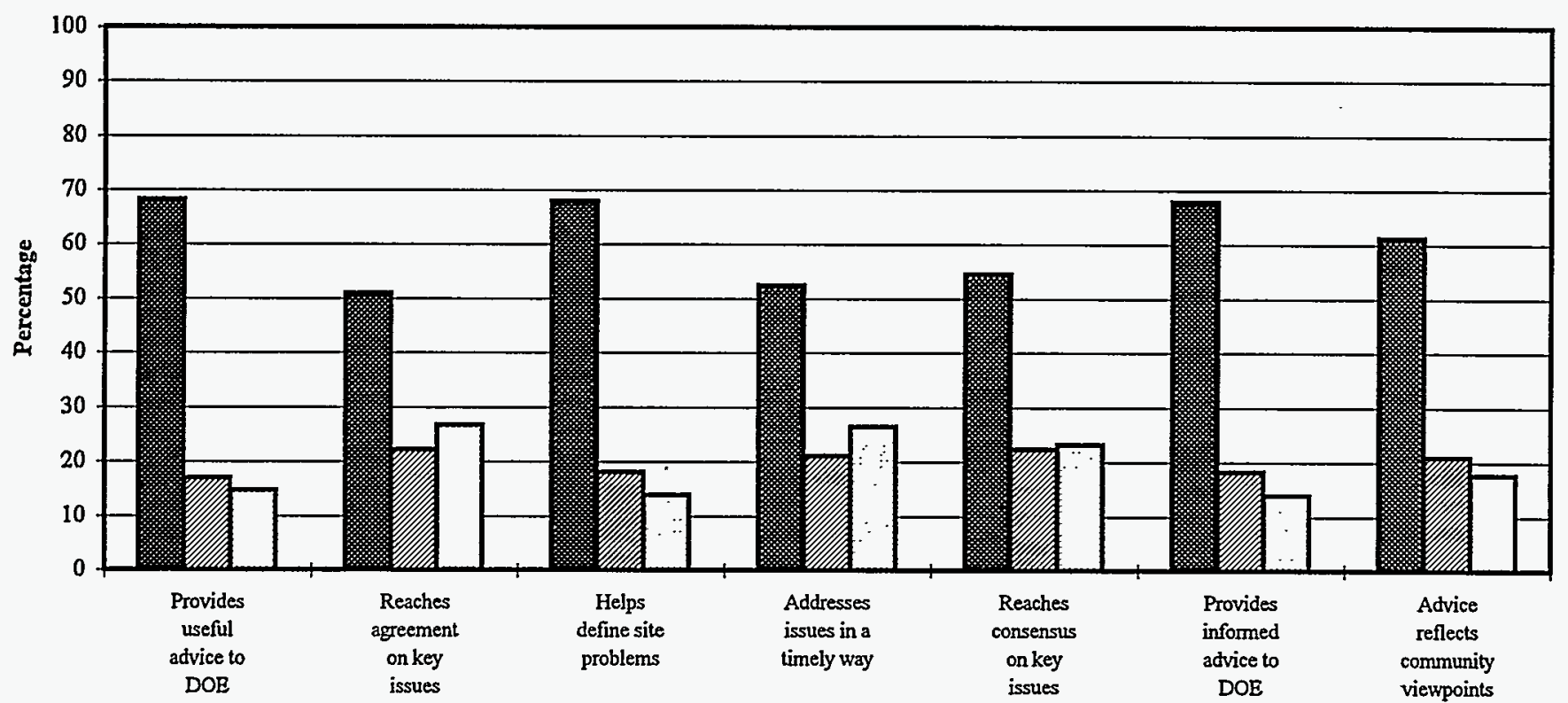

Agree (\%) ENeither Agree Nor Disagree/Don't Know (\%) DDisagree (\%) 
Figure 4a. Nevada

Goal 4. Improve DOE's (and where applicable, regulators') site decisions and decision making process

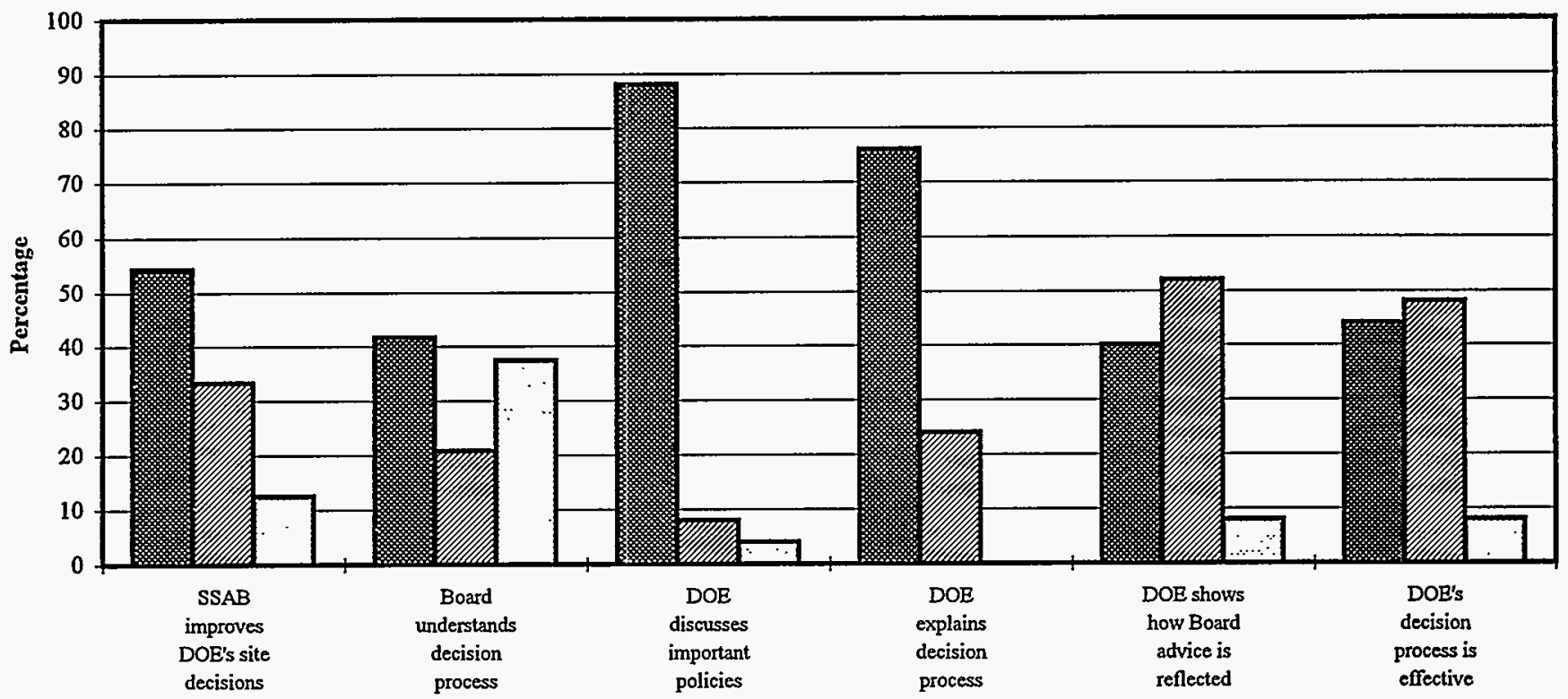

F Agree (\%) Deither Agree Nor Disagree/Don't Know (\%) DDisagree (\%)

Figure 4b. Long Survey: Entire Sample

Goal 4. Improve DOE's (and where applicable, regulators') site decisions and decision making process

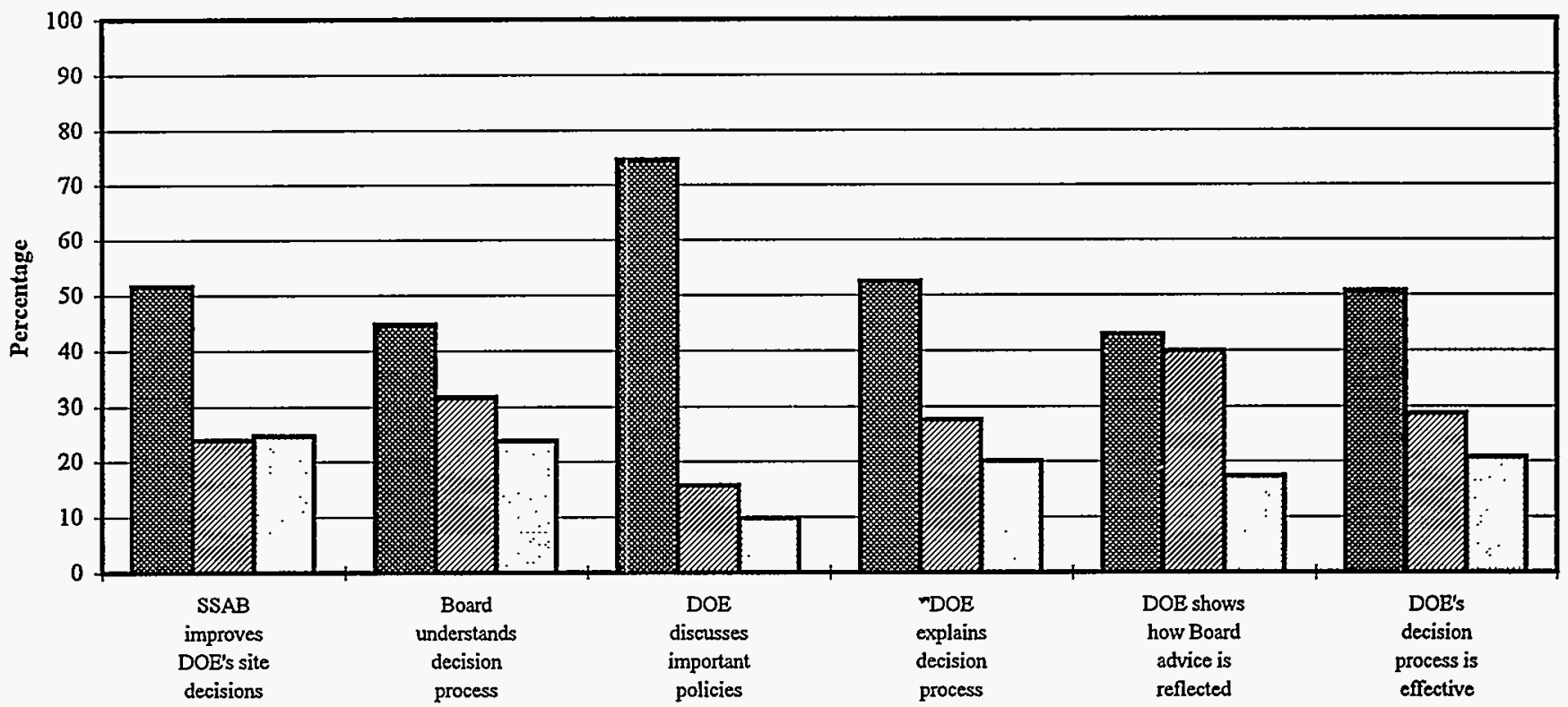

Agree (\%) ENeither Agree Nor Disagree/Dont Know (\%) DDisagree (\%)

SSAB Supplementary Appendix

August 1996 
Figure 5a. Nevada

Goal 5. Lead to more acceptable actions

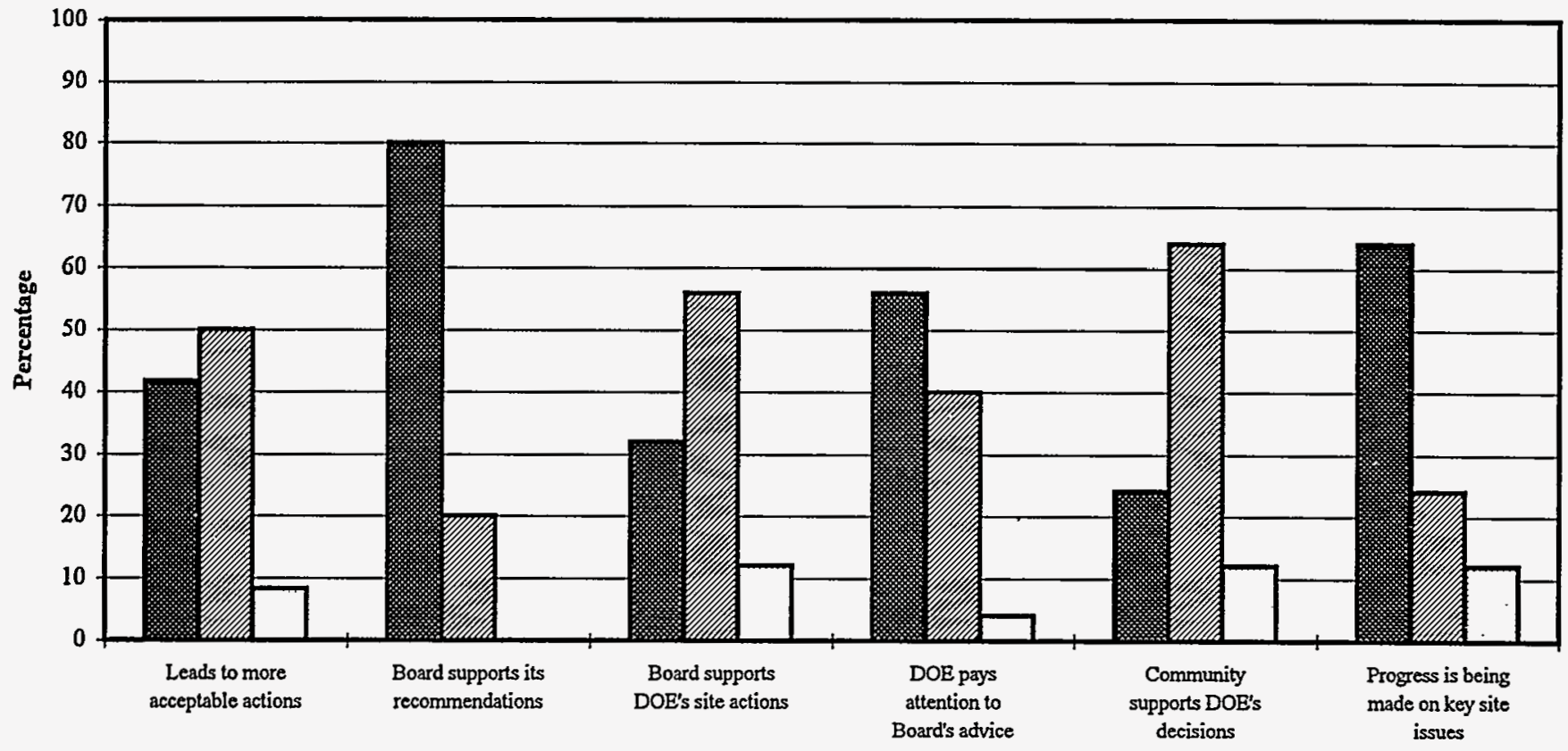

Agrce (\%) Weither Agree Nor Disagree/Dont Know (\%) DDisagree (\%)

Figure 5b. Long Survey: Entire Sample

Goal 5. Lead to more acceptable actions

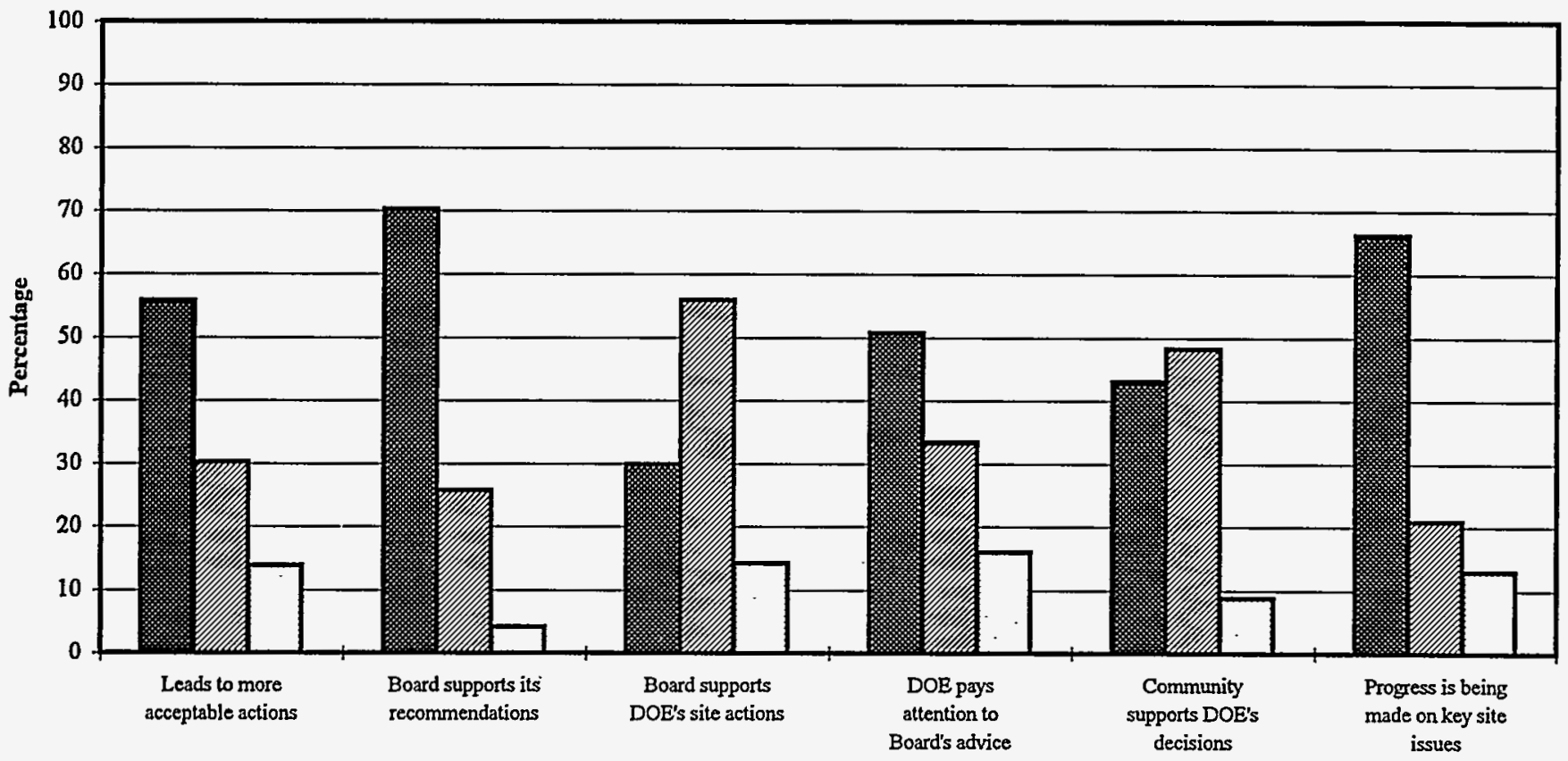

Agree (\%) ENeither Agree Nor Disagree/Dont Know (\%) DDisagree (\%)

SSAB Supplementary Appendix

August 1996 
Figure 6a. Nevada

Goal 6. Contribute to trust and confidence in DOE

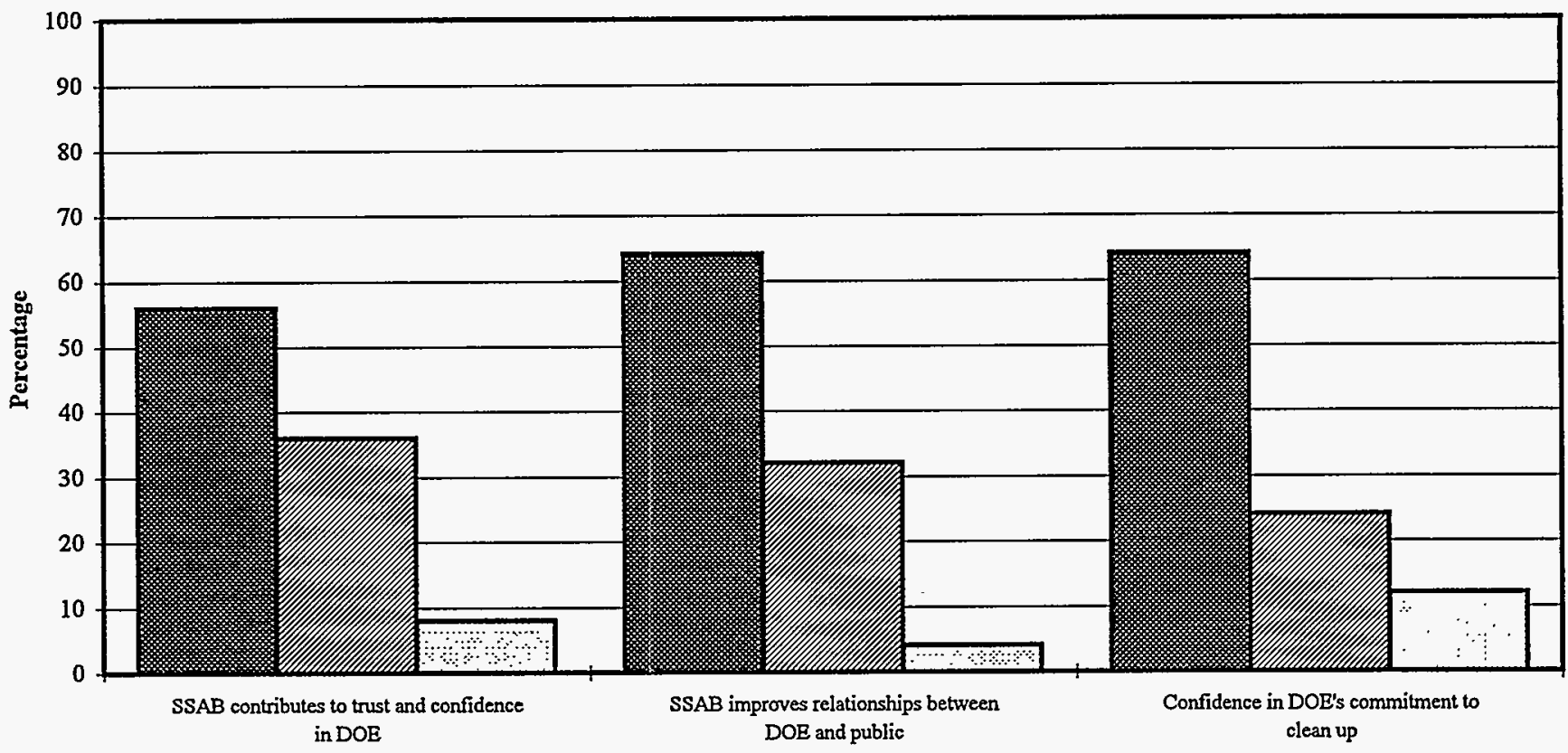

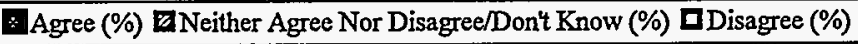

Figure 6b. Long Survey: Entire Sample

Goal 6. Contribute to trust and confidence in DOE

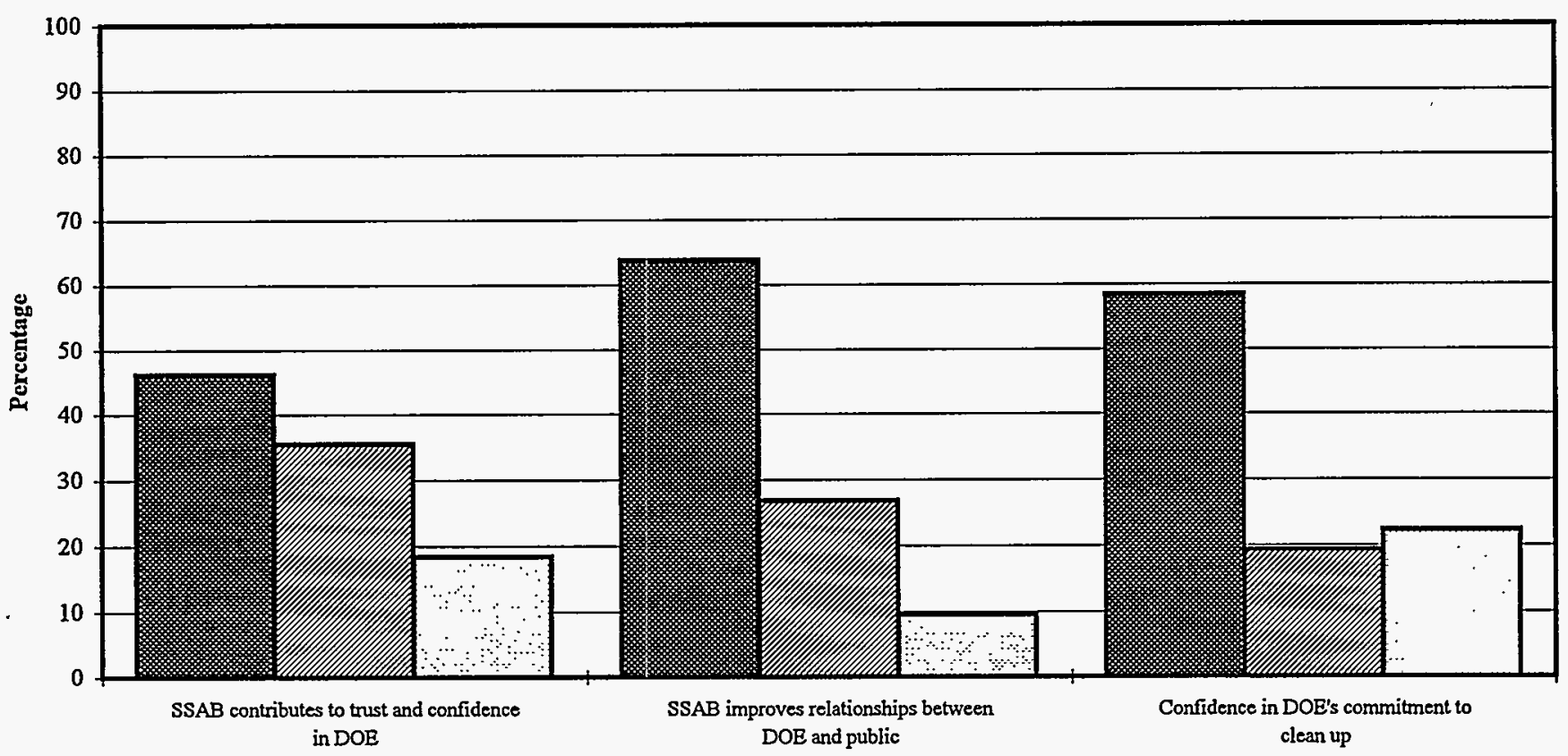

Agree (\%) GNeither Agree Nor Disagree/Don't Know (\%) DDisagree (\%)

SSAB Supplementary Appendix 
Figure 7a. Nevada

Public awareness

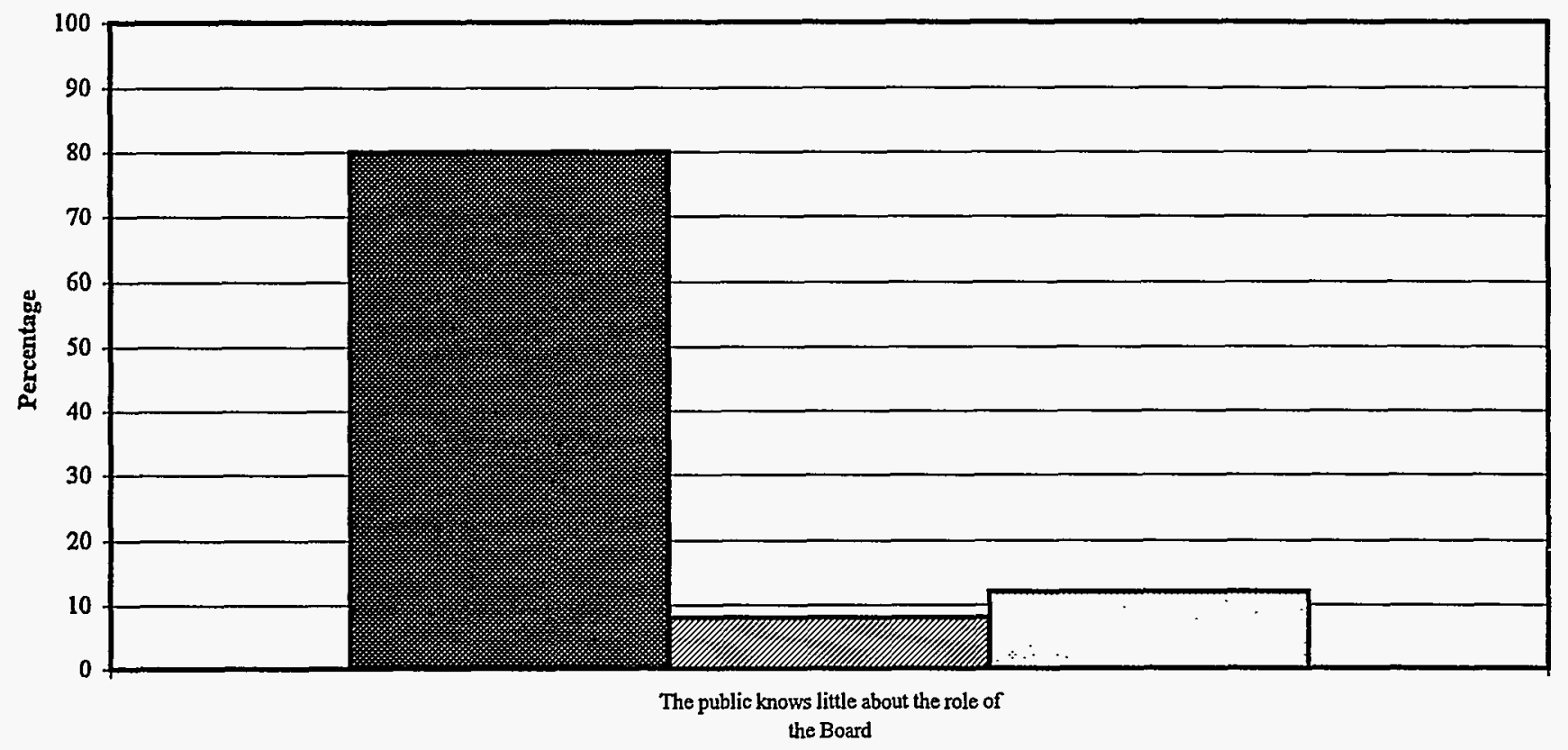

Egree (\%) Deither Agree Nor Disagree/Don't Know (\%) DDisagree (\%)

Figure 7b. Long Survey: Entire Sample

Public awareness

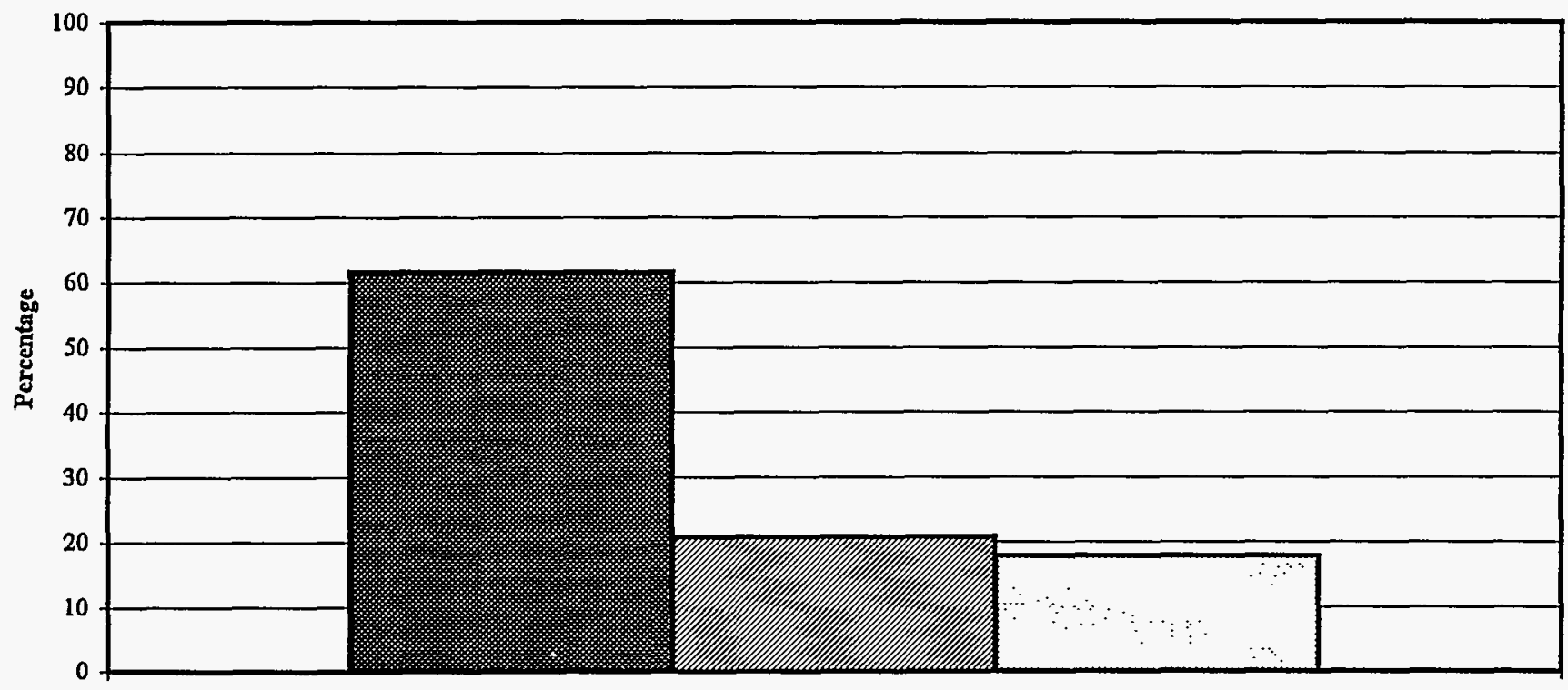

The public knows little about the role of the Board

Agree (\%) שN Neither Agree Nor Disagree/Don't Know (\%) DDisagree (\%)

SSAB Supplementary Appendix

August 1996

S-139 
Figure 8a. Nevada

DOE-HQ involvement with the SSAB Initiative

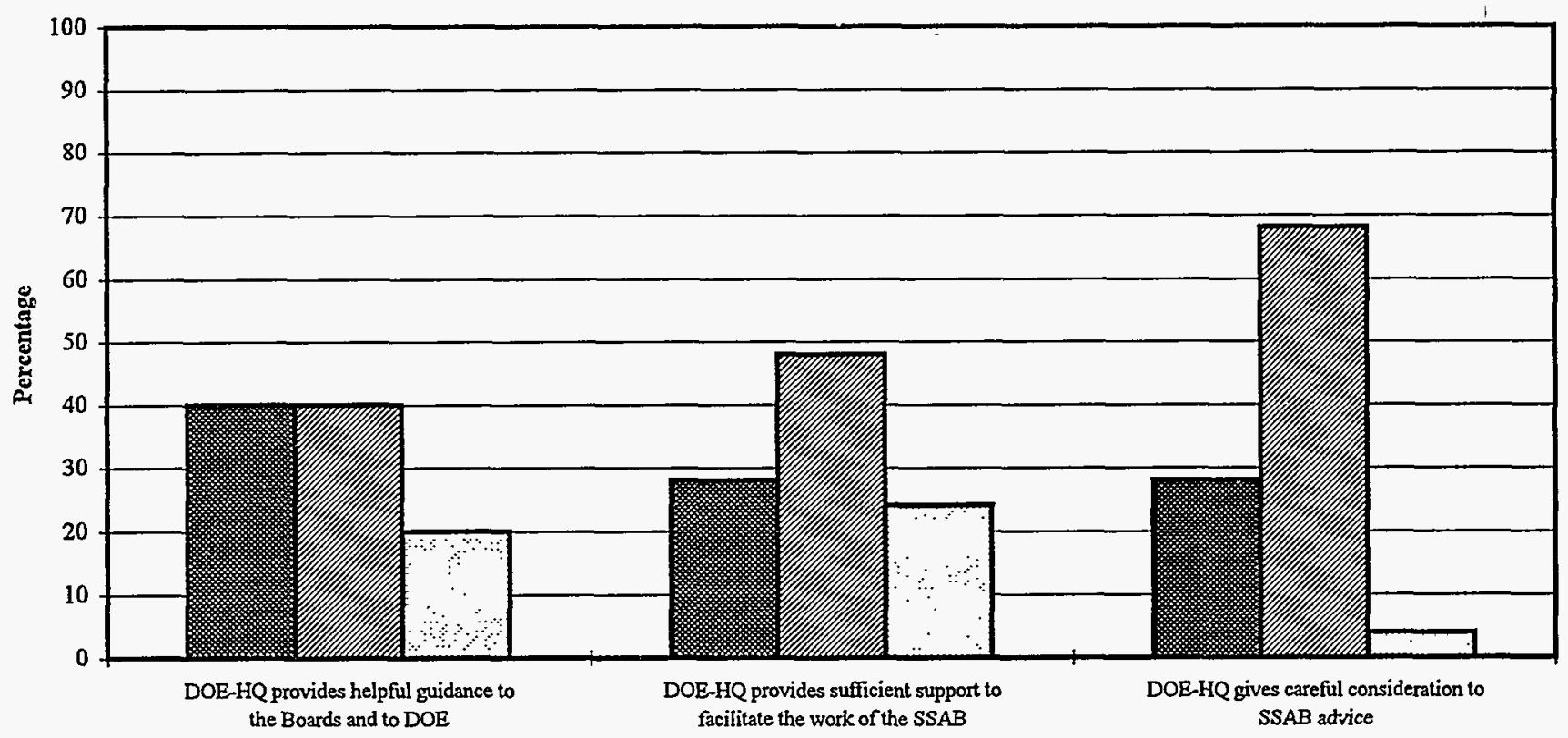

Agree (\%) DNeither Agree Nor Disagree/Don't Know (\%) DDisagree (\%)

Figure 8b. Long Survey: Entire Sample DOE-HQ involvement with the SSAB Initiative

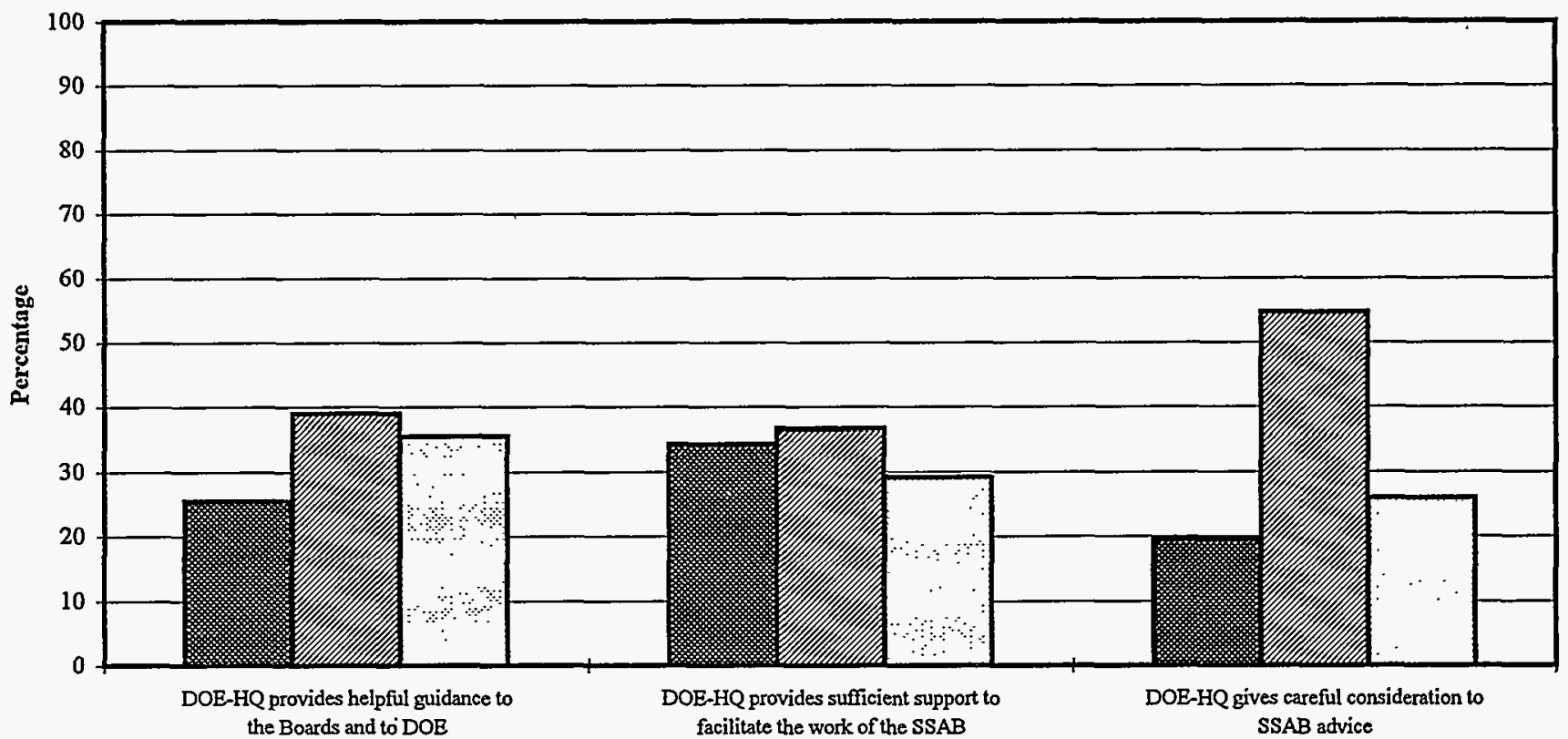

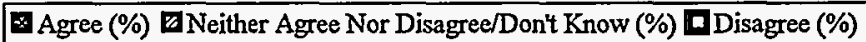

SSAB Supplementary Appendix 
Figure 9a. Nevada

Personal experience with the SSAB Initiative

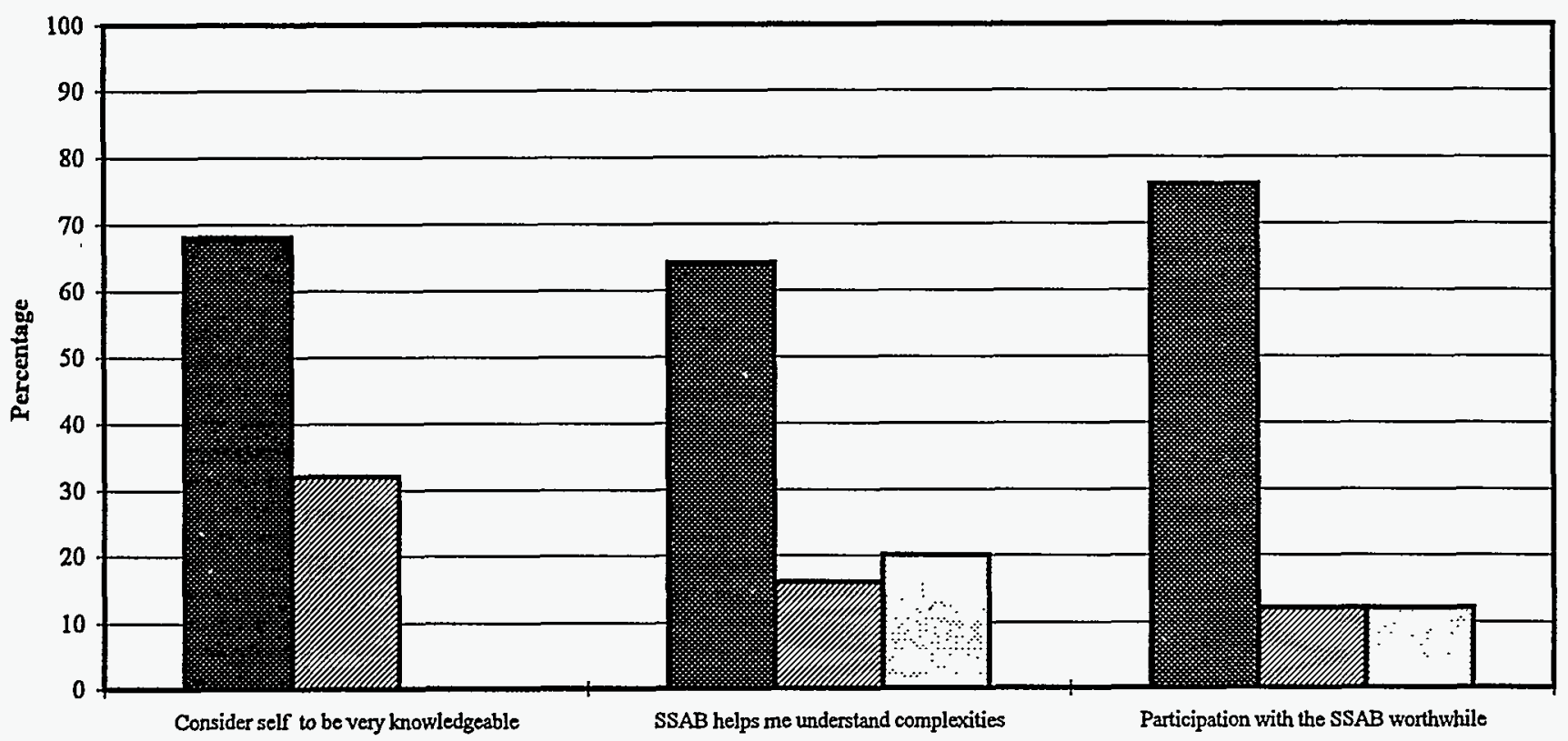

Agree (\%) Weither Agree Nor Disagree/Don't Know (\%) DDisagree (\%)

Figure 9b. Long Survey: Entire Sample

Personal experience with the SSAB Initiative

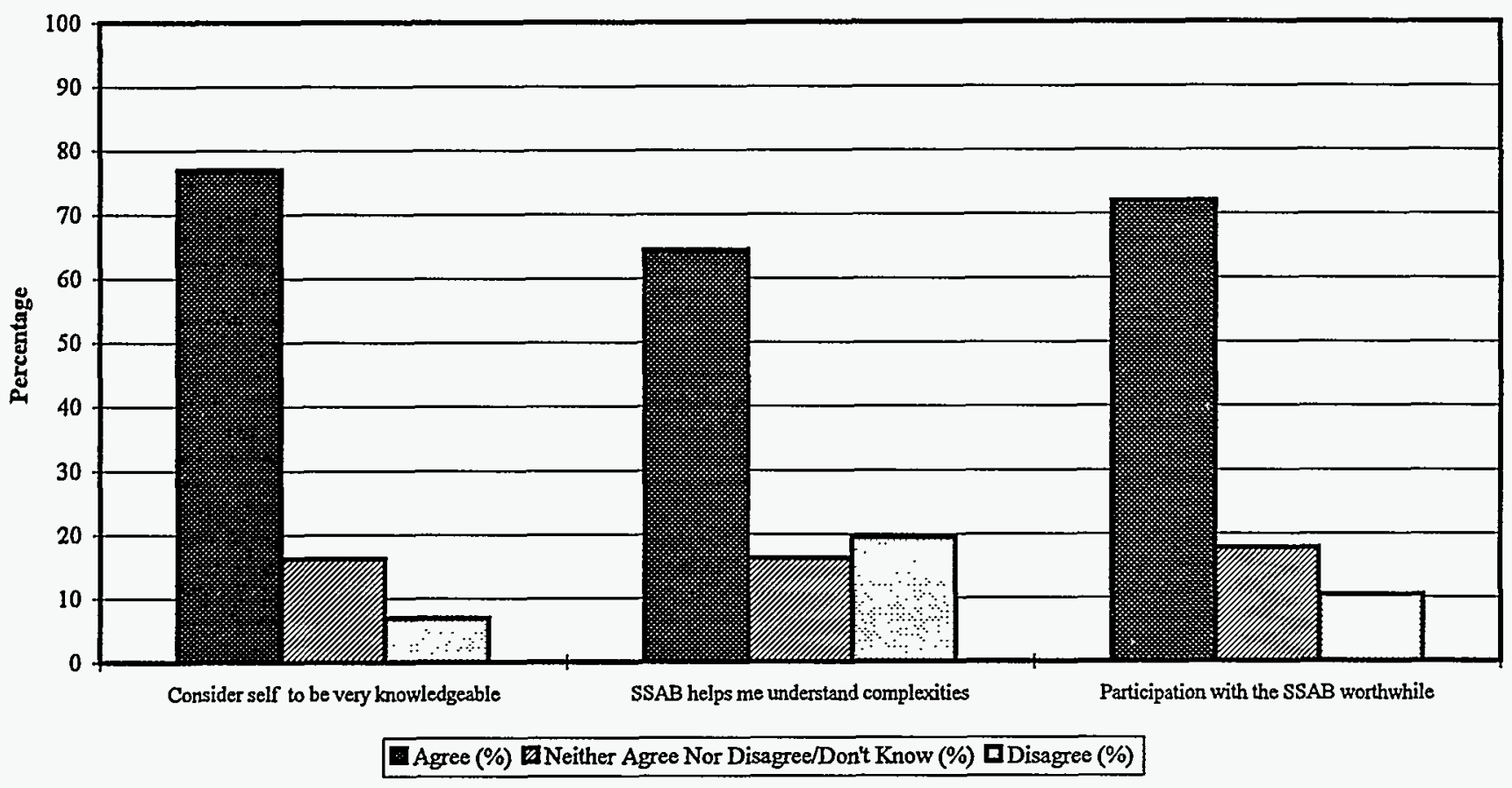

SSAB Supplementary Appendix

August 1996 
Figure 10a. Nevada

Group/Team work skills

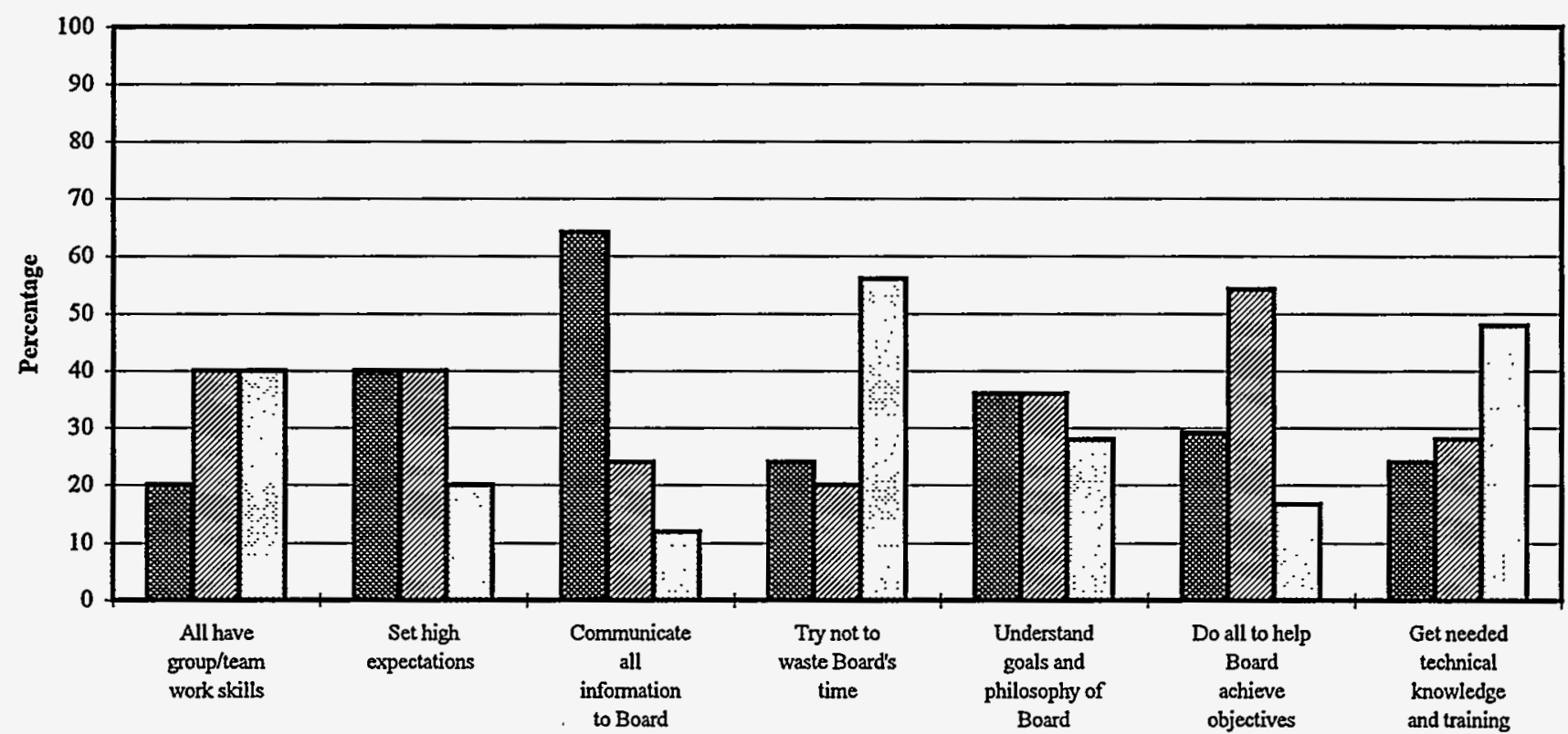

Agree (\%) DNeither Agree Nor Disagree/Don't Know (\%) DDisagree (\%)

Figure 10b. Long Survey: Entire Sample Group/Team work skills

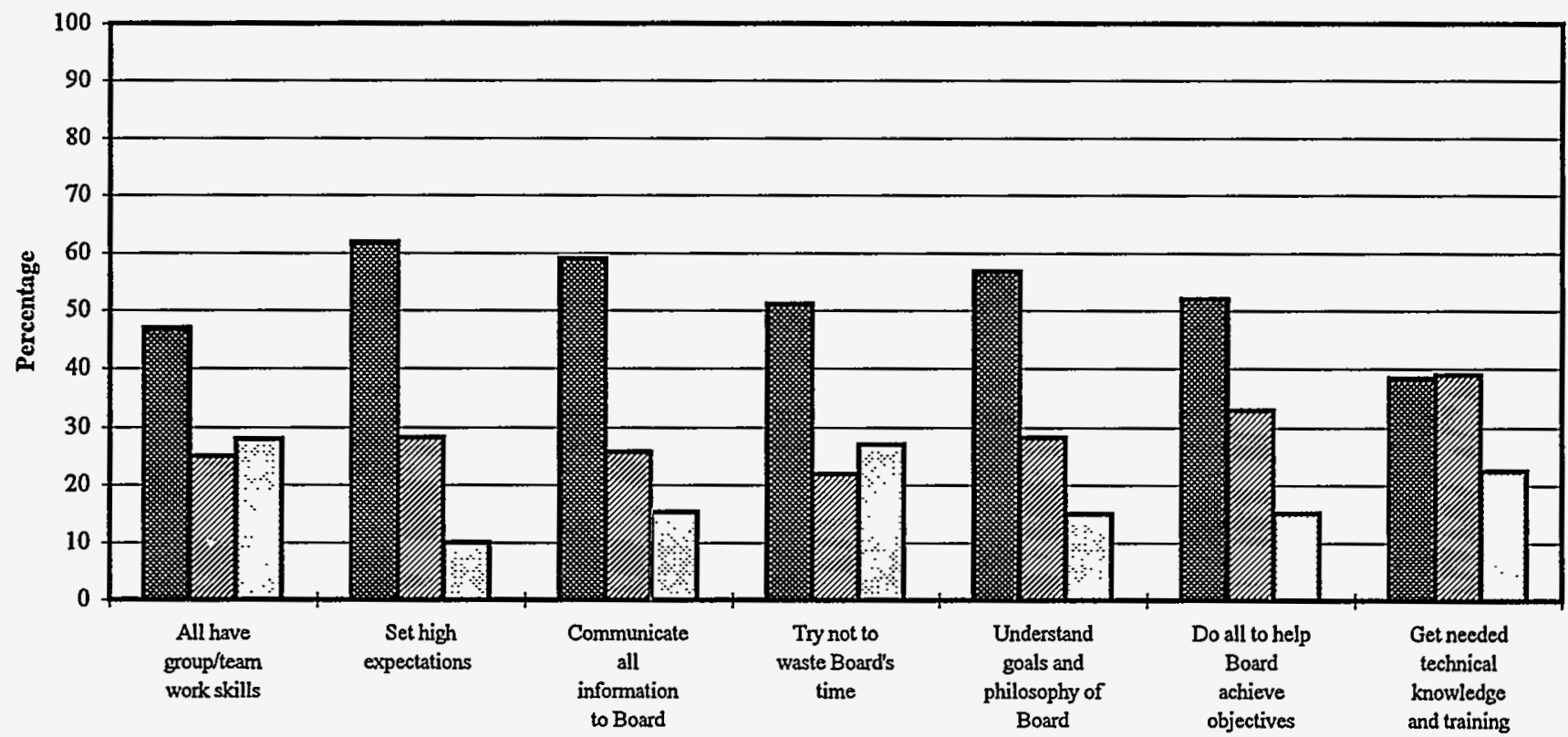

Agree (\%) ENeither Agree Nor Disagree/Don't Know (\%) DDisagree (\%)

SSAB Supplementary Appendix

August 1996

S-142 
Figure 11a. Nevada

Working relations

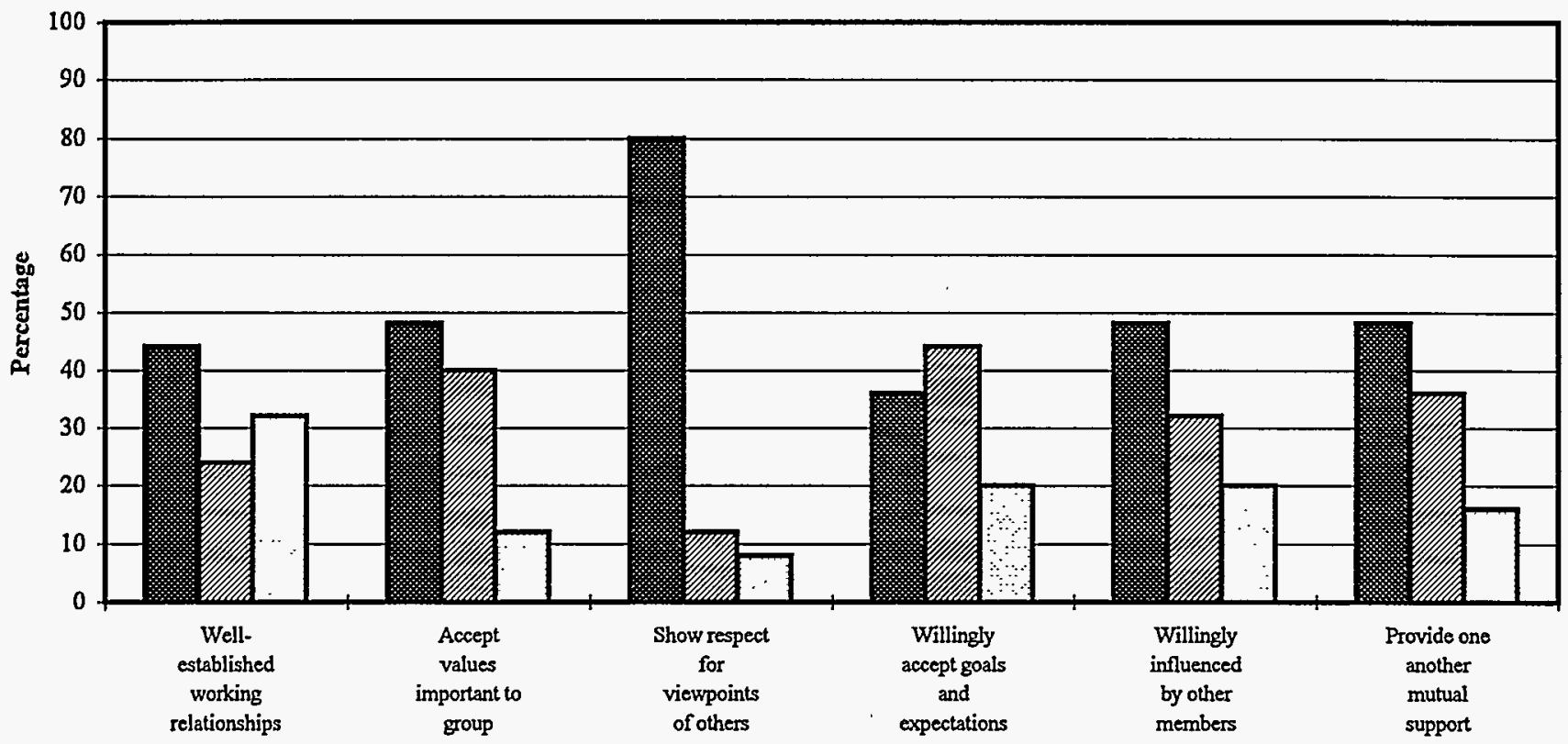

Agree (\%) ENeither Agree Nor Disagree/Don't Know (\%) DDisagree (\%)

Figure 11b. Long Survey: Entire Sample

Working relations

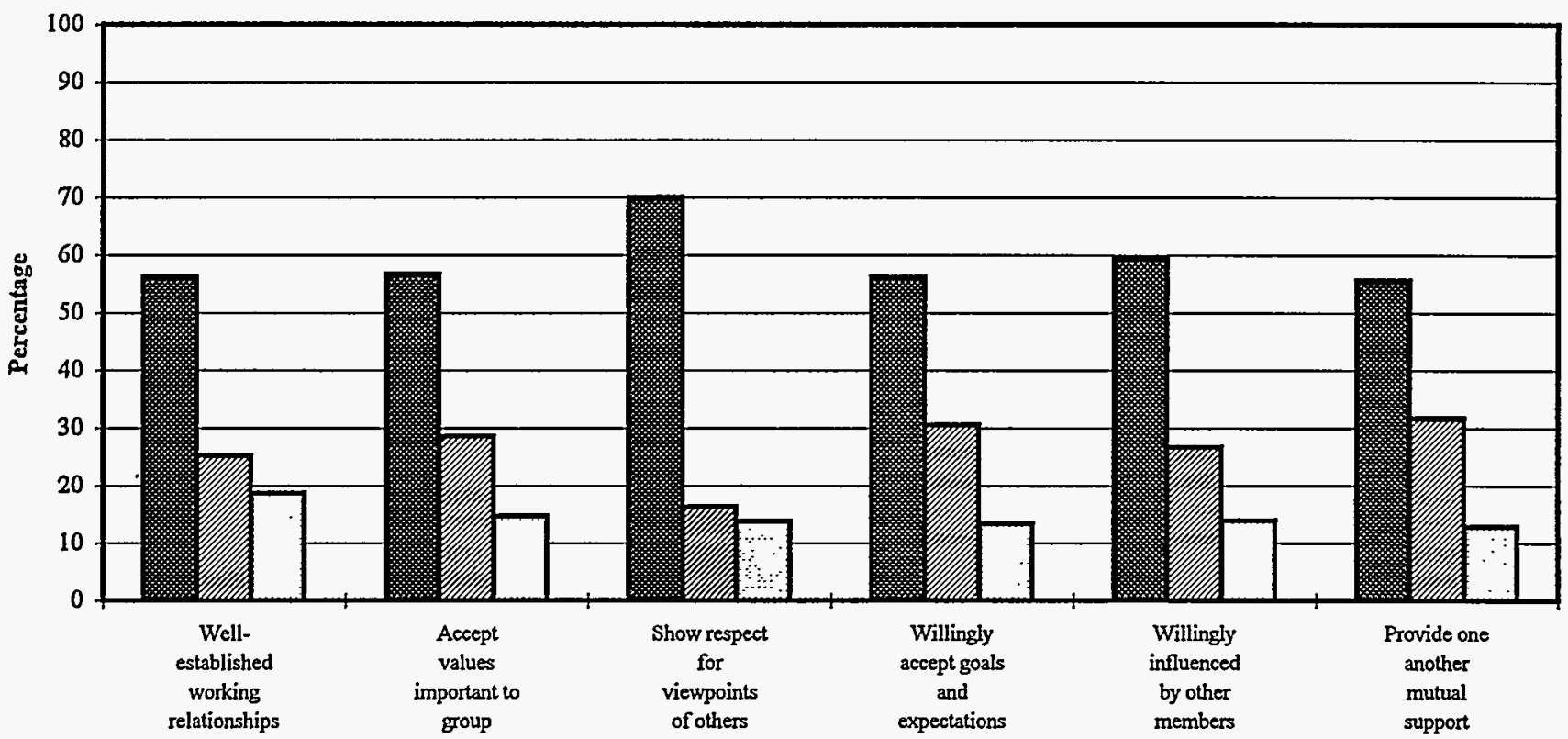

E Agree (\%) Neither Agree Nor Disagree/Don't Know (\%) DDisagree (\%)

SSAB Supplementary Appendix

August 1996 
Figure 12a. Nevada

Member affect

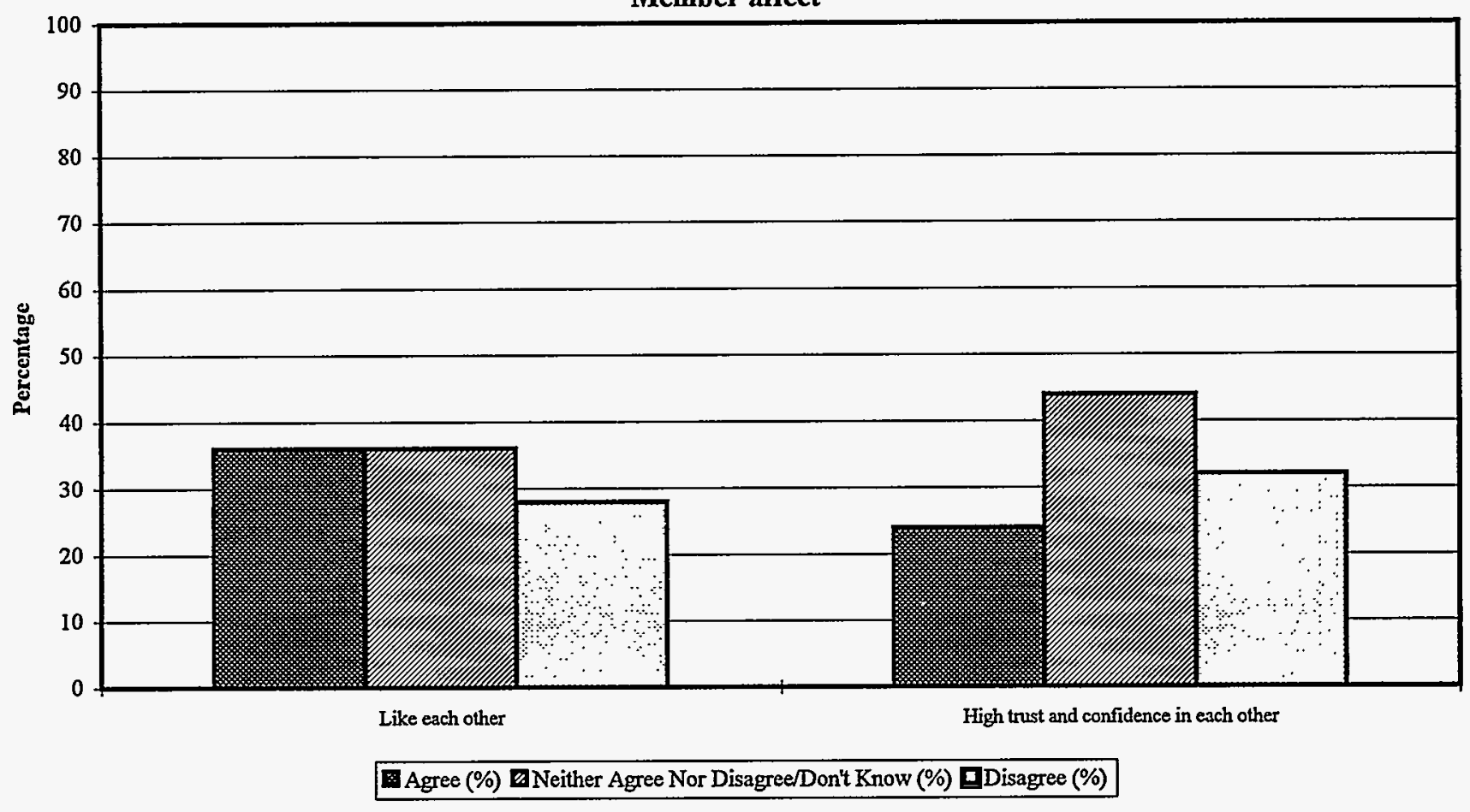

Figure 12b. Long Survey: Entire Sample

\section{Member affect}

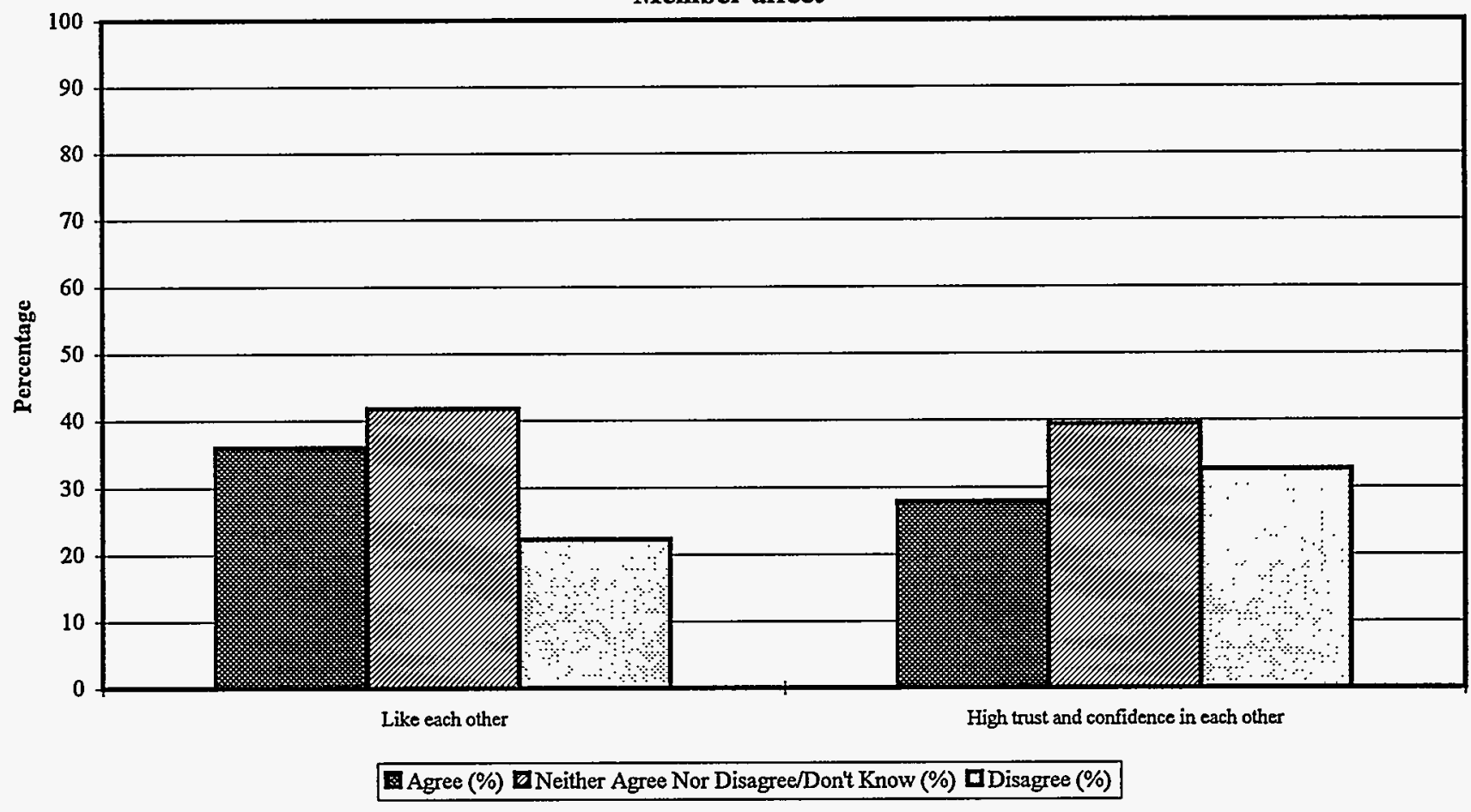

SSAB Supplementary Appendix August 1996 
Figure 13a. Nevada

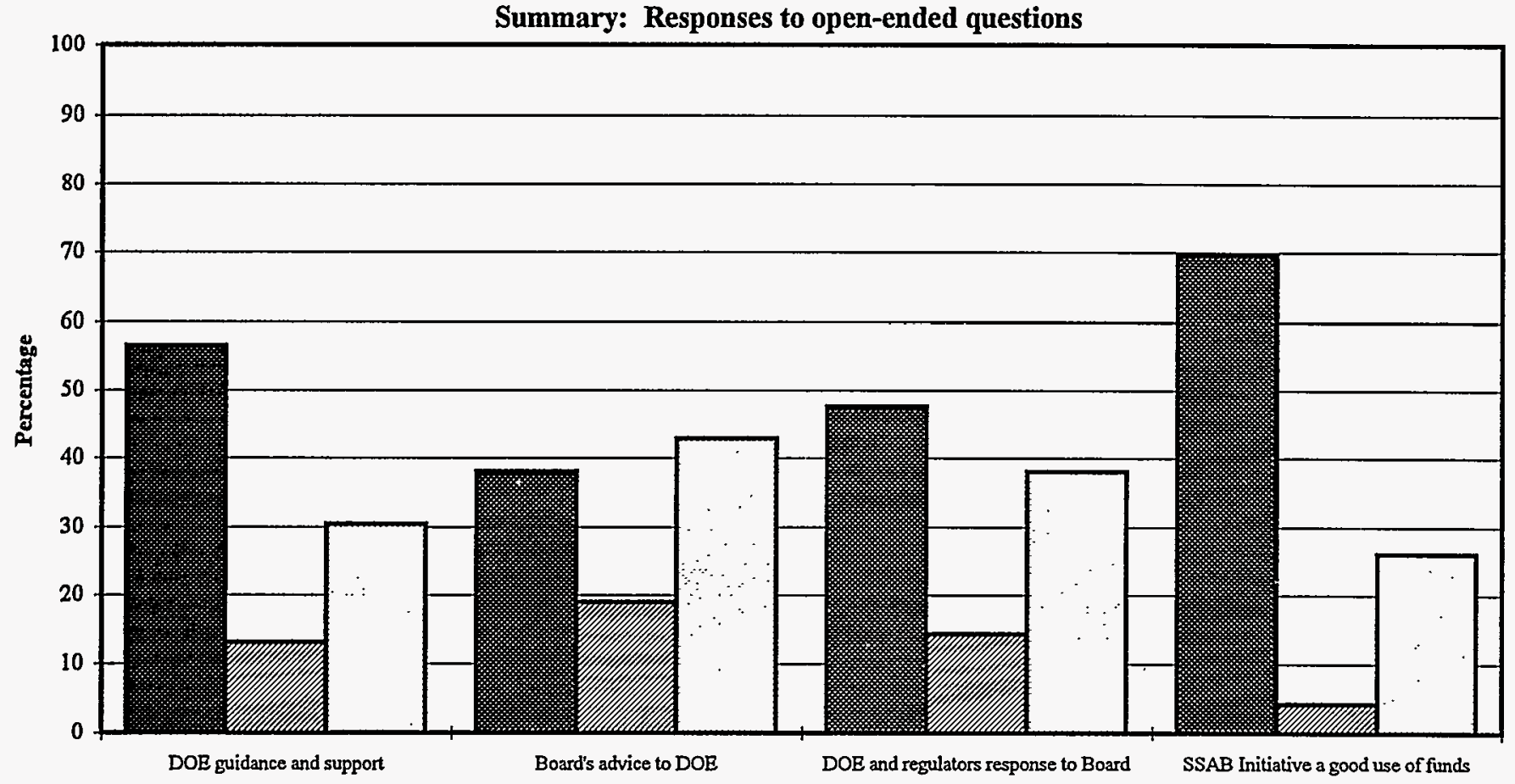

Yes, Good (\%) DNeutral, Conditional, No Opinion (\%) םNo, Not Good (\%)

Figure 13b. Long Survey: Entire Sample

Summary: Responses to open-ended questions

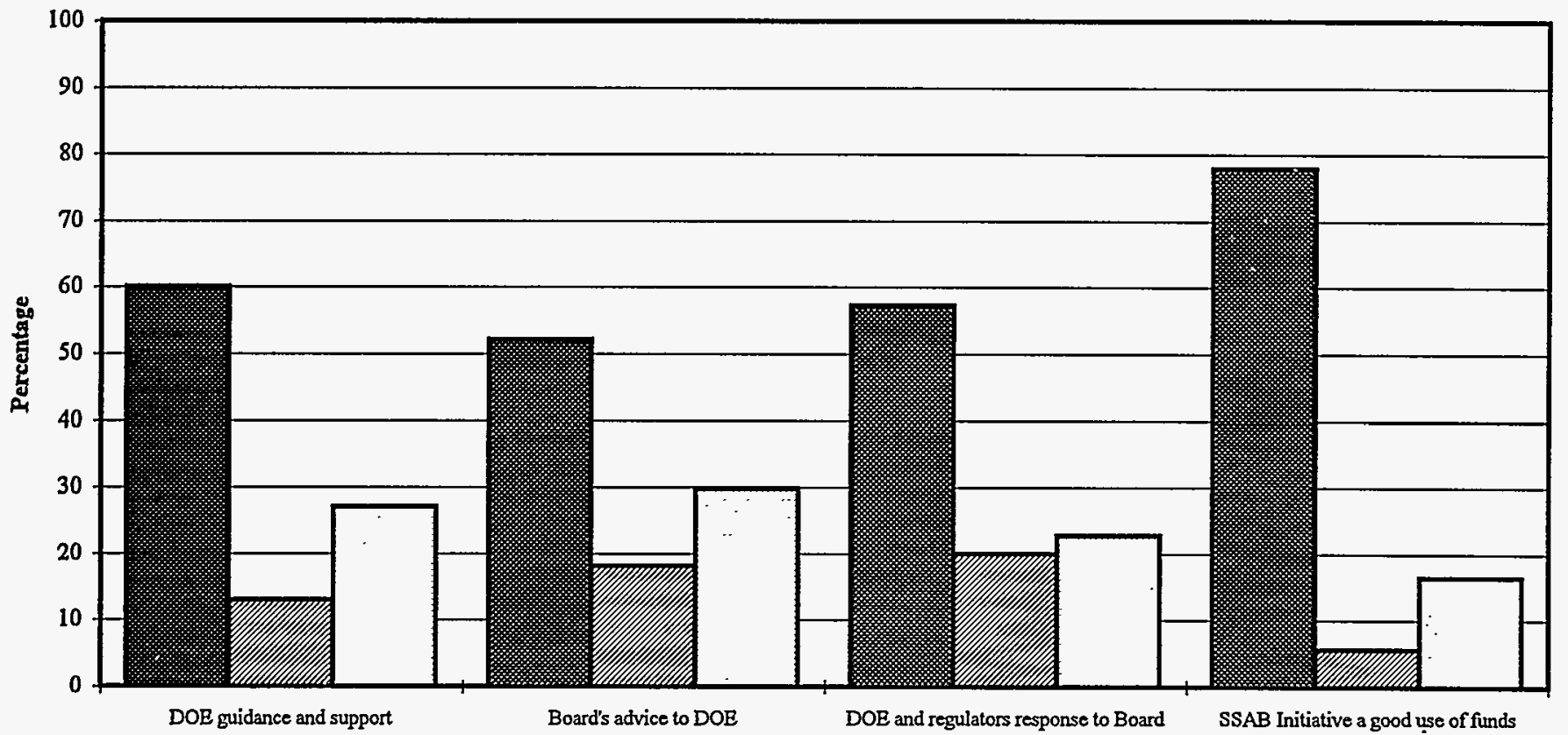

PYes, Good (\%) DNeutral, Conditional, No Opinion (\%) DNo, Not Good (\%)

SSAB Supplementary Appendix

August 1996 
Figure 14a. Nevada

Summary: Combined responses for SSAB Initiative goals (Means)

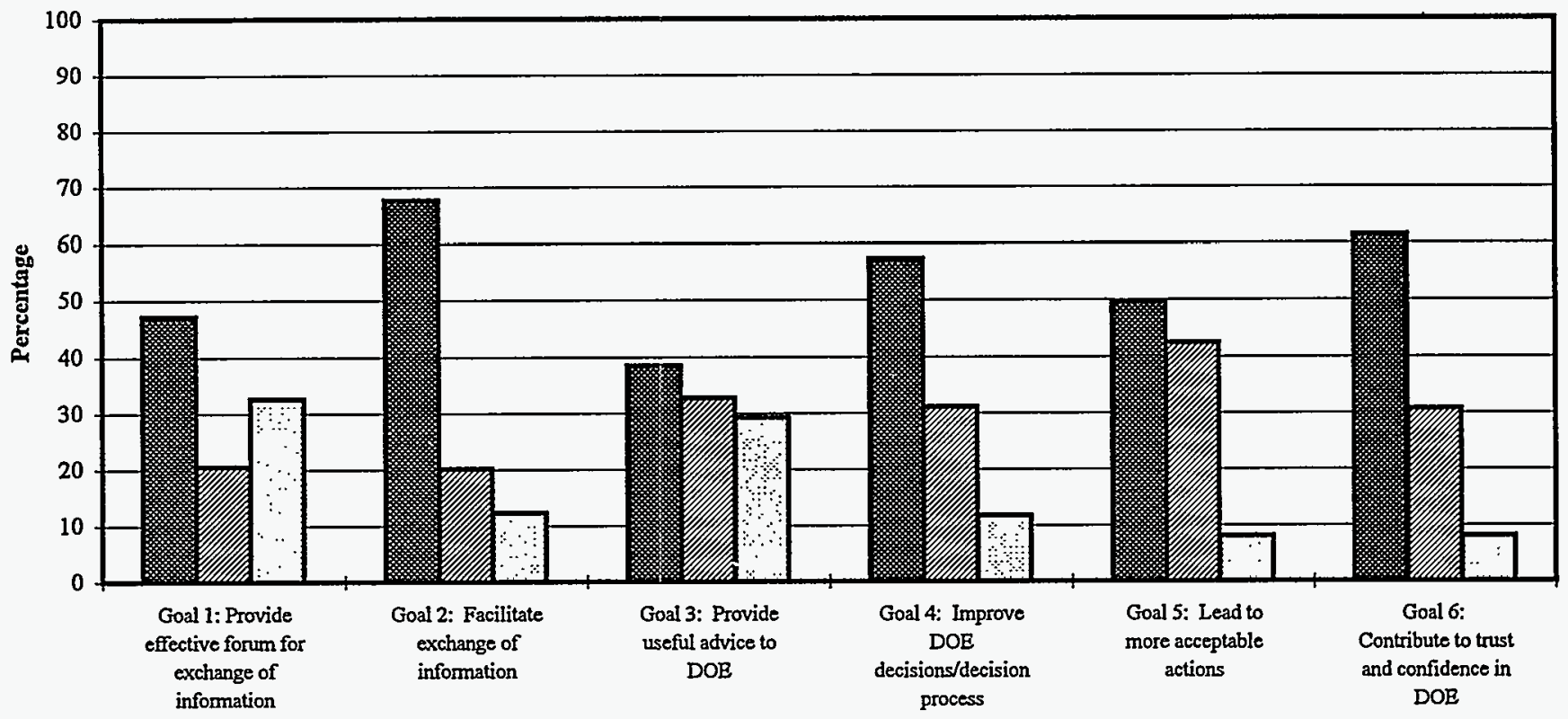

Agree (\%) WNeither Agree Nor Disagree/Don't Know (\%) DDisagree (\%)

Figure 14b. Long Survey: Entire Sample

Summary: Combined responses for SSAB Initiative goals (Means)

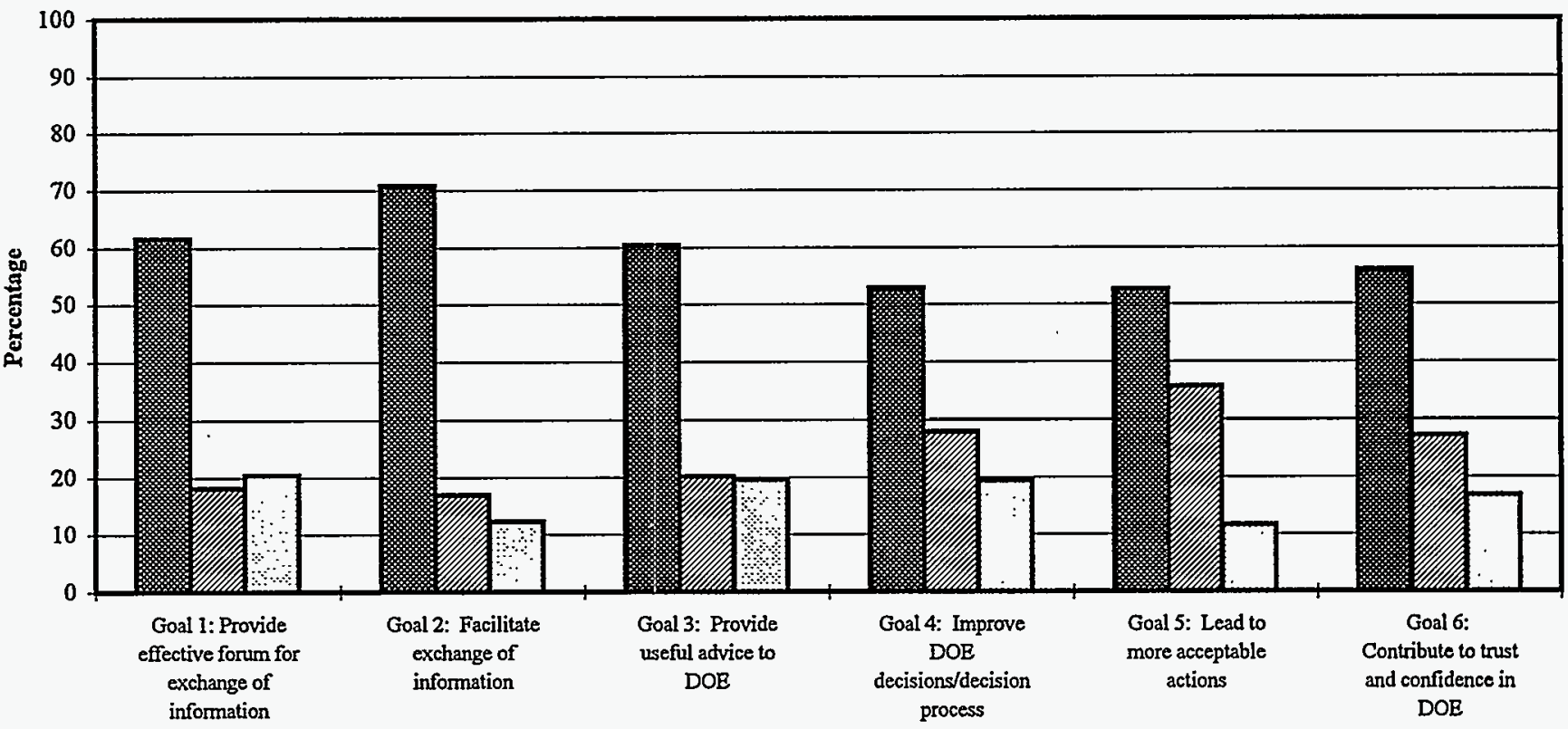

Agree (\%) DNeither Agree Nor Disagree/Don't Know (\%) DDisagree (\%)

SSAB Supplementary Appendix

August 1996 
Figure 15a. Nevada

Summary: Combined responses on DOE-HQ involvement,

Personal experience, Group/Team work skills,

Working relations, Member affect (Means)

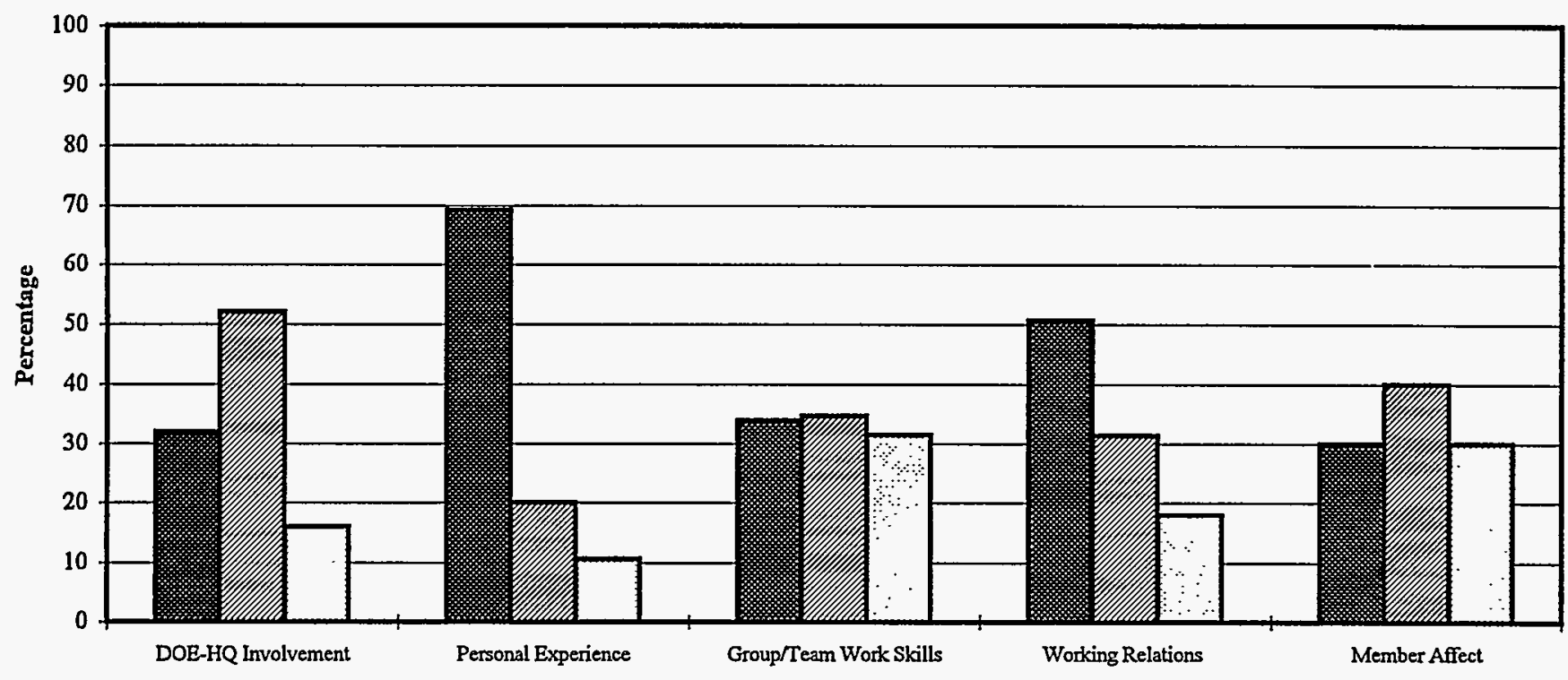

Agree (\%) WNeither Agree Nor Disagree/Don't Know (\%) DDisagree (\%)

Figure 15b. Long Survey: Entire Sample

Summary: Combined responses on DOE-HQ involvement,

Personal experience, Group/Team work skills,

Working relations, Member affect (Means)

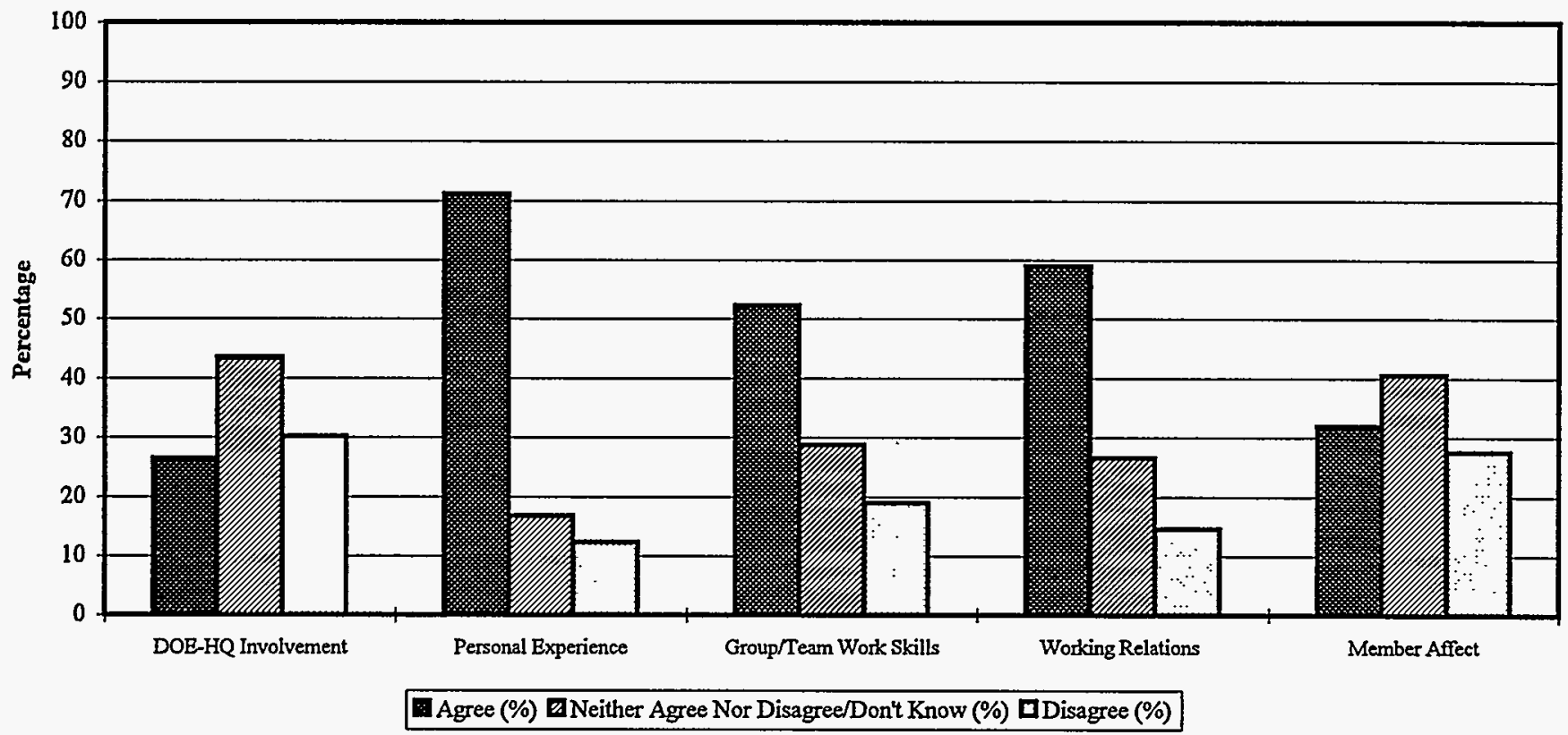

SSAB Supplementary Appendix 
Table 1.

Goal 1. Establish processes and procedures to provide an effective forum for exchange of information and viewpoints regarding DOE site issues

\begin{tabular}{|c|c|c|c|c|c|c|c|c|}
\hline \multirow[t]{2}{*}{ Nevada } & \multicolumn{2}{|c|}{ Agree } & \multicolumn{2}{|c|}{$\begin{array}{l}\text { Neither/ } \\
\text { Don't Know }\end{array}$} & \multicolumn{2}{|c|}{ Disagree } & \multicolumn{2}{|c|}{ Total } \\
\hline & $\mathrm{N}$ & $\%$ & $\mathrm{~N}$ & $\%$ & $\mathrm{~N}$ & $\%$ & $\mathbf{N}$ & $\%$ \\
\hline $\begin{array}{l}\text { The Board has established processes and } \\
\text { procedures for the effective exchange of } \\
\text { information }\end{array}$ & 17 & $68.0 \%$ & 3 & $12.0 \%$ & 5 & $20.0 \%$ & 25 & $100 \%$ \\
\hline $\begin{array}{l}\text { The Board has agreed-upon procedures to } \\
\text { operate the board }\end{array}$ & 17 & $68.0 \%$ & 3 & $12.0 \%$ & 5 & $20.0 \%$ & 25 & $100 \%$ \\
\hline $\begin{array}{l}\text { The Board has adequate support to allow } \\
\text { it to focus on substantive issues (R) } *\end{array}$ & 8 & $32.0 \%$ & 4 & $16.0 \%$ & 13 & $52.0 \%$ & 25 & $100 \%$ \\
\hline $\begin{array}{l}\text { The Board provides sufficient time for } \\
\text { discussion of issues }\end{array}$ & 12 & $48.0 \%$ & 5 & $20.0 \%$ & 8 & $32.0 \%$ & 25 & $100 \%$ \\
\hline The Board has effective leadership & 13 & $52.0 \%$ & 6 & $24.0 \%$ & 6 & $24.0 \%$ & 25 & $100 \%$ \\
\hline $\begin{array}{l}\text { DOE requests Board advice far enough } \\
\text { ahead of decision deadlines }(R) *\end{array}$ & 1 & $4.0 \%$ & 4 & $16.0 \%$ & 20 & $80.0 \%$ & 25 & $100 \%$ \\
\hline $\begin{array}{l}\text { DOE handles Board administrative issues } \\
\text { expeditiously (R) * }\end{array}$ & 17 & $68.0 \%$ & 7 & $28.0 \%$ & 1 & $4.0 \%$ & 25 & $100 \%$ \\
\hline $\begin{array}{l}\text { The SSAB staff provide satisfactory } \\
\text { support }\end{array}$ & 11 & $44.0 \%$ & 6 & $24.0 \%$ & 8 & $32.0 \%$ & 25 & $100 \%$ \\
\hline $\begin{array}{l}\text { The facilitator for the Board has helped } \\
\text { the Board function effectively }\end{array}$ & 10 & $40.0 \%$ & 8 & $32.0 \%$ & 7 & $28.0 \%$ & 25 & $100 \%$ \\
\hline
\end{tabular}

* (R) denotes a question that was asked in the negative form. For ease of review, these questions and data have been reversed in this table and are stated in positive forms.

SSAB Supplementary Appendix

August 1996 
Table 2.

Goal 2. Facilitate interaction and exchange of information and viewpoints regarding DOE site íssues

\begin{tabular}{|c|c|c|c|c|c|c|c|c|}
\hline \multirow[t]{2}{*}{ Nevada } & \multicolumn{2}{|c|}{ Agree } & \multicolumn{2}{|c|}{$\begin{array}{l}\text { Neither/ } \\
\text { Don't Know }\end{array}$} & \multicolumn{2}{|c|}{ Disagree } & \multicolumn{2}{|c|}{ Total } \\
\hline & $\mathrm{N}$ & $\%$ & $\mathrm{~N}$ & $\%$ & $N$. & $\%$ & $N$ & $\%$ \\
\hline $\begin{array}{l}\text { The SSAB facilitates effective exchange } \\
\text { of viewpoints on site issues }\end{array}$ & 16 & $64.0 \%$ & 5 & $20.0 \%$ & 4 & $16.0 \%$ & 25 & $100 \%$ \\
\hline $\begin{array}{l}\text { The SSAB contributes to Board members' } \\
\text { understanding of the basis for key site } \\
\text { decisions }\end{array}$ & 16 & $640 \%$ & 5 & $20.0 \%$ & 4 & $16.0 \%$ & 25 & $100 \%$ \\
\hline $\begin{array}{l}\text { The SSAB contributes to DOE and } \\
\text { regulators' understanding of the public's } \\
\text { viewpoints on key site decisions }\end{array}$ & 19 & $76.0 \%$ & 3 & $12.0 \%$ & 3 & $12.0 \%$ & 25 & $100 \%$ \\
\hline $\begin{array}{l}\text { The SSAB contributes to a constructive } \\
\text { working relationship among the } \\
\text { participants }\end{array}$ & 19 & $76.0 \%$ & 6 & $24.0 \%$ & 0 & $0 \%$ & 25 & $100 \%$ \\
\hline The SSAB strives for consensus & 19 & $79.2 \%$ & 5 & $20.8 \%$ & 0 & $0 \%$ & 24 & $100 \%$ \\
\hline $\begin{array}{l}\text { The SSAB gives fair consideration to } \\
\text { dissenting opinions }\end{array}$ & 19 & $76.0 \%$ & 3 & $12.0 \%$ & 3 & $12.0 \%$ & 25 & $100 \%$ \\
\hline $\begin{array}{l}\text { The Board has made the effort needed to } \\
\text { learn about site issues }\end{array}$ & 18 & $72.0 \%$ & 3 & $12.0 \%$ & 4 & $16.0 \%$ & 25 & $100 \%$ \\
\hline $\begin{array}{l}\text { The Board invites expert advice into its } \\
\text { discussion on key policy issues }\end{array}$ & 21 & $84.0 \%$ & 2 & $8.0 \%$ & 2 & $8.0 \%$ & 25 & $100 \%$ \\
\hline $\begin{array}{l}\text { The Board responds to public inquiries } \\
\text { and comments about its decisions }\end{array}$ & 10 & $40.0 \%$ & 11 & $44.0 \%$ & 4 & $16.0 \%$ & 25 & $100 \%$ \\
\hline $\begin{array}{l}\text { The Board solicits feedback from the } \\
\text { community on its work }\end{array}$ & 9 & $36.0 \%$ & 8 & $32.0 \%$ & 8 & $32.0 \%$ & 25 & $100 \%$ \\
\hline $\begin{array}{l}\text { DOE makes information on key site issues } \\
\text { readily available to the Board }\end{array}$ & 19 & $76.0 \%$ & 3 & $12.0 \%$ & 3 & $12.0 \%$ & 25 & $100 \%$ \\
\hline $\begin{array}{l}\text { The regulators make important } \\
\text { contributions to the Board's work }\end{array}$ & 17 & $68.0 \%$ & 6 & $24.0 \%$ & 2 & $8.0 \%$ & 25 & $100 \%$ \\
\hline $\begin{array}{l}\text { The SSAB creates a climate supportive of } \\
\text { differing viewpoints }(\mathrm{R})^{*}\end{array}$ & 17 & $68.0 \%$ & 5 & $20.0 \%$ & 3 & $12.0 \%$ & 25 & $100 \%$ \\
\hline
\end{tabular}

* (R) denotes a question that was asked in the negative form. For ease of review, these questions and data have been reversed in this table and are stated in positive forms.

SSAB Supplementary Appendix

August 1996 
Table 3.

Goal 3. Provide useful advice and/or recommendations to DOE (and regulators, where appropriate)

\begin{tabular}{|c|c|c|c|c|c|c|c|c|}
\hline \multirow[t]{2}{*}{ Nevada } & \multicolumn{2}{|c|}{ Agree } & \multicolumn{2}{|c|}{$\begin{array}{l}\text { Neither/ } \\
\text { Don't Know }\end{array}$} & \multicolumn{2}{|c|}{ Disagree } & \multicolumn{2}{|c|}{ Total } \\
\hline & $\mathrm{N}$ & $\%$ & $N$ & $\%$ & $\mathrm{~N}$ & $\%$ & $\mathrm{~N}$ & $\%$ \\
\hline $\begin{array}{l}\text { The Board provides useful advice to DOE } \\
\text { (and regulators, where appropriate)(R) }{ }^{*}\end{array}$ & 11 & $440 \%$ & 9 & $36.0 \%$ & 5 & $20.0 \%$ & 25 & $100 \%$ \\
\hline $\begin{array}{l}\text { The SSAB reaches agreement about } \\
\text { prioritization of key site issues for which } \\
\text { advice is sought }(\mathrm{R})^{*}\end{array}$ & 11 & $44.0 \%$ & 5 & $20.0 \%$ & 9 & $36.0 \%$ & 25 & $100 \%$ \\
\hline The Board helps define site problems & 10 & $40.0 \%$ & 8 & $32.0 \%$ & 7 & $28.0 \%$ & 25 & $100 \%$ \\
\hline $\begin{array}{l}\text { The Board addresses issues in a timely } \\
\text { manner }(R) *\end{array}$ & 2 & $8.0 \%$ & 8 & $32.0 \%$ & 15 & $60.0 \%$ & 25 & $100 \%$ \\
\hline $\begin{array}{l}\text { The Board reaches consensus on key site } \\
\text { issues (R)* }\end{array}$ & 8 & $32.0 \%$ & 10 & $40.0 \%$ & 7 & $28.0 \%$ & 25 & $100 \%$ \\
\hline $\begin{array}{l}\text { The Board provides informed advice to } \\
\text { DOE (and regulators, where appropriate) }\end{array}$ & 14 & $56.0 \%$ & 8 & $32.0 \%$ & 3 & $12.0 \%$ & 25 & $100 \%$ \\
\hline $\begin{array}{l}\text { The Board provides advice that reflects } \\
\text { the viewpoints and priorities of the } \\
\text { community }\end{array}$ & 11 & $44.0 \%$ & 9 & $36.0 \%$ & 5 & $20.0 \%$ & 25 & $100 \%$ \\
\hline
\end{tabular}

* (R) denotes a question that was asked in the negative form. For ease of review, these questions and data have been reversed in this table and are stated in positive forms.

SSAB Supplementary Appendix 
Table 4.

Goal 4. Improve DOE's (and where applicable, regulators') site decisions and decision making process

\begin{tabular}{|c|c|c|c|c|c|c|c|c|}
\hline \multirow[t]{2}{*}{ Nevada } & \multicolumn{2}{|c|}{ Agree } & \multicolumn{2}{|c|}{$\begin{array}{c}\text { Neither/ } \\
\text { Don't Know }\end{array}$} & \multicolumn{2}{|c|}{ Disagree } & \multicolumn{2}{|c|}{ Total } \\
\hline & $\mathrm{N}$ & $\%$ & $\mathrm{~N}$ & $\%$ & $\mathrm{~N}$ & $\%$ & $\mathrm{~N}$ & $\%$ \\
\hline $\begin{array}{l}\text { The SSAB has improved DOE's site } \\
\text { decisions (R) * }\end{array}$ & 13 & $54.2 \%$ & 8 & $33.3 \%$ & 3 & $12.5 \%$ & 24 & $100 \%$ \\
\hline $\begin{array}{l}\text { The Board understands the decision } \\
\text { making process at [a particular] site }\end{array}$ & 10 & $41.7 \%$ & 5 & $20.8 \%$ & 9 & $37.5 \%$ & 24 & $100 \%$ \\
\hline $\begin{array}{l}\text { DOE discusses important policies } \\
\text { affecting site decisions with the Board }\end{array}$ & 22 & $88.0 \%$ & 2 & $8.0 \%$ & 1 & $4.0 \%$ & 25 & $100 \%$ \\
\hline $\begin{array}{l}\text { DOE has explained to the Board its site } \\
\text { decision making process }\end{array}$ & 19 & $76.0 \%$ & 6 & $24.0 \%$ & 0 & $0 \%$ & 25 & $100 \%$ \\
\hline $\begin{array}{l}\text { The DOE shows how Board advice is } \\
\text { reflected in site decisions }\end{array}$ & 10 & $40.0 \%$ & 13 & $52.0 \%$ & 2 & $8.0 \%$ & 25 & $100 \%$ \\
\hline $\begin{array}{l}\text { The decision making process used by } \\
\text { DOE at [a particular] site is effective in } \\
\text { furthering site clean-up (R) }{ }^{*}\end{array}$ & 11 & $44.0 \%$ & 12 & $48.0 \%$ & 2 & $8.0 \%$ & 25 & $100 \%$ \\
\hline
\end{tabular}

* (R) denotes a question that was asked in the negative form. For ease of review, these questions and data have been reversed in this table and are stated in positive forms.

SSAB Supplementary Appendix 
Table 5.

Goal 5. Lead to more acceptable actions

\begin{tabular}{|c|c|c|c|c|c|c|c|c|}
\hline \multirow[t]{2}{*}{ Nevada } & \multicolumn{2}{|c|}{ Agree } & \multicolumn{2}{|c|}{$\begin{array}{c}\text { Neither/ } \\
\text { Don't Know }\end{array}$} & \multicolumn{2}{|c|}{ Disagree } & \multicolumn{2}{|c|}{ Total } \\
\hline & $N$ & $\%$ & $N$ & $\%$ & $N$ & $\%$ & $\mathrm{~N}$ & $\%$ \\
\hline $\begin{array}{l}\text { The SSAB leads to more acceptable site } \\
\text { decisions }\end{array}$ & 10 & $41.7 \%$ & 12 & $50.0 \%$ & 2 & $8.3 \%$ & 24 & $100 \%$ \\
\hline $\begin{array}{l}\text { The Board supports the recommendations } \\
\text { it gives DOE }\end{array}$ & 20 & $80.0 \%$ & 5 & $20.0 \%$ & 0 & $0 \%$ & 25 & $100 \%$ \\
\hline The Board supports DOE's site actions & 8 & $32.0 \%$ & 14 & $56.0 \%$ & 3 & $12.0 \%$ & 25 & $100 \%$ \\
\hline $\begin{array}{l}\text { The DOE pays attention to the Board's } \\
\text { advice on key site issues (R) * }\end{array}$ & 14 & $56.0 \%$ & 10 & $40.0 \%$ & 1 & $4.0 \%$ & 25 & $100 \%$ \\
\hline $\begin{array}{l}\text { There is support in the community for } \\
\text { DOE's site decisions that have SSAB } \\
\text { input }\end{array}$ & 6 & $24.0 \%$ & 16 & $64.0 \%$ & 3 & $12.0 \%$ & 25 & $100 \%$ \\
\hline Progress is being made on key site issues & 16 & $64.0 \%$ & 6 & $24.0 \%$ & 3 & $12.0 \%$ & 25 & $100 \%$ \\
\hline
\end{tabular}

* (R) denotes a question that was asked in the negative form. For ease of review, these questions and data have been reversed in this table and are stated in positive forms.

SSAB Supplementary Appendix 
Table 6.

Goal 6. Contribute to trust and confidence in DOE

\begin{tabular}{|c|c|c|c|c|c|c|c|c|}
\hline \multirow[t]{2}{*}{ Nevada } & \multicolumn{2}{|c|}{ Agree } & \multicolumn{2}{|c|}{$\begin{array}{c}\text { Neither/ } \\
\text { Don't Know }\end{array}$} & \multicolumn{2}{|c|}{ Disagree } & \multicolumn{2}{|c|}{ Total } \\
\hline & $\mathrm{N}$ & $\%$ & $\mathrm{~N}$ & $\%$ & $\mathbf{N}$ & $\%$ & $\mathrm{~N}$ & $\%$ \\
\hline $\begin{array}{l}\text { The SSAB contributes to trust and } \\
\text { confidence in DOE }\end{array}$ & 14 & $56.0 \%$ & 9 & $36.0 \%$ & 2 & $8.0 \%$ & 25 & $100 \%$ \\
\hline $\begin{array}{l}\text { Relationships between DOE and the public } \\
\text { have improved since the formation of the } \\
\text { SSAB }\end{array}$ & 16 & $64.0 \%$ & 8 & $32.0 \%$ & 1 & $4.0 \%$ & 25 & $100 \%$ \\
\hline $\begin{array}{l}\mathrm{DOE} \text { is committed to clean up [a } \\
\text { particular] site }\end{array}$ & 16 & $64.0 \%$ & 6 & $24.0 \%$ & 3 & $12.0 \%$ & 25 & $100 \%$ \\
\hline
\end{tabular}


Table 7.

Public awareness

\begin{tabular}{llllllllll}
\hline \multicolumn{1}{c}{ Nevada } & Agree & $\begin{array}{c}\text { Neither/ } \\
\text { Don't Know }\end{array}$ & Disagree & \multicolumn{2}{c}{ Total } \\
& $\mathrm{N}$ & $\%$ & $\mathrm{~N}$ & $\%$ & $\mathrm{~N}$ & $\%$ & $\mathrm{~N}$ & $\%$ \\
\hline $\begin{array}{l}\text { The public knows little about the role of } \\
\text { the Board }\end{array}$ & 20 & $80.0 \%$ & 2 & $8.0 \%$ & 3 & $12.0 \%{ }^{\prime}$ & 25 & $100 \%$ \\
\hline
\end{tabular}

Table 8.

SSAB Initiative is a good use of funds

\begin{tabular}{cccccccccc}
\hline \multirow{2}{*}{ Nevada } & Yes, Good & $\begin{array}{c}\text { Neutral, } \\
\text { Conditional, } \\
\text { No Opinion }\end{array}$ & $\begin{array}{c}\text { No, } \\
\text { Not Good }\end{array}$ & Total \\
& $\mathrm{N}$ & $\%$ & $\mathrm{~N}$ & $\%$ & $\mathrm{~N}$ & $\%$ & $\mathrm{~N}$ & $\%$ \\
\hline SSAB Initiative is a good use of funds & 17 & $73.9 \%$ & 1 & $4.3 \%$ & 5 & $21.7 \%$ & 23 & $100 \%$ \\
\hline
\end{tabular}


Table 9.

DOE-HQ involvement with the SSAB Initiative

\begin{tabular}{|c|c|c|c|c|c|c|c|c|}
\hline \multirow[t]{2}{*}{ Nevada } & \multicolumn{2}{|c|}{ Agree } & \multicolumn{2}{|c|}{$\begin{array}{c}\text { Neither/ } \\
\text { Don't Know }\end{array}$} & \multicolumn{2}{|c|}{ Disagree } & \multicolumn{2}{|c|}{ Total } \\
\hline & $N$ & $\%$ & $\mathbf{N}$ & $\%$ & $N$ & $\%$ & $N$ & $\%$ \\
\hline $\begin{array}{l}\text { DOE-HQ provides helpful guidance to the } \\
\text { Boards and to DOE }\end{array}$ & 10 & $40.0 \%$ & 10 & $40.0 \%$ & 5 & $20.0 \%$ & 25 & $100 \%$ \\
\hline $\begin{array}{l}\text { DOE-HQ provides sufficient support to } \\
\text { facilitate the work of the SSAB }\end{array}$ & 7 & $28.0 \%$ & 12 & $48.0 \%$ & 6 & $24.0 \%$ & 25 & $100 \%$ \\
\hline $\begin{array}{l}\text { DOE-HQ gives careful consideration to } \\
\text { SSAB advice in its decisions }\end{array}$ & 7 & 28.0 & 17 & $68.0 \%$ & 1 & $4.0 \%$ & 25 & $100 \%$ \\
\hline
\end{tabular}


Table 10.

Personal experience with the SSAB Initiative

\begin{tabular}{|c|c|c|c|c|c|c|c|c|}
\hline \multirow[t]{2}{*}{ Nevada } & \multicolumn{2}{|c|}{ Agree } & \multicolumn{2}{|c|}{$\begin{array}{c}\text { Neither/ } \\
\text { Don't Know }\end{array}$} & \multicolumn{2}{|c|}{ Disagree } & \multicolumn{2}{|c|}{ Total } \\
\hline & $N$ & $\%$ & $N$ & $\%$ & $\mathrm{~N}$ & $\%$ & $\mathrm{~N}$ & $\%$ \\
\hline $\begin{array}{l}\text { I consider myself to be very } \\
\text { knowledgeable about site issues }\end{array}$ & 17 & $68.0 \%$ & 8 & $32.0 \%$ & 0 & $0 \%$ & 25 & $100 \%$ \\
\hline $\begin{array}{l}\text { Because of the SSAB, I better understand } \\
\text { the complexities of site clean-up }\end{array}$ & 16 & $64.0 \%$ & 4 & $16.0 \%$ & 5 & $20.0 \%$ & 25 & $100 \%$ \\
\hline $\begin{array}{l}\text { I feel that my participation in the SSAB } \\
\text { has been worthwhile }(R) *\end{array}$ & 19 & $76.0 \%$ & 3 & $12.0 \%$ & 3 & $12.0 \%$ & 25 & $100 \%$ \\
\hline
\end{tabular}

* (R) denotes a question that was asked in the negative form. For ease of review, these questions and data have been reversed in this table and are stated in positive forms. 
Table 11.

Group/Team work skills

\begin{tabular}{|c|c|c|c|c|c|c|c|c|}
\hline \multirow[t]{2}{*}{ Nevada } & \multicolumn{2}{|c|}{ Agree } & \multicolumn{2}{|c|}{$\begin{array}{l}\text { Neither/ } \\
\text { Don't Know }\end{array}$} & \multicolumn{2}{|c|}{ Disagree } & \multicolumn{2}{|c|}{ Total } \\
\hline & $N$ & $\%$ & $\mathrm{~N}$ & $\%$ & $\mathrm{~N}$ & $\%$ & $\mathrm{~N}$ & $\%$ \\
\hline All have group/team work skills & 5 & $20.0 \%$ & 10 & $40.0 \%$ & 10 & $40.0 \%$ & 25 & $100 \%$ \\
\hline $\begin{array}{l}\text { Have developed well-established working } \\
\text { relationships with each other }\end{array}$ & 11 & $44.0 \%$ & 6 & $24.0 \%$ & 8 & $32.0 \%$ & 25 & $100 \%$ \\
\hline Like each other & 9 & $36.0 \%$ & 9 & $36.0 \%$ & 7 & $28.0 \%$ & 25 & $100 \%$ \\
\hline $\begin{array}{l}\text { Have a high degree of trust and } \\
\text { confidence in each other }\end{array}$ & 6 & $24.0 \%$ & 11 & $44.0 \%$ & 8 & $32.0 \%$ & 25 & $100 \%$ \\
\hline $\begin{array}{l}\text { Accept the values that are important to the } \\
\text { group }\end{array}$ & 12 & $48.0 \%$ & 10 & $40.0 \%$ & 3 & $12.0 \%$ & 25 & $100 \%$ \\
\hline Show respect for the viewpoints of others & 20 & $80.0 \%$ & 3 & $12.0 \%$ & 2 & $8.0 \%$ & 25 & $100 \%$ \\
\hline $\begin{array}{l}\text { Accept willingly the goals and } \\
\text { expectations for the Board }\end{array}$ & 9 & $36.0 \%$ & 11 & $44.0 \%$ & 5 & $20.0 \%$ & 25 & $100 \%$ \\
\hline $\begin{array}{l}\text { Set high expectations for what they } \\
\text { believe they can accomplish }\end{array}$ & 10 & $40.0 \%$ & 10 & $40.0 \%$ & 5 & $20.0 \%$ & 25 & $100 \%$ \\
\hline $\begin{array}{l}\text { Communicate fully and frankly to the } \\
\text { Board all relevant and valuable } \\
\text { information }\end{array}$ & 16 & $64.0 \%$ & 6 & $24.0 \%$ & 3 & $12.0 \%$ & 25 & $100 \%$ \\
\hline $\begin{array}{l}\text { Try not to waste the Board's time with } \\
\text { irrelevant material or communications }\end{array}$ & 6 & $24.0 \%$ & 5 & $20.0 \%$ & 14 & $56.0 \%$ & 25 & $100 \%$ \\
\hline $\begin{array}{l}\text { Are willing to be influenced by other } \\
\text { Board members about new ideas and } \\
\text { methods }\end{array}$ & 12 & $48.0 \%$ & 8 & $32.0 \%$ & 5 & $20.0 \%$ & 25 & $100 \%$ \\
\hline $\begin{array}{l}\text { Clearly understand the goals and } \\
\text { philosphy of the Board's operation }\end{array}$ & 9 & $36.0 \%$ & 9 & $36.0 \%$ & 7 & $28.0 \%$ & 25 & $100 \%$ \\
\hline $\begin{array}{l}\text { Do all they can to help the Board achieve } \\
\text { its objectives }\end{array}$ & 7 & $29.2 \%$ & 13 & $54.2 \%$ & 4 & $16.7 \%$ & 24 & $100 \%$ \\
\hline $\begin{array}{l}\text { Get the technical knowledge and training } \\
\text { in group skills they need }\end{array}$ & 6 & $24.0 \%$ & 7 & $28.0 \%$ & 12 & $48.0 \%$ & 25 & $100 \%$ \\
\hline Provide one another mutual help & 12 & $48.0 \%$ & 9 & $36.0 \%$ & 4 & $16.0 \%$ & 25 & $100 \%$ \\
\hline
\end{tabular}


Pantex Site Specific Advisory Board

Evaluation Survey Results

SSAB Supplementary Appendix

August 1996

S-158 
Figure 1a. Pantex

Goal 1. Establish processes and procedures to provide an effective forum for exchange of information and viewpoints regarding DOE site issues

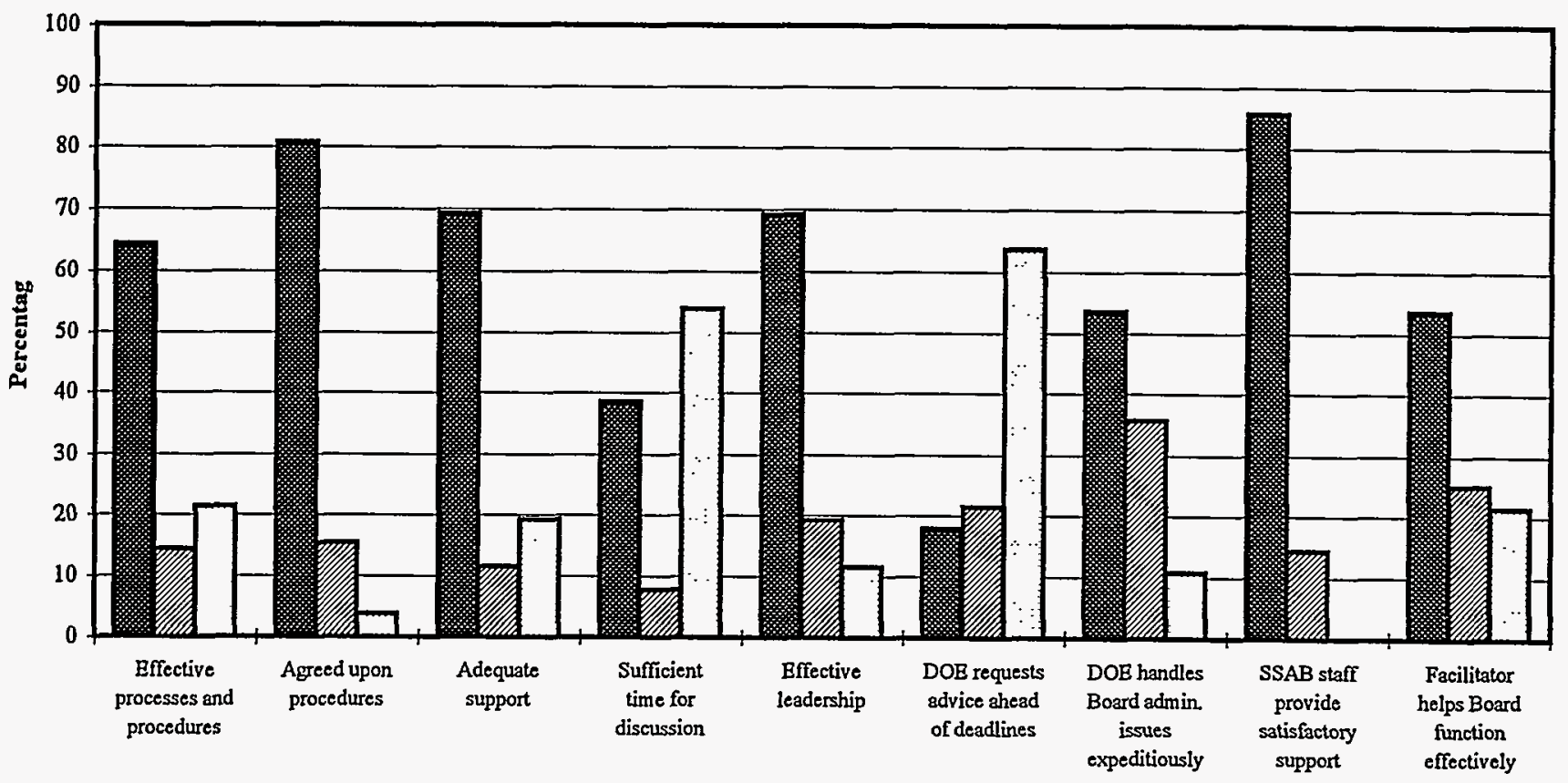

Agree (\%) aNeither Agree Nor Disagree/Don't Know (\%) Disagree (\%)

Figure 1b. Long Survey: Entire Sample

Goal 1. Establish processes and procedures to provide an effective forum for exchange of information and viewpoints regarding DOE site issues

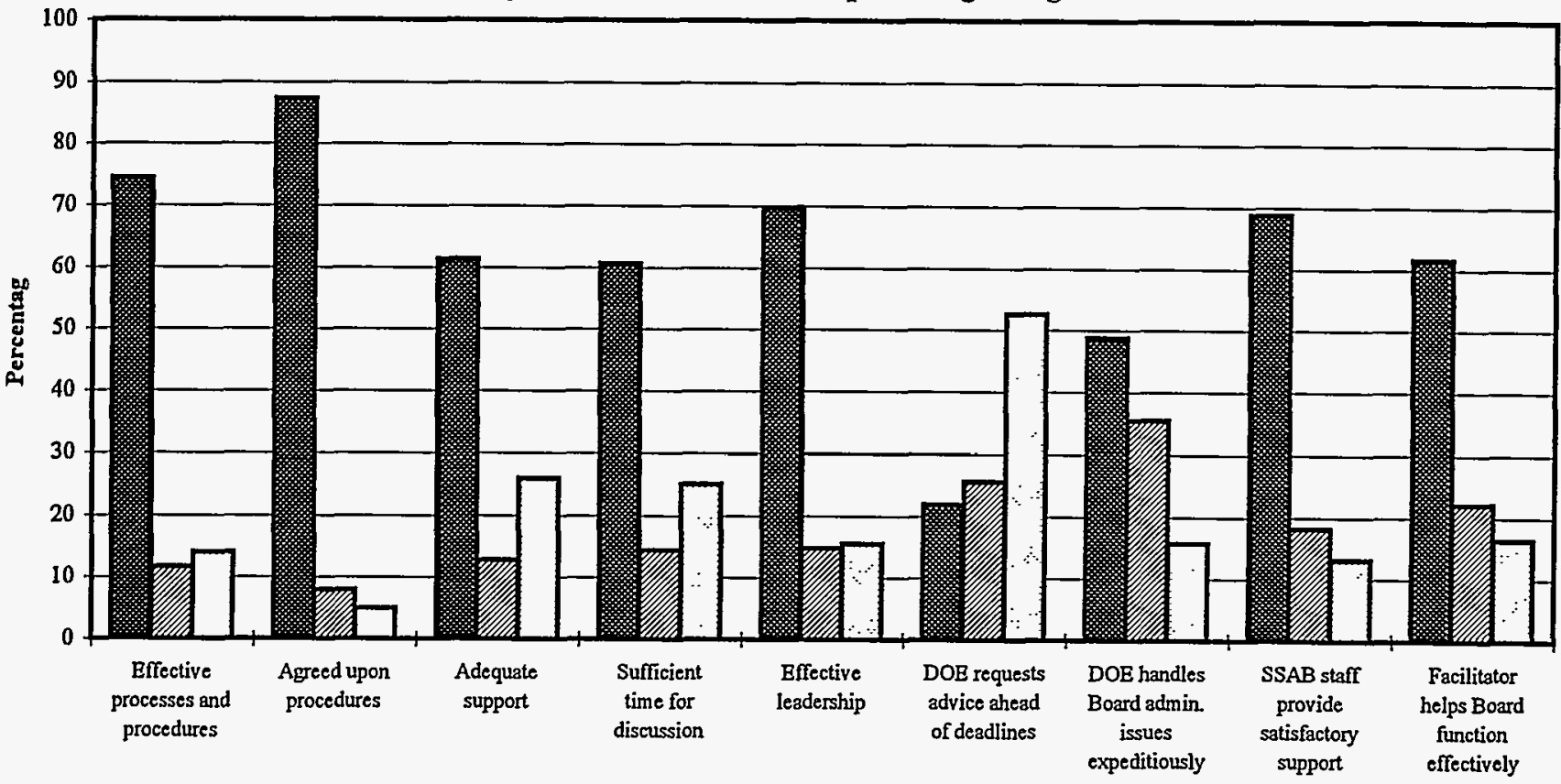

Agree (\%) Deither Agree Nor Disagree/Don't Know (\%)DDisagree (\%)

SSAB Supplementary Appendix

August 1996 
Figure 2a. Pantex

Goal 2. Facilitate interaction and exchange of information and viewpoints regarding DOE site issues

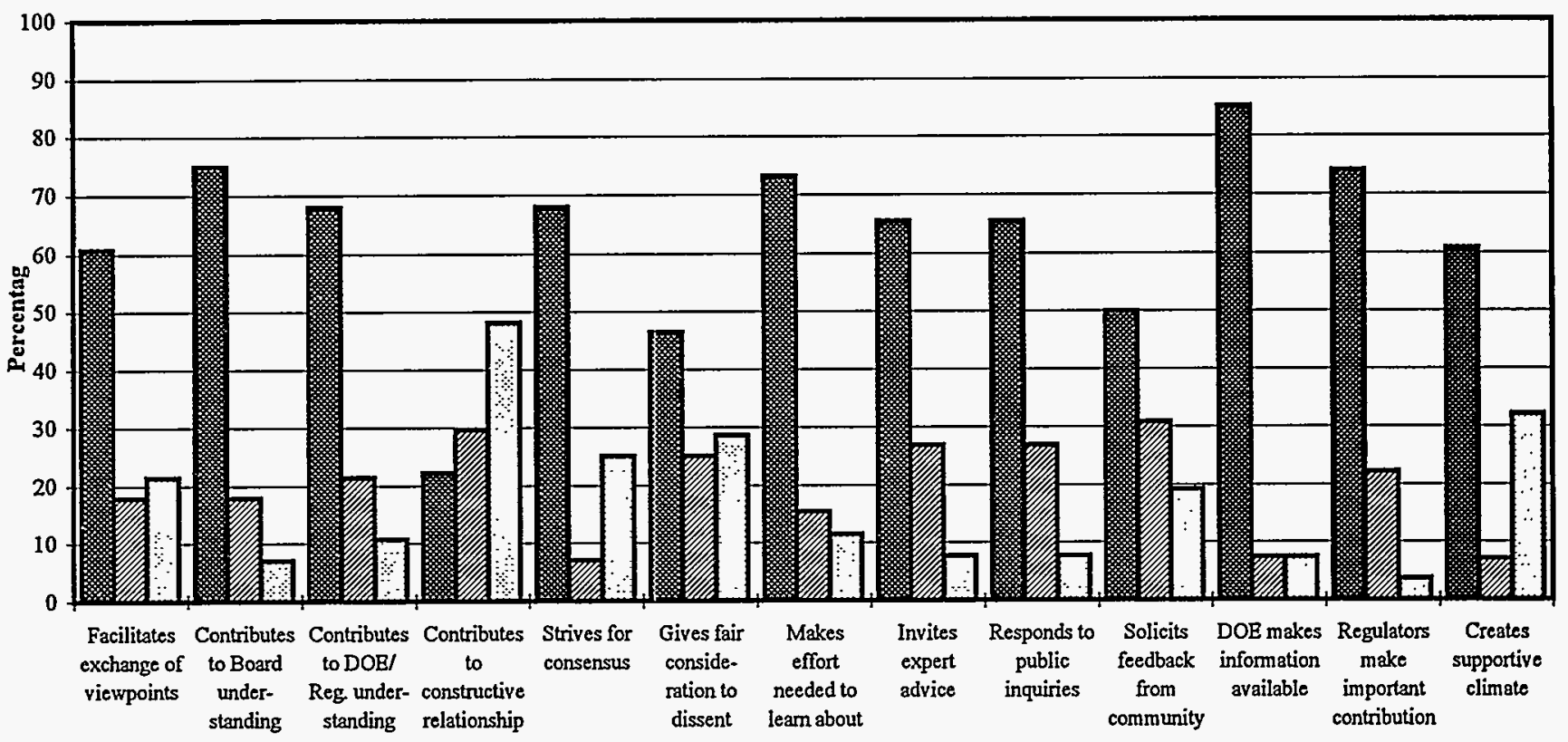

A Agree (\%) BNeither Agree Nor Disagree/Don't Know (\%)DDisagree (\%)

Figure 2b. Long Survey: Entire Sample

Goal 2. Facilitate interaction and exchange of information and viewpoints regarding DOE site issues

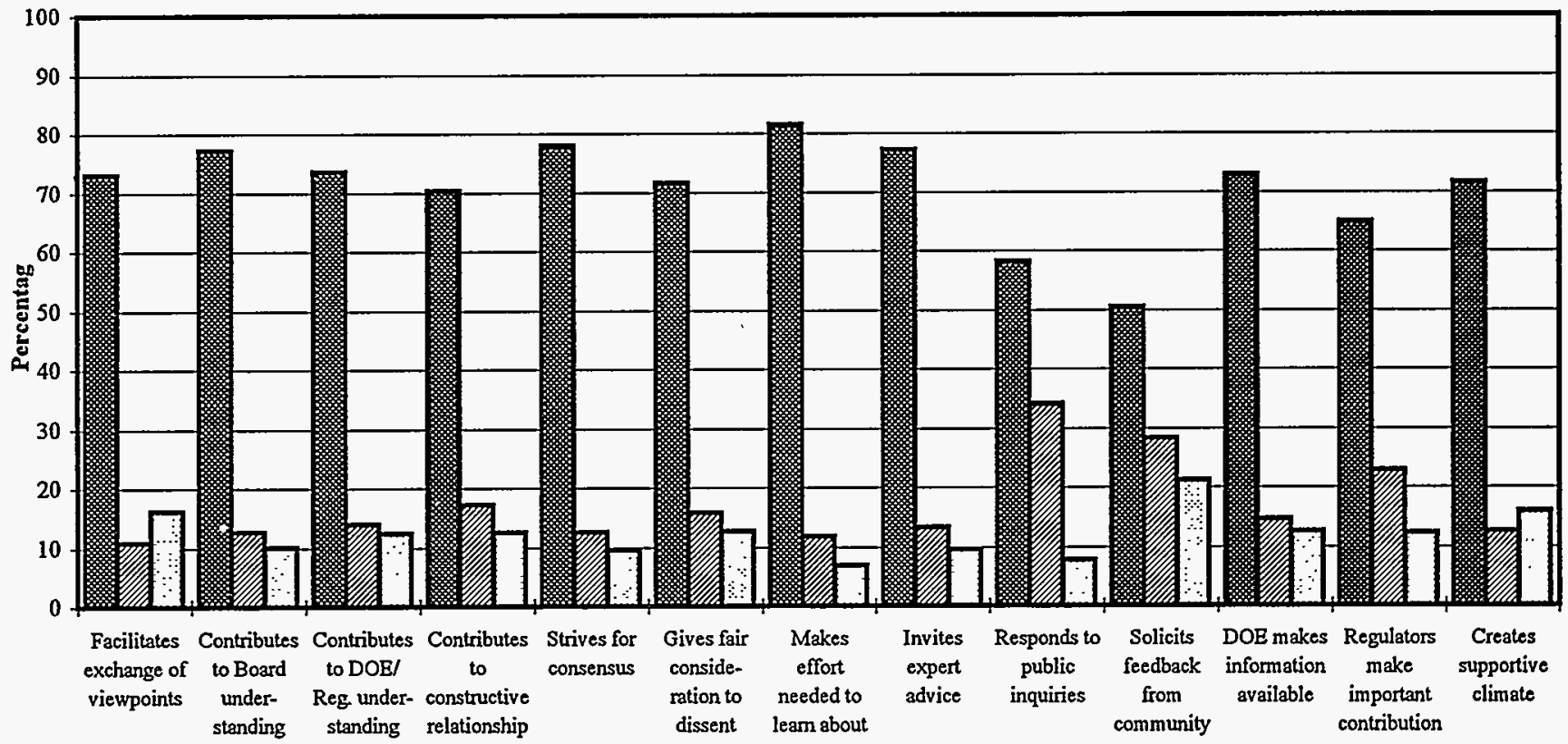

Agree (\%) DNeither Agree Nor Disagree/Don't Know (\%)DDisagree (\%)

SSAB Supplementary Appendix

August 1996 
Figure 3a. Pantex

Goal 3. Provide useful advice and/or recommendations to DOE

(and regulators, where appropriate)

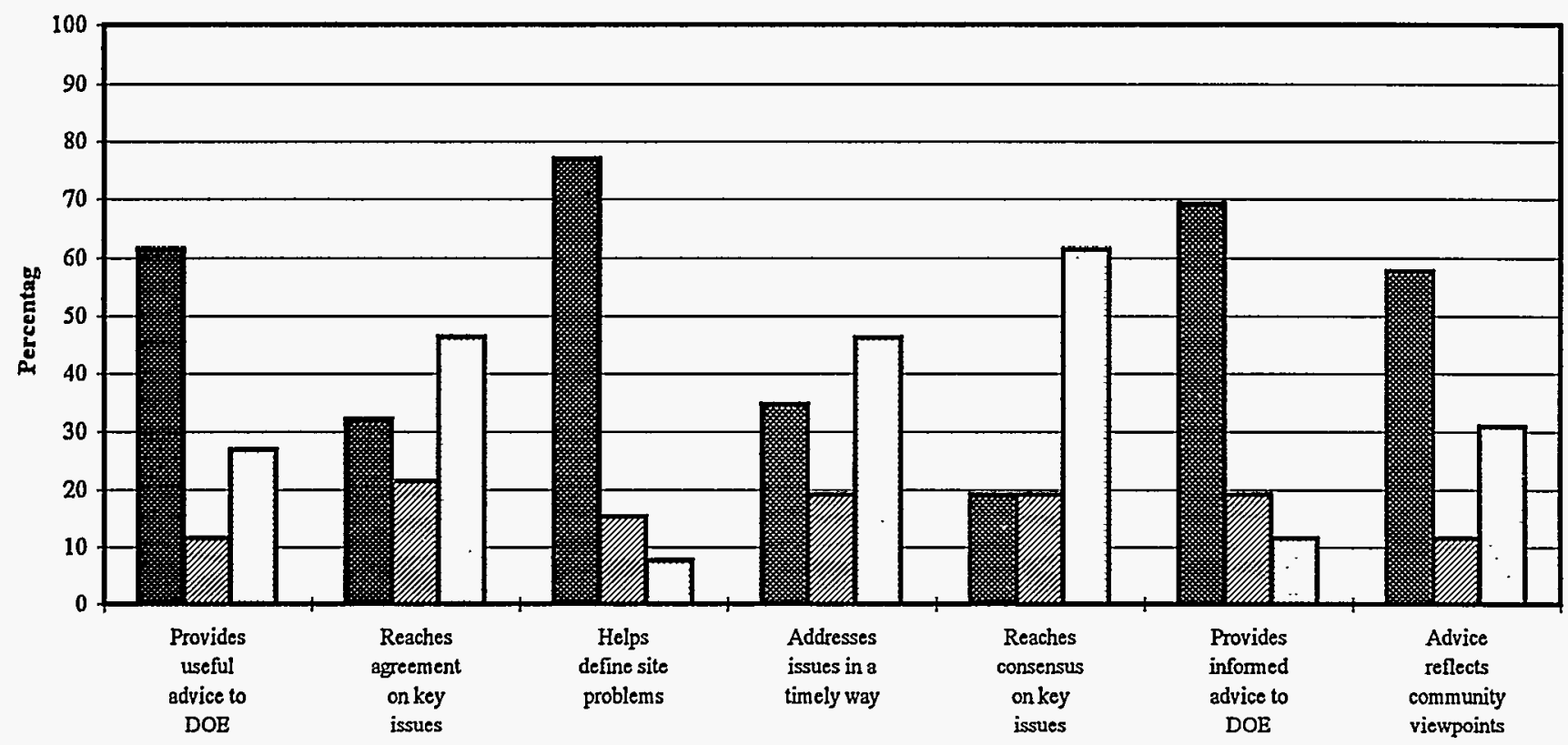

Agree (\%) Deither Agree Nor Disagree/Don't Know (\%)DDisagree (\%)

Figure 3b. Long Survey: Entire Sample

Goal 3. Provide useful advice and/or recommendations to DOE (and regulators, where appropriate)

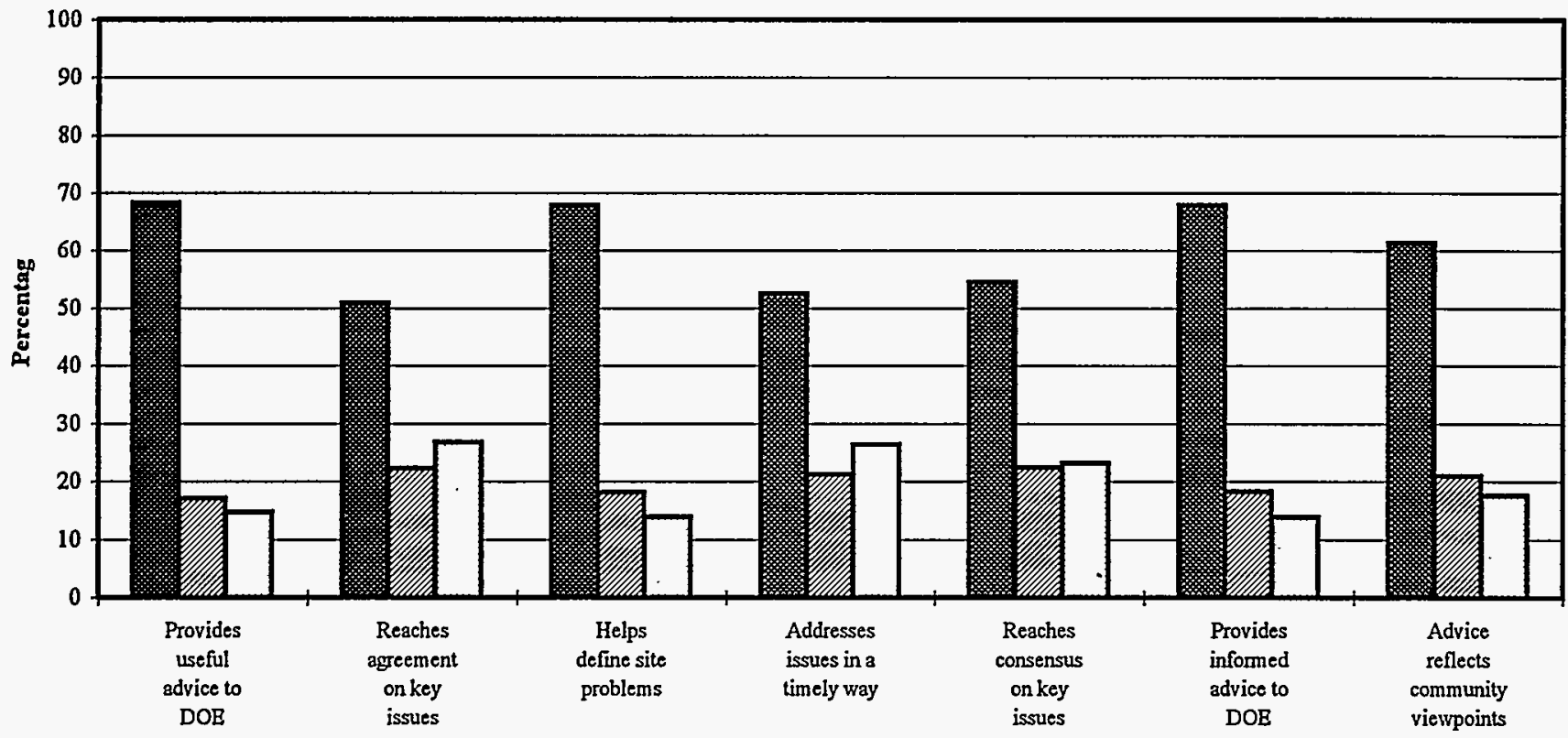

Agree (\%) Neither Agree Nor Disagree/Don't Know (\%)DDisagree (\%)

SSAB Supplementary Appendix

August 1996 
Figure 4a. Pantex

Goal 4. Improve DOE's (and where applicable, regulators') site decisions and decision making process

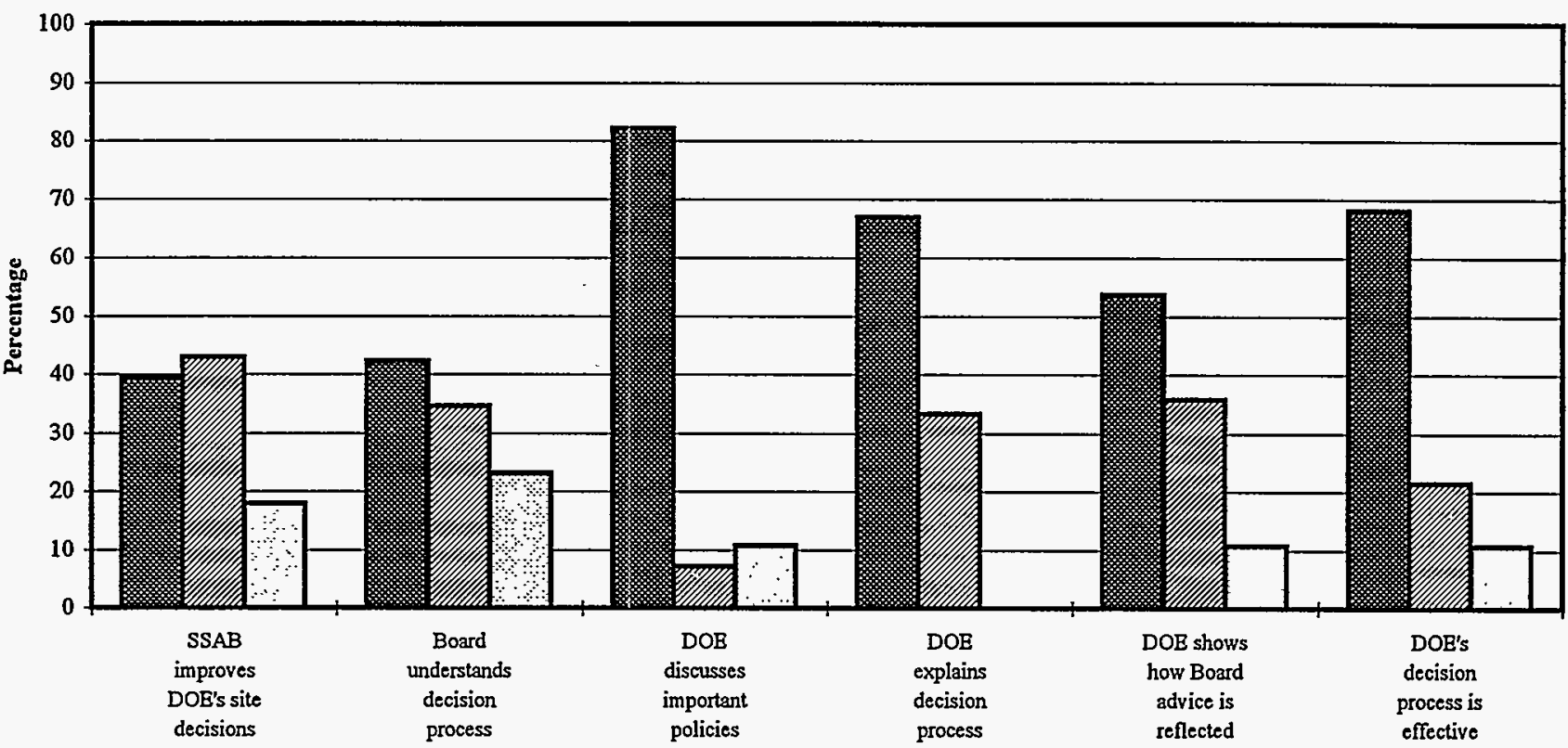

QAgree (\%) DNeither Agree Nor Disagree/Don't Know (\%) DDisagree (\%)

Figure 4b. Long Survey: Entire Sample

Goal 4. Improve DOE's (and where applicable, regulators') site decisions and decision making process

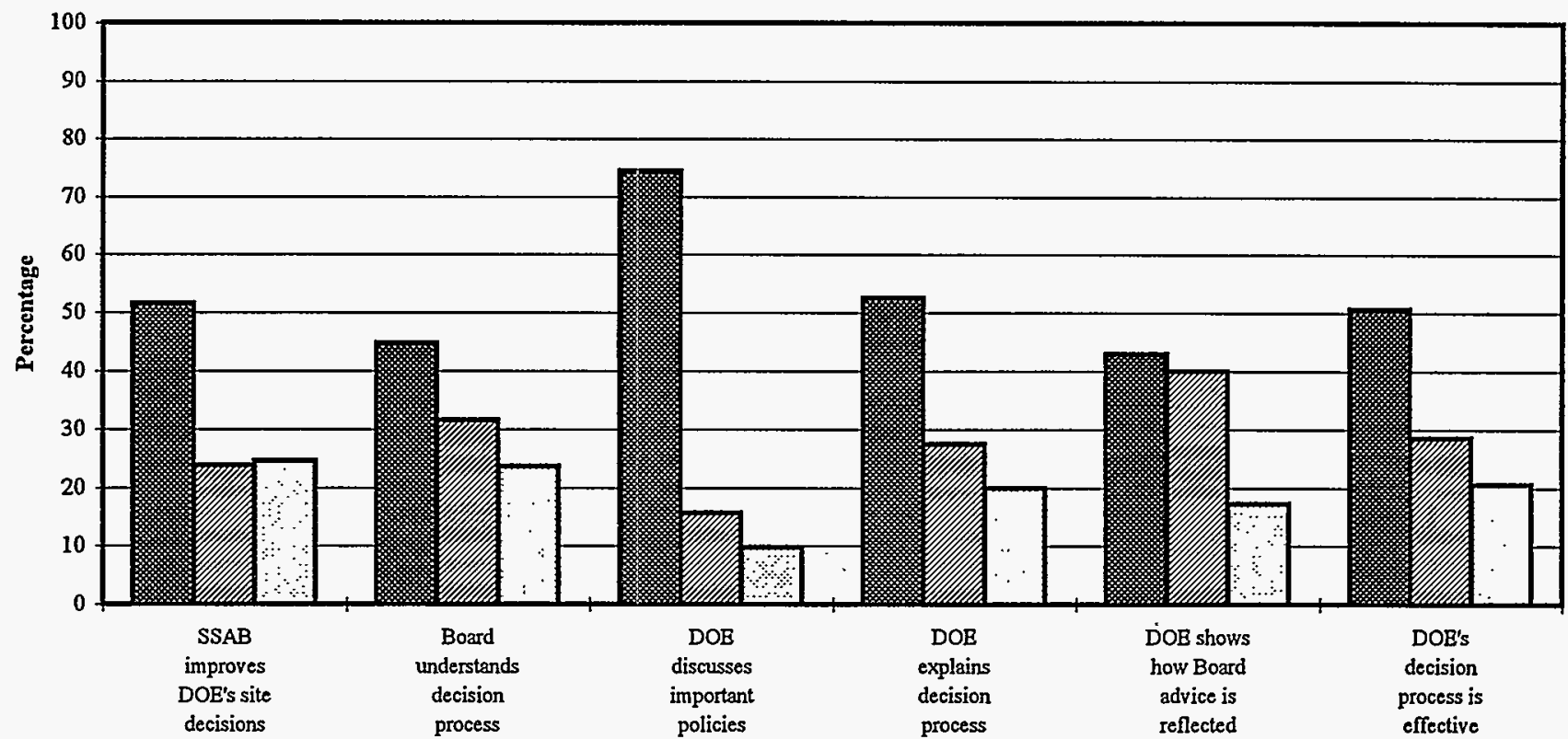

EAgree (\%) DNeither Agree Nor Disagree/Don't Know (\%)Disagree (\%)

SSAB Supplementary Appendix 
Figure 5a. Pantex

Goal 5. Lead to more acceptable actions

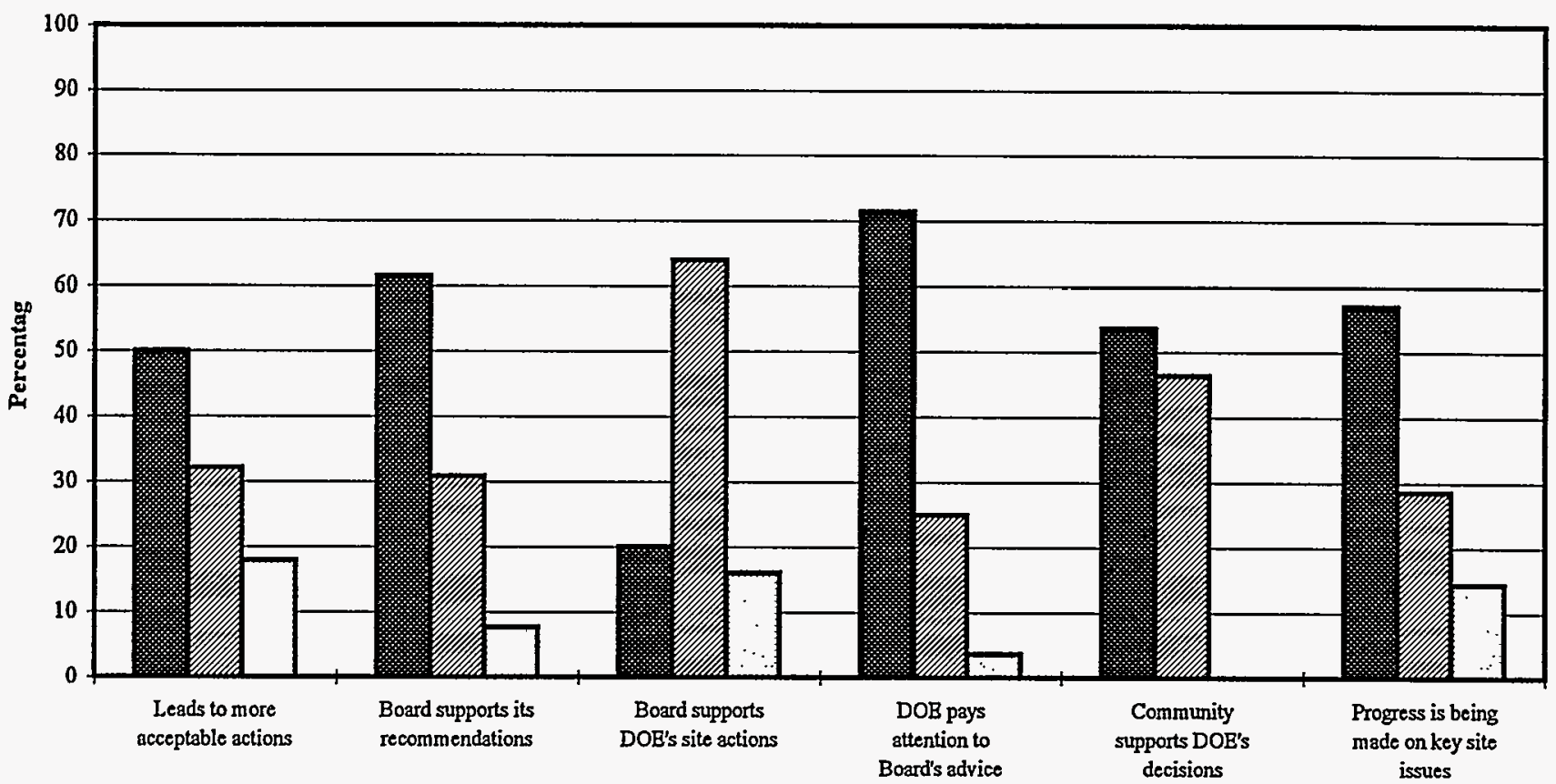

Agree (\%) DNeither Agree Nor Disagree/Don't Know (\%)DDisagree (\%)

Figure 5b. Long Survey: Entire Sample

Goal 5. Lead to more acceptable actions

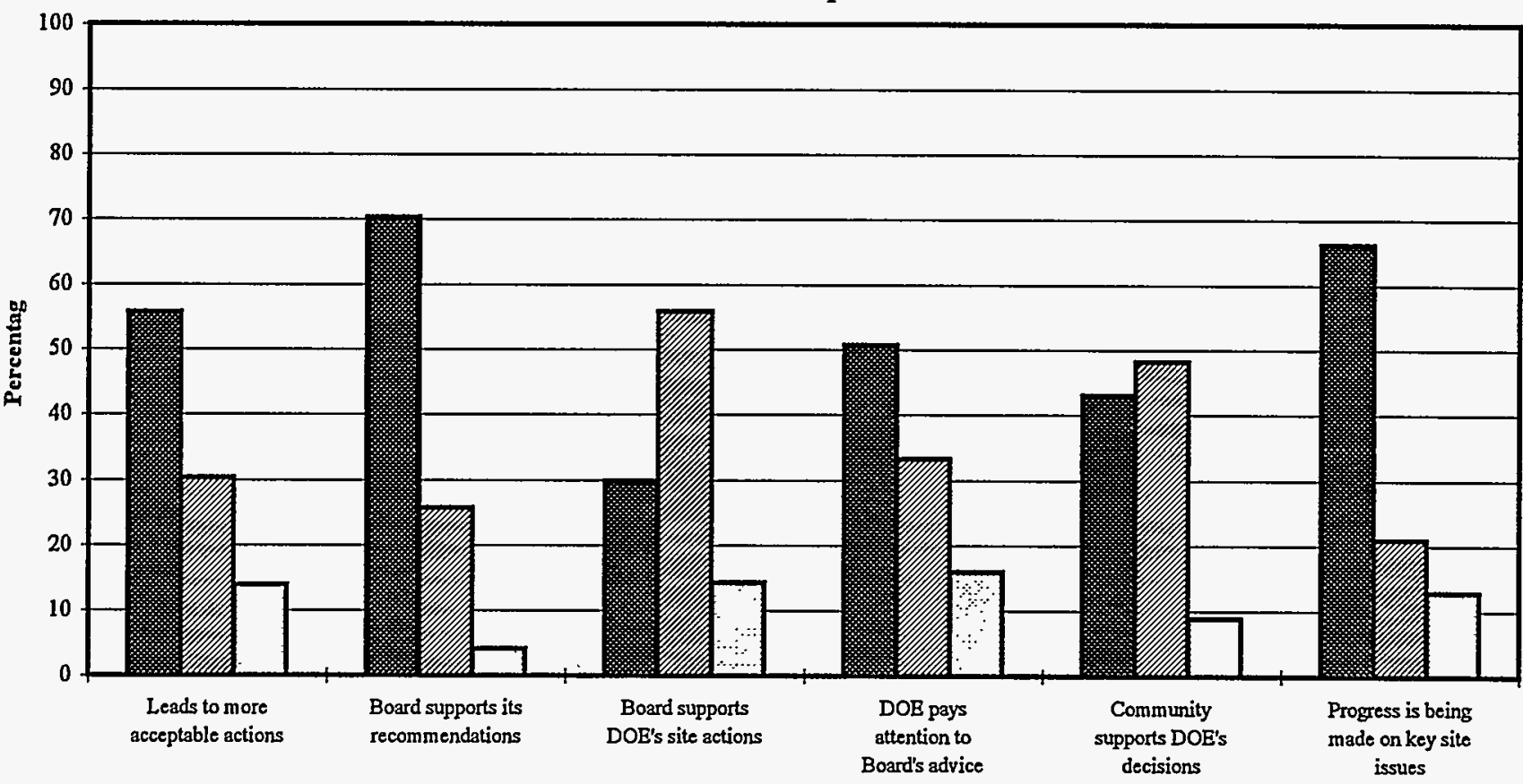

A Agree (\%) Neither Agree Nor Disagree/Don't Know (\%)DDisagree (\%)

SSAB Supplementary Appendix 
Figure 6a. Pantex

Goal 6. Contribute to trust and confidence in DOE

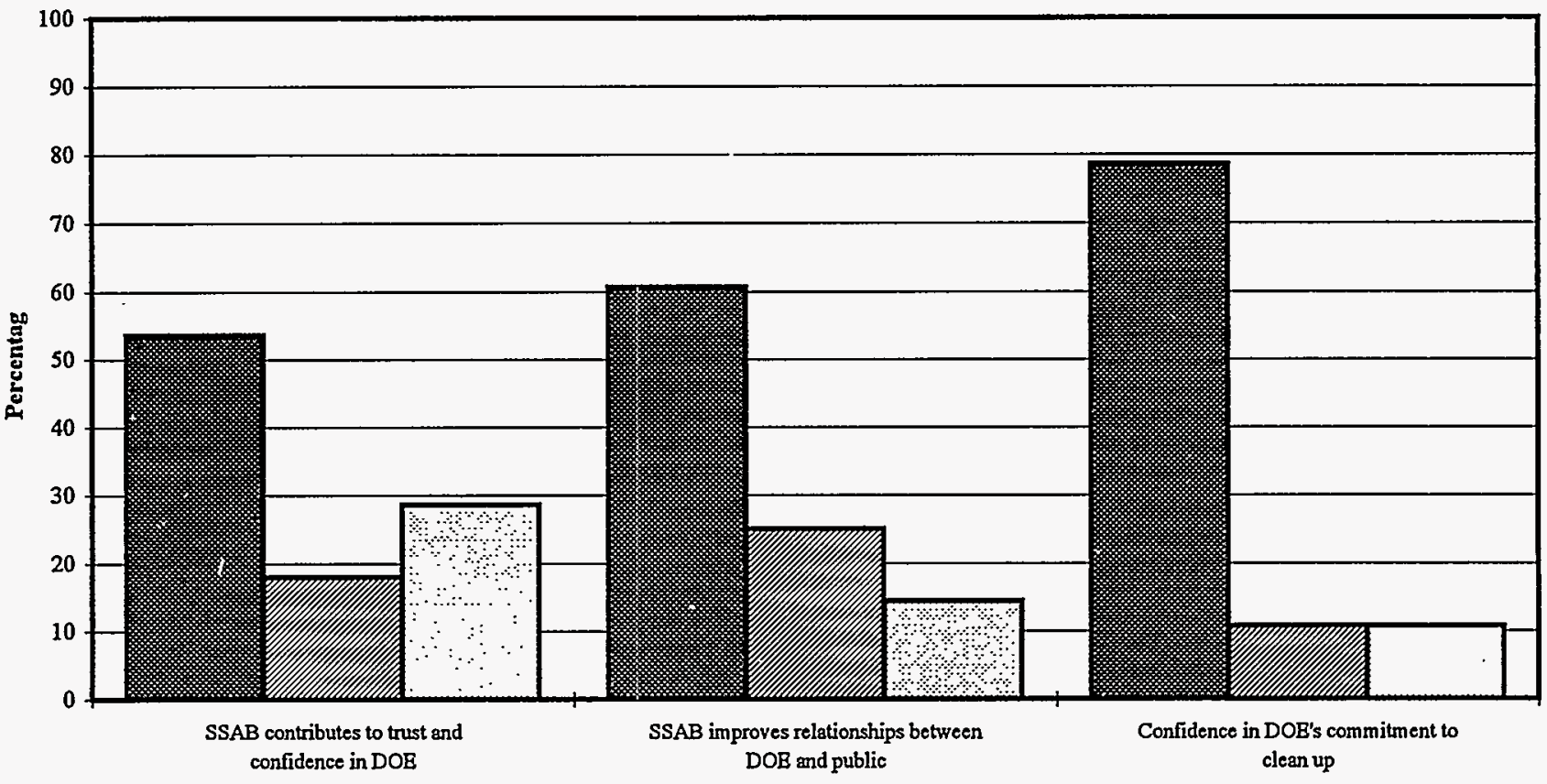

Q Agree (\%) Neither Agree Nor Disagree/Don't Know (\%) Disagree (\%)

Figure 6b. Long Survey: Entire Sample

Goal 6. Contribute to trust and confidence in DOE

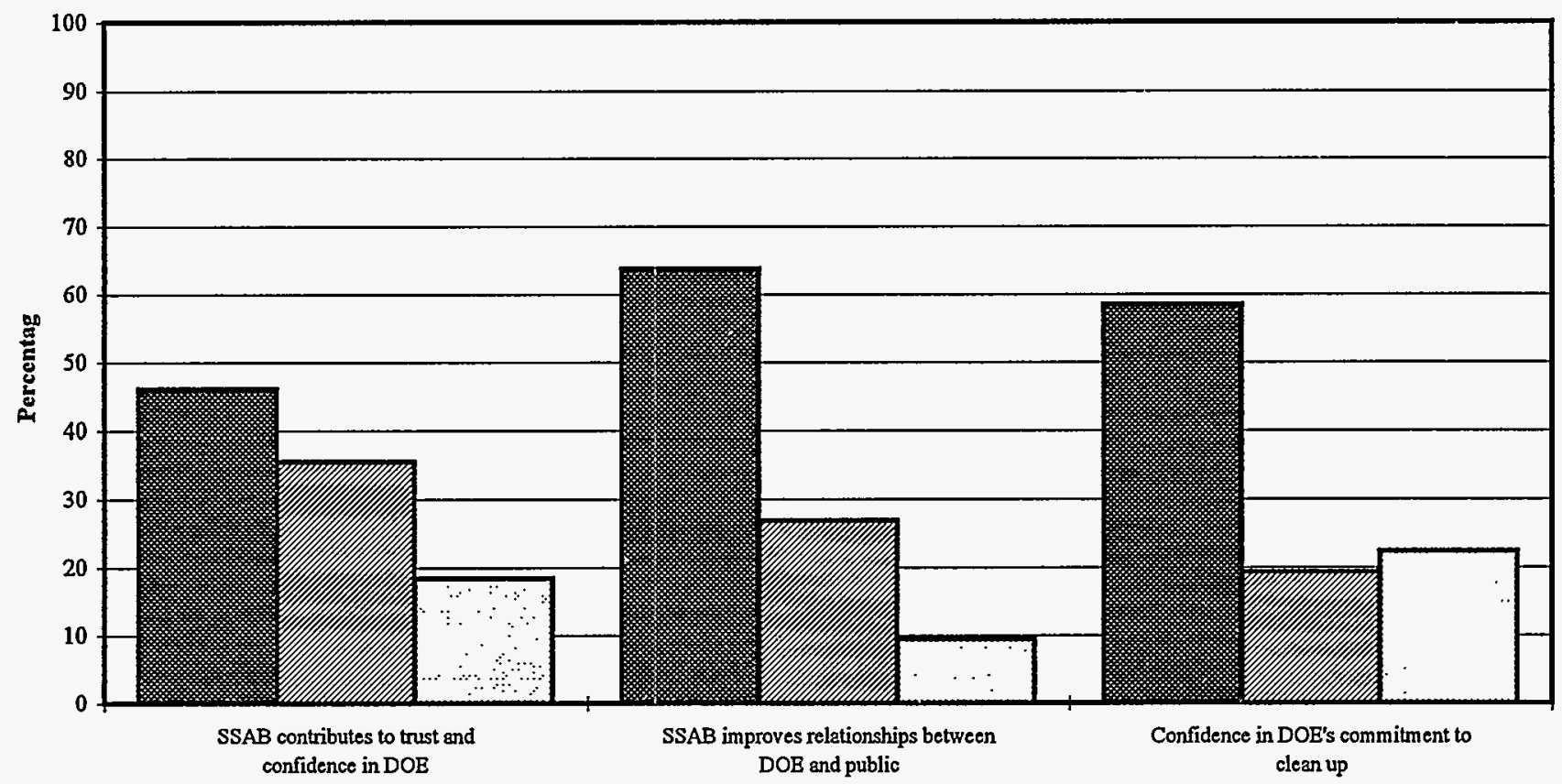

Agree (\%) Neither Agree Nor Disagree/Don't Know (\%)Disagree (\%)

SSAB Supplementary Appendix

August 1996 
Figure 7a. Pantex

Public awareness

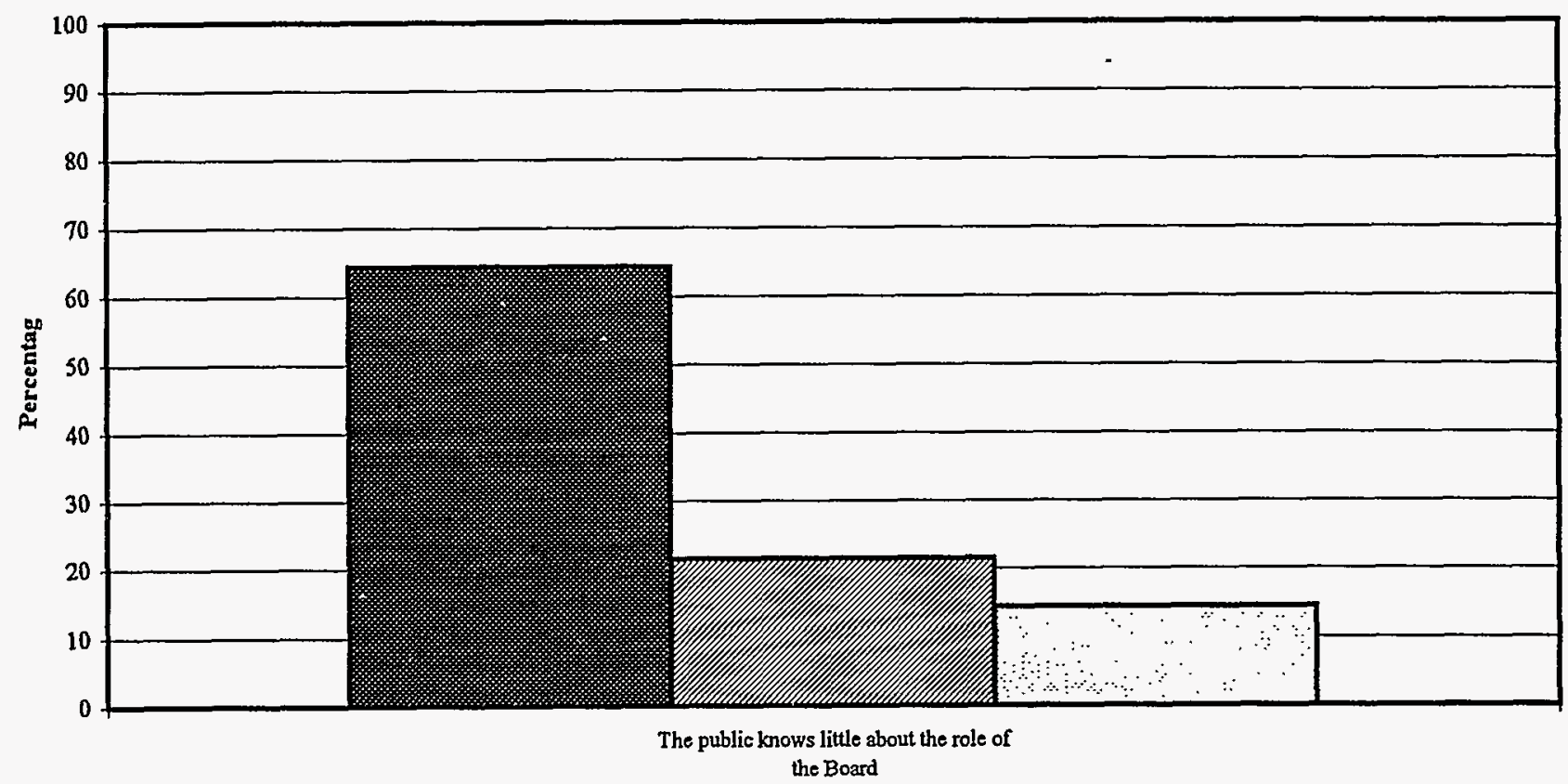

Q Agree (\%) Neither Agree Nor Disagree/Don't Know (\%)DDisagree (\%)

Figure 7b. Long Survey: Entire Sample

Public awareness

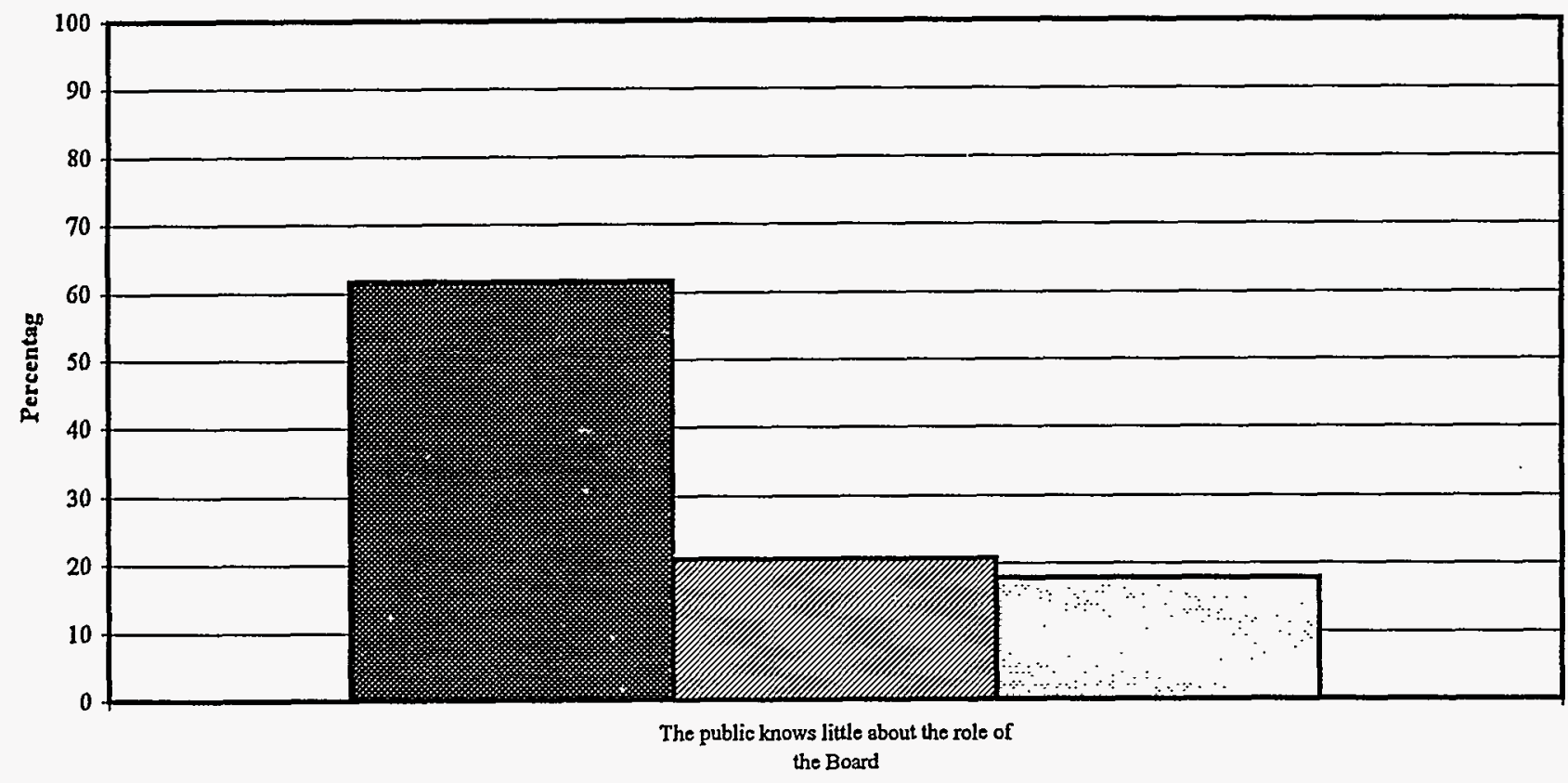

Agree (\%) DNeither Agree Nor Disagree/Don't Know (\%)DDisagree (\%)

SSAB Supplementary Appendix

August 1996 
Figure 8a. Pantex

DOE-HQ involvement with the SSAB Initiative

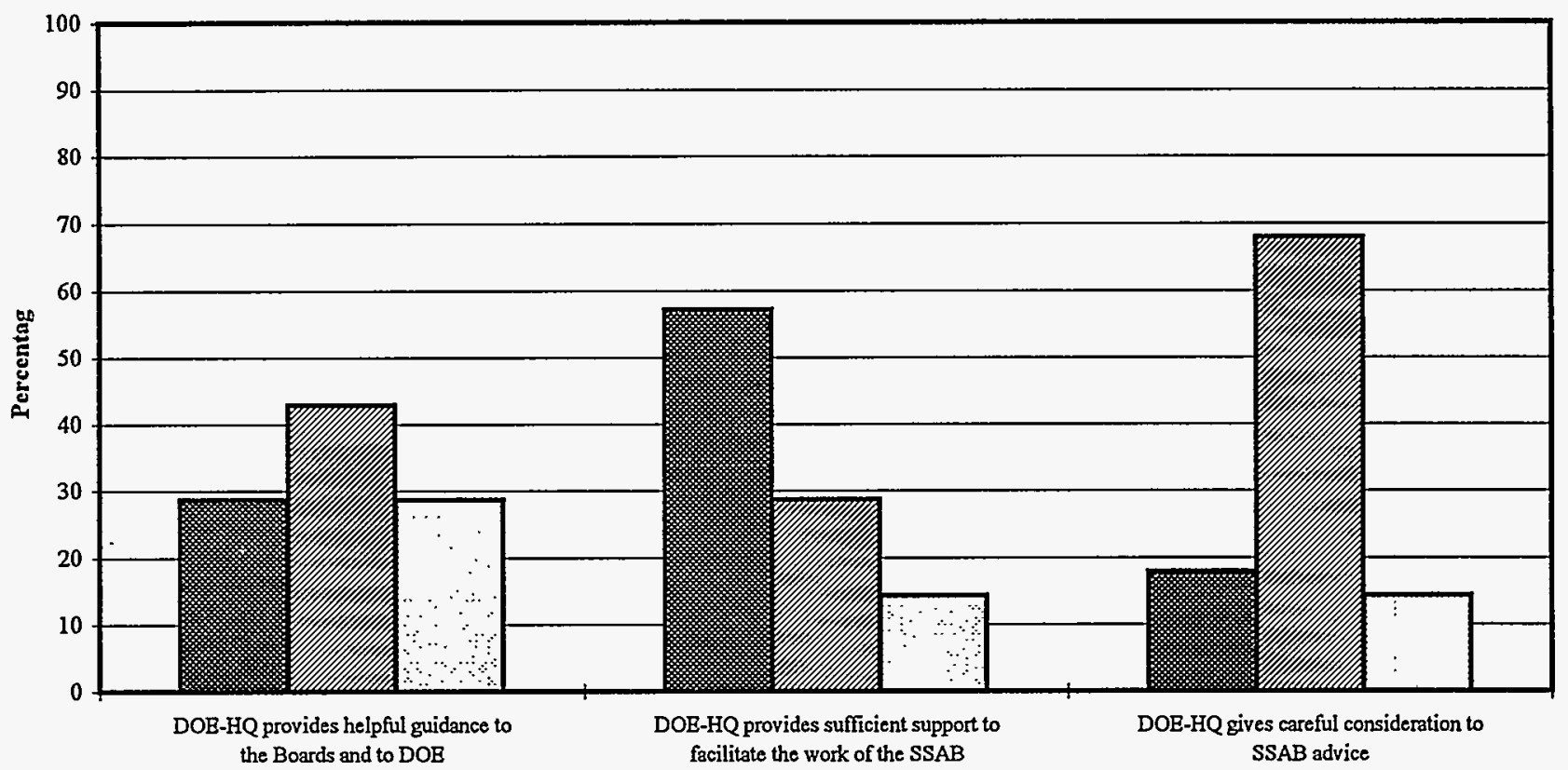

E Agree (\%) ENeither Agree Nor Disagree/Don't Know (\%)DDisagree (\%)

Figure 8b. Long Survey: Entire Sample DOE-HQ involvement with the SSAB Initiative

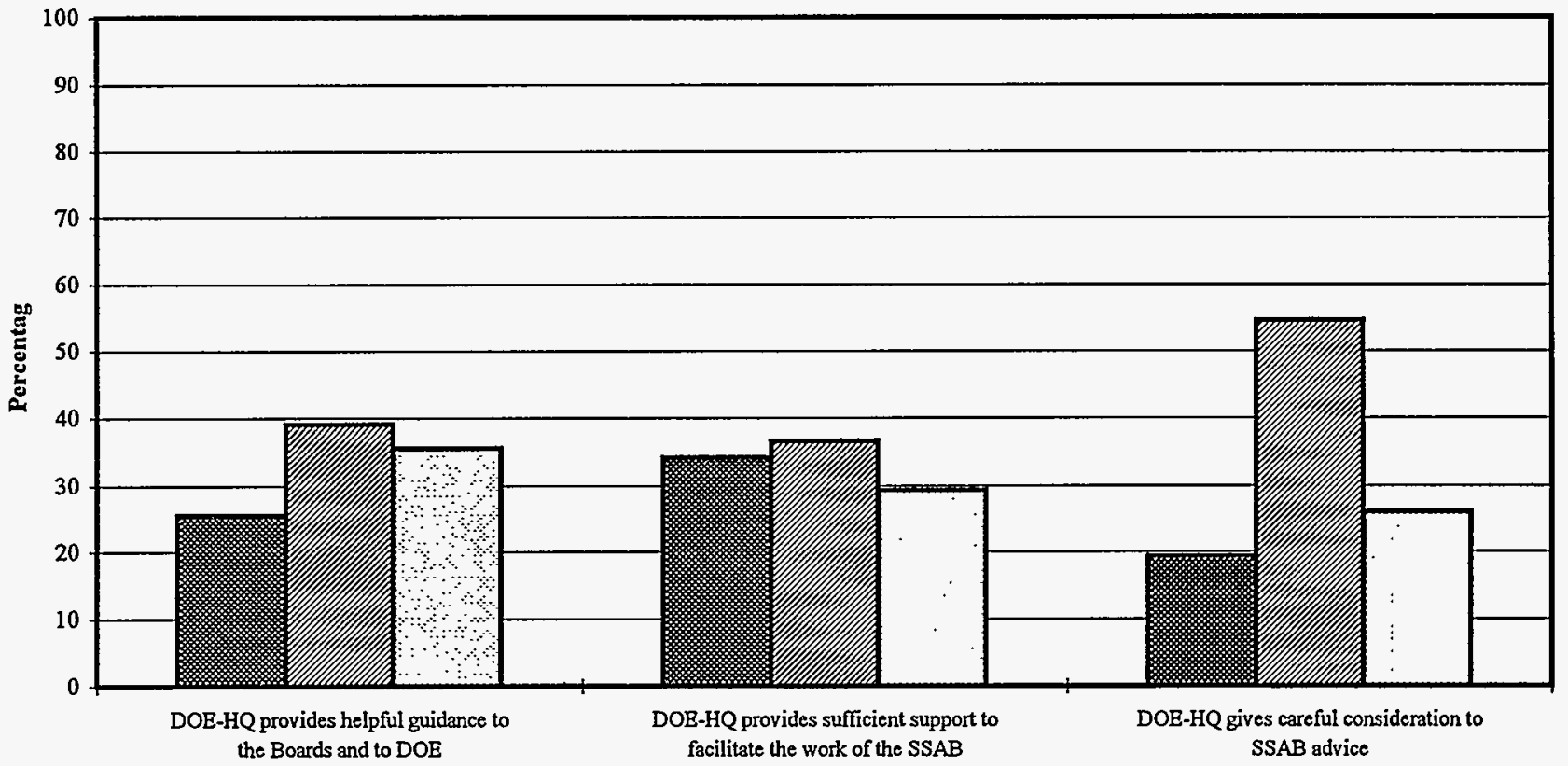

四Agree (\%) QNeither Agree Nor Disagree/Don't Know (\%)Disagree (\%)

SSAB Supplementary Appendix August 1996 
Figure 9a. Pantex

Personal experience with the SSAB Initiative

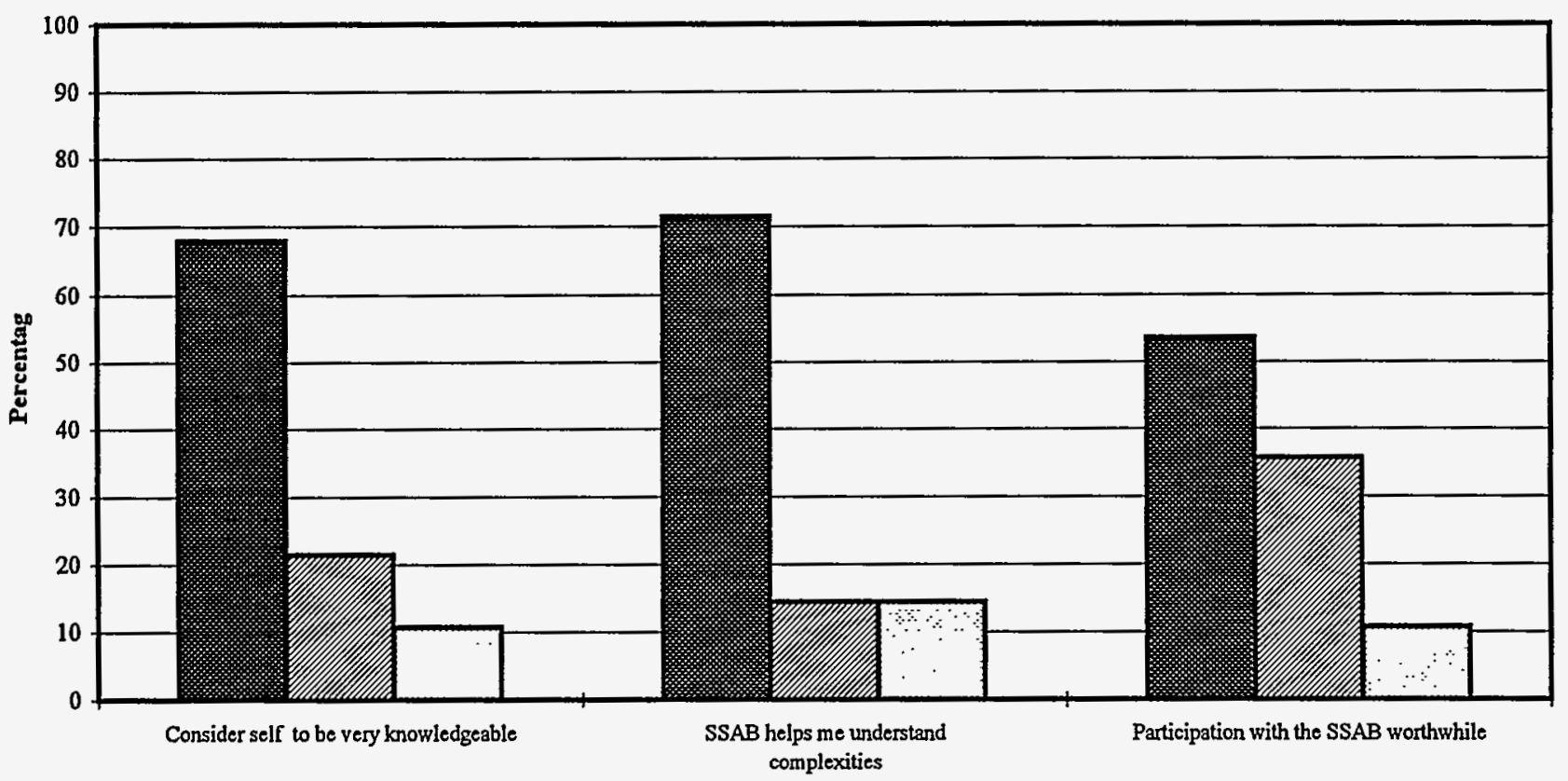

Agree (\%) Deither Agree Nor Disagree/Don't Know (\%)DDisagree (\%)

Figure 9b. Long Survey: Entire Sample

Personal experience with the SSAB Initiative

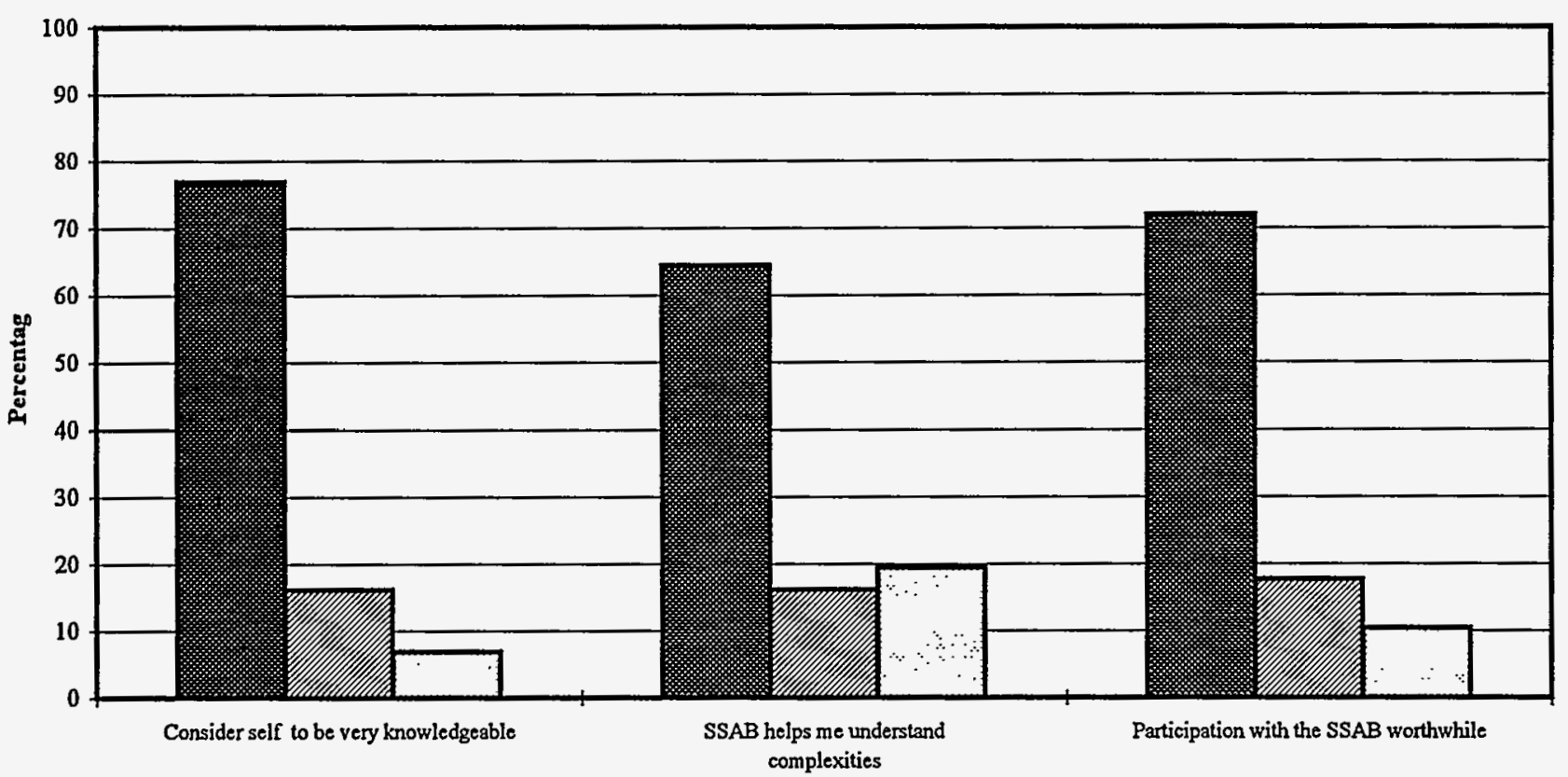

Agree (\%) Neither Agree Nor Disagree/Don't Know (\%) Disagree (\%)

SSAB Supplementary Appendix

August 1996 
Figure 10a. Pantex .

Group/Team work skills

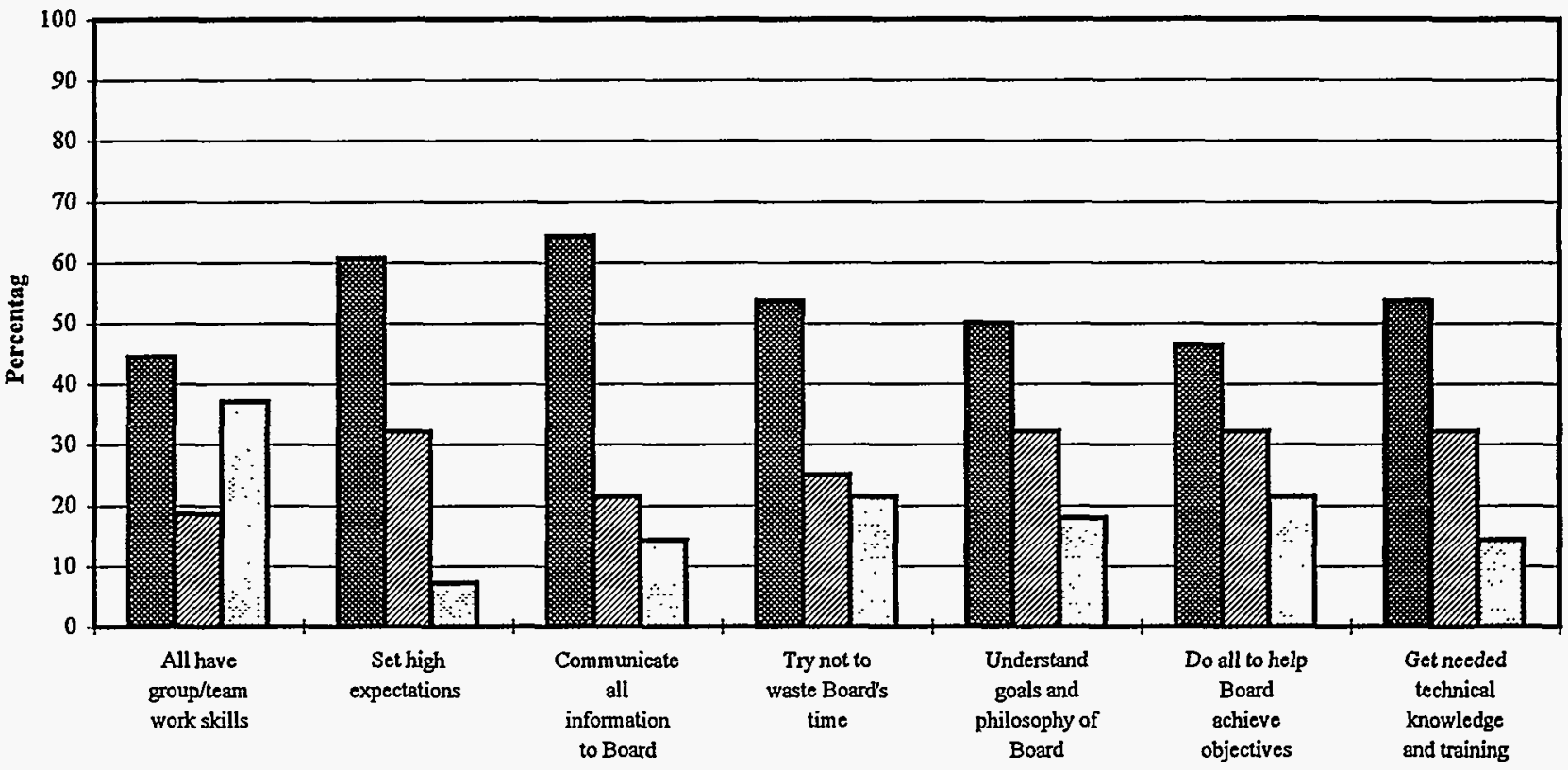

Agree (\%) DNeither Agree Nor Disagree/Don't Know (\%)DDisagree (\%)

Figure 10b. Long Survey: Entire Sample

Group/Team work skills

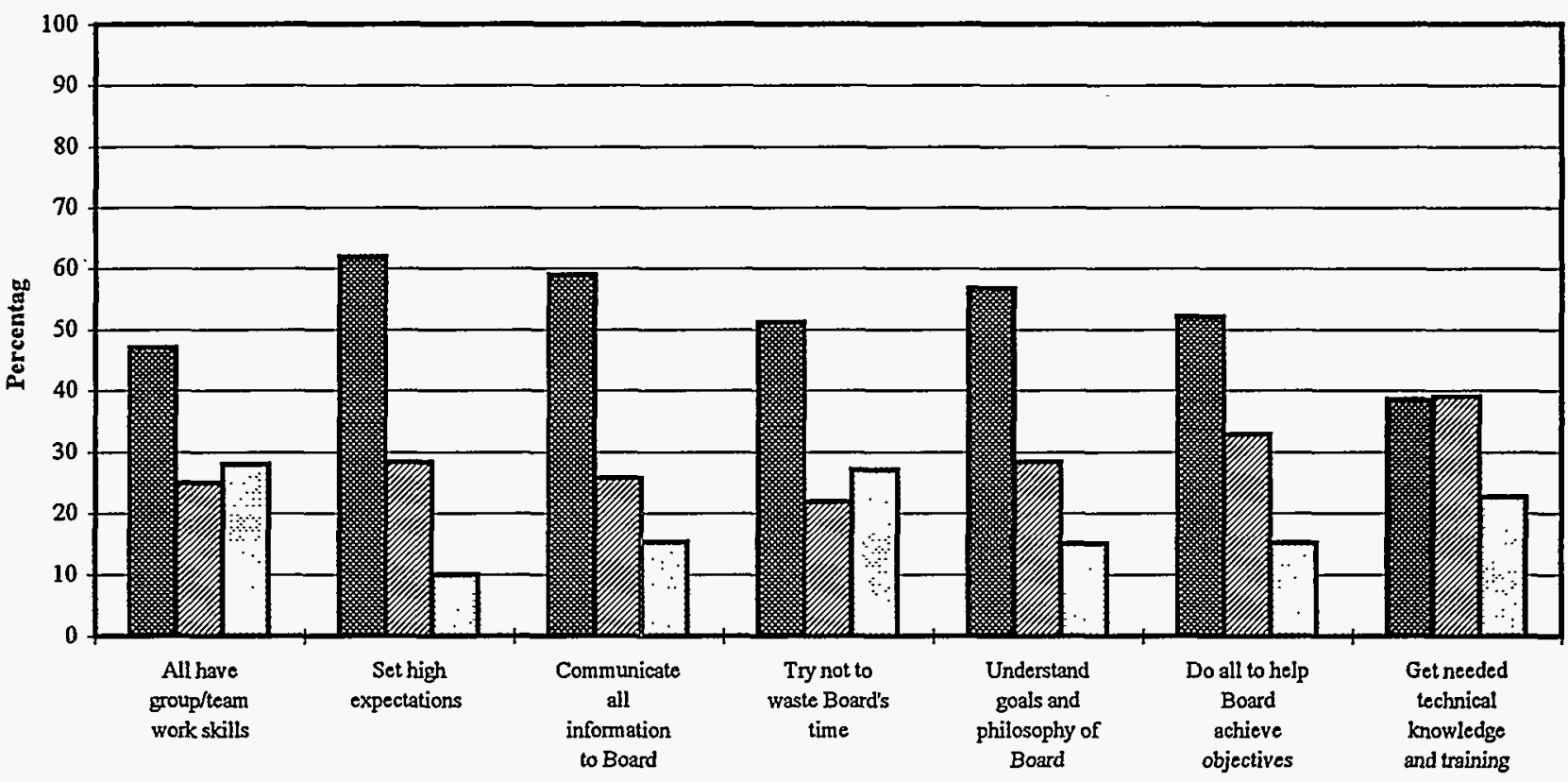

A Agree (\%) ENeither Agree Nor Disagree/Don't Know (\%)Disagree (\%)

SSAB Supplementary Appendix

August 1996 
Figure 11a. Pantex

Working relations

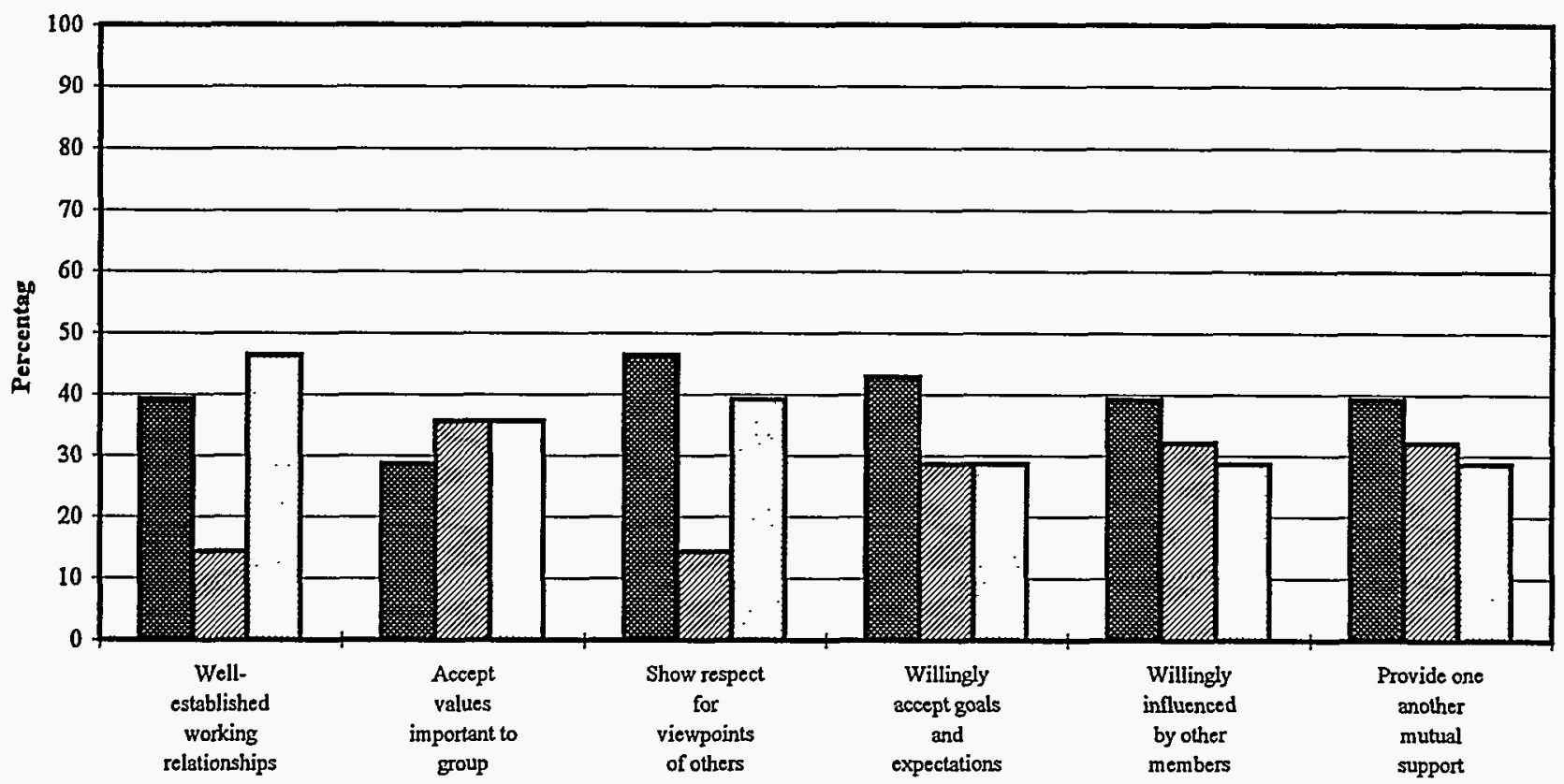

A Agree (\%) ⿴囗大 Neither Agree Nor Disagree/Don't Know (\%) DDisagree (\%)

Figure 11b. Long Survey: Entire Sample

Working relations

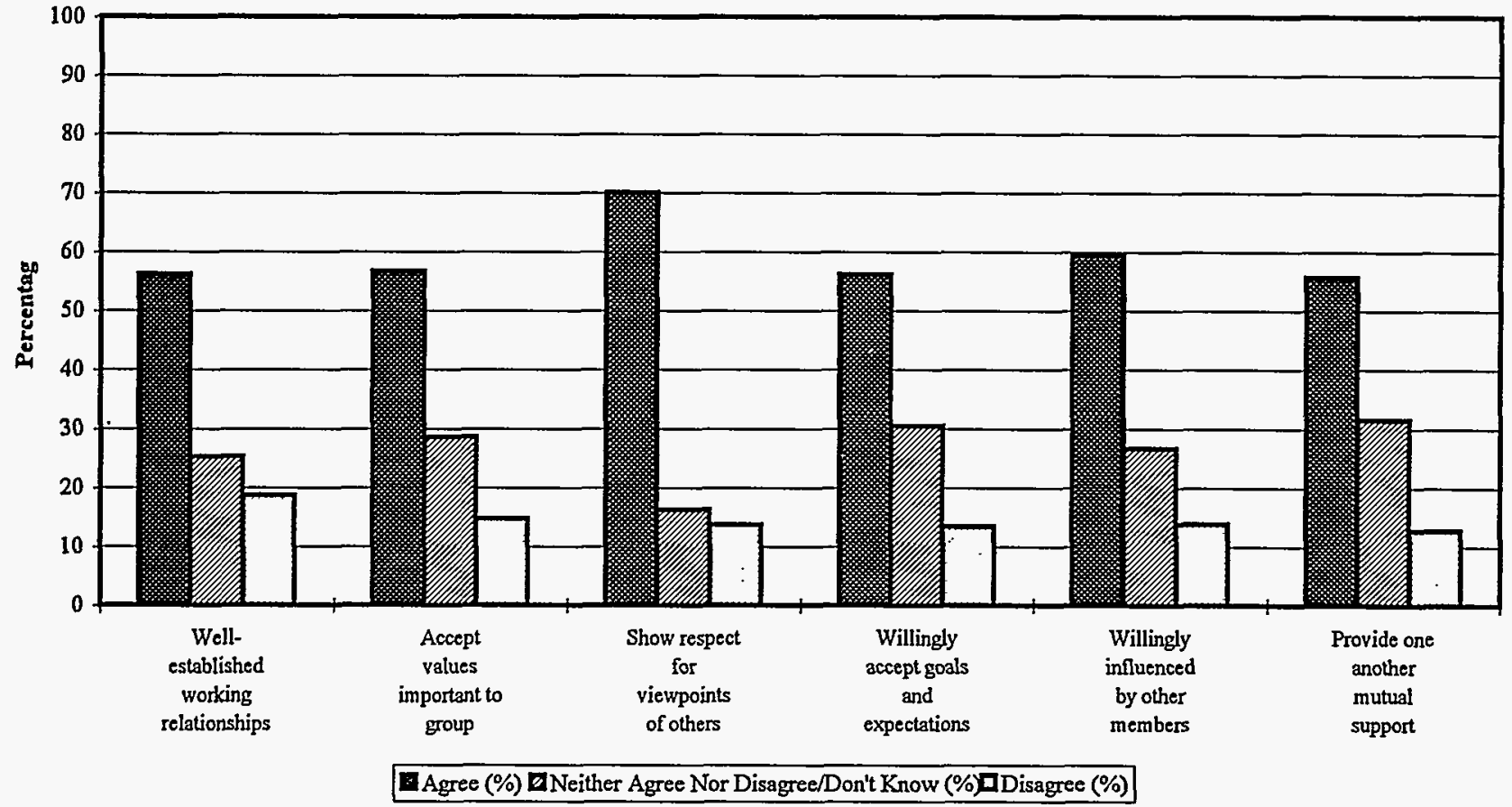

SSAB Supplementary Appendix

August 1996 
Figure 12a. Pantex

Member affect

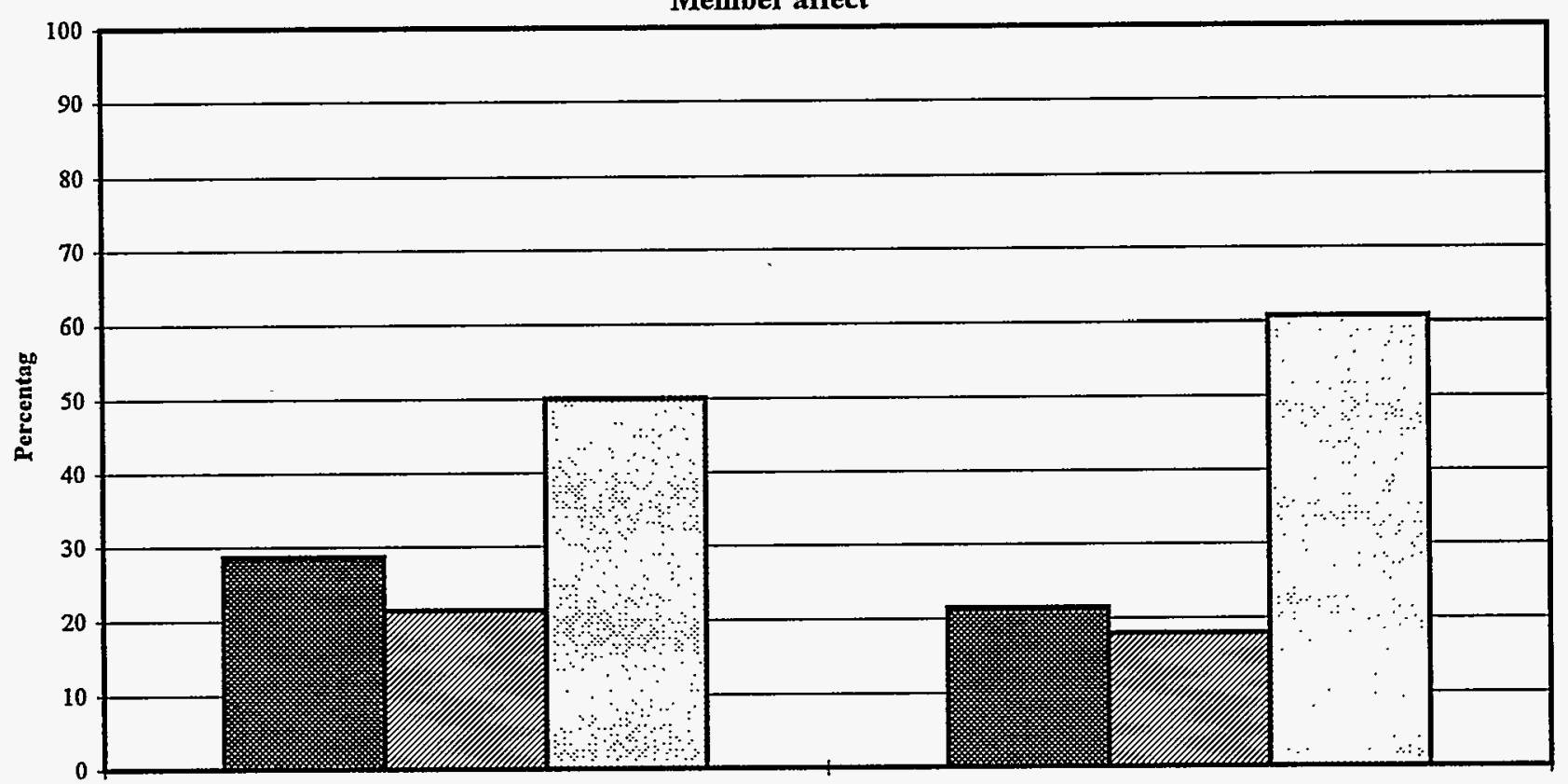

Like each other

High trust and confidence in each other

PAgree (\%) DNeither Agree Nor Disagree/Don't Know (\%)DDisagree (\%)

Figure 12b. Long Survey: Entire Sample Member affect

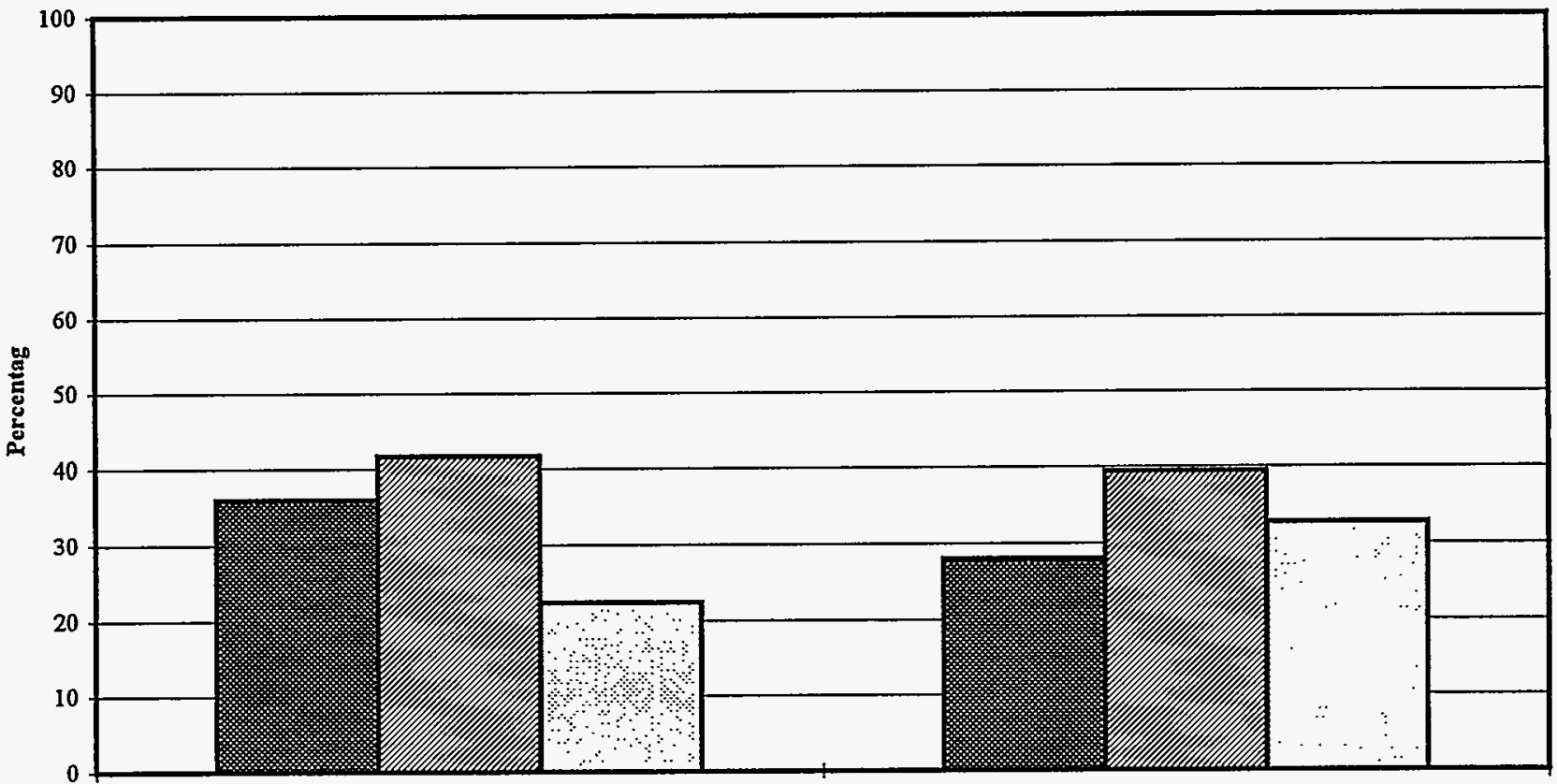

Like each other

High trust and confidence in each other

Agree (\%) Weither Agree Nor Disagree/Don't Know (\%)Disagree (\%)

SSAB Supplementary Appendix

August 1996 
Figure 13a. Pantex

Summary: Responses to open-ended questions

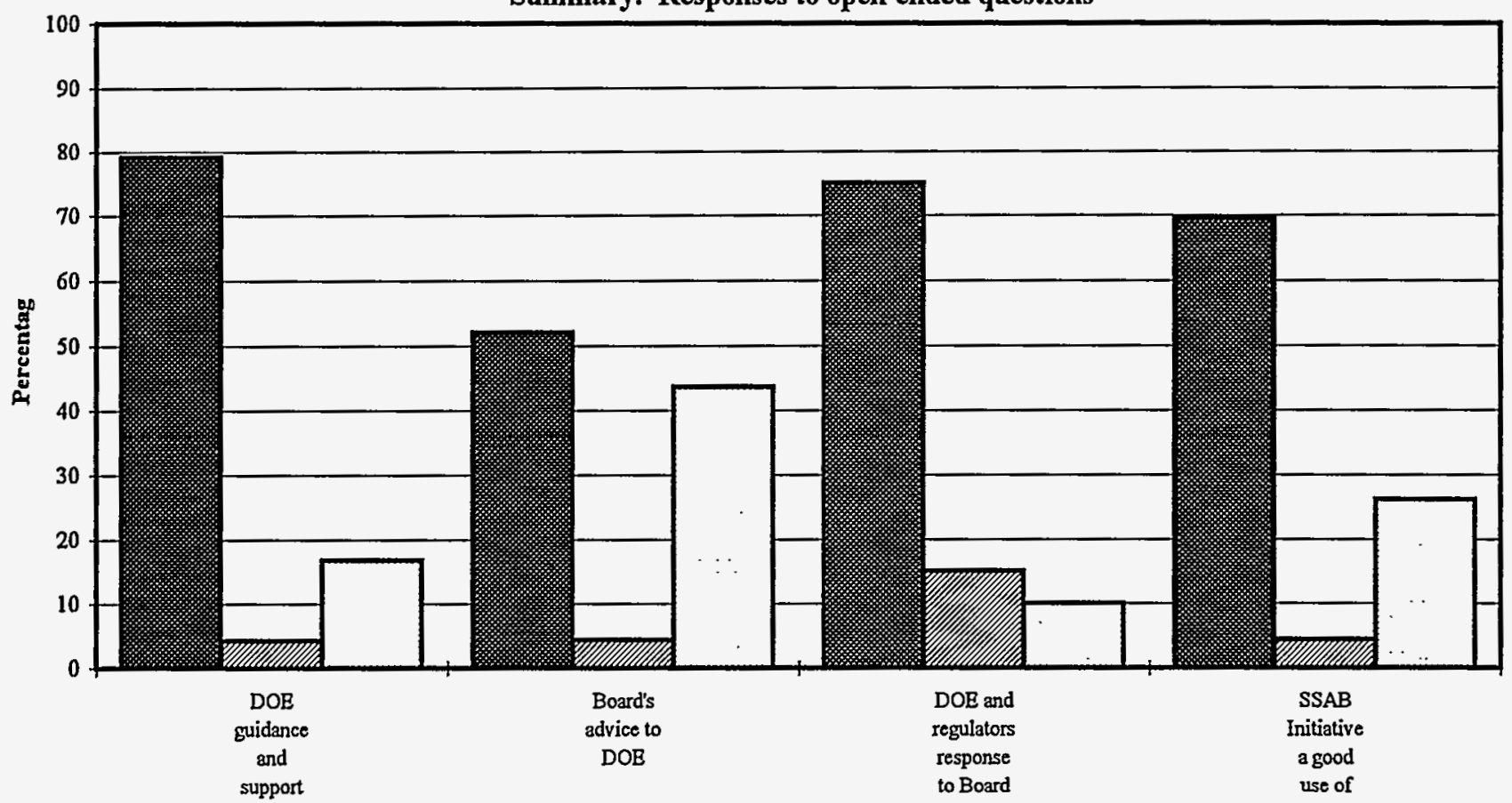

Y Yes, Good (\%) ENeutral, Conditional, No Opinion (\%) $\square$ No, Not Good (\%)

Figure 13b. Long Survey: Entire Sample

Summary: Responses to open-ended questions

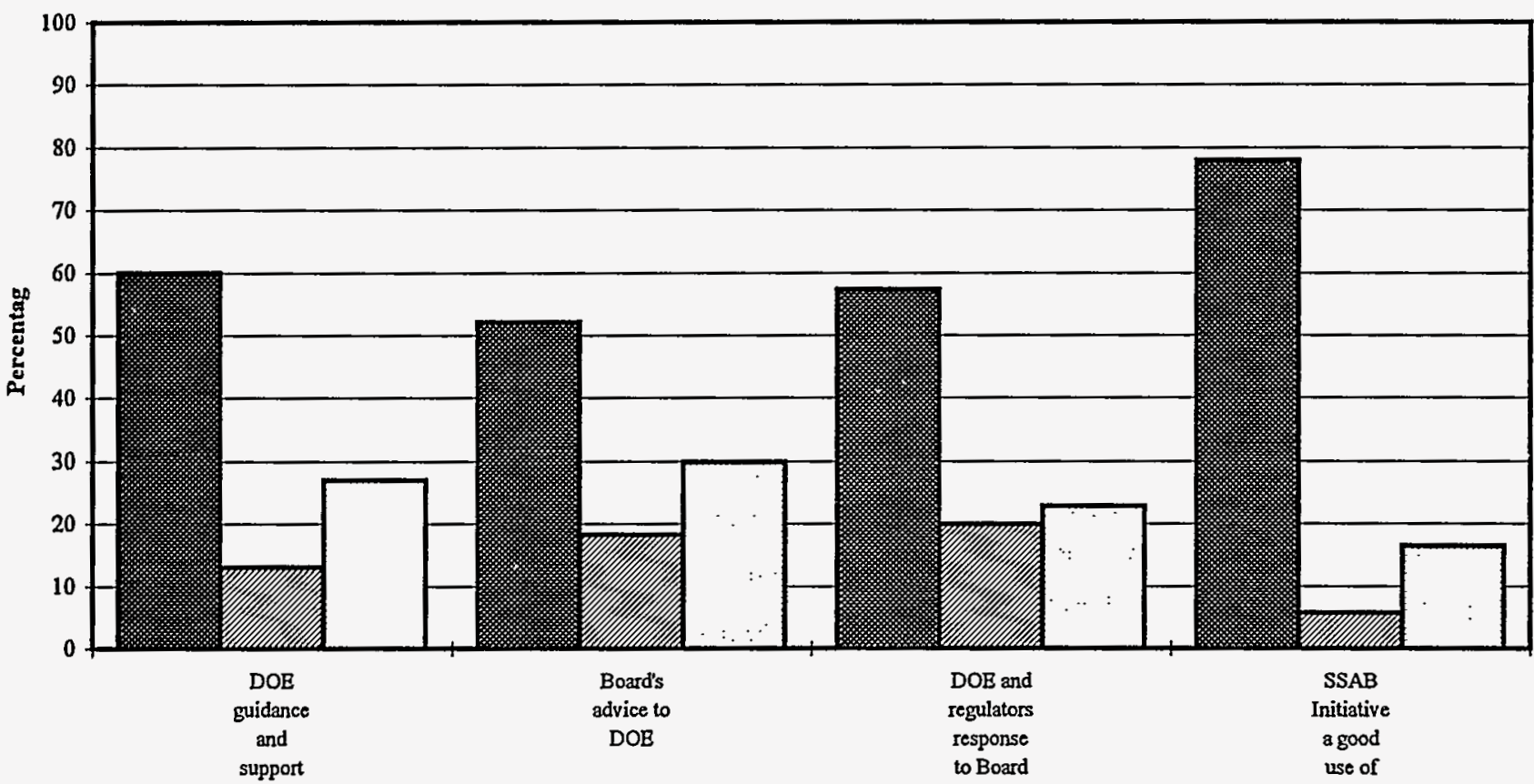

QYes, Good (\%) ENeutral, Conditional, No Opinion (\%) DNo, Not Good (\%)

SSAB Supplementary Appendix

August 1996 
Figure 14a. Pantex

Summary: Combined responses for SSAB Initiative goals (Means)

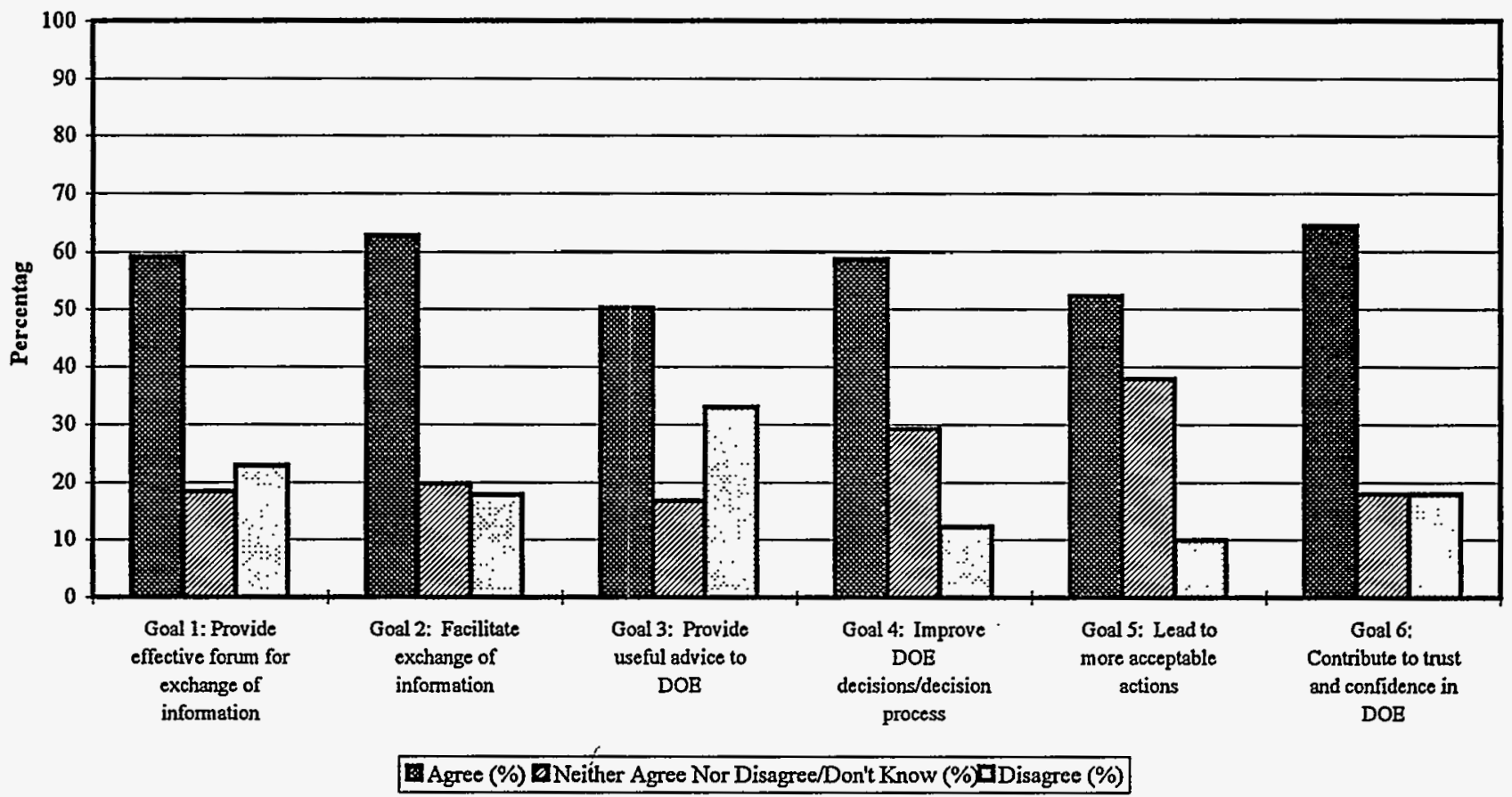

Figure 14b. Long Survey: Entire Sample

Summary: Combined responses for SSAB Initiative goals (Means)

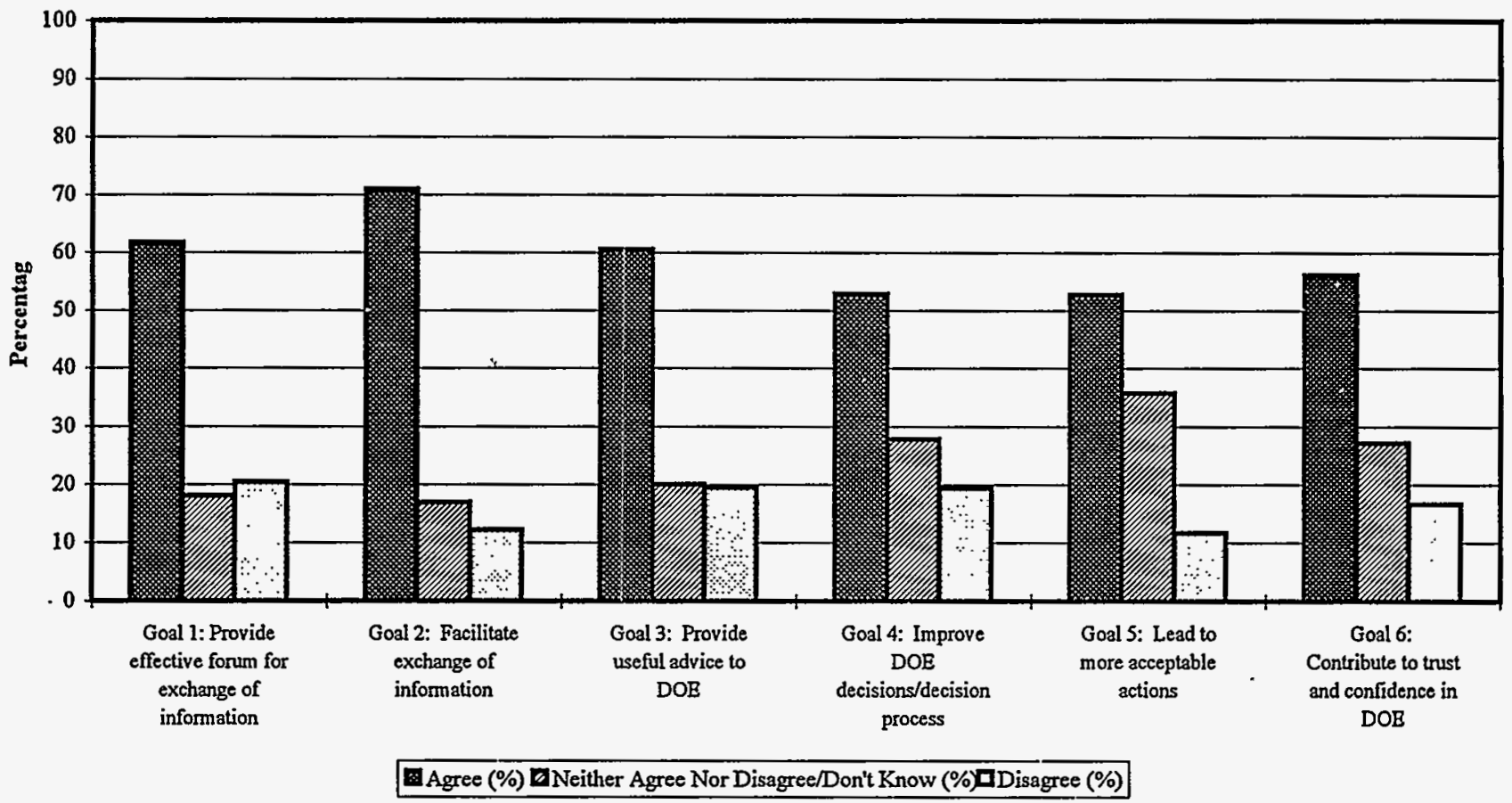

SSAB Supplementary Appendix

August 1996 
Figure 15a. Pantex

Summary: Combined responses on DOE-HQ involvement,

Personal experience, Group/Team work skills,

Working relations, Member affect (Means)

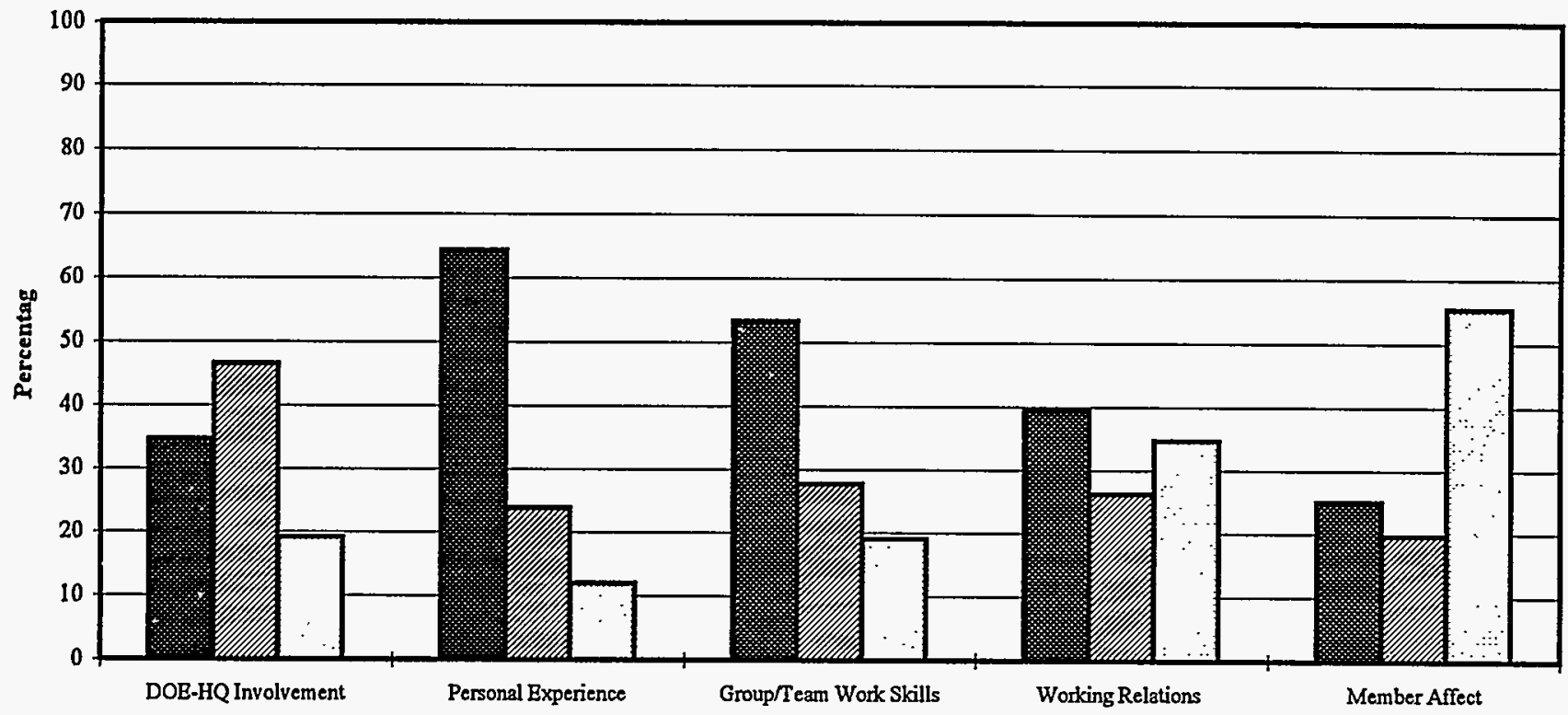

DAgree (\%) W Neither Agree Nor Disagree/Don't Know (\%)DDisagree (\%)

Figure 15b. Long Survey: Entire Sample

Summary: Combined responses on DOE-HQ involvement,

Personal experience, Group/Team work skills,

Working relations, Member affect (Means)

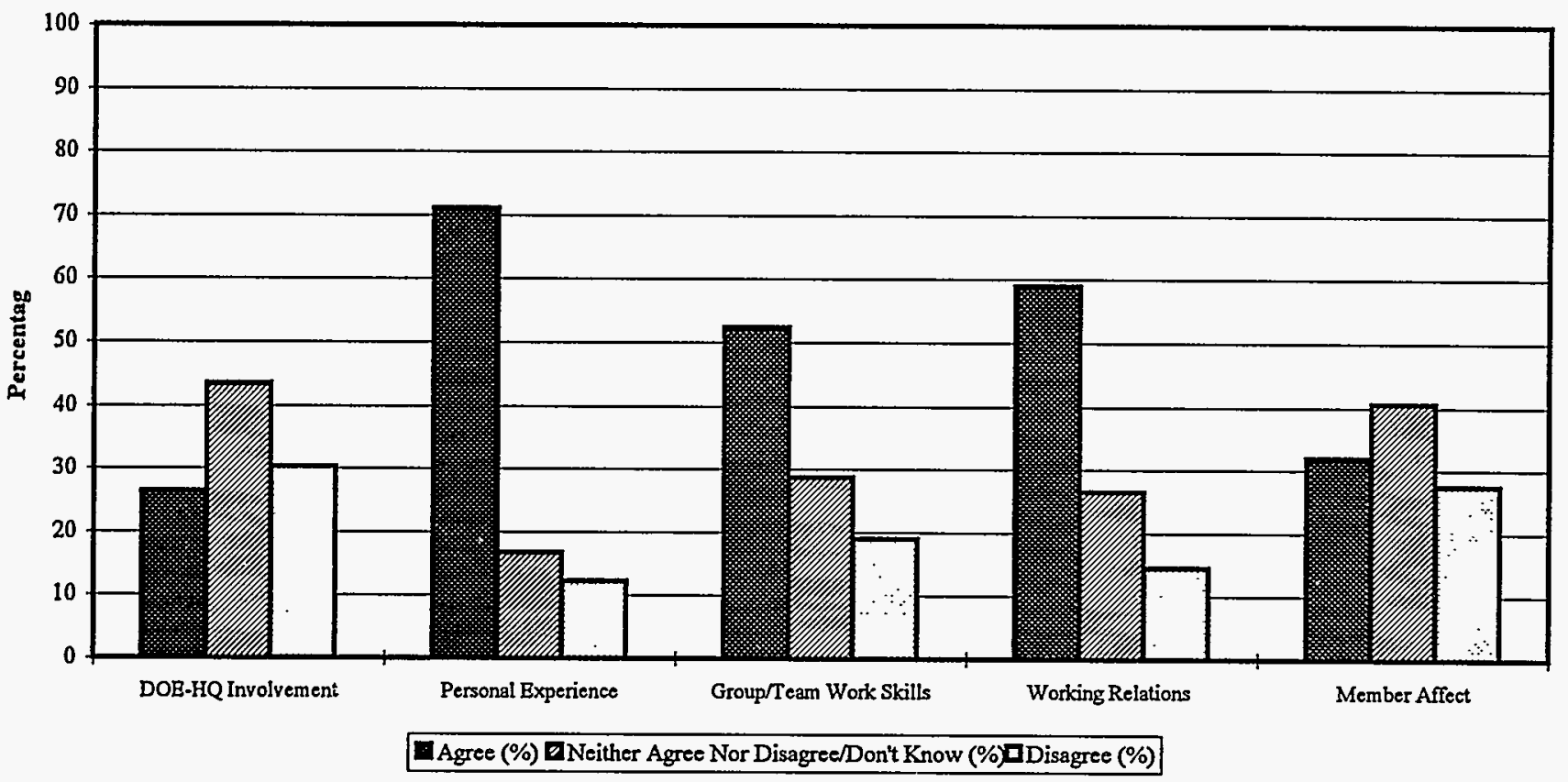

SSAB Supplementary Appendix

August 1996 
Table 1.

Goal 1. Establish processes and procedures to provide an effective forum for exchange of information and viewpoints regarding DOE site issues

\begin{tabular}{|c|c|c|c|c|c|c|c|c|}
\hline \multirow[t]{2}{*}{ Pantex } & \multicolumn{2}{|c|}{ Agree } & \multicolumn{2}{|c|}{$\begin{array}{c}\text { Neither/ } \\
\text { Don't Know }\end{array}$} & \multicolumn{2}{|c|}{ Disagree } & \multicolumn{2}{|c|}{ Total } \\
\hline & $N$ & $\%$ & $\mathrm{~N}$ & $\%$ & $N$ & $\%$ & $\mathrm{~N}$ & $\%$ \\
\hline $\begin{array}{l}\text { The Board has established processes and } \\
\text { procedures for the effective exchange of } \\
\text { information }\end{array}$ & 18 & $64.3 \%$ & 4 & $14.3 \%$ & 6 & $21.4 \%$ & 28 & $100 \%$ \\
\hline $\begin{array}{l}\text { The Board has agreed-upon procedures to } \\
\text { operate the board }\end{array}$ & 21 & $80.8 \%$ & 4 & $15.4 \%$ & 1 & $3.8 \%$ & 26 & $100 \%$ \\
\hline $\begin{array}{l}\text { The Board has adequate support to allow } \\
\text { it to focus on substantive issues (R) } *\end{array}$ & 18 & $69.2 \%$ & 3 & $11.5 \%$ & 5 & $19.2 \%$ & 26 & $100 \%$ \\
\hline $\begin{array}{l}\text { The Board provides sufficient time for } \\
\text { discussion of issues }\end{array}$ & 10 & $38.5 \%$ & 2 & $7.7 \%$ & 14 & $53.8 \%$ & 26 & $100 \%$ \\
\hline The Board has effective leadership & 18 & $69.2 \%$ & 5 & $19.2 \%$ & 3 & $11.5 \%$ & 26 & $100 \%$ \\
\hline $\begin{array}{l}\text { DOE requests Board advice far enough } \\
\text { ahead of decision deadlines }(\mathrm{R}) *\end{array}$ & 5 & $17.9 \%$ & 6 & $21.4 \%$ & 17 & $60.7 \%$ & 28 & $100 \%$ \\
\hline $\begin{array}{l}\text { DOE handles Board administrative issues } \\
\text { expeditiously (R)* }\end{array}$ & 15 & $53.6 \%$ & 10 & $35.7 \%$ & 3 & $10.7 \%$ & 28 & $100 \%$ \\
\hline $\begin{array}{l}\text { The SSAB staff provide satisfactory } \\
\text { support }\end{array}$ & 24 & $85.7 \%$ & 4 & $14.3 \%$ & 0 & $0 \%$ & 28 & $100 \%$ \\
\hline $\begin{array}{l}\text { The facilitator for the Board has helped } \\
\text { the Board function effectively }\end{array}$ & 15 & $53.6 \%$ & 7 & $25.0 \%$ & 6 & $21.4 \%$ & 28 & $100 \%$ \\
\hline
\end{tabular}

* (R) denotes a question that was asked in the negative form. For ease of review, these questions and data have been reversed in this table and are stated in positive forms.

SSAB Supplementary Appendix

August 1996 
Table 2.

Goal 2. Facilitate interaction and exchange of information and viewpoints regarding DOE site issues

\begin{tabular}{|c|c|c|c|c|c|c|c|c|}
\hline \multirow[t]{2}{*}{ Pantex } & \multicolumn{2}{|c|}{ Agree } & \multicolumn{2}{|c|}{$\begin{array}{c}\text { Neither/ } \\
\text { Don't Know }\end{array}$} & \multicolumn{2}{|c|}{ Disagree } & \multicolumn{2}{|c|}{ Total } \\
\hline & $N$ & $\%$ & $\mathbf{N}$ & $\%$ & $\mathrm{~N}$ & $\%$ & $\mathbb{N}$ & $\%$ \\
\hline $\begin{array}{l}\text { The SSAB facilitates effective exchange } \\
\text { of viewpoints on site issues }\end{array}$ & 17 & $60.7 \%$ & 5 & $17.9 \%$ & 6 & $21.4 \%$ & 28 & $100 \%$ \\
\hline $\begin{array}{l}\text { The SSAB contributes to Board members' } \\
\text { understanding of the basis for key site } \\
\text { decisions }\end{array}$ & 21 & $75.0 \%$ & 5 & $17.9 \%$ & 2 & $7.1 \%$ & 28 & $100 \%$ \\
\hline $\begin{array}{l}\text { The SSAB contributes to DOE and } \\
\text { regulators' understanding of the public's } \\
\text { viewpoints on key site decisions }\end{array}$ & 19 & $67.9 \%$ & 6 & $21.4 \%$ & 3 & $10.7 \%$ & 28 & $100 \%$ \\
\hline $\begin{array}{l}\text { The SSAB contributes to a constructive } \\
\text { working relationship among the } \\
\text { participants }\end{array}$ & 6 & $22.2 \%$ & 8 & $29.6 \%$ & 13 & $48.1 \%$ & 27 & $100 \%$ \\
\hline The SSAB strives for consensus & 19 & $67.9 \%$ & 2 & $7.1 \%$ & 7 & $25.0 \%$ & 28 & $100 \%$ \\
\hline $\begin{array}{l}\text { The SSAB gives fair consideration to } \\
\text { dissenting opinions }\end{array}$ & 13 & $46.4 \%$ & 7 & $25.0 \%$ & 8 & $28.6 \%$ & 28 & $100 \%$ \\
\hline $\begin{array}{l}\text { The Board has made the effort needed to } \\
\text { learn about site issues }\end{array}$ & 19 & $73.1 \%$ & 4 & $15.4 \%$ & 3 & $11.5 \%$ & 26 & $100 \%$ \\
\hline $\begin{array}{l}\text { The Board invites expert advice into its } \\
\text { discussion on key policy issues }\end{array}$ & 17 & $65.4 \%$ & 7 & $26.9 \%$ & 2 & $7.7 \%$ & 26 & $100 \%$ \\
\hline $\begin{array}{l}\text { The Board responds to public inquiries } \\
\text { and comments about its decisions }\end{array}$ & 17 & $65.4 \%$ & 7 & $26.9 \%$ & 2 & $7.7 \%$ & 26 & $100 \%$ \\
\hline $\begin{array}{l}\text { The Board solicits feedback from the } \\
\text { community on its work }\end{array}$ & 13 & $50.0 \%$ & 8 & $30.8 \%$ & 5 & $19.2 \%$ & 26 & $100 \%$ \\
\hline $\begin{array}{l}\text { DOE makes information on key site issues } \\
\text { readily available to the Board }\end{array}$ & 23 & $85.2 \%$ & 2 & $7.4 \%$ & 2 & $7.4 \%$ & 27 & $100 \%$ \\
\hline $\begin{array}{l}\text { The regulators make important } \\
\text { contributions to the Board's work }\end{array}$ & 20 & $74.1 \%$ & 6 & $22.2 \%$ & 1 & $3.7 \%$ & 27 & $100 \%$ \\
\hline $\begin{array}{l}\text { The SSAB creates a climate supportive of } \\
\text { differing viewpoints }(\mathrm{R})^{*}\end{array}$ & 17 & $60.7 \%$ & 2 & $7.1 \%$ & 9 & $32.1 \%$ & 28 & $100 \%$ \\
\hline
\end{tabular}

* (R) denotes a question that was asked in the negative form. For ease of review, these questions and data have been reversed in this table and are stated in positive forms.

SSAB Supplementary Appendix

August 1996 
Table 3.

Goal 3. Provide useful advice and/or recommendations to DOE

(and regulators, where appropriate)

\begin{tabular}{|c|c|c|c|c|c|c|c|c|}
\hline \multirow[t]{2}{*}{ Pantex } & \multicolumn{2}{|c|}{ Agree } & \multicolumn{2}{|c|}{$\begin{array}{l}\text { Neither/ } \\
\text { Don't Know }\end{array}$} & \multicolumn{2}{|c|}{ Disagree } & \multicolumn{2}{|c|}{ Total } \\
\hline & $\mathbf{N}$ & $\%$ & $\mathbf{N}$ & $\%$ & $\mathbf{N}$ & $\%$ & $\mathbf{N}$ & $\%$ \\
\hline $\begin{array}{l}\text { The Board provides useful advice to DOE } \\
\text { (and regulators, where appropriate) }(R)^{*}\end{array}$ & 16 & $61.5 \%$ & 3 & $11.5 \%$ & 7 & $26.9 \%$ & 26 & $100 \%$ \\
\hline $\begin{array}{l}\text { The SSAB reaches agreement about } \\
\text { prioritization of key site issues for which } \\
\text { advice is sought }(\mathrm{R})^{*}\end{array}$ & 9 & $32.1 \%$ & 6 & $21.4 \%$ & 13 & $46.4 \%$ & 28 & $100 \%$ \\
\hline The Board helps define site problems & 20 & $76.9 \%$ & 4 & $15.4 \%$ & 2 & $7.7 \%$ & 26 & $100 \%$ \\
\hline $\begin{array}{l}\text { The Board addresses issues in a timely } \\
\text { manner }(\mathrm{R}){ }^{*}\end{array}$ & 9 & $34.6 \%$ & 5 & $19.2 \%$ & 12 & $46.2 \%$ & 26 & $100 \%$ \\
\hline $\begin{array}{l}\text { The Board reaches consensus on key site } \\
\text { issues }(R)^{*}\end{array}$ & 5 & $19.2 \%$ & 5 & $19.2 \%$ & 16 & $61.5 \%$ & 26 & $100 \%$ \\
\hline $\begin{array}{l}\text { The Board provides informed advice to } \\
\text { DOE (and regulators, where appropriate) }\end{array}$ & 18 & $69.2 \%$ & 5 & $19.2 \%$ & 3 & $11.5 \%$ & 26 & $100 \%$ \\
\hline $\begin{array}{l}\text { The Board provides advice that reflects } \\
\text { the viewpoints and priorities of the } \\
\text { community }\end{array}$ & 15 & $57.7 \%$ & 3 & $11.5 \%$ & 8 & $30.8 \%$ & 26 & $100 \%$ \\
\hline
\end{tabular}

* (R) denotes a question that was asked in the negative form. For ease of review, these questions and data have been reversed in this table and are stated in positive forms. 
Table 4.

Goal 4. Improve DOE's (and where applicable, regulators') site decisions and decision making process

\begin{tabular}{|c|c|c|c|c|c|c|c|c|}
\hline \multirow[t]{2}{*}{ Pantex } & \multicolumn{2}{|c|}{ Agree } & \multicolumn{2}{|c|}{$\begin{array}{l}\text { Neither/ } \\
\text { Don't Know }\end{array}$} & \multicolumn{2}{|c|}{ Disagree } & \multicolumn{2}{|c|}{ Total } \\
\hline & $\mathrm{N}$ & $\%$ & $N$ & $\%$ & $\mathrm{~N}$ & $\%$ & $N$ & $\%$ \\
\hline $\begin{array}{l}\text { The SSAB has improved DOE's site } \\
\text { decisions (R) * }\end{array}$ & 11 & $39.3 \%$ & 12 & $42.9 \%$ & 5 & $17.9 \%$ & 28 & $100 \%$ \\
\hline $\begin{array}{l}\text { The Board understands the decision } \\
\text { making process at [a particular] site }\end{array}$ & 11 & $42.3 \%$ & 9 & $34.6 \%$ & 6 & $23.1 \%$ & 26 & $100 \%$ \\
\hline $\begin{array}{l}\text { DOE discusses important policies } \\
\text { affecting site decisions with the Board }\end{array}$ & 23 & $82.1 \%$ & 2 & $7.1 \%$ & 3 & $10.7 \%$ & 28 & $100 \%$ \\
\hline $\begin{array}{l}\text { DOE has explained to the Board its site } \\
\text { decision making process }\end{array}$ & 18 & $66.7 \% \cdot$ & 9 & $33.3 \%$ & 0 & $0 \%$ & 27 & $100 \%$ \\
\hline $\begin{array}{l}\text { The DOE shows how Board advice is } \\
\text { reflected in site decisions }\end{array}$ & 15 & $53.6 \%$ & 10 & $35.7 \%$ & 3 & $10.7 \%$ & 28 & $100 \%$ \\
\hline $\begin{array}{l}\text { The decision making process used by } \\
\text { DOE at [a particular] site is effective in } \\
\text { furthering site clean-up (R) }{ }^{*}\end{array}$ & 19 & $67.9 \%$ & 6 & $21.4 \%$ & 3 & $10.7 \%$ & 28 & $100 \%$ \\
\hline
\end{tabular}

* (R) denotes a question that was asked in the negative form. For ease of review, these questions and data have been reversed in this table and are stated in positive forms. 
Table 5.

Goal 5. Lead to more acceptable actions

\begin{tabular}{|c|c|c|c|c|c|c|c|c|}
\hline \multirow[t]{2}{*}{ Pantex } & \multicolumn{2}{|c|}{ Agree } & \multicolumn{2}{|c|}{$\begin{array}{l}\text { Neither/ } \\
\text { Don't Know }\end{array}$} & \multicolumn{2}{|c|}{ Disagree } & \multicolumn{2}{|c|}{ Total } \\
\hline & $\mathrm{N}$ & $\%$ & $\mathrm{~N}$ & $\%$ & $\mathrm{~N}$ & $\%$ & $\mathrm{~N}$ & $\%$ \\
\hline $\begin{array}{l}\text { The SSAB leads to more acceptable site } \\
\text { decisions }\end{array}$ & 14 & $50.0 \%$ & 9 & $32.1 \%$ & 5 & $17.9 \%$ & 28 & $100 \%$ \\
\hline $\begin{array}{l}\text { The Board supports the recommendations } \\
\text { it gives DOE }\end{array}$ & 16 & $61.5 \%$ & 8 & $30.8 \%$ & 2 & $7.7 \%$ & 26 & $100 \%$ \\
\hline The Board supports DOE's site actions & 5 & $20.0 \%$ & 16 & $64.0 \%$ & 4 & $16.0 \%$ & $2 \dot{5}$ & $100 \%$ \\
\hline $\begin{array}{l}\text { The DOE pays attention to the Board's } \\
\text { advice on key site issues }(\mathrm{R}){ }^{*}\end{array}$ & 20 & $71.4 \%$ & 7 & $25.0 \%$ & 1 & $3.6 \%$ & 28 & $100 \%$ \\
\hline $\begin{array}{l}\text { There is support in the community for } \\
\text { DOE's site decisions that have SSAB } \\
\text { input }\end{array}$ & 15 & $53.6 \%$ & 13 & $46.4 \%$ & 0 & $0 \%$ & 28 & $100 \%$ \\
\hline Progress is being made on key site issues & 16 & $57.1 \%$ & 8 & $28.6 \%$ & 4 & $14.3 \%$ & 28 & $100 \%$ \\
\hline
\end{tabular}

* (R) denotes a question that was asked in the negative form. For ease of review, these questions and data have been reversed in this table and are stated in positive forms. 
Table 6.

Goal 6. Contribute to trust and confidence in DOE

\begin{tabular}{|c|c|c|c|c|c|c|c|c|}
\hline \multirow[t]{2}{*}{ Pantex } & \multicolumn{2}{|c|}{ Agree } & \multicolumn{2}{|c|}{$\begin{array}{c}\text { Neither/ } \\
\text { Don't Know }\end{array}$} & \multicolumn{2}{|c|}{ Disagree } & \multicolumn{2}{|c|}{ Total } \\
\hline & $N$ & $\%$ & $\mathbf{N}$ & $\%$ & $\mathrm{~N}$ & $\%$ & $N$ & $\%$ \\
\hline $\begin{array}{l}\text { The SSAB contributes to trust and } \\
\text { confidence in DOE }\end{array}$ & 15 & $53.6 \%$ & 5 & $17.9 \%$ & 8 & $28.6 \%$ & 28 & $100 \%$ \\
\hline $\begin{array}{l}\text { Relationships between DOE and the public } \\
\text { have improved since the formation of the } \\
\text { SSAB }\end{array}$ & 17 & $60.7 \%$ & 7 & $25.0 \%$ & 4 & $14.3 \%$ & 28 & $100 \%$ \\
\hline $\begin{array}{l}\text { DOE is committed to clean up [a } \\
\text { particular] site }\end{array}$ & 22 & $78.6 \%$ & 3 & $10.7 \%$ & 3 & $10.7 \%$ & 28 & $100 \%$ \\
\hline
\end{tabular}


Table 7.

Public awareness

\begin{tabular}{|c|c|c|c|c|c|c|c|c|}
\hline \multirow[t]{2}{*}{ Pantex } & \multicolumn{2}{|c|}{ Agree } & \multicolumn{2}{|c|}{$\begin{array}{c}\text { Neither/ } \\
\text { Don't Know }\end{array}$} & \multicolumn{2}{|c|}{ Disagree } & \multicolumn{2}{|c|}{ Total } \\
\hline & $\mathrm{N}$ & $\%$ & $\mathbf{N}$ & $\%$ & $N$ & $\%$ & $N$ & $\%$ \\
\hline $\begin{array}{l}\text { The public knows little about the role of } \\
\text { the Board }\end{array}$ & 18 & $64.3 \%$ & 6 & $21.4 \%$ & 4 & $14.3 \%$ & 28 & $100 \%$ \\
\hline
\end{tabular}

Table 8.

SSAB Initiative is a good use of funds

\begin{tabular}{ccccccccc}
\hline \multirow{2}{*}{ Pantex } & Yes, Good & $\begin{array}{c}\text { Neutral, } \\
\text { Conditional, } \\
\text { No Opinion }\end{array}$ & $\begin{array}{c}\text { No, } \\
\text { Not Good }\end{array}$ & Total \\
& $\mathrm{N}$ & $\%$ & $\mathrm{~N}$ & $\%$ & $\mathrm{~N}$ & $\%$ & $\mathrm{~N}$ & $\%$ \\
\hline SSAB Initiative is a good use of funds & 16 & $69.6 \%$ & 1 & $4.3 \%$ & 6 & $26.1 \%$ & 23 & $100 \%$ \\
\hline
\end{tabular}


Table 9.

DOE-HQ involvement with the SSAB Initiative

\begin{tabular}{|c|c|c|c|c|c|c|c|c|}
\hline \multirow[t]{2}{*}{ Pantex } & \multicolumn{2}{|c|}{ Agree } & \multicolumn{2}{|c|}{$\begin{array}{l}\text { Neither/ } \\
\text { Don't Know }\end{array}$} & \multicolumn{2}{|c|}{ Disagree } & \multicolumn{2}{|c|}{ Total } \\
\hline & $N$ & $\%$ & $N$ & $\%$ & $N$ & $\%$ & $N$ & $\%$ \\
\hline $\begin{array}{l}\text { DOE-HQ provides helpful guidance to the } \\
\text { Boards and to DOE }\end{array}$ & 8 & $28.6 \%$ & 12 & $42.9 \%$ & 8 & $28.6 \%$ & 28 & $100 \%$ \\
\hline $\begin{array}{l}\text { DOE-HQ provides sufficient support to } \\
\text { facilitate the work of the SSAB }\end{array}$ & 16 & $57.1 \%$ & 8 & $28.6 \%$ & 4 & $14.3 \%$ & 28 & $100 \%$ \\
\hline $\begin{array}{l}\text { DOE-HQ gives careful consideration to } \\
\text { SSAB advice in its decisions }\end{array}$ & 5 & $17.9 \%$ & 19 & $67.9 \%$ & 4 & $14.3 \%$ & 28 & $100 \%$ \\
\hline
\end{tabular}


Table 10.

Personal experience with the SSAB Initiative

\begin{tabular}{|c|c|c|c|c|c|c|c|c|}
\hline \multirow[t]{2}{*}{ Pantex } & \multicolumn{2}{|c|}{ Agree } & \multicolumn{2}{|c|}{$\begin{array}{l}\text { Neither/ } \\
\text { Don't Know }\end{array}$} & \multicolumn{2}{|c|}{ Disagree } & \multicolumn{2}{|c|}{ Total } \\
\hline & $\mathrm{N}$ & $\%$ & $N$ & $\%$ & $N$ & $\%$ & $\mathrm{~N}$ & $\%$ \\
\hline $\begin{array}{l}\text { I consider myself to be very } \\
\text { knowledgeable about site issues }\end{array}$ & 19 & $67.9 \%$ & 6 & $21.4 \%$ & 3 & $10.7 \%$ & 28 & $100 \%$ \\
\hline $\begin{array}{l}\text { Because of the SSAB, I better understand } \\
\text { the complexities of site clean-up }\end{array}$ & 20 & $71.4 \%$ & 4 & $14.3 \%$ & 4 & $14.3 \%$ & 28 & $100 \%$ \\
\hline $\begin{array}{l}\text { I feel that my participation in the SSAB } \\
\text { has been worthwhile }(\mathrm{R}) *\end{array}$ & 15 & $53.6 \%$ & 10 & $35.7 \%$ & 3 & $10.7 \%$ & 28 & $100 \%$ \\
\hline
\end{tabular}

* (R) denotes a question that was asked in the negative form. For ease of review, these questions and data have been reversed in this table and are stated in positive forms. 
Table 11.

Group/Team work skills

\begin{tabular}{|c|c|c|c|c|c|c|c|c|}
\hline \multirow[t]{2}{*}{ Pantex } & \multicolumn{2}{|c|}{ Agree } & \multicolumn{2}{|c|}{$\begin{array}{l}\text { Neither/ } \\
\text { Don't Know }\end{array}$} & \multicolumn{2}{|c|}{ Disagree } & \multicolumn{2}{|c|}{ Total } \\
\hline & $\mathrm{N}$ & $\%$ & $\mathbf{N}$ & $\%$ & $\mathbf{N}$ & $\%$ & $\mathbf{N}$ & $\%$ \\
\hline All have group/team work skills & 12 & $44.4 \%$ & 5 & $18.5 \%$ & 10 & $37.0 \%$ & 27 & $100 \%$ \\
\hline $\begin{array}{l}\text { Have developed well-established working } \\
\text { relationships with each other }\end{array}$ & 11 & $39.3 \%$ & 4 & $14.3 \%$ & 13 & $46.4 \%$ & 28 & $100 \%$ \\
\hline Like each other & 8 & $28.6 \%$ & 6 & $21.4 \%$ & 14 & $50.0 \%$ & 28 & $100 \%$ \\
\hline $\begin{array}{l}\text { Have a high degree of trust and } \\
\text { confidence in each other }\end{array}$ & 6 & $21.4 \%$ & 5 & $17.9 \%$ & 17 & $60.7 \%$ & 28 & $100 \%$ \\
\hline $\begin{array}{l}\text { Accept the values that are important to the } \\
\text { group }\end{array}$ & 8 & $28.6 \%$ & 10 & $35.7 \%$ & 10 & $35.7 \%$ & 28 & $100 \%$ \\
\hline Show respect for the viewpoints of others & 13 & $46.4 \%$ & 4 & $14.3 \%$ & 11 & $39.3 \%$ & 28 & $100 \%$ \\
\hline $\begin{array}{l}\text { Accept willingly the goals and } \\
\text { expectations for the Board }\end{array}$ & 12 & $42.9 \%$ & 8 & $28.6 \%$ & 8 & $28.6 \%$ & 28 & $100 \%$ \\
\hline $\begin{array}{l}\text { Set high expectations for what they } \\
\text { believe they can accomplish }\end{array}$ & 17 & $60.7 \%$ & 9 & $32.1 \%$ & 2 & $7.1 \%$ & 28 & $100 \%$ \\
\hline $\begin{array}{l}\text { Communicate fully and frankly to the } \\
\text { Board all relevant and valuable } \\
\text { information }\end{array}$ & 18 & $64.3 \%$ & 6 & $21.4 \%$ & 4 & $14.3 \%$ & 28 & $100 \%$ \\
\hline $\begin{array}{l}\text { Try not to waste the Board's time with } \\
\text { irrelevant material or communications }\end{array}$ & 15 & $53.6 \%$ & 7 & $25.0 \%$ & 6 & $21.4 \%$ & 28 & $100 \%$ \\
\hline $\begin{array}{l}\text { Are willing to be influenced by other } \\
\text { Board members about new ideas and } \\
\text { methods }\end{array}$ & 11 & $39.3 \%$ & 9 & $32.1 \%$ & 8 & $28.6 \%$ & 28 & $100 \%$ \\
\hline $\begin{array}{l}\text { Clearly understand the goals and } \\
\text { philosphy of the Board's operation }\end{array}$ & 14 & $50.0 \%$ & 9 & $32.1 \%$ & 5 & $17.9 \%$ & 28 & $100 \%$ \\
\hline $\begin{array}{l}\text { Do all they can to help the Board achieve } \\
\text { its objectives }\end{array}$ & 13 & $46.4 \%$ & 9 & $32.1 \%$ & 6 & $21.4 \%$ & 28 & $100 \%$ \\
\hline $\begin{array}{l}\text { Get the technical knowledge and training } \\
\text { in group skills they need }\end{array}$ & 15 & $53.6 \%$ & 9 & $32.1 \%$ & 4 & $14.3 \%$ & 28 & $100 \%$ \\
\hline Provide one another mutual help & 11 & $39.3 \%$ & 9 & $32.1 \%$ & 8 & $28.6 \%$ & 28 & $100 \%$ \\
\hline
\end{tabular}

SSAB Supplementary Appendix

August 1996 
Rocky Flats Site Specific Advisory Board

Evaluation Survey Results 
Figure 1a. Rocky Flats

Goal 1. Establish processes and procedures to provide an effective forum

for exchange of information and viewpoints regarding DOE site issues

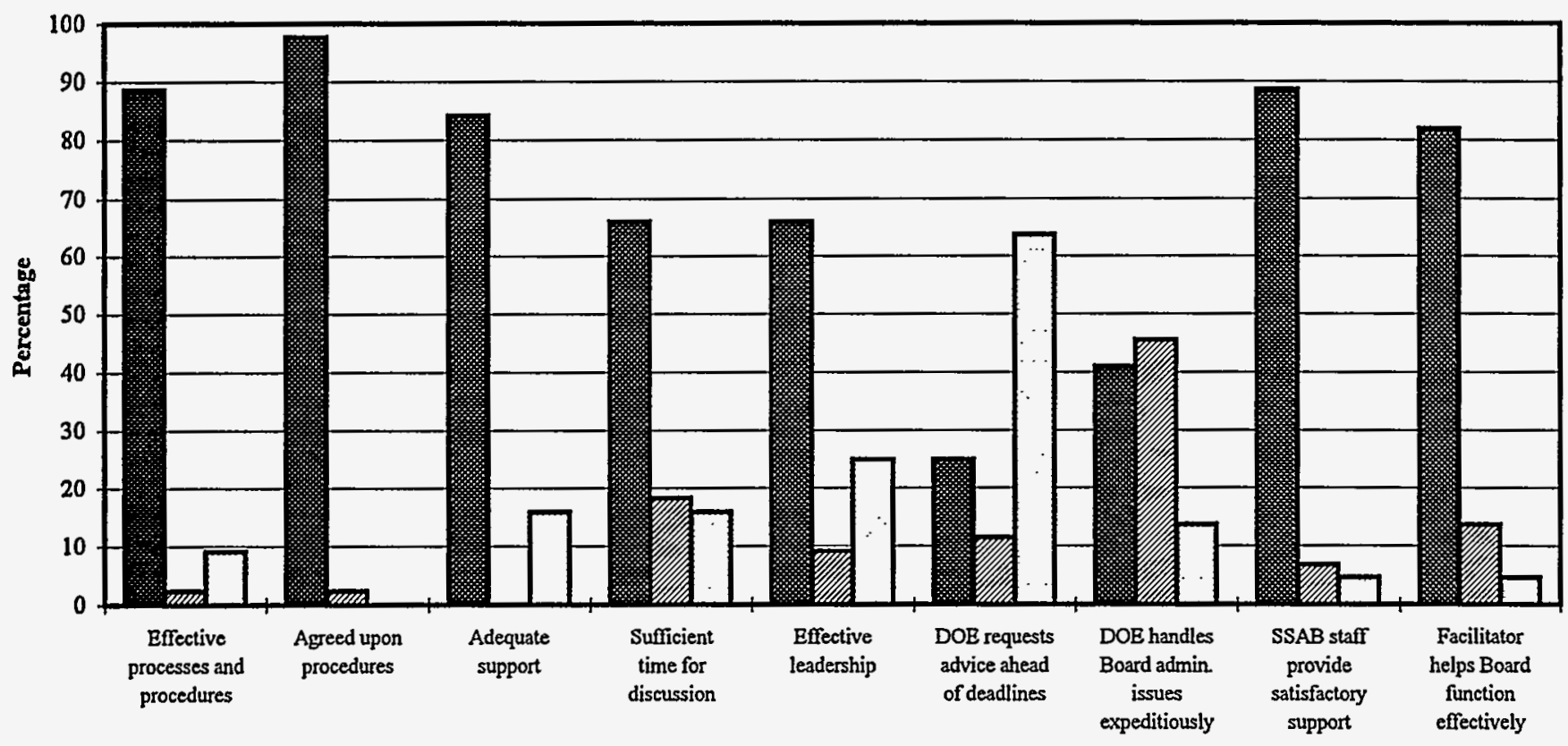

Agree (\%) Ed Neither Agree Nor Disagree/Don't Know (\%) DDisagree (\%)

Figure 1b. Long Survey: Entire Sample

Goal 1. Establish processes and procedures to provide an effective forum for exchange of information and viewpoints regarding DOE site issues

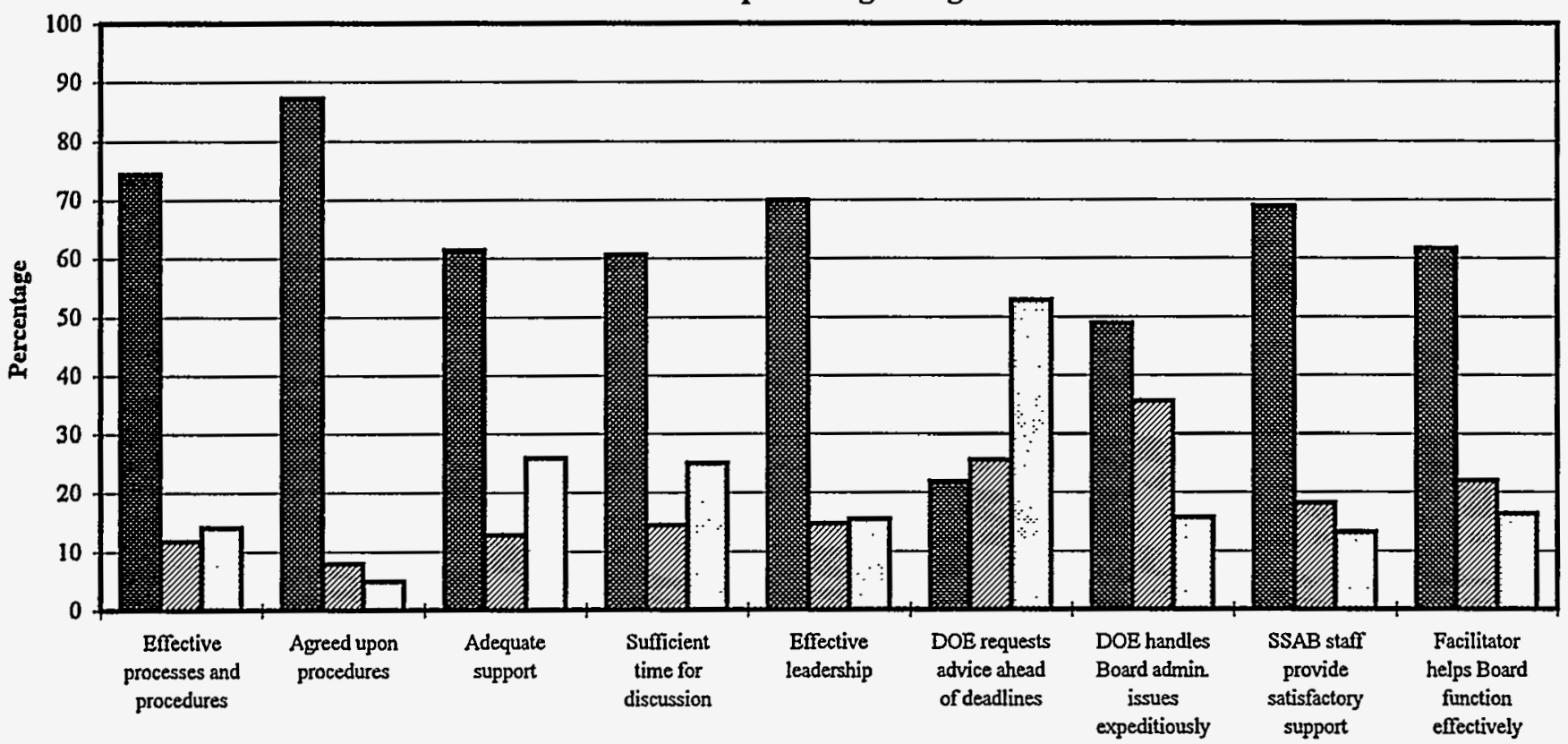

P. Agree (\%) Q Neither Agree Nor Disagree/Don't Know (\%) DDisagree (\%)

SSAB Supplementary Appendix

August 1996 
Figure 2a. Rocky Flats

Goal 2. Facilitate interaction and exchange of information and viewpoints regarding DOE site issues

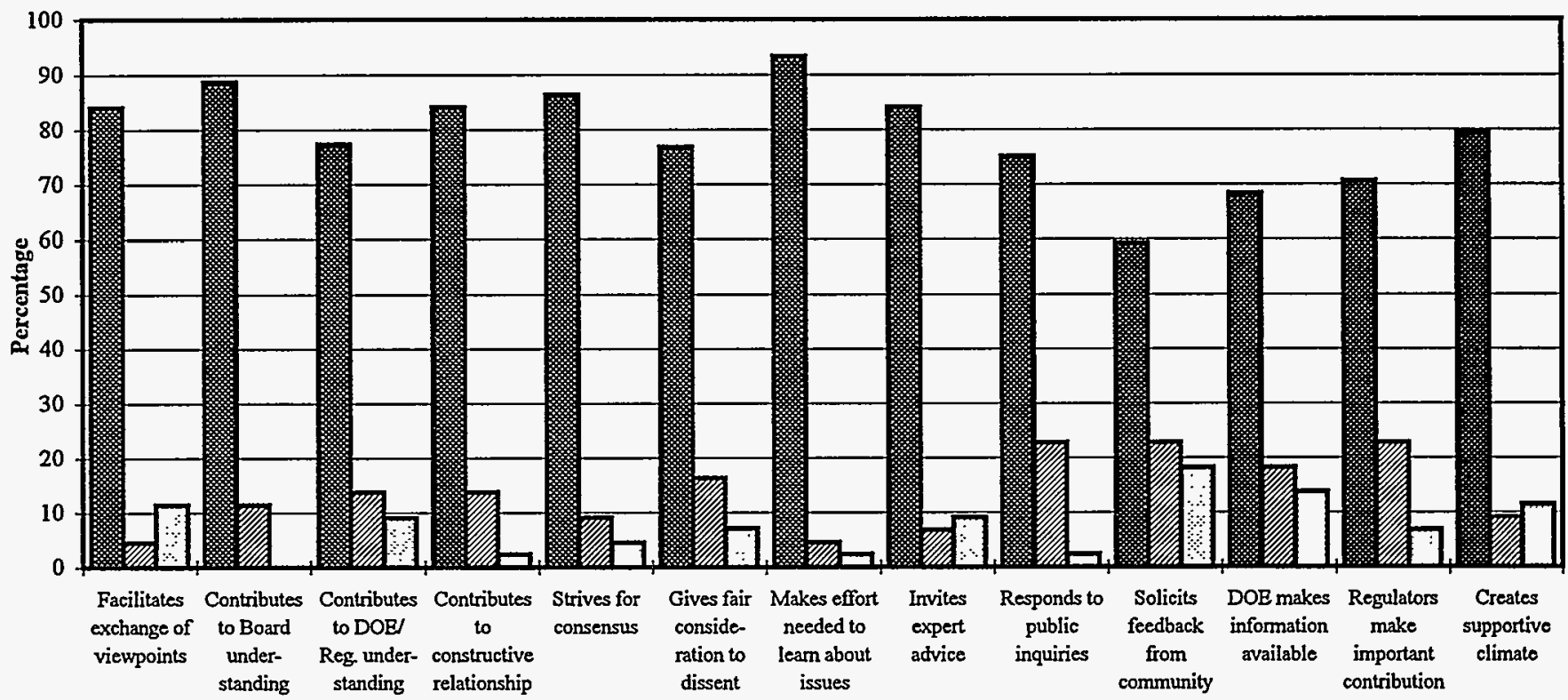

Agree (\%) DNeither Agree Nor Disagree/Don't Know (\%) DDisagree (\%)

Figure 2b. Long Survey: Entire Sample

Goal 2. Facilitate interaction and exchange of information and viewpoints regarding $D O E$ site issues

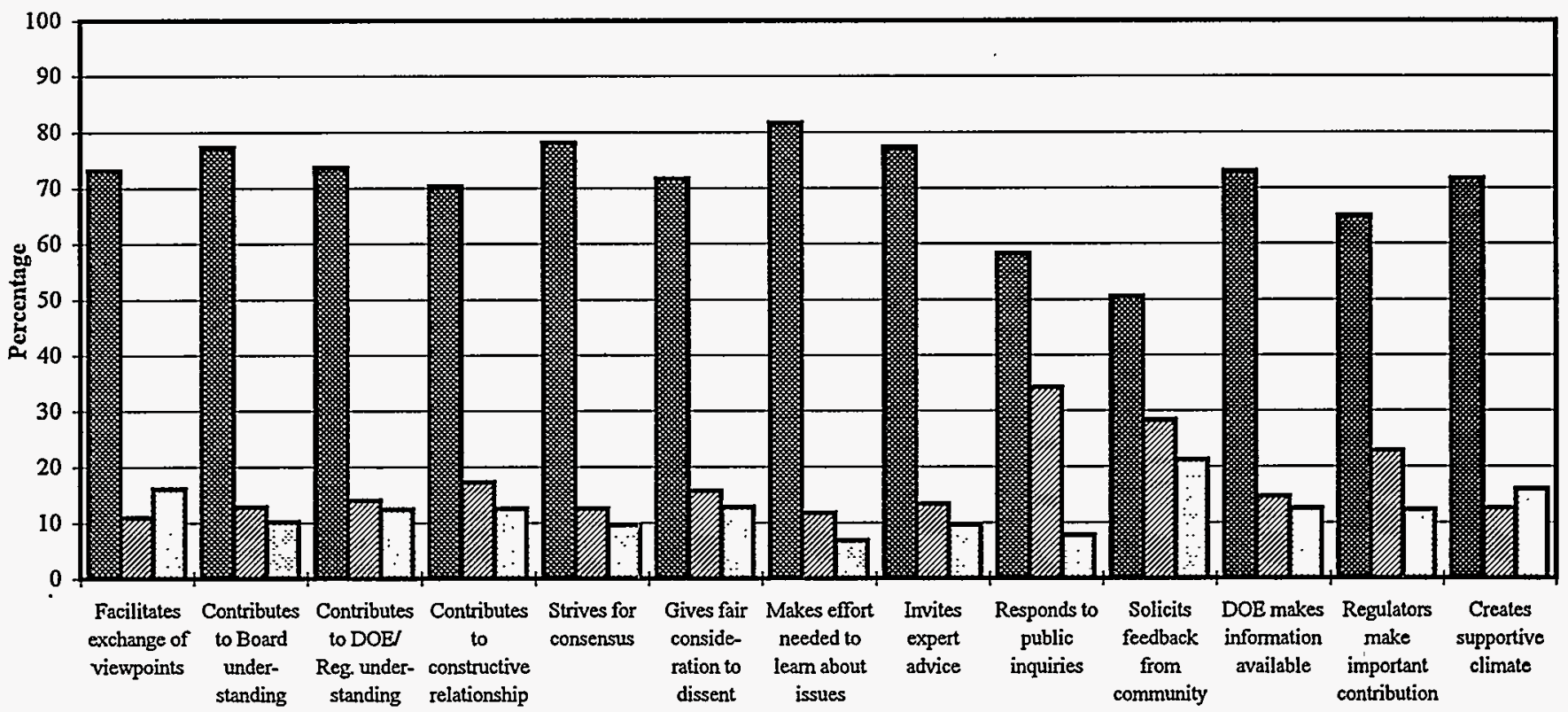

Agree (\%) Deither Agree Nor Disagree/Don't Know (\%) DDisagree (\%)

SSAB Supplementary Appendix

August 1996 
Figure 3a. Rocky Flats

Goal 3. Provide useful advice and/or recommendations to DOE

(and regulators, where appropriate)

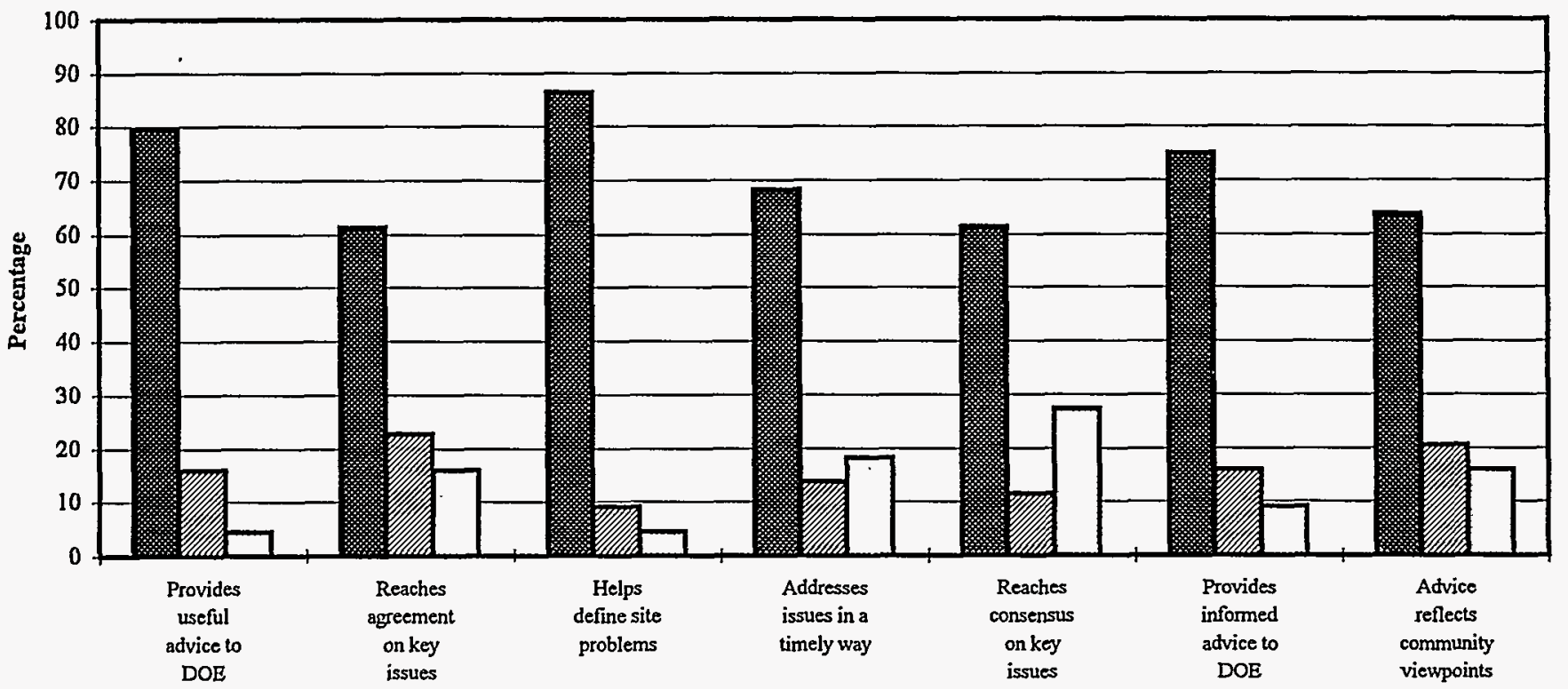

D. Agree (\%) प Neither Agree Nor Disagree/Don't Know (\%) DDisagree (\%)

Figure 3b. Long Survey: Entire Sample

Goal 3. Provide useful advice and/or recommendations to DOE

(and regulators, where appropriate)

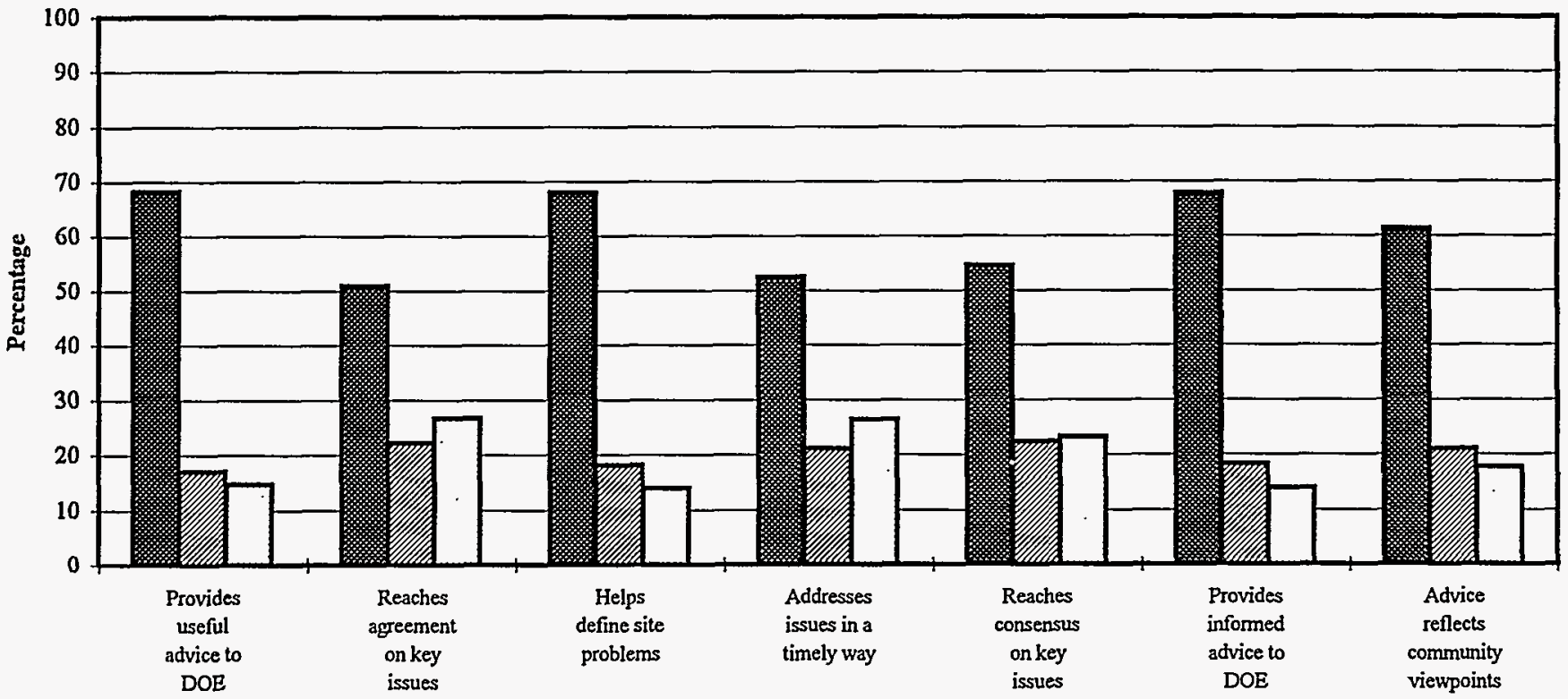

Agree (\%) 7 Neither Agree Nor Disagree/Don't Know (\%) DDisagree (\%)

SSAB Supplementary Appendix

August 1996 
Figure 4a. Rocky Flats

Goal 4. Improve DOE's (and where applicable, regulators') site decisions and decision making process

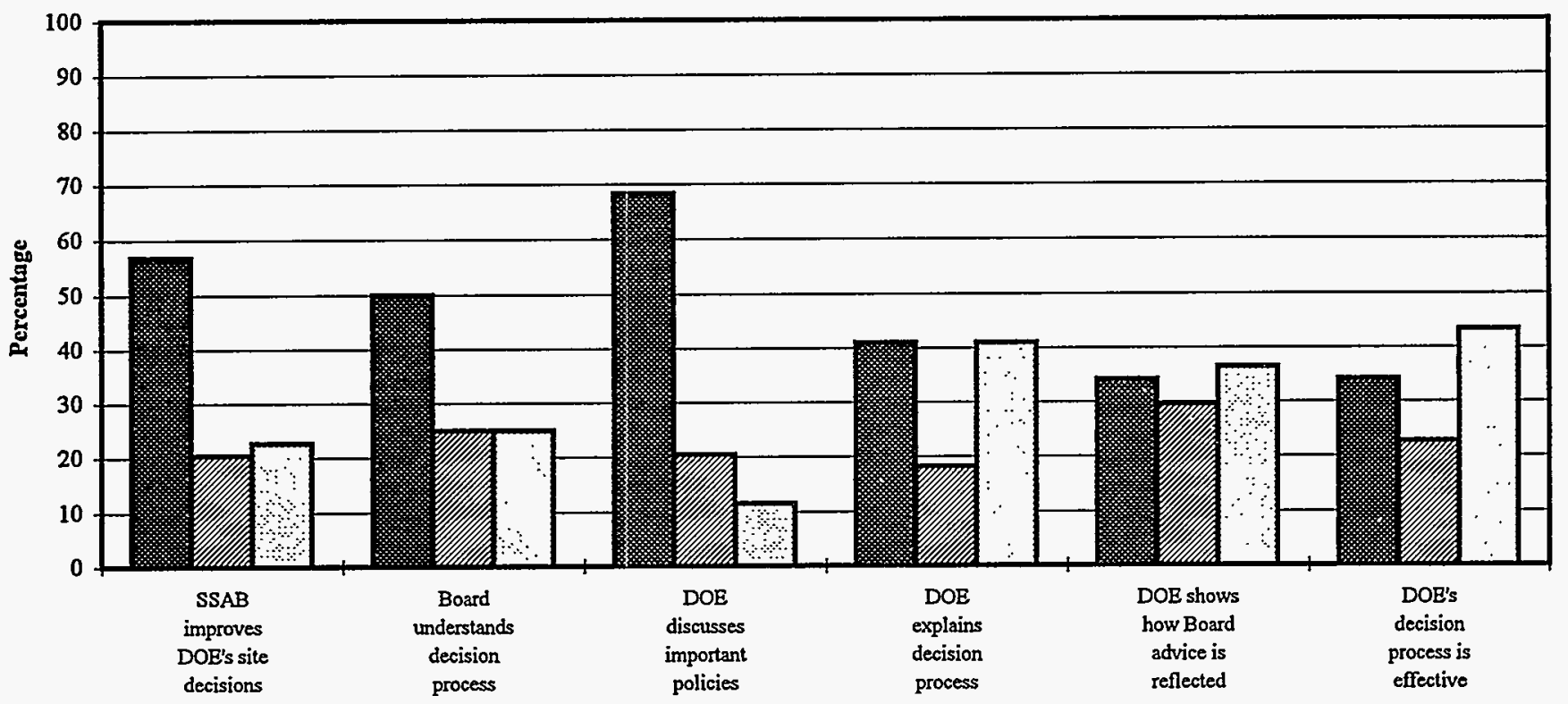

Agree (\%) GNeither Agree Nor Disagree/Don't Know (\%) DDisagree (\%)

Figure 4b. Long Survey: Entire Sample

Goal 4. Improve DOE's (and where applicable, regulators') site decisions and decision making process

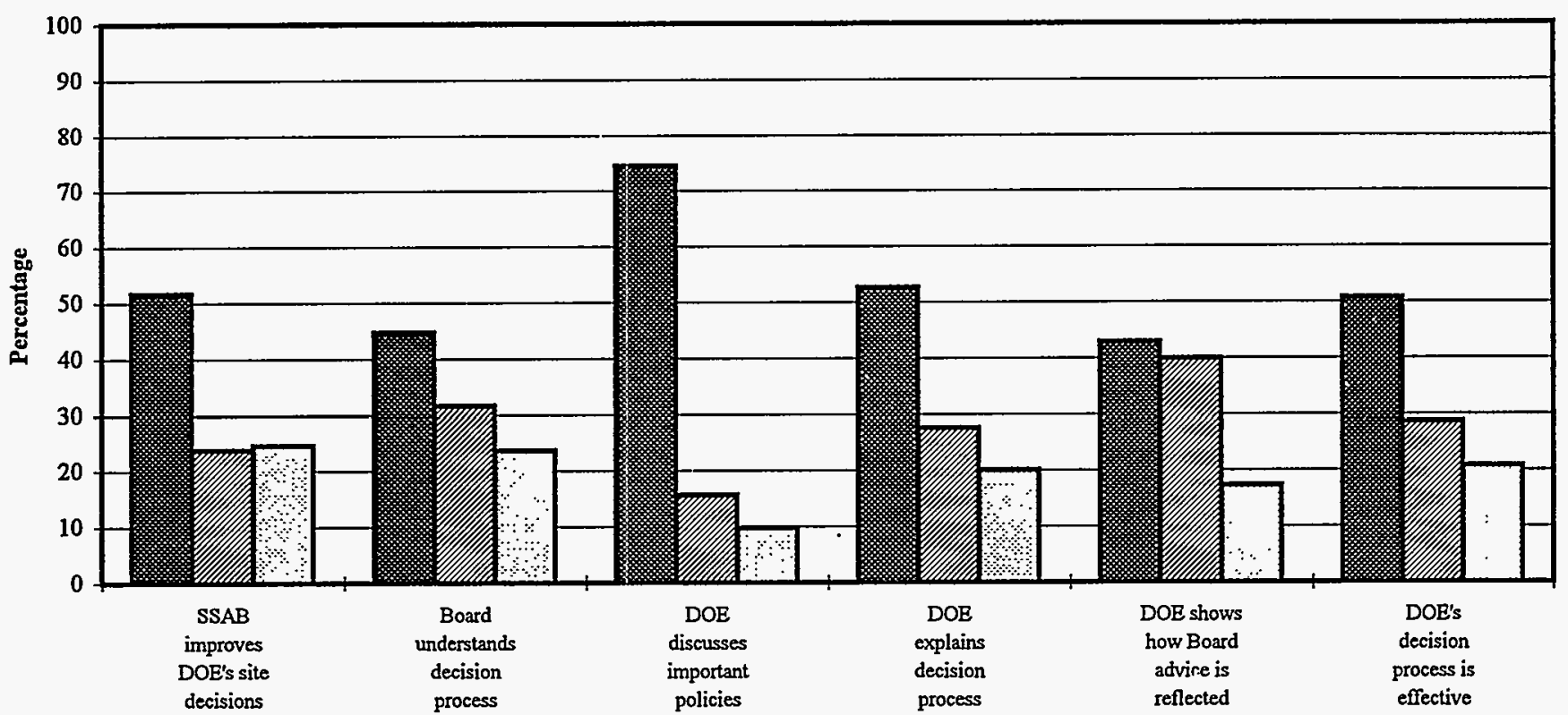

Agree (\%) DNeither Agree Nor Disagree/Don't Know (\%) DDisagree (\%)

SSAB Supplementary Appendix 
Figure 5a. Rocky Flats

Goal 5. Lead to more acceptable actions

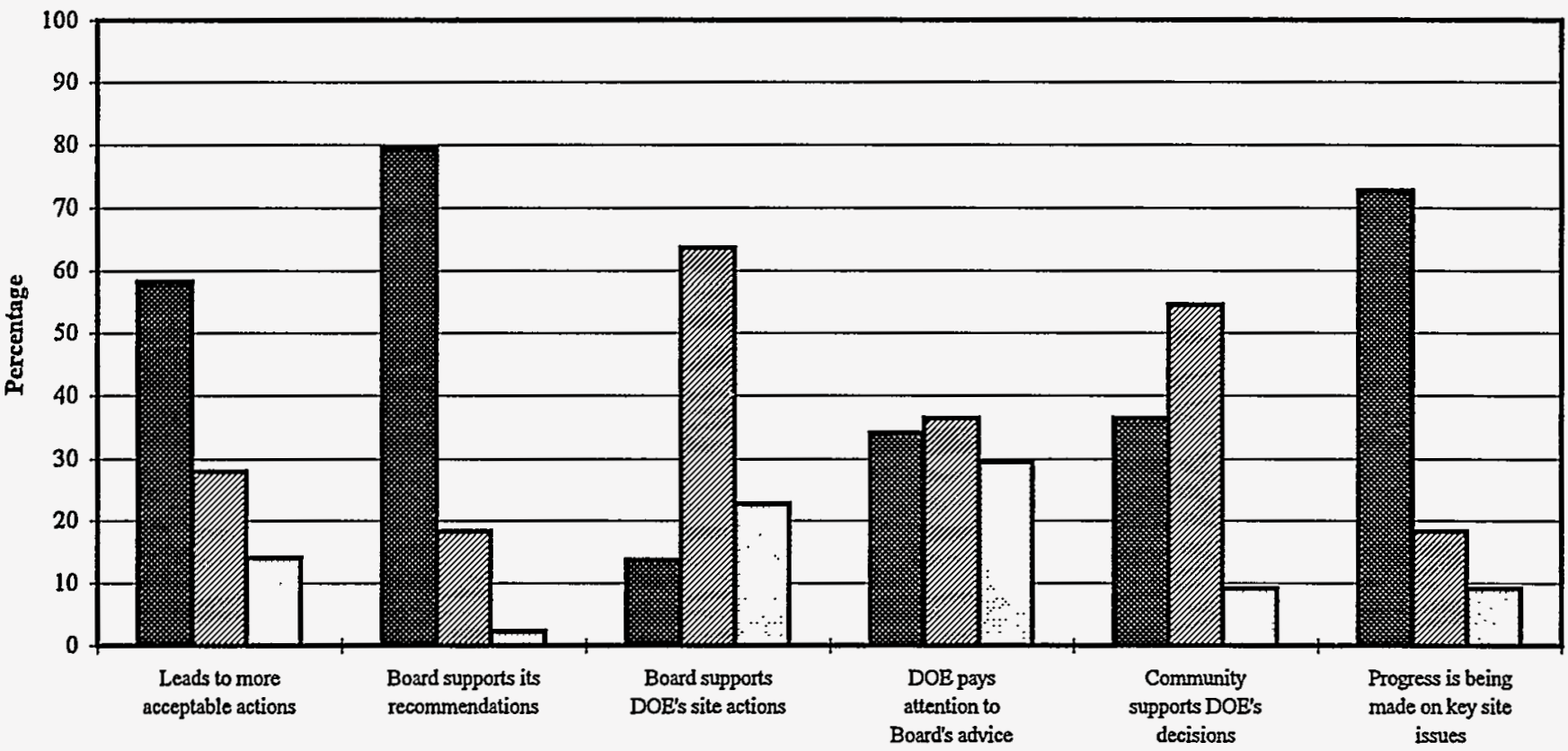

F Agree (\%) Eeither Agree Nor Disagree/Don't Know (\%) D Disagree (\%)

Figure 5b. Long Survey: Entire Sample

Goal 5. Lead to more acceptable actions

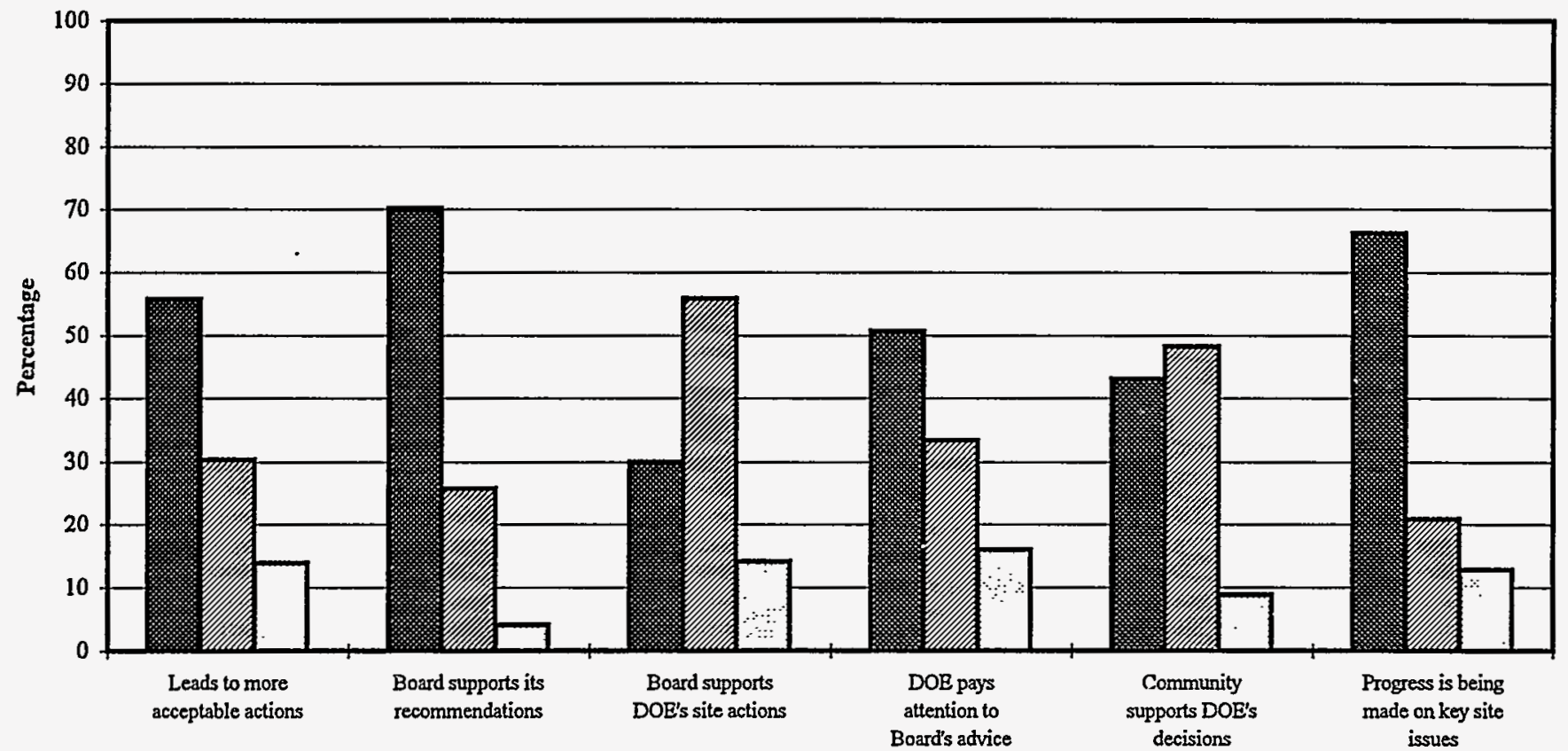

Agree (\%) DNeither Agree Nor Disagree/Don't Know (\%) DDisagree (\%)

SSAB Supplementary Appendix

August 1996 
Figure 6a. Rocky Flats

Goal 6. Contribute to trust and confidence in DOE

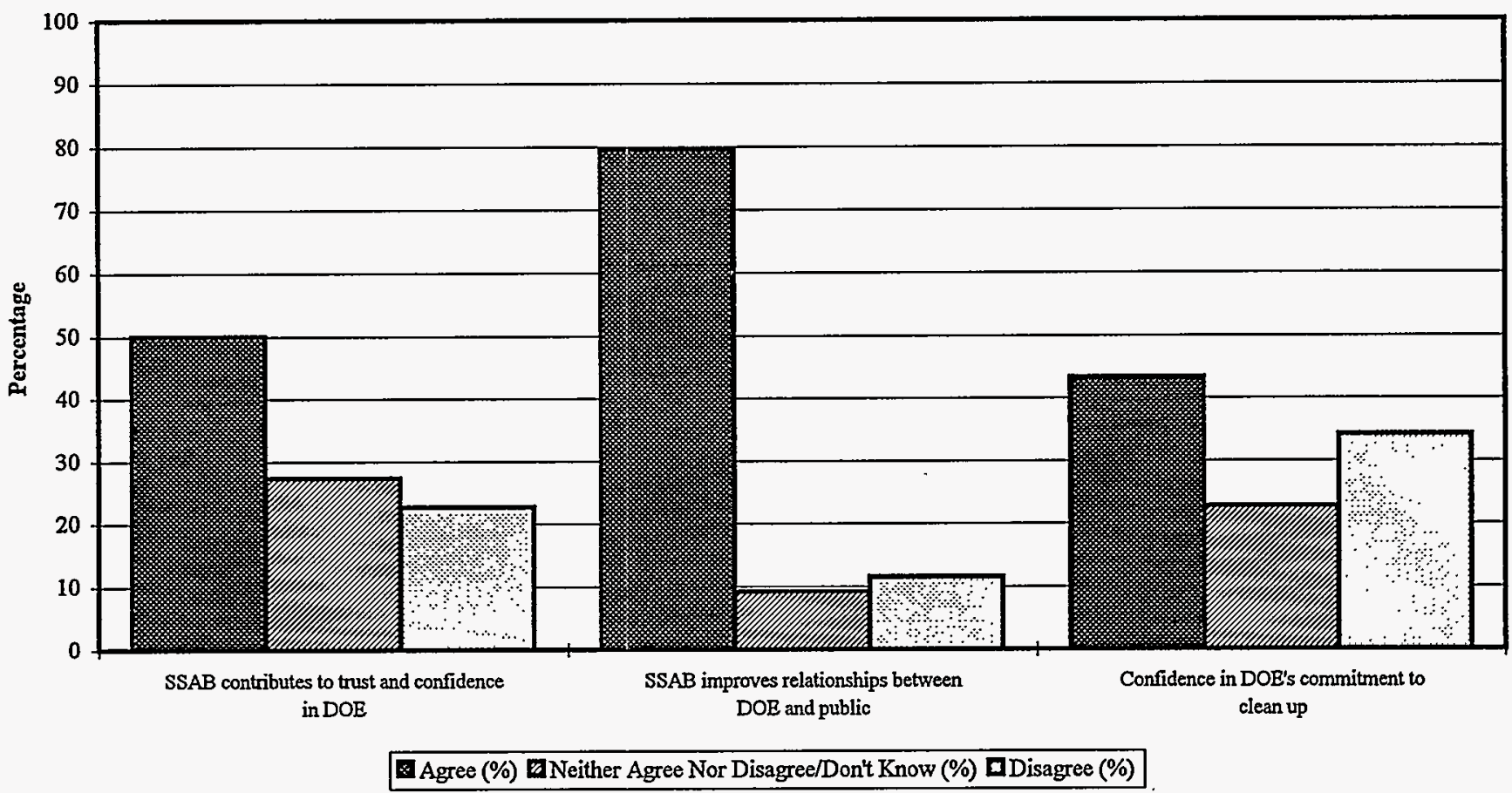

Figure 6 . Long Survey: Entire Sample

Goal 6. Contribute to trust and confidence in DOE

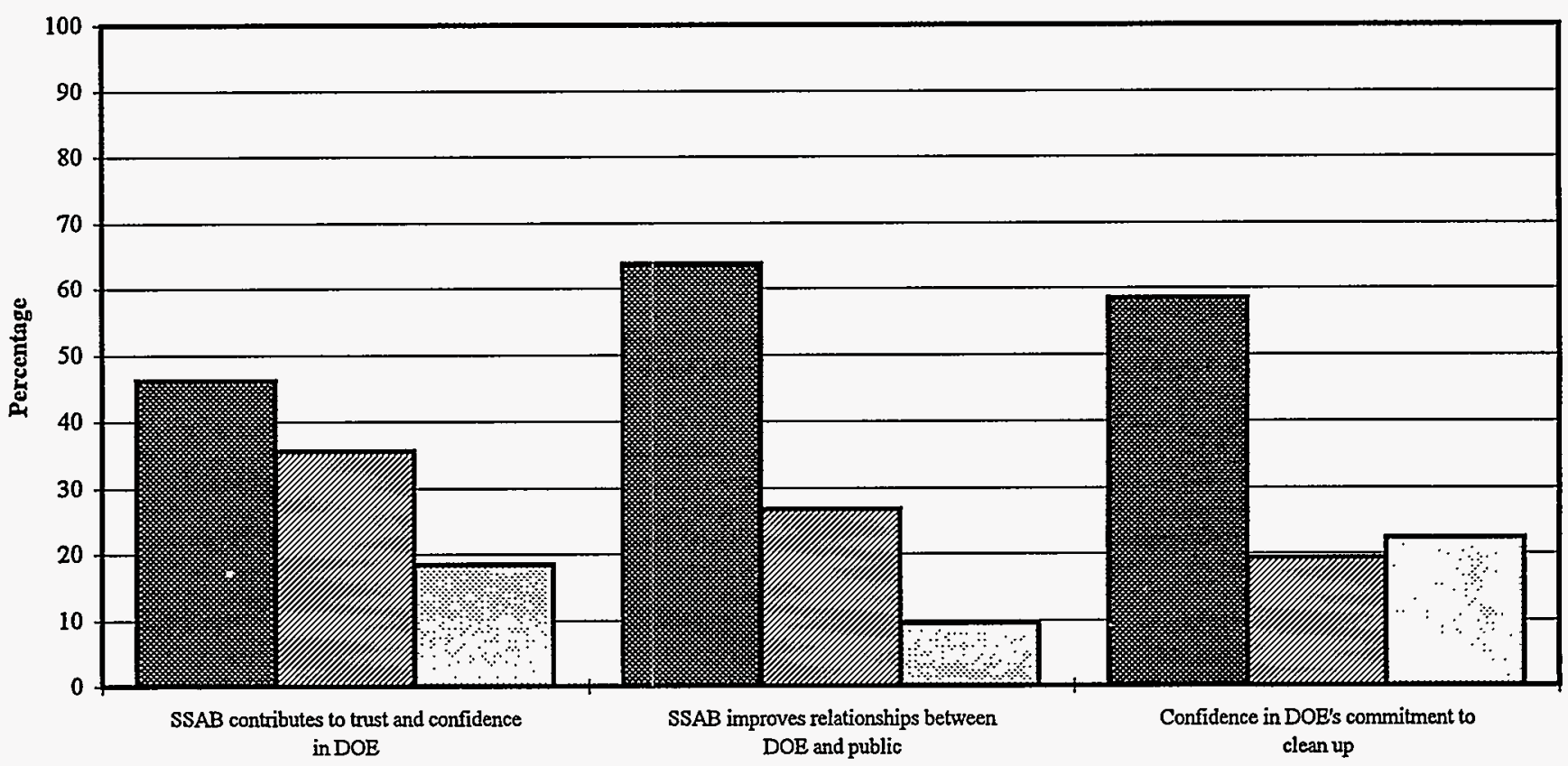

Agree (\%) Deither Agree Nor Disagree/Don't Know (\%) DDisagree (\%)

SSAB Supplementary Appendix

August 1996 
Figure 7a. Rocky Flats

Public awareness

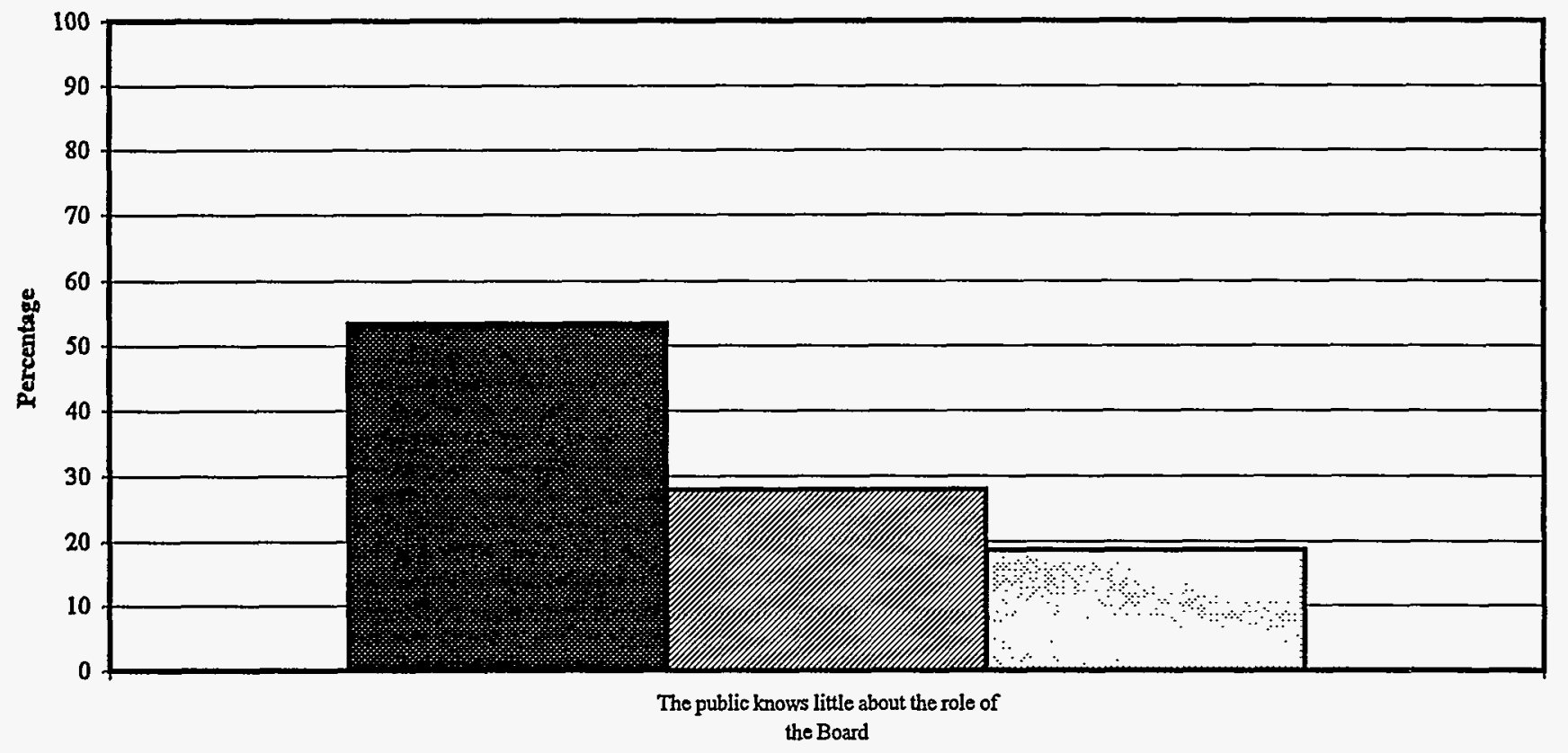

Agree (\%) ENeither Agree Nor Disagree/Don't Know (\%) DDisagree (\%)

Figure 7b. Long Survey: Entire Sample

Public awareness

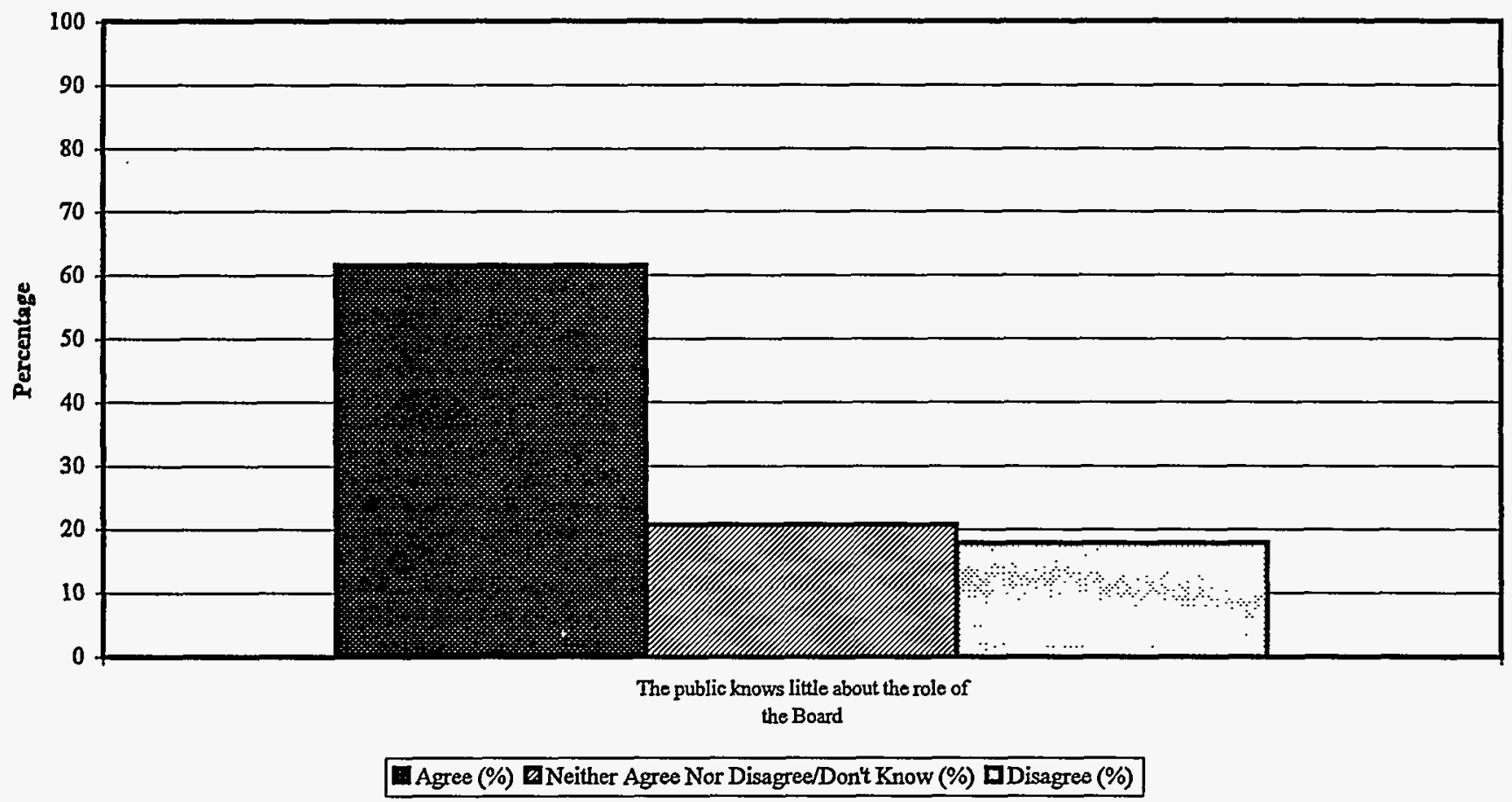

SSAB Supplementary Appendix

August 1996 
Figure 8a. Rocky Flats

DOE-HQ involvement with the SSAB Initiative

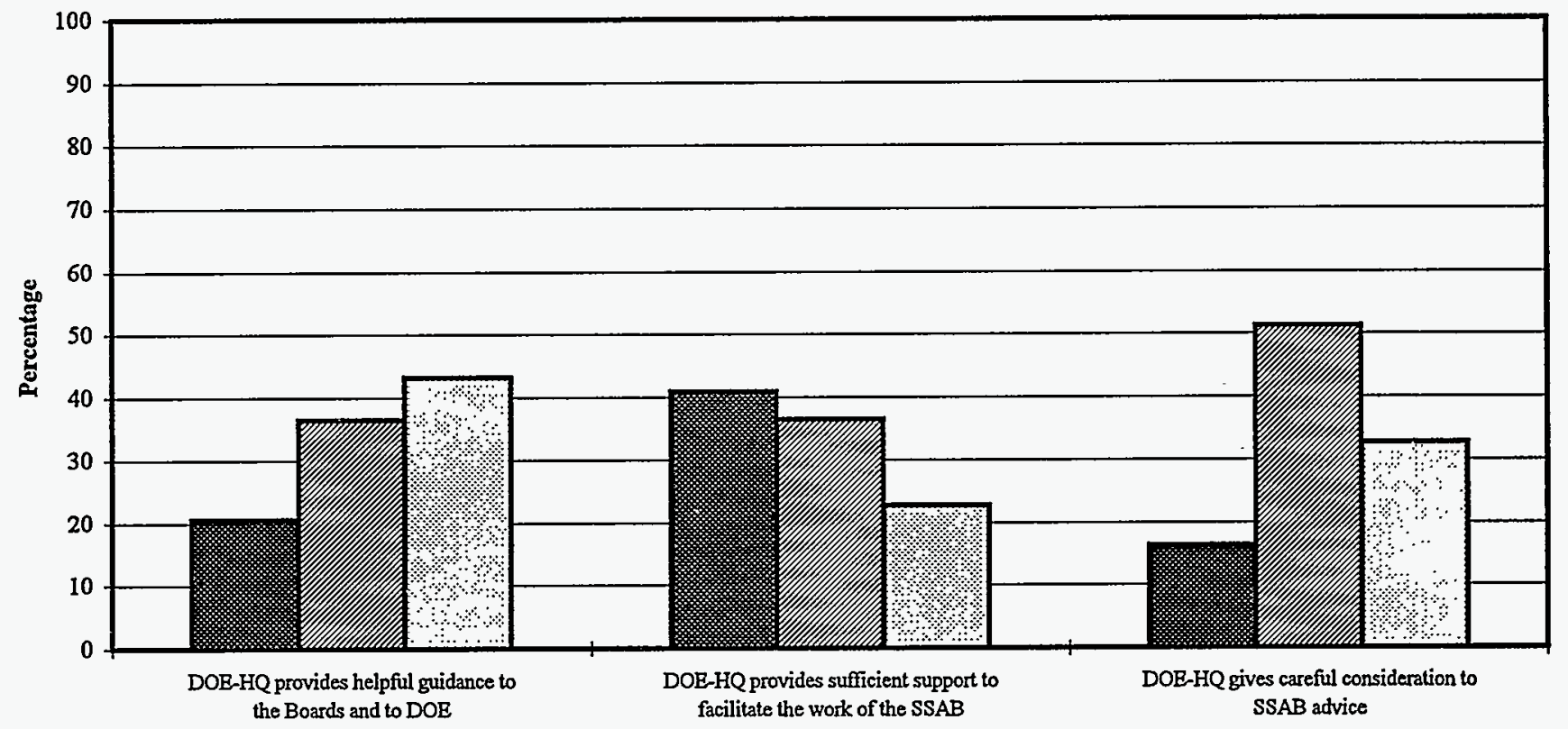

Agree (\%) Neither Agree Nor Disagree/Don't Know (\%) DDisagree (\%)

Figure 8b. Long Survey: Entire Sample

DOE-HQ involvement with the SSAB Initiative

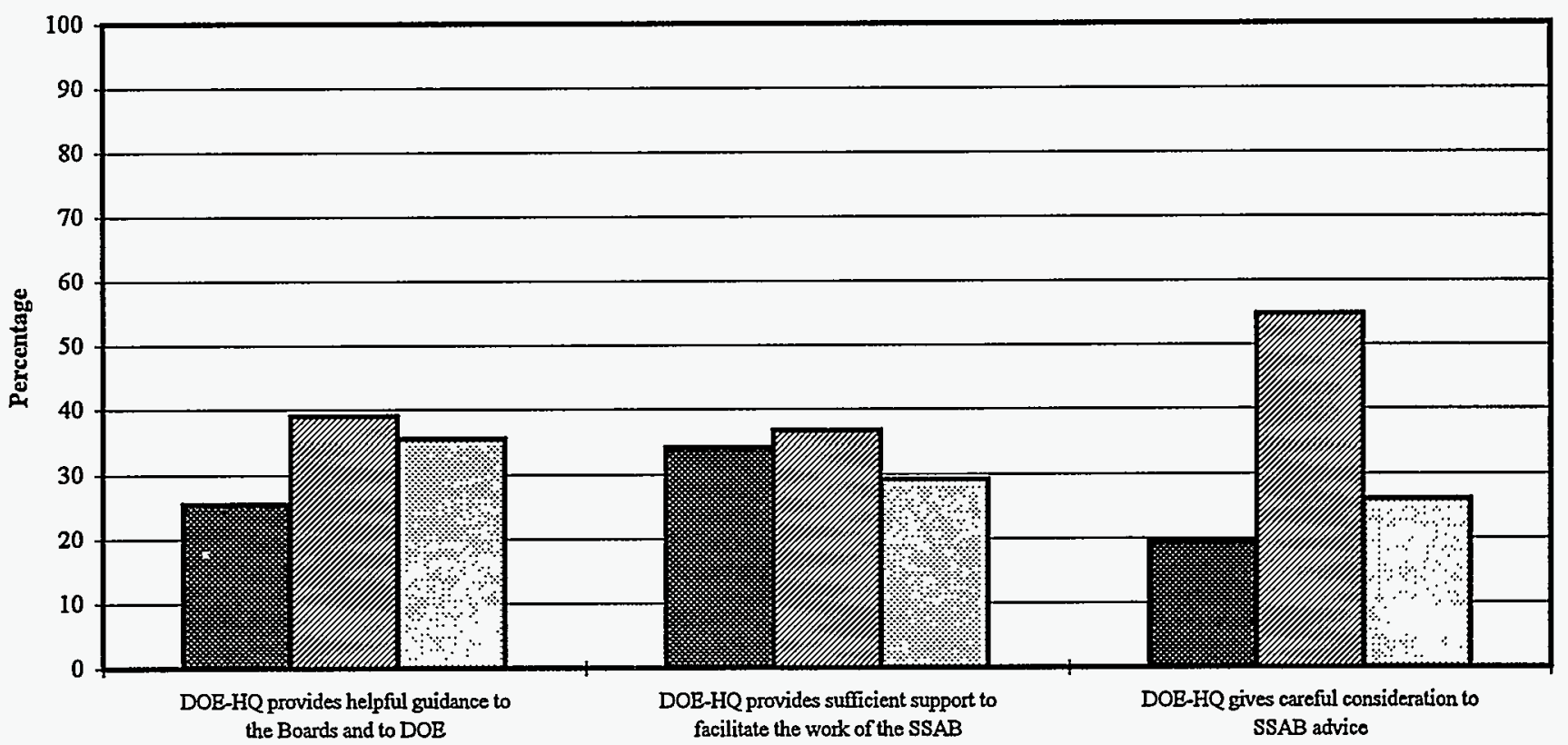

D Agree (\%) ENeither Agree Nor Disagree/Don't Know (\%) DDisagree (\%)

SSAB Supplementary Appendix

August 1996 
Figure 9a. Rocky Flats

Personal experience with the SSAB Initiative

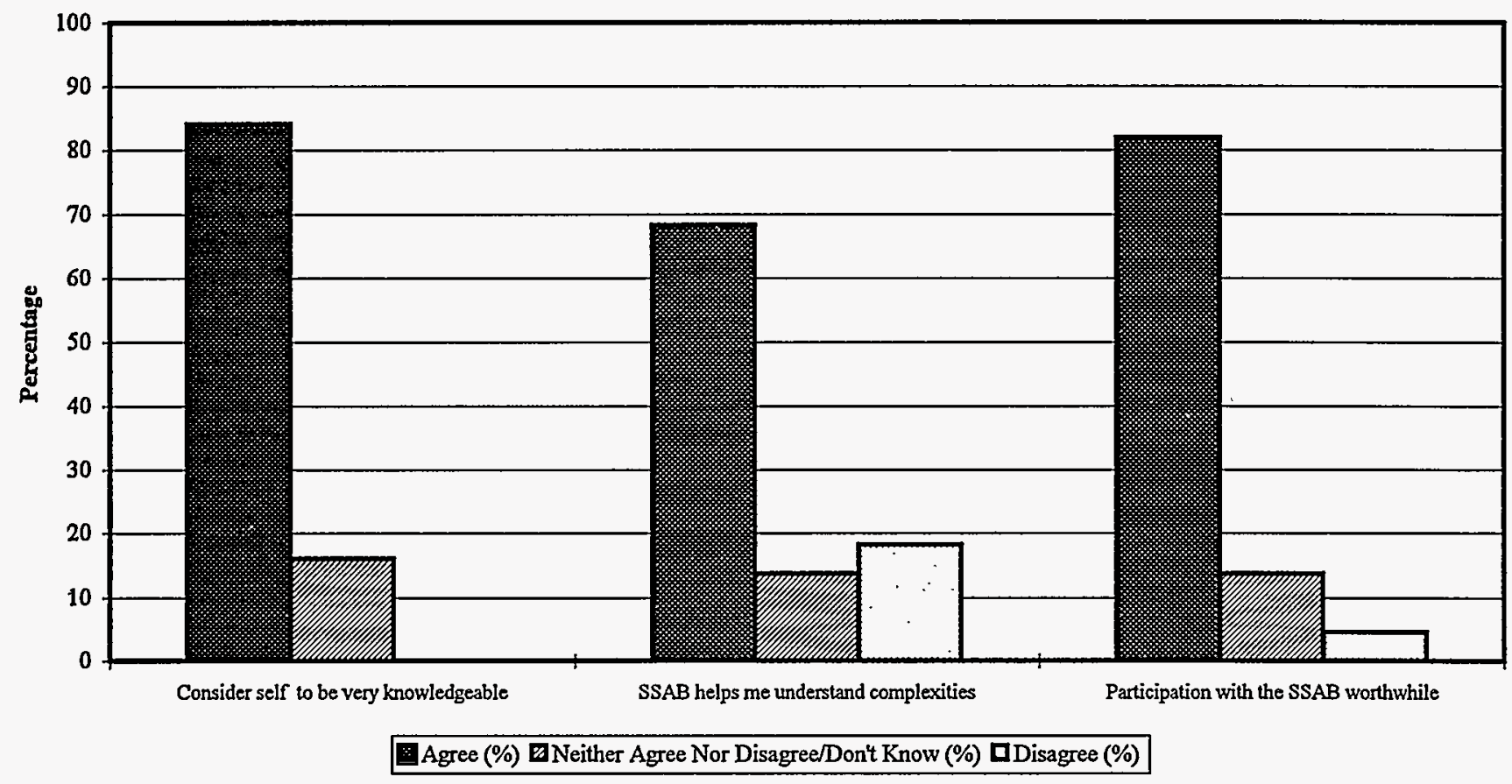

Figure 9b. Long Survey: Entire Sample Personal experience with the SSAB Initiative

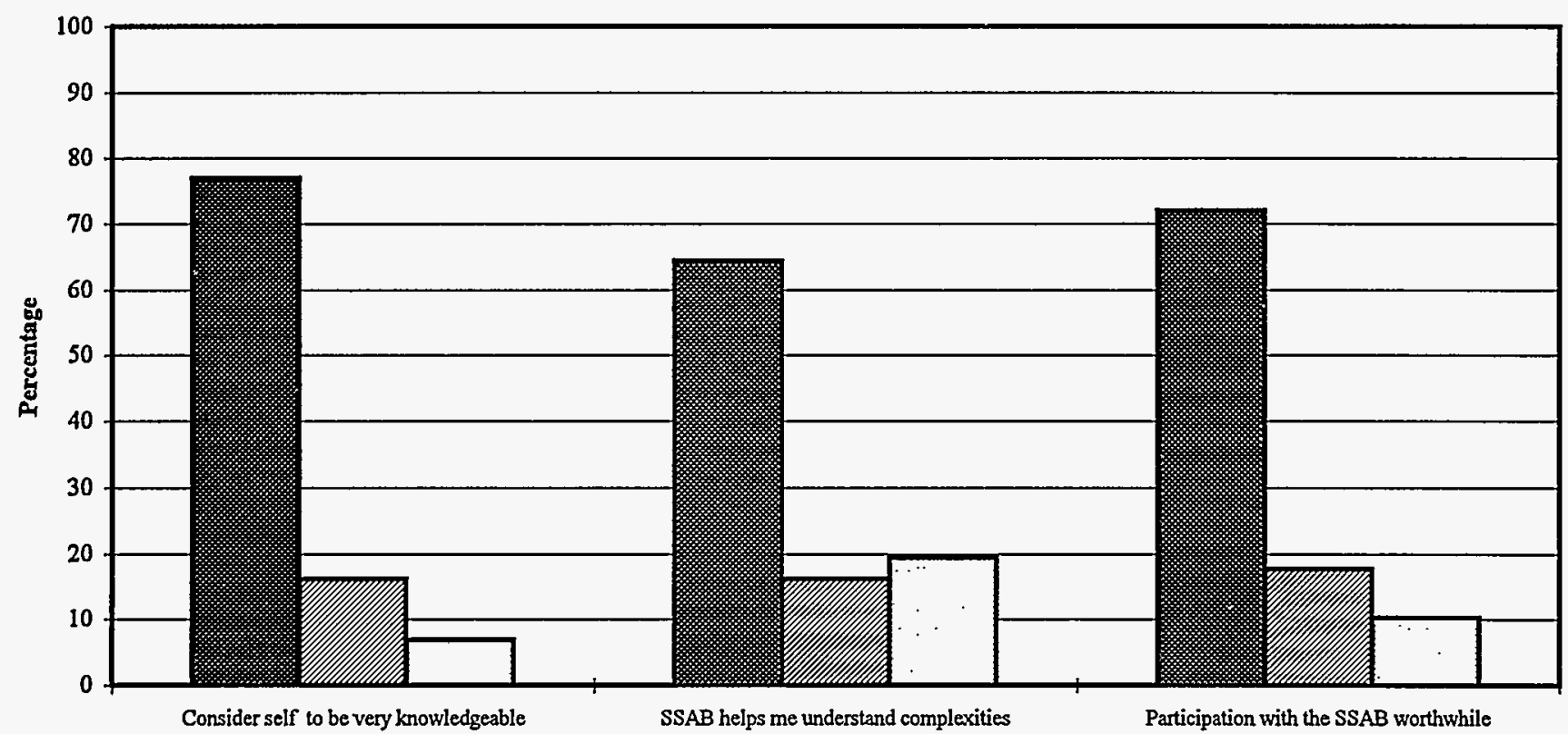

Agree (\%) Neither Agree Nor Disagree/Don't Know (\%) DDisagree (\%)

SSAB Supplementary Appendix 
Figure 10a. Rocky Flats

Group/Team work skills

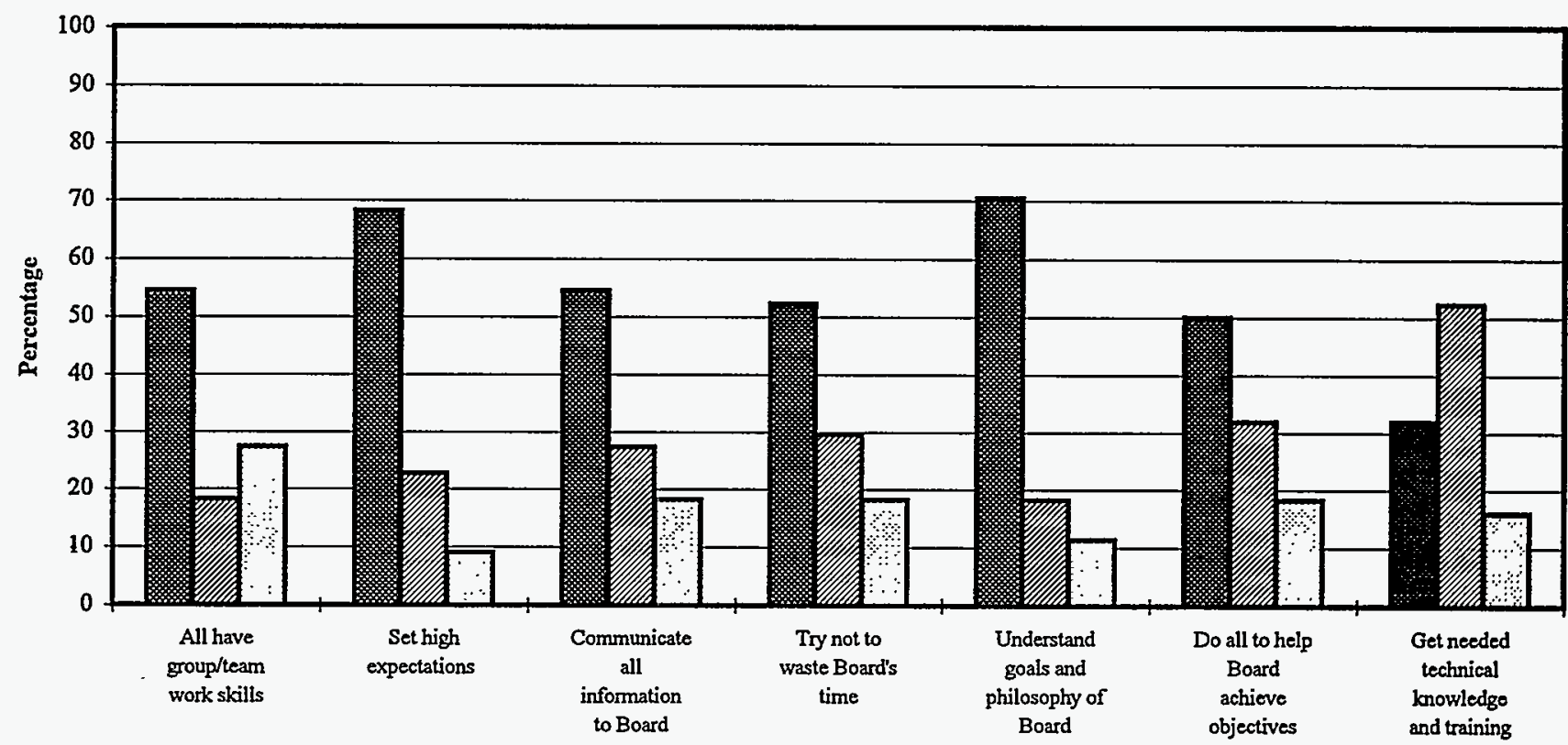

Agree (\%) Weither Agree Nor Disagree/Don't Know (\%) DDisagree (\%)

Figure 10b. Long Survey: Entire Sample Group/Team work skills

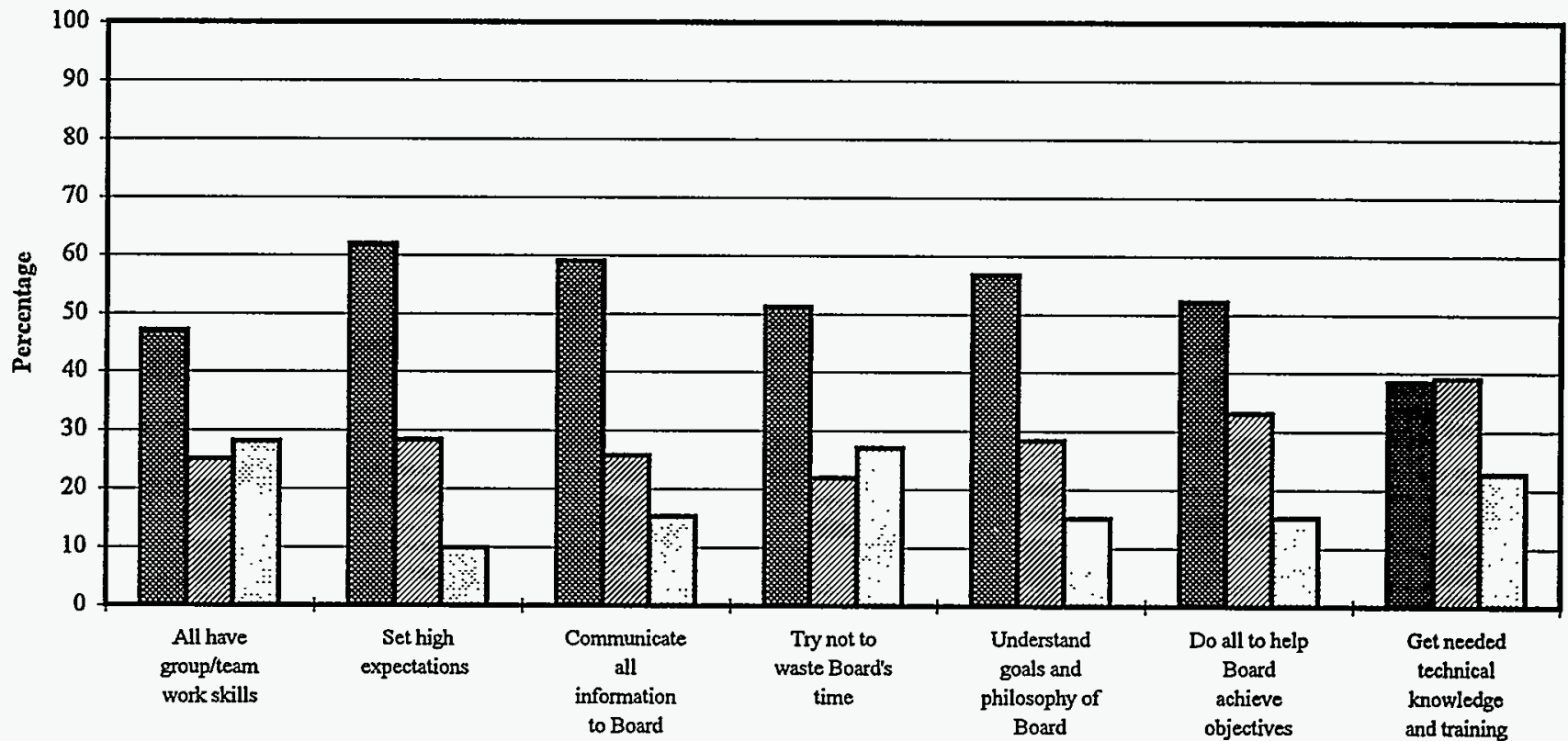

Agree (\%) Weither Agree Nor Disagree/Don't Know (\%) DDisagree (\%)

SSAB Supplementary Appendix

August 1996 
Figure 11a. Rocky Flats

Working relations

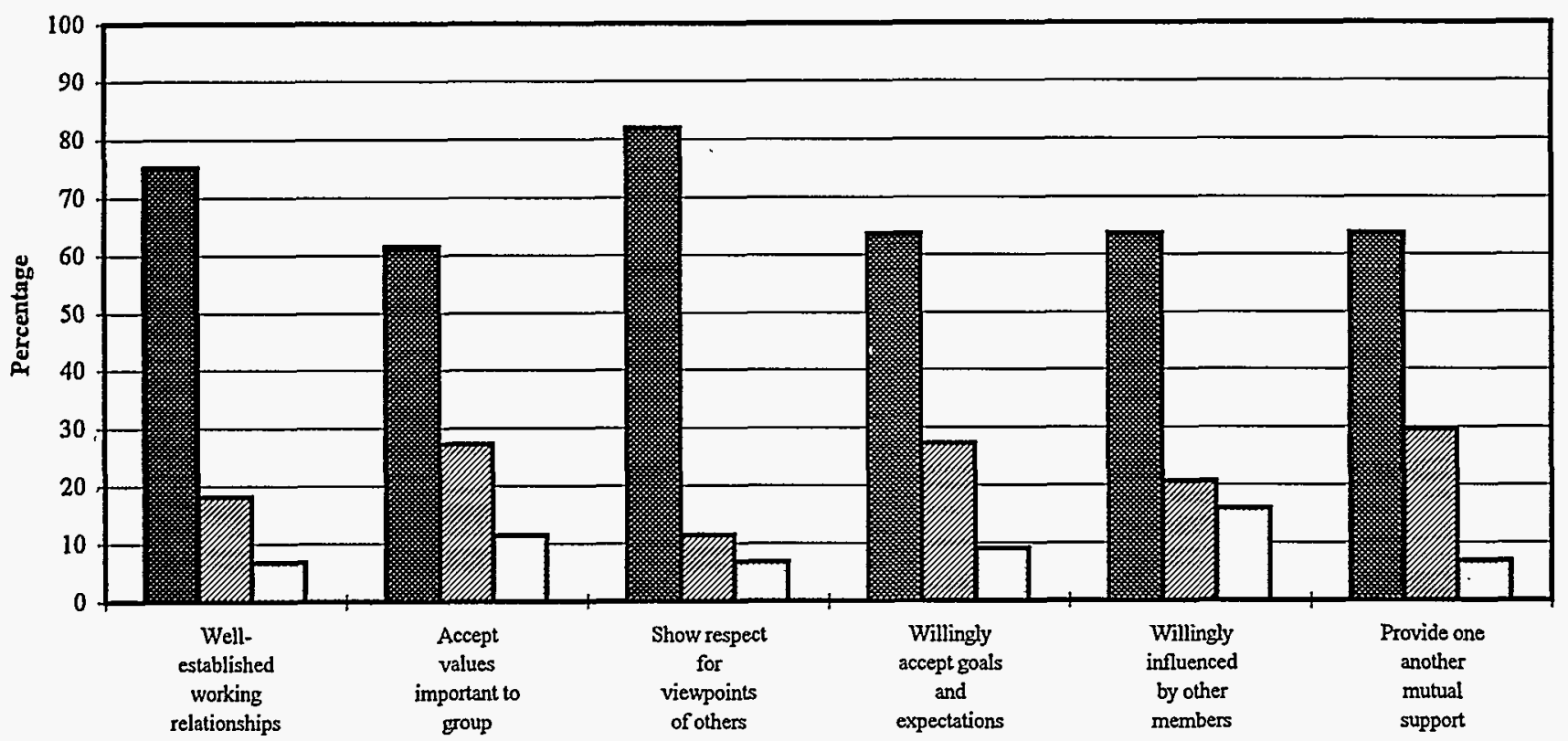

E Agree (\%) E Neither Agree Nor Disagree/Don't Know (\%) QDisagree (\%)

Figure 11b. Long Survey: Entire Sample Working relations

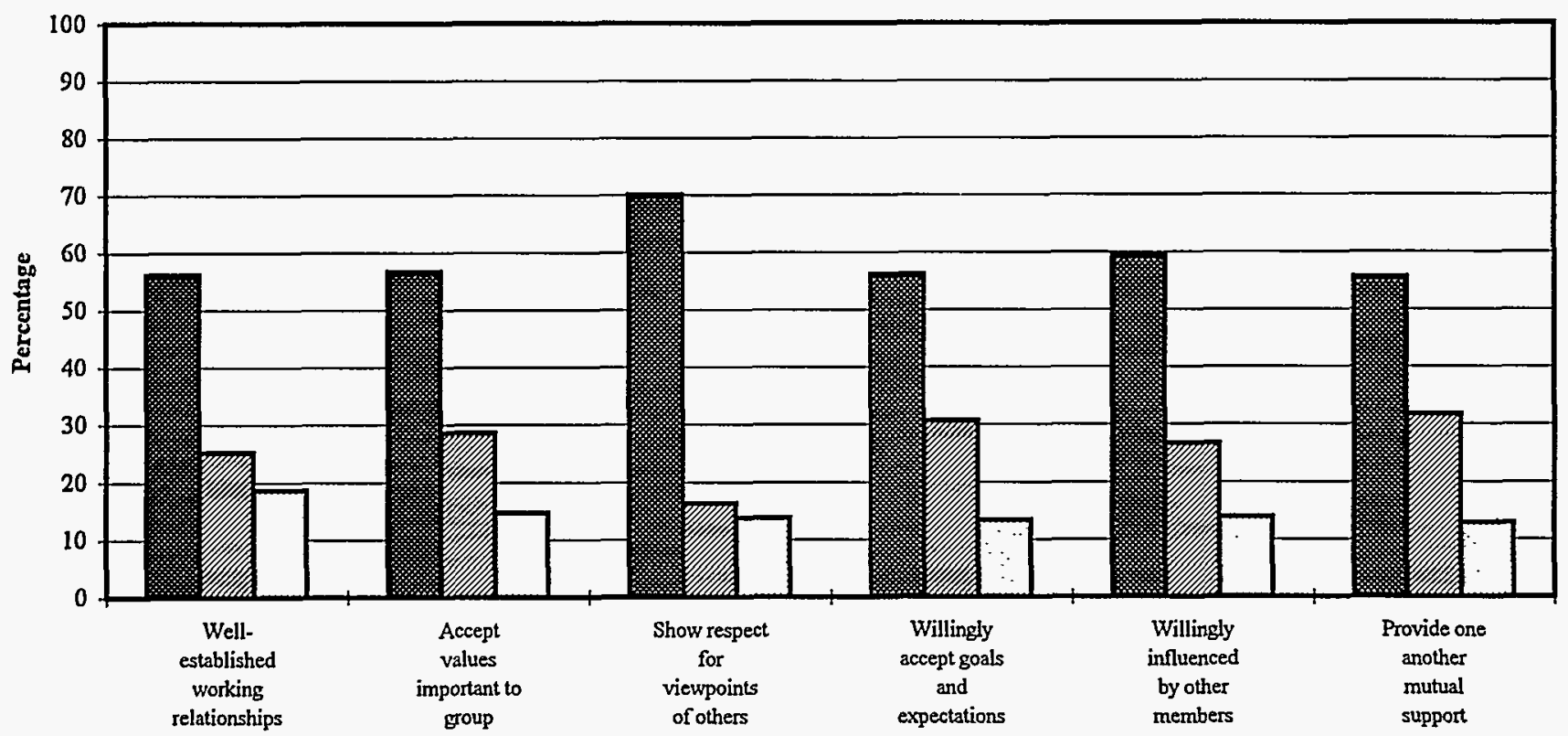

Agree (\%) Deither Agree Nor Disagree/Dont Know (\%) DDisagree (\%)

SSAB Supplementary Appendix 
Figure 12a. Rocky Flats

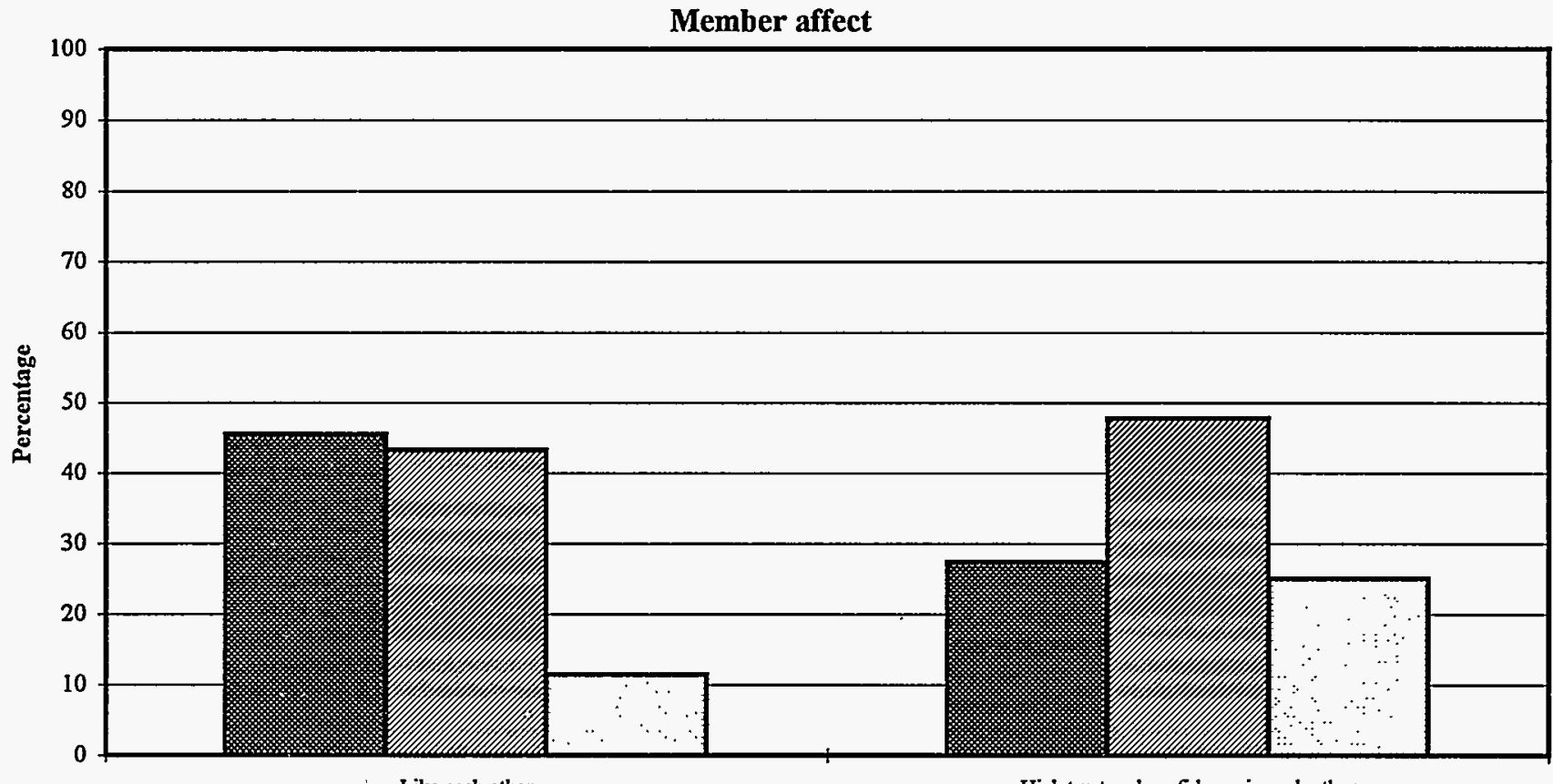

Like each other

High trust and confidence in each other

Agree (\%) Neither Agree Nor Disagree/Don't Know (\%) DDisagree (\%)

Figure 12b. Long Survey: Entire Sample

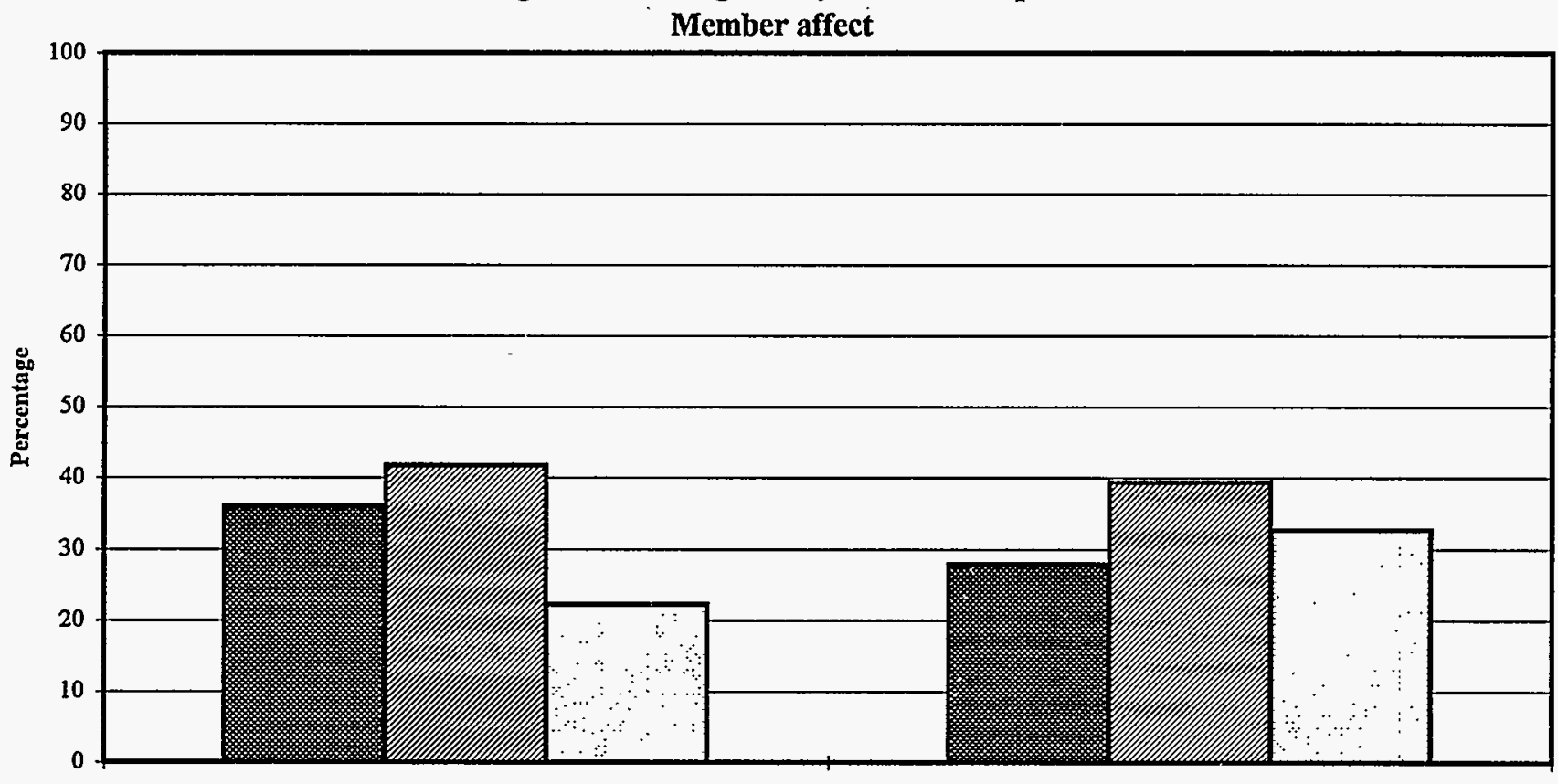

Like each other

High trust and confidence in each other

Agree (\%) Neither Agree Nor Disagree/Don't Know (\%) DDisagree (\%)

SSAB Supplementary Appendix

August 1996 
Figure 13a. Rocky Flats

Summary: Responses to open-ended questions

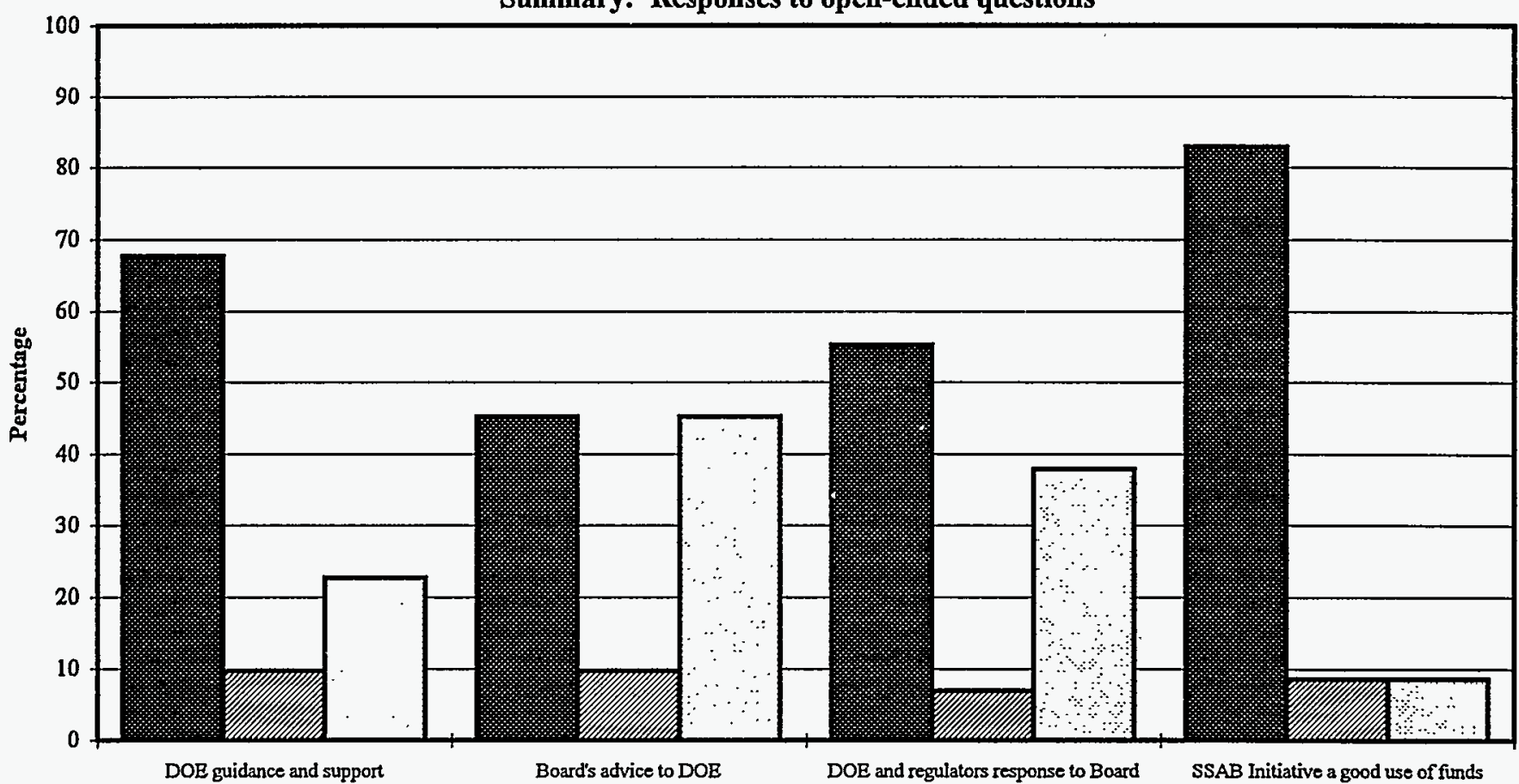

FYes, Good (\%) $\mathbf{\square}$ Neutral, Conditional, No Opinion (\%) घNo, Not Good (\%)

Figure 13b. Long Survey: Entire Sample

Summary: Responses to open-ended questions

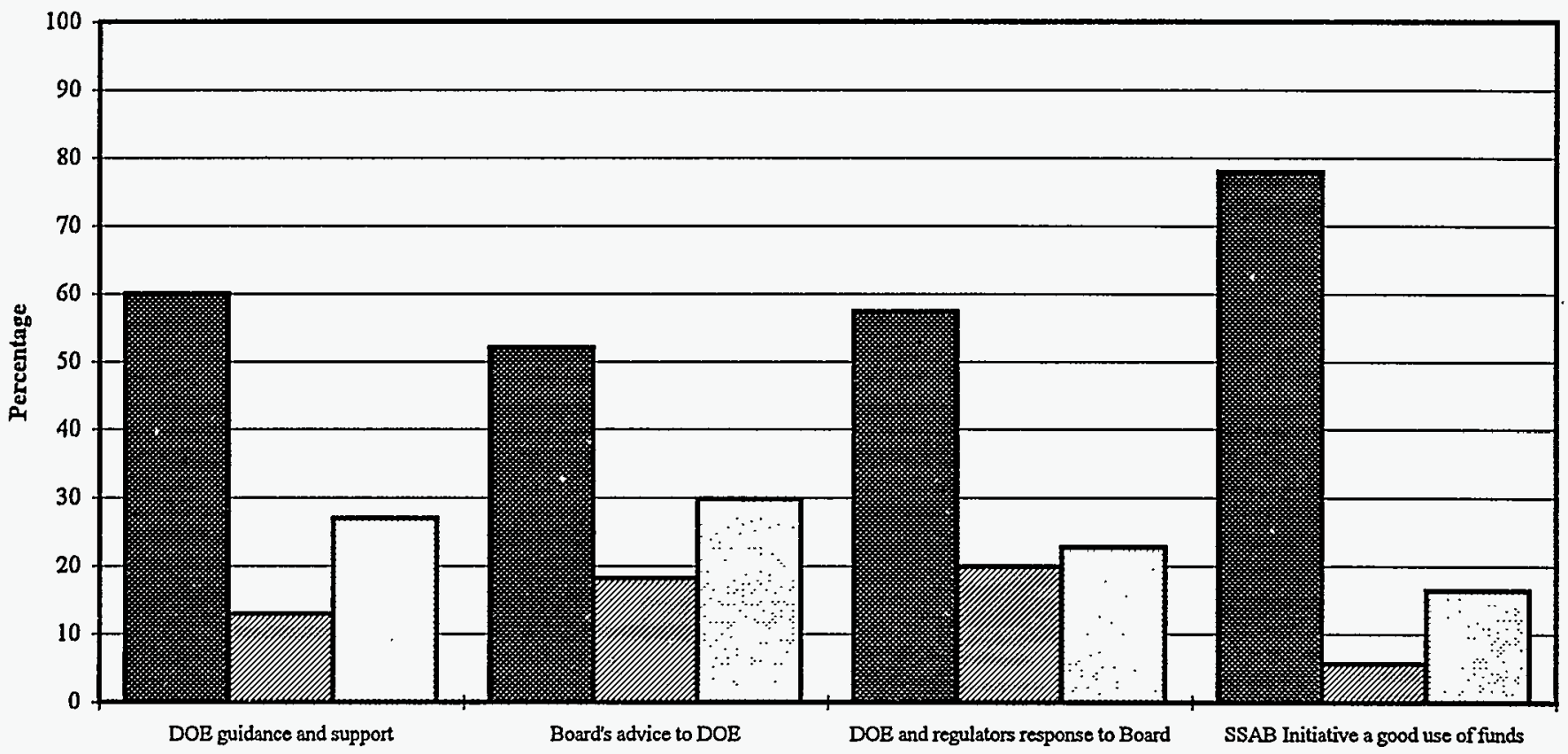

Yes, Good (\%) ENeutral, Conditional, No Opinion (\%) DNo, Not Good (\%)

SSAB Supplementary Appendix

August 1996 
Figure 14a. Rocky Flats

Summary: Combined responses for SSAB Initiative goals (Means)

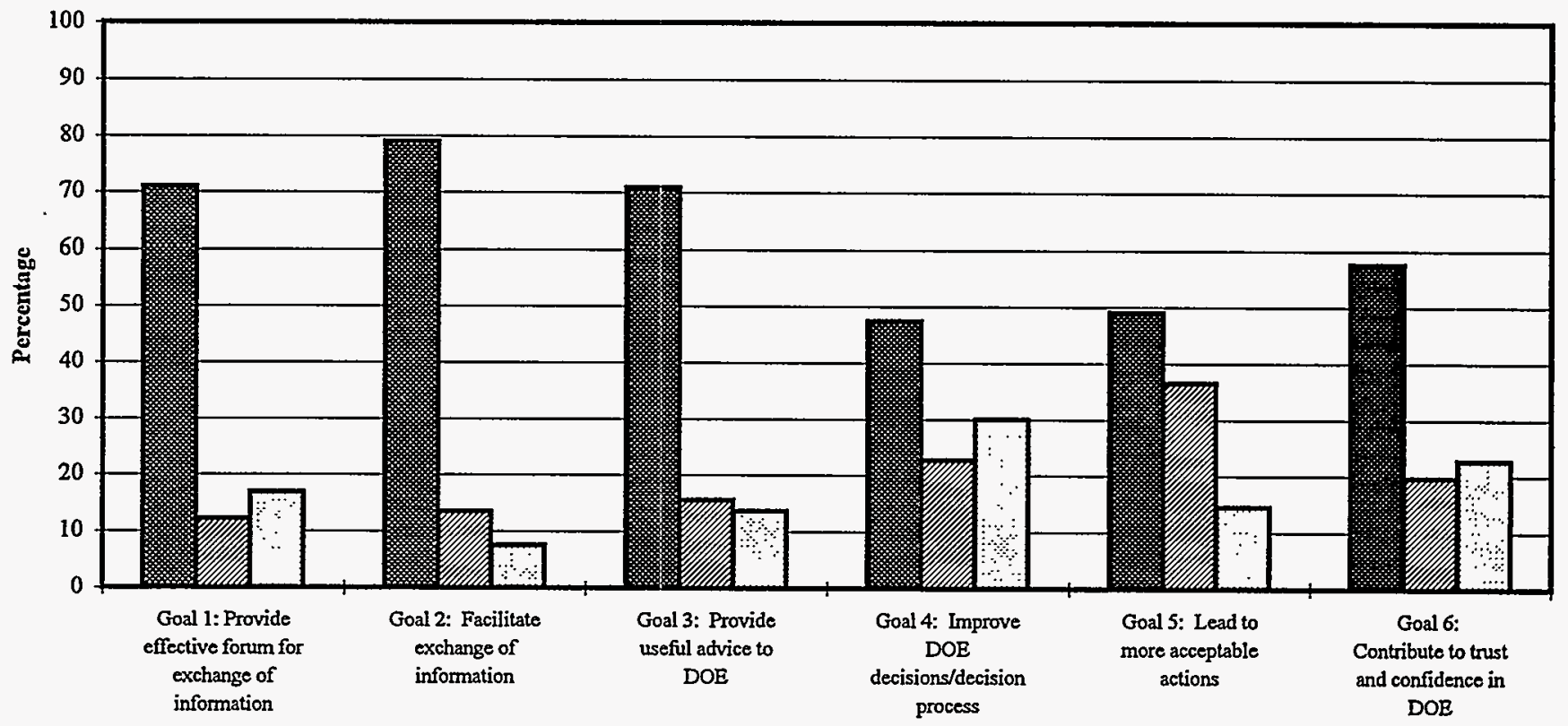

Agree (\%) ENeither Agree Nor Disagree/Don't Know (\%) DDisagree (\%)

Figure 14b. Long Survey: Entire Sample

Summary: Combined responses for SSAB Initiative goals (Means)

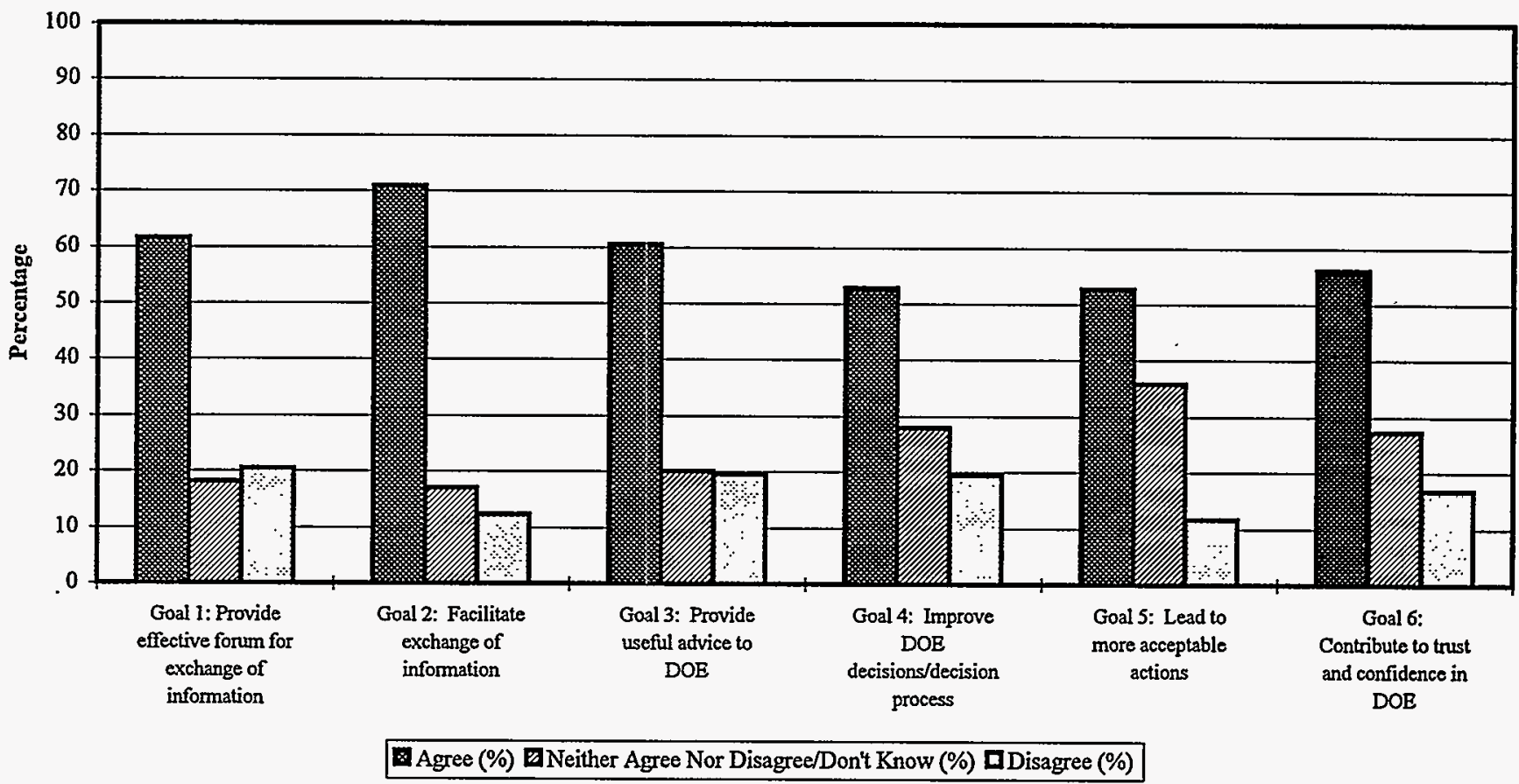

SSAB Supplementary Appendix

August 1996 
Figure 15a. Rocky Flats

Summary: Combined responses on DOE-HQ involvement,

Personal experience, Group/Team work skills,

Working relations, Member affect (Means)

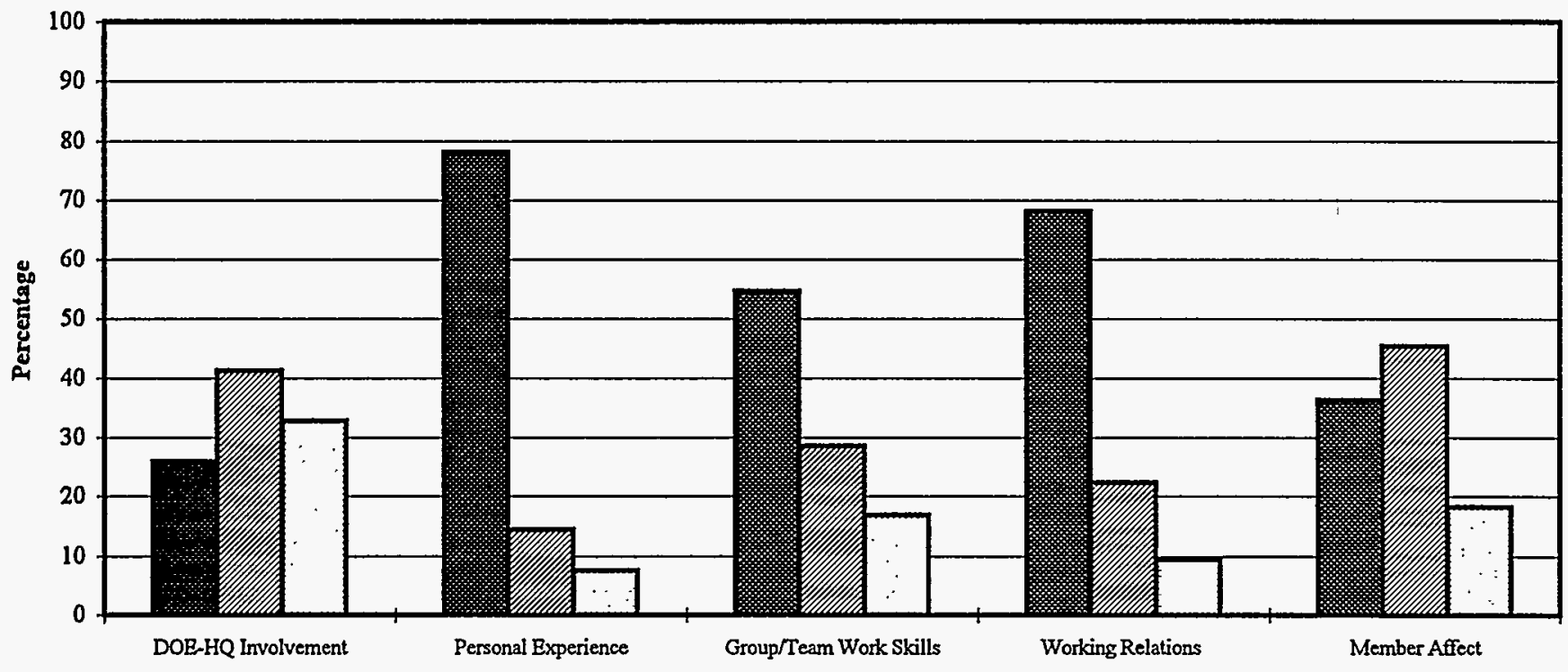

Agree (\%) ENeither Agree Nor Disagree/Don't Know (\%) DDisagree (\%)

Figure 15b. Long Survey: Entire Sample

Summary: Combined responses on DOE-HQ involvement,

Personal experience, Group/Team work skills,

Working relations, Member affect (Means)

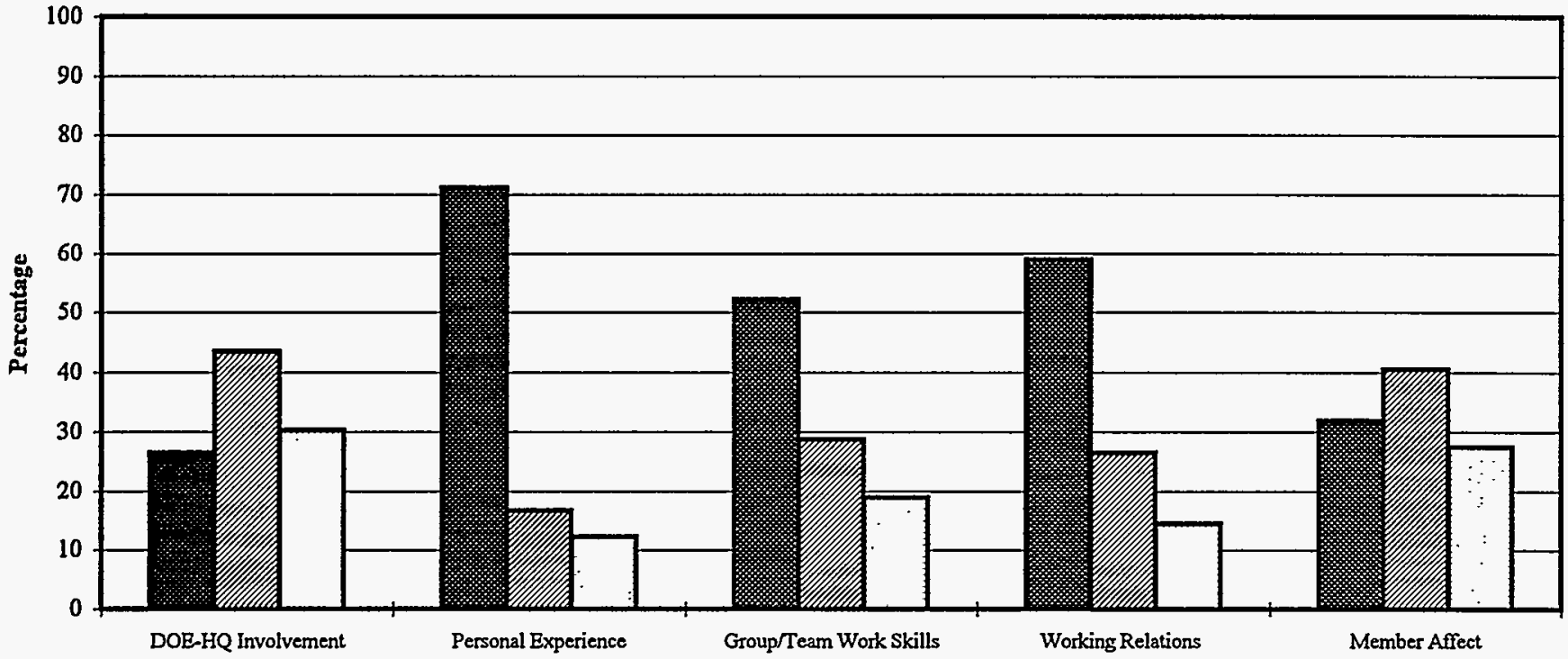

Agree (\%) ENeither Agree Nor Disagree/Don't Know (\%) DDisagree (\%)

SSAB Supplementary Appendix 
Table 1.

Goal 1. Establish processes and procedures to provide an effective forum for exchange of information and viewpoints regarding DOE site issues

\begin{tabular}{|c|c|c|c|c|c|c|c|c|}
\hline \multirow[t]{2}{*}{ Rocky Flats } & \multicolumn{2}{|c|}{ Agree } & \multicolumn{2}{|c|}{$\begin{array}{l}\text { Neither/ } \\
\text { Don't Know }\end{array}$} & \multicolumn{2}{|c|}{ Disagree } & \multicolumn{2}{|c|}{ Total } \\
\hline & $\mathbf{N}$ & $\%$ & $N$ & $\%$ & $\mathrm{~N}$ & $\%$ & $\mathrm{~N}$ & $\%$ \\
\hline $\begin{array}{l}\text { The Board has established processes and } \\
\text { procedures for the effective exchange of } \\
\text { information }\end{array}$ & 39 & $88.6 \%$ & 1 & $2.3 \%$ & 4 & $9.1 \%$ & 44 & $100 \%$ \\
\hline $\begin{array}{l}\text { The Board has agreed-upon procedures to } \\
\text { operate the board }\end{array}$ & 43 & $97.7 \%$ & 1 & $2.3 \%$ & 0 & $0 \%$ & 44 & $100 \%$ \\
\hline $\begin{array}{l}\text { The Board has adequate support to allow } \\
\text { it to focus on substantive issues (R) * }\end{array}$ & 37 & $84.1 \%$ & 0 & $0 \%$ & 7 & $15.9 \%$ & 44 & $100 \%$ \\
\hline $\begin{array}{l}\text { The Board provides sufficient time for } \\
\text { discussion of issues }\end{array}$ & 29 & $65.9 \%$ & 8 & $18.2 \%$ & 7 & $15.9 \%$ & 44 & $100 \%$ \\
\hline The Board has effective leadership & 29 & $65.9 \%$ & 4 & $9.1 \%$ & 11 & $25.0 \%$ & 44 & $100 \%$ \\
\hline $\begin{array}{l}\text { DOE requests Board advice far enough } \\
\text { ahead of decision deadlines }(R) *\end{array}$ & 11 & $25.0 \%$ & 5 & $11.4 \%$ & 28 & $63.6 \%$ & 44 & $100 \%$ \\
\hline $\begin{array}{l}\text { DOE handles Board administrative issues } \\
\text { expeditiously (R) * }\end{array}$ & 18 & $40.9 \%$ & 20 & $45.5 \%$ & 6 & $13.6 \%$ & 44 & $100 \%$ \\
\hline $\begin{array}{l}\text { The SSAB staff provide satisfactory } \\
\text { support }\end{array}$ & 39 & $88.6 \%$ & 3 & $6.8 \%$ & 2 & $4.5 \%$ & 44 & $100 \%$ \\
\hline $\begin{array}{l}\text { The facilitator for the Board has helped } \\
\text { the Board function effectively }\end{array}$ & 36 & $81.8 \%$ & 6 & $13.6 \%$ & 2 & $4.5 \%$ & 44 & $100 \%$ \\
\hline
\end{tabular}

* (R) denotes a question that was asked in the negative form. For ease of review, these questions and data have been reversed in this table and are stated in positive forms.

SSAB Supplementary Appendix

August 1996 
Table 2.

Goal 2. Facilitate interaction and exchange of information and viewpoints regarding DOE site issues

\begin{tabular}{|c|c|c|c|c|c|c|c|c|}
\hline \multirow[t]{2}{*}{ Rocky Flats } & \multicolumn{2}{|c|}{ Agree } & \multicolumn{2}{|c|}{$\begin{array}{l}\text { Neither/ } \\
\text { Don't Know }\end{array}$} & \multicolumn{2}{|c|}{ Disagree } & \multicolumn{2}{|c|}{ Total } \\
\hline & $\mathrm{N}$ & $\%$ & $N$ & $\%$ & $\mathrm{~N}$ & $\%$ & $\mathrm{~N}$ & $\%$ \\
\hline $\begin{array}{l}\text { The SSAB facilitates effective exchange } \\
\text { of viewpoints on site issues }\end{array}$ & 37 & $84.1 \%$ & 2 & $4.5 \%$ & 5 & $11.4 \%$ & 44 & $100 \%$ \\
\hline $\begin{array}{l}\text { The SSAB contributes to Board members' } \\
\text { understanding of the basis for key site } \\
\text { decisions }\end{array}$ & 39 & $88.6 \%$ & 5 & $11.4 \%$ & 0 & $0 \%$ & 44 & $100 \%$ \\
\hline $\begin{array}{l}\text { The SSAB contributes to DOE and } \\
\text { regulators' understanding of the public's } \\
\text { viewpoints on key site decisions }\end{array}$ & 34 & $77.3 \%$ & 6 & $13.6 \%$ & 4 & $9.1 \%$ & 44 & $100 \%$ \\
\hline $\begin{array}{l}\text { The SSAB contributes to a constructive } \\
\text { working relationship among the } \\
\text { participants }\end{array}$ & 37 & $84.1 \%$ & 6 & $13.6 \%$ & 1 & $2.3 \%$ & 44 & $100 \%$ \\
\hline The SSAB strives for consensus & 38 & $86.4 \%$ & 4 & $9.1 \%$ & 2 & $4.5 \%$ & 44 & $100 \%$ \\
\hline $\begin{array}{l}\text { The SSAB gives fair consideration to } \\
\text { dissenting opinions }\end{array}$ & 33 & $76.7 \%$ & 7 & $16.3 \%$ & 3 & $7.0 \%$ & 43 & $100 \%$ \\
\hline $\begin{array}{l}\text { The Board has made the effort needed to } \\
\text { learn about site issues }\end{array}$ & 41 & $93.2 \%$ & 2 & $4.5 \%$ & 1 & $2.3 \%$ & 44 & $100 \%$ \\
\hline $\begin{array}{l}\text { The Board invites expert advice into its } \\
\text { discussion on key policy issues }\end{array}$ & 37 & $84.1 \%$ & 3 & $6.8 \%$ & 4 & $9.1 \%$ & 44 & $100 \%$ \\
\hline $\begin{array}{l}\text { The Board responds to public inquiries } \\
\text { and comments about its decisions }\end{array}$ & 33 & $75.0 \%$ & 10 & $22.7 \%$ & 1 & $2.3 \%$ & 44 & $100 \%$ \\
\hline $\begin{array}{l}\text { The Board solicits feedback from the } \\
\text { community on its work }\end{array}$ & 26 & $59.1 \%$ & 10 & $22.7 \%$ & 8 & $18.2 \%$ & 44 & $100 \%$ \\
\hline $\begin{array}{l}\text { DOE makes information on key site issues } \\
\text { readily available to the Board }\end{array}$ & 30 & $68.2 \%$ & 8 & $18.2 \%$ & 6 & $13.6 \%$ & 44 & $100 \%$ \\
\hline $\begin{array}{l}\text { The regulators make important } \\
\text { contributions to the Board's work }\end{array}$ & 31 & $70.5 \%$ & 10 & $22.7 \%$ & 3 & $6.8 \%$ & 44 & $100 \%$ \\
\hline $\begin{array}{l}\text { The SSAB creates a climate supportive of } \\
\text { differing viewpoints }(\mathrm{R})^{*}\end{array}$ & 35 & $79.5 \%$ & 4 & $9.1 \%$ & 5 & $11.4 \%$ & 44 & $100 \%$ \\
\hline
\end{tabular}

* (R) denotes a question that was asked in the negative form. For ease of review, these questions and data have been reversed in this table and are stated in positive forms.

SSAB Supplementary Appendix

August 1996 


\section{Table 3.}

Goal 3. Provide useful advice and/or recommendations to DOE

(and regulators, where appropriate)

\begin{tabular}{|c|c|c|c|c|c|c|c|c|}
\hline \multirow[t]{2}{*}{ Rocky Flats } & \multicolumn{2}{|c|}{ Agree } & \multicolumn{2}{|c|}{$\begin{array}{l}\text { Neither/ } \\
\text { Don't Know }\end{array}$} & \multicolumn{2}{|c|}{ Disagree } & \multicolumn{2}{|c|}{ Total } \\
\hline & $\mathrm{N}$ & $\%$ & $\mathrm{~N}$ & $\%$ & $\mathrm{~N}$ & $\%$ & $\mathrm{~N}$ & $\%$ \\
\hline $\begin{array}{l}\text { The Board provides useful advice to } \mathrm{DOE} \\
\text { (and regulators, where appropriate) }(\mathrm{R})^{*}\end{array}$ & 35 & $79.5 \%$ & 7 & $15.9 \%$ & 2 & $4.5 \%$ & 44 & $100 \%$ \\
\hline $\begin{array}{l}\text { The SSAB reaches agreement about } \\
\text { prioritization of key site issues for which } \\
\text { advice is sought }(\mathrm{R}) *\end{array}$ & 27 & $61.4 \%$ & 10 & $22.7 \%$ & 7 & $15.9 \%$ & 44 & $100 \%$ \\
\hline The Board helps define site problems & 38 & $86.4 \%$ & 4 & $9.1 \%$ & 2 & $4.5 \%$ & 44 & $100 \%$ \\
\hline $\begin{array}{l}\text { The Board addresses issues in a timely } \\
\text { manner }(\mathrm{R}) \text { * }\end{array}$ & 30 & $68.2 \%$ & 6 & $13.6 \%$ & 8 & $18.2 \%$ & 44 & $100 \%$ \\
\hline $\begin{array}{l}\text { The Board reaches consensus on key site } \\
\text { issues }(\mathrm{R})^{*}\end{array}$ & 27 & $61.4 \%$ & 5 & $11.4 \%$ & 12 & $27.3 \%$ & 44 & $100 \%$ \\
\hline $\begin{array}{l}\text { The Board provides informed advice to } \\
\text { DOE (and regulators, where appropriate) }\end{array}$ & 33 & $75.0 \%$ & 7 & $15.9 \%$ & 4 & $9.1 \%$ & 44 & $100 \%$ \\
\hline $\begin{array}{l}\text { The Board provides advice that reflects } \\
\text { the viewpoints and priorities of the } \\
\text { community }\end{array}$ & 28 & $63.6 \%$ & 9 & $20.5 \%$ & 7 & $15.9 \%$ & 44 & $100 \%$ \\
\hline
\end{tabular}

* (R) denotes a question that was asked in the negative form. For ease of review, these questions and data have been reversed in this table and are stated in positive forms.

SSAB Supplementary Appendix 
Table 4.

Goal 4. Improve DOE's (and where applicable, regulators') site decisions and decision making process

\begin{tabular}{|c|c|c|c|c|c|c|c|c|}
\hline \multirow[t]{2}{*}{ Rocky Flats } & \multicolumn{2}{|c|}{ Agree } & \multicolumn{2}{|c|}{$\begin{array}{c}\text { Neither/ } \\
\text { Don't Know }\end{array}$} & \multicolumn{2}{|c|}{ Disagree } & \multicolumn{2}{|c|}{ Total } \\
\hline & $N$ & $\%$ & $\mathrm{~N}$ & $\%$ & $\mathrm{~N}$ & $\%$ & $N$ & $\%$ \\
\hline $\begin{array}{l}\text { The SSAB has improved DOE's site } \\
\text { decisions (R) * }\end{array}$ & 25 & $56.8 \%$ & 9 & $20.5 \%$ & 10 & $22.7 \%$ & 44 & $100 \%$ \\
\hline $\begin{array}{l}\text { The Board understands the decision } \\
\text { making process at [a particular] site }\end{array}$ & 22 & $50.0 \%$ & 11 & $25.0 \%$ & 11 & $25.0 \%$ & 44 & $100 \%$ \\
\hline $\begin{array}{l}\text { DOE discusses important policies } \\
\text { affecting site decisions with the Board }\end{array}$ & 30 & $68.2 \%$ & 9 & $20.5 \%$ & 5 & $11.4 \%$ & 44 & $100 \%$ \\
\hline $\begin{array}{l}\text { DOE has explained to the Board its site } \\
\text { decision making process }\end{array}$ & 18 & $40.9 \%$ & 8 & $18.2 \%$ & 18 & $40.9 \%$ & 44 & $100 \%$ \\
\hline $\begin{array}{l}\text { The DOE shows how Board advice is } \\
\text { reflected in site decisions }\end{array}$ & 15 & $34.1 \%$ & 13 & $29.5 \%$ & 16 & $36.4 \%$ & 44 & $100 \%$ \\
\hline $\begin{array}{l}\text { The decision making process used by } \\
\text { DOE at [a particular] site is effective in } \\
\text { furthering site clean-up (R) }{ }^{*}\end{array}$ & 15 & $34.1 \%$ & 10 & $22.7 \%$ & 19 & $43.2 \%$ & 44 & $100 \%$ \\
\hline
\end{tabular}

* (R) denotes a question that was asked in the negative form. For ease of review, these questions and data have been reversed in this table and are stated in positive forms.

SSAB Supplementary Appendix

August 1996 
Table 5.

Goal 5. Lead to more acceptable actions

\begin{tabular}{|c|c|c|c|c|c|c|c|c|}
\hline \multirow[t]{2}{*}{ Rocky Flats } & \multicolumn{2}{|c|}{ Agree } & \multicolumn{2}{|c|}{$\begin{array}{c}\text { Neither/ } \\
\text { Don't Know }\end{array}$} & \multicolumn{2}{|c|}{ Disagree } & \multicolumn{2}{|c|}{ Total } \\
\hline & $\mathrm{N}$ & $\%$ & $N$ & $\%$ & $\mathrm{~N}$ & $\%$ & $\mathrm{~N}$ & $\%$ \\
\hline $\begin{array}{l}\text { The SSAB leads to more acceptable site } \\
\text { decisions }\end{array}$ & 25 & $58.1 \%$ & 12 & $27.9 \%$ & 6 & $14.0 \%$ & 43 & $100 \%$ \\
\hline $\begin{array}{l}\text { The Board supports the recommendations } \\
\text { it gives DOE }\end{array}$ & 35 & $79.5 \%$ & 8 & $18.2 \%$ & 1 & $2.3 \%$ & 44 & $100 \%$ \\
\hline The Board supports DOE's site actions & 6 & $13.6 \%$ & 28 & $63.6 \%$ & 10 & $22.7 \%$ & 44 & $100 \%$ \\
\hline $\begin{array}{l}\text { The DOE pays attention to the Board's } \\
\text { advice on key site issues (R) * }\end{array}$ & 15 & $34.1 \%$ & 16 & $36.4 \%$ & 13 & $29.5 \%$ & 44 & $100 \%$ \\
\hline $\begin{array}{l}\text { There is support in the community for } \\
\text { DOE's site decisions that have SSAB } \\
\text { input }\end{array}$ & 16 & $36.4 \%$ & 24 & $54.5 \%$ & 4 & $9.1 \%$ & 44 & $100 \%$ \\
\hline Progress is being made on key site issues & 32 & $72.7 \%$ & 8 & $18.2 \%$ & 4 & $9.1 \%$ & 44 & $100 \%$ \\
\hline
\end{tabular}

* (R) denotes a question that was asked in the negative form. For ease of review, these questions and data have been reversed in this table and are stated in positive forms.

SSAB Supplementary Appendix

August 1996 
Table 6.

Goal 6. Contribute to trust and confidence in DOE

\begin{tabular}{|c|c|c|c|c|c|c|c|c|}
\hline \multirow[t]{2}{*}{ Rocky Flats } & \multicolumn{2}{|c|}{ Agree } & \multicolumn{2}{|c|}{$\begin{array}{c}\text { Neither/ } \\
\text { Don't Know }\end{array}$} & \multicolumn{2}{|c|}{ Disagree } & \multicolumn{2}{|c|}{ Total } \\
\hline & $N$ & $\%$ & $\mathrm{~N}$ & $\%$ & $N$ & $\%$ & $\mathrm{~N}$ & $\%$ \\
\hline $\begin{array}{l}\text { The SSAB contributes to trust and } \\
\text { confidence in DOE }\end{array}$ & 22 & $50.0 \%$ & 12 & $27.3 \%$ & 10 & $22.7 \%$ & 44 & $100 \%$ \\
\hline $\begin{array}{l}\text { Relationships between DOE and the public } \\
\text { have improved since the formation of the } \\
\text { SSAB }\end{array}$ & 35 & $79.5 \%$ & 4 & $9.1 \%$ & 5 & $11.4 \%$ & 44 & $100 \%$ \\
\hline $\begin{array}{l}\text { DOE is committed to clean up [a } \\
\text { particular] site }\end{array}$ & 19 & $43.2 \%$ & 10 & $22.7 \%$ & 15 & $34.1 \%$ & 44 & $100 \%$ \\
\hline
\end{tabular}


Table 7.

$\therefore$ Public awareness

\begin{tabular}{lllllllllll}
\hline \multicolumn{1}{l}{ Rocky Flats } & $\cdots$ & Agree & $\begin{array}{c}\text { Neither/ } \\
\text { Don't Know }\end{array}$ & Disagree & Total \\
& & $\mathrm{N}$ & $\%$ & $\mathrm{~N}$ & $\%$ & $\mathrm{~N}$ & $\%$ & $\mathrm{~N}$ & $\%$ \\
\hline $\begin{array}{l}\text { The public knows little about the role of } \\
\text { the Board }\end{array}$ & 23 & $53.5 \%$ & 12 & $27.9 \%$ & 8 & $18.6 \%$ & 43 & $100 \%$ \\
\hline
\end{tabular}

Table 8.

SSAB Initiative is a good use of funds

\begin{tabular}{cccccccccc}
\hline \multirow{2}{*}{ Rocky Flats } & Yes, Good & $\begin{array}{c}\text { Neutral, } \\
\text { Conditional, } \\
\text { No Opinion }\end{array}$ & $\begin{array}{c}\text { No, } \\
\text { Not Good }\end{array}$ & Total \\
& $\mathrm{N}$ & $\%$ & $\mathrm{~N}$ & $\%$ & $\mathrm{~N}$ & $\%$ & $\mathrm{~N}$ & $\%$ \\
\hline SSAB Initiative is a good use of funds & 29 & $82.9 \%$ & 3 & $8.6 \%$ & 3 & $8.6 \%$ & 35 & $100 \%$ \\
\hline
\end{tabular}


Table 9.

DOE-HQ involvement with the SSAB Initiative

\begin{tabular}{|c|c|c|c|c|c|c|c|c|}
\hline \multirow[t]{2}{*}{ Rocky Flats } & \multicolumn{2}{|c|}{ Agree } & \multicolumn{2}{|c|}{$\begin{array}{l}\text { Neither/ } \\
\text { Don't Know }\end{array}$} & \multicolumn{2}{|c|}{ Disagree } & \multicolumn{2}{|c|}{ Total } \\
\hline & $\mathbf{N}$ & $\%$ & $N^{\circ}$ & $\%$ & $N$ & $\%$ & $N$ & $\%$ \\
\hline $\begin{array}{l}\text { DOE-HQ provides helpful guidance to the } \\
\text { Boards and to DOE }\end{array}$ & 9 & $20.5 \%$ & 16 & $36.4 \%$ & 19 & $43.2 \%$ & 44 & $100 \%$ \\
\hline $\begin{array}{l}\text { DOE-HQ provides sufficient support to } \\
\text { facilitate the work of the SSAB }\end{array}$ & 18 & $40.9 \%$ & 16 & $36.4 \%$ & 10 & $22.7 \%$ & 44 & $100 \%$ \\
\hline $\begin{array}{l}\text { DOE-HQ gives careful consideration to } \\
\text { SSAB advice in its decisions }\end{array}$ & 7 & $16.3 \%$ & 22 & $51.2 \%$ & 14 & $32.6 \%$ & 44 & $100 \%$ \\
\hline
\end{tabular}


Table 10.

Personal experience with the SSAB Initiative

\begin{tabular}{|c|c|c|c|c|c|c|c|c|}
\hline \multirow[t]{2}{*}{ Rocky Flats } & \multicolumn{2}{|c|}{ Agree } & \multicolumn{2}{|c|}{$\begin{array}{c}\text { Neither/ } \\
\text { Don't Know }\end{array}$} & \multicolumn{2}{|c|}{ Disagree } & \multicolumn{2}{|c|}{ Total } \\
\hline & $N$ & $\%$ & $N$ & $\%$ & $N$ & $\%$ & $N$ & $\%$ \\
\hline $\begin{array}{l}\text { I consider myself to be very } \\
\text { knowledgeable about site issues }\end{array}$ & 37 & $84.1 \%$ & 7 & $15.9 \%$ & 0 & $0 \%$ & 44 & $100 \%$ \\
\hline $\begin{array}{l}\text { Because of the SSAB, I better understand } \\
\text { the complexities of site clean-up }\end{array}$ & 30 & $68.2 \%$ & 6 & $13.6 \%$ & 8 & $18.2 \%$ & 44 & $100 \%$ \\
\hline $\begin{array}{l}\text { I feel that my participation in the SSAB } \\
\text { has been worthwhile }(\mathrm{R}) *\end{array}$ & 36 & $81.8 \%$ & 6 & $13.6 \%$ & 2 & $4.5 \%$ & 44 & $100 \%$ \\
\hline
\end{tabular}

* (R) denotes a question that was asked in the negative form. For ease of review, these questions and data have been reversed in this table and are stated in positive forms.

SSAB Supplementary Appendix 
Table 11.

Group/Team work skills

\begin{tabular}{|c|c|c|c|c|c|c|c|c|}
\hline \multirow[t]{2}{*}{ Rocky Flats } & \multicolumn{2}{|c|}{ Agree } & \multicolumn{2}{|c|}{$\begin{array}{c}\text { Neither/ } \\
\text { Don't Know }\end{array}$} & \multicolumn{2}{|c|}{ Disagree } & \multicolumn{2}{|c|}{ Total } \\
\hline & $\mathrm{N}$ & $\%$ & $\mathbf{N}$ & $\%$ & $N$ & $\%$ & $\mathbf{N}$ & $\%$ \\
\hline All have group/team work skills & 24 & $54.5 \%$ & 8 & $18.2 \%$ & 12 & $27.3 \%$ & 44 & $100 \%$ \\
\hline $\begin{array}{l}\text { Have developed well-established working } \\
\text { relationships with each other }\end{array}$ & 33 & $75.0 \%$ & 8 & $18.2 \%$ & 3 & $6.8 \%$ & 44 & $100 \%$ \\
\hline Like each other & 20 & $45.5 \%$ & 19 & $43.2 \%$ & 5 & $11.4 \%$ & 44 & $100 \%$ \\
\hline $\begin{array}{l}\text { Have a high degree of trust and } \\
\text { confidence in each other }\end{array}$ & 12 & $27.3 \%$ & 21 & $47.7 \%$ & 11 & $25.0 \%$ & 44 & $100 \%$ \\
\hline $\begin{array}{l}\text { Accept the values that are important to the } \\
\text { group }\end{array}$ & 27 & $61.4 \%$ & 12 & $27.3 \%$ & 5 & $11.4 \%$ & 44 & $100 \%$ \\
\hline Show respect for the viewpoints of others & 36 & $81.8 \%$ & 5 & $11.4 \%$ & 3 & $6.8 \%$ & 44 & $100 \%$ \\
\hline $\begin{array}{l}\text { Accept willingly the goals and } \\
\text { expectations for the Board }\end{array}$ & 28 & $63.6 \%$ & 12 & $27.3 \%$ & 4 & $9.1 \%$ & 44 & $100 \%$ \\
\hline $\begin{array}{l}\text { Set high expectations for what they } \\
\text { believe they can accomplish }\end{array}$ & 30 & $68.2 \%$ & 10 & $22.7 \%$ & 4 & $9.1 \%$ & 44 & $100 \%$ \\
\hline $\begin{array}{l}\text { Communicate fully and frankly to the } \\
\text { Board all relevant and valuable } \\
\text { information }\end{array}$ & 24 & $54.5 \%$ & 12 & $27.3 \%$ & 8 & $18.2 \%$ & 44 & $100 \%$ \\
\hline $\begin{array}{l}\text { Try not to waste the Board's time with } \\
\text { irrelevant material or communications }\end{array}$ & 23 & $52.3 \%$ & 13 & $29.5 \%$ & 8 & $18.2 \%$ & 44 & $100 \%$ \\
\hline $\begin{array}{l}\text { Are willing to be influenced by other } \\
\text { Board members about new ideas and } \\
\text { methods }\end{array}$ & 28 & $63.6 \%$ & 9 & $20.5 \%$ & 7 & $15.9 \%$ & 44 & $100 \%$ \\
\hline $\begin{array}{l}\text { Clearly understand'the goals and } \\
\text { philosphy of the Board's operation }\end{array}$ & 31 & $70.5 \%$ & 8 & $18.2 \%$ & 5 & $11.4 \%$ & 44 & $100 \%$ \\
\hline $\begin{array}{l}\text { Do all they can to help the Board achieve } \\
\text { its objectives }\end{array}$ & 22 & $50.0 \%$ & 14 & $31.8 \%$ & 8 & $18.2 \%$ & 44 & $100 \%$ \\
\hline $\begin{array}{l}\text { Get the technical knowledge and training } \\
\text { in group skills they need }\end{array}$ & 14 & $31.8 \%$ & 23 & $52.3 \%$ & 7 & $15.9 \%$ & 44 & $100 \%$ \\
\hline Provide one another mutual help & 28 & $63.6 \%$ & 13 & $29.5 \%$ & 3 & $6.8 \%$ & 44 & $100 \%$ \\
\hline
\end{tabular}

SSAB Supplementary Appendix 
St. Louis Site Specific Advisory Board

Evaluation Survey Results

SSAB Supplementary Appendix 
Figure 1a. St. Louis

Goal 1. Establish processes and procedures to provide an effective forum for exchange of information and viewpoints regarding DOE site issues

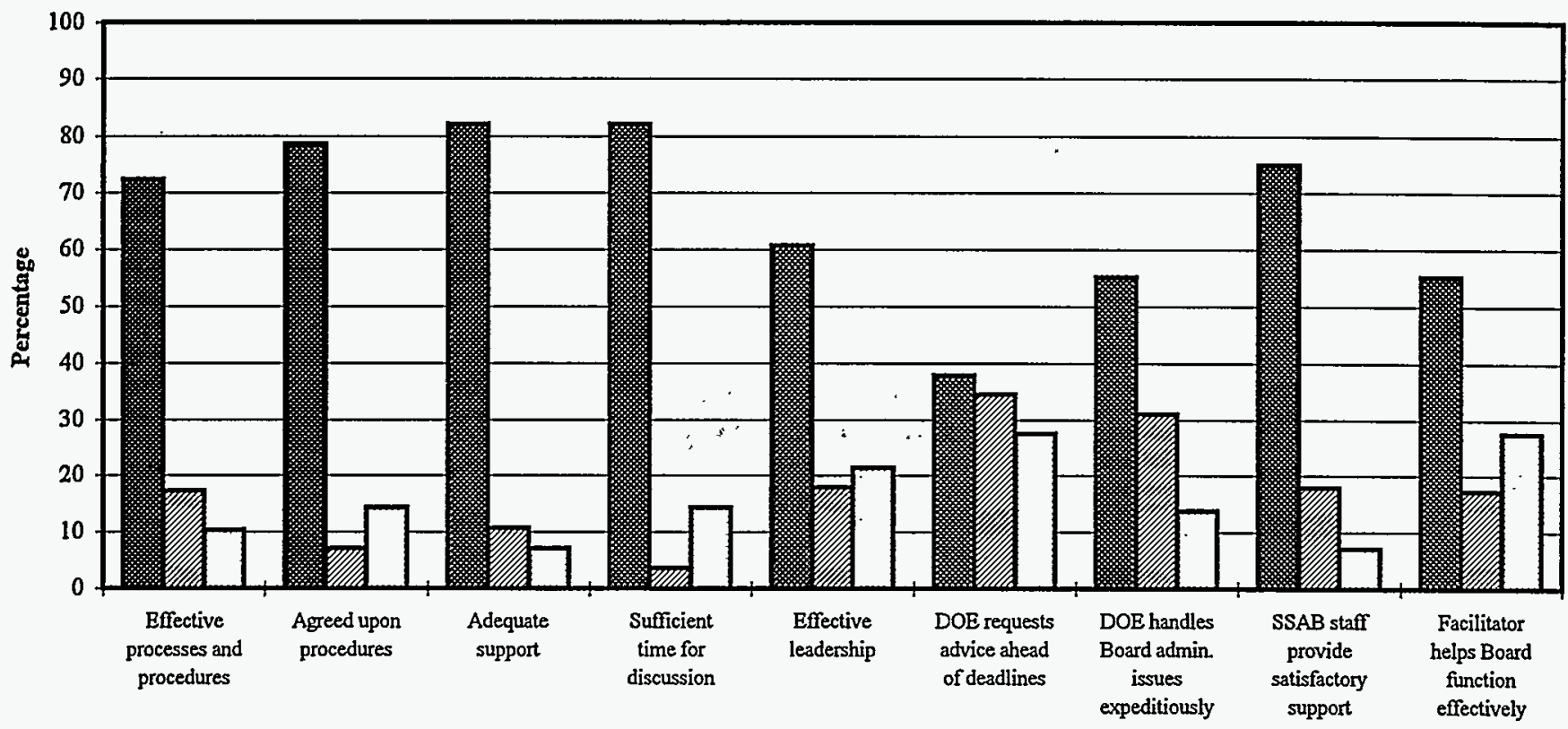

2Agree (\%) שNeither Agree Nor Disagree/Don't Know (\%) पDisagree (\%)

Figure 1b. Long Survey: Entire Sample

Goal 1. Establish processes and procedures to provide an effective forum for exchange of information and viewpoints regarding DOE site issues

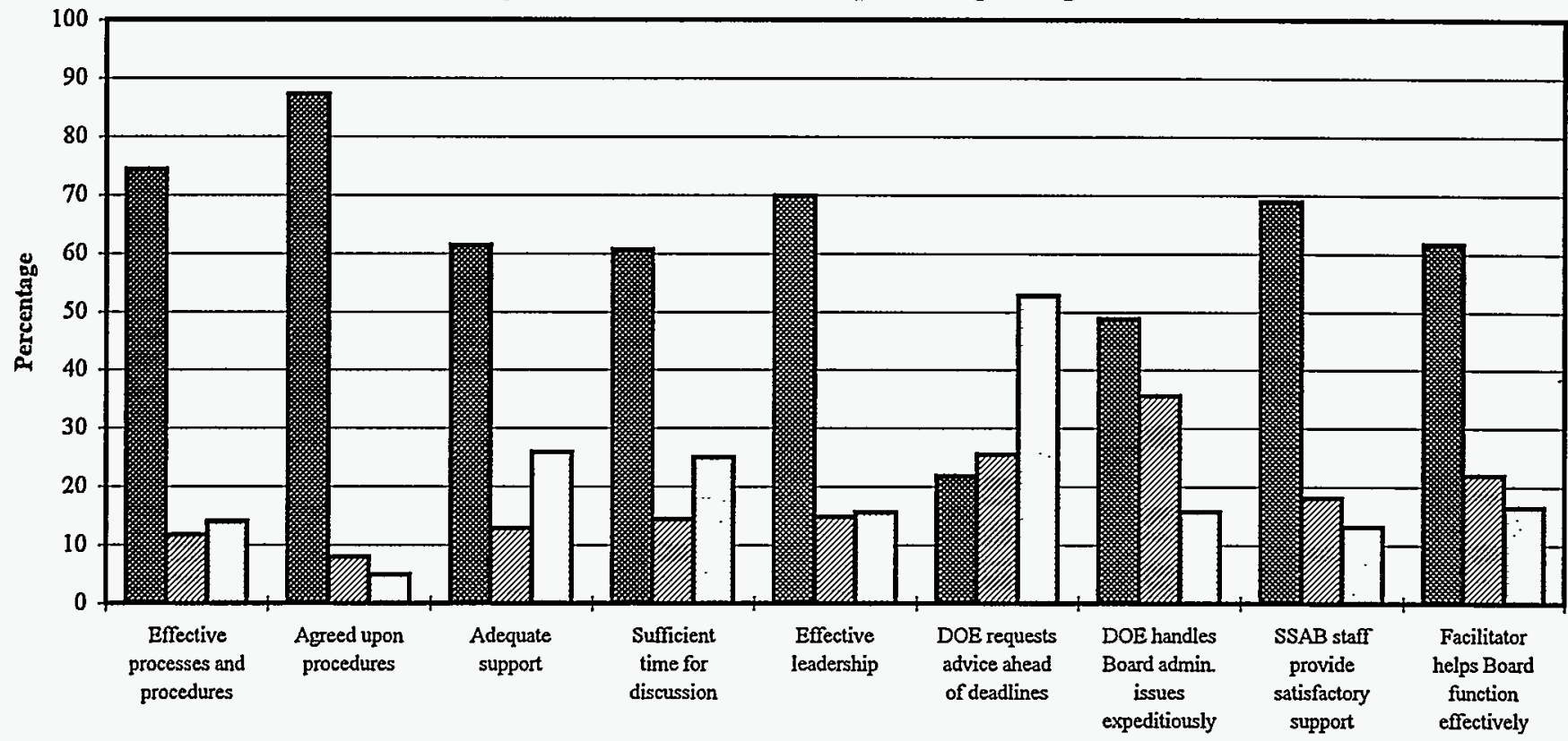

甸Agree (\%) Neither Agree Nor Disagree/Don't Know (\%) DDisagree (\%)

SSAB Supplementary Appendix

August 1996 
Figure 2a. St. Louis

Goal 2. Facilitate interaction and exchange of information and viewpoints regarding DOE site issues

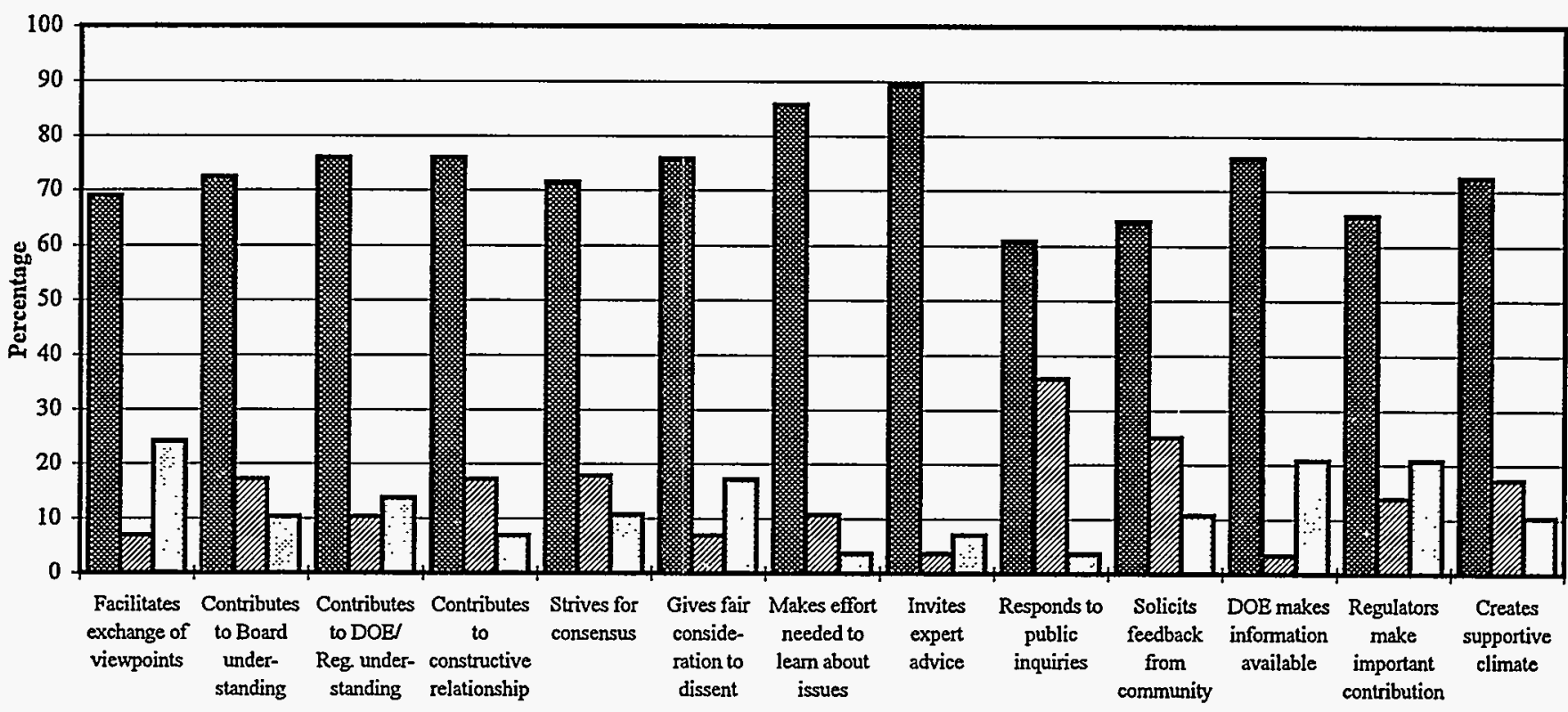

A Agree (\%) Neither Agree Nor Disagree/Don't Know (\%) DDisagree (\%)

Figure 2b. Long Survey: Entire Sample

Goal 2. Facilitate interaction and exchange of information and viewpoints regarding DOE site issues

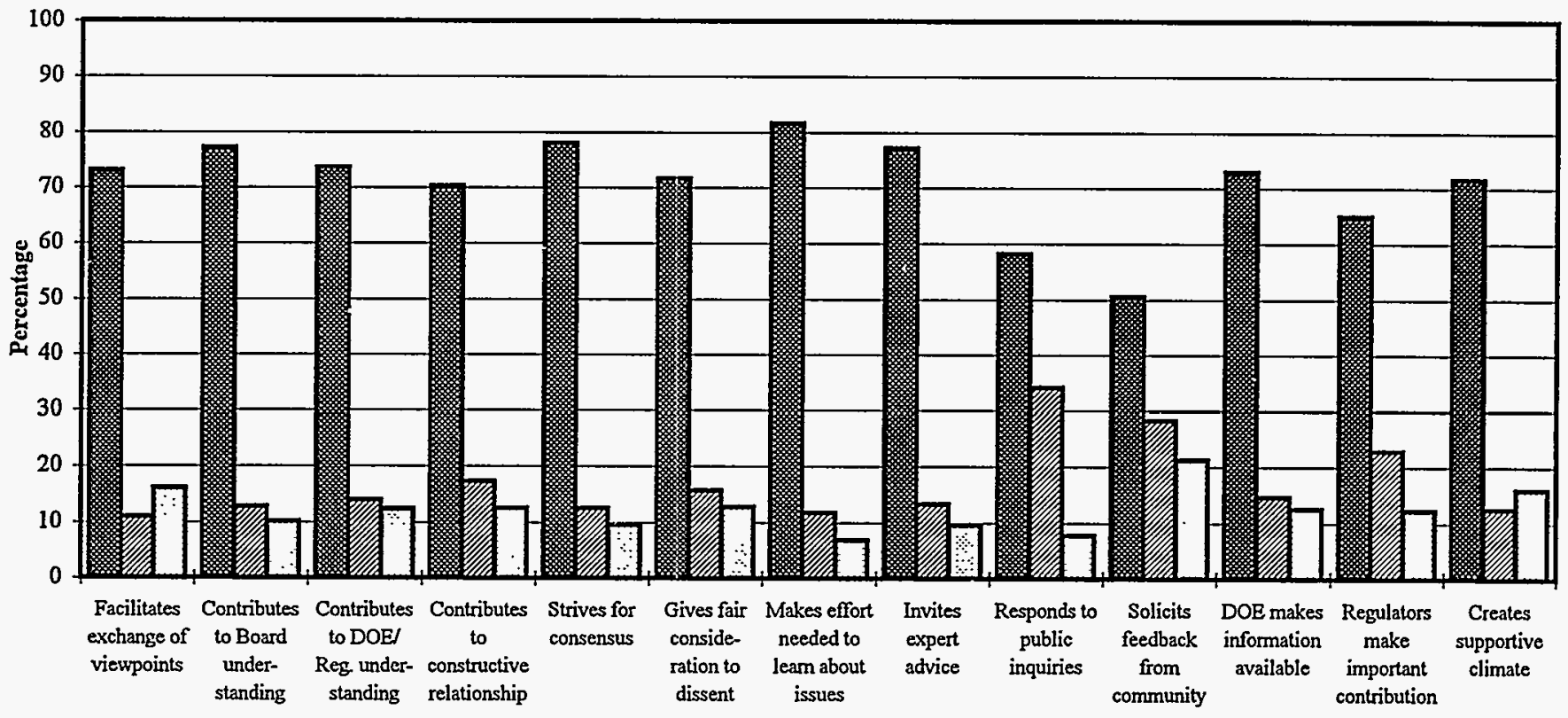

Agree (\%) Neither Agree Nor Disagree/Don't Know (\%) 口Disagree (\%)

SSAB Supplementary Appendix

August 1996 
Figure 3a. St. Louis

Goal 3. Provide useful advice and/or recommendations to DOE

(and regulators, where appropriate)

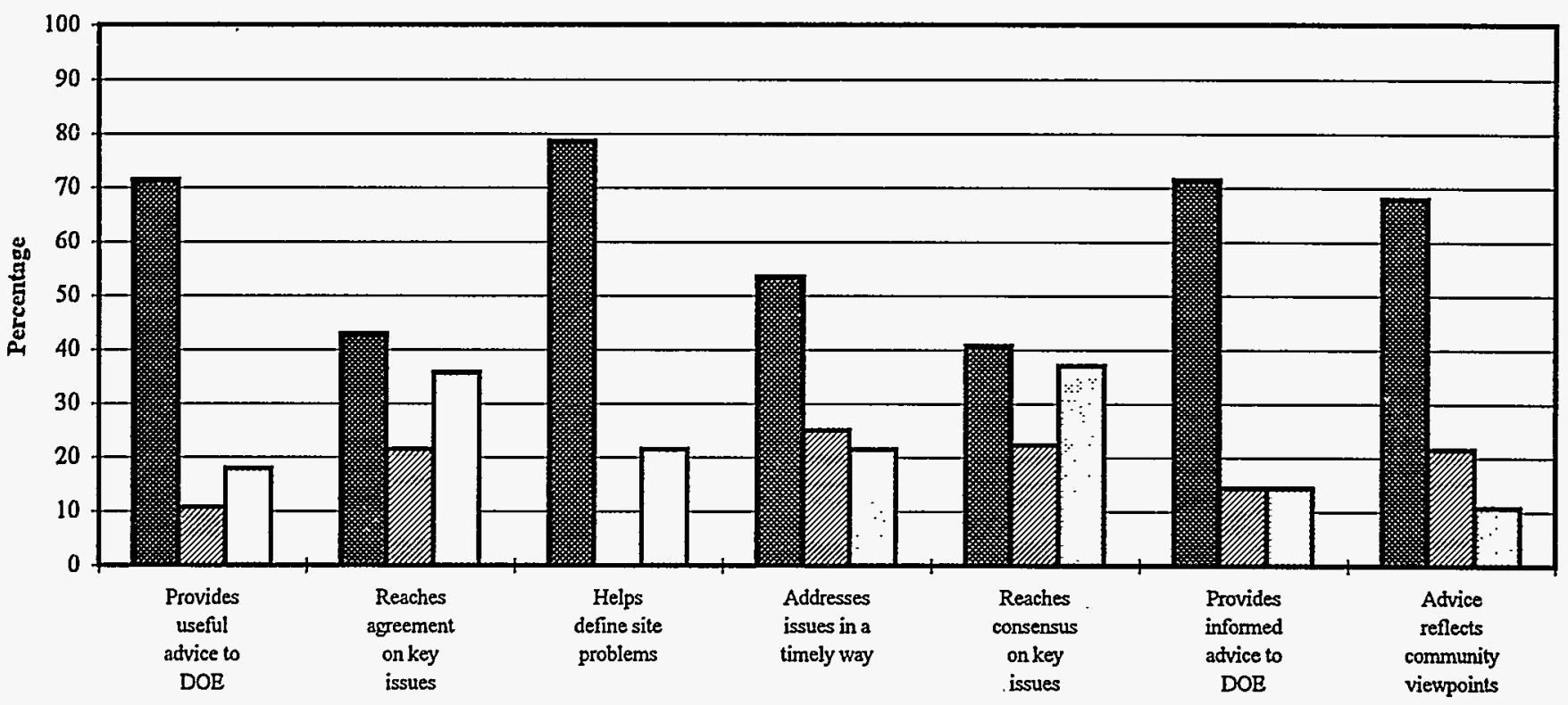

Agree (\%) Neither Agree Nor Disagree/Don't Know (\%) DDisagree (\%)

Figure 3b. Long Survey: Entire Sample

Goal 3. Provide useful advice and/or recommendations to DOE

(and regulators, where appropriate)

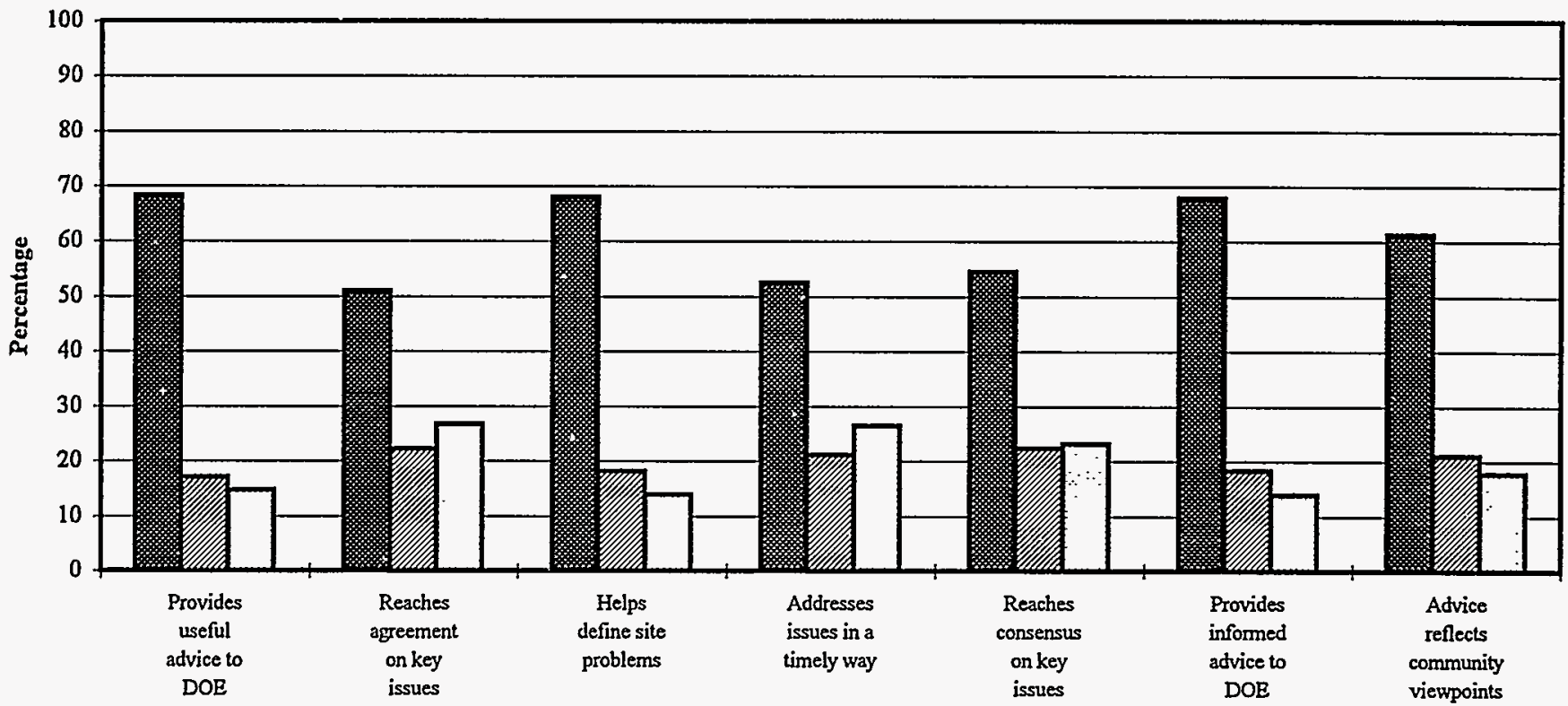

Agree (\%) Deither Agree Nor Disagree/Don't Know (\%) DDisagree (\%) 
Figure 4a. St. Louis

Goal 4. Improve DOE's (and where applicable, regulators') site decisions and decision making process

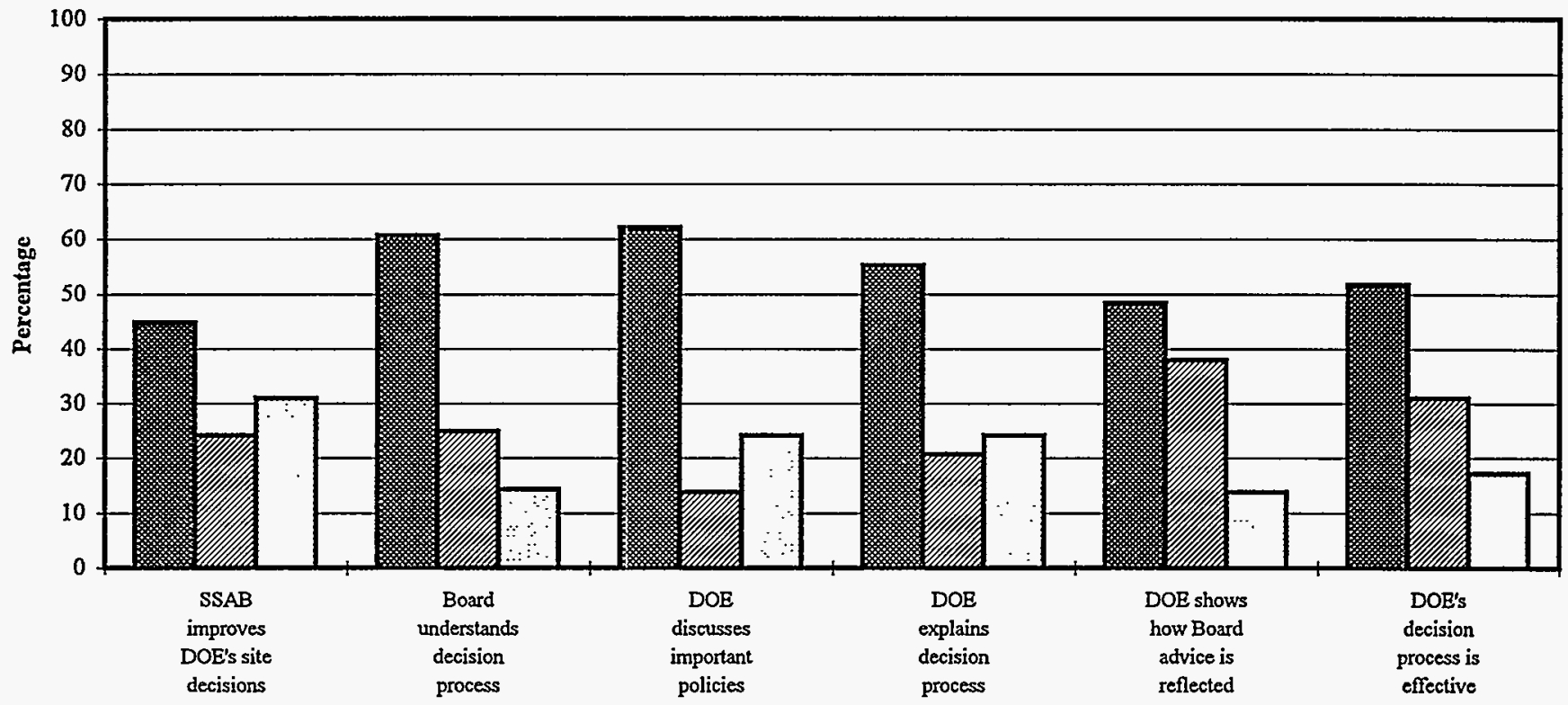

Agree (\%) Neither Agree Nor Disagree/Don't Know (\%) DDisagree (\%)

Figure 4b. Long Survey: Entire Sample

Goal 4. Improve DOE's (and where applicable, regulators')

site decisions and decision making process

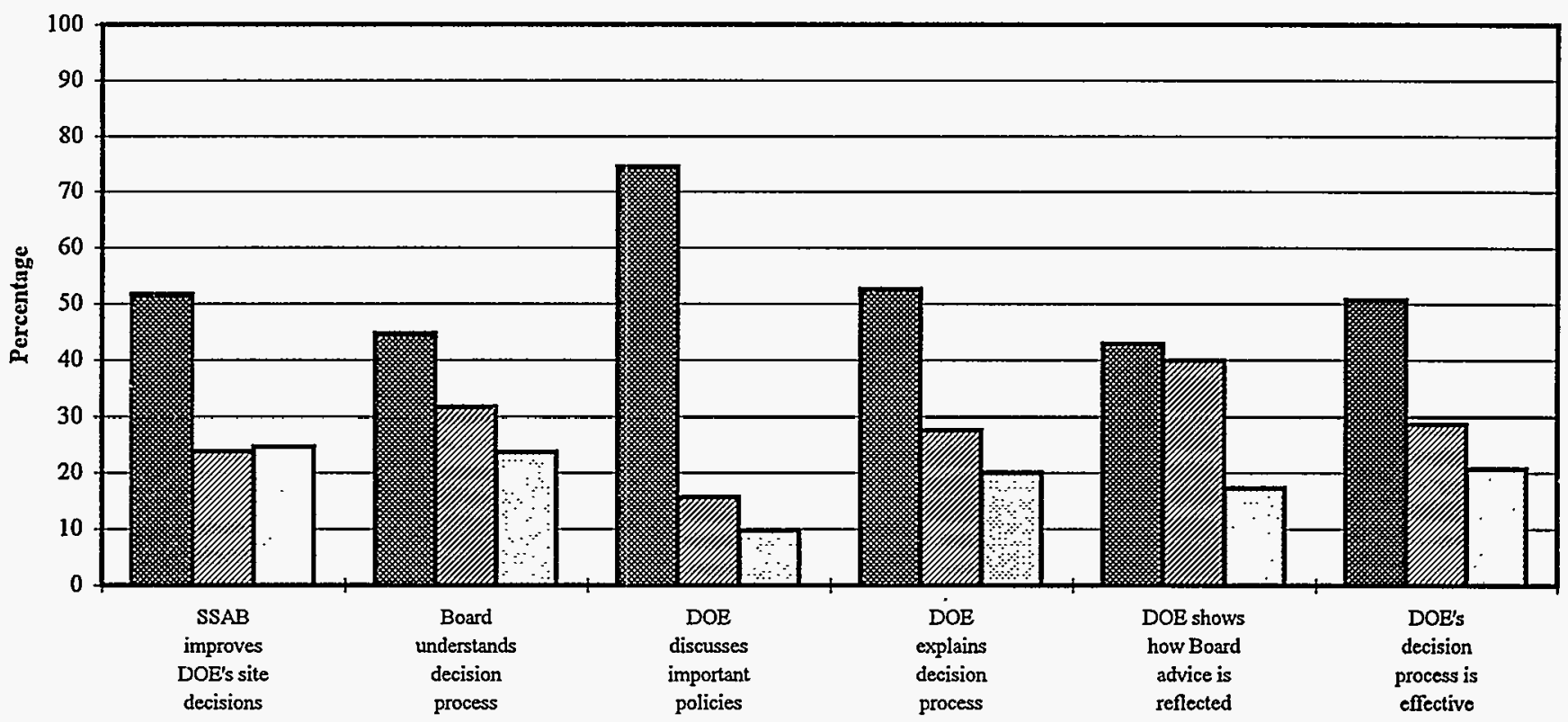

Agree (\%) ENeither Agree Nor Disagree/Don't Know (\%) DDisagree (\%)

SSAB Supplementary Appendix

August 1996 
Figure 5a. St. Louis

Goal 5. Lead to more acceptable actions

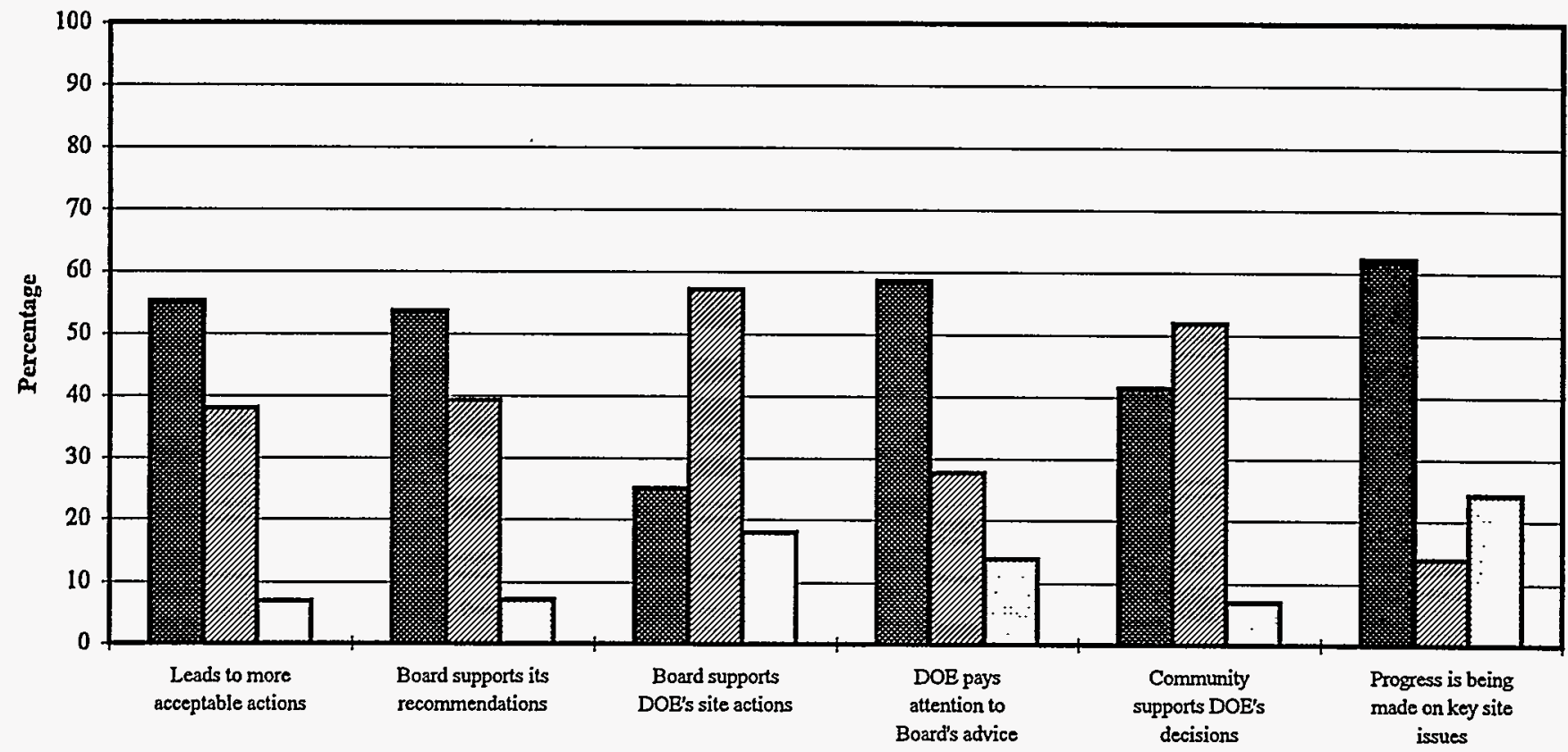

Agree (\%) Weither Agree Nor Disagree/Don't Know (\%) DDisagree (\%)

Figure 5b. Long Survey: Entire Sample

Goal 5. Lead to more acceptable actions

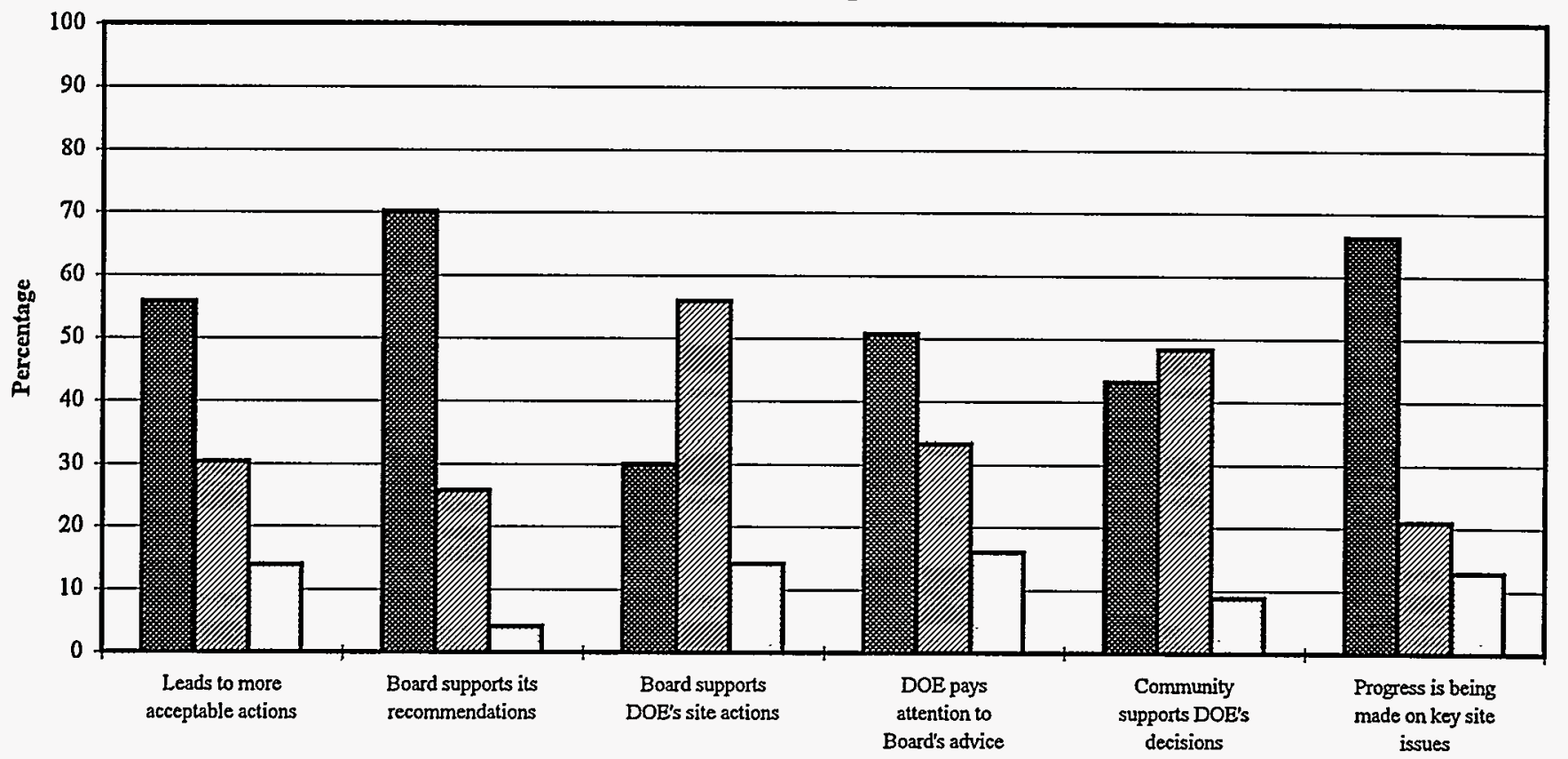

A Agree (\%) ENeither Agree Nor Disagree/Don't Know (\%) DDisagree (\%)

SSAB Supplementary Appendix

August 1996 
Figure 6a. St. Louis

Goal 6. Contribute to trust and confidence in DOE

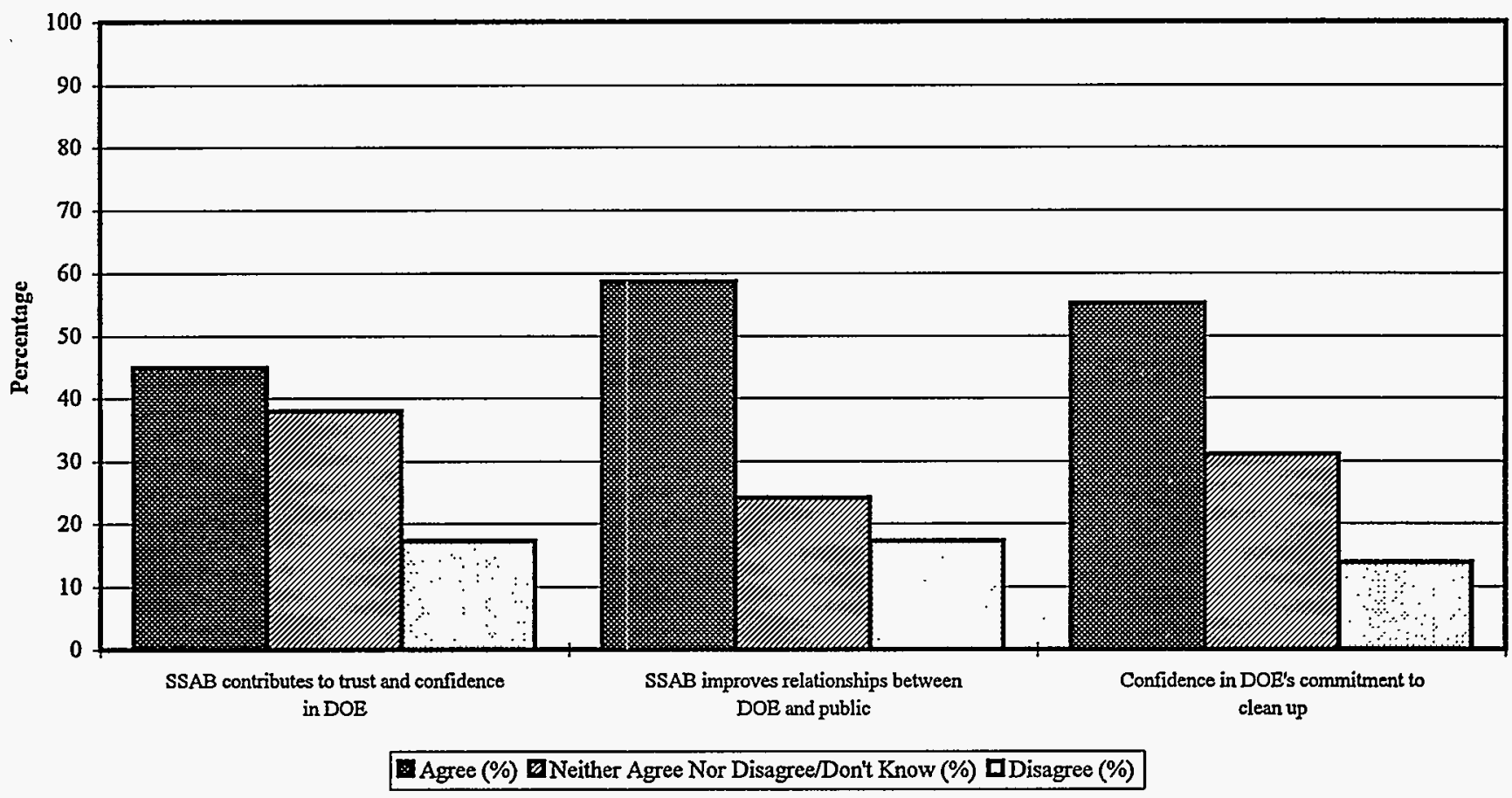

Figure 6b. Long Survey: Entire Sample

Goal 6. Contribute to trust and confidence in DOE

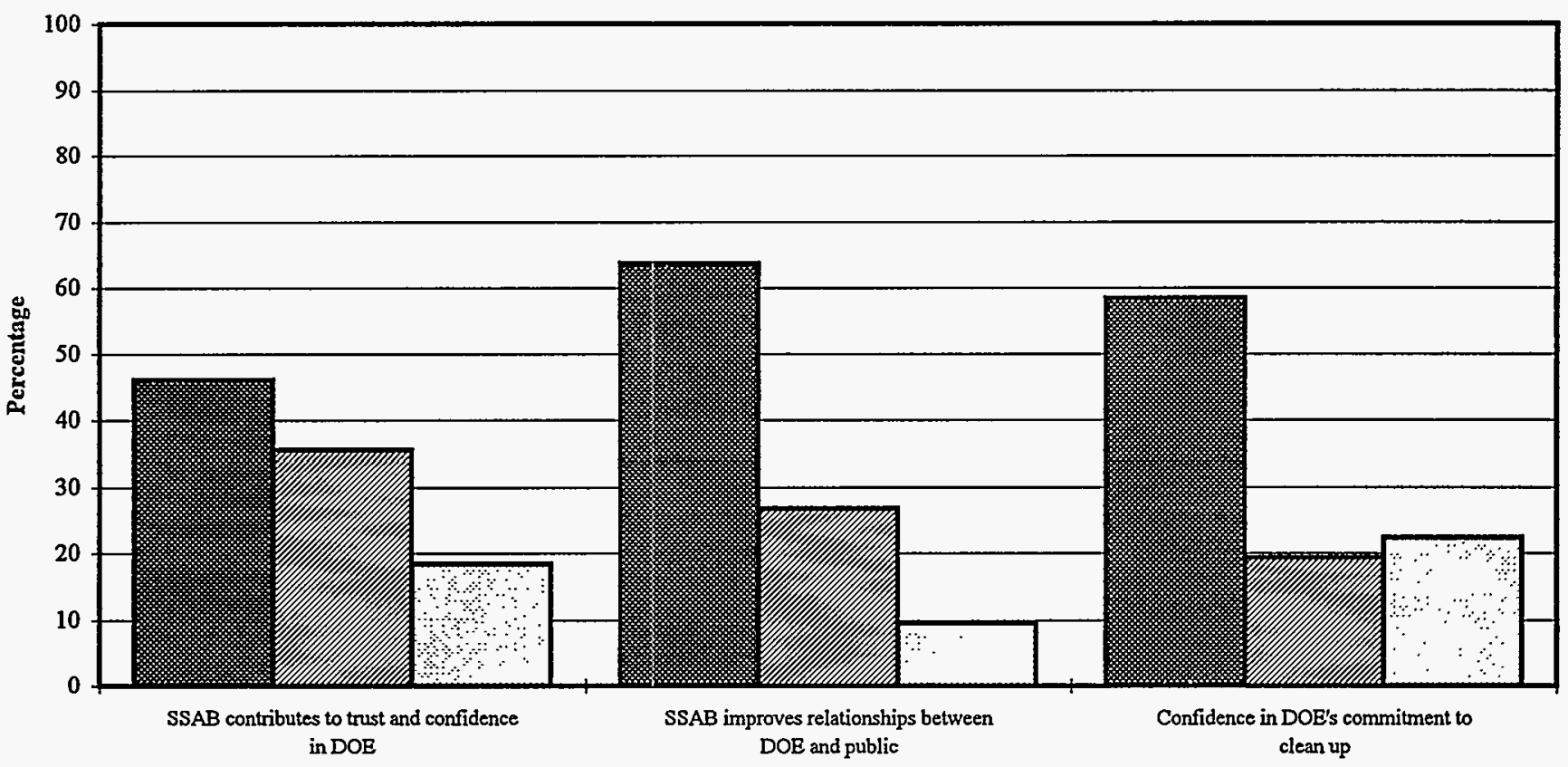

Agree (\%) Deither Agree Nor Disagree/Don't Know (\%) DDisagree (\%)

SSAB Supplementary Appendix

August 1996 
Figure 7a. St. Louis

Public awareness

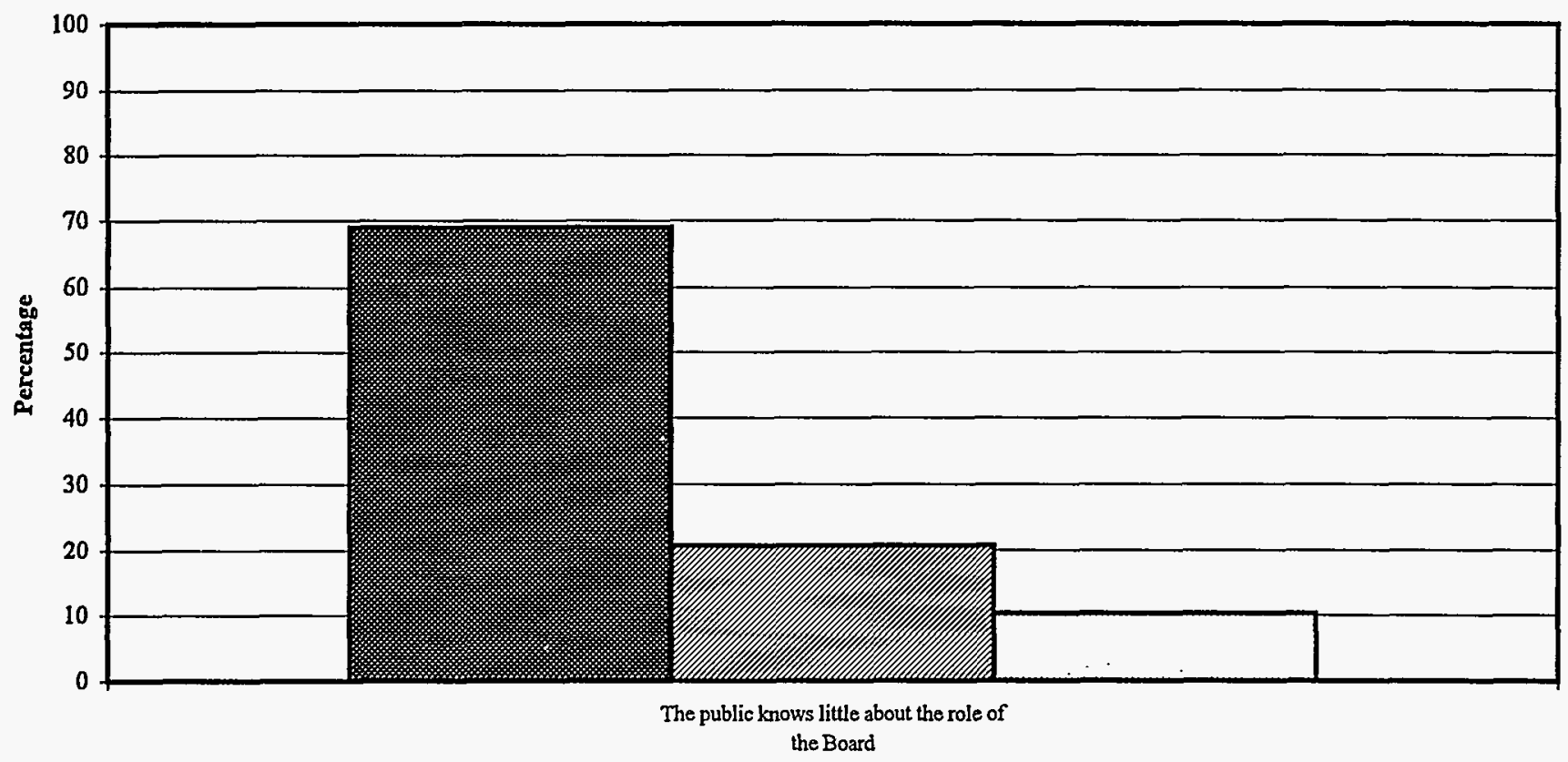

Agree (\%) DNeither Agree Nor Disagree/Don't Know (\%) DDisagree (\%)

Figure 7b. Long Survey: Entire Sample

Public awareness

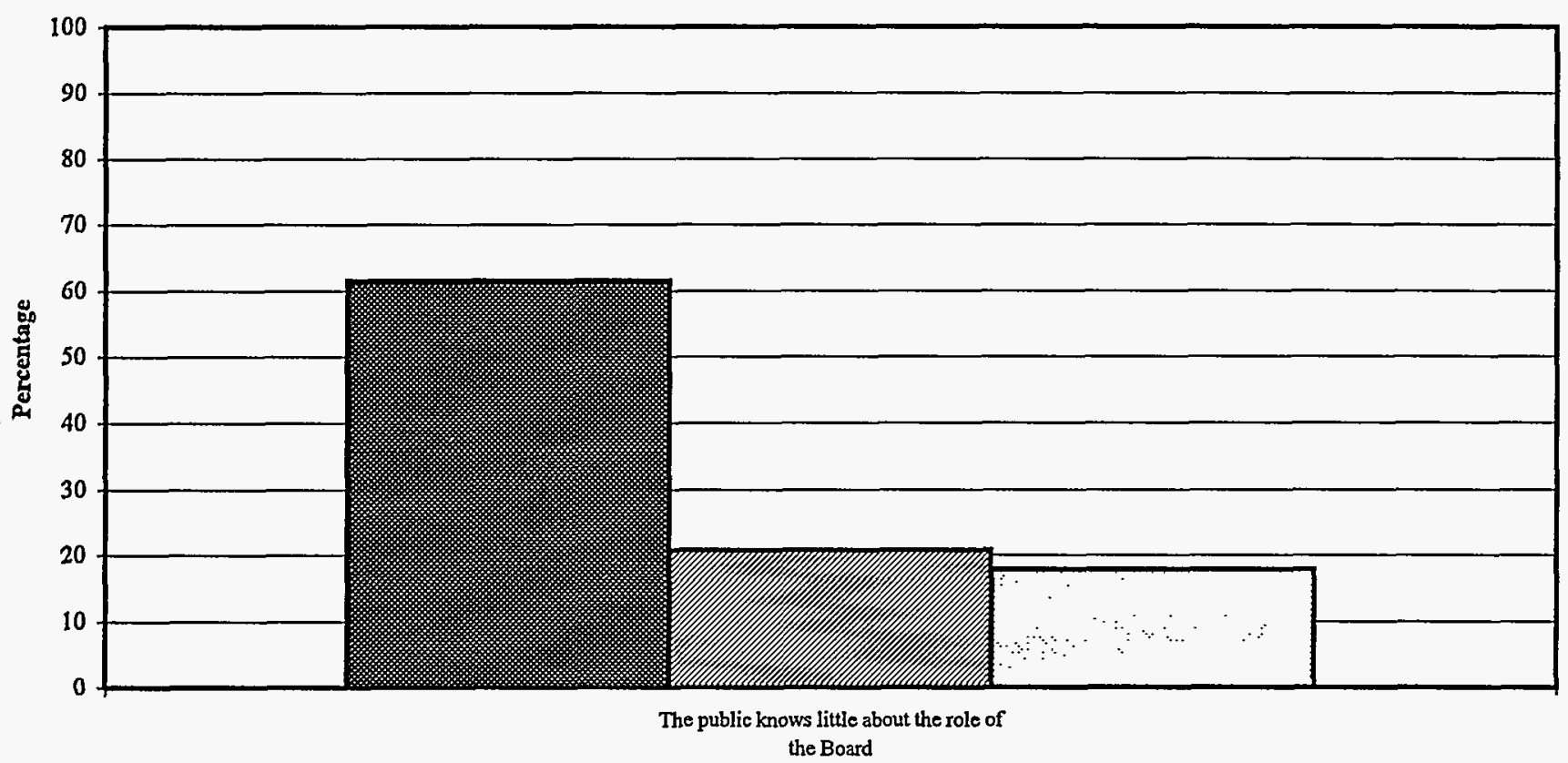

⿴囗大 Agree (\%) $\mathbf{N}$ Neither Agree Nor Disagree/Don't Know (\%) 口Disagree (\%)

SSAB Supplementary Appendix

August 1996 
Figure 8a. St. Louis

DOE-HQ involvement with the SSAB Initiative

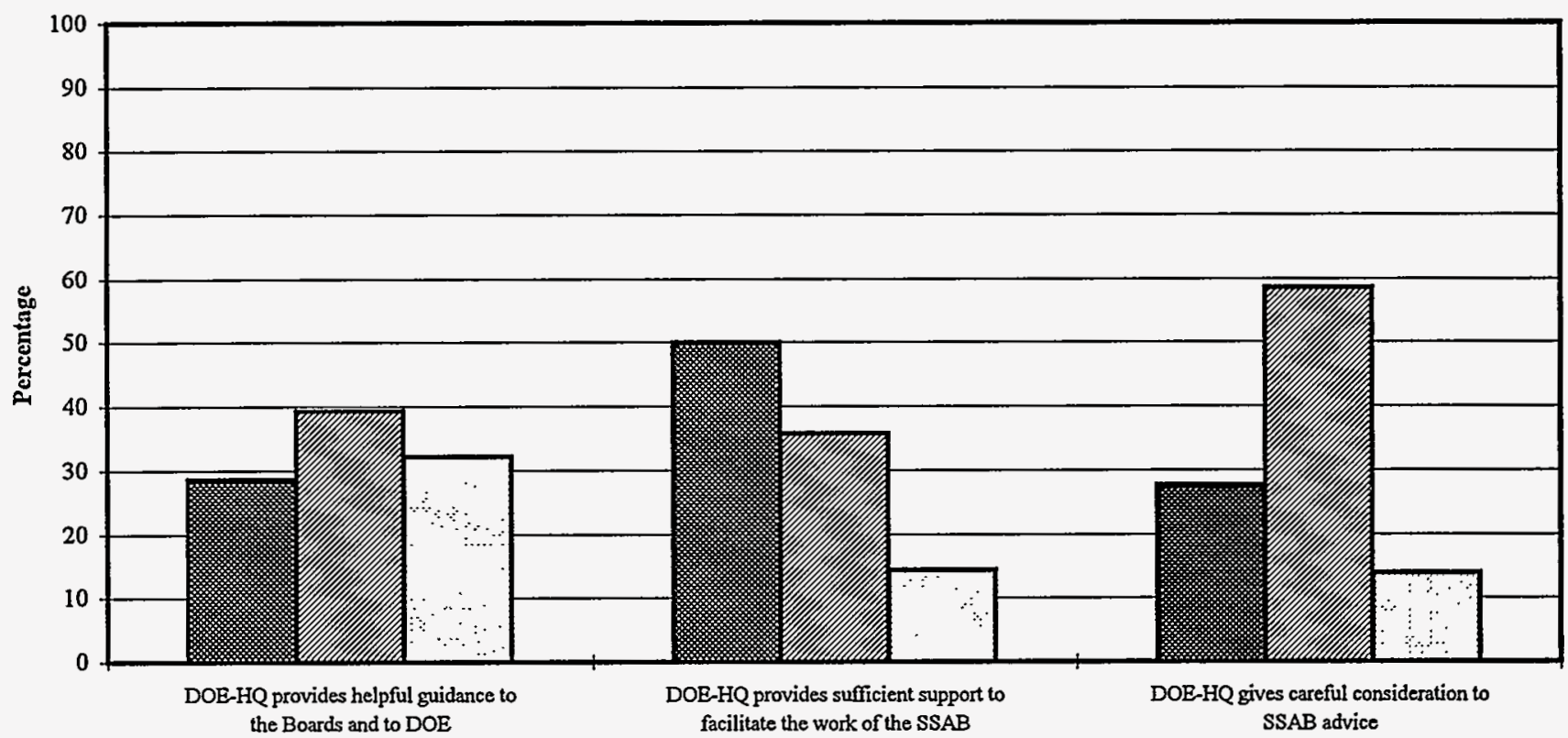

Agree (\%) Neither Agree Nor Disagree/Don't Know (\%) DDisagree (\%)

Figure 8b. Long Survey: Entire Sample

DOE-HQ involvement with the SSAB Initiative

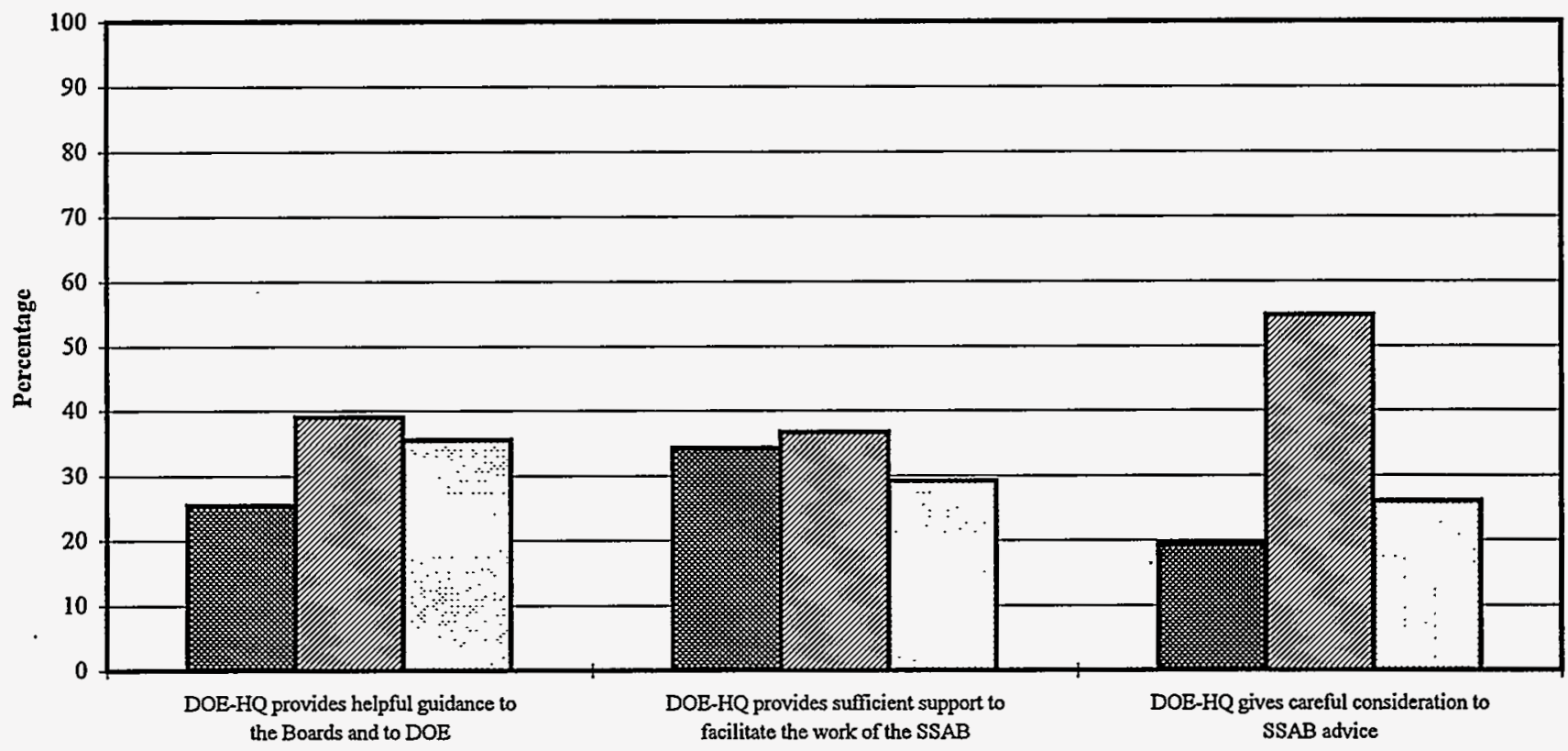

A Agree (\%) Neither Agree Nor Disagree/Don't Know (\%) DDisagree (\%)

SSAB Supplementary Appendix

August 1996 
Figure 9a. St. Louis

Personal experience with the SSAB Initiative

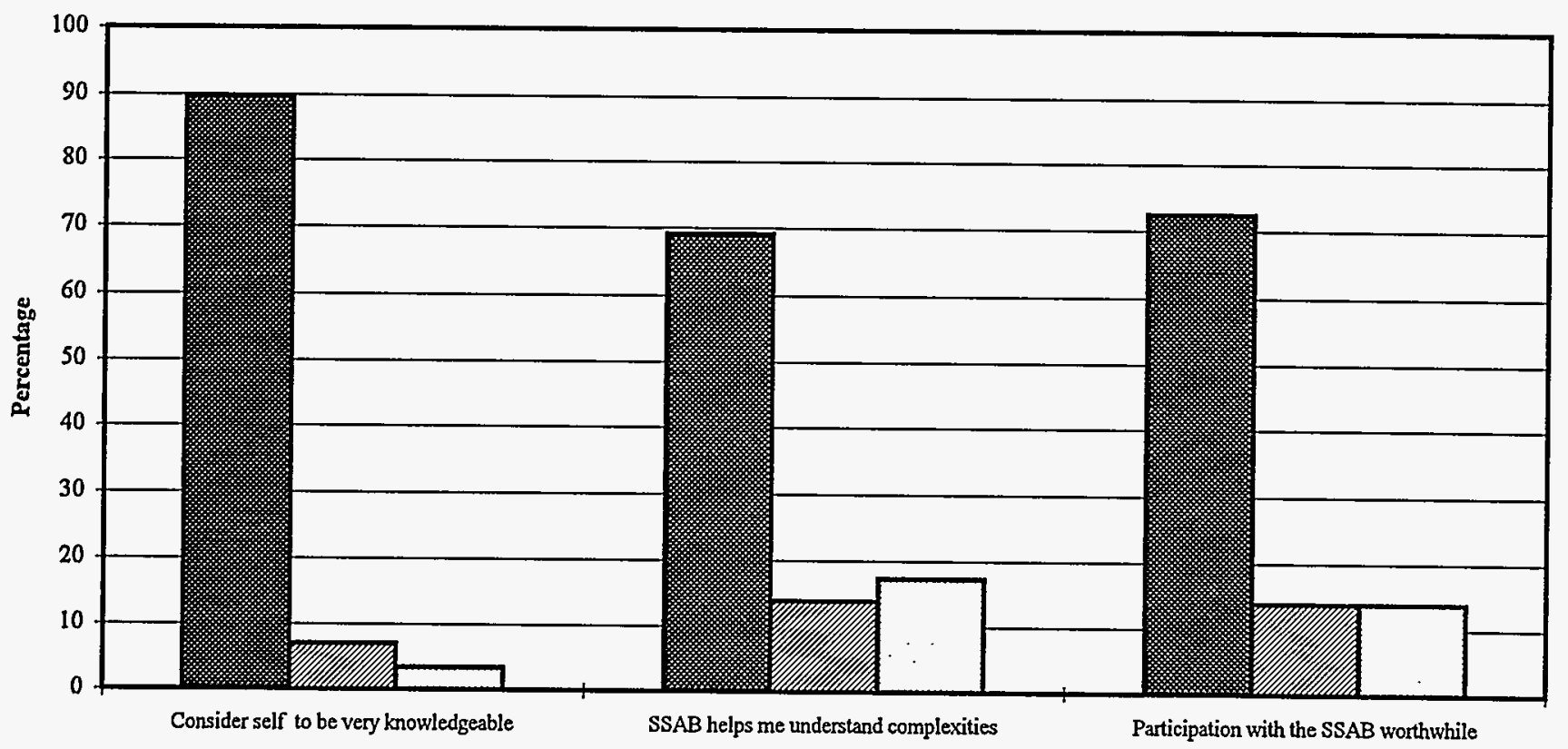

Agree (\%) Weither Agree Nor Disagree/Don't Know (\%) DDisagree (\%)

Figure 9b. Long Survey: Entire Sample

Personal experience with the SSAB Initiative

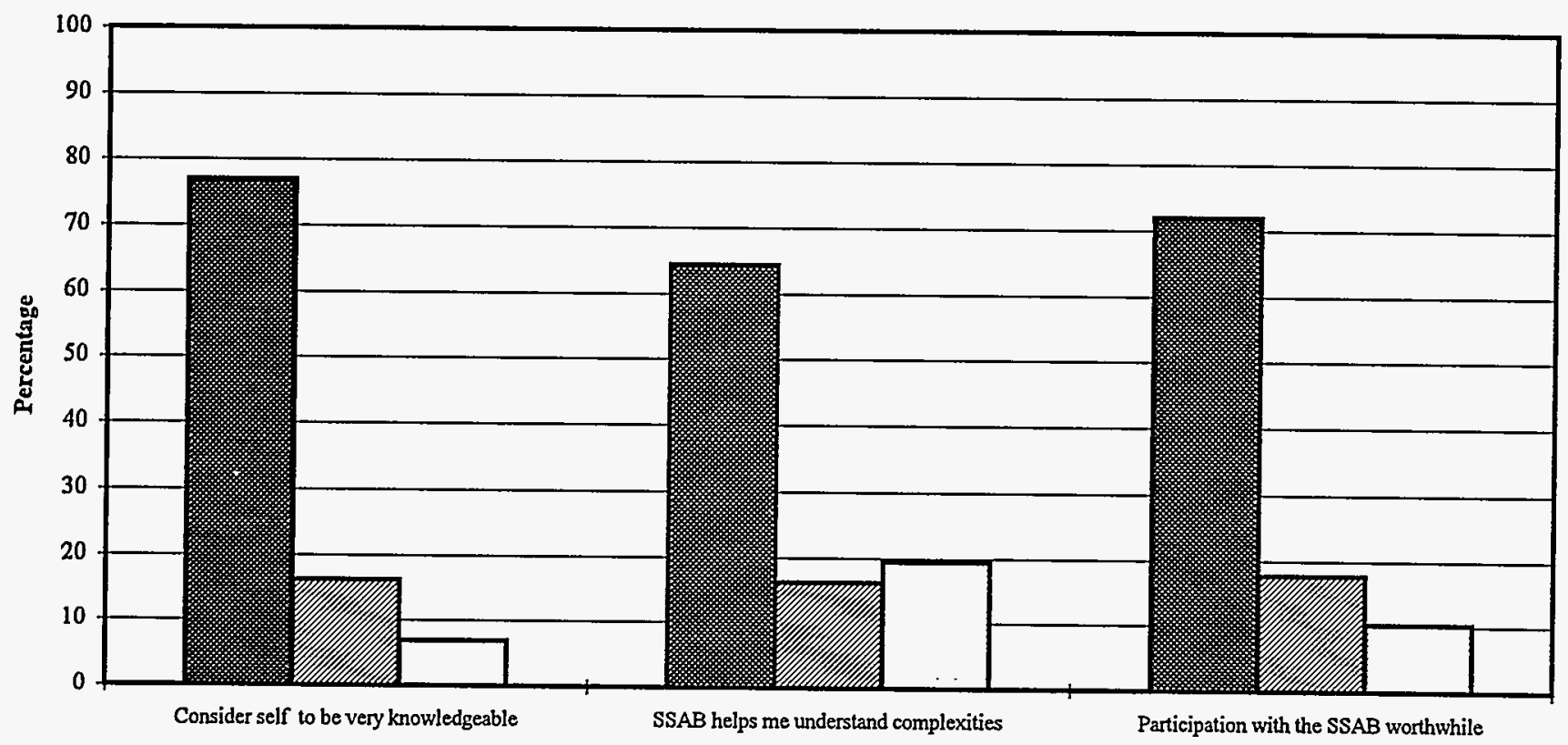

国 Agree (\%) Neither Agree Nor Disagree/Don't Know (\%) DDisagree (\%)

SSAB Supplementary Appendix 
Figure 10a. St. Louis

Group/Team work skills

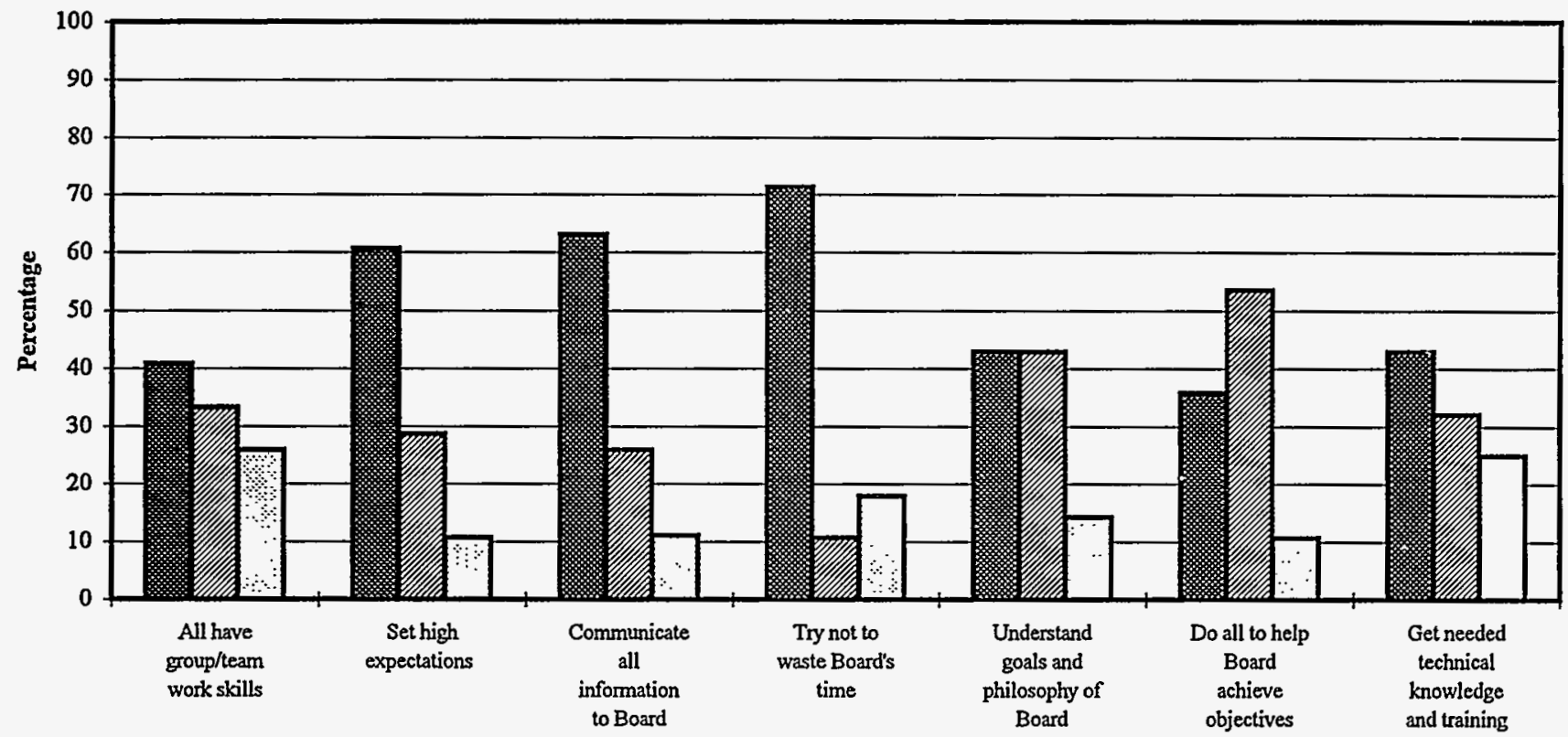

Agree (\%) Deither Agree Nor Disagree/Don't Know (\%) Disagree (\%)

Figure 10b. Long Survey: Entire Sample

Group/Team work skills

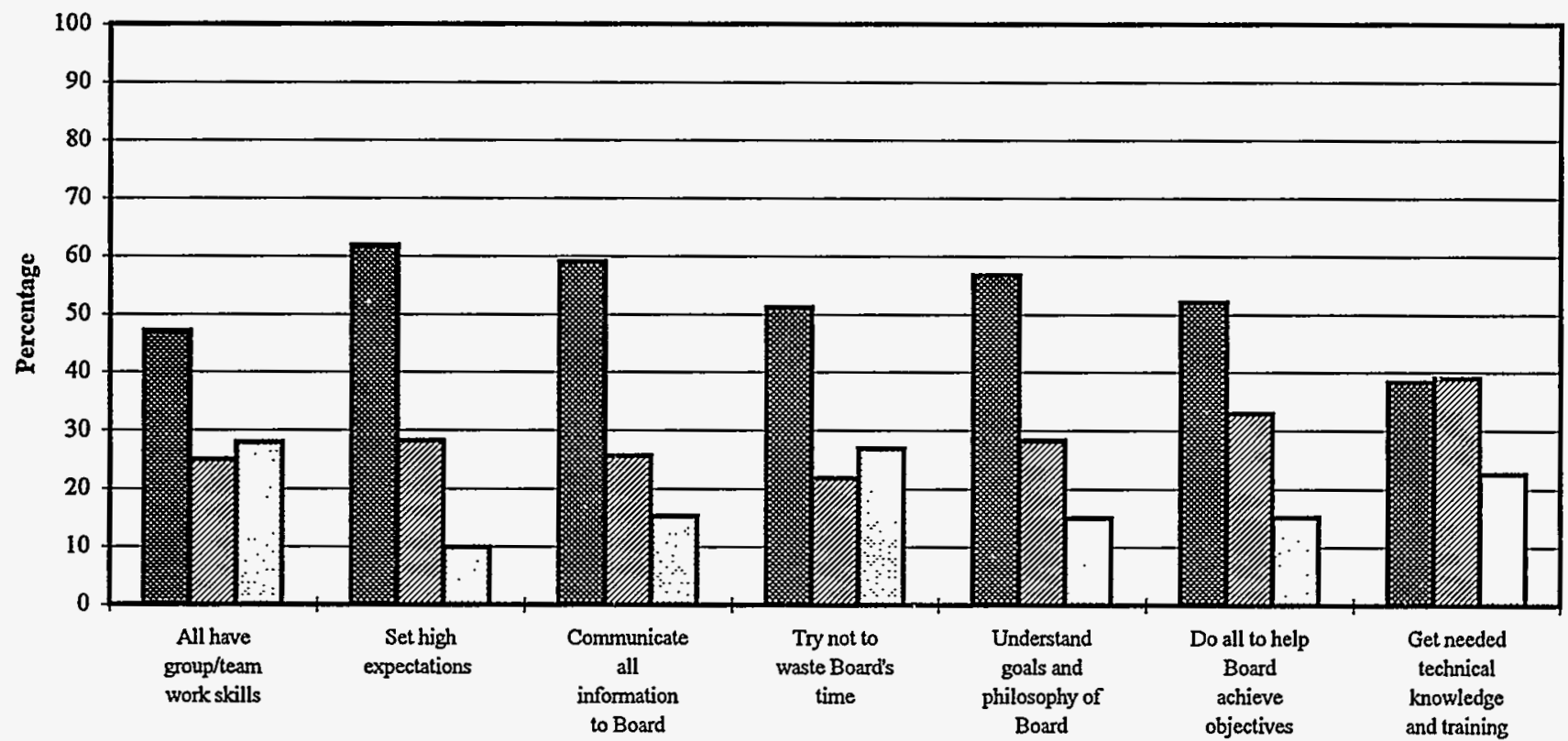

Agree (\%) E Neither Agree Nor Disagree/Dont Know (\%) DDisagree (\%)

SSAB Supplementary Appendix

August 1996 
Figure 11a. St. Louis

Working relatiọns

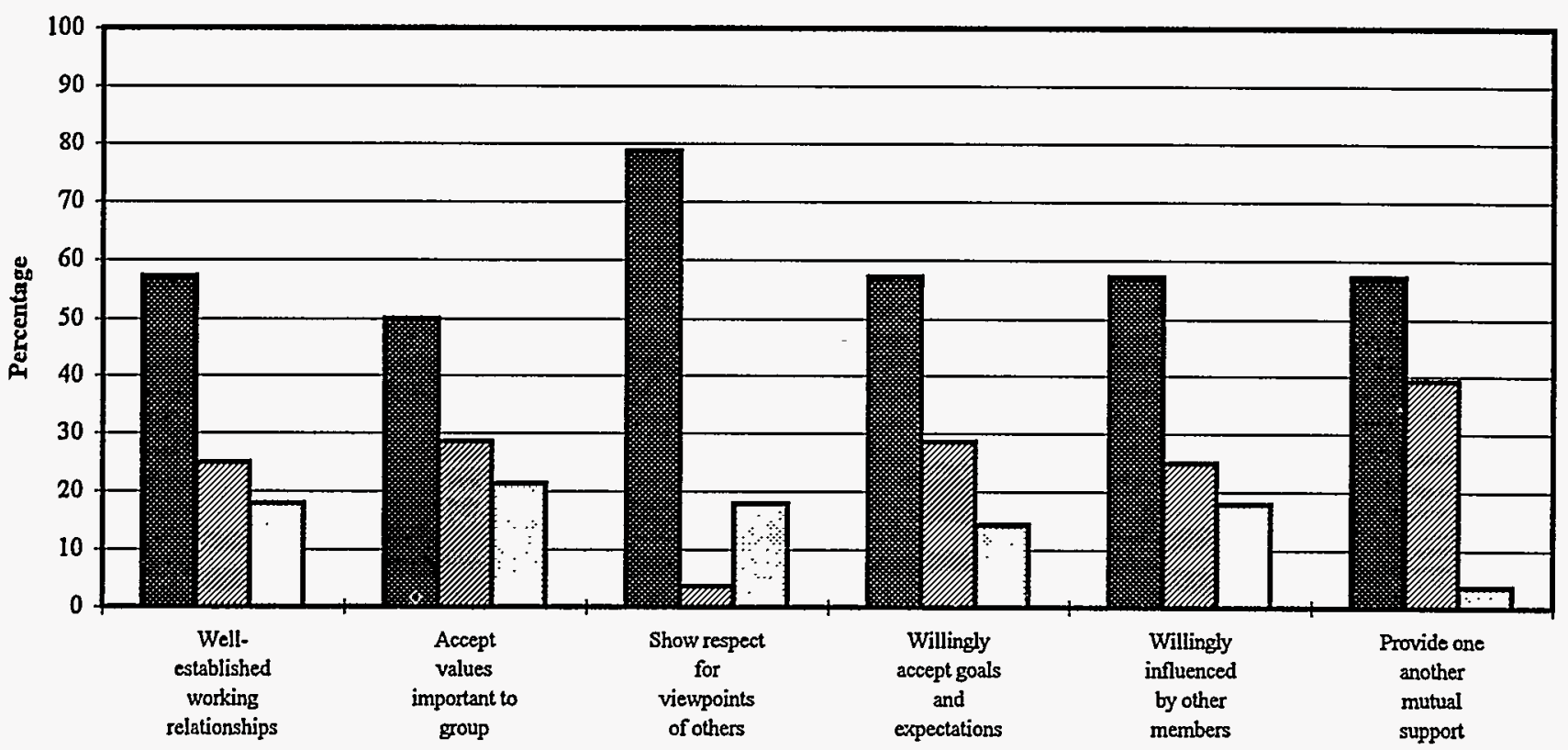

Agree (\%) Deither Agree Nor Disagree/Don't Know (\%) DDisagree (\%)

Figure 11b. Long Survey: Entire Sample

Working relations

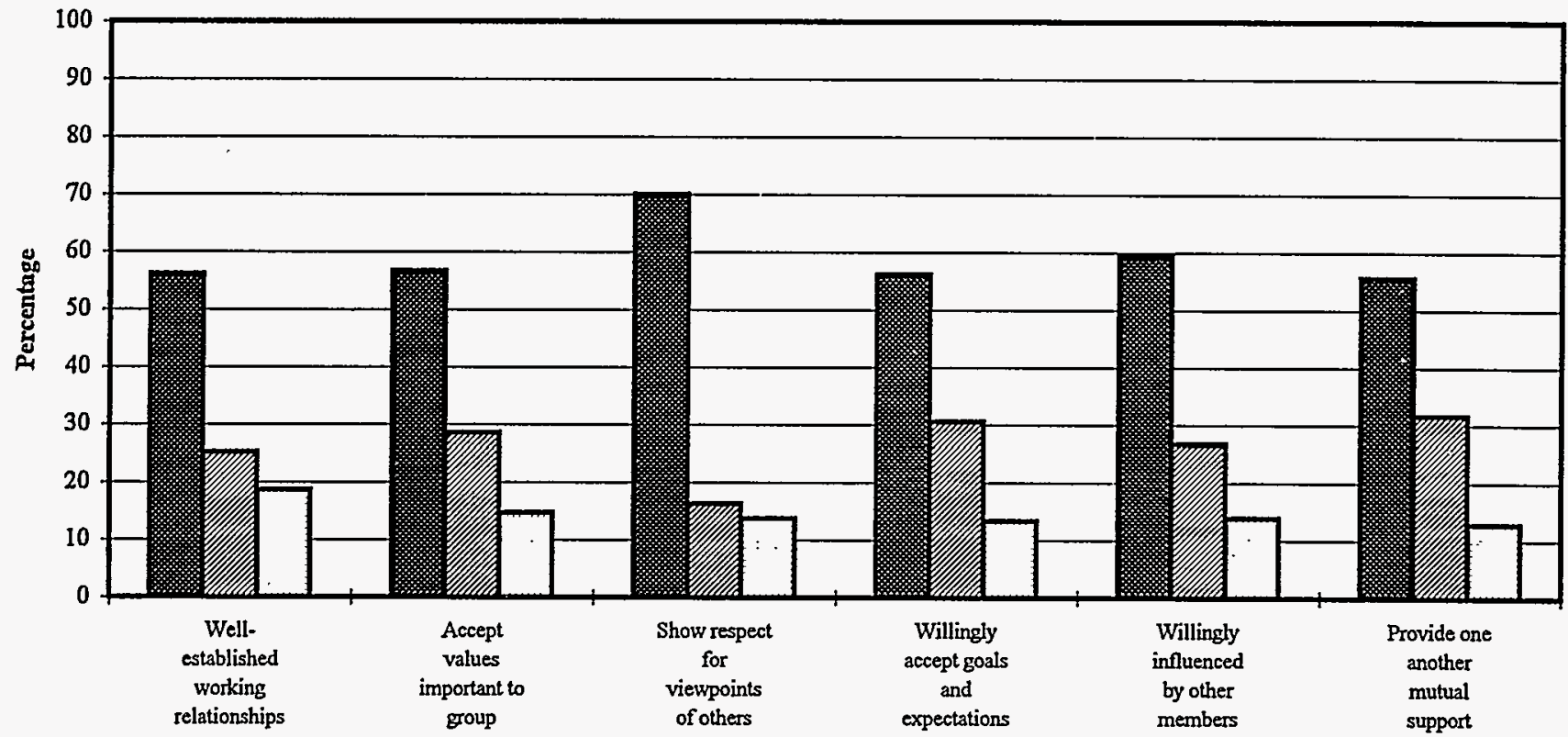

$E_{\text {Agree (\%) }}$ Neither Agree Nor Disagree/Don't Know (\%) DDisagree (\%)

SSAB Supplementary Appendix

August 1996 
Figure 12a. St. Louis

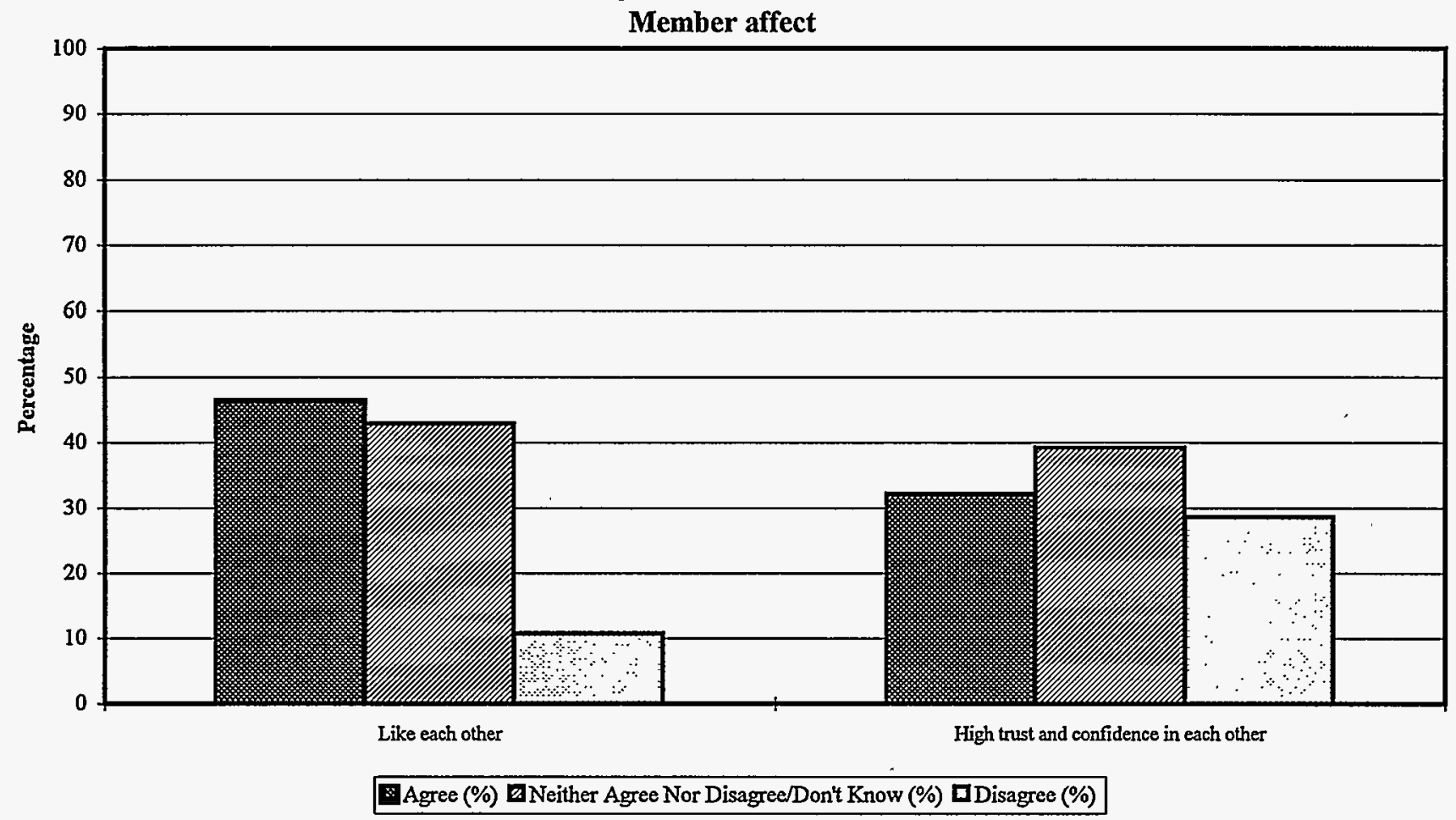

Figure 12b. Long Survey: Entire Sample

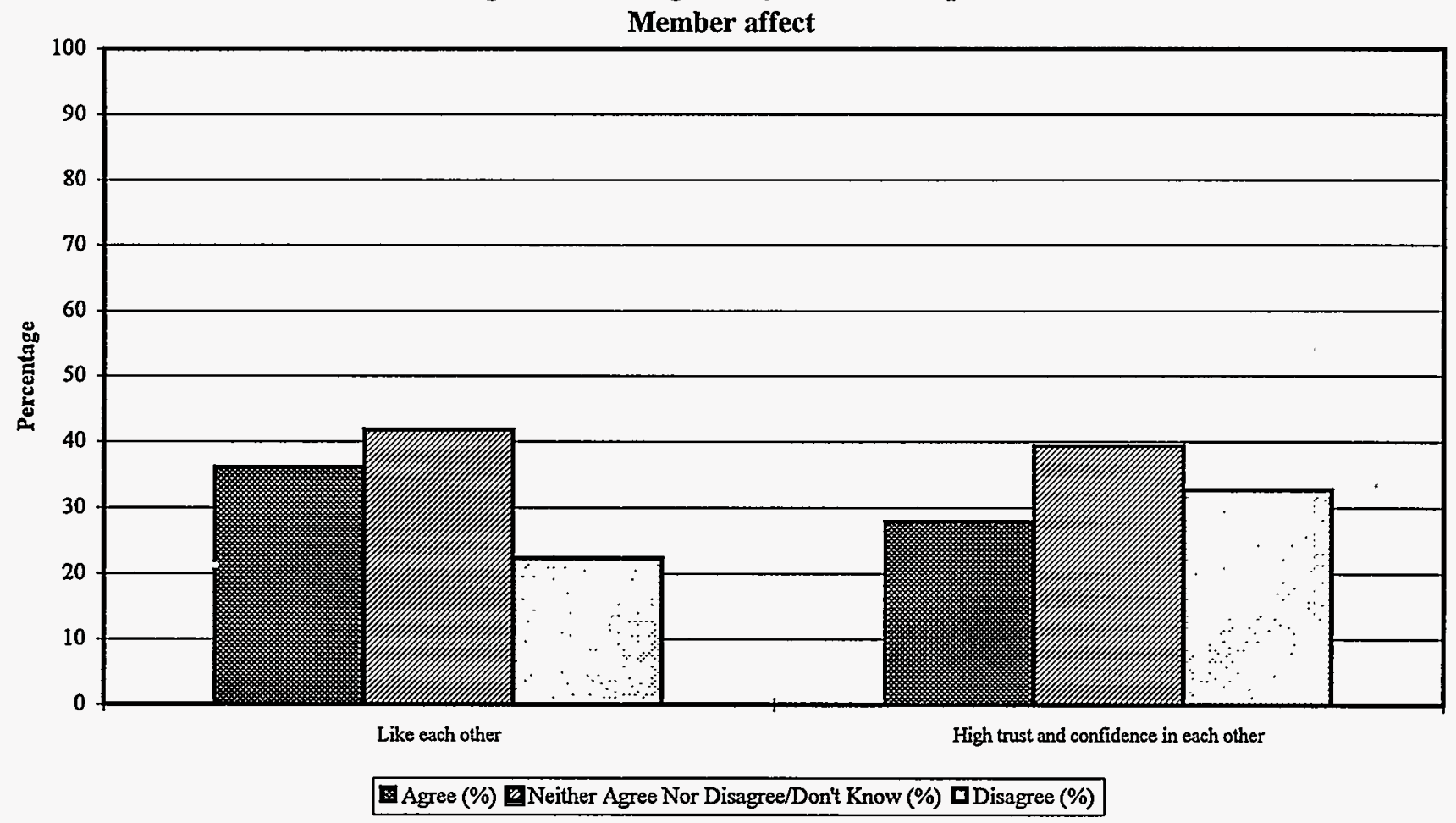

SSAB Supplementary Appendix 
Figure 13a. St. Louis

Summary: Responses to open-ended questions

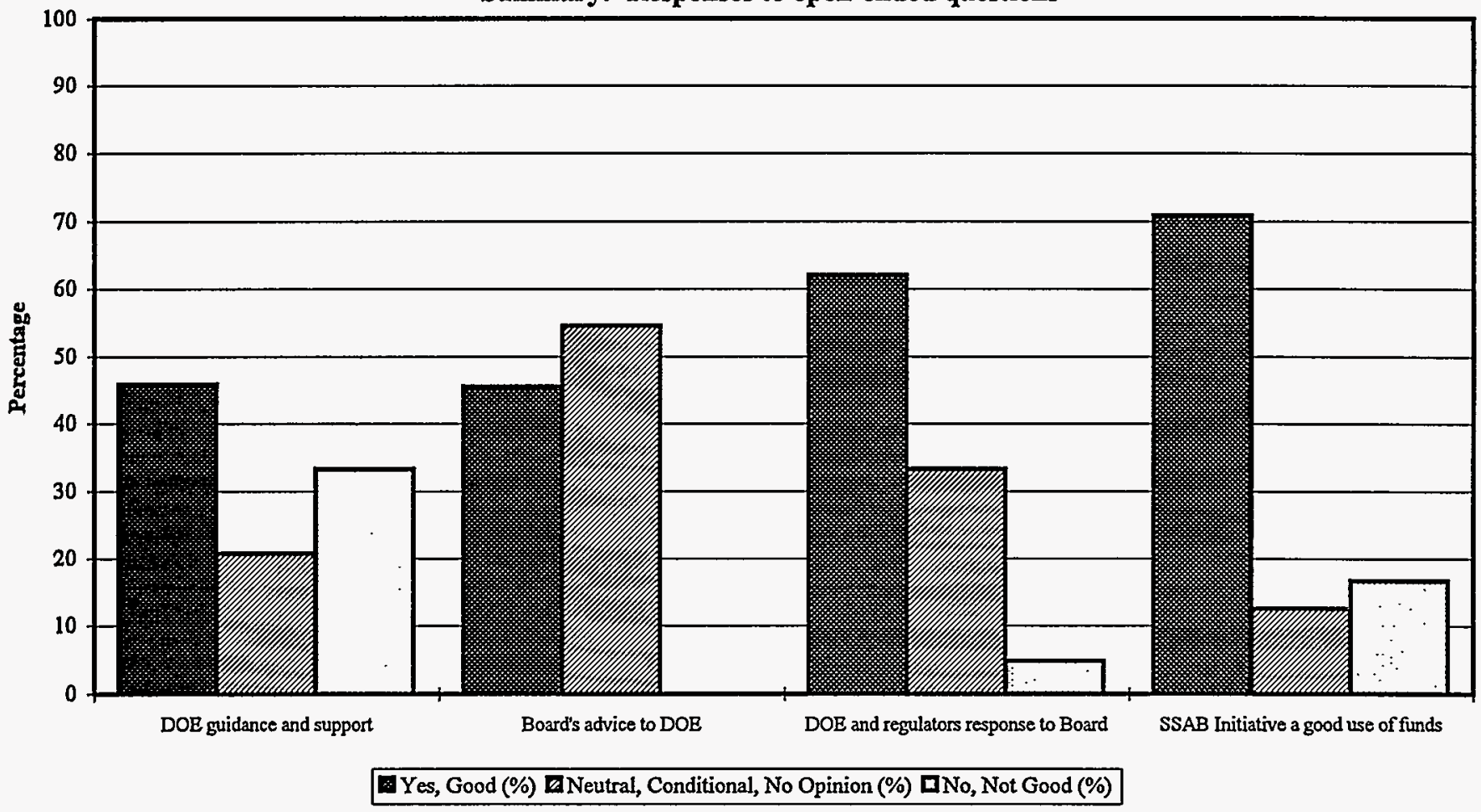

Figure 13b. Long Survey: Entire Sample

Summary: Responses to open-ended questions

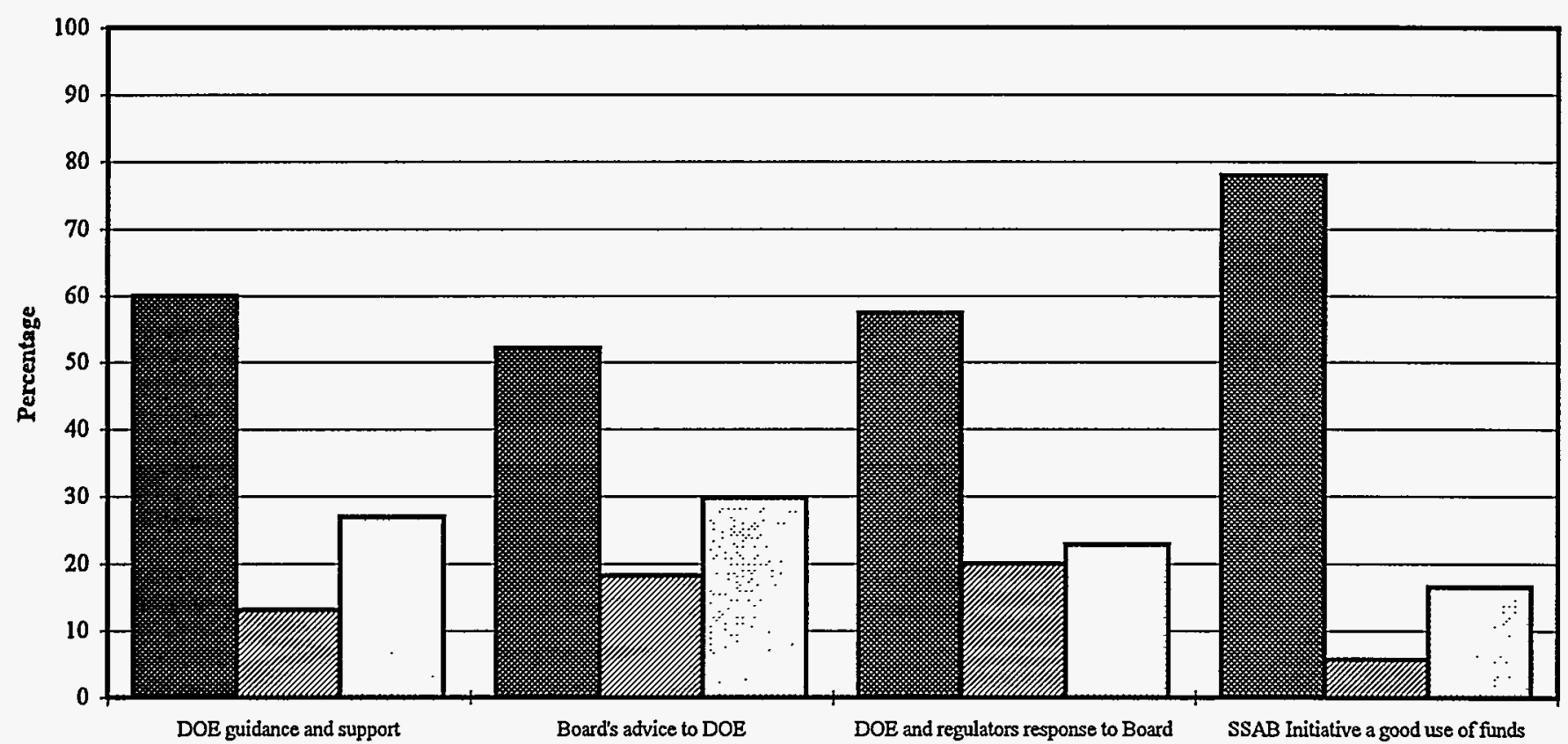

YYes, Good (\%) Neutral, Conditional, No Opinion (\%) DNo, Not Good (\%)

SSAB Supplementary Appendix August 1996 
Figure 14a. St. Louis

Summary: Combined responses for SSAB Initiative goals (Means)

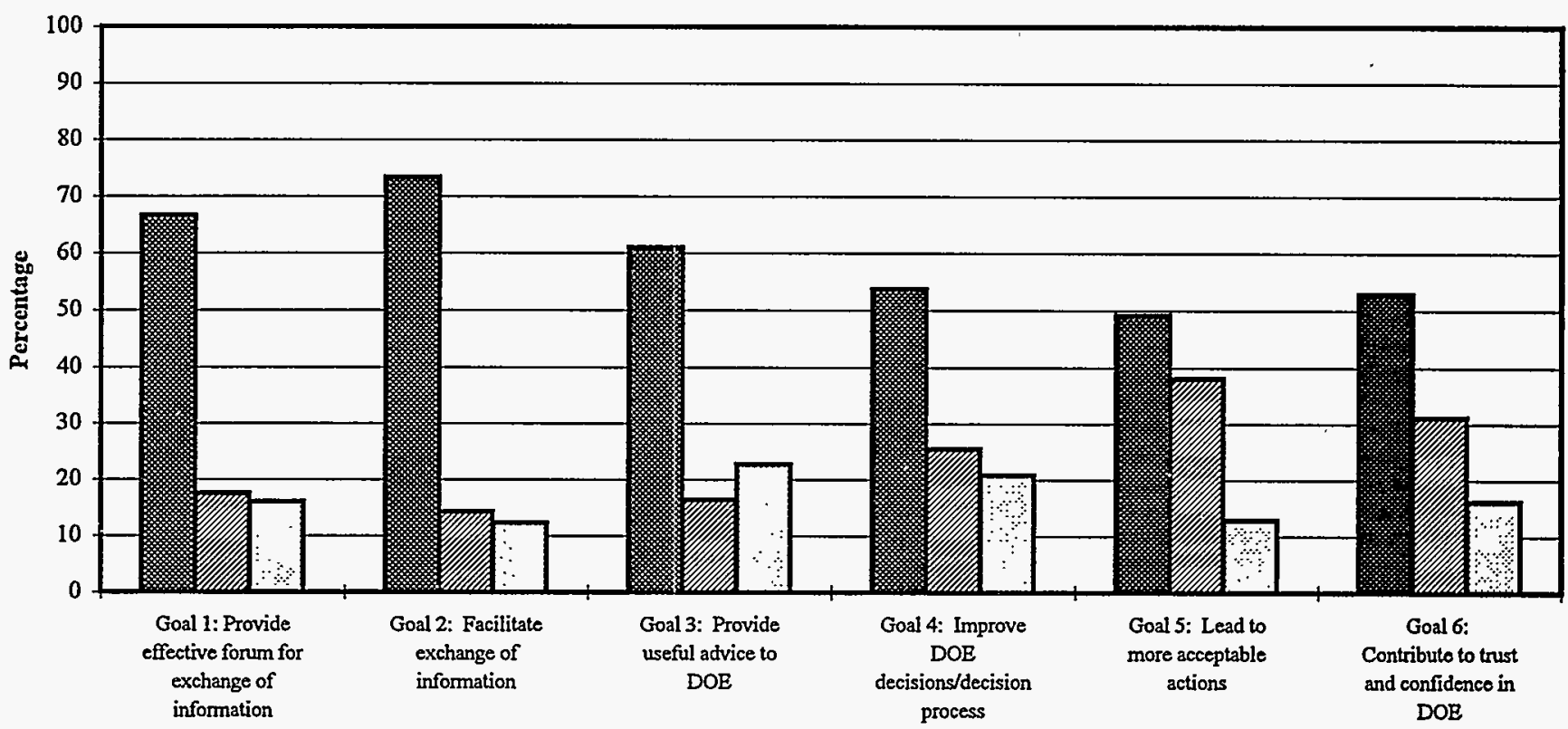

EAgree (\%) $\boldsymbol{Q}$ Neither Agree Nor Disagree/Don't Know (\%) DDisagree (\%)

Figure 14b. Long Survey: Entire Sample

Summary: Combined responses for SSAB Initiative goals (Means)

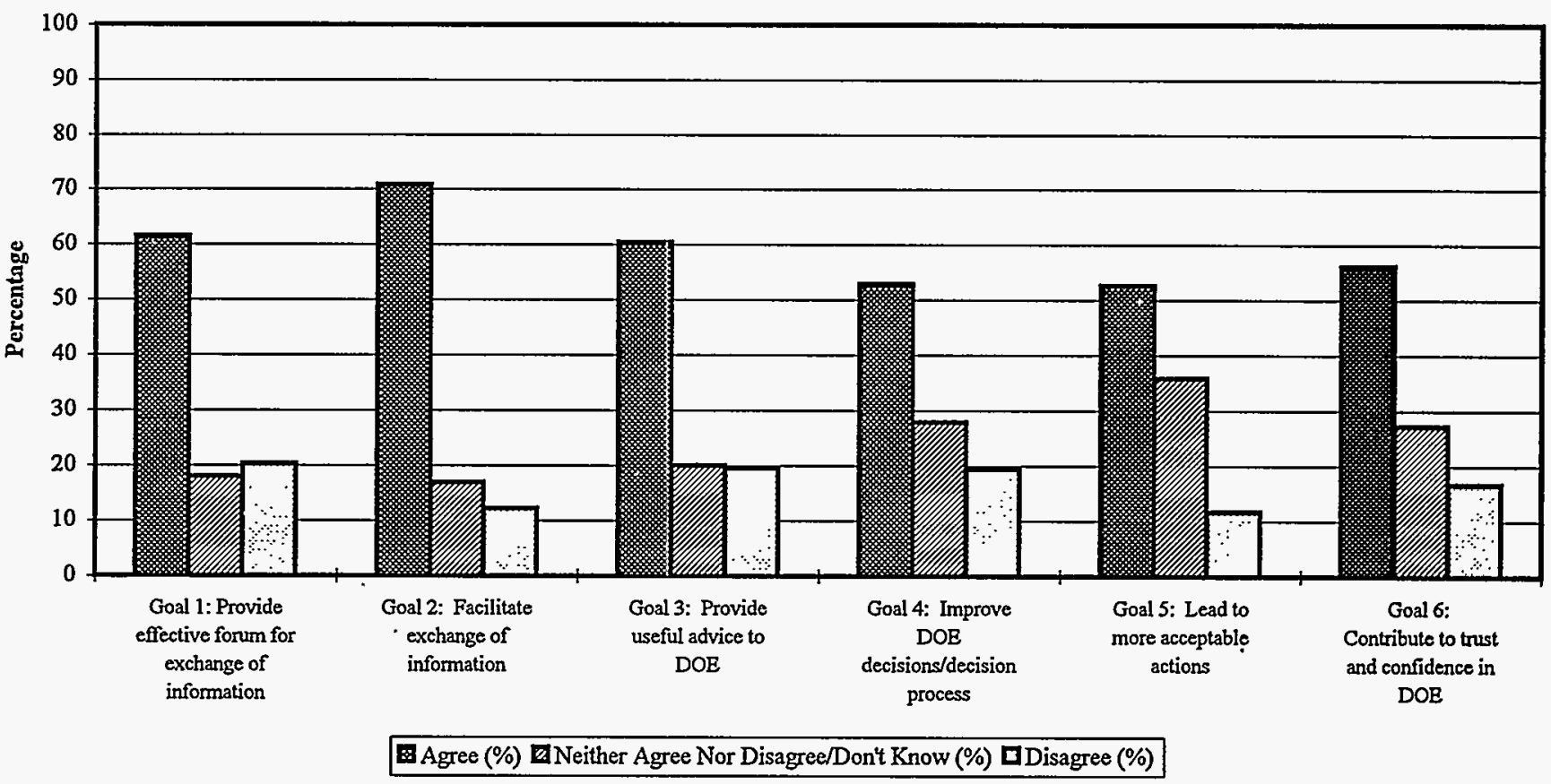

SSAB Supplementary Appendix

August 1996 
Figure 15a. St. Louis

Summary: Combined responses on DOE-HQ involvement,

Personal experience, Group/Team work skills,

Working relations, Member affect (Means)

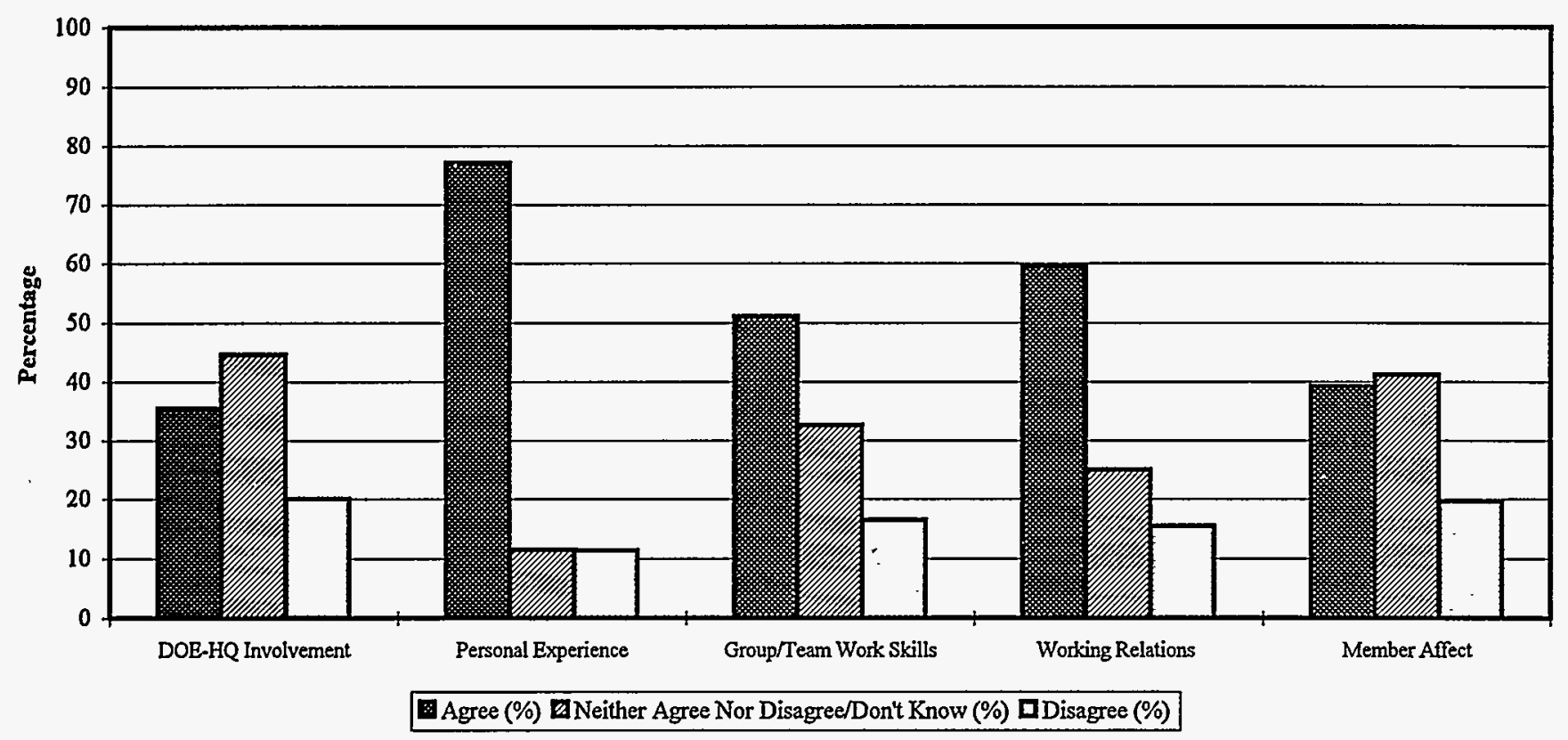

Figure 15b. Long Survey: Entire Sample

Summary: Combined responses on DOE-HQ involvement,

Personal experience, Group/Team work skills,

Working relations, Member affect (Means)

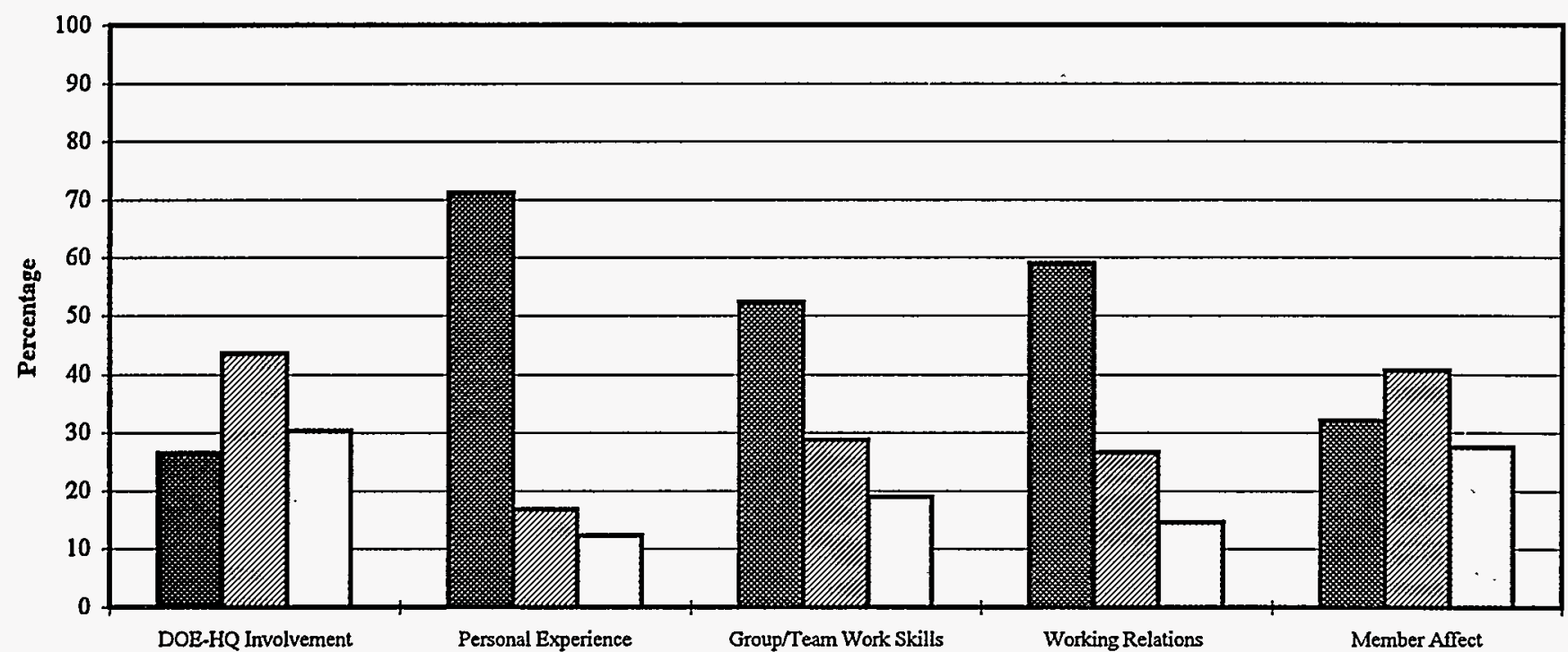

Agree (\%) Weither Agree Nor Disagree/Don't Know (\%) DDisagree (\%)

SSAB Supplementary Appendix 
Table 1.

Goal 1. Establish processes and procedures to provide an effective forum for exchange of information and viewpoints regarding DOE site issues

\begin{tabular}{|c|c|c|c|c|c|c|c|c|}
\hline \multirow[t]{2}{*}{ St. Louis } & \multicolumn{2}{|c|}{ Agree } & \multicolumn{2}{|c|}{$\begin{array}{l}\text { Neither/ } \\
\text { Don't Know }\end{array}$} & \multicolumn{2}{|c|}{ Disagree } & \multicolumn{2}{|c|}{ Total } \\
\hline & $\mathrm{N}$ & $\%$ & $\mathrm{~N}$ & $\%$ & $\mathrm{~N}$ & $\%$ & $N$ & $\%$ \\
\hline $\begin{array}{l}\text { The Board has established processes and } \\
\text { procedures for the effective exchange of } \\
\text { information }\end{array}$ & 21 & $72.4 \%$ & 5 & $17.2 \%$ & 3 & $10.3 \%$ & 29 & $100 \%$ \\
\hline $\begin{array}{l}\text { The Board has agreed-upon procedures to } \\
\text { operate the board }\end{array}$ & 22 & $78.6 \%$ & 2 & $7.1 \%$ & 4 & $14.3 \%$ & 28 & $100 \%$ \\
\hline $\begin{array}{l}\text { The Board has adequate support to allow } \\
\text { it to focus on substantive issues }(R)^{*}\end{array}$ & 23 & $82.1 \%$ & 3 & $10.7 \%$ & 2 & $7.1 \%$ & 28 & $100 \%$ \\
\hline $\begin{array}{l}\text { The Board provides sufficient time for } \\
\text { discussion of issues }\end{array}$ & 23 & $82.1 \%$ & 1 & $3.6 \%$ & 4 & $14.3 \%$ & 28 & $100 \%$ \\
\hline The Board has effective leadership & 17 & $60.7 \%$ & 5 & $17.9 \%$ & 6 & $21.4 \%$ & 28 & $100 \%$ \\
\hline $\begin{array}{l}\text { DOE requests Board advice far enough } \\
\text { ahead of decision deadlines }(\mathrm{R})^{*}\end{array}$ & 11 & $37.9 \%$ & 10 & $34.5 \%$ & 8 & $27.6 \%$ & 29 & $100 \%$ \\
\hline $\begin{array}{l}\text { DOE handles Board administrative issues } \\
\text { expeditiously (R) * }\end{array}$ & 16 & $55.2 \%$ & 9 & $31.0 \%$ & 4 & $13.8 \%$ & 29 & $100 \%$ \\
\hline $\begin{array}{l}\text { The SSAB staff provide satisfactory } \\
\text { support }\end{array}$ & 21 & $75.0 \%$ & 5 & $17.9 \%$ & 2 & $7.1 \%$ & 28 & $100 \%$ \\
\hline $\begin{array}{l}\text { The facilitator for the Board has helped } \\
\text { the Board function effectively }\end{array}$ & 16 & $55.2 \%$ & 5 & $17.2 \%$ & 8 & $27.6 \%$ & 29 & $100 \%$ \\
\hline
\end{tabular}

* (R) denotes a question that was asked in the negative form. For ease of review, these questions and data have been reversed in this table and are stated in positive forms.

SSAB Supplementary Appendix

August 1996 
Table 2. Goal 2. Facilitate interaction and exchange of information and viewpoints
regarding DOE site issues

\begin{tabular}{|c|c|c|c|c|c|c|c|c|}
\hline \multirow[t]{2}{*}{ St. Louis } & \multicolumn{2}{|c|}{ Agree } & \multicolumn{2}{|c|}{$\begin{array}{l}\text { Neither/ } \\
\text { Don't Know }\end{array}$} & \multicolumn{2}{|c|}{ Disagree } & \multicolumn{2}{|c|}{ Total } \\
\hline & $\mathrm{N}$ & $\%$ & $\mathrm{~N}$ & $\%$ & $\mathrm{~N}$ & $\%$ & $\mathrm{~N}$ & $\%$ \\
\hline $\begin{array}{l}\text { The SSAB facilitates effective exchange } \\
\text { of viewpoints on site issues }\end{array}$ & 20 & $69.0 \%$ & 2 & $6.9 \%$ & 7 & $24.1 \%$ & 29 & $100 \%$ \\
\hline $\begin{array}{l}\text { The SSAB contributes to Board members' } \\
\text { understanding of the basis for key site } \\
\text { decisions }\end{array}$ & 21 & $72.4 \%$ & 5 & $17.2 \%$ & 3 & $10.3 \%$ & 29 & $100 \%$ \\
\hline $\begin{array}{l}\text { The SSAB contributes to DOE and } \\
\text { regulators' understanding of the public's } \\
\text { viewpoints on key site decisions }\end{array}$ & 22 & $75.9 \%$ & 3 & $10.3 \%$ & 4 & $13.8 \%$ & 29 & $100 \%$ \\
\hline $\begin{array}{l}\text { The SSAB contributes to a constructive } \\
\text { working relationship among the } \\
\text { participants }\end{array}$ & 22 & $75.9 \%$ & 5 & $17.2 \%$ & 2 & $6.9 \%$ & 29 & $100 \%$ \\
\hline The SSAB strives for consensus & 20 & $71.4 \%$ & 5 & $17.9 \%$ & 3 & $10.7 \%$ & 28 & $100 \%$ \\
\hline $\begin{array}{l}\text { The SSAB gives fair consideration to } \\
\text { dissenting opinions }\end{array}$ & 22 & $75.9 \%$ & 2 & $6.9 \%$ & 5 & $17.2 \%$ & 29 & $100 \%$ \\
\hline $\begin{array}{l}\text { The Board has made the effort needed to } \\
\text { learn about site issues }\end{array}$ & 24 & $85.7 \%$ & 3 & $10.7 \%$ & 1 & $3.6 \%$ & 28 & $100 \%$ \\
\hline $\begin{array}{l}\text { The Board invites expert advice into its } \\
\text { discussion on key policy issues }\end{array}$ & 25 & $89.3 \%$ & 1 & $3.6 \%$ & 2 & $7.1 \%$ & 28 & $100 \%$ \\
\hline $\begin{array}{l}\text { The Board responds to public inquiries } \\
\text { and comments about its decisions }\end{array}$ & 17 & $60.7 \%$ & 10 & $35.7 \%$ & 1 & $3.6 \%$ & 28 & $100 \%$ \\
\hline $\begin{array}{l}\text { The Board solicits feedback from the } \\
\text { community on its work }\end{array}$ & 18 & $64.3 \%$ & 7 & $25.0 \%$ & 3 & $10.7 \%$ & 28 & $100 \%$ \\
\hline $\begin{array}{l}\mathrm{DOE} \text { makes information on key site issues } \\
\text { readily available to the Board }\end{array}$ & 22 & $75.9 \%$ & 1 & $3.4 \%$ & 6 & $20.7 \%$ & 29 & $100 \%$ \\
\hline $\begin{array}{l}\text { The regulators make important } \\
\text { contributions to the Board's work }\end{array}$ & 19 & $65.5 \%$ & 4 & $13.8 \%$ & 6 & $20.7 \%$ & 29 & $100 \%$ \\
\hline $\begin{array}{l}\text { The SSAB creates a climate supportive of } \\
\text { differing viewpoints }(\mathrm{R}){ }^{*}\end{array}$ & 21 & $72.4 \%$ & 5 & $17.2 \%$ & 3 & $10.3 \%$ & 29 & $100 \%$ \\
\hline
\end{tabular}

* (R) denotes a question that was asked in the negative form. For ease of review, these questions and data have been reversed in this table and are stated in positive forms.

SSAB Supplementary Appendix 
Table 3.

Goal 3. Provide useful advice and/or recommendations to DOE (and regulators, where appropriate)

\begin{tabular}{|c|c|c|c|c|c|c|c|c|}
\hline \multirow[t]{2}{*}{ St. Louis } & \multicolumn{2}{|c|}{ Agree } & \multicolumn{2}{|c|}{$\begin{array}{l}\text { Neither/ } \\
\text { Don't Know }\end{array}$} & \multicolumn{2}{|c|}{ Disagree } & \multicolumn{2}{|c|}{ Total } \\
\hline & $\mathrm{N}$ & $\%$ & $\mathrm{~N}$ & $\%$ & $N$ & $\%$ & $\mathbf{N}$ & $\%$ \\
\hline $\begin{array}{l}\text { The Board provides useful advice to DOE } \\
\text { (and regulators, where appropriate) (R) * }\end{array}$ & 20 & $71.4 \%$ & 3 & $10.7 \%$ & 5 & $17.9 \%$ & 28 & $100 \%$ \\
\hline $\begin{array}{l}\text { The SSAB reaches agreement about } \\
\text { prioritization of key site issues for which } \\
\text { advice is sought (R) * }\end{array}$ & 12 & $42.9 \%$ & 6 & $21.4 \%$ & 10 & $35.7 \%$ & 28 & $100 \%$ \\
\hline The Board helps define site problems & 22 & $78.6 \%$ & 0 & $0 \%$ & 6 & $21.4 \%$ & 28 & $100 \%$ \\
\hline $\begin{array}{l}\text { The Board addresses issues in a timely } \\
\text { manner }(\mathrm{R}) *\end{array}$ & 15 & $53.6 \%$ & 7 & $25.0 \%$ & 6 & $21.4 \%$ & 28 & $100 \%$ \\
\hline $\begin{array}{l}\text { The Board reaches consensus on key site } \\
\text { issues }(R) *\end{array}$ & 11 & $40.7 \%$ & 6 & $22.2 \%$ & 10 & $37.0 \%$ & 27 & $100 \%$ \\
\hline $\begin{array}{l}\text { The Board provides informed advice to } \\
\text { DOE (and regulators, where appropriate) }\end{array}$ & 20 & $71.4 \%$ & 4 & $14.3 \%$ & 4 & $14.3 \%$ & 28 & $100 \%$ \\
\hline $\begin{array}{l}\text { The Board provides advice that reflects } \\
\text { the viewpoints and priorities of the } \\
\text { community }\end{array}$ & 19 & $67.9 \%$ & 6 & $21.4 \%$ & 3 & $10.7 \%$ & 28 & $100 \%$ \\
\hline
\end{tabular}

* (R) denotes a question that was asked in the negative form. For ease of review, these questions and data have been reversed in this table and are stated in positive forms. 


\section{Table 4.}

Goal 4. Improve DOE's (and where applicable, regulators') site decisions and decision making process

\begin{tabular}{|c|c|c|c|c|c|c|c|c|}
\hline \multirow[t]{2}{*}{ St. Louis } & \multicolumn{2}{|c|}{ Agree } & \multicolumn{2}{|c|}{$\begin{array}{l}\text { Neither/ } \\
\text { Don't Know }\end{array}$} & \multicolumn{2}{|c|}{ Disagree } & \multicolumn{2}{|c|}{ Total } \\
\hline & $N$ & $\%$ & $\mathrm{~N}$ & $\%$ & $N$ & $\%$ & $\mathrm{~N}$ & $\%$ \\
\hline $\begin{array}{l}\text { The SSAB has improved DOE's site } \\
\text { decisions }(\mathrm{R})^{*}\end{array}$ & 13 & $44.8 \%$ & 7 & $24.1 \%$ & 9 & $31.0 \%$ & 29 & $100 \%$ \\
\hline $\begin{array}{l}\text { The Board understands the decision } \\
\text { making process at [a particular] site }\end{array}$ & 17 & $60.7 \%$ & 7 & $25.0 \%$ & 4 & $14.3 \%$ & 28 & $100 \%$ \\
\hline $\begin{array}{l}\text { DOE discusses important policies } \\
\text { affecting site decisions with the Board }\end{array}$ & 18 & $62.1 \%$ & 4 & $13.8 \%$ & 7 & $24.1 \%$ & 29 & $100 \%$ \\
\hline $\begin{array}{l}\text { DOE has explained to the Board its site } \\
\text { decision making process }\end{array}$ & 16 & $55.2 \%$ & 6 & $20.7 \%$ & 7 & $24.1 \%$ & 29 & $100 \%$ \\
\hline $\begin{array}{l}\text { The DOE shows how Board advice is } \\
\text { reflected in site decisions }\end{array}$ & 14 & $48.3 \%$ & 11 & $37.9 \%$ & 4 & $13.8 \%$ & 29 & $100 \%$ \\
\hline $\begin{array}{l}\text { The decision making process used by } \\
\text { DOE at [a particular] site is effective in } \\
\text { furthering site clean-up (R) * }\end{array}$ & 15 & $51.7 \%$ & 9 & $31.0 \%$ & 5 & $17.2 \%$ & 29 & $100 \%$ \\
\hline
\end{tabular}

* (R) denotes a question that was asked in the negative form. For ease of review, these questions and data have been reversed in this table and are stated in positive forms. 
Table 5.

Goal 5. Lead to more acceptable actions

\begin{tabular}{|c|c|c|c|c|c|c|c|c|}
\hline \multirow[t]{2}{*}{ St. Louis } & \multicolumn{2}{|c|}{ Agree } & \multicolumn{2}{|c|}{$\begin{array}{l}\text { Neither/ } \\
\text { Don't Know }\end{array}$} & \multicolumn{2}{|c|}{ Disagree } & \multicolumn{2}{|c|}{ Total } \\
\hline & $\mathrm{N}$ & $\%$ & $N$ & $\%$ & $\mathbf{N}$ & $\%$ & $\mathrm{~N}$ & $\%$ \\
\hline $\begin{array}{l}\text { The SSAB leads to more acceptable site } \\
\text { decisions }\end{array}$ & 16 & $55.2 \%$ & 11 & $37.9 \%$ & 2 & $6.9 \%$ & 29 & $100 \%$ \\
\hline $\begin{array}{l}\text { The Board supports the recommendations } \\
\text { it gives DOE }\end{array}$ & 15 & $53.6 \%$ & 11 & $39.3 \%$ & 2 & $7.1 \%$ & 28 &, $100 \%$ \\
\hline The Board supports DOE's site actions & 7 & $25.0 \%$ & 16 & $57.1 \%$ & 5 & $17.9 \%$ & 28 & $100 \%$ \\
\hline $\begin{array}{l}\text { The DOE pays attention to the Board's } \\
\text { advice on key site issues }(R)^{*}\end{array}$ & 17 & $58.6 \%$ & 8 & $27.6 \%$ & 4 & $13.8 \%$ & 29 & $100 \%$ \\
\hline $\begin{array}{l}\text { There is support in the community for } \\
\text { DOE's site decisions that have SSAB } \\
\text { input }\end{array}$ & 12 & $41.4 \%$ & 15 & $51.7 \%$ & 2 & $6.9 \%$ & 29 & $100 \%$ \\
\hline Progress is being made on key site issues & 18 & $62.1 \%$ & 4 & $13.8 \%$ & 7 & $24.1 \%$ & 29 & $100 \%$ \\
\hline
\end{tabular}

* (R) denotes a question that was asked in the negative form. For ease of review, these questions and data have been reversed in this table and are stated in positive forms.

SSAB Supplementary Appendix 
Table 6.

Goal 6. Contribute to trust and confidence in DOE

\begin{tabular}{|c|c|c|c|c|c|c|c|c|}
\hline \multirow[t]{2}{*}{ St. Louis } & \multicolumn{2}{|c|}{ Agree } & \multicolumn{2}{|c|}{$\begin{array}{c}\text { Neither/ } \\
\text { Don't Know }\end{array}$} & \multicolumn{2}{|c|}{ Disagree } & \multicolumn{2}{|c|}{ Total } \\
\hline & $\mathrm{N}$ & $\%$ & $\mathrm{~N}$ & $\%$ & $\mathrm{~N}$ & $\%$ & $\mathrm{~N}$ & $\%$ \\
\hline $\begin{array}{l}\text { The SSAB contributes to trust and } \\
\text { confidence in DOE }\end{array}$ & 13 & $44.8 \%$ & 11 & $37.9 \%$ & 5 & $17.2 \%$ & 29 & $100 \%$ \\
\hline $\begin{array}{l}\text { Relationships between DOE and the public } \\
\text { have improved since the formation of the } \\
\text { SSAB }\end{array}$ & 17 & $58.6 \%$ & 7 & $24.1 \%$ & 5 & $17.2 \%$ & 29 & $100 \%$ \\
\hline $\begin{array}{l}\text { DOE is committed to clean up [a } \\
\text { particular] site }\end{array}$ & 16 & $55.2 \%$ & 9 & $31.0 \%$ & 4 & $13.8 \%$ & 29 & $100 \%$ \\
\hline
\end{tabular}


Table 7.

Public awareness

\begin{tabular}{llllllllll}
\hline \multicolumn{1}{c}{ St. Louis } & Agree & $\begin{array}{c}\text { Neither/ } \\
\text { Don't Know }\end{array}$ & Disagree & Total \\
& $\mathrm{N}$ & $\%$ & $\mathrm{~N}$ & $\%$ & $\mathrm{~N}$ & $\%$ & $\mathrm{~N}$ & $\%$ \\
\hline $\begin{array}{l}\text { The public knows little about the role of } \\
\text { the Board }\end{array}$ & 20 & $69.0 \%$ & 6 & $20.7 \%$ & 3 & $10.3 \%$ & 29 & $100 \%$ \\
\hline
\end{tabular}

Table 8.

SSAB Initiative is a good use of funds

\begin{tabular}{ccccccccc}
\hline St. Louis & Yes, Good & $\begin{array}{c}\text { Neutral, } \\
\text { Conditional, } \\
\text { No Opinion }\end{array}$ & $\begin{array}{c}\text { No, } \\
\text { Not Good }\end{array}$ & Total \\
& $\mathrm{N}$ & $\%$ & $\mathrm{~N}$ & $\%$ & $\mathrm{~N}$ & $\%$ & $\mathrm{~N}$ & $\%$ \\
\hline SSAB Initiative is a good use of funds & 17 & $70.8 \%$ & 3 & $12.5 \%$ & 4 & $16.7 \%$ & 24 & $100 \%$ \\
\hline
\end{tabular}

SSAB Supplementary Appendix 
Table 9.

DOE-HQ involvement with the SSAB Initiative

\begin{tabular}{|c|c|c|c|c|c|c|c|c|}
\hline \multirow[t]{2}{*}{ St. Louis } & \multicolumn{2}{|c|}{ Agree } & \multicolumn{2}{|c|}{$\begin{array}{l}\text { Neither/ } \\
\text { Don't Know }\end{array}$} & \multicolumn{2}{|c|}{ Disagree } & \multicolumn{2}{|c|}{ Total } \\
\hline & $N$ & $\%$ & $N$ & $\%$ & $\mathrm{~N}$ & $\%$ & $\mathrm{~N}$ & $\%$ \\
\hline $\begin{array}{l}\text { DOE-HQ provides helpful guidance to the } \\
\text { Boards and to DOE }\end{array}$ & 8 & $28.6 \%$ & 11 & $39.3 \%$ & 9 & $32.1 \%$ & 28 & $100 \%$ \\
\hline $\begin{array}{l}\text { DOE-HQ provides sufficient support to } \\
\text { facilitate the work of the SSAB }\end{array}$ & 14 & $50.0 \%$ & 10 & $35.7 \%$ & 4 & $14.3 \%$ & 28 & $100 \%$ \\
\hline $\begin{array}{l}\text { DOE-HQ gives careful consideration to } \\
\text { SSAB advice in its decisions }\end{array}$ & 8 & $27.6 \%$ & 17 & $58.6 \%$ & 4 & $13.8 \%$ & 29 & $100 \%$ \\
\hline
\end{tabular}


Table 10.

Personal experience with the SSAB Initiative

\begin{tabular}{|c|c|c|c|c|c|c|c|c|}
\hline \multirow[t]{2}{*}{ St. Louis } & \multicolumn{2}{|c|}{ Agree } & \multicolumn{2}{|c|}{$\begin{array}{c}\text { Neither/ } \\
\text { Don't Know }\end{array}$} & \multicolumn{2}{|c|}{ Disagree } & \multicolumn{2}{|c|}{ Total } \\
\hline & $\mathbf{N}$ & $\%$ & $\mathrm{~N}$ & $\%$ & $N$ & $\%$ & $\mathbf{N}$ & $\%$ \\
\hline $\begin{array}{l}\text { I consider myself to be very } \\
\text { knowledgeable about site issues }\end{array}$ & 26 & $89.7 \%$ & 2 & $6.9 \%$ & 1 & $3.4 \%$ & 29 & $100 \%$ \\
\hline $\begin{array}{l}\text { Because of the SSAB, I better understand } \\
\text { the complexities of site clean-up }\end{array}$ & 20 & $69.0 \%$ & 4 & $13.8 \%$ & 5 & $17.2 \%$ & 29 & $100 \%$ \\
\hline $\begin{array}{l}\text { I feel that my participation in the SSAB } \\
\text { has been worthwhile }(R)^{*}\end{array}$ & 21 & $72.4 \%$ & 4 & $13.8 \%$ & 4 & $13.8 \%$ & 29 & $100 \%$ \\
\hline
\end{tabular}

* (R) denotes a question that was asked in the negative form. For ease of review, these questions and data have been reversed in this table and are stated in positive forms.

SSAB Supplementary Appendix

August 1996 
Table 11.

Group/Team work skills

\begin{tabular}{|c|c|c|c|c|c|c|c|c|}
\hline \multirow[t]{2}{*}{ St. Louis } & \multicolumn{2}{|c|}{ Agree } & \multicolumn{2}{|c|}{$\begin{array}{l}\text { Neither/ } \\
\text { Don't Know }\end{array}$} & \multicolumn{2}{|c|}{ Disagree } & \multicolumn{2}{|c|}{ Total } \\
\hline & $\mathrm{N}$ & $\%$ & $\mathrm{~N}$ & $\%$ & $N$ & $\%$ & $N$ & $\%$ \\
\hline All have group/team work skills & 11 & $40.7 \%$ & 9 & $33.3 \%$ & 7 & $25.9 \%$ & 27 & $100 \%$ \\
\hline $\begin{array}{l}\text { Have developed well-established working } \\
\text { relationships with each other }\end{array}$ & 16 & $57.1 \%$ & 7 & $25.0 \%$ & 5 & $17.9 \%$ & 28 & $100 \%$ \\
\hline Like each other & 13 & $46.4 \%$ & 12 & $42.9 \%$ & 3 & $10.7 \%$ & 28 & $100 \%$ \\
\hline $\begin{array}{l}\text { Have a high degree of trust and } \\
\text { confidence in each other }\end{array}$ & 9 & $32.1 \%$ & 11 & $39.3 \%$ & 8 & $28.6 \%$ & 28 & $100 \%$ \\
\hline $\begin{array}{l}\text { Accept the values that are important to the } \\
\text { group }\end{array}$ & 14 & $50.0 \%$ & 8 & $28.6 \%$ & 6 & $21.4 \%$ & 28 & $100 \%$ \\
\hline $\begin{array}{l}\text { Show respepct for the viewpoints of } \\
\text { others }\end{array}$ & 22 & $78.6 \%$ & 1 & $3.6 \%$ & 5 & $17.9 \%$ & 28 & $100 \%$ \\
\hline $\begin{array}{l}\text { Accept willingly the goals and } \\
\text { expectations for the Board }\end{array}$ & 16 & $57.1 \%$ & 8 & $28.6 \%$ & 4 & $14.3 \%$ & 28 & $100 \%$ \\
\hline $\begin{array}{l}\text { Set high expectations for what they } \\
\text { believe they can accomplish }\end{array}$ & 17 & $60.7 \%$ & 8 & $28.6 \%$ & 3 & $10.7 \%$ & 28 & $100 \%$ \\
\hline $\begin{array}{l}\text { Communicate fully and frankly to the } \\
\text { Board all relevant and valuable } \\
\text { information }\end{array}$ & 17 & $63.0 \%$ & 7 & $25.9 \%$ & 3 & $11.1 \%$ & 27 & $100 \%$ \\
\hline $\begin{array}{l}\text { Try not to waste the Board's time with } \\
\text { irrelevant material or communications }\end{array}$ & 20 & $71.4 \%$ & 3 & $10.7 \%$ & 5 & $17.9 \%$ & 28 & $100 \%$ \\
\hline $\begin{array}{l}\text { Are willing to be influenced by other } \\
\text { Board members about new ideas and } \\
\text { methods }\end{array}$ & 16 & $57.1 \%$ & 7 & $25.0 \%$ & 5 & $17.9 \%$ & 28 & $100 \%$ \\
\hline $\begin{array}{l}\text { Clearly understand the goals and } \\
\text { philosphy of the Board's operation }\end{array}$ & 12 & $42.9 \%$ & 12 & $42.9 \%$ & 4 & $14.3 \%$ & 28 & $100 \%$ \\
\hline $\begin{array}{l}\text { Do all they can to help the Board achieve } \\
\text { its objectives }\end{array}$ & 10 & $35.7 \%$ & 15 & $53.6 \%$ & 3 & $10.7 \%$ & 28 & $100 \%$ \\
\hline $\begin{array}{l}\text { Get the technical knowledge and training } \\
\text { in group skills they need }\end{array}$ & 12 & $42.9 \%$ & 9 & $32.1 \%$ & 7 & $25.0 \%$ & 28 & $100 \%$ \\
\hline Provide one another mutual help & 16 & $57.1 \%$ & 11 & $39.3 \%$ & 1 & $3.6 \%$ & 28 & $100 \%$ \\
\hline
\end{tabular}

SSAB Supplementary Appendix

August 1996 
Sandia Site Specific Advisory Board Evaluation Survey Results

SSAB Supplementary Appendix August 1996 
Figure 1a. Sandia

Goal 1. Establish processes and procedures to provide an effective forum for exchange of information and viewpoints regarding DOE site issues

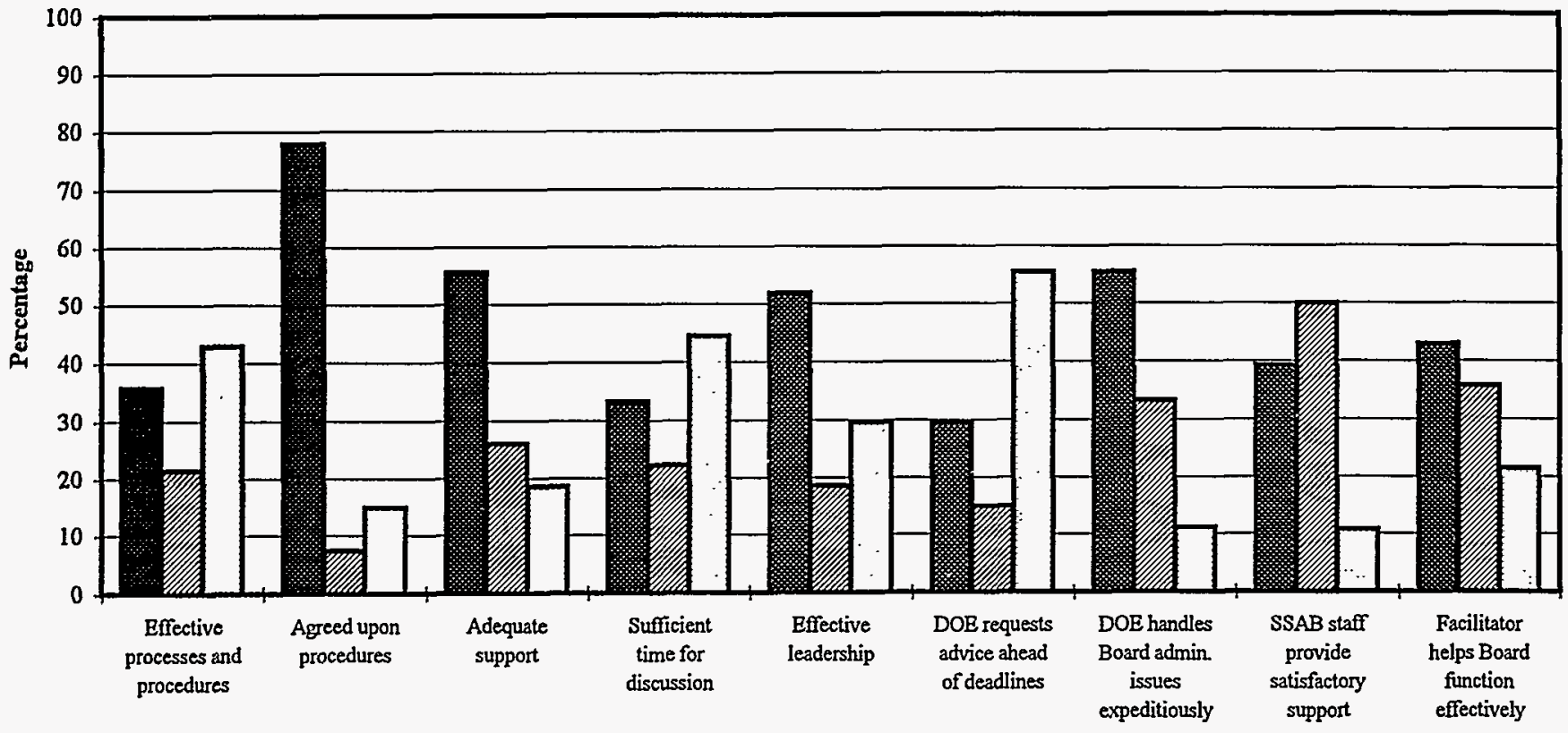

Agree (\%) $\square$ Neither Agree Nor Disagree/Don't Know (\%) DDisagree (\%)

Figure 1b. Long Survey: Entire Sample

Goal 1. Establish processes and procedures to provide an effective forum for exchange of information and viewpoints regarding DOE site issues

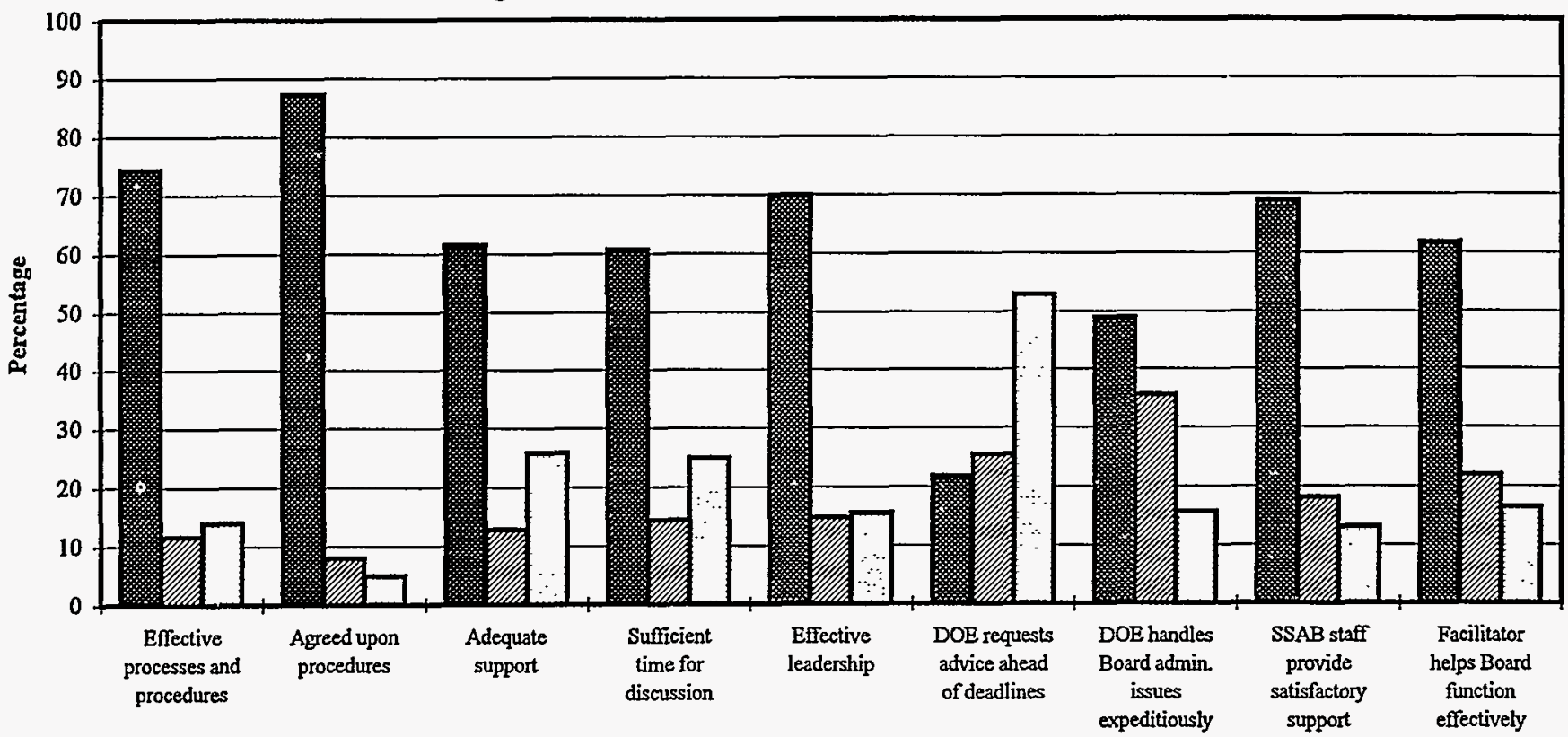

Agree (\%) Deither Agree Nor Disagree/Don't Know (\%) DDisagree (\%)

SSAB Supplementary Appendix

August 1996 
Figure 2a. Sandia

Goal 2. Facilitate interaction and exchange of information and viewpoints regarding DOE site issues

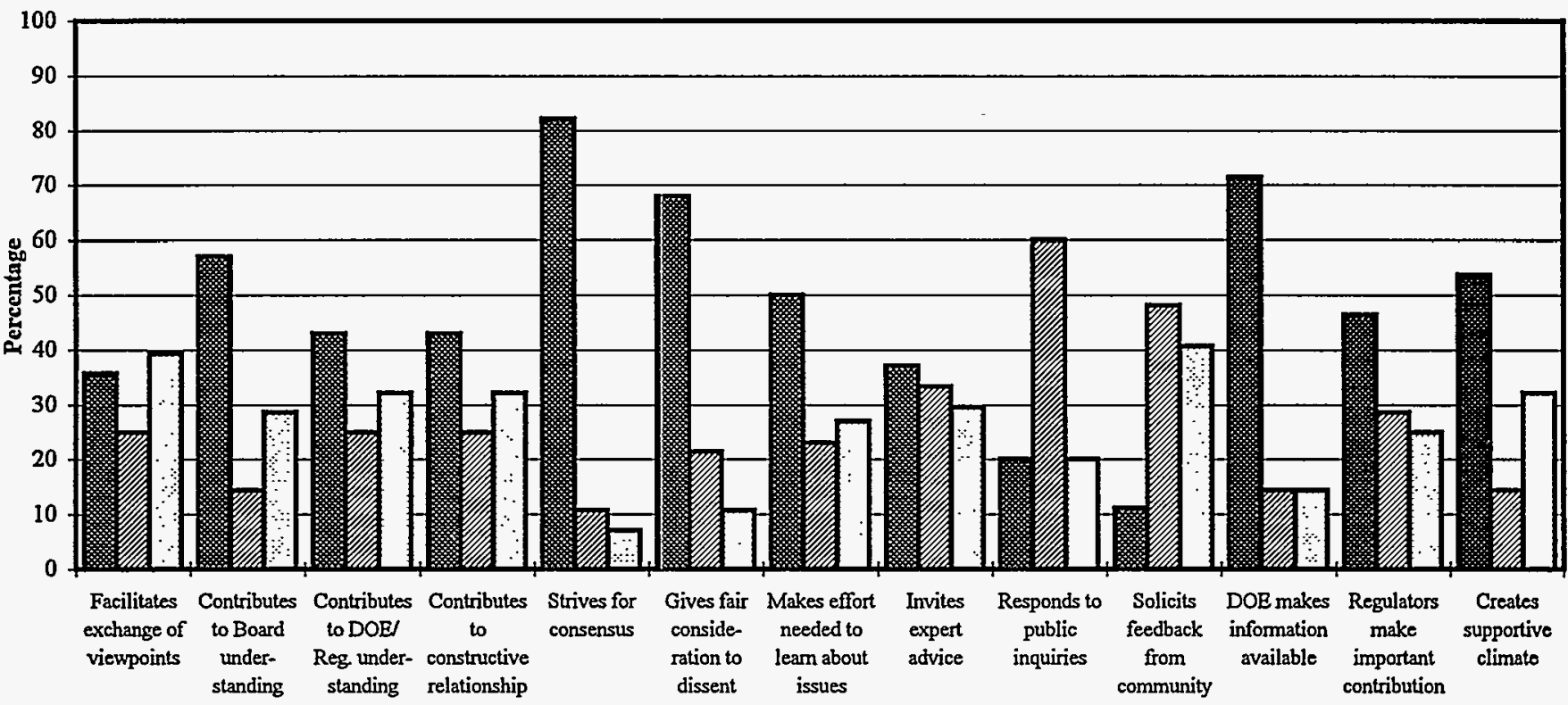

Agree (\%) ENeither Agree Nor Disagree/Don't Know (\%) DDisagree (\%)

Figure 2b. Long Survey: Entire Sample

Goal 2. Facilitate interaction and exchange of information and viewpoints regarding $\mathrm{DOE}$ site issues

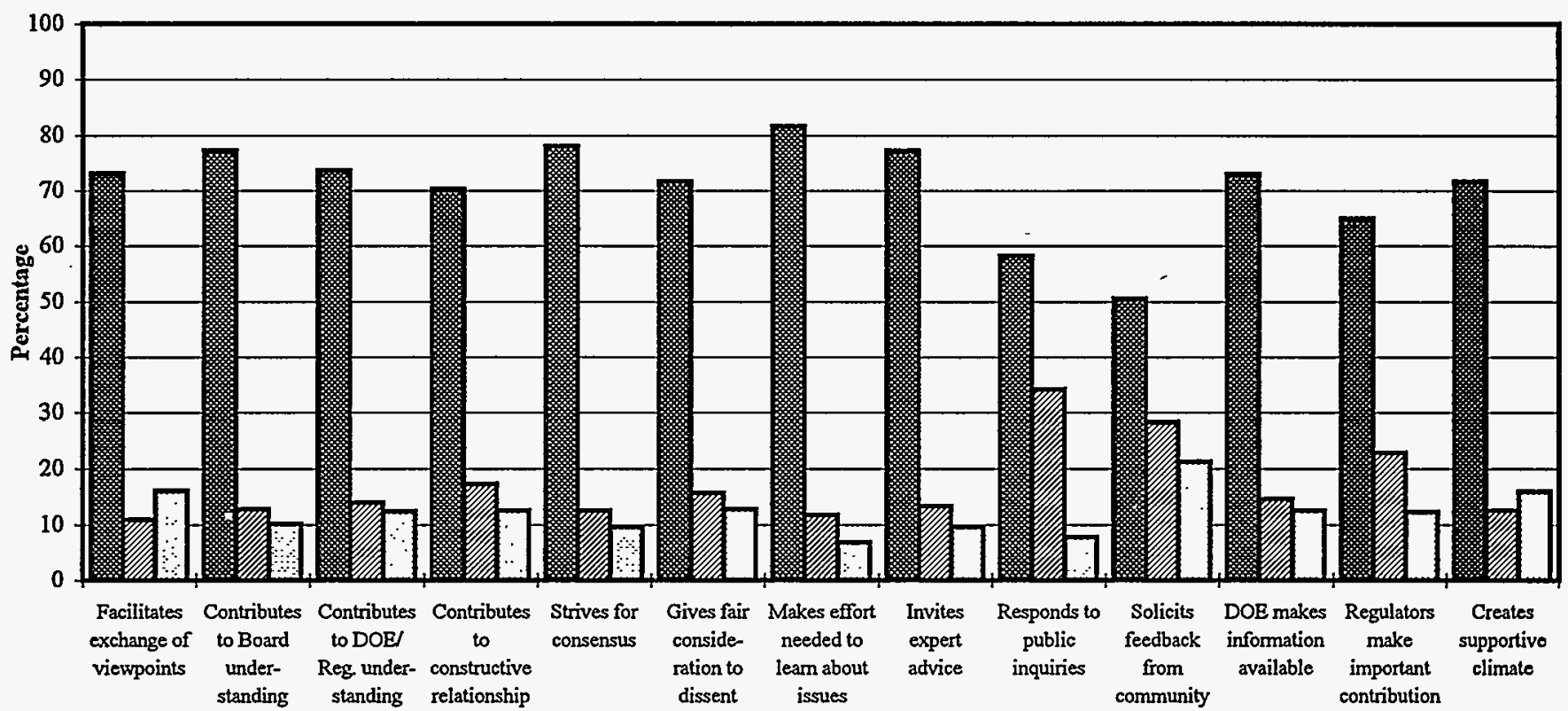

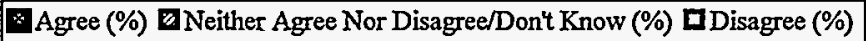

SSAB Supplementary Appendix

August 1996 
Figure 3a. Sandia

Goal 3. Provide useful advice and/or recommendations to DOE

(and regulators, where appropriate)

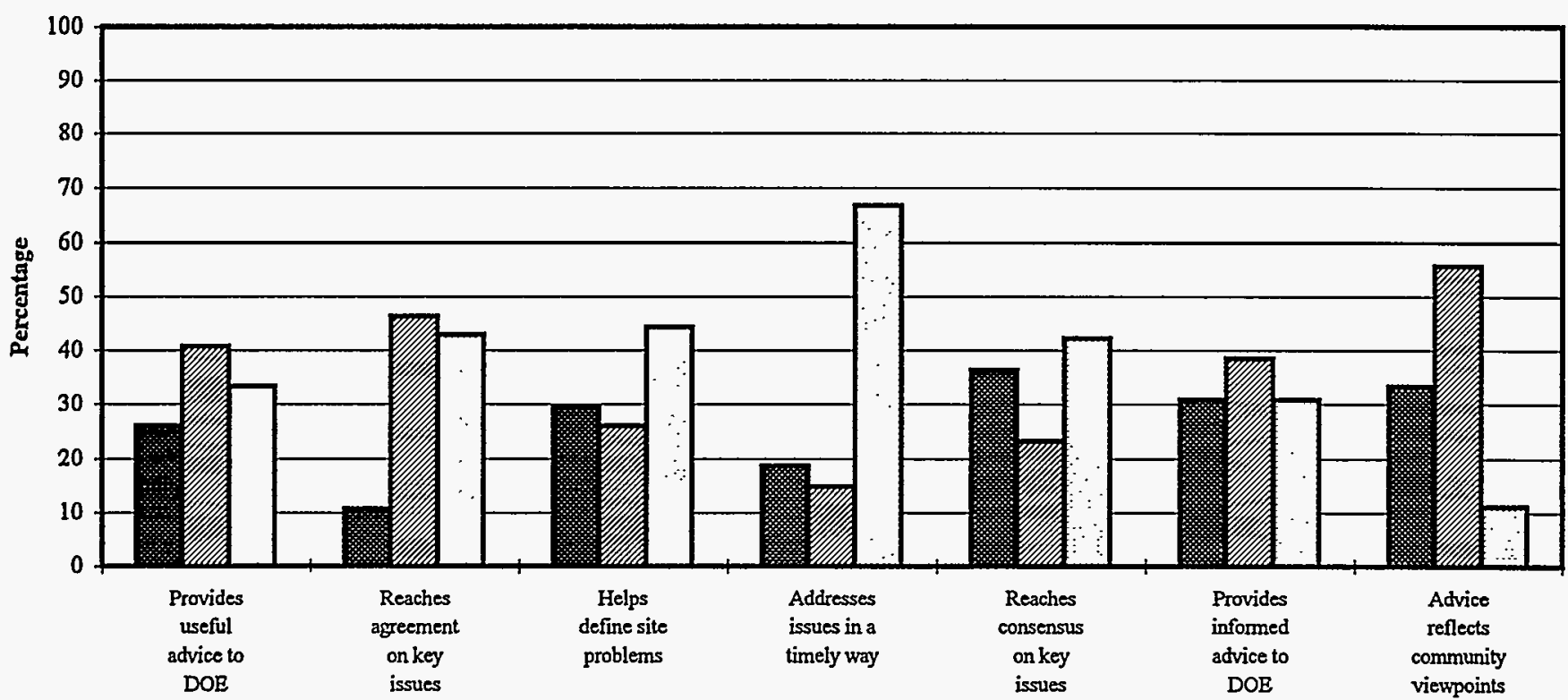

Agree (\%) Deither Agree Nor Disagree/Dont Know (\%) DDisagree (\%)

Figure 3b. Long Survey: Entire Sample

Goal 3. Provide useful advice and/or recommendations to DOE

(and regulators, where appropriate)

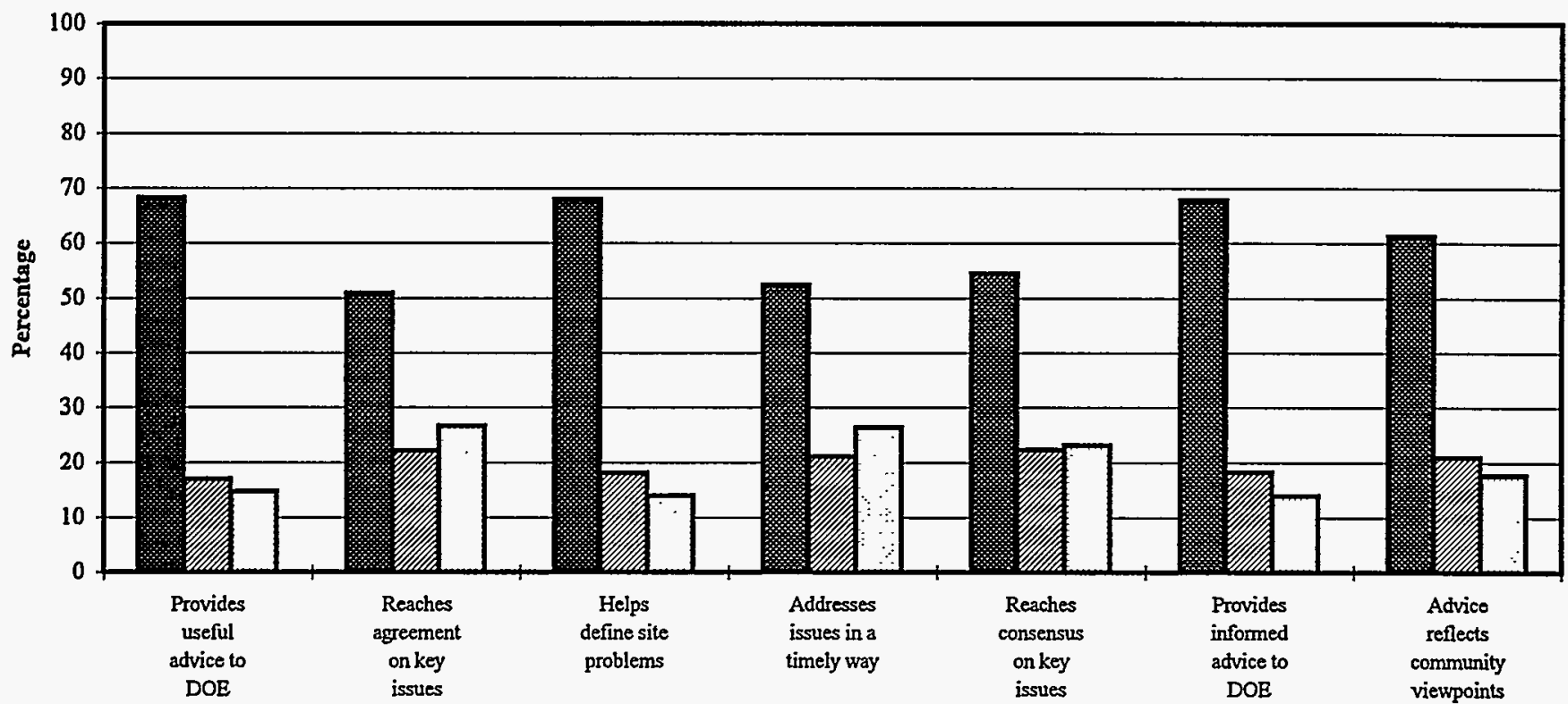

Agree (\%) EDeither Agree Nor Disagree/Don't Know (\%) DDisagree (\%)

SSAB Supplementary Appendix

August 1996 
Figure 4a. Sandia

Goal 4. Improve DOE's (and where applicable, regulators') site decisions and decision making process

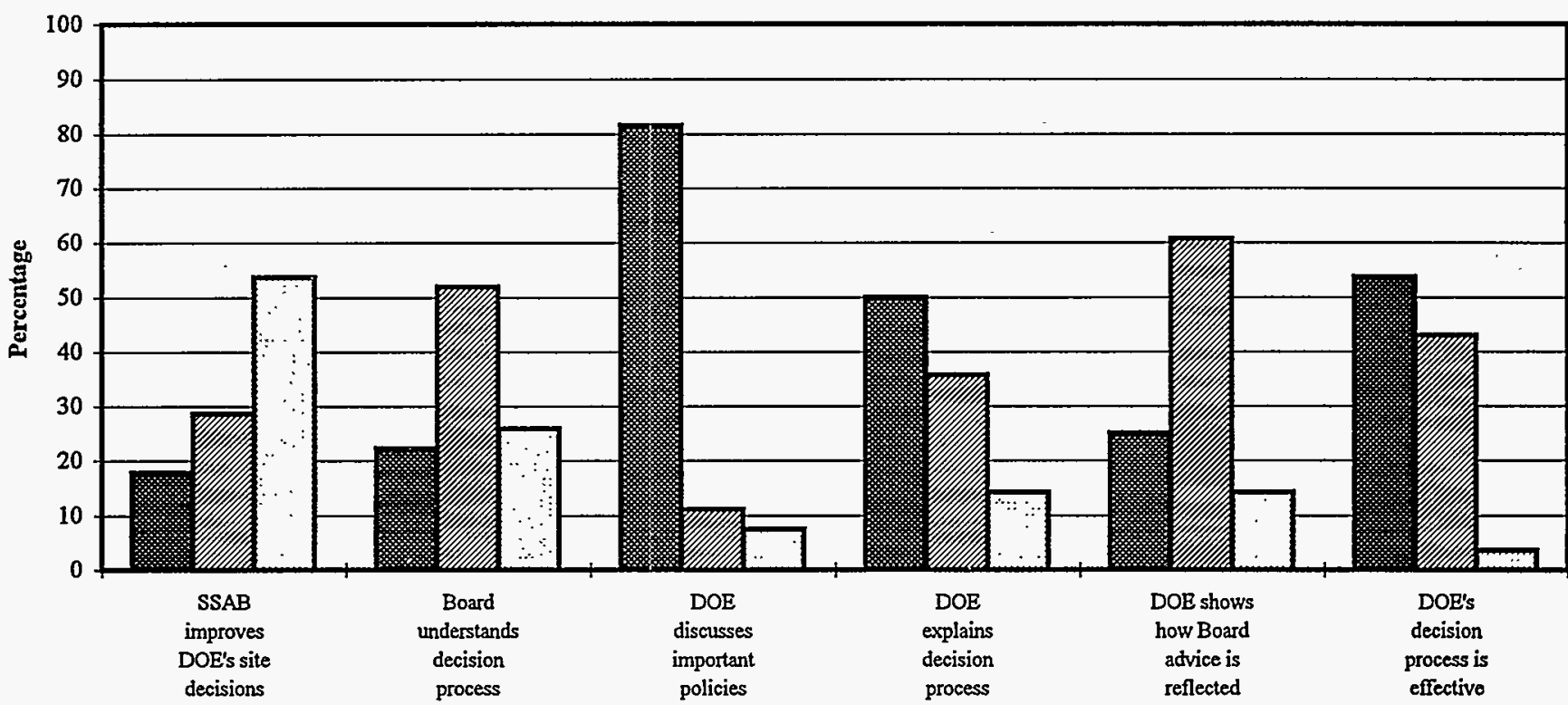

Agree (\%) Neither Agree Nor Disagree/Don't Know (\%) DDisagree (\%)

Figure 4b. Long Survey: Entire Sample

Goal 4. Improve DOE's (and where applicable, regulators')

site decisions and decision making process

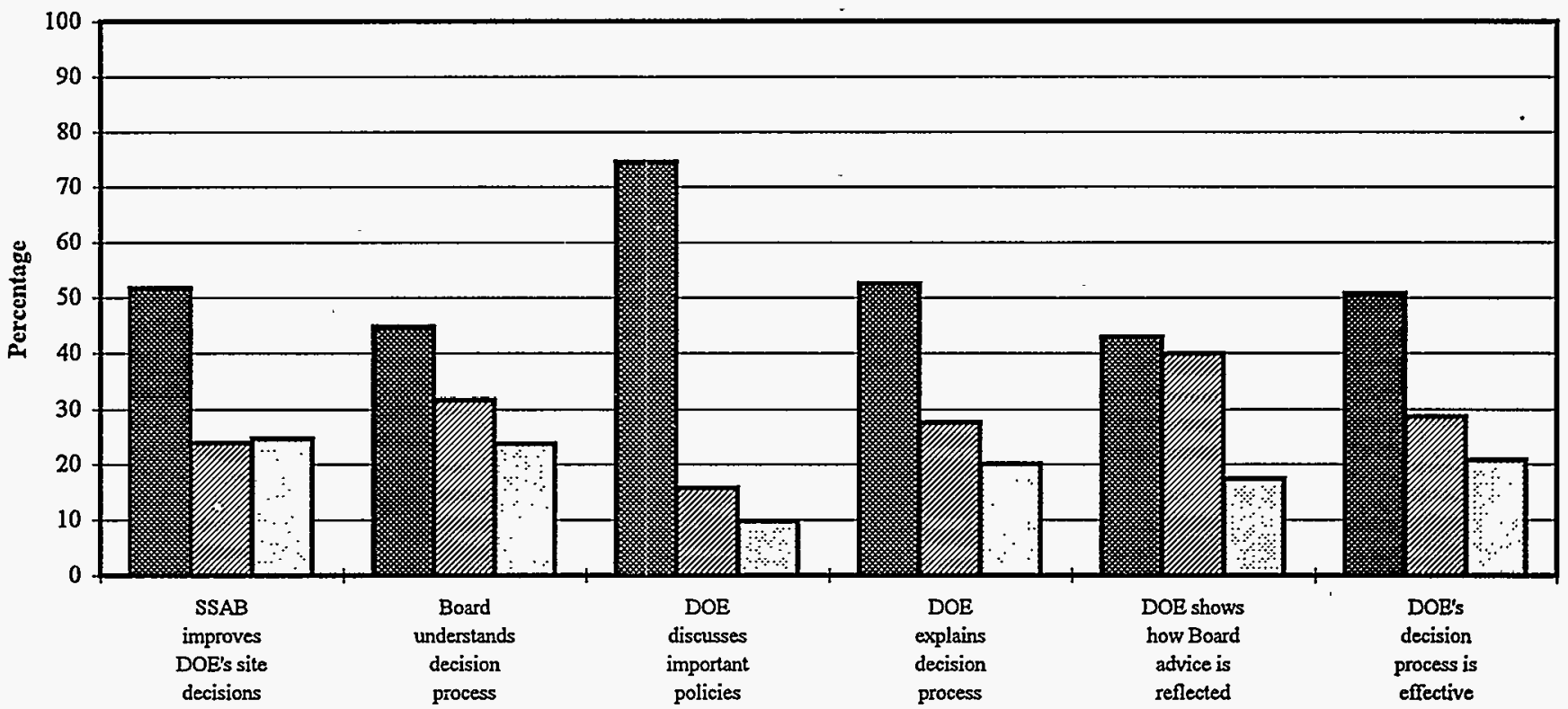

$\checkmark$ Agree (\%) DNeither Agree Nor Disagree/Don't Know (\%) DDisagree (\%)

SSAB Supplementary Appendix

August 1996 
Figure 5a. Sandia

Goal 5. Lead to more acceptable actions

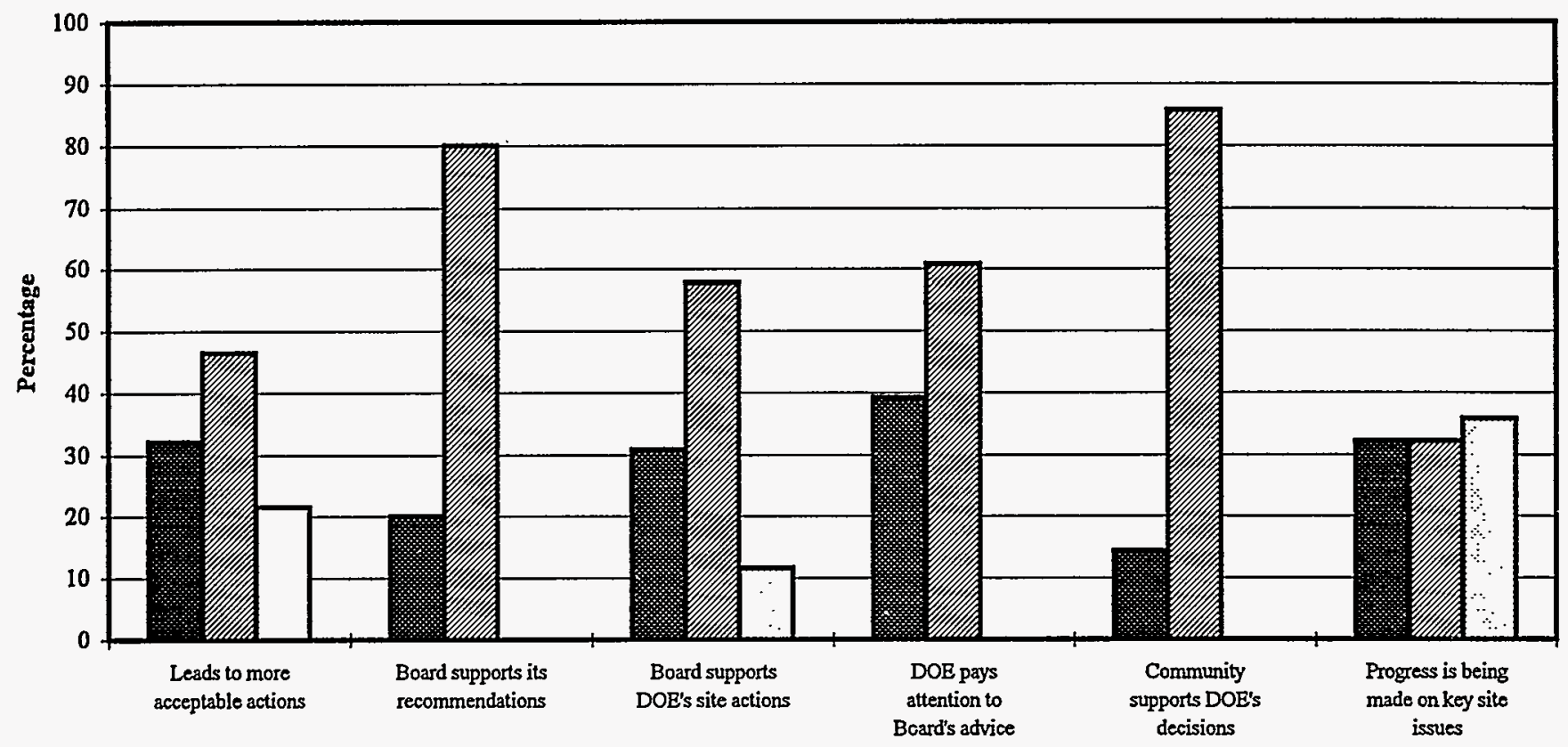

EAgree (\%) ENeither Agree Nor Disagree/Don't Know (\%) DDisagree (\%)

Figure 5b. Long Survey: Entire Sample

Goal 5. Lead to more acceptable actions

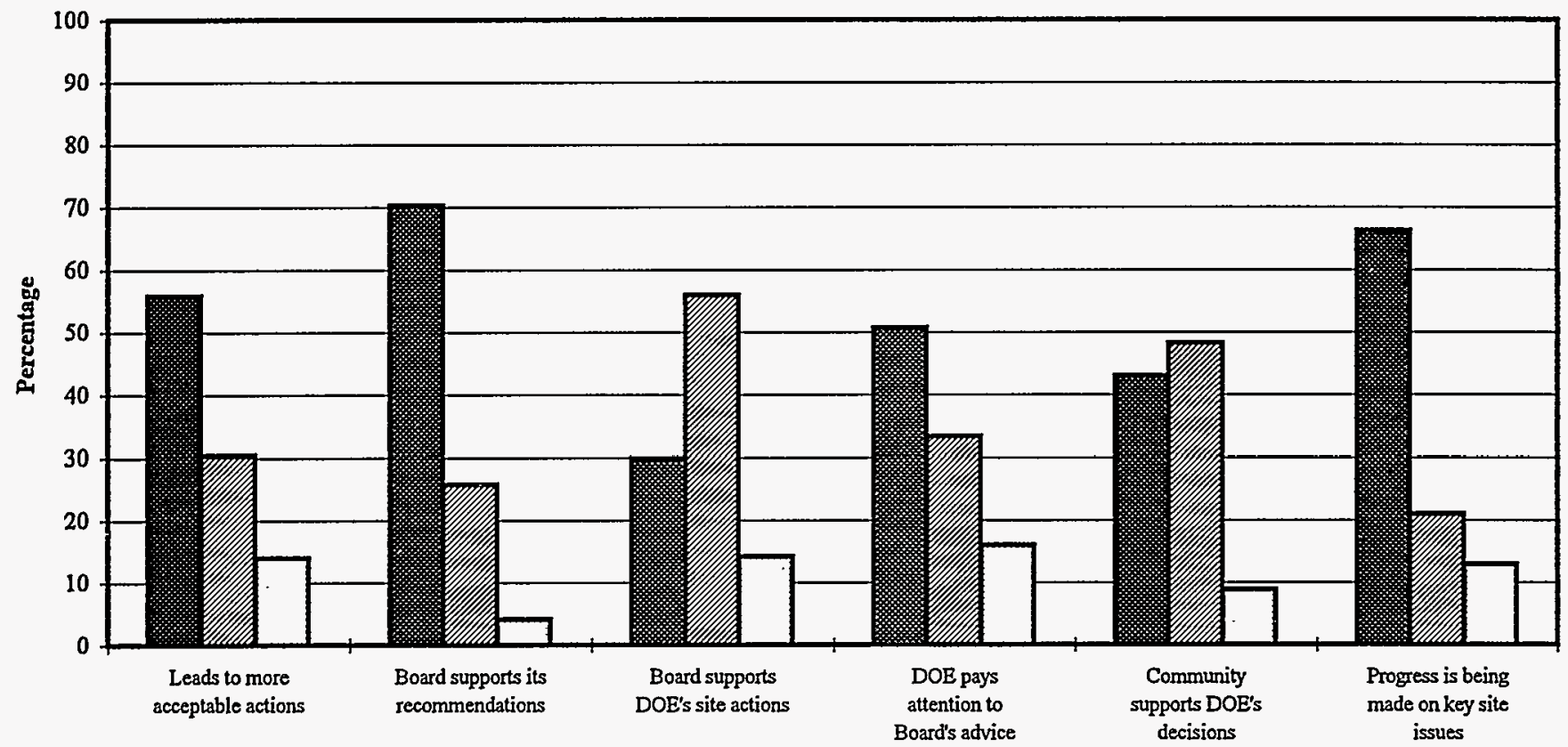

Agree (\%) Q Neither Agree Nor Disagree/Don't Know (\%) DDisagree (\%)

SSAB Supplementary Appendix

August 1996 
Figure 6a. Sandia

Goal 6. Contribute to trust and confidence in DOE

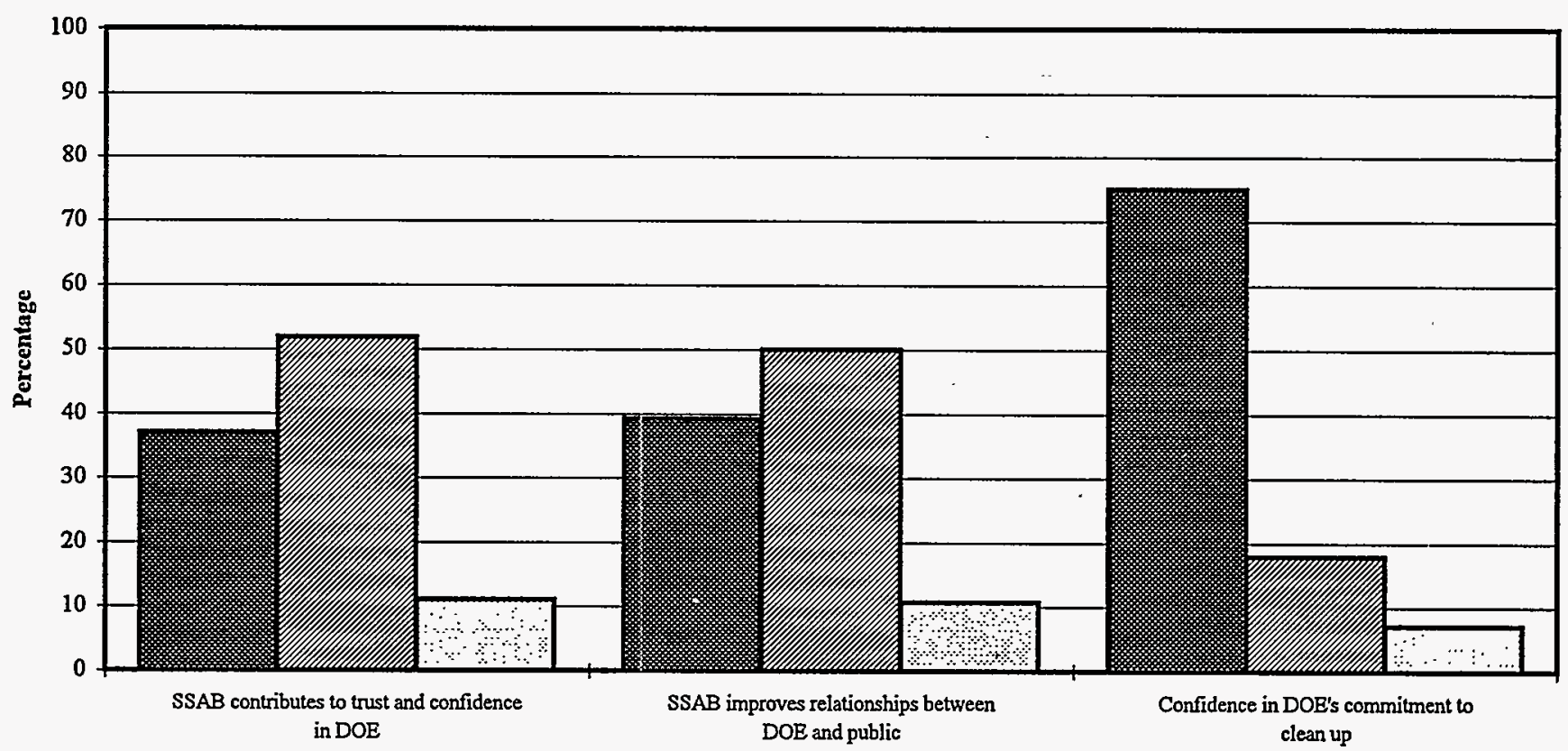

D Agree (\%) Deither Agree Nor Disagree/Don't Know (\%) DDisagree (\%)

Figure 6b. Long Survey: Entire Sample

Goal 6. Contribute to trust and confidence in DOE

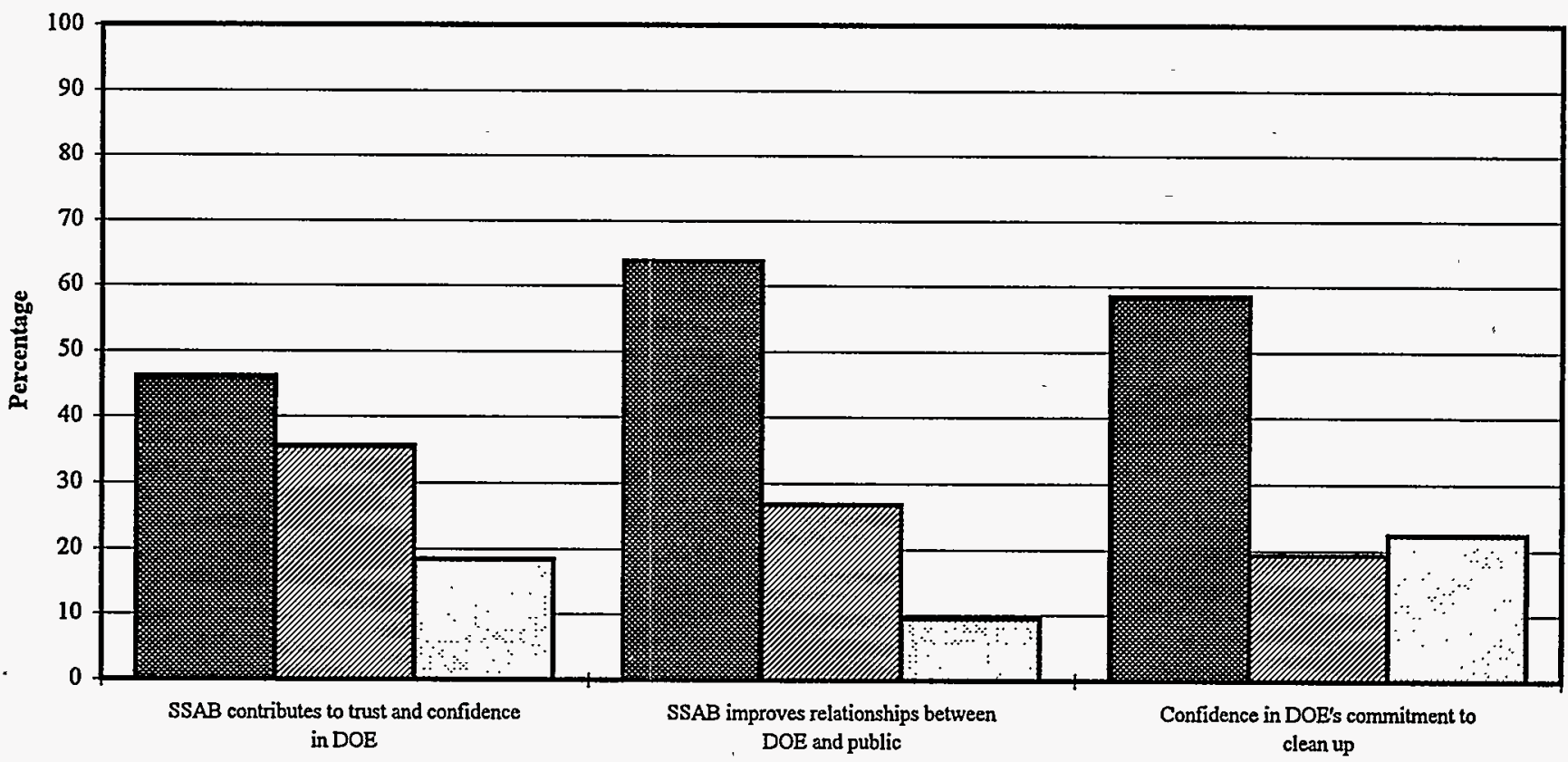

Agree (\%) Weither Agree Nor Disagree/Don't Know (\%) DDisagree (\%)

SSAB Supplementary Appendix

August 1996 
Figure 7a. Sandia

Public awareness

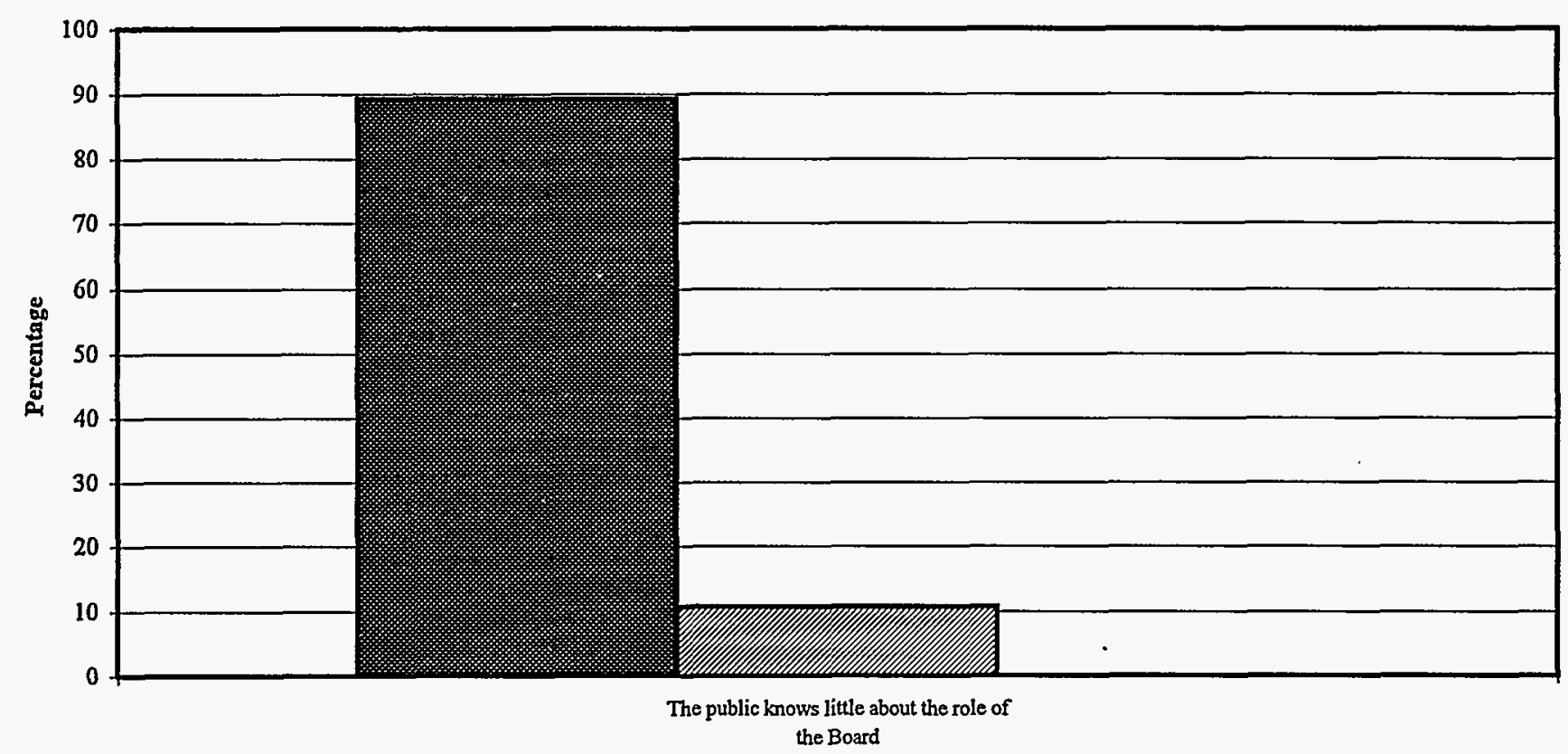

Agree (\%) Deither Agree Nor Disagree/Don't Know (\%) DDisagree (\%)

Figure 7b. Long Survey: Entire Sample

Public awareness

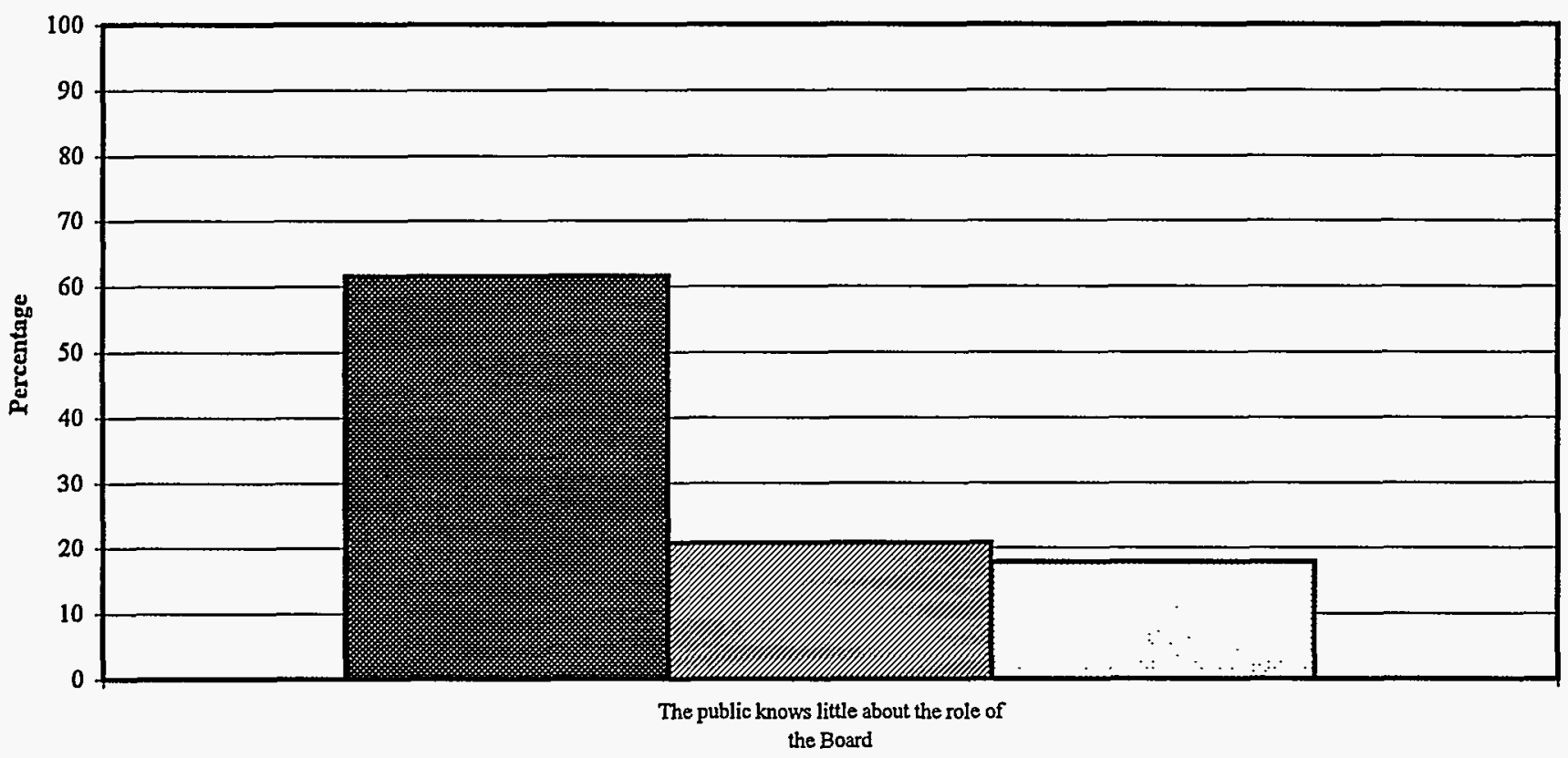

Agree (\%) 0 Neither Agree Nor Disagree/Dont Know (\%) DDisagree (\%)

SSAB Supplementary Appendix

August 1996 
Figure 8a. Sandia

DOE-HQ involvement with the SSAB Initiative

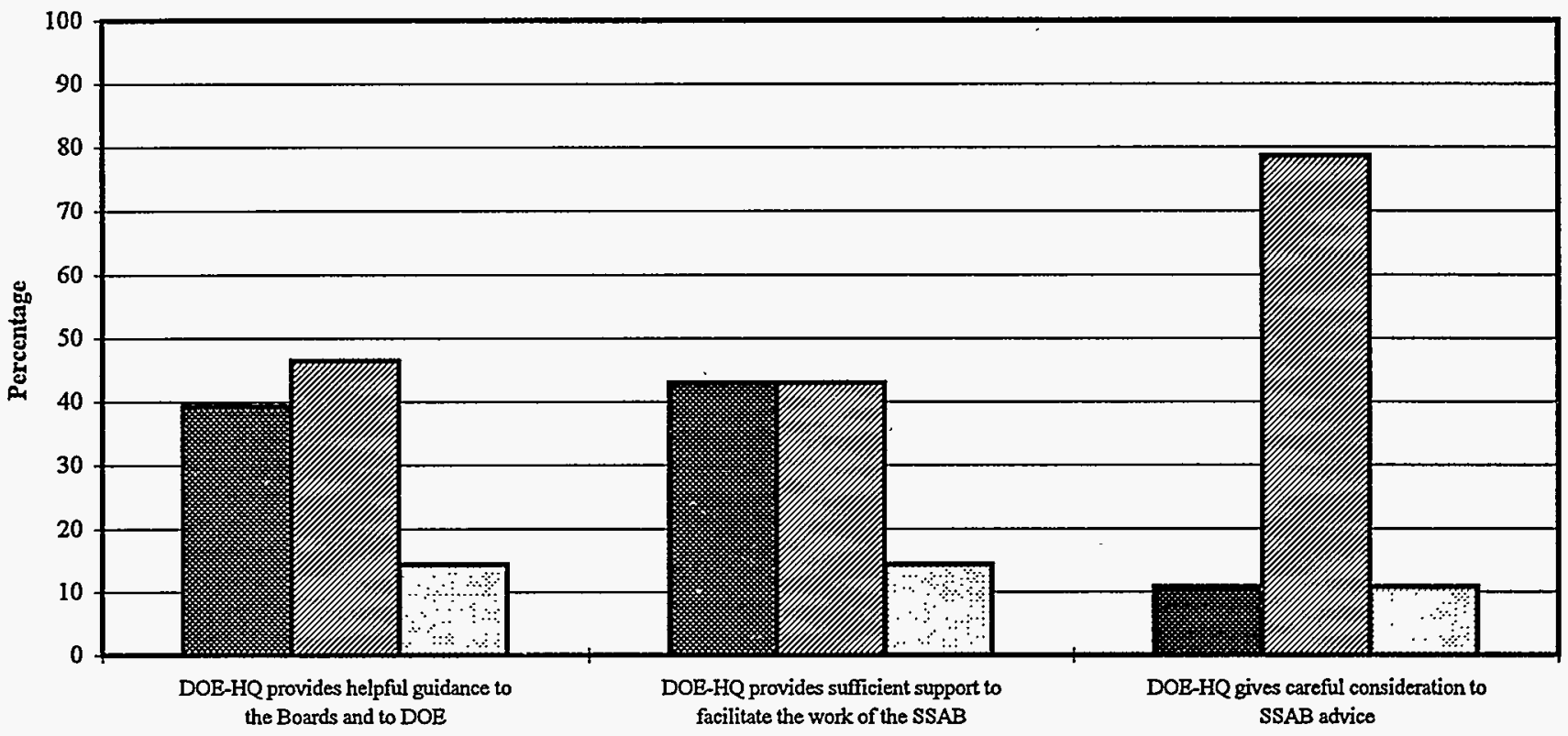

Agree (\%) Weither Agree Nor Disagree/Don't Know (\%) EDisagree (\%)

Figure 8b. Long Survey: Entire Sample DOE-HQ involvement with the SSAB Initiative

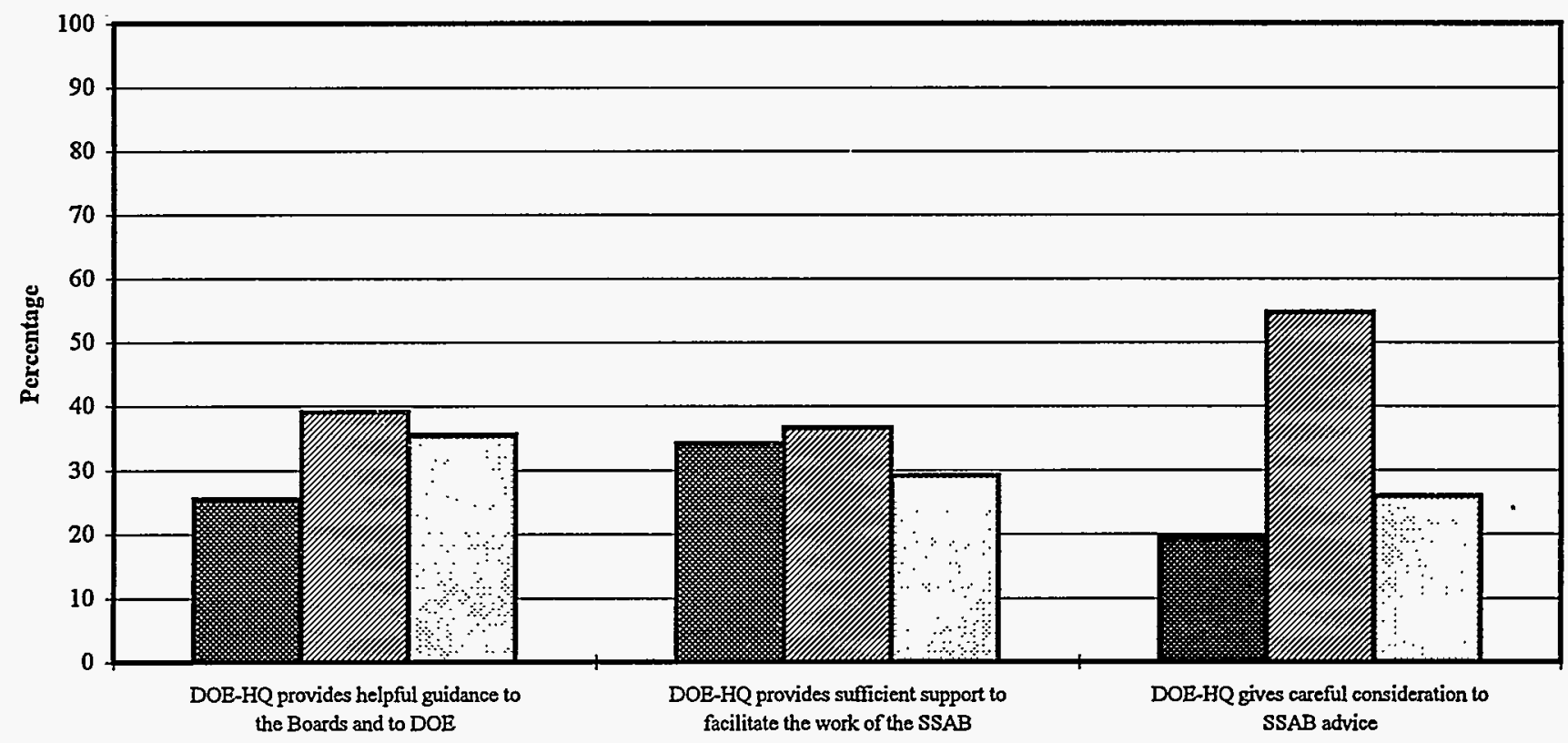

Agree (\%) E Neither Agree Nor Disagree/Don't Know (\%) DDisagree (\%)

SSAB Supplementary Appendix August 1996 
Figure 9a. Sandia

Personal experience with the SSAB Initiative

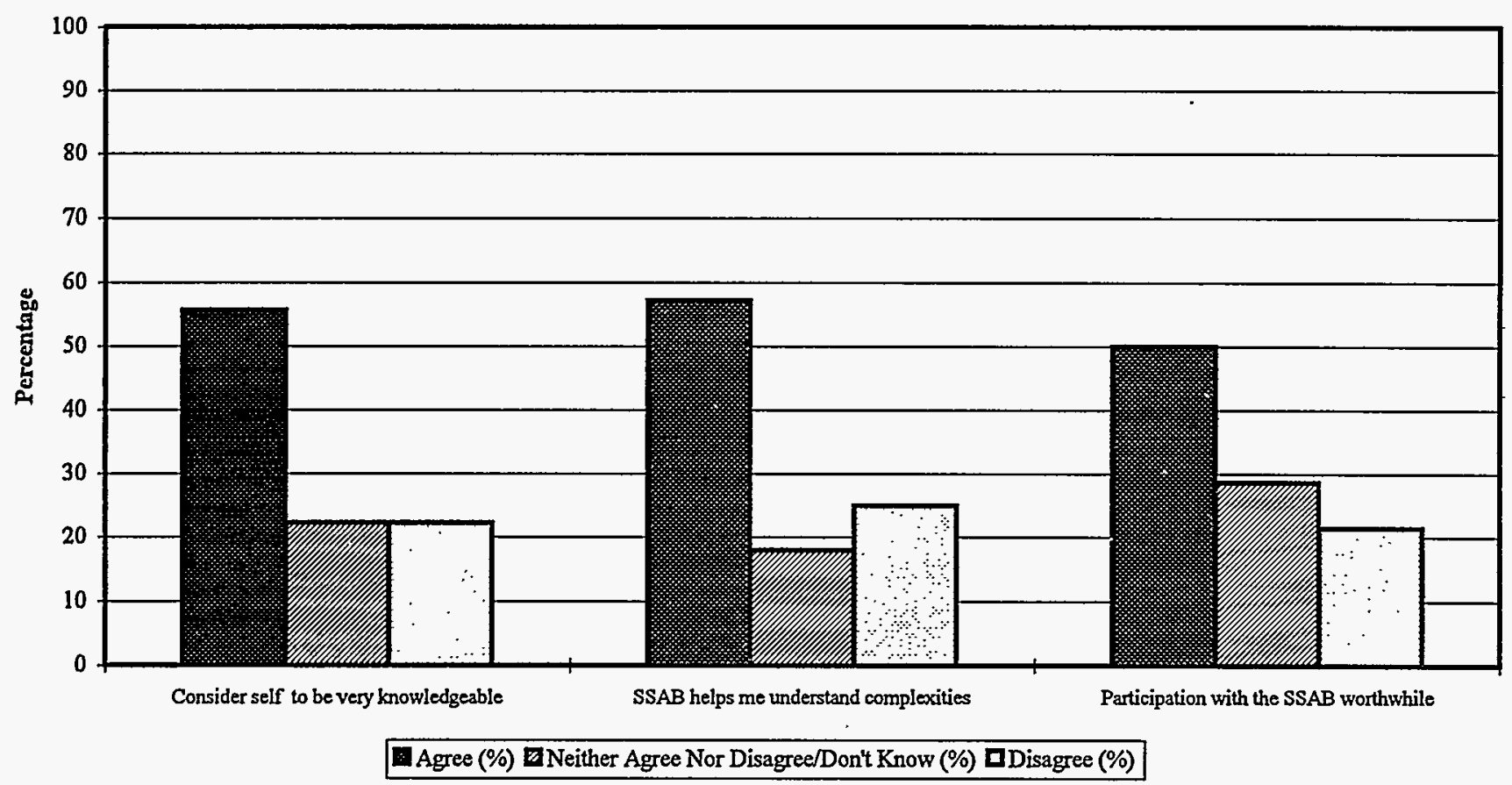

Figure 9b. Long Survey: Entire Sample Personal experience with the SSAB Initiative

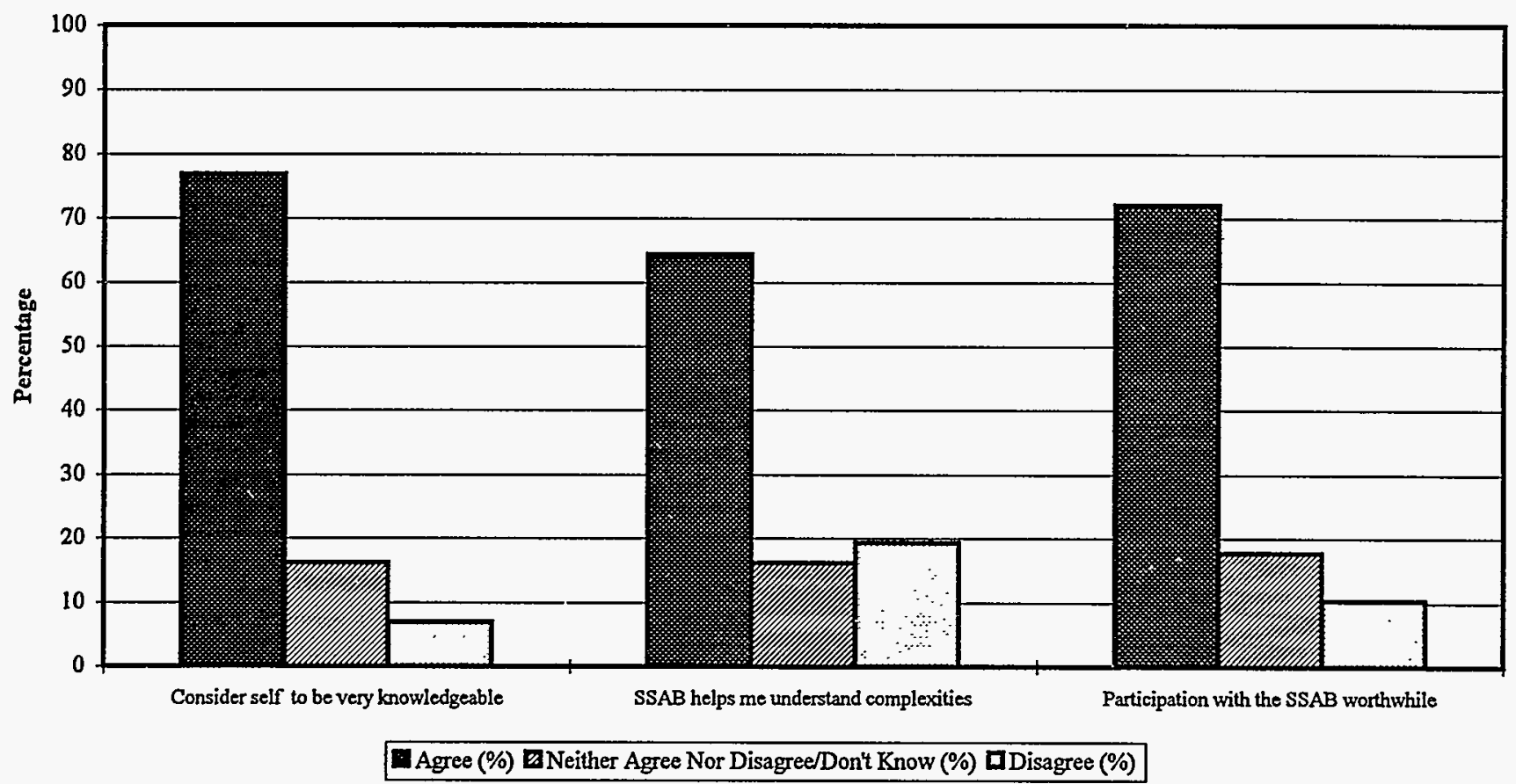

SSAB Supplementary Appendix August 1996 
Figure 10a. Sandia

Group/Team work skills

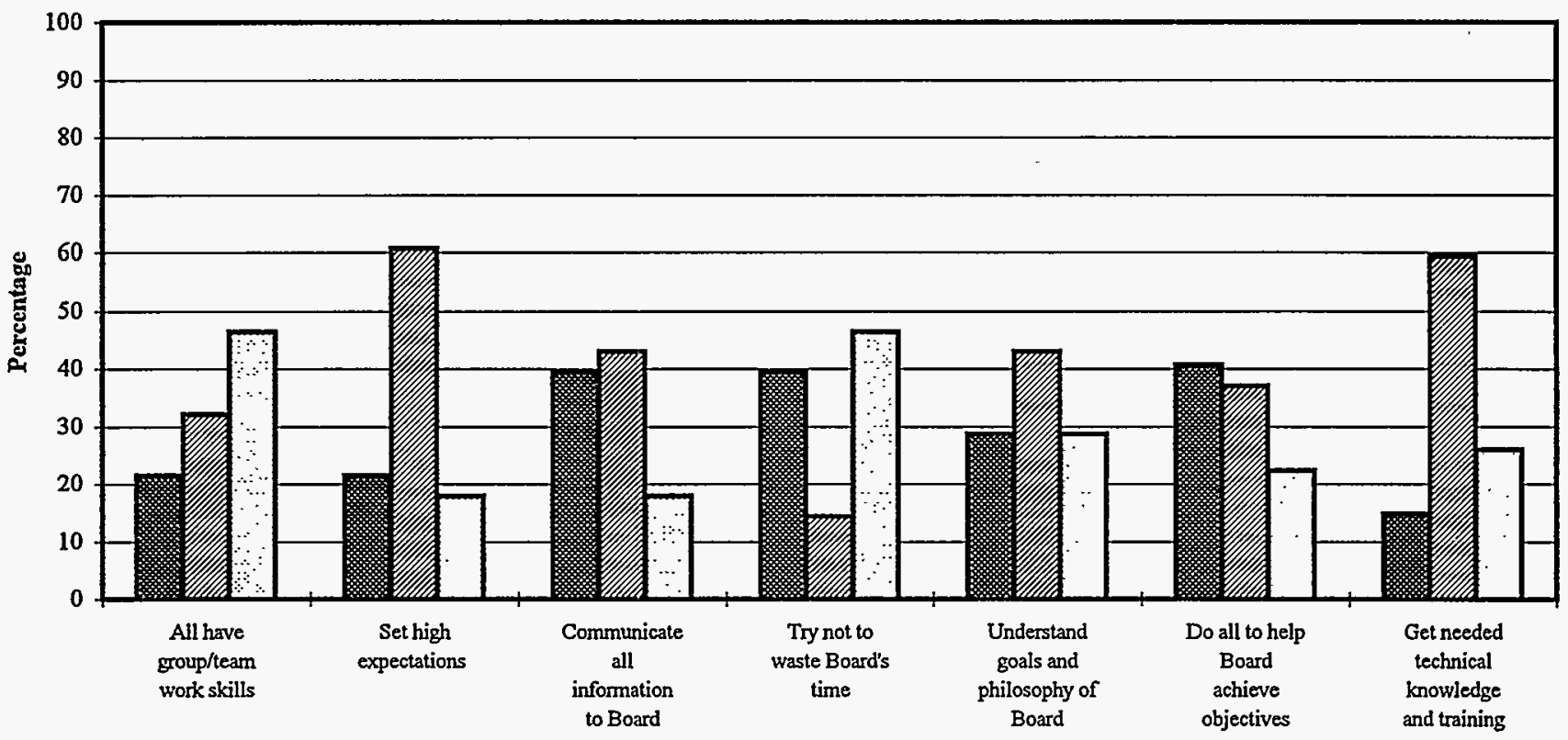

EAgree (\%) $\boldsymbol{D}$ Neither Agree Nor Disagree/Don't Know (\%) DDisagree (\%)

Figure 10b. Long Survey: Entire Sample Group/Team work skills

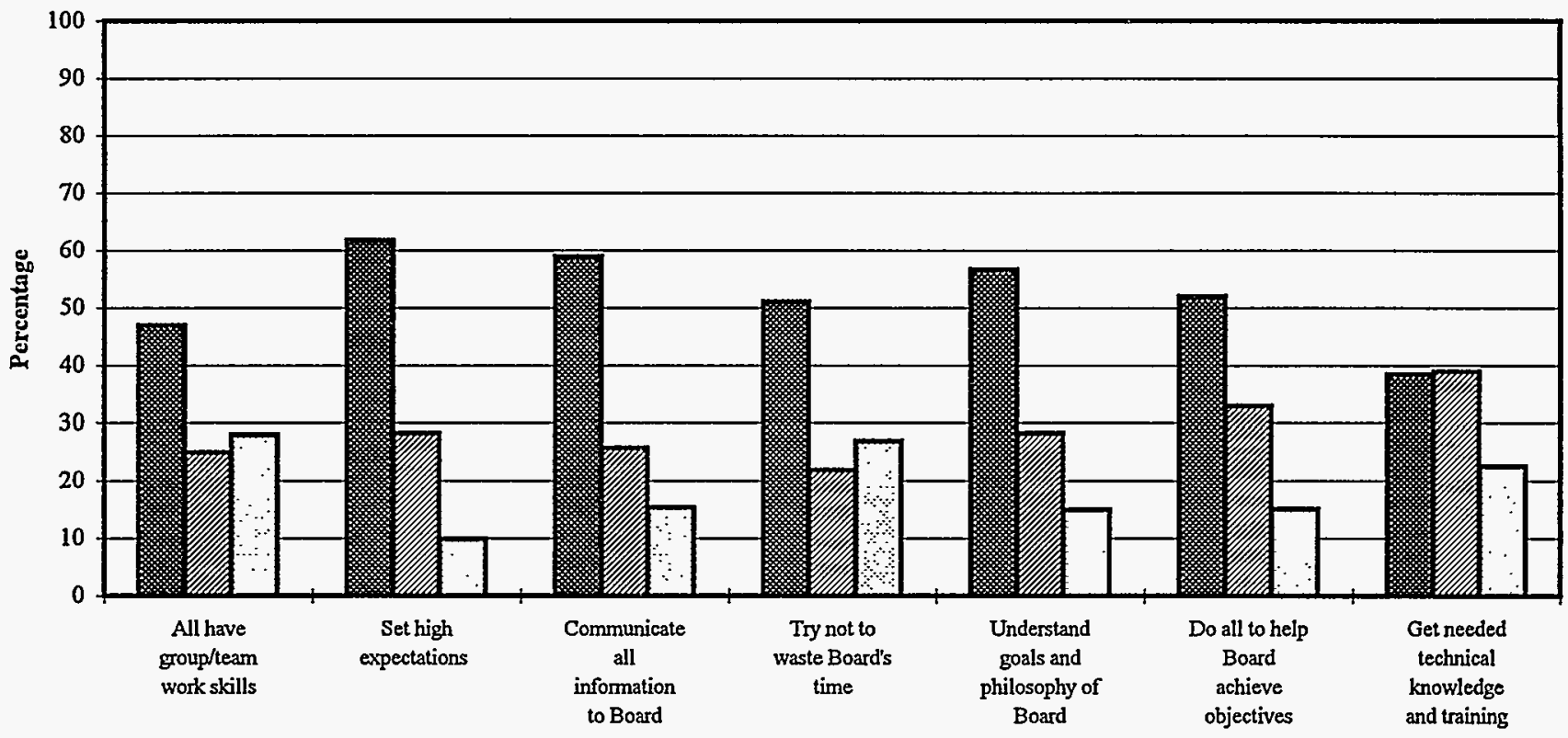

Agree (\%) Neither Agree Nor Disagree/Don't Know (\%) DDisagree (\%)

SSAB Supplementary Appendix 
Figure 11a. Sandia

Working relations

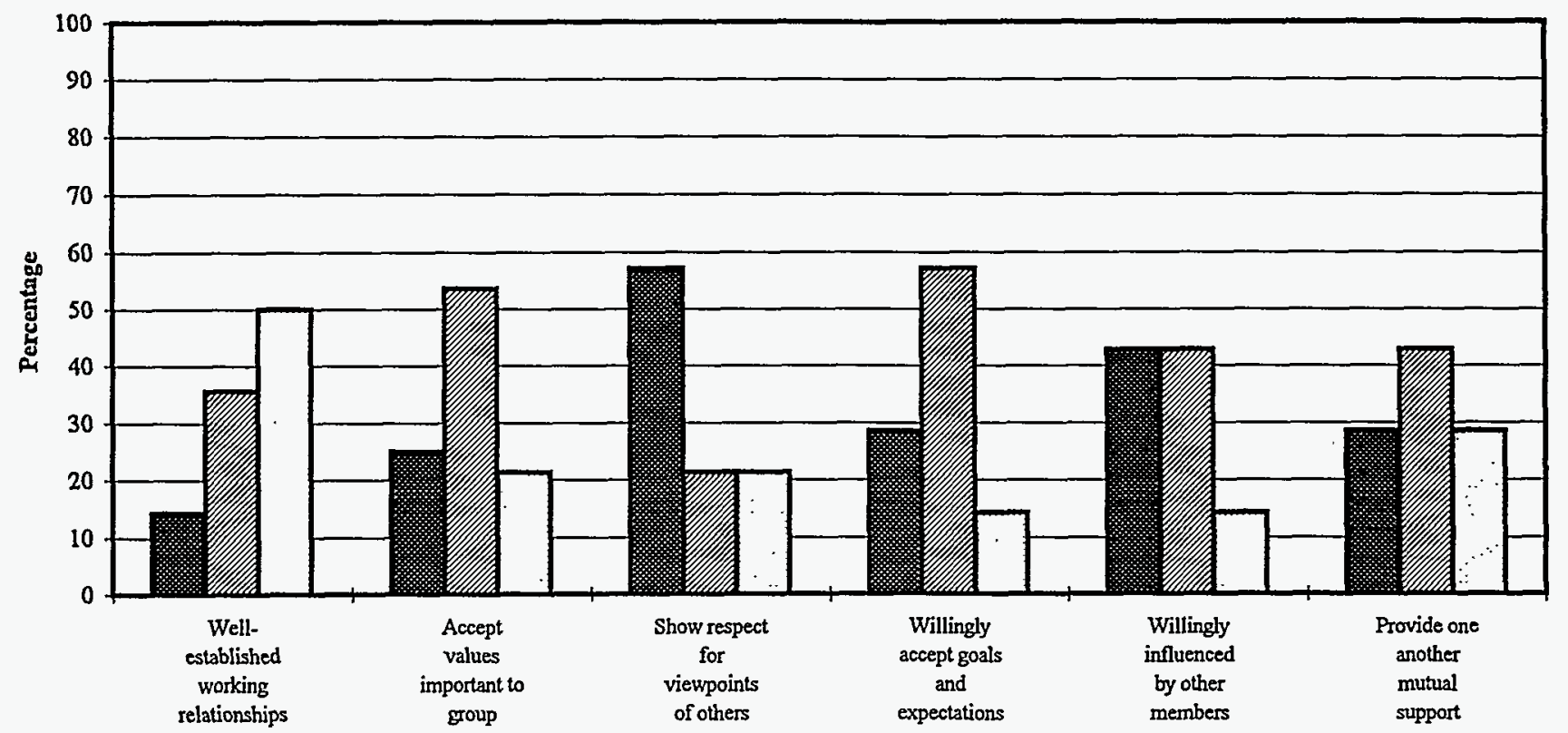

Agree (\%) ENeither Agree Nor Disagree/Don't Know (\%) QDisagree (\%)

Figure 11b. Long Survey: Entire Sample Working relations

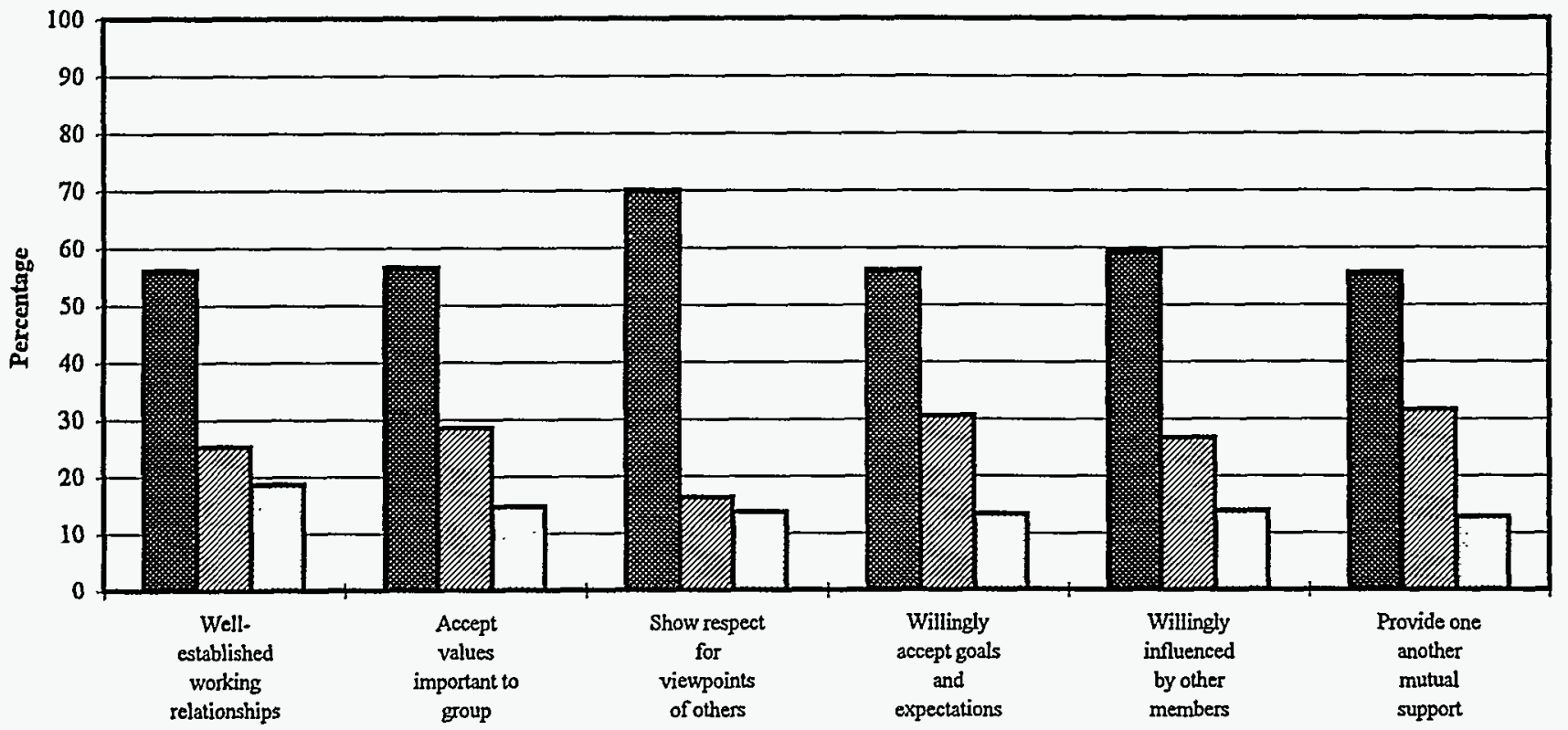

Agree (\%) Deither Agree Nor Disagree/Don't Know (\%) DDisagree (\%)

SSAB Supplementary Appendix 
Figure 12a. Sandia

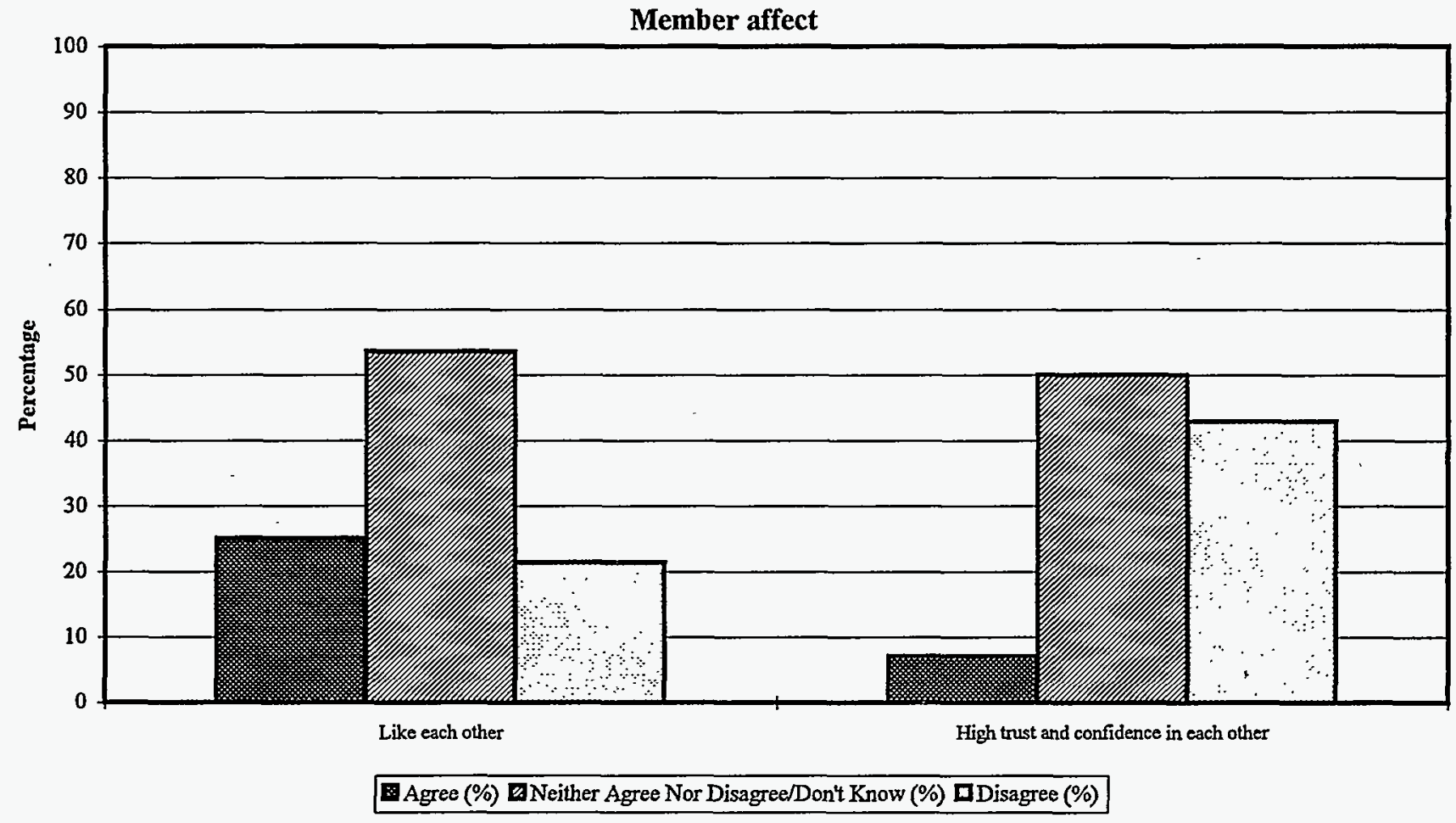

Figure 12b. Long Survey: Entire Sample

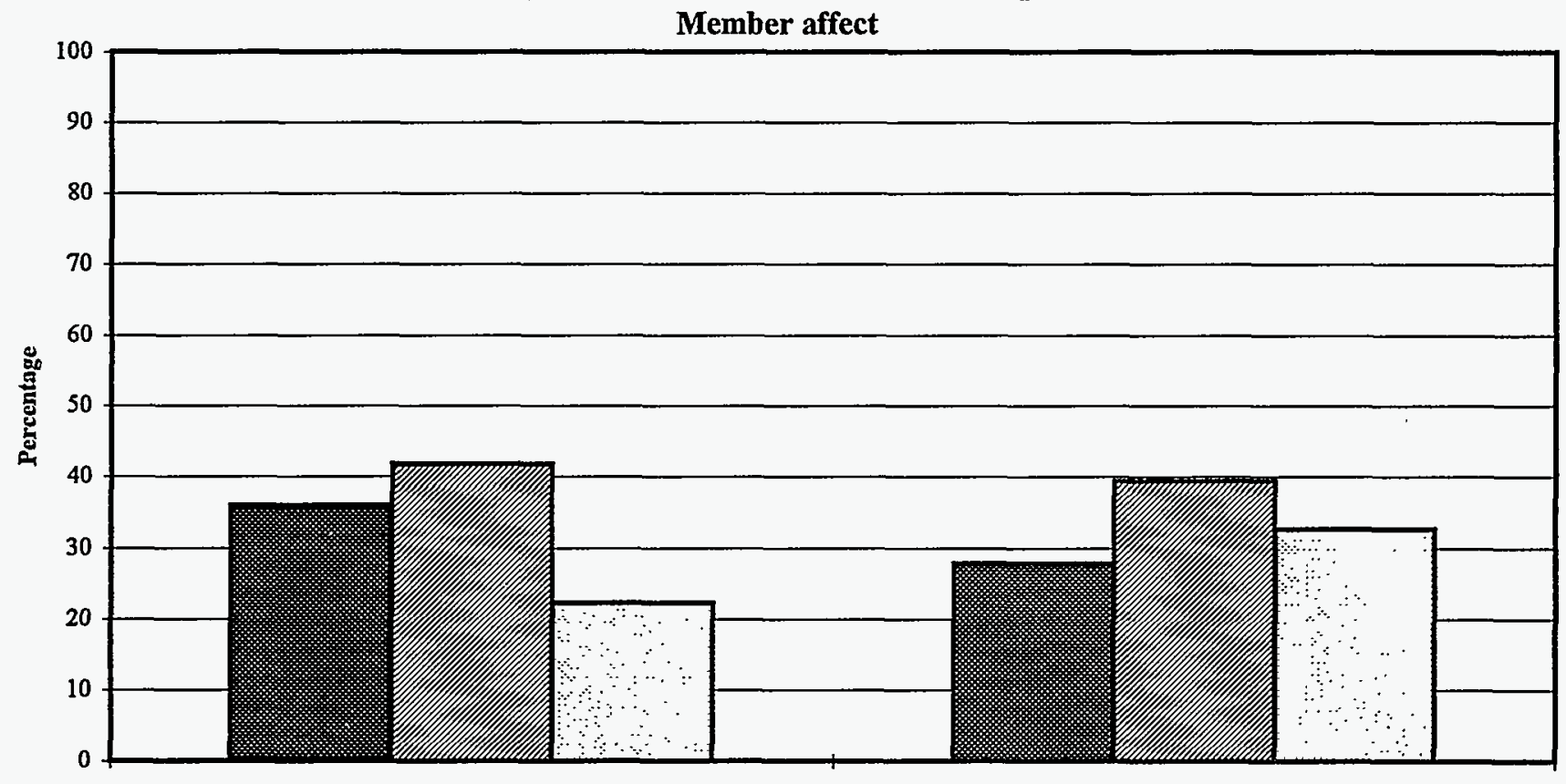

Like each other

High trust and confidence in each other

Agree (\%) Deither Agree Nor Disagree/Don't Know (\%) DDisagree (\%)

SSAB Supplementary Appendix

August 1996 
.Figure 13a. Sandia

Summary: Responses to open-ended questions

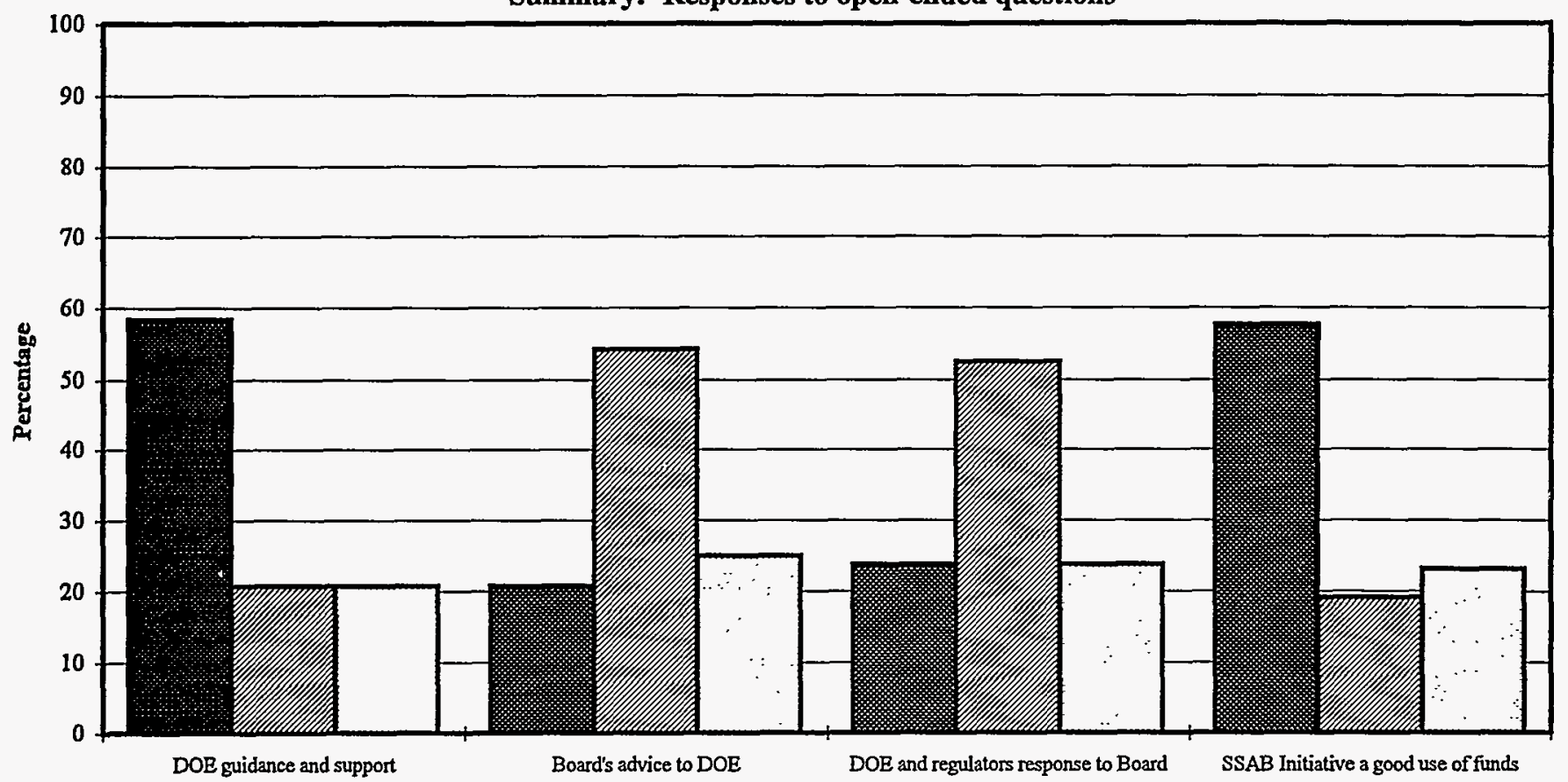

YYes, Good (\%) Neutral, Conditional, No Opinion (\%) DNo, Not Good (\%)

Figure 13b. Long Survey: Entire Sample

Summary: Responses to open-ended questions

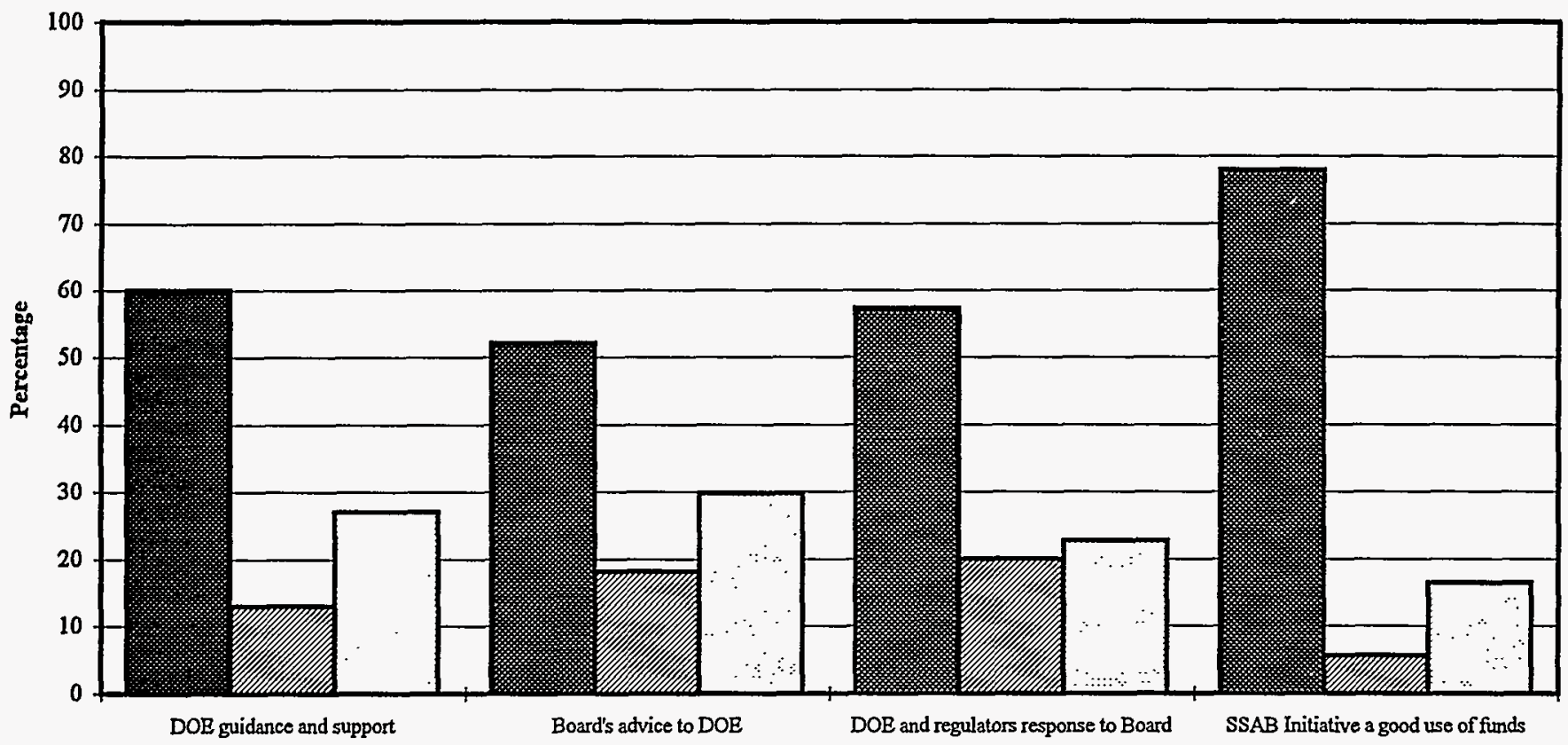

Y Yes, Good (\%) ENeutral, Conditional, No Opinion (\%) DNo, Not Good (\%)

SSAB Supplementary Appendix

August 1996 
Figure 14a. Sandia

Summary: Combined responses for SSAB Initiative goals (Means)

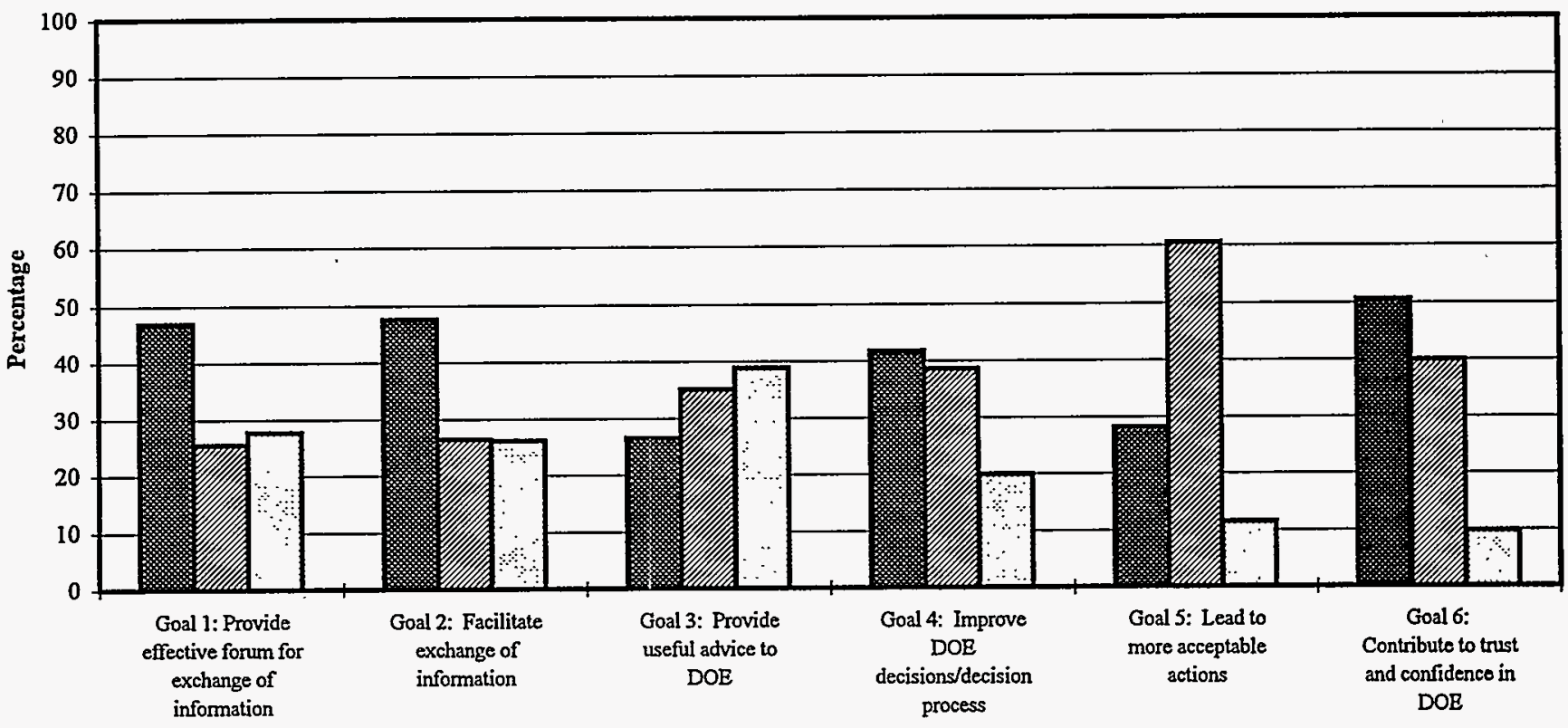

ब Agree (\%) ENeither Agree Nor Disagree/Don't Know (\%) DDisagree (\%)

Figure 14b. Long Survey: Entire Sample

Summary: Combined responses for SSAB Initiative goals (Means)

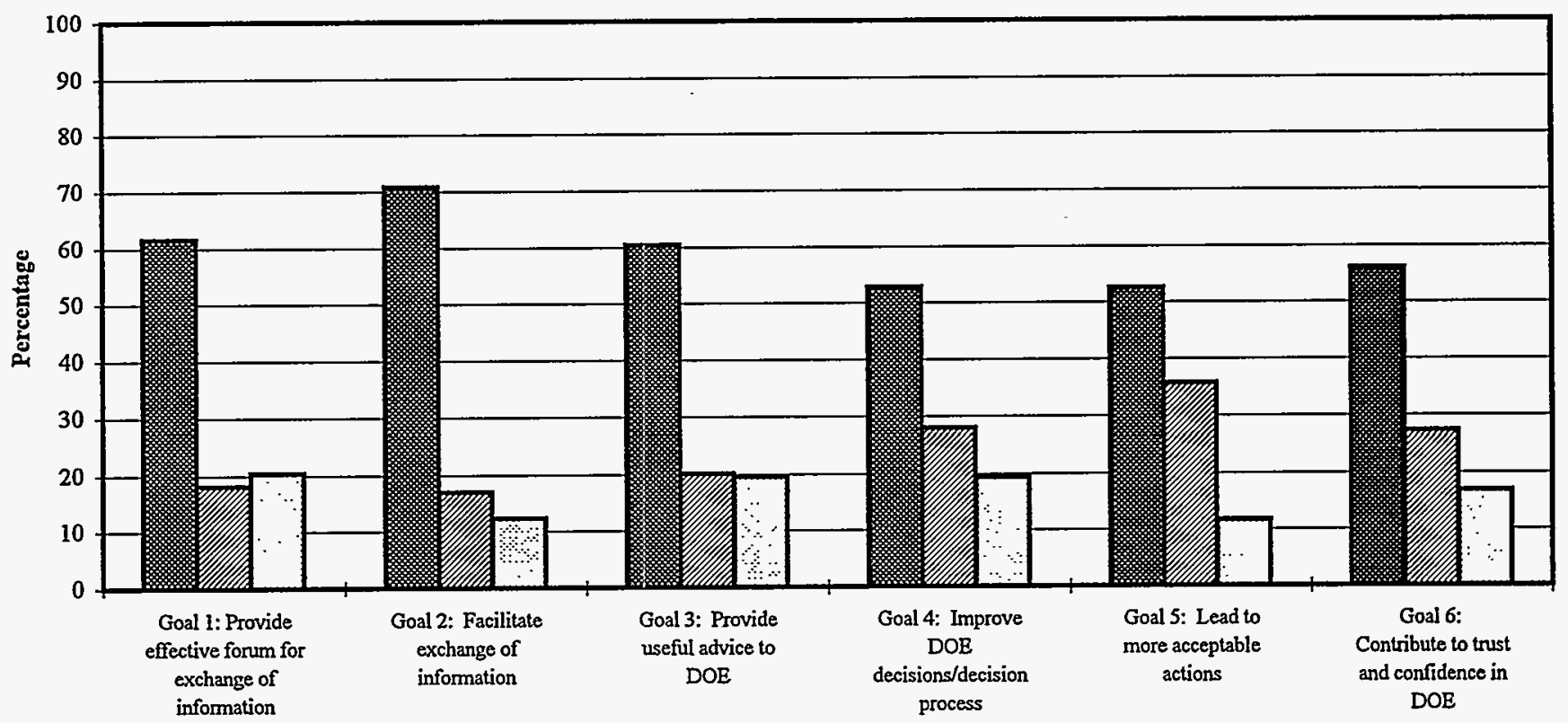

QAgree (\%) שNeither Agree Nor Disagree/Don't Know (\%) DDisagree (\%)

SSAB Supplementary Appendix

August 1996 
Figure 15a. Sandia

Summary: Combined responses on DOE-HQ involvement,

Personal experience, Group/Team work skills,

Working relations, Member affect (Means)

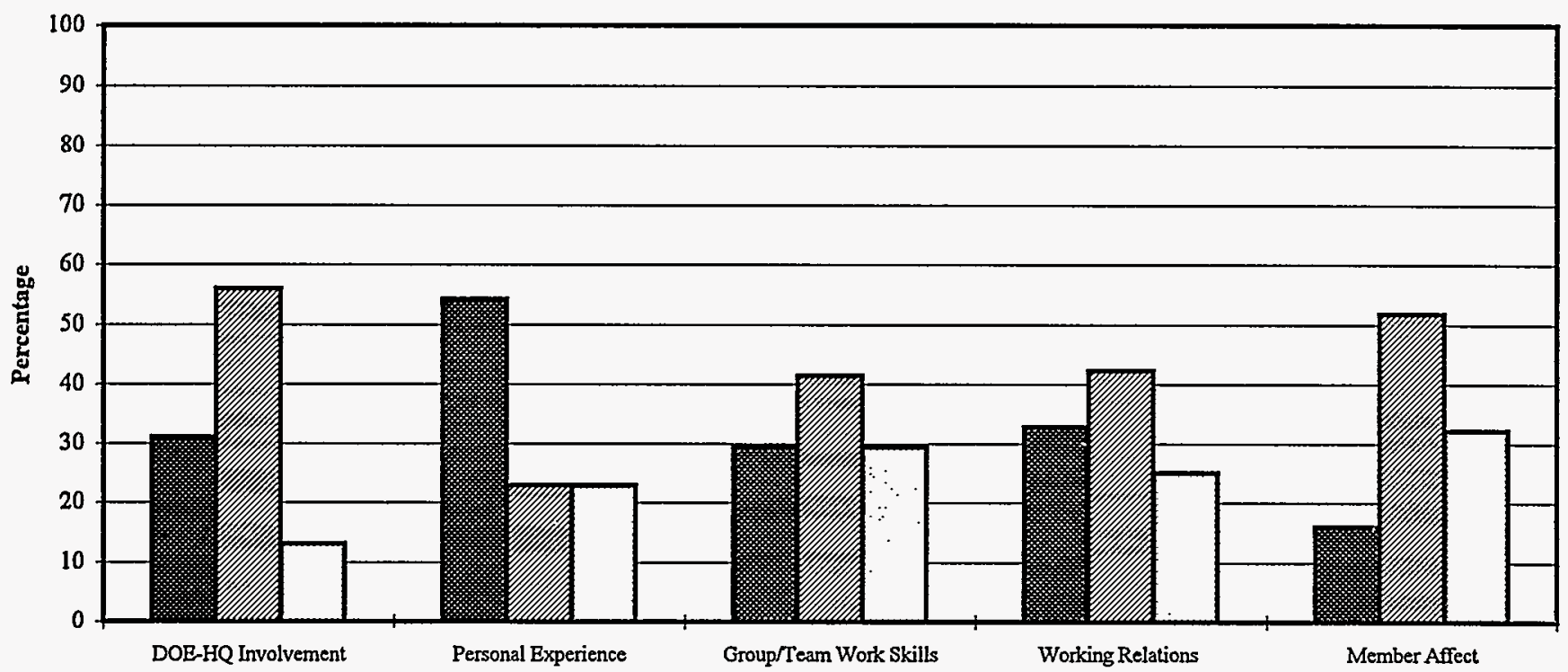

Agree (\%) W Neither Agree Nor Disagree/Don't Know (\%) DDisagree (\%)

Figure 15b. Long Survey: Entire Sample

Summary: Combined responses on DOE-HQ involvement,

Personal experience, Group/Team work skills,

Working relations, Member affect (Means)

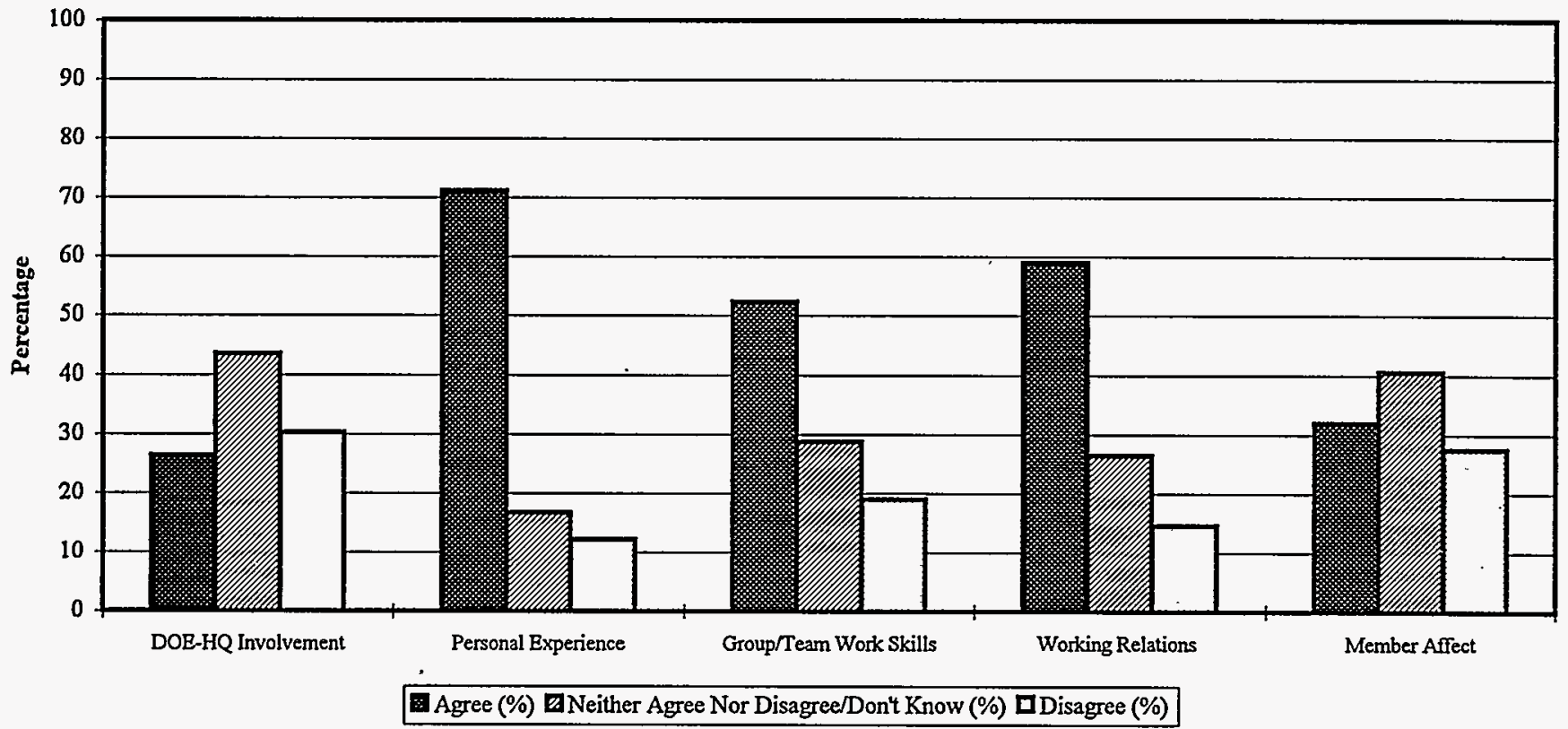

SSAB Supplementary Appendix 
Table 1.

Goal 1. Establish processes and procedures to provide an effective forum for exchange of information and viewpoints regarding DOE site issues

\begin{tabular}{|c|c|c|c|c|c|c|c|c|}
\hline \multirow[t]{2}{*}{ Sandia } & \multicolumn{2}{|c|}{ Agree } & \multicolumn{2}{|c|}{$\begin{array}{l}\text { Neither/ } \\
\text { Don't Know }\end{array}$} & \multicolumn{2}{|c|}{ Disagree } & \multicolumn{2}{|c|}{ Total } \\
\hline & $\mathrm{N}$ & $\%$ & $N$ & $\%$ & $N$ & $\%$ & $\mathrm{~N}$ & $\%$ \\
\hline $\begin{array}{l}\text { The Board has established processes and } \\
\text { procedures for the effective exchange of } \\
\text { information }\end{array}$ & 10 & $35.7 \%$ & 6 & $21.4 \%$ & 12 & $42.9 \%$ & 28 & $100 \%$ \\
\hline $\begin{array}{l}\text { The Board has agreed-upon procedures to } \\
\text { operate the board }\end{array}$ & 21 & $77.8 \%$ & 2 & $7.4 \%$ & 4 & $14.8 \%$ & 27 & $100 \%$ \\
\hline $\begin{array}{l}\text { The Board has adequate support to allow } \\
\text { it to focus on substantive issues }(\mathrm{R}) *\end{array}$ & 15 & $55.6 \%$ & 7 & $25.9 \%$ & 5 & $18.5 \%$ & 27 & $100 \%$ \\
\hline $\begin{array}{l}\text { The Board provides sufficient time for } \\
\text { discussion of issues }\end{array}$ & 9 & $33.3 \%$ & 6 & $22.2 \%$ & 12 & $44.4 \%$ & 27 & $100 \%$ \\
\hline The Board has effective leadership & 14 & $51.9 \%$ & 5 & $18.5 \%$ & 8 & $29.6 \%$ & 27 & $100 \%$ \\
\hline $\begin{array}{l}\text { DOE requests Board advice far enough } \\
\text { ahead of decision deadlines }(\mathrm{R}){ }^{*}\end{array}$ & 8 & $29.6 \%$ & 4 & $14.8 \%$ & 15 & $55.6 \%$ & 27 & $100 \%$ \\
\hline $\begin{array}{l}\text { DOE handles Board administrative issues } \\
\text { expeditiously (R)* }\end{array}$ & 15 & $55.6 \%$ & 9 & $33.3 \%$ & 3 & $11.1 \%$ & 27 & $100 \%$ \\
\hline $\begin{array}{l}\text { The SSAB staff provide satisfactory } \\
\text { support }\end{array}$ & 11 & $39.3 \%$ & 14 & $50.0 \%$ & 3 & $10.7 \%$ & 28 & $100 \%$ \\
\hline $\begin{array}{l}\text { The facilitator for the Board has helped } \\
\text { the Board function effectively }\end{array}$ & 12 & $42.9 \%$ & 10 & $35.7 \%$ & 6 & $21.4 \%$ & 28 & $100 \%$ \\
\hline
\end{tabular}

* (R) denotes a question that was asked in the negative form. For ease of review, these questions and data have been reversed in this table and are stated in positive forms.

SSAB Supplementary Appendix 
Table 2.

Goal 2. Facilitate interaction and exchange of information and viewpoints regarding DOE site issues

\begin{tabular}{|c|c|c|c|c|c|c|c|c|}
\hline \multirow[t]{2}{*}{ Sandia } & \multicolumn{2}{|c|}{ Agree } & \multicolumn{2}{|c|}{$\begin{array}{l}\text { Neither/ } \\
\text { Don't Know }\end{array}$} & \multicolumn{2}{|c|}{ Disagree } & \multicolumn{2}{|c|}{ Total } \\
\hline & $N$ & $\%$ & $\mathrm{~N}$ & $\%$ & $\mathrm{~N}$ & $\%$ & $\mathrm{~N}$ & $\%$ \\
\hline $\begin{array}{l}\text { The SSAB facilitates effective exchange } \\
\text { of viewpoints on site issues }\end{array}$ & 10 & $35.7 \%$ & 7 & 25.0 & 11 & $39.3 \%$ & 28 & $100 \%$ \\
\hline $\begin{array}{l}\text { The SSAB contributes to Board members' } \\
\text { understanding of the basis for key site } \\
\text { decisions }\end{array}$ & 16 & $57.1 \%$ & 4 & $14.3 \%$ & 8 & $28.6 \%$ & 28 & $100 \%$ \\
\hline $\begin{array}{l}\text { The SSAB contributes to DOE and } \\
\text { regulators' understanding of the public's } \\
\text { viewpoints on key site decisions }\end{array}$ & 12 & $42.9 \%$ & 7 & $25.0 \%$ & 9 & $32.1 \%$ & 28 & $100 \%$ \\
\hline $\begin{array}{l}\text { The SSAB contributes to a constructive } \\
\text { working relationship among the } \\
\text { participants }\end{array}$ & 12 & $42.9 \%$ & 7 & $25.0 \%$ & 9 & $32.1 \%$ & 28 & $100 \%$ \\
\hline The SSAB strives for consensus & 23 & $82.1 \%$ & 3 & $10.7 \%$ & 2 & $7.1 \%$ & 28 & $100 \%$ \\
\hline $\begin{array}{l}\text { The SSAB gives fair consideration to } \\
\text { dissenting opinions }\end{array}$ & 19 & $67.9 \%$ & 6 & $21.4 \%$ & 3 & $10.7 \%$ & 28 & $100 \%$ \\
\hline $\begin{array}{l}\text { The Board has made the effort needed to } \\
\text { learn about site issues }\end{array}$ & 13 & $50.0 \%$ & 6 & $23.1 \%$ & 7 & $26.9 \%$ & 26 & $100 \%$ \\
\hline $\begin{array}{l}\text { The Board invites expert advice into its } \\
\text { discussion on key policy issues }\end{array}$ & 10 & $37.0 \%$ & 9 & $33.3 \%$ & 8 & $29.6 \%$ & 27 & $100 \%$ \\
\hline $\begin{array}{l}\text { The Board responds to public inquiries } \\
\text { and comments about its decisions }\end{array}$ & 5 & $20.0 \%$ & 15 & $60.0 \%$ & 5 & $20.0 \%$ & 25 & $100 \%$ \\
\hline $\begin{array}{l}\text { The Board solicits feedback from the } \\
\text { community on its work }\end{array}$ & 3 & $11.1 \%$ & 13 & $48.1 \%$ & 11 & $40.7 \%$ & 27 & $100 \%$ \\
\hline $\begin{array}{l}\text { DOE makes information on key site issues } \\
\text { readily available to the Board }\end{array}$ & 20 & $71.4 \%$ & 4 & $14.3 \%$ & 4 & $14.3 \%$ & 28 & $100 \%$ \\
\hline $\begin{array}{l}\text { The regulators make important } \\
\text { contributions to the Board's work }\end{array}$ & 13 & $46.4 \%$ & 8 & $28.6 \%$ & 7 & $25.0 \%$ & 28 & $100 \%$ \\
\hline $\begin{array}{l}\text { The SSAB creates a climate supportive of } \\
\text { differing viewpoints }(\mathrm{R}){ }^{*}\end{array}$ & 15 & $53.6 \%$ & 4 & $14.3 \%$ & 9 & $32.1 \%$ & 28 & $100 \%$ \\
\hline
\end{tabular}

* (R) denotes a question that was asked in the negative form. For ease of review, these questions and data have been reversed in this table and are stated in positive forms.

SSAB Supplementary Appendix

August 1996 
Table 3.

Goal 3. Provide useful advice and/or recommendations to DOE (and regulators, where appropriate)

\begin{tabular}{|c|c|c|c|c|c|c|c|c|}
\hline \multirow[t]{2}{*}{ Sandia } & \multicolumn{2}{|c|}{ Agree } & \multicolumn{2}{|c|}{$\begin{array}{l}\text { Neither/ } \\
\text { Don't Know }\end{array}$} & \multicolumn{2}{|c|}{ Disagree } & \multicolumn{2}{|c|}{ Total } \\
\hline & $\mathrm{N}$ & $\%$ & $\mathbf{N}$ & $\%$ & $\mathbf{N}$ & $\%$ & $\mathbf{N}$ & $\%$ \\
\hline $\begin{array}{l}\text { The Board provides useful advice to DOE } \\
\text { (and regulators, where appropriate) (R) }\end{array}$ & 7 & $25.9 \%$ & 11 & $40.7 \%$ & 9 & $33.3 \%$ & 27 & $100 \%$ \\
\hline $\begin{array}{l}\text { The SSAB reaches agreement about } \\
\text { prioritization of key site issues for which } \\
\text { advice is sought }(\mathrm{R})^{*}\end{array}$ & 3 & $10.7 \%$ & 13 & $46.4 \%$ & 12 & $42.9 \%$ & 28 & $100 \%$ \\
\hline The Board helps define site problems & 8 & $29.6 \%$ & 7 & $25.9 \%$ & 12 & $44.4 \%$ & 27 & $100 \%$ \\
\hline $\begin{array}{l}\text { The Board addresses issues in a timely } \\
\text { manner }(\mathrm{R})^{*}\end{array}$ & 5 & $18.5 \%$ & 4 & $14.8 \%$ & 18 & $66.7 \%$ & 27 & $100 \%$ \\
\hline $\begin{array}{l}\text { The Board reaches consensus on key site } \\
\text { issues (R)* }\end{array}$ & 9 & $34.6 \%$ & 6 & $23.1 \%$ & 11 & $42.3 \%$ & 26 & $100 \%$ \\
\hline $\begin{array}{l}\text { The Board provides informed advice to } \\
\text { DOE (and regulators, where appropriate) }\end{array}$ & 8 & $30.8 \%$ & 10 & $38.5 \%$ & 8 & $30.8 \%$ & 26 & $100 \%$ \\
\hline $\begin{array}{l}\text { The Board provides advice that reflects } \\
\text { the viewpoints and priorities of the } \\
\text { community }\end{array}$ & 9 & $33.3 \%$ & 15 & $55.6 \%$ & 3 & $11.1 \%$ & 27 & $100 \%$ \\
\hline
\end{tabular}

* (R) denotes a question that was asked in the negative form. For ease of review, these questions and data have been reversed in this table and are stated in positive forms. 
Table 4.

Goal 4. Improve DOE's (and where applicable, regulators') site decisions and decision making process

\begin{tabular}{|c|c|c|c|c|c|c|c|c|}
\hline \multirow[t]{2}{*}{ Sandia } & \multicolumn{2}{|c|}{ Agree } & \multicolumn{2}{|c|}{$\begin{array}{c}\text { Neither/ } \\
\text { Don't Know }\end{array}$} & \multicolumn{2}{|c|}{ Disagree } & \multicolumn{2}{|c|}{ Total } \\
\hline & $\mathrm{N}$ & $\%$ & $\mathrm{~N}$ & $\%$ & $\mathrm{~N}$ & $\%$ & $N$ & $\%$, \\
\hline $\begin{array}{l}\text { The SSAB has improved DOE's site } \\
\text { decisions }(\mathrm{R})^{*}\end{array}$ & 5 & $17.9 \%$ & 8 & $28.6 \%$ & 15 & $53.6 \%$ & 28 & $100 \%$ \\
\hline $\begin{array}{l}\text { The Board understands the decision } \\
\text { making process at [a particular] site }\end{array}$ & 6 & $22.2 \%$ & 14 & $51.9 \%$ & 7 & $25.9 \%$ & 27 & $100 \%$ \\
\hline $\begin{array}{l}\text { DOE discusses important policies } \\
\text { affecting site decisions with the Board }\end{array}$ & 22 & $81.5 \%$ & 3 & $11.1 \%$ & 2 & $7.4 \%$ & 27 & $100 \%$ \\
\hline $\begin{array}{l}\text { DOE has explained to the Board its site } \\
\text { decision making process }\end{array}$ & 14 & $50.0 \%$ & 10 & $35.7 \%$ & 4 & $14.3 \%$ & 28 & $100 \%$ \\
\hline $\begin{array}{l}\text { The DOE shows how Board advice is } \\
\text { reflected in site decisions }\end{array}$ & 7 & 25.0 & 17 & $60.7 \%$ & 4 & $14.3 \%$ & 28 & $100 \%$ \\
\hline $\begin{array}{l}\text { The decision making process used by } \\
\text { DOE at [a particular] site is effective in } \\
\text { furthering site clean-up }(R)^{*}\end{array}$ & 15 & $53.6 \%$ & 12 & $42.9 \%$ & 1 & $3.6 \%$ & 28 & $100 \%$ \\
\hline
\end{tabular}

* (R) denotes a question that was asked in the negative form. For ease of review, these questions and data have been reversed in this table and are stated in positive forms. 
Table 5.

Goal 5. Lead to more acceptable actions

\begin{tabular}{|c|c|c|c|c|c|c|c|c|}
\hline \multirow[t]{2}{*}{ Sandia } & \multicolumn{2}{|c|}{ Agree } & \multicolumn{2}{|c|}{$\begin{array}{c}\text { Neither/ } \\
\text { Don't Know }\end{array}$} & \multicolumn{2}{|c|}{ Disagree } & \multicolumn{2}{|c|}{ Total } \\
\hline & $N$ & $\%$ & $\mathrm{~N}$ & $\%$ & $N$ & $\%$ & $\mathrm{~N}$ & $\%$ \\
\hline $\begin{array}{l}\text { The SSAB leads to more acceptable site } \\
\text { decisions }\end{array}$ & 9 & $32.1 \%$ & 13 & $46.4 \%$ & 6 & $21.4 \%$ & 28 & $100 \%$ \\
\hline $\begin{array}{l}\text { The Board supports the recommendations } \\
\text { it gives DOE }\end{array}$ & 5 & $20.0 \%$ & 20 & $80.0 \%$ & 0 & $.0 \%$ & 25 & $100 \%$ \\
\hline The Board supports DOE's site actions & 8 & $30.8 \%$ & 15 & $57.7 \%$ & 3 & $11.5 \%$ & 26 & $100 \%$ \\
\hline $\begin{array}{l}\text { The DOE pays attention to the Board's } \\
\text { advice on key site issues (R) * }\end{array}$ & 11 & $39.3 \%$ & 17 & $60.7 \%$ & 0 & $0 \%$ & 28 & $100 \%$ \\
\hline $\begin{array}{l}\text { There is support in the community for } \\
\text { DOE's site decisions that have SSAB } \\
\text { input }\end{array}$ & 4 & $14.3 \%$ & 24 & $85.7 \%$ & 0 & $0 \%$ & 28 & $100 \%$ \\
\hline Progress is being made on key site issues & 9 & $32.1 \%$ & 9 & $32.1 \%$ & 10 & $35.7 \%$ & 28 & $100 \%$ \\
\hline
\end{tabular}

* (R) denotes a question that was asked in the negative form. For ease of review, these questions and data have been reversed in this table and are stated in positive forms.

SSAB Supplementary Appendix 
Table 6.

Goal 6. Contribute to trust and confidence in DOE

\begin{tabular}{|c|c|c|c|c|c|c|c|c|}
\hline \multirow[t]{2}{*}{ Sandia } & \multicolumn{2}{|c|}{ Agree } & \multicolumn{2}{|c|}{$\begin{array}{c}\text { Neither/ } \\
\text { Don't Know }\end{array}$} & \multicolumn{2}{|c|}{ Disagree } & \multicolumn{2}{|c|}{ Total } \\
\hline & $\mathrm{N}$ & $\%$ & $\mathbf{N}$ & $\%$ & $\mathbf{N}$ & $\%$ & $\mathbf{N}$ & $\%$ \\
\hline $\begin{array}{l}\text { The SSAB contributes to trust and } \\
\text { confidence in DOE }\end{array}$ & 10 & $37.0 \%$ & 14 & $51: 9 \%$ & 3 & $11.1 \%$ & 27 & $100 \%$ \\
\hline $\begin{array}{l}\text { Relationships between DOE and the public } \\
\text { have improved since the formation of the } \\
\text { SSAB }\end{array}$ & 11 & $39.3 \%$ & 14 & $50.0 \%$ & 3 & $10.7 \%$ & 28 & $100 \%$ \\
\hline $\begin{array}{l}\text { DOE is committed to clean up [a } \\
\text { particular] site }\end{array}$ & 21 & $75.0 \%$ & 5 & $17.9 \%$ & 2 & $7.1 \%$ & 28 & $100 \%$ \\
\hline
\end{tabular}


Table 7.

Public awareness

\begin{tabular}{|c|c|c|c|c|c|c|c|c|}
\hline \multirow[t]{2}{*}{ Sandia } & \multicolumn{2}{|c|}{ Agree } & \multicolumn{2}{|c|}{$\begin{array}{c}\text { Neither/ } \\
\text { Don't Know }\end{array}$} & \multicolumn{2}{|c|}{ Disagree } & \multicolumn{2}{|c|}{ Total } \\
\hline & $N$ & $\%$ & $\mathrm{~N}$ & $\%$ & $\mathbf{N}$ & $\%$ & $\mathrm{~N}$ & $\%$ \\
\hline $\begin{array}{l}\text { The public knows little about the role of } \\
\text { the Board }\end{array}$ & 25 & $89.3 \%$ & 3 & $10.7 \%$ & 0 & $0 \%$ & 28 & $100 \%$ \\
\hline
\end{tabular}

Table 8.

SSAB Initiative is a good use of funds

\begin{tabular}{ccccccccc}
\hline \multirow{2}{*}{ Sandia } & Yes, Good & $\begin{array}{c}\text { Neutral, } \\
\text { Conditional, } \\
\text { No Opinion }\end{array}$ & $\begin{array}{c}\text { No, } \\
\text { Not Good }\end{array}$ & Total \\
& $\mathrm{N}$ & $\%$ & $\mathrm{~N}$ & $\%$ & $\mathrm{~N}$ & $\%$ & $\mathrm{~N}$ & $\%$ \\
\hline SSAB Initiative is a good use of funds & 15 & $57.7 \%$ & 5 & $19.2 \%$ & 6 & $23.1 \%$ & 26 & $100 \%$ \\
\hline
\end{tabular}

SSAB Supplementary Appendix 


\section{Table 9.}

DOE-HQ involvement with the SSAB Initiative

\begin{tabular}{|c|c|c|c|c|c|c|c|c|}
\hline \multirow[t]{2}{*}{ Sandia } & \multicolumn{2}{|c|}{ Agree } & \multicolumn{2}{|c|}{$\begin{array}{l}\text { Neither/ } \\
\text { Don't Know }\end{array}$} & \multicolumn{2}{|c|}{ Disagree } & \multicolumn{2}{|c|}{ Total } \\
\hline & $\mathrm{N}$ & $\%$ & $\mathbf{N}$ & $\%$ & $N$ & $\%$ & $N$ & $\%$ \\
\hline $\begin{array}{l}\text { DOE-HQ provides helpful guidance to the } \\
\text { Boards and to DOE }\end{array}$ & 11 & $39.3 \%$ & 13 & $46.4 \%$ & 4 & $14.3 \%$ & 28 & $100 \%$ \\
\hline $\begin{array}{l}\text { DOE-HQ provides sufficient support to } \\
\text { facilitate the work of the SSAB }\end{array}$ & 12 & $42.9 \%$ & 12 & $42.9 \%$ & 4 & $14.3 \%$ & 28 & $100 \%$ \\
\hline $\begin{array}{l}\text { DOE-HQ gives careful consideration to } \\
\text { SSAB advice in its decisions }\end{array}$ & 3 & $10.7 \%$ & 22 & $78.6 \%$ & 3 & $10.7 \%$ & 28 & $100 \%$ \\
\hline
\end{tabular}


Table 10.

Personal experience with the SSAB Initiative

\begin{tabular}{|c|c|c|c|c|c|c|c|c|}
\hline \multirow[t]{2}{*}{ Sandia } & \multicolumn{2}{|c|}{ Agree } & \multicolumn{2}{|c|}{$\begin{array}{l}\text { Neither/ } \\
\text { Don't Know }\end{array}$} & \multicolumn{2}{|c|}{ Disagree } & \multicolumn{2}{|c|}{ Total } \\
\hline & $\mathbf{N}$ & $\%$ & $\mathrm{~N}$ & $\%$ & $N$ & $\%$ & $\mathrm{~N}$ & $\%$ \\
\hline $\begin{array}{l}\text { I consider myself to be very } \\
\text { knowledgeable about site issues }\end{array}$ & 15 & $55.6 \%$ & 6 & $22.2 \%$ & 6 & $22.2 \%$ & 27 & $100 \%$ \\
\hline $\begin{array}{l}\text { Because of the SSAB, I better understand } \\
\text { the complexities of site clean-up }\end{array}$ & 16 & $57.1 \%$ & 5 & $17.9 \%$ & 7 & $25.0 \%$ & 28 & $100 \%$ \\
\hline $\begin{array}{l}\text { I feel that my participation in the SSAB } \\
\text { has been worthwhile }(\mathrm{R}) *\end{array}$ & 14 & $50.0 \%$ & 8 & $28.6 \%$ & 6 & $21.4 \%$ & 28 & $100 \%$ \\
\hline
\end{tabular}

* (R) denotes a question that was asked in the negative form. For ease of review, these questions and data have been reversed in this table and are stated in positive forms. 
Table 11.

Group/Team work skills

\begin{tabular}{|c|c|c|c|c|c|c|c|c|}
\hline \multirow[t]{2}{*}{ Sandia } & \multicolumn{2}{|c|}{ Agree } & \multicolumn{2}{|c|}{$\begin{array}{l}\text { Neither/ } \\
\text { Don't Know }\end{array}$} & \multicolumn{2}{|c|}{ Disagree } & \multicolumn{2}{|c|}{ Total } \\
\hline & $N$ & $\%$ & $\mathrm{~N}$ & $\%$ & $\mathrm{~N}$ & $\%$ & $\mathrm{~N}$ & $\%$ \\
\hline All have group/team work skills & 6 & $2.1 .4 \%$ & 9 & $32.1 \%$ & 13 & $46.4 \%$ & 28 & $100 \%$ \\
\hline $\begin{array}{l}\text { Have developed well-established working } \\
\text { relationships with each other }\end{array}$ & 4 & $14.3 \%$ & 10 & $35.7 \%$ & 14 & $50.0 \%$ & 28 & $100 \%$ \\
\hline Like each other & 7 & $25.0 \%$ & 15 & $53.6 \%$ & 6 & $21.4 \%$ & 28 & $100 \%$ \\
\hline $\begin{array}{l}\text { Have a high degree of trust and } \\
\text { confidence in each other }\end{array}$ & 2 & $7.1 \%$ & 14 & $50.0 \%$ & 12 & $42.9 \%$ & 28 & $100 \%$ \\
\hline $\begin{array}{l}\text { Accept the values that are important to the } \\
\text { group }\end{array}$ & 7 & $25.0 \%$ & 15 & $53.6 \%$ & 6 & $21.4 \%$ & 28 & $100 \%$ \\
\hline Show respect for the viewpoints of others & 16 & $57.1 \%$ & 6 & $21.4 \%$ & 6 & $21.4 \%$ & 28 & $100 \%$ \\
\hline $\begin{array}{l}\text { Accept willingly the goals and } \\
\text { expectations for the Board }\end{array}$ & 8 & $28.6 \%$ & 16 & $57.1 \%$ & 4 & $14.3 \%$ & 28 & $100 \%$ \\
\hline $\begin{array}{l}\text { Set high expectations for what they } \\
\text { believe they can accomplish }\end{array}$ & 6 & $21.4 \%$ & 17 & $60.7 \%$ & 5 & $17.9 \%$ & 28 & $100 \%$ \\
\hline $\begin{array}{l}\text { Communicate fully and frankly to the } \\
\text { Board all relevant and valuable } \\
\text { information }\end{array}$ & 11 & $39.3 \%$ & 12 & $42.9 \%$ & 5 & $17.9 \%$ & 28 & $100 \%$ \\
\hline $\begin{array}{l}\text { Try not to waste the Board's time with } \\
\text { irrelevant material or communications }\end{array}$ & 11 & $39.3 \%$ & 4 & $14.3 \%$ & 13 & $46.4 \%$ & 28 & $100 \%$ \\
\hline $\begin{array}{l}\text { Are willing to be influenced by other } \\
\text { Board members about new ideas and } \\
\text { methods }\end{array}$ & 12 & $42.9 \%$ & 12 & $42.9 \%$ & 4 & $14.3 \%$ & 28 & $100 \%$ \\
\hline $\begin{array}{l}\text { Clearly understand the goals and } \\
\text { philosphy of the Board's operation }\end{array}$ & 8 & $28.6 \%$ & 12 & $42.9 \%$ & 8 & $28.6 \%$ & 28 & $100 \%$ \\
\hline $\begin{array}{l}\text { Do all they can to help the Board achieve } \\
\text { its objectives }\end{array}$ & 11 & $40.7 \%$ & 10 & $37.0 \%$ & 6 & $22.2 \%$ & 27 & $100 \%$ \\
\hline $\begin{array}{l}\text { Get the technical knowledge and training } \\
\text { in group skills they need }\end{array}$ & 4 & $14.8 \%$ & 16 & $59.3 \%$ & 7 & $25.9 \%$ & 27 & $100 \%$ \\
\hline Provide one another mutual help & 8 & $28.6 \%$ & 12 & $42.9 \%$ & 8 & $28.6 \%$ & 28 & $100 \%$ \\
\hline
\end{tabular}

SSAB Supplementary Appendix 
Savannah River Site Specific Advisory Board Evaluation Survey Results

SSAB Supplementary Appendix August 1996 
Figure 1a. Savannah River

Goal 1. Establish processes and procedures to provide an effective forum for exchange of information and viewpoints regarding DOE site issues

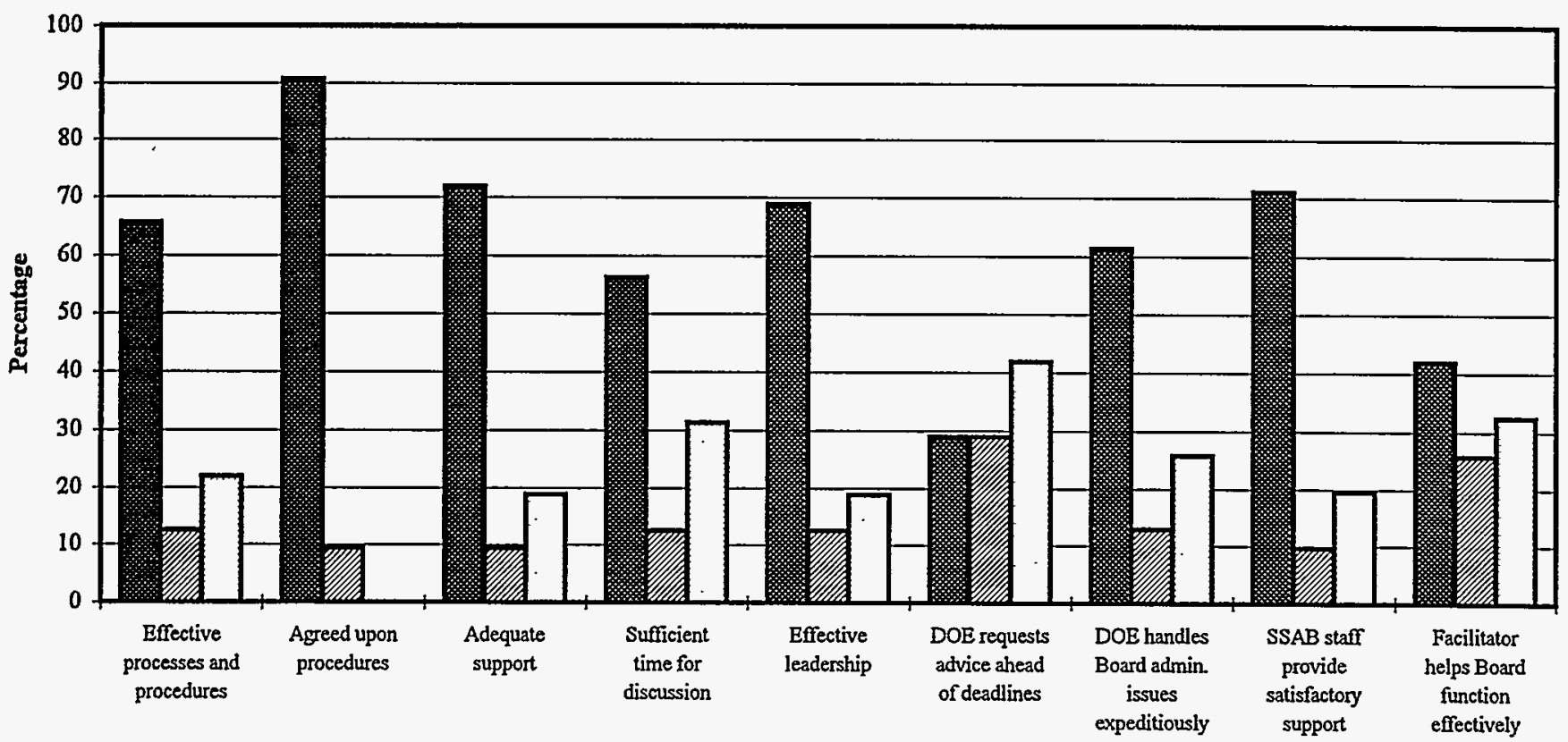

Agree (\%) DNeither Agree Nor Disagree/Don't Know (\%) DDisagree (\%)

Figure 1b. Long Survey: Entire Sample

Goal 1. Establish processes and procedures to provide an effective forum for exchange of information and viewpoints regarding DOE site issues

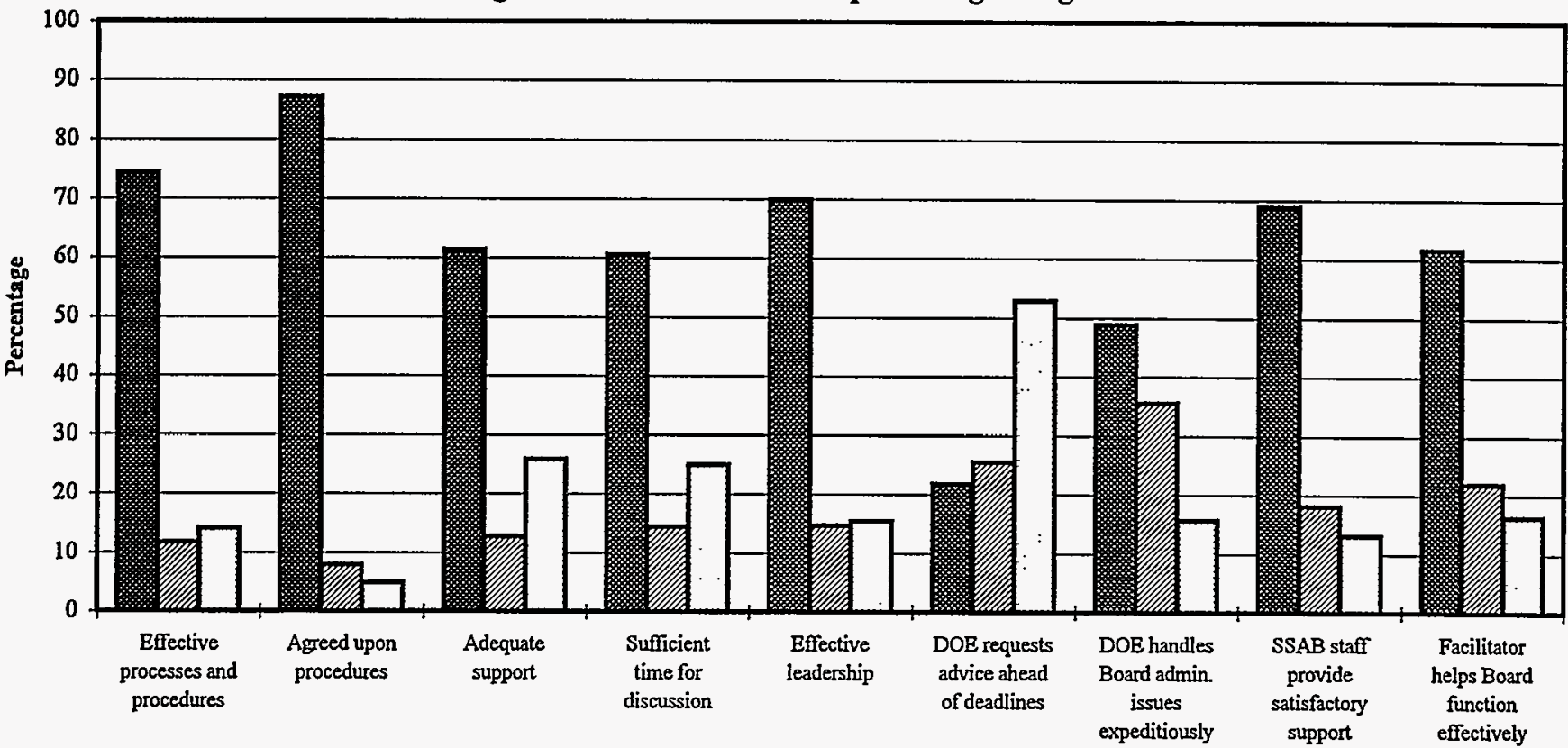

⿴囗大 Agree (\%) ENeither Agree Nor Disagree/Don't Know (\%) DDisagree (\%)

SSAB Supplementary Appendix 
Figure 2a. Savannah River

Goal 2. Facilitate interaction and exchange of information and viewpoints regarding DOE site issues

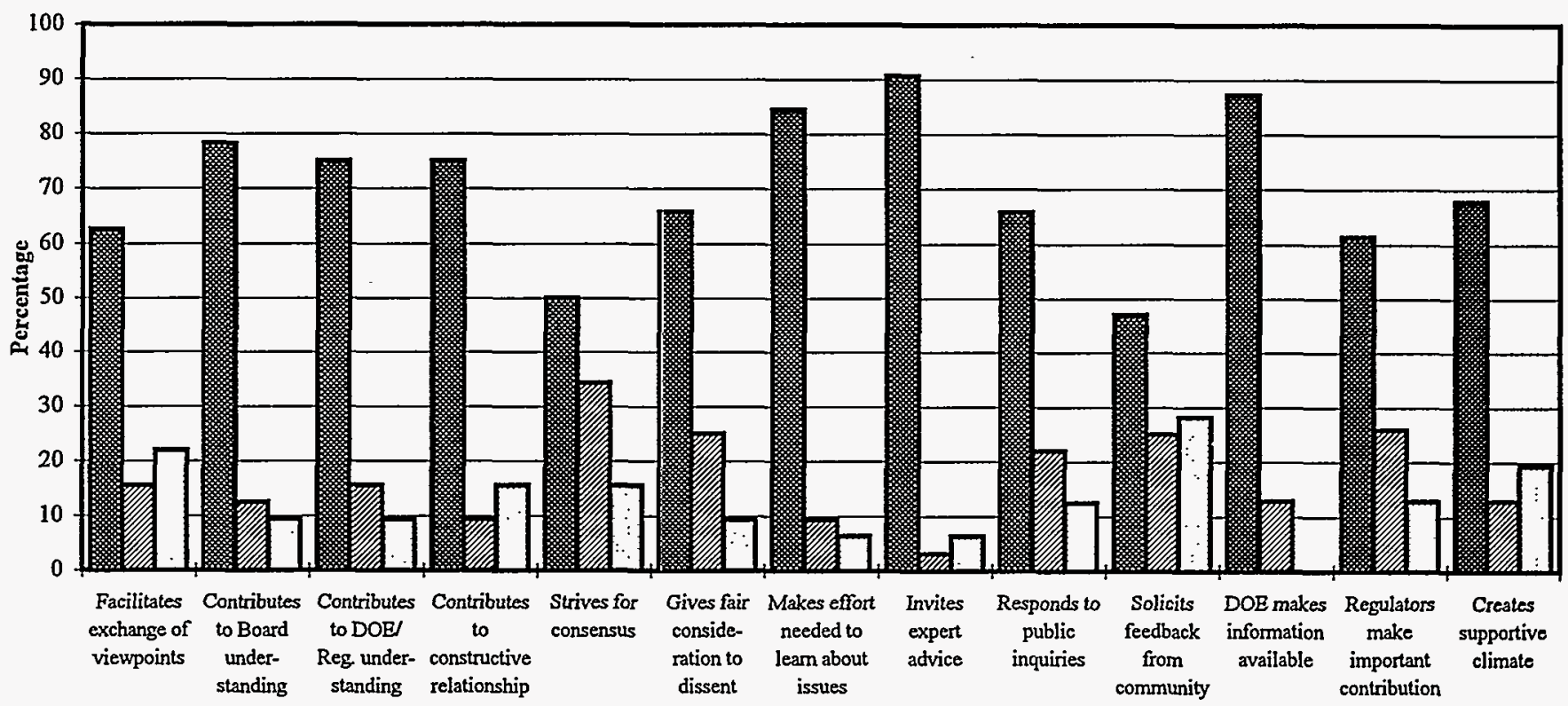

E Agree (\%) Neither Agree Nor Disagree/Don't Know (\%) DDisagree (\%)

Figure 2b. Long Survey: Entire Sample

Goal 2. Facilitate interaction and exchange of information and viewpoints regarding $\mathrm{DOE}$ site issues

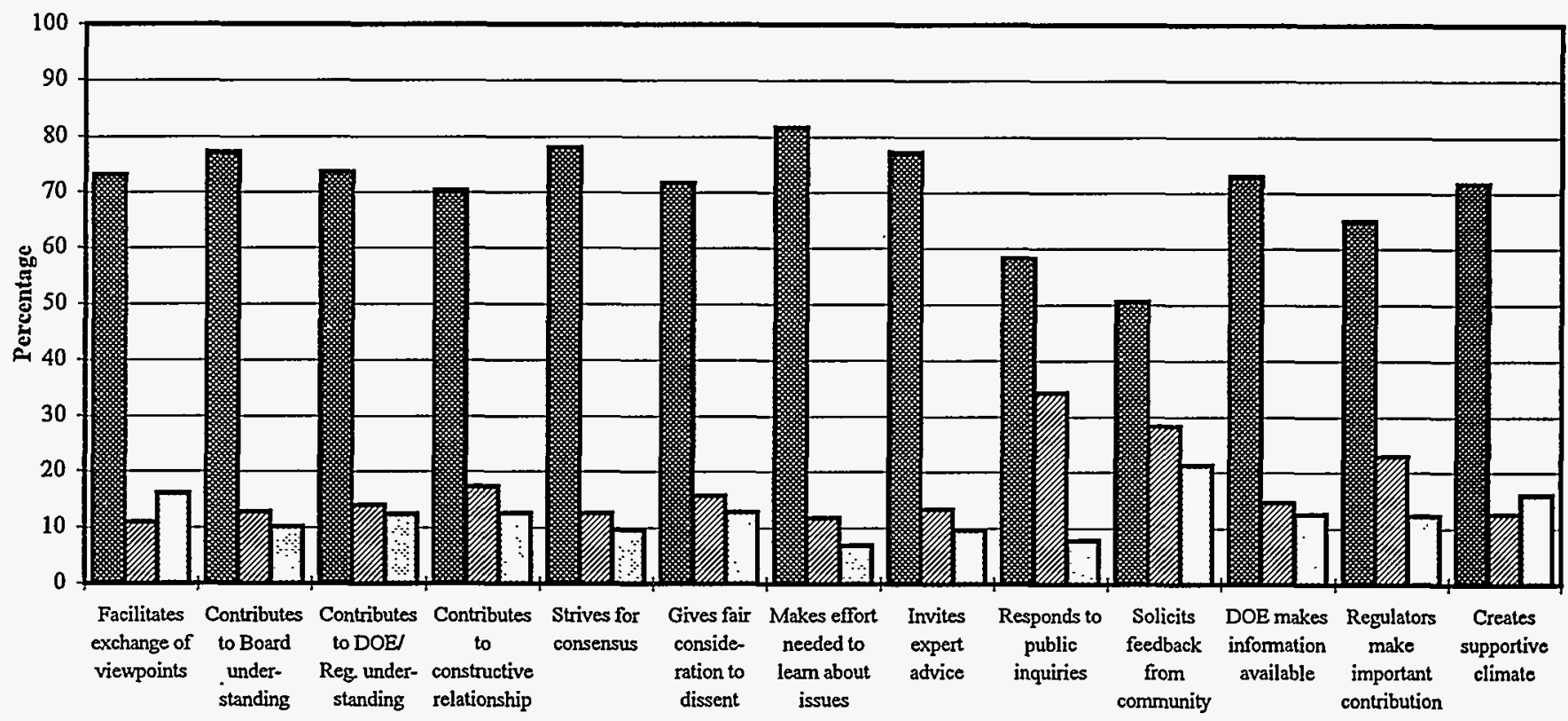

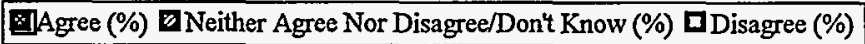

SSAB Supplementary Appendix

August 1996 
Figure 3a. Savannah River

Goal 3. Provide useful advice and/or recommendations to DOE

(and regulators, where appropriate)

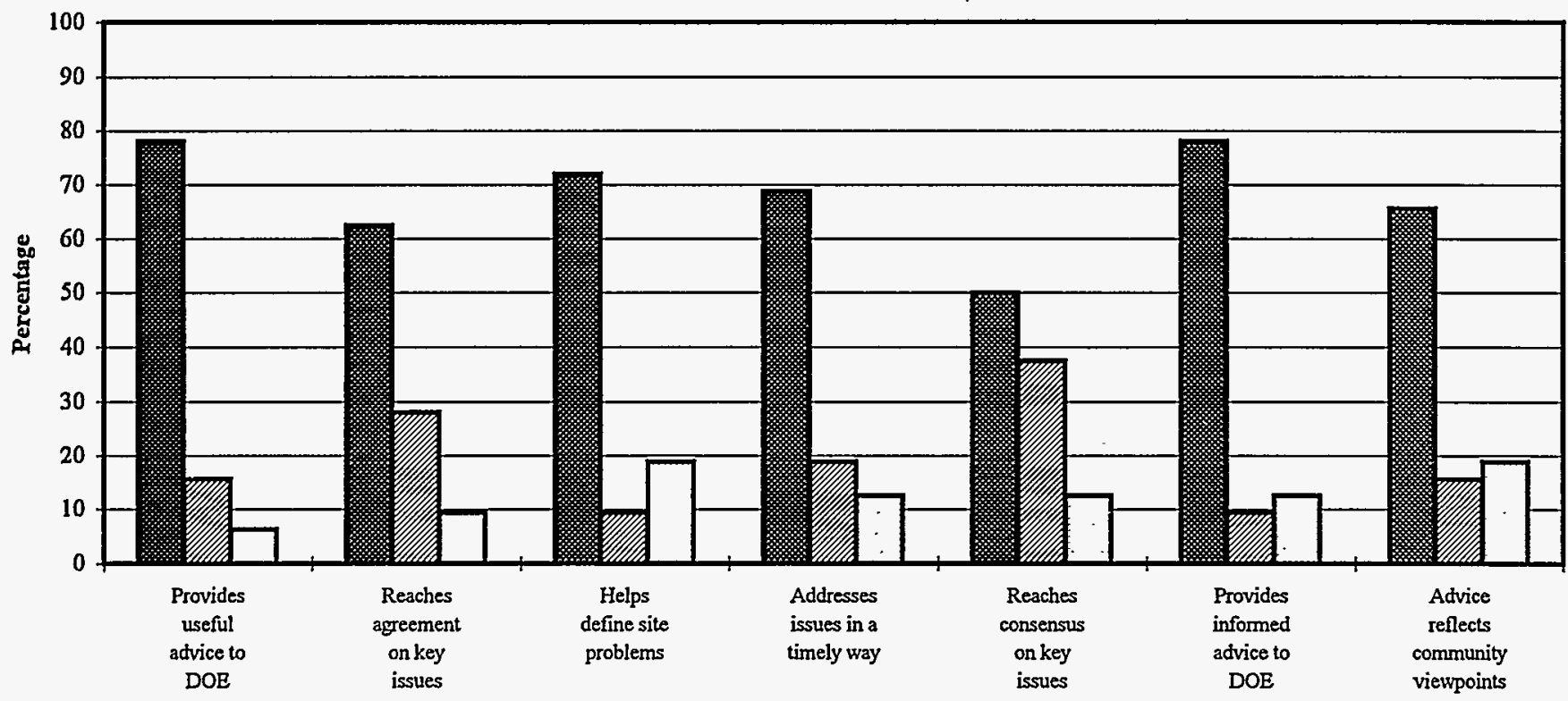

实Agree (\%) Neither Agree Nor Disagree/Don't Know (\%) DDisagree (\%)

Figure 3b. Long Survey: Entire Sample

Goal 3. Provide useful advice and/or recommendations to DOE

(and regulators, where appropriate)

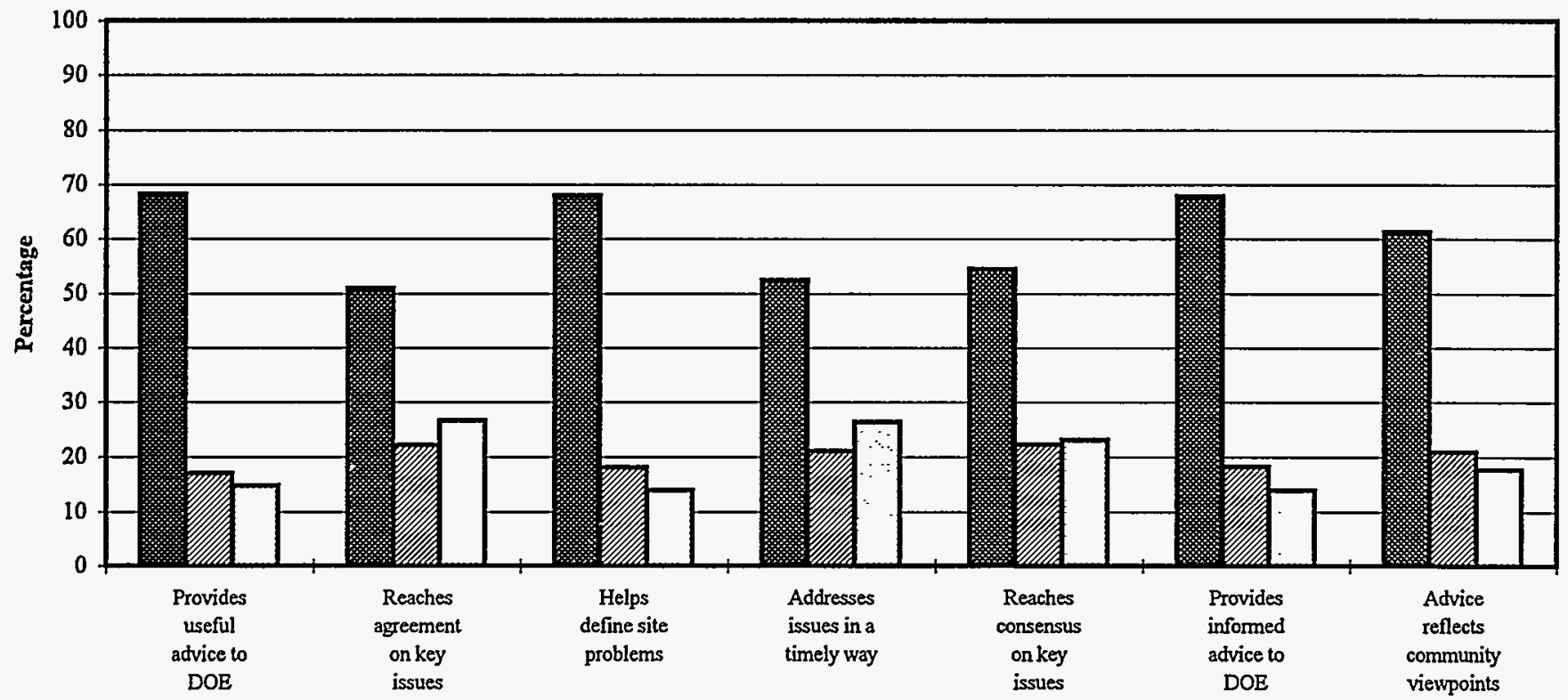

Agree (\%) Weither Agree Nor Disagree/Don't Know (\%) DDisagree (\%)

SSAB Supplementary Appendix

August 1996 
Figure 4a. Savannah River

Goal 4. Improve DOE's (and where applicable, regulators') site decisions and decision making process

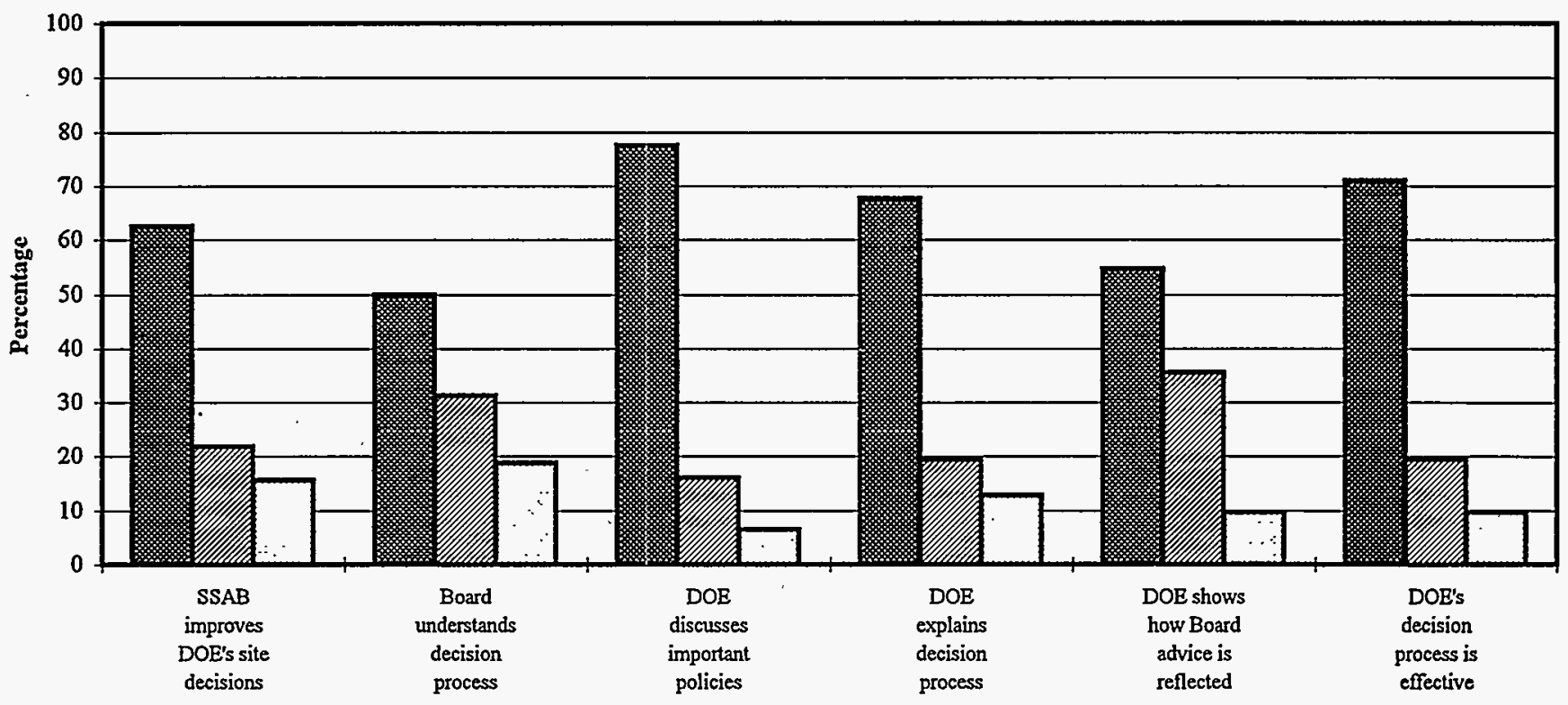

F Agree (\%) Neither Agree Nor Disagree/Don't Know (\%) DDisagree (\%)

Figure 4b. Long Survey: Entire Sample

Goal 4. Improve DOE's (and where applicable, regulators') site decisions and decision making process

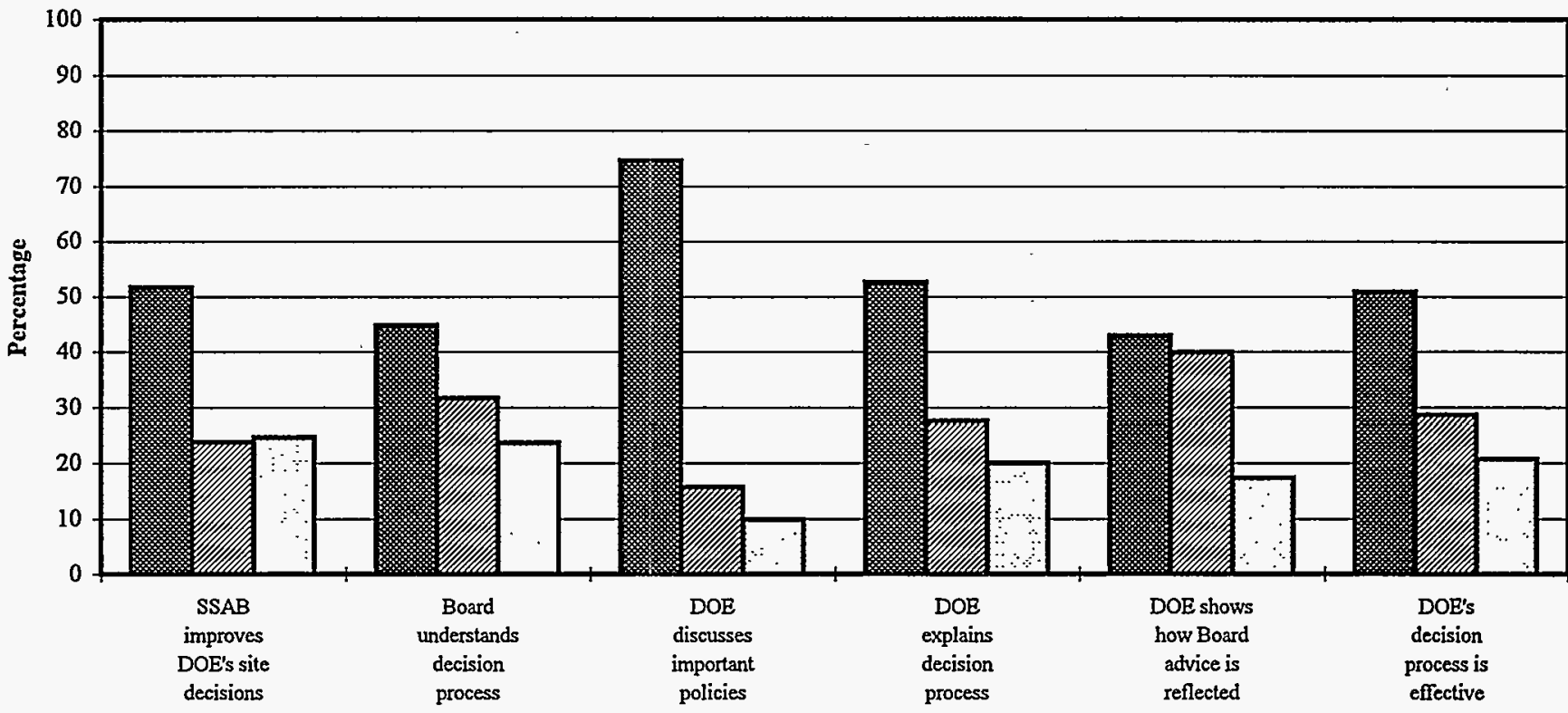

Agree (\%) Deither Agree Nor Disagree/Don't Know (\%) DDisagree (\%)

SSAB Supplementary Appendix

August 1996 
Figure 5a. Savannah River

Goal 5. Lead to more acceptable actions

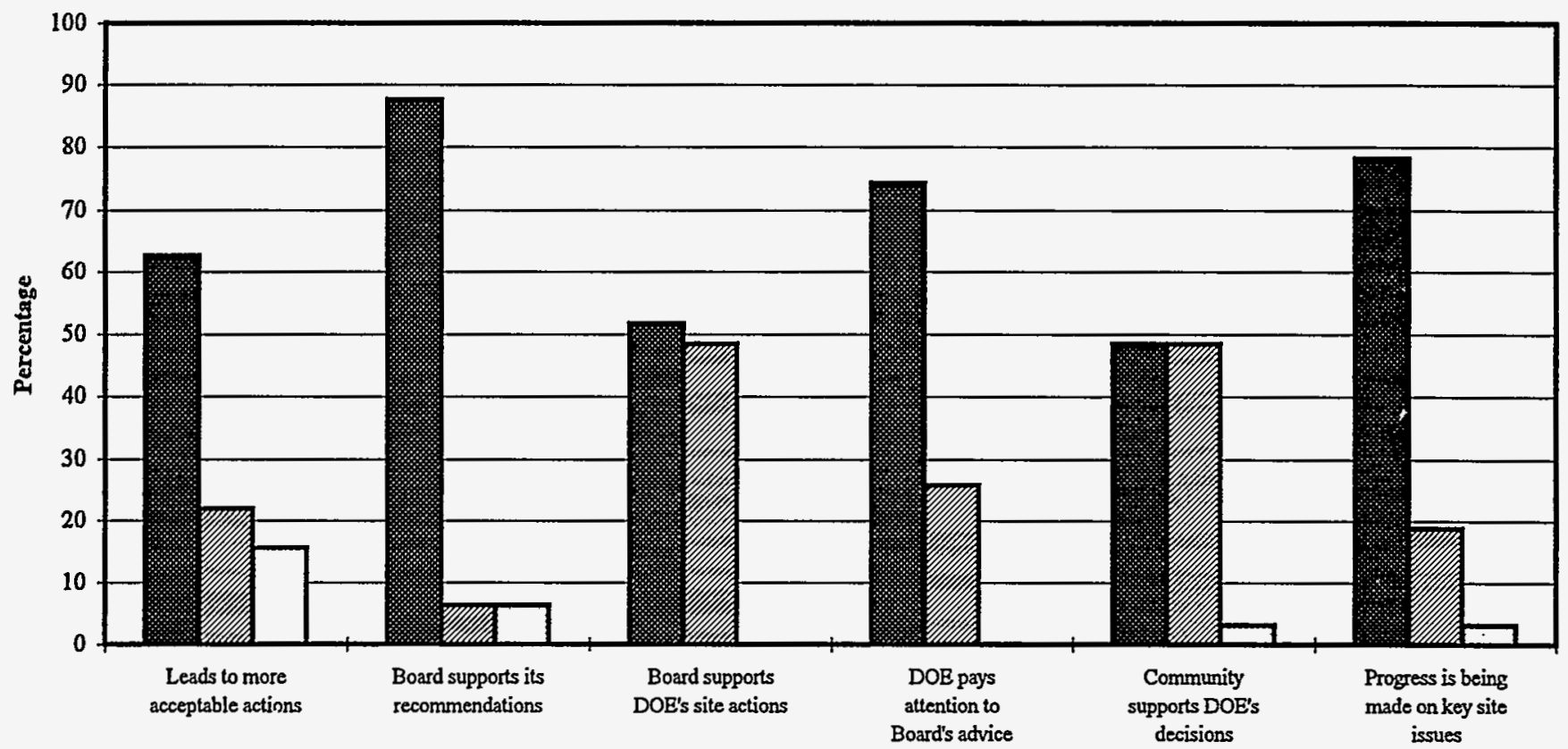

Agree (\%) QNeither Agree Nor Disagree/Don't Know (\%) DDisagree (\%)

Figure 5b. Long Survey: Entire Sample

Goal 5. Lead to more acceptable actions

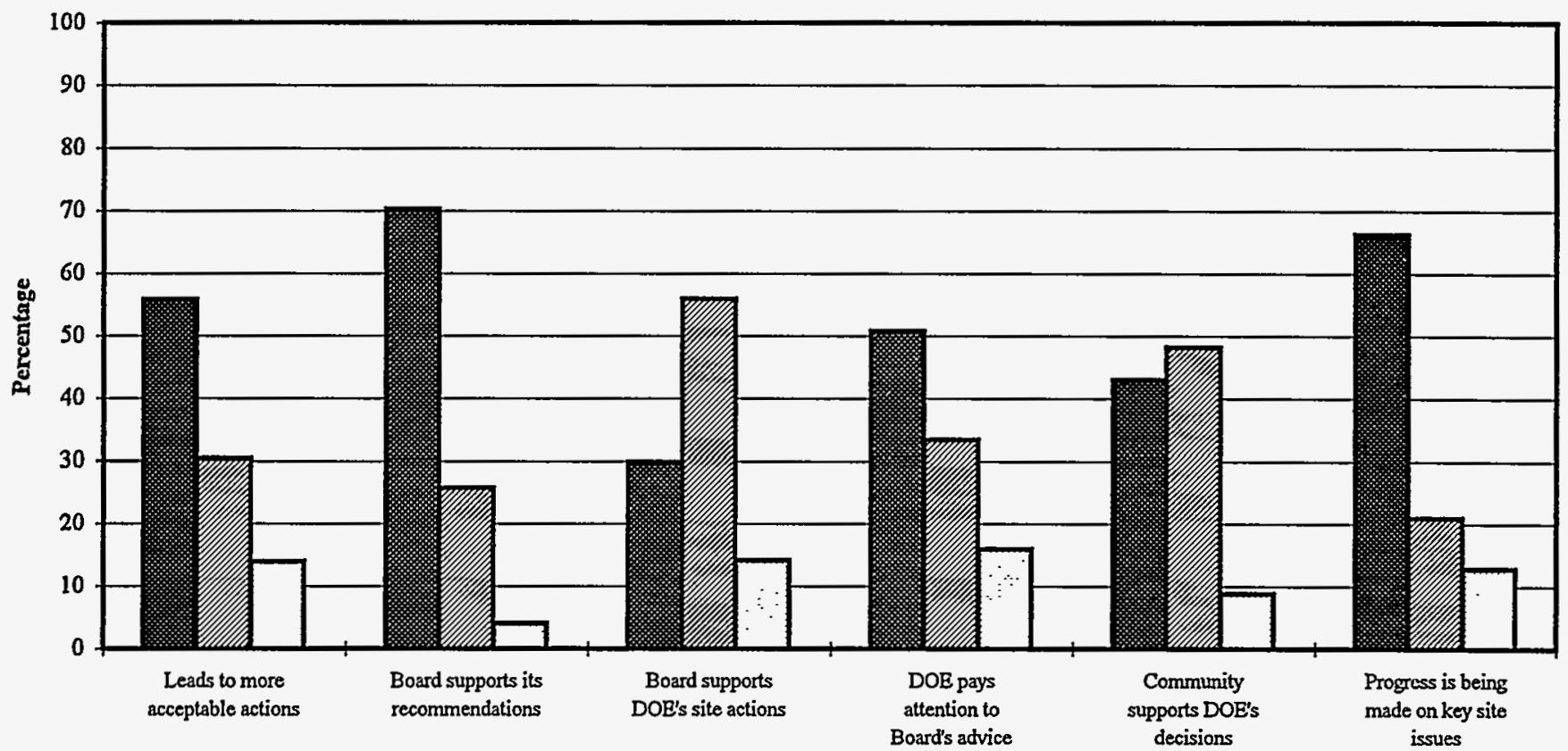

Agree (\%) Deither Agree Nor Disagree/Don't Know (\%) QDisagree (\%)

SSAB Supplementary Appendix

August 1996 
Figure 6a. Savannah River

Goal 6. Contribute to trust and confidence in DOE

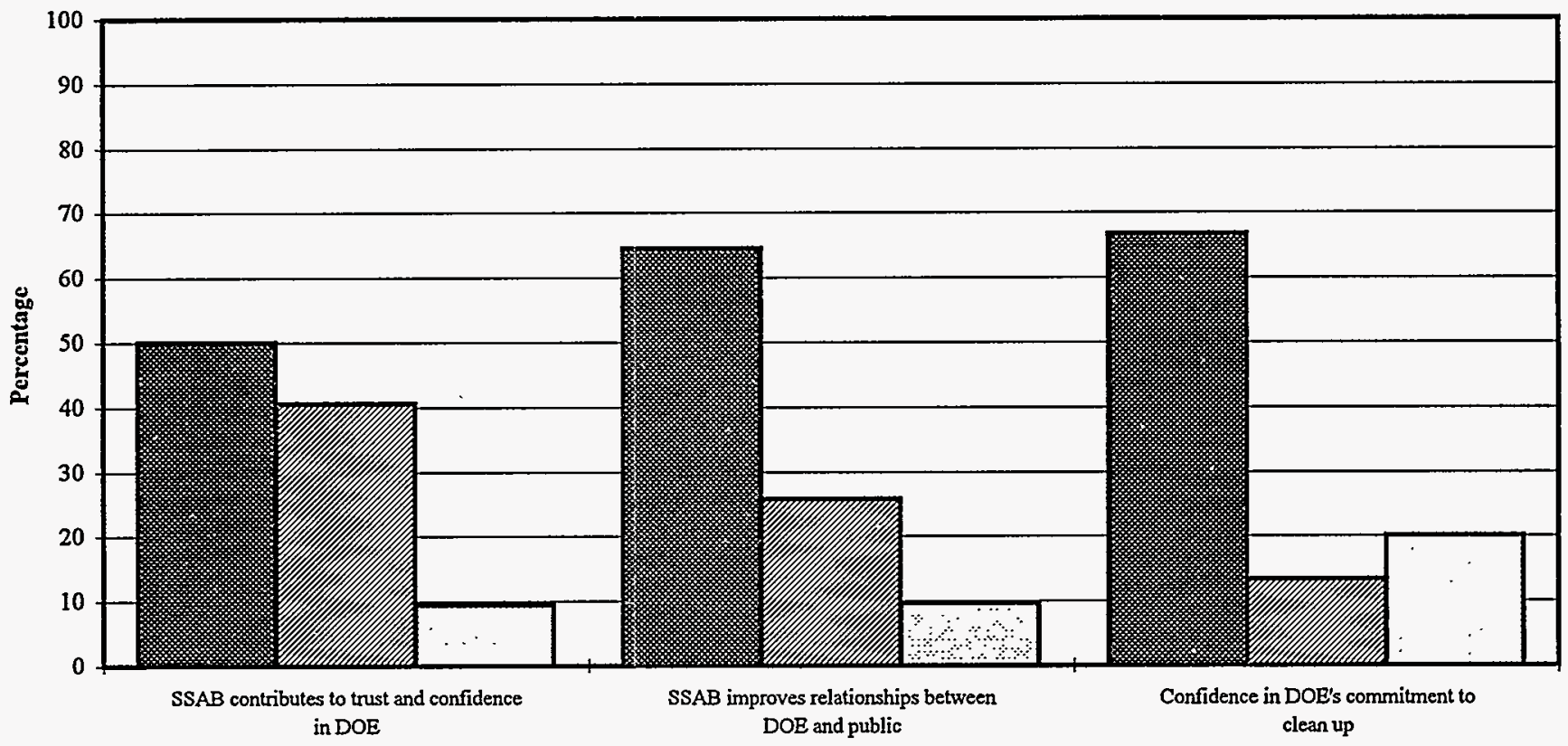

E Agree (\%) DNeither Agree Nor Disagree/Don't Know (\%) DDisagree (\%)

Figure 6b. Long Survey: Entire Sample

Goal 6. Contribute to trust and confidence in DOE

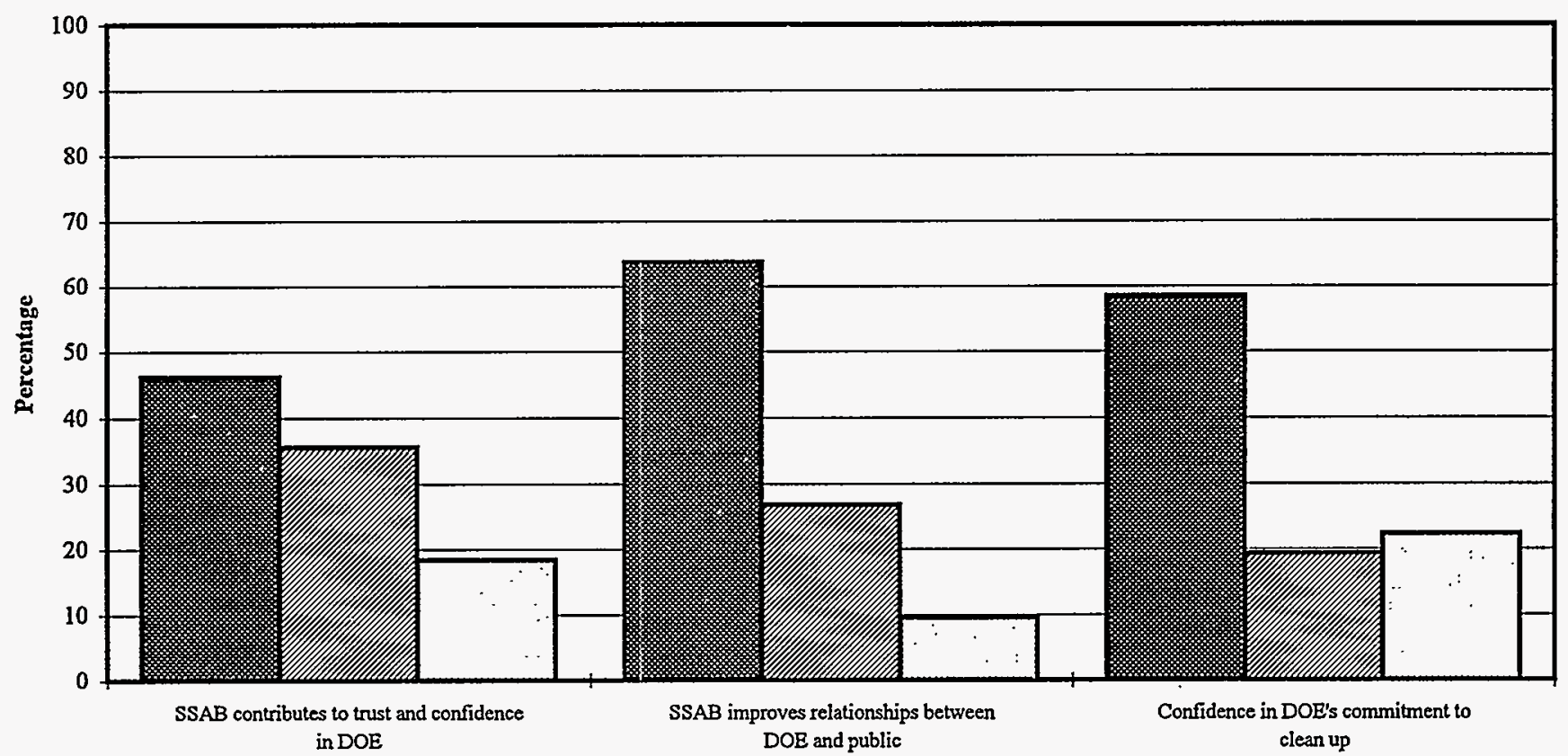

Agree (\%) Deither Agree Nor Disagree/Don't Know (\%) GDisagree (\%)

SSAB Supplementary Appendix

August 1996 
Figure 7a. Savannah River

Public awareness

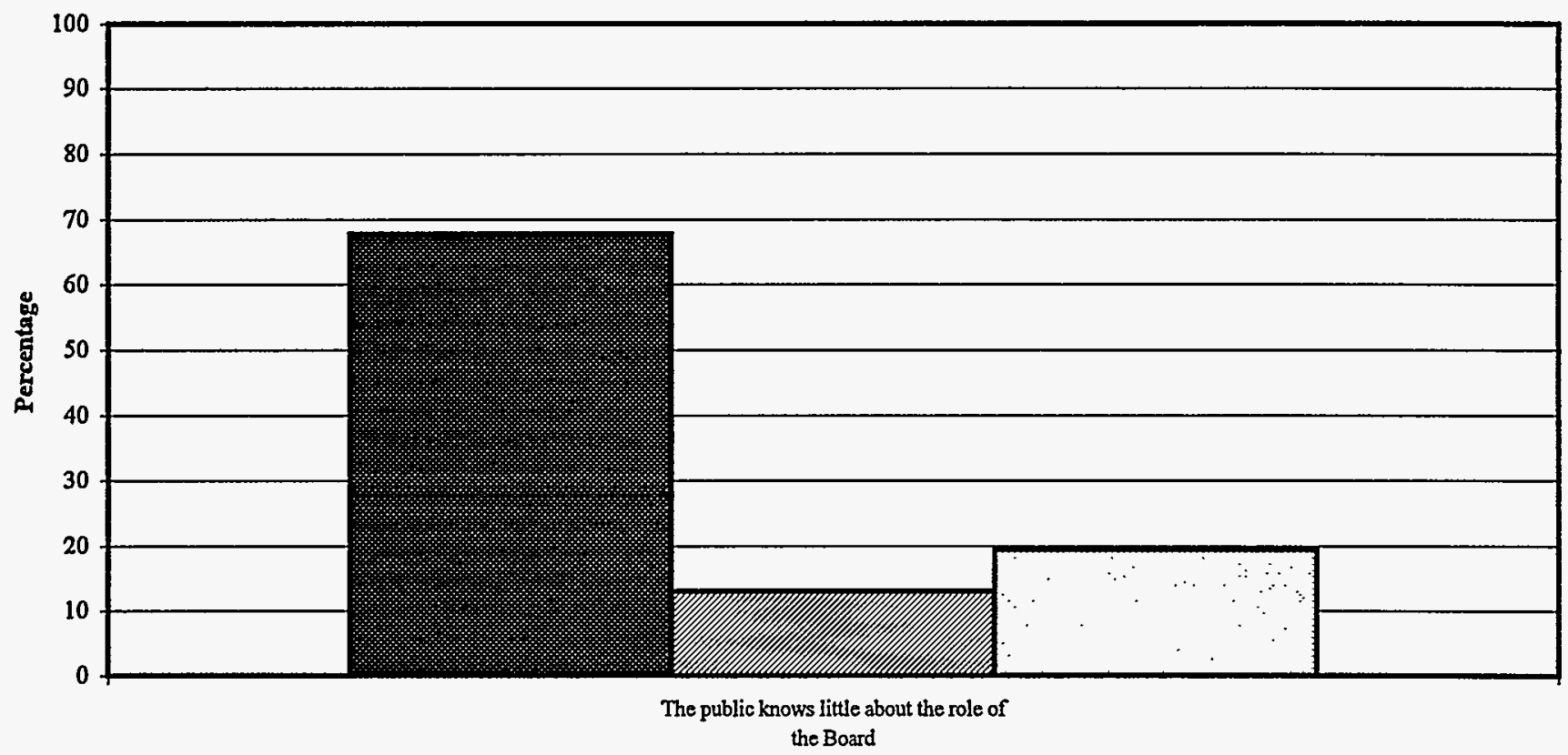

E Agree (\%) Deither Agree Nor Disagree/Don't Know (\%) DDisagree (\%)

Figure 7b. Long Survey: Entire Sample

Public awareness

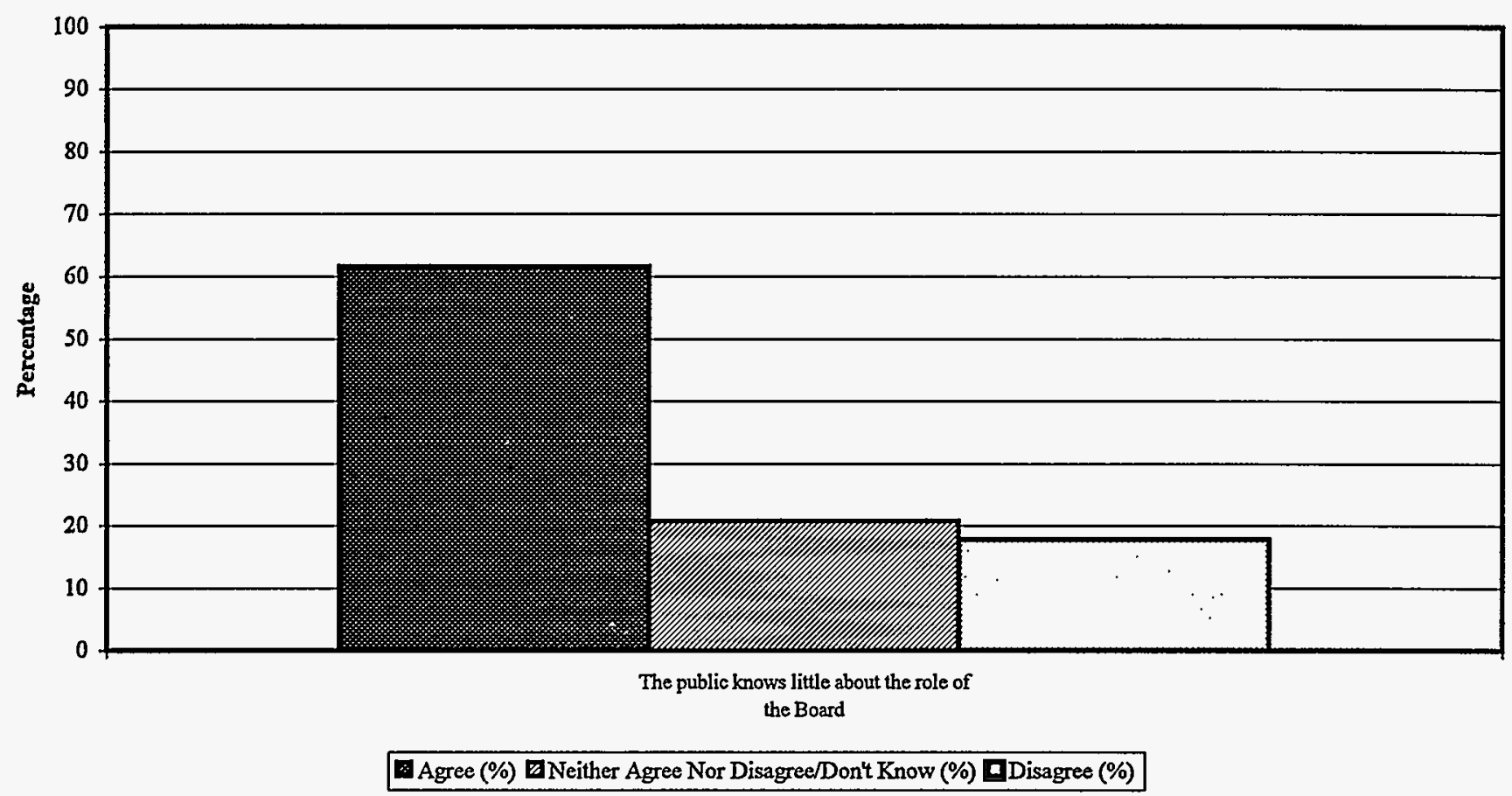

SSAB Supplementary Appendix

August 1996 
Figure 8a. Savannah River

DOE-HQ involvement with the SSAB Initiative

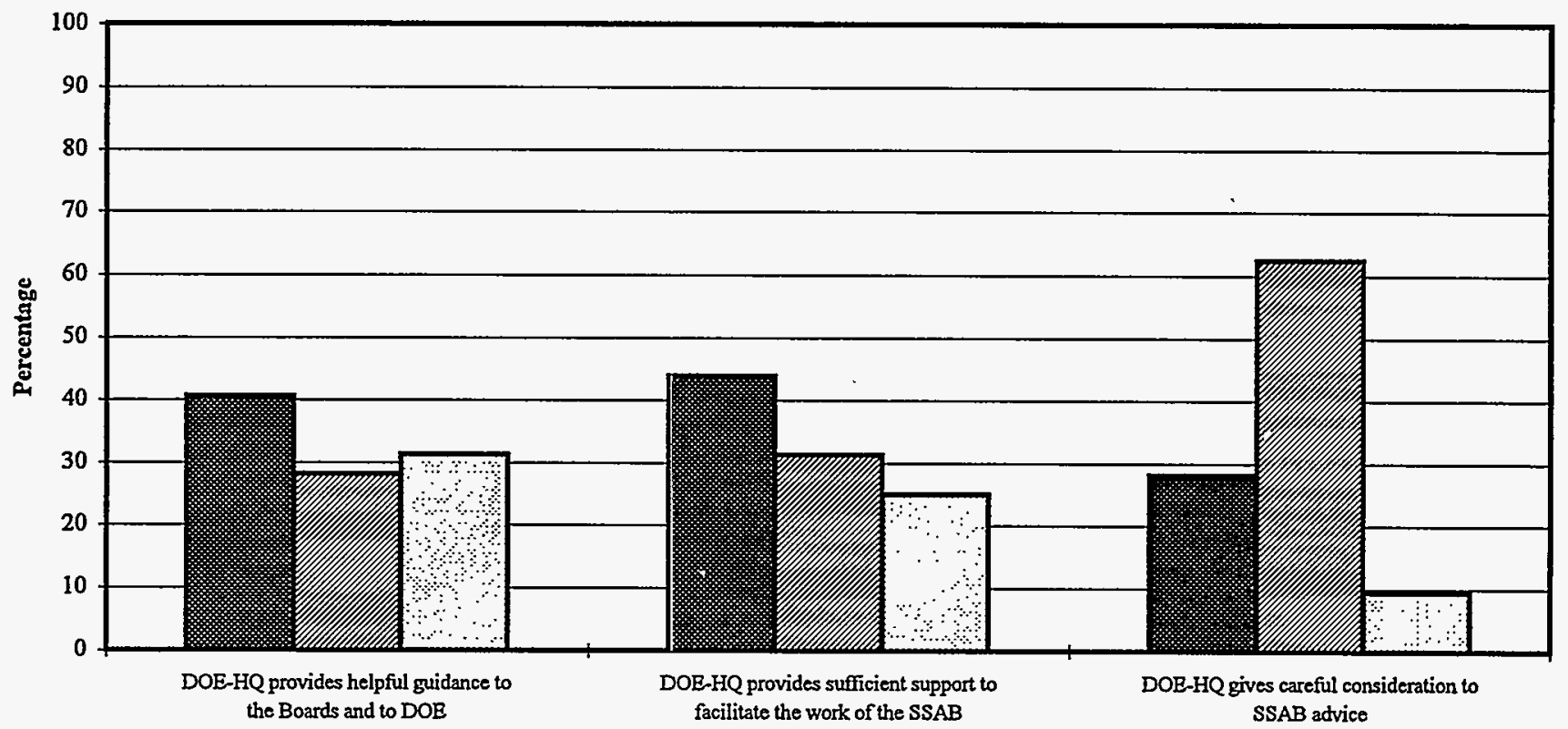

Agree (\%) ENeither Agree Nor Disagree/Don't Know (\%) DDisagree (\%)

Figure 8b. Long Survey: Entire Sample DOE-HQ involvement with the SSAB Initiative

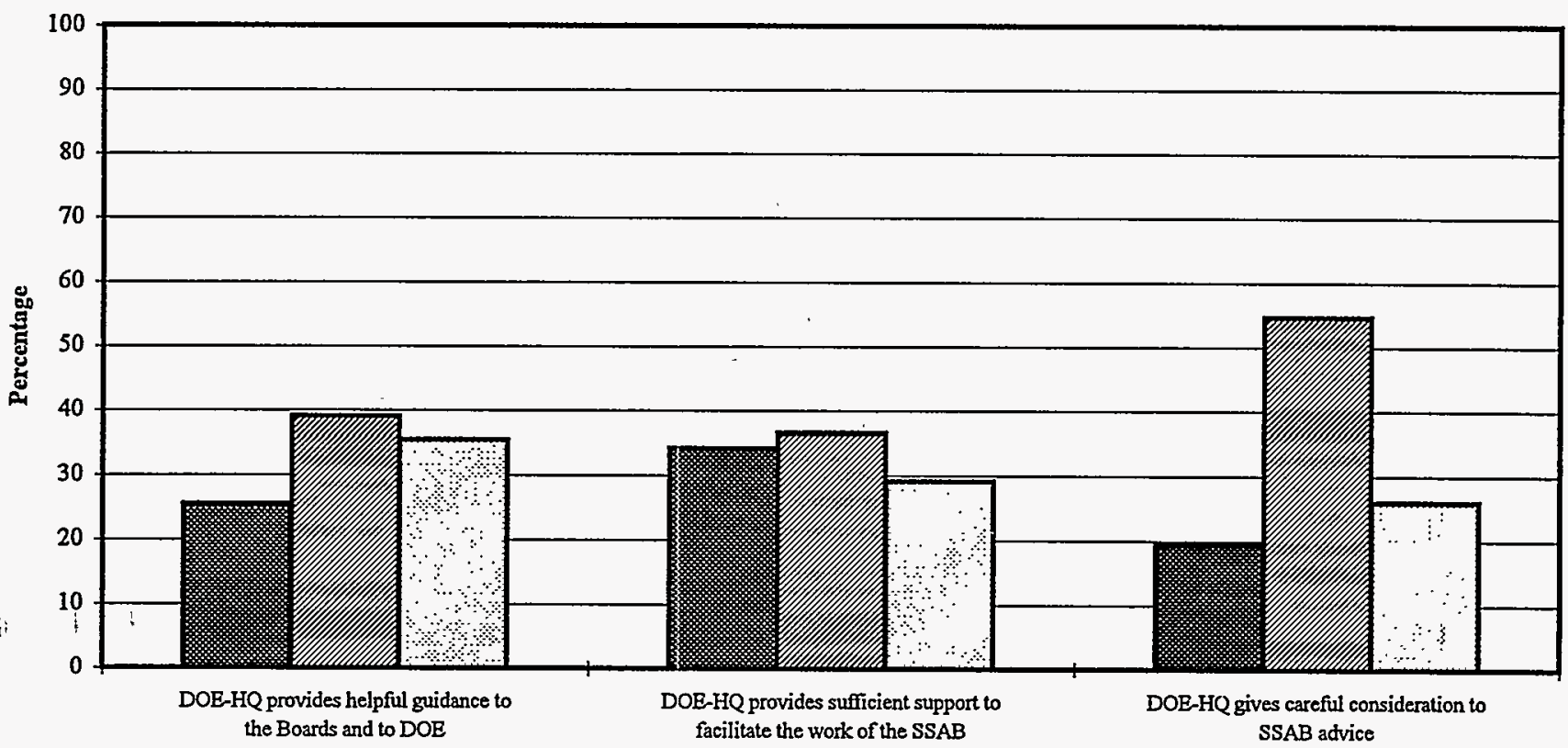

Agree (\%) ENeither Agree Nor Disagree/Don't Know (\%) DDisagree (\%)

SSAB Supplementary Appendix

August 1996 
Figure 9a. Savannah River

Personal experience with the SSAB Initiative

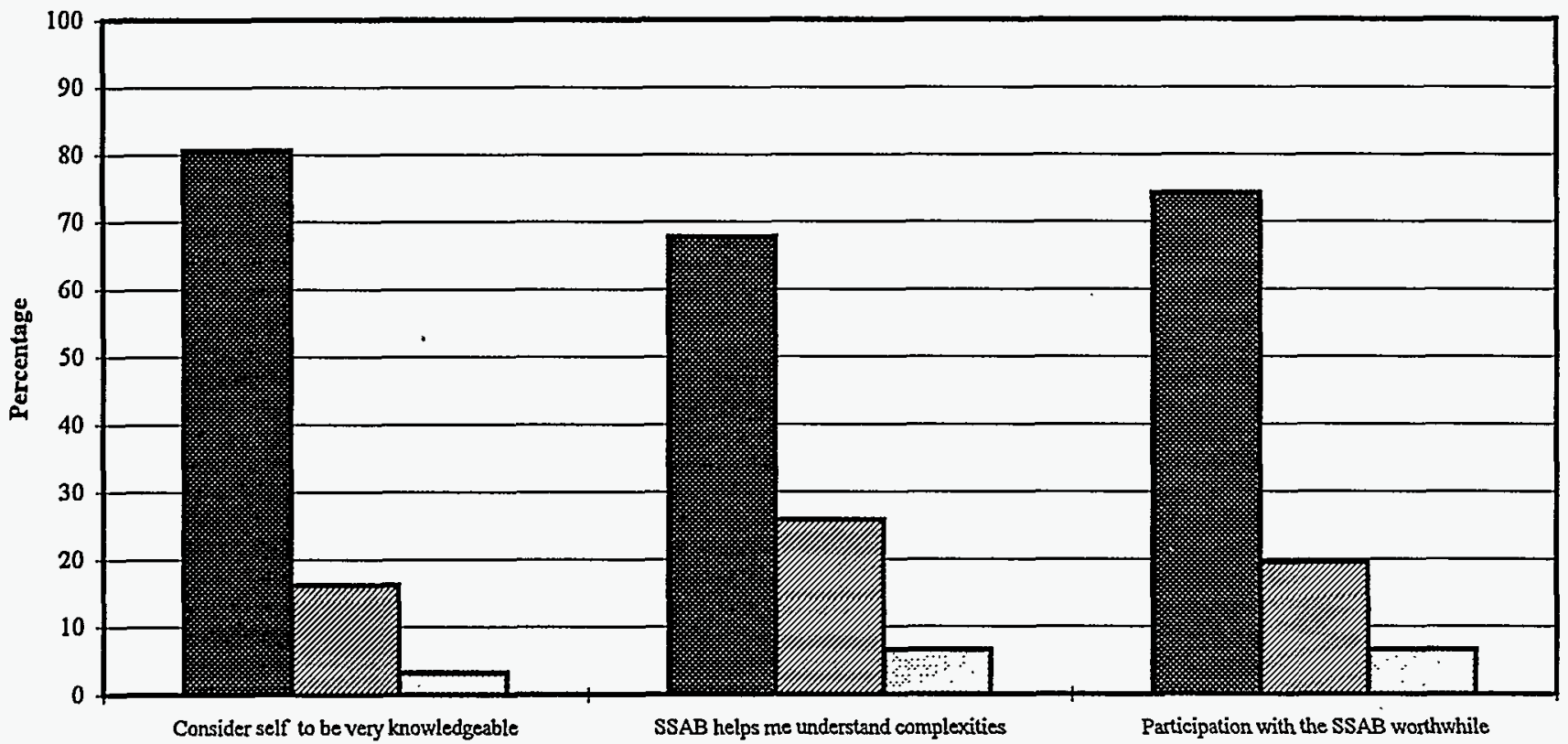

Agree (\%) DNeither Agree Nor Disagree/Don't Know (\%) DDisagree (\%)

Figure 9b. Long Survey: Entire Sample Personal experience with the SSAB Initiative

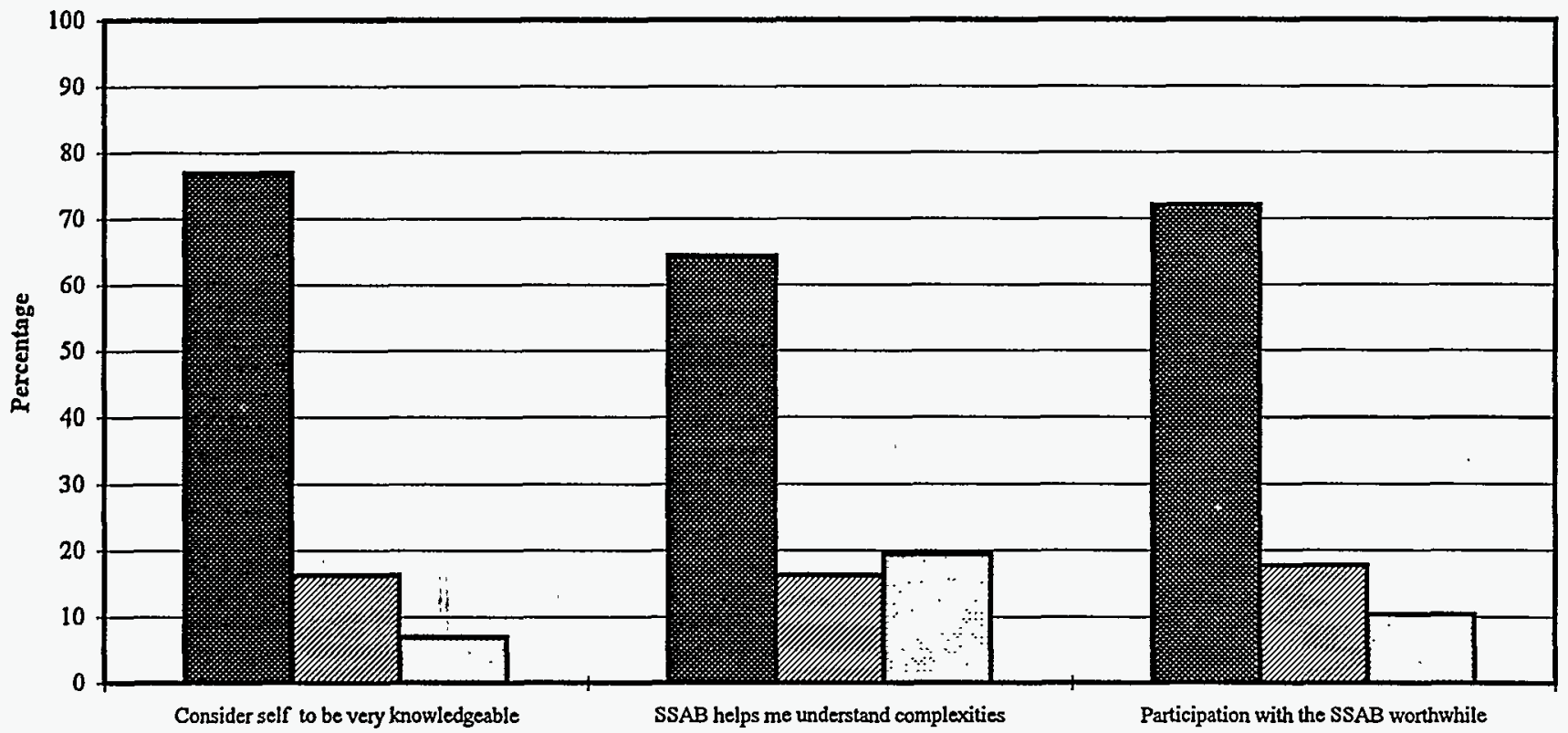

Agree (\%) DNeither Agree Nor Disagree/Don't Know (\%) DDisagree (\%)

SSAB Supplementary Appendix 
Figure 10a. Savannah River

Group/Team work skills

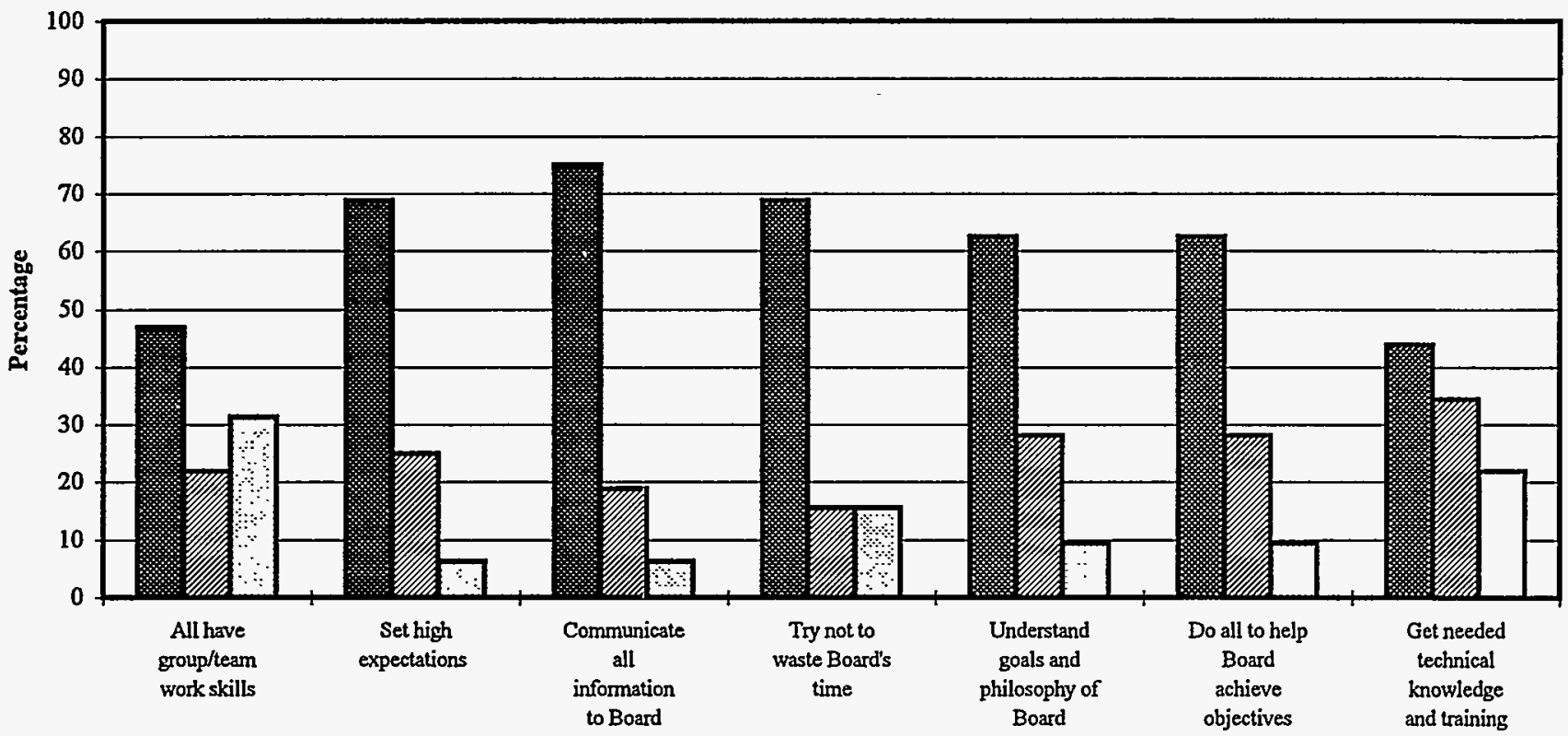

Agree (\%) Deither Agree Nor Disagree/Don't Know (\%) DDisagree (\%)

Figure 10b. Long Survey: Entire Sample

Group/Team work skills

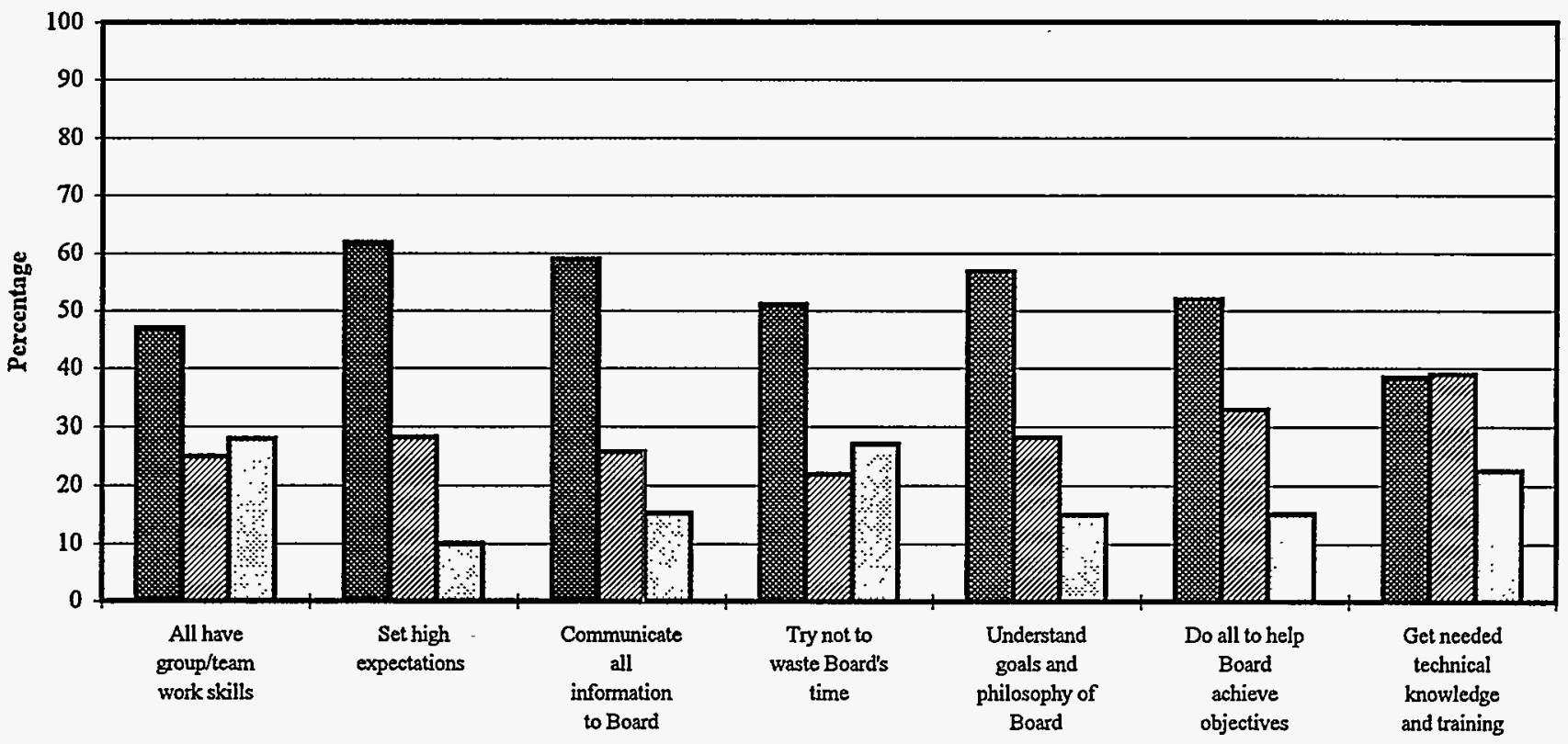

Agree (\%) DNeither Agree Nor Disagree/Don't Know (\%) पDisagree (\%)

SSAB Supplementary Appendix

August 1996 
Figure 11a. Savannah River

Working relatiọns

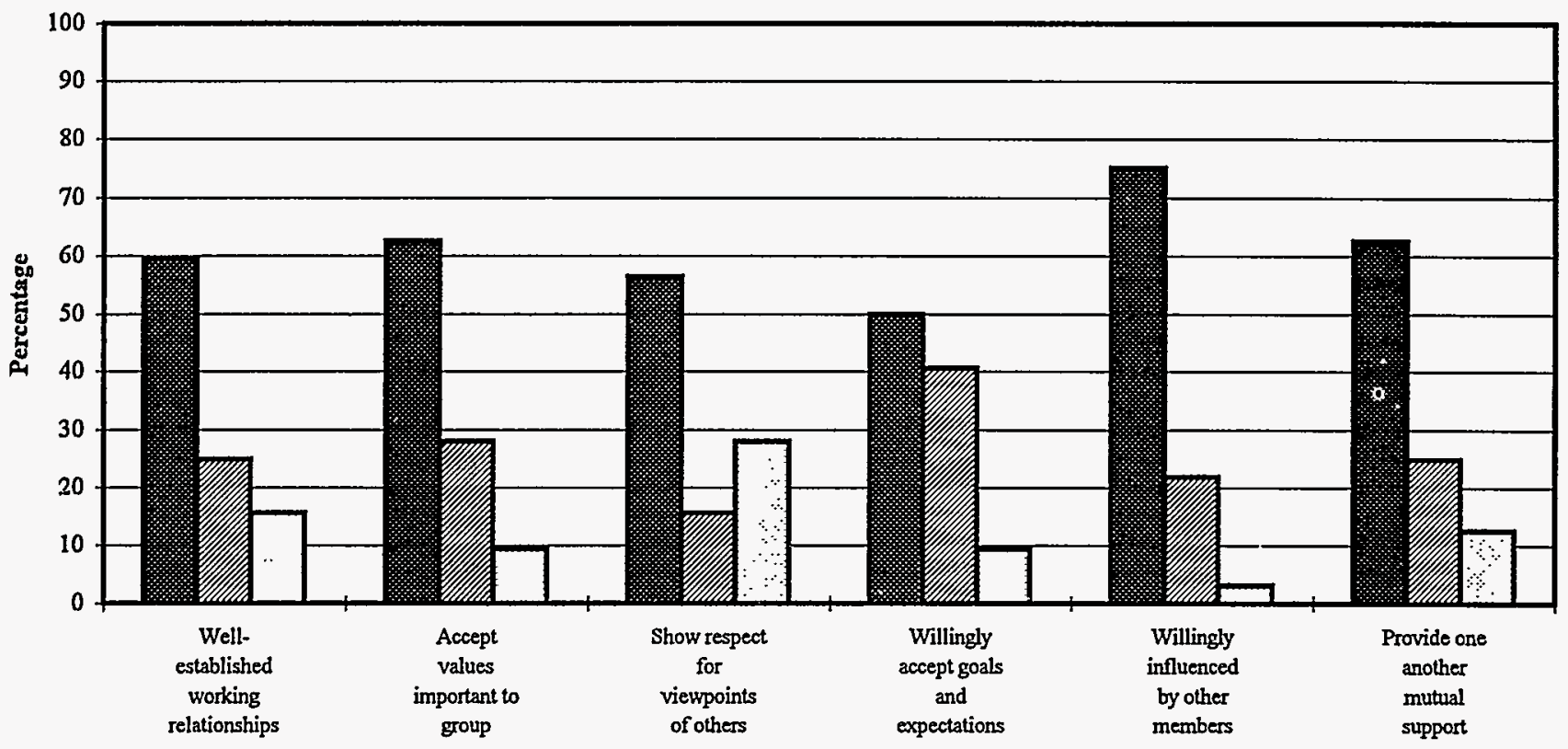

Agree (\%) ENeither Agree Nor Disagree/Don't Know (\%) DDisagree (\%)

Figure 11b. Long Survey: Entire Sample Working relations

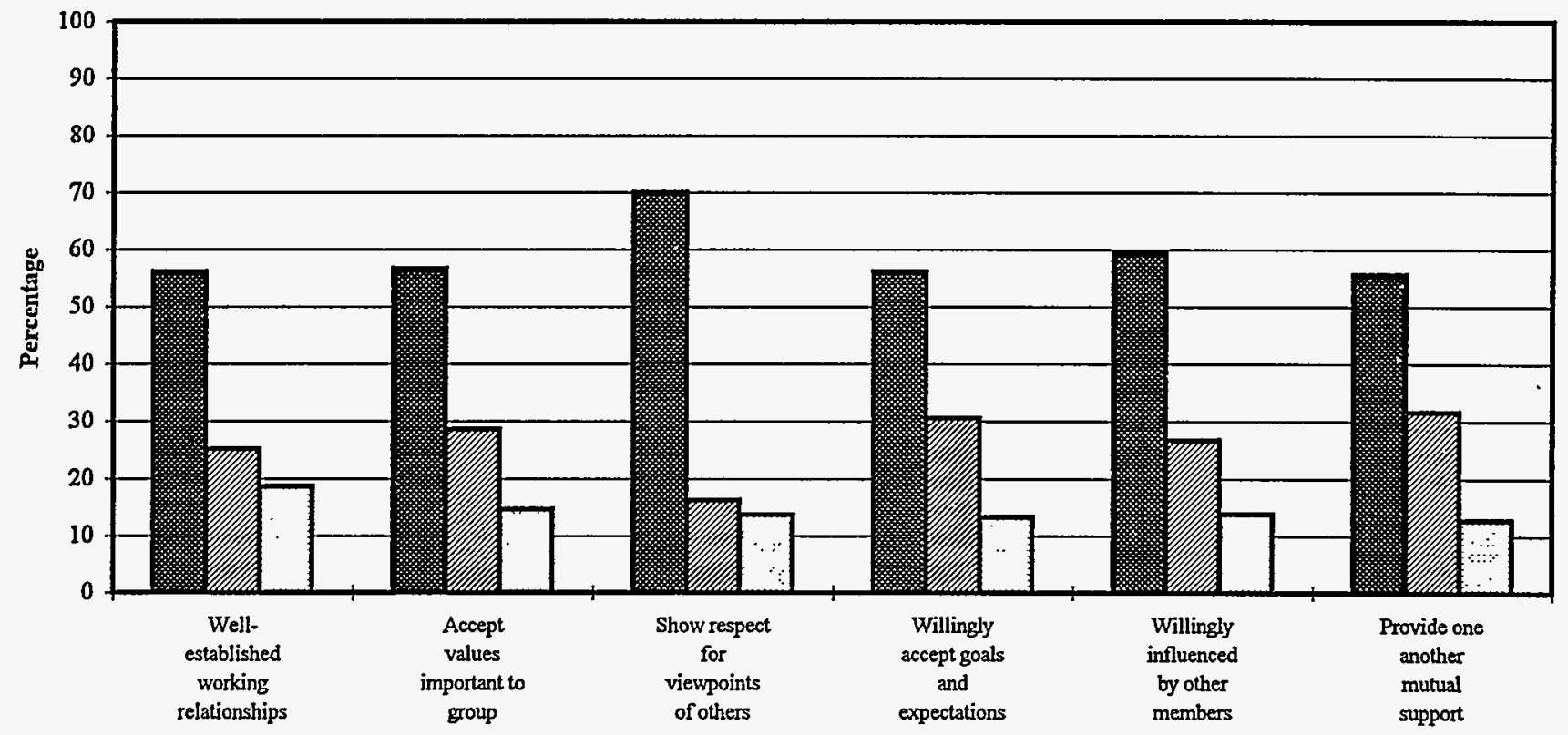

A Agree (\%) DNeither Agree Nor Disagree/Don't Know (\%) DDisagree (\%)

SSAB Supplementary Appendix

August 1996 
Figure 12a. Savannah River

Member affect

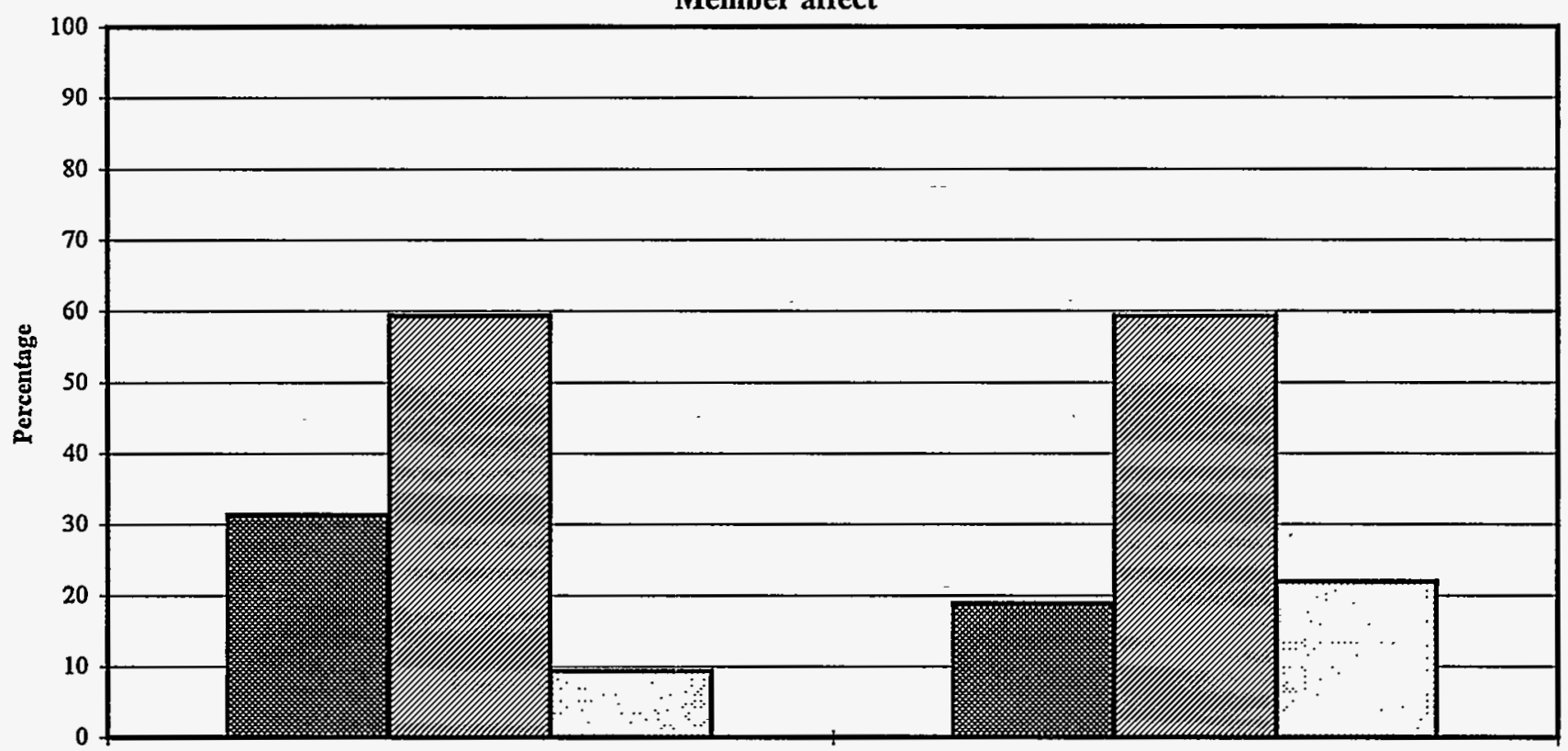

Like each other

High trust and confidence, in each other

Agree (\%) ENeither Agree Nor Disagree/Don't Know (\%) DDisagree (\%)

Figure 12b. Long:Survey: Entire Sample

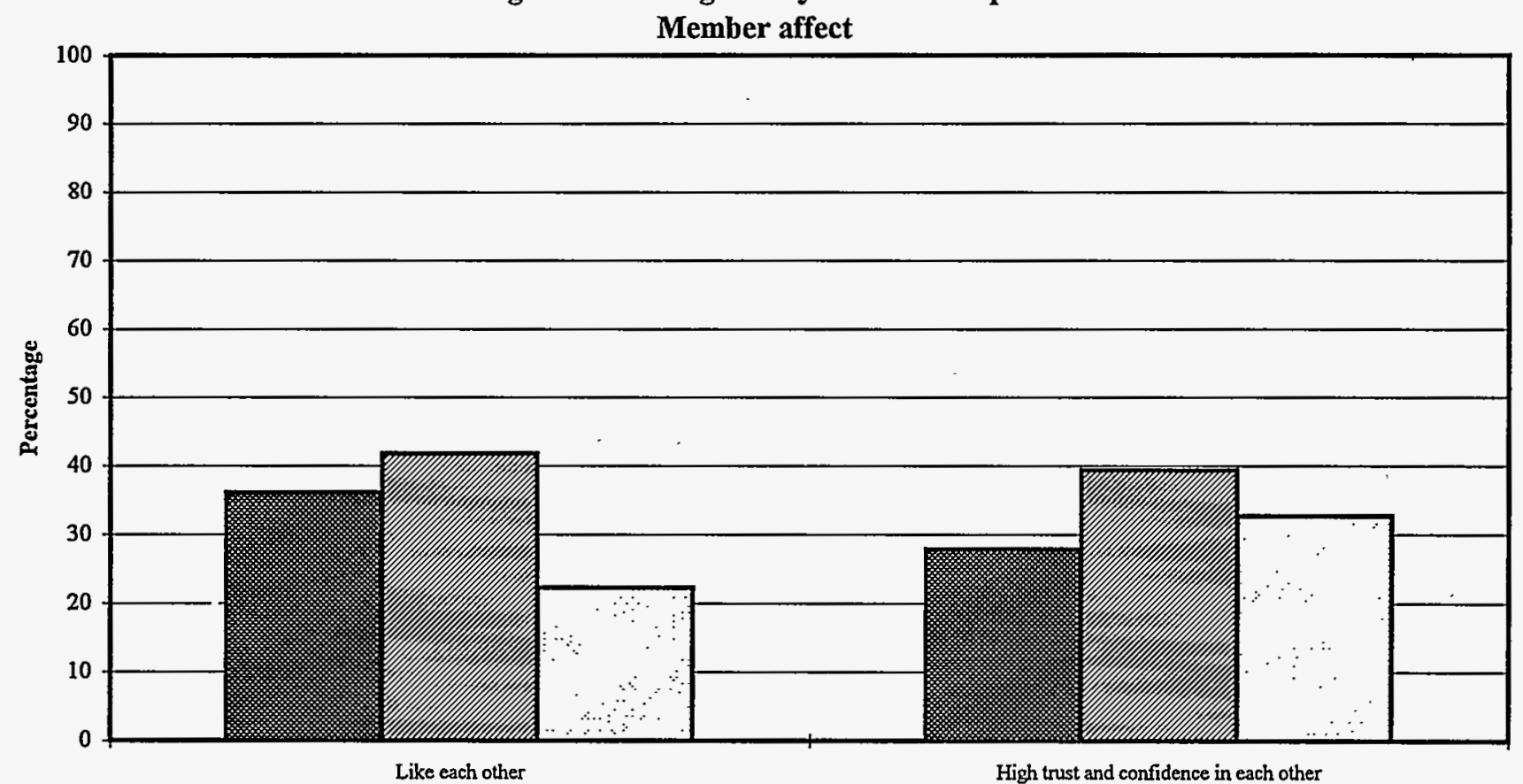

Like each other

High trust and confidence in each other

⿶Agree (\%) Neither Agree Nor Disagree/Don't Know (\%) DDisagree (\%)

SSAB Supplementary Appendix 
Figure 13a. Savannah River

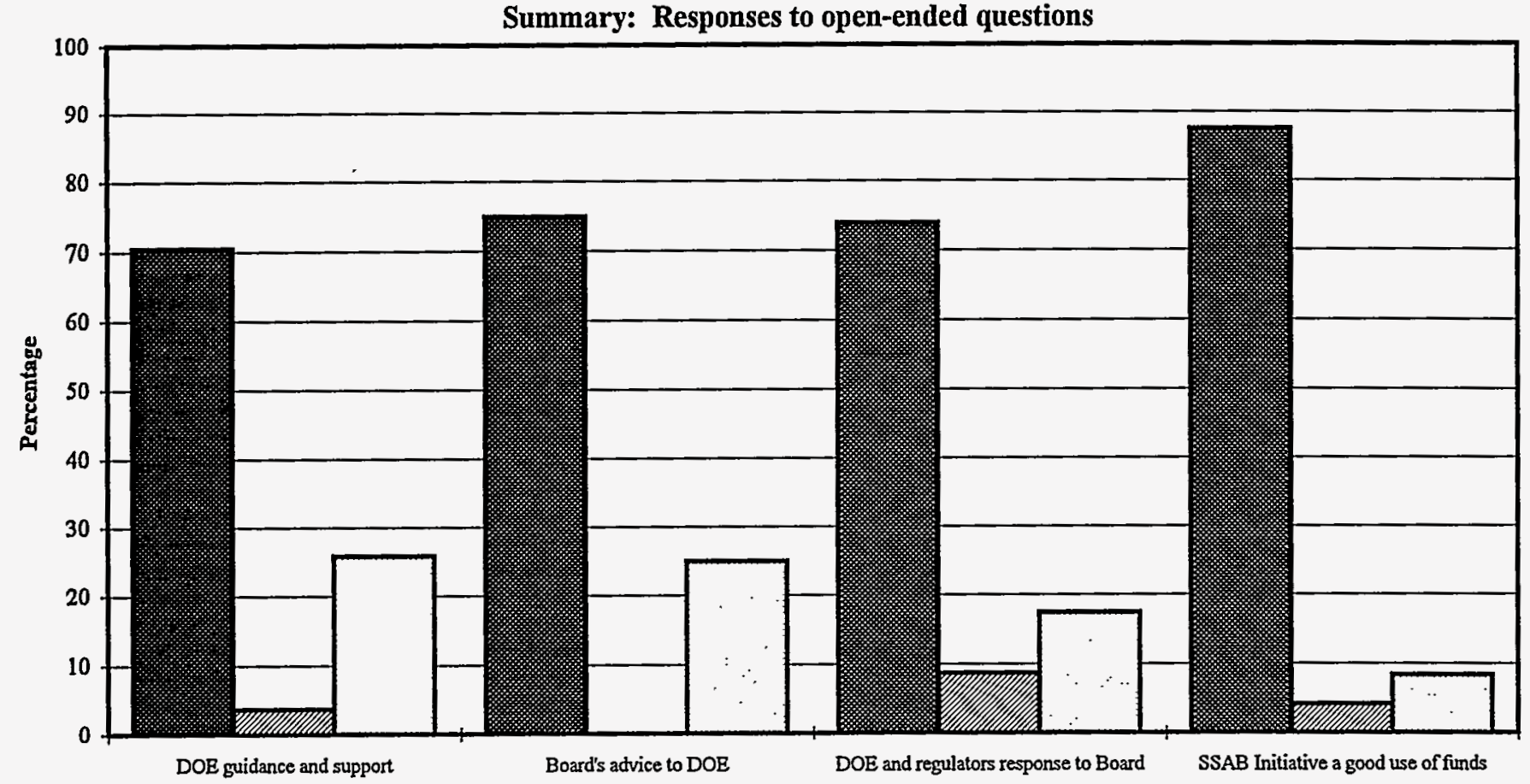

Ges, Good (\%) DNeutral, Conditional, No Opinion (\%) DNo, Not Good (\%)

Figure 13b. Long Survey: Entire Sample

Summary: Responses to open-ended questions

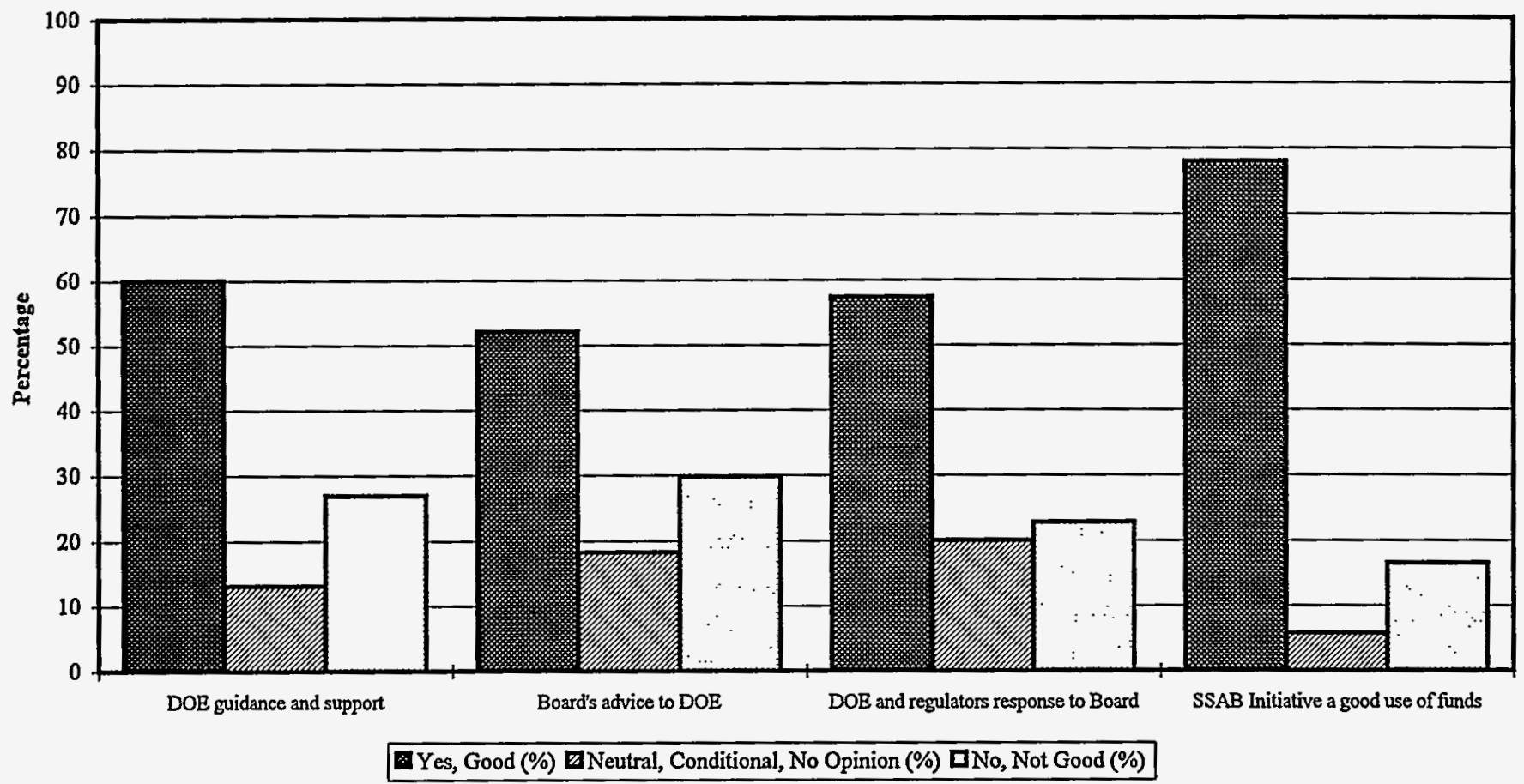

SSAB Supplementary Appendix

August 1996 
Figure 14a. Savannah River

Summary: Combined responses for SSAB Initiative goals (Means)

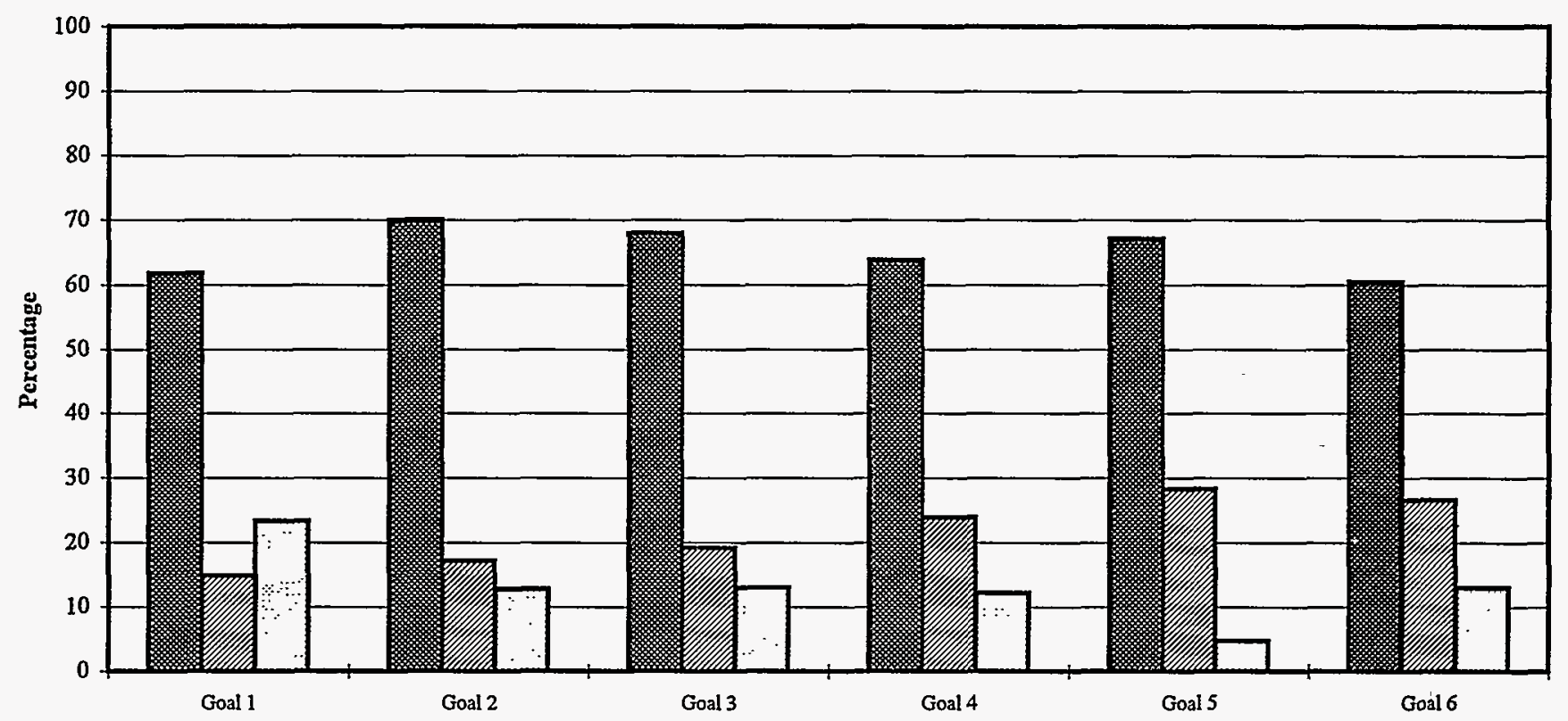

区 Agree (\%) Neither Agree Nor Disagree/Don't Know (\%) DDisagree (\%)

Figure 14b. Long Survey: Entire Sample

Summary: Combined responses for SSAB Initiative goals (Means)

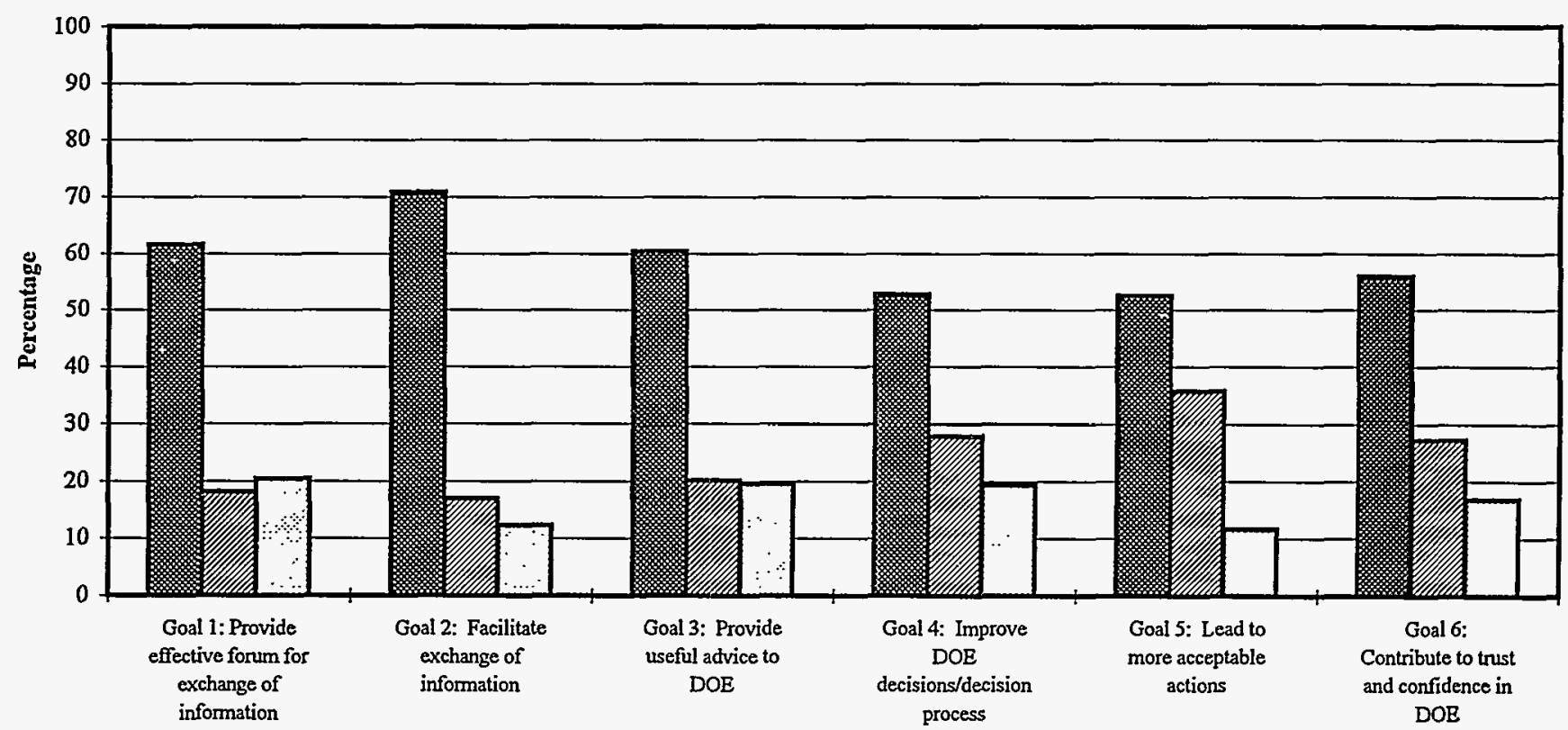

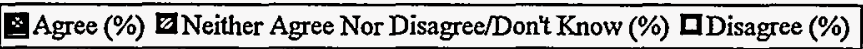

SSAB Supplementary Appendix

August 1996 
Figure 15a. Savannah River

Summary: Combined responses on DOE-HQ involvement, Personal experience, Group/Team work skills,

Working relations, Member affect (Means)

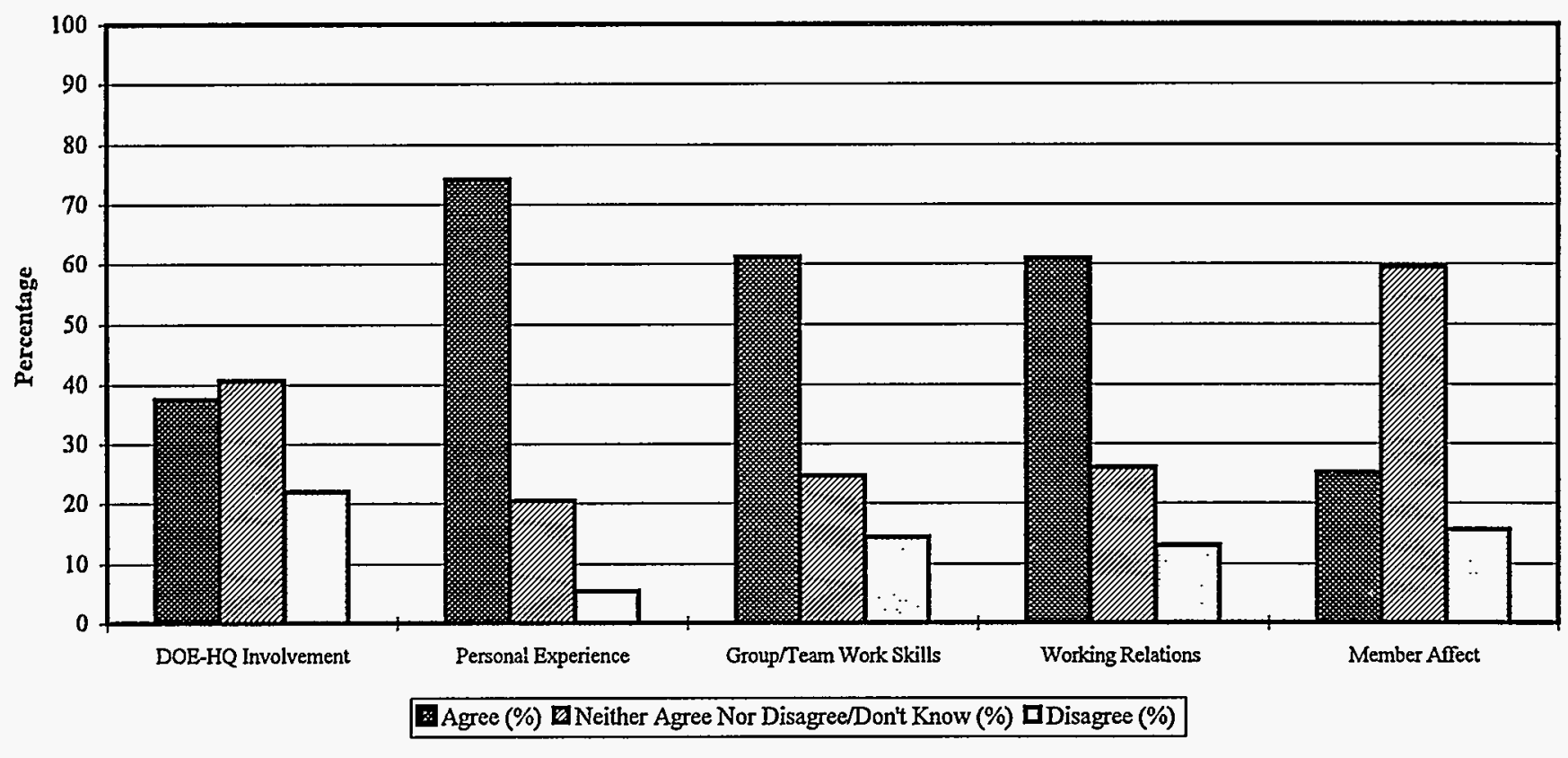

Figure 15b. Long Survey: Entire Sample

Summary: Combined responses on DOE-HQ involvement,

Personal experience, Group/Team work skills,

Working relations, Member affect (Means)

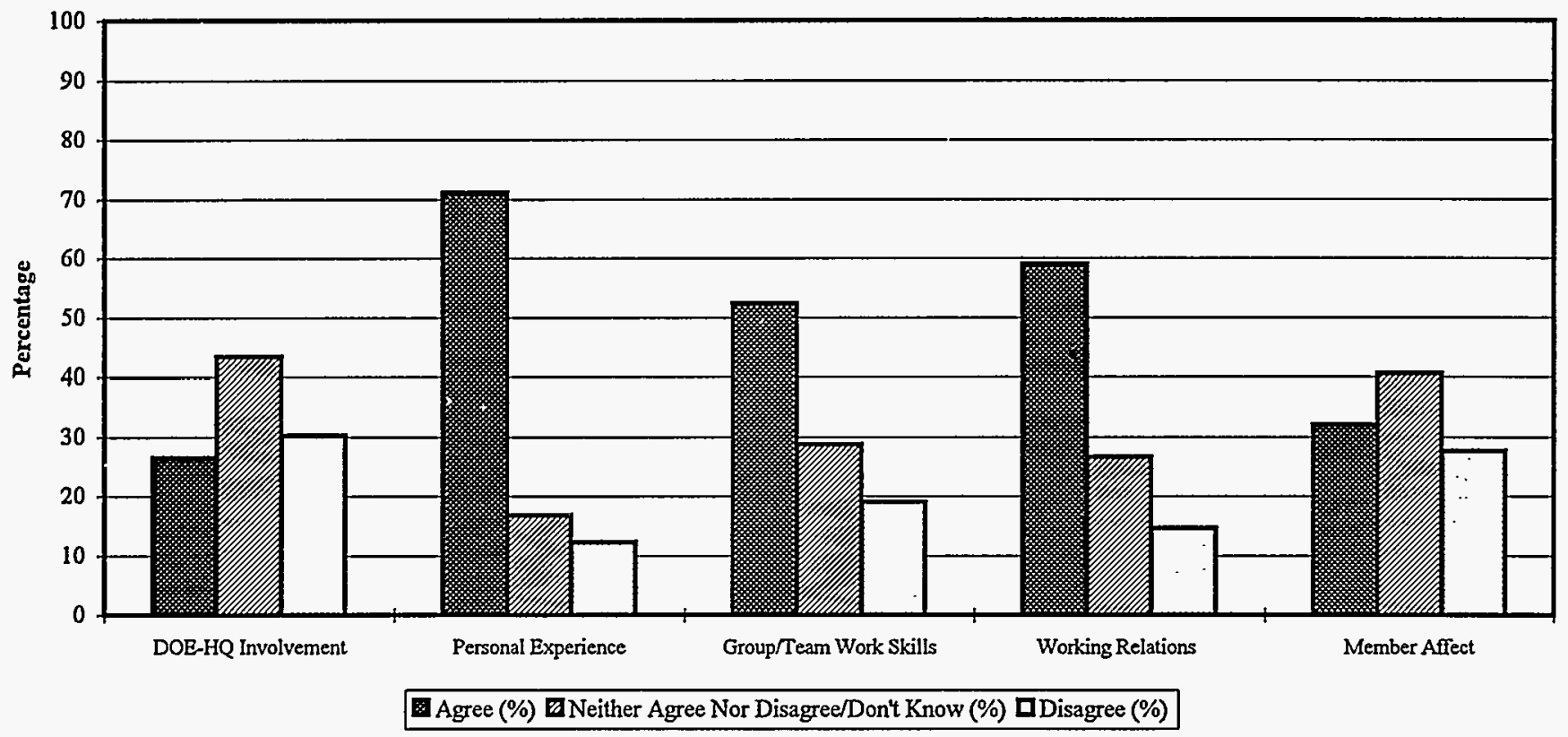

SSAB Supplementary Appendix 
Table 1.

Goal 1. Establish processes and procedures to provide an effective forum for exchange of information and viewpoints regarding DOE site issues

\begin{tabular}{|c|c|c|c|c|c|c|c|c|}
\hline \multirow[t]{2}{*}{ Savannah River } & \multicolumn{2}{|c|}{ Agree } & \multicolumn{2}{|c|}{$\begin{array}{c}\text { Neither/ } \\
\text { Don't Know }\end{array}$} & \multicolumn{2}{|c|}{ Disagree } & \multicolumn{2}{|c|}{ Total } \\
\hline & $\mathrm{N}$ & $\%$ & $\mathrm{~N}$ & $\%$ & $\mathrm{~N}$ & $\%$ & $\mathrm{~N}$ & $\%$ \\
\hline $\begin{array}{l}\text { The Board has established processes and } \\
\text { procedures for the effective exchange of } \\
\text { information }\end{array}$ & 21 & $65.6 \%$ & 4 & $12.5 \%$ & 7 & $21.9 \%$ & 32 & $100 \%$ \\
\hline $\begin{array}{l}\text { The Board has agreed-upon procedures to } \\
\text { operate the board }\end{array}$ & 29 & $90.6 \%$ & 3 & $9.4 \%$ & 0 & $0 \%$ & 32 & $100 \%$ \\
\hline $\begin{array}{l}\text { The Board has adequate support to allow } \\
\text { it to focus on substantive issues }(R)^{*}\end{array}$ & 23 & $71.9 \%$ & 3 & $9.4 \%$ & 6 & $18.8 \%$ & 32 & $100 \%$ \\
\hline $\begin{array}{l}\text { The Board provides sufficient time for } \\
\text { discussion of issues }\end{array}$ & 18 & $56.3 \%$ & 4 & $12.5 \%$ & 10 & $31.3 \%$ & 32 & $100 \%$ \\
\hline The Board has effective leadership & 22 & $68.8 \%$ & 4 & $12.5 \%$ & 6 & $18.8 \%$ & 32 & $100 \%$ \\
\hline $\begin{array}{l}\text { DOE requests Board advice far enough } \\
\text { ahead of decision deadlines }(R)^{*}\end{array}$ & 9 & $29.0 \%$ & 9 & $29.0 \%$ & 13 & $41.9 \%$ & 31 & $100 \%$ \\
\hline $\begin{array}{l}\text { DOE handles Board administrative issues } \\
\text { expeditiously (R) * }\end{array}$ & 19 & $61.3 \%$ & 4 & $12.9 \%$ & 8 & $25.8 \%$ & 31 & $100 \%$ \\
\hline $\begin{array}{l}\text { The SSAB staff provide satisfactory } \\
\text { support }\end{array}$ & 22 & $71.0 \%$ & 3 & $9.7 \%$ & 6 & $19.4 \%$ & 31 & $100 \%$ \\
\hline $\begin{array}{l}\text { The facilitator for the Board has helped } \\
\text { the Board function effectively }\end{array}$ & 13 & $41.9 \%$ & 8 & $25.8 \%$ & 10 & $32.3 \%$ & 31 & $100 \%$ \\
\hline
\end{tabular}

* (R) denotes a question that was asked in the negative form. For ease of review, these questions and data have been reversed in this table and are stated in positive forms.

SSAB Supplementary Appendix

August 1996 
Table 2.

Goal 2. Facilitate interaction and exchange of information and viewpoints regarding DOE site issues

\begin{tabular}{|c|c|c|c|c|c|c|c|c|}
\hline \multirow[t]{2}{*}{ Savannah River } & \multicolumn{2}{|c|}{ Agree } & \multicolumn{2}{|c|}{$\begin{array}{l}\text { Neither/ } \\
\text { Don't Know }\end{array}$} & \multicolumn{2}{|c|}{ Disagree } & \multicolumn{2}{|c|}{ Total } \\
\hline & $N$ & $\%$ & $\mathbf{N}$ & $\%$ & $\mathrm{~N}$ & $\%$ & $N$ & $\%$ \\
\hline $\begin{array}{l}\text { The SSAB facilitates effective exchange } \\
\text { of viewpoints on site issues }\end{array}$ & 20 & $62.5 \%$ & 5 & $15.6 \%$ & 7 & $21.9 \%$ & 32 & $100 \%$ \\
\hline $\begin{array}{l}\text { The SSAB contributes to Board members' } \\
\text { understanding of the basis for key site } \\
\text { decisions }\end{array}$ & 25 & $78.1 \%$ & 4 & $12.5 \%$ & 3 & $9.4 \%$ & 32 & $100 \%$ \\
\hline $\begin{array}{l}\text { The SSAB contributes to DOE and } \\
\text { regulators' understanding of the public's } \\
\text { viewpoints on key site decisions }\end{array}$ & 24 & $75.0 \%$ & 5 & $15.6 \%$ & 3 & $9.4 \%$ & 32 & $100 \%$ \\
\hline $\begin{array}{l}\text { The SSAB contributes to a constructive } \\
\text { working relationship among the } \\
\text { participants }\end{array}$ & 24 & $75.0 \%$ & 3 & $9.4 \%$ & 5 & $15.6 \%$ & 32 & $100 \%$ \\
\hline The SSAB strives for consensus & 16 & $50.0 \%$ & 11 & $34.4 \%$ & 5 & $15.6 \%$ & 32 & $100 \%$ \\
\hline $\begin{array}{l}\text { The SSAB gives fair consideration to } \\
\text { dissenting opinions }\end{array}$ & 21 & $65.6 \%$ & 8 & $25.0 \%$ & 3 & $9.4 \%$ & 32 & $100 \%$ \\
\hline $\begin{array}{l}\text { The Board has made the effort needed to } \\
\text { learn about site issues }\end{array}$ & 27 & $84.4 \%$ & 3 & $9.4 \%$ & 2 & $6.3 \%$ & 32 & $100 \%$ \\
\hline $\begin{array}{l}\text { The Board invites expert advice into its } \\
\text { discussion on key policy issues }\end{array}$ & 29 & $90.6 \%$ & 1 & $3.1 \%$ & 2 & $6.3 \%$ & 32 & $100 \%$ \\
\hline $\begin{array}{l}\text { The Board responds to public inquiries } \\
\text { and comments about its decisions }\end{array}$ & 21 & $65.6 \%$ & 7 & $21.9 \%$ & 4 & $12.5 \%$ & 32 & $100 \%$ \\
\hline $\begin{array}{l}\text { The Board solicits feedback from the } \\
\text { community on its work }\end{array}$ & 15 & $46.9 \%$ & 8 & $25.0 \%$ & 9 & $28.1 \%$ & 32 & $100 \%$ \\
\hline $\begin{array}{l}\text { DOE makes information on key site issues } \\
\text { readily available to the Board }\end{array}$ & 27 & $87.1 \%$ & 4 & $12.9 \%$ & 0 & $0 \%$ & 31 & $100 \%$ \\
\hline $\begin{array}{l}\text { The regulators make important } \\
\text { contributions to the Board's work }\end{array}$ & 19 & $61.3 \%$ & 8 & $25.8 \%$ & 4 & $12.9 \%$ & 31 & $100 \%$ \\
\hline $\begin{array}{l}\text { The SSAB creates a climate supportive of } \\
\text { differing viewpoints }(\mathrm{R}) *\end{array}$ & 21 & $67.7 \%$ & 4 & $12.9 \%$ & 6 & $19.4 \%$ & 31 & $100 \%$ \\
\hline
\end{tabular}

* (R) denotes a question that was asked in the negative form. For ease of review, these questions and data have been reversed in this table and are stated in positive forms.

SSAB Supplementary Appendix

August 1996 
Table 3.

Goal 3. Provide useful advice and/or recommendations to DOE (and regulators, where appropriate).

\begin{tabular}{|c|c|c|c|c|c|c|c|c|}
\hline \multirow[t]{2}{*}{ Savannah River } & \multicolumn{2}{|c|}{ Agree } & \multicolumn{2}{|c|}{$\begin{array}{l}\text { Neither/ } \\
\text { Don't Know }\end{array}$} & \multicolumn{2}{|c|}{ Disagree } & \multicolumn{2}{|c|}{ Total } \\
\hline & $\mathrm{N}$ & $\%$ & $\mathrm{~N}$ & $\%$ & $\hat{N}$ & $\%$ & $N$ & $\%$ \\
\hline $\begin{array}{l}\text { The Board provides useful advice to DOE } \\
\text { (and regulators, where appropriate) (R) * }\end{array}$ & 25 & $78.1 \%$ & 5 & $15.6 \%$ & 2 & $6.3 \%$ & 32 & $100 \%$ \\
\hline $\begin{array}{l}\text { The SSAB reaches agreement about } \\
\text { prioritization of key site issues for which } \\
\text { advice is sought }(\mathrm{R})^{*}\end{array}$ & 20 & $62.5 \%$ & 9 & $28.1 \%$ & 3 & $9.4 \%$ & 32 & $100 \%$ \\
\hline The Board helps define site problems & 23 & $71.9 \%$ & 3 & $9.4 \%$ & 6 & $18.8 \%$ & 32 & $100 \%$ \\
\hline $\begin{array}{l}\text { The Board addresses issues in a timely } \\
\text { manner }(\mathrm{R})^{*}\end{array}$ & 22 & $68.8 \%$ & 6 & $18.8 \%$ & 4 & $12.5 \%$ & 32 & $100 \%$ \\
\hline $\begin{array}{l}\text { The Board reaches consensus on key site } \\
\text { issues (R) * }\end{array}$ & 16 & $50.0 \%$ & 12 & $37.5 \%$ & 4 & $12.5 \%$ & 32 & $100 \%$ \\
\hline $\begin{array}{l}\text { The Board provides informed advice to } \\
\text { DOE (and regulators, where appropriate) }\end{array}$ & 25 & $78.1 \%$ & 3 & $9.4 \%$ & 4 & $12.5 \%$ & 32 & $100 \%$ \\
\hline $\begin{array}{l}\text { The Board provides advice that reflects } \\
\text { the viewpoints and priorities of the } \\
\text { community }\end{array}$ & 21 & $65.6 \%$ & 5 & $15.6 \%$ & 6 & $18.8 \%$ & 32 & $100 \%$ \\
\hline
\end{tabular}

* (R) denotes a question that was asked in the negative form. For ease of review, these questions and data have been reversed in this table and are stated in positive forms.

SSAB Supplementary Appendix

August 1996 
Table 4.

Goal 4. Improve DOE's (and where applicable, regulators') site decisions and decision making process

\begin{tabular}{|c|c|c|c|c|c|c|c|c|}
\hline \multirow[t]{2}{*}{ Savannah River } & \multicolumn{2}{|c|}{ Agree } & \multicolumn{2}{|c|}{$\begin{array}{l}\text { Neither/ } \\
\text { Don't Know }\end{array}$} & \multicolumn{2}{|c|}{ Disagree } & \multicolumn{2}{|c|}{ Total } \\
\hline & $\mathrm{N}$ & $\%$ & $\dot{\mathrm{N}}$ & $\%$ & $\stackrel{\mathrm{N}}{*}$ & $\therefore \quad \therefore$ & $\mathrm{N}$ & $\%$ \\
\hline $\begin{array}{l}\text { The SSAB has improved DOE's site } \\
\text { decisions }(\mathrm{R})^{*}\end{array}$ & 20 & $62.5 \%$ & 7 & $21.9 \%$ & 5 & $15.6 \%$ & 32 & $100 \%$ \\
\hline $\begin{array}{l}\text { The Board understands the decision } \\
\text { making process at [a particular] site }\end{array}$ & 16 & $50.0 \%$ & 10 & $31.3 \%$ & 6 & $18.8 \%$ & 32 & $100 \%$ \\
\hline $\begin{array}{l}\text { DOE discusses important policies } \\
\text { affecting site decisions with the Board }\end{array}$ & 24 & $77.4 \%$ & 5 & $16.1 \%$ & 2 & $6.5 \%$ & 31 & $100 \%$ \\
\hline $\begin{array}{l}\text { DOE has explained to the Board its site } \\
\text { decision making process }\end{array}$ & 21 & $67.7 \%$ & 6 & $19.4 \%$ & 4 & $12.9 \%$ & 31 & $100 \%$ \\
\hline $\begin{array}{l}\text { The DOE shows how Board advice is } \\
\text { reflected in site decisions }\end{array}$ & 17 & $54.8 \%$ & 11 & $35.5 \%$ & 3 & $9.7 \%$ & 31 & $100 \%$ \\
\hline $\begin{array}{l}\text { The decision making process used by } \\
\text { DOE at [a particular] site is effective in } \\
\text { furthering site clean-up (R) * }\end{array}$ & 22 & $71.0 \%$ & 6 & $19.4 \%$ & 3 & $9.7 \%$ & 31 & $100 \%$ \\
\hline
\end{tabular}

* (R) denotes a question that was asked in the negative form. For ease of review, these questions and data have been reversed in this table and are stated in positive forms. 
Table 5.

Goal 5. Lead to more acceptable actions

\begin{tabular}{|c|c|c|c|c|c|c|c|c|}
\hline \multirow[t]{2}{*}{ Savannah River } & \multicolumn{2}{|c|}{ Agree } & \multicolumn{2}{|c|}{$\begin{array}{c}\text { Neither/ } \\
\text { Don't Know }\end{array}$} & \multicolumn{2}{|c|}{ Disagree } & \multicolumn{2}{|c|}{ Total } \\
\hline & $N$ & $\%$ & $\mathrm{~N}$ & $\%$ & $\mathrm{~N}$ & $\%$ & $\mathrm{~N}$ & $\%$ \\
\hline $\begin{array}{l}\text { The SSAB leads to more acceptable site } \\
\text { decisions }\end{array}$ & 20 & $62.5 \%$ & 7 & $21.9 \%$ & 5 & $15.6 \%$ & 32 & $100 \%$ \\
\hline $\begin{array}{l}\text { The Board supports the recommendations } \\
\text { it gives } D O E\end{array}$ & 28 & $87.5 \%$ & 2 & $6.3 \%$ & 2 & $6.3 \%$ & 32 & $100 \%$ \\
\hline The Board supports DOE's site actions & 16 & $51.6 \%$ & 15 & $48.4 \%$ & 0 & $0 \%$ & 31 & $100 \%$ \\
\hline $\begin{array}{l}\text { The DOE pays attention to the Board's } \\
\text { advice on key site issues }(R)^{*}\end{array}$ & 23 & $74.2 \%$ & 8 & $25.8 \%$ & 0 & $0 \%$ & 31 & $100 \%$ \\
\hline $\begin{array}{l}\text { There is support in the community for } \\
\text { DOE's site decisions that have SSAB } \\
\text { input }\end{array}$ & 15 & $48.4 \%$ & 15 & $48.4 \%$ & 1 & $3.2 \%$ & 31 & $100 \%$ \\
\hline Progress is being made on key site issues & 25 & $78.1 \%$ & 6 & $18.8 \%$ & 1 & $3.1 \%$ & 32 & $100 \%$ \\
\hline
\end{tabular}

* (R) denotes a question that was asked in the negative form. For ease of review, these questions and data have been reversed in this table and are stated in positive forms.

SSAB Supplementary Appendix 
Table 6.

Goal 6. Contribute to trust and confidence in DOE

\begin{tabular}{|c|c|c|c|c|c|c|c|c|}
\hline \multirow[t]{2}{*}{ Savannah River } & \multicolumn{2}{|c|}{ Agree } & \multicolumn{2}{|c|}{$\begin{array}{c}\text { Neither/ } \\
\text { Don't Know }\end{array}$} & \multicolumn{2}{|c|}{ Disagree } & \multicolumn{2}{|c|}{ Total } \\
\hline & $\mathrm{N}$ & $\%$ & $\mathrm{~N}$ & $\%$ & $\mathrm{~N}$ & $\%$ & $\mathbf{N}_{\text {, }}$ & $\%$ \\
\hline $\begin{array}{l}\text { The SSAB contributes to trust and } \\
\text { confidence in DOE }\end{array}$ & 16 & $50.0 \%$ & 13 & $40.6 \%$ & 3 & $9.4 \%$ & 32 & $100 \%$ \\
\hline $\begin{array}{l}\text { Relationships between DOE and the public } \\
\text { have improved since the formation of the } \\
\text { SSAB }\end{array}$ & 20 & $64.5 \%$ & 8 & $25.8 \%$ & 3 & $9.7 \%$ & $31^{\circ}$ & $.100 \%$ \\
\hline $\begin{array}{l}\text { DOE is committed to clean up [a } \\
\text { particular] site }\end{array}$ & 20 & $66.7 \%$ & 4 & $13.3 \%$ & 6 & $20.0 \%$ & 30 & $100 \%$ \\
\hline
\end{tabular}


Table 7.

Public awareness

\begin{tabular}{|c|c|c|c|c|c|c|c|c|}
\hline \multirow[t]{2}{*}{ Savannah River } & \multicolumn{2}{|c|}{ Agree } & \multicolumn{2}{|c|}{$\begin{array}{c}\text { Neither/ } \\
\text { Don't Know }\end{array}$} & \multicolumn{2}{|c|}{ Disagree } & \multicolumn{2}{|c|}{ Total } \\
\hline & $\mathrm{N}$ & $\%$ & $\mathbf{N}$ & $\%$ & $\mathrm{~N}$ & $\%$ & $N$ & $\%$ \\
\hline $\begin{array}{l}\text { The public knows little about the role of } \\
\text { the Board }\end{array}$ & 21 & $67.7 \%$ & 4 & $12.9 \%$ & 6 & $19.4 \%$ & 31 & $100 \%$ \\
\hline
\end{tabular}

Table 8.

SSAB Initiative is a good use of funds

\begin{tabular}{cccccccccc}
\hline Savannah River & Yes, Good & $\begin{array}{c}\text { Neutral, } \\
\text { Conditional, } \\
\text { No Opinion }\end{array}$ & $\begin{array}{c}\text { No, } \\
\text { Not Good }\end{array}$ & Total \\
& $\mathrm{N}$ & $\%$ & $\mathrm{~N}$ & $\%$ & $\mathrm{~N}$ & $\%$ & $\mathrm{~N}$ & $\%$ \\
\hline SSAB Initiative is a good use of funds & 21 & $87.5 \%$ & 1 & $4.2 \%$ & 2 & $8.3 \%$ & 24 & $100 \%$ \\
\hline
\end{tabular}


Table 9.

DOE-HQ involvement with the SSAB Initiative

\begin{tabular}{|c|c|c|c|c|c|c|c|c|}
\hline \multirow[t]{2}{*}{ Savannah River } & \multicolumn{2}{|c|}{ Agree } & \multicolumn{2}{|c|}{$\begin{array}{c}\text { Neither/ } \\
\text { Don't Know }\end{array}$} & \multicolumn{2}{|c|}{ Disagree } & \multicolumn{2}{|c|}{ Total } \\
\hline & $\mathrm{N}$ & $\%$ & $\mathrm{~N}$ & $\%$ & $N$ & $\% \ldots$ & $N$ & $\%$ \\
\hline $\begin{array}{l}\text { DOE-HQ provides helpful guidance to the } \\
\text { Boards and to DOE }\end{array}$ & 13 & $40.6 \%$ & 9 & $28.1 \%$ & 10 & $31.3 \%$ & 32 & $100 \%$ \\
\hline $\begin{array}{l}\text { DOE-HQ provides sufficient support to } \\
\text { facilitate the work of the SSAB }\end{array}$ & 14 & $43.8 \%$ & 10 & $31.3 \%$ & 8 & $25.0 \%$ & 32 & $100 \%$ \\
\hline $\begin{array}{l}\text { DOE-HQ gives careful consideration to } \\
\text { SSAB advice in its decisions }\end{array}$ & 9 & $28.1 \%$ & 20 & $62.5 \%$ & 3 & $9.4 \%$ & 32 & $100 \%$ \\
\hline
\end{tabular}


Table 10.

Personal experience with the SSAB Initiative

\begin{tabular}{|c|c|c|c|c|c|c|c|c|}
\hline \multirow[t]{2}{*}{ Savannah River } & \multicolumn{2}{|c|}{ Agree } & \multicolumn{2}{|c|}{$\begin{array}{c}\text { Neither/ } \\
\text { Don't Know }\end{array}$} & \multicolumn{2}{|c|}{ Disagree } & \multicolumn{2}{|c|}{ Total } \\
\hline & $N$ & $\%$ & $\mathrm{~N}$ & $\%$ & $\mathrm{~N}$ & $\%$ & $\mathrm{~N}$ & $\%$ \\
\hline $\begin{array}{l}\text { I consider myself to be very } \\
\text { knowledgeable about site issues }\end{array}$ & 25 & $80.6 \%$ & 5 & $16.1 \%$ & 1 & $3.2 \%$ & 31 & $100 \%$ \\
\hline $\begin{array}{l}\text { Because of the SSAB, I better understand } \\
\text { the complexities of site clean-up }\end{array}$ & 21 & $67.7 \%$ & 8 & $25.8 \%$ & 2 & $6.5 \%$ & 31 & $100 \%$ \\
\hline $\begin{array}{l}\text { I feel that my participation in the SSAB } \\
\text { has been worthwhile }(R)^{*}\end{array}$ & 23 & $74.2 \%$ & 6 & $19.4 \%$ & 2 & $6.5 \%$ & 31 & $100 \%$ \\
\hline
\end{tabular}

* (R) denotes a question that was asked in the negative form. For ease of review, these questions and data have been reversed in this table and are stated in positive forms.

SSAB Supplementary Appendix 
Table 11.

Group/Team work skills

\begin{tabular}{|c|c|c|c|c|c|c|c|c|}
\hline \multirow[t]{2}{*}{ Savannah River } & \multicolumn{2}{|c|}{ Agree } & \multicolumn{2}{|c|}{$\begin{array}{l}\text { Neither/ } \\
\text { Don't Know }\end{array}$} & \multicolumn{2}{|c|}{ Disagree } & \multicolumn{2}{|c|}{ Total } \\
\hline & $\mathrm{N}$ & $\%$ & $\mathrm{~N}$ & $\%$ & $\mathbf{N}$ & $\%$ & $\mathbf{N}$ & $\%$ \\
\hline All have group/team work skills & 15 & $46.9 \%$ & 7 & $21.9 \%$ & 10 & $31.3 \%$ & 32 & $100 \%$ \\
\hline $\begin{array}{l}\text { Have developed well-established working } \\
\text { relationships with each other }\end{array}$ & 19 & $59.4 \%$ & 8 & $25.0 \%$ & 5 & $15.6 \%$ & 32 & $100 \%$ \\
\hline Like each other & 10 & $31.3 \%$ & 19 & $59.4 \%$ & 3 & $9.4 \%$ & 32 & $100 \%$ \\
\hline $\begin{array}{l}\text { Have a high degree of trust and } \\
\text { confidence in each other }\end{array}$ & 6 & $18.8 \%$ & 19 & $59.4 \%$ & 7 & $21.9 \%$ & 32 & $100 \%$ \\
\hline $\begin{array}{l}\text { Accept the values that are important to the } \\
\text { group }\end{array}$ & 20 & $62.5 \%$ & 9 & $28.1 \%$ & 3 & $9.4 \%$ & 32 & $100 \%$ \\
\hline $\begin{array}{l}\text { Show respect for the viewpoints of } \\
\text { others }\end{array}$ & 18 & $56.3 \%$ & 5 & $15.6 \%$ & 9 & $28.1 \%$ & 32 & $100 \%$ \\
\hline $\begin{array}{l}\text { Accept willingly the goals and } \\
\text { expectations for the Board }\end{array}$ & 16 & $50.0 \%$ & 13 & $40.6 \%$ & 3 & $9.4 \%$ & 32 & $100 \%$ \\
\hline $\begin{array}{l}\text { Set high expectations for what they } \\
\text { believe they can accomplish }\end{array}$ & 22 & $68.8 \%$ & 8 & $25.0 \%$ & 2 & $6.3 \%$ & 32 & $100 \%$ \\
\hline $\begin{array}{l}\text { Communicate fully and frankly to the } \\
\text { Board all relevant and valuable } \\
\text { information }\end{array}$ & 24 & $75.0 \%$ & 6 & $18.8 \%$ & 2 & $6.3 \%$ & 32 & $100 \%$ \\
\hline $\begin{array}{l}\text { Try not to waste the Board's time with } \\
\text { irrelevant material or communications }\end{array}$ & 22 & $68.8 \%$ & 5 & $15.6 \%$ & 5 & $15.6 \%$ & 32 & $100 \%$ \\
\hline $\begin{array}{l}\text { Are willing to be influenced by other } \\
\text { Board members about new ideas and } \\
\text { methods }\end{array}$ & 24 & $75.0 \%$ & 7 & $21.9 \%$ & 1 & $3.1 \%$ & 32 & $100 \%$ \\
\hline $\begin{array}{l}\text { Clearly understand the goals and } \\
\text { philosphy of the Board's operation }\end{array}$ & 20 & $62.5 \%$ & 9 & $28.1 \%$ & 3 & $9.4 \%$ & 32 & $100 \%$ \\
\hline $\begin{array}{l}\text { Do all they can to help the Board achieve } \\
\text { its objectives }\end{array}$ & 20 & $62.5 \%$ & 9 & $28.1 \%$ & 3 & $9.4 \%$ & 32 & $100 \%$ \\
\hline $\begin{array}{l}\text { Get the technical knowledge and training } \\
\text { in group skills they need }\end{array}$ & 14 & $43.8 \%$ & 11 & $34.4 \%$ & 7 & $21.9 \%$ & 32 & $100 \%$ \\
\hline Provide one another mutual help & 20 & $62.5 \%$ & 8 & $25.0 \%$ & 4 & $12.5 \%$ & 32 & $100 \%$ \\
\hline
\end{tabular}

